\title{
ஸ்ரீாாம் வெங்கட்ராமன்
}
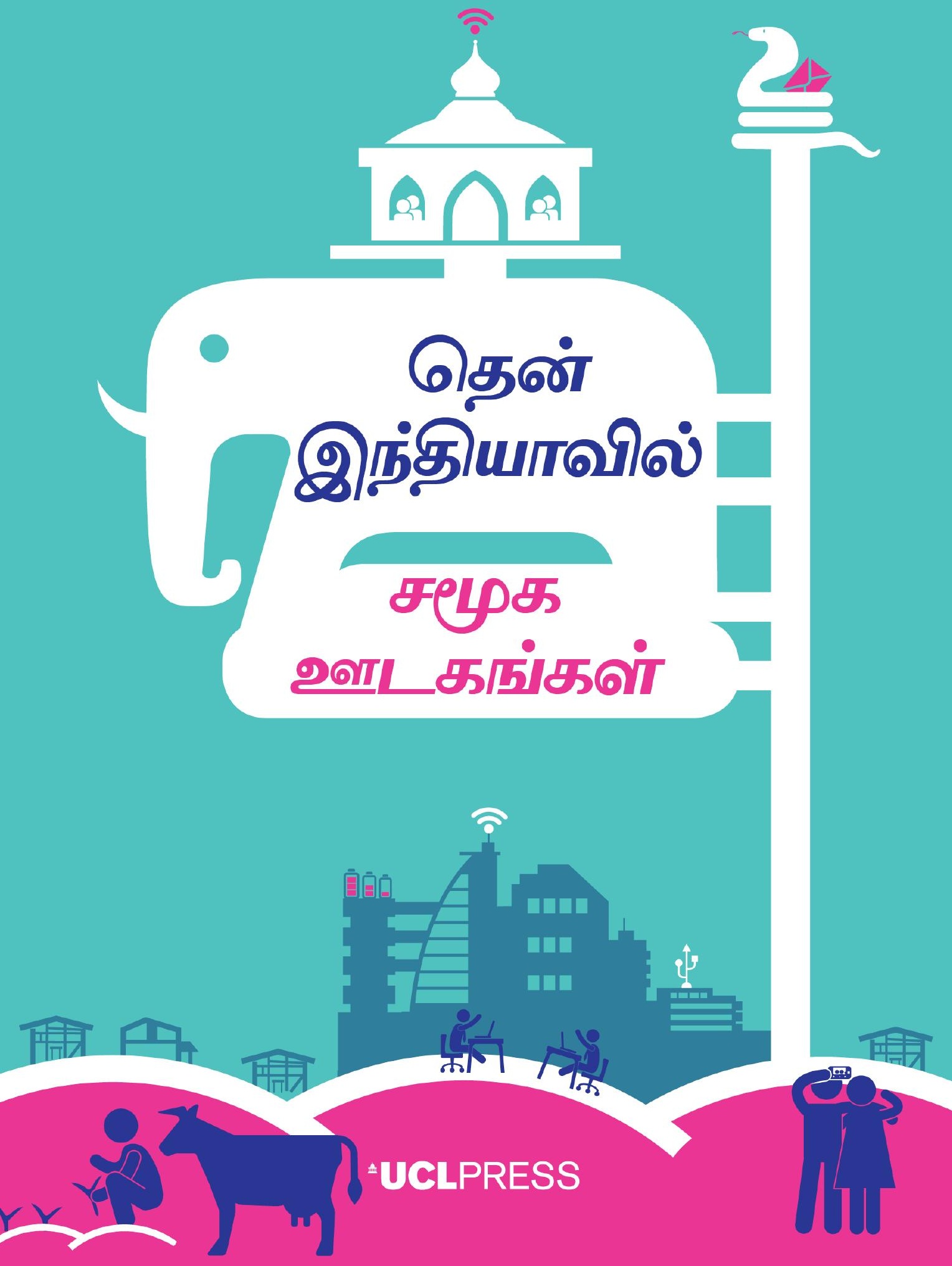


\section{தென்னிந்தியாவில் சமூக ஊடகங்கள்}




\section{நாம் ஏன் பதிவிடுகிறோம்}

\section{பிரசுரிக்கப்பட்ட மற்றும் எதிர்வரும் தலைப்புகள்}

தென்கிழக்கு துருக்கியில் சமூக ஊடகங்கள் எலிசபெட்டா கோஸ்டா

வடக்கு சிலியில் சமூக ஊடகங்கள்

நெல் ஹெய்ன்ஸ்

கிராமப்புற சீனாவில் சமூக ஊடகங்கள்

டாம் மெக்டொனால்ட்

ஆங்கிலேய கிராமத்தில் சமூக ஊடகங்கள்

டேனியல் மில்லர்

மனக்கண்ணில் தோற்றுவிக்கப்படும் முகநூல்

டேனியல் மில்லர் மற்றும் ஜோலின்னா சினனன்

உலகம் சமூக ஊடகங்களை எப்படி மாற்றியிருக்கிறது

டேனியல் மில்லர் மற்றும் பலர்

தென் இத்தாலியில் சமூக ஊடகங்கள்

ரஸ்வான் நிகோலஸ்கு

ட்ரினிடாடில் சமூக ஊடகங்கள்

ஜோலின்னா சினனன்

வளர்ந்துவரும் பிரேசிலில் சமூக ஊடகங்கள்

ஜூலியானோ ஸ்பையர்

தென்னிந்தியாவில் சமூக ஊடகங்கள்

ஸ்ரீராம் வெங்கட்ராமன்

தொழில்துறை சீனாவில் சமூக ஊடகங்கள் க்சின்யுவான் வாங்

இலவசமாக பதிவிறக்க: www.UCL.ac.uk/ucl-press

நாம் ஏன் பதிவிடுகிறோம் 


\section{தென்னிந்தியாவில் சமூக ஊடகங்கள்}

ஸ்ரீராம் வெங்கட்ராமன் 
முதல் பிரசுரம் 2019

யுசிஎல் பதிப்பகம்

பல்கலைக்கழக கல்லூரி லண்டன்

கோவெர் தெரு,

லண்டன் WC1E 6BT

இலவசமாக பதிவிறக்கம் கிடைக்கிறது: www.ucl.ac.uk/ucl-press

எழுத்துக்கள் (C) ஸ்ரீராம் வெங்கட்ராமன் 2018

படங்கள் (c) நூலாசிரியர்கள் மற்றும் தலைப்பில் குறிப்பிடப்பட்டுள்ள காப்புரிமை பெற்றவர்கள் 2018

இந்தப்புத்தகத்திற்கு CIP பட்டியல் குறிப்பேட்டுப் பதிவேடு பிரிட்டிஷ் நூலகத்தில் கிடைக்கும்

இந்தப்புத்தகம், Creative Commons Attribution Non-commercial Non-derivative 4.0 International license (CC BY-NC-ND 4.0) என்ற உரிமத்தின் கீழ் பிரசுரிக்கப்பட்டது. இந்த உரிமம் வணிகம் அல்லாத தனிப்பட்ட உபயோகத்திற்காக, ஆசிரியர்கள் மற்றும் பதிப்பாளர்கள் ஆகியோரின் பண்புக்கூற்றுடன் இந்தப் புத்தகத்தை பகிரவும், பிரதியெடுக்கவும், விநியோகிக்கவும், ஒலிபரப்பவும் உங்களுக்கு அனுமதி அளிக்கிறது.

மேலும் விவரங்களுக்கு: http://creativecommons.org/licenses/

ISBN: 978-1-78735-490-6

DOI: https://doi.org/10.14324/111.9781787354906 


\section{மொழிபெயர்ப்பாளரைப் பற்றி...}

இந்தப் புத்தகத்தின் தமிழ் மொழிபெயர்ப்பாளர் செல்வி பிரியதர்ஷினி. இவர் தமிழ்நாட்டீல் கோவையில் வசித்து வருபவர். முதுகலை வணிகத்தில் பட்டம் மற்றும் கணினி விஞ்ஞானத்தில் முதுகலைப் பட்டயத்துடன் தமிழ், ஹிந்தி மற்றும் ஆங்கிலம் ஆகிய மொழிகளில் புலமையும், மொழி பெயர்க்கும் திறமையும் பெற்ற இவர் தம் வாழ்வை வணிகவியல் உதவிப் பேராசிரியராகத் தொடங்கினார். பின்னர் பாரத ஸ்டேட் வங்கியில் பணியில் சேர்ந்து பல இடங்களில் பல நிலைகளில் பணி புரிந்துள்ளார்.

வங்கிப் பணியிலிருந்து விருப்ப ஓய்வு பெற்ற பின்னர் இவர் தமது பன்மொழித் திறமையைக் கொண்டு மொழி பெயர்த்தல் (Translation), வசனம் அமைத்தல் (Sub-titling) மற்றும் வாய்மொழிப் பொருள் விளக்கம் (Interpretation) ஆகியவற்றில் ஈடுபட்டுள்ளார். தமது சீரிய பணி காரணமாக யுனிவர்சிடி காலேஜ் ஆஃப் லண்டனின் இந்த மொழிபெயர்ப்பு மற்றும் வசனம் அமைத்தல் பணி தவிர கேட்ஸ் ஃபவுண்டேஷன், மலேசியாவில் உள்ள பூஜ்ய ஸ்ரீ ஸ்ரீ ரவிசங்கர் குருஜியின் ஆசிரமமான சம்கியா, மற்றும் மலேசியா தமிழ் சங்கம் போன்ற உலகளாவிய நிறுவனங்களை வாடிக்கையாளராகப் பெற்றுள்ளார். இவரைத் தொடர்பு கொள்ள: priyakm16@gmail.com 



\section{நாம் ஏன் பதிவிடுகிறோம் என்ற தொடருக்கான அறிமுகவுரை}

இந்தப்புத்தகம், 11 தலைப்புகளைக் கொண்ட தொடரில் ஒன்று. இவற்றில் ஒன்பது தலைப்புகள், தனித்தனியே, பிரேசில், சிலி, சீனா, இங்கிலாந்து, இந்தியா, இத்தாலி, டிரினிடாட் மற்றும் துருக்கி ஆகிய ஒன்பது ஆய்வுக்களங்களுக்கான, தனிவரைவாகும். இவை 2016-2017 ஆண்டு வரிசையில் பிரசுரிக்கப்படும். இந்தத்தொடர், எங்களது அனைத்து கண்டுபிடிப்புகளின் ஒப்பீட்டுப் புத்தகமான "உலகம் சமூக ஊடகங்களை எப்படி மாற்றியிருக்கிறது" என்ற புத்தகத்தையும், ஆங்கிலேய மற்றும் ட்ரினிடாடிய ஆய்வுக்களங்களில் மக்கள் முகநூலில் பதிவேற்றிய காட்சியமைப்புகளின் மாறுபாடு பற்றிய புத்தகமான, "மனக்கண்ணில் தோற்றுவிக்கப்படும் முகநூல்" என்ற இறுதி புத்தகத்தையும் உள்ளடக்கியதாகும்.

நாங்கள் மக்களிடம், ஒன்பது ஆய்வுக்களங்களைப் பற்றி ஒரே அத்தியாய தலைப்புகள் கொண்ட (அத்தியாயம் 5 தவிர) ஒன்பது தனிவரைவுகள் உருவாக்கியிருக்கிறோம் என்று கூறினால், அவர்கள், இவற்றில் ஒரே விஷயம் மீண்டும் மீண்டும் கூறப்பட்டிருக்கும் சாத்தியக்கூறுகள் இருக்கும் என்று விசாரப்படுகின்றனர். இருப்பினும், நீங்கள் இவற்றில் பல புத்தகங்களை படிக்க முடிவு செய்தால் (நீங்கள் படிக்க முடிவு செய்யவேண்டும் என்று நாங்கள், வேண்டுகிறோம்), மேற்கூறிய விசாரத்திற்கு முற்றிலும் மாறுபாடாக இந்தப் புத்தகம் விளங்குவதை நீங்கள் பார்க்க முடியும். ஒவ்வொரு புத்தகமும், வெவ்வேறு தலைப்புகள் கொண்டது போல ஒவ்வொன்றிலிருந்து முற்றிலும் மாறுபட்டு விளங்குகிறது.

அநேகமாக, இதுவே எங்களது முக்கியமான ஒரு கண்டுபிடிப்பாக இருக்கலாம். இணையதளம் மற்றும் சமூக ஊடகங்கள் 
பற்றிய பல ஆய்வுகள், பல குழுக்களிடையே பொதுமைப்படுத்த முடியும் என்று நாம் அனுமானிக்க கூடிய ஆராய்ச்சி முறைகளின் அடிப்படையில் அமைந்தவை. நாம் ஒரு இடத்தில காணப்படும் குறும்பதிவுகளை வைத்து டுவிட்டரை பற்றி எழுதுகிறோம். நாம் ஒரு மக்கட்தொகையினரிடையே சமூக ஊடகங்கள் மற்றும் நட்புறவைப் பற்றிய சோதனைகள் செய்து அனைத்து மக்கட்தொகையினர் மத்தியிலும் விளைவுகள் அதேபோலவே இருக்கும் என்ற அனுமானத்தில் அதைப்பற்றி எழுதுகிறோம். ஒரே அத்தியாய தலைப்புகளுடன் ஒன்பது புத்தகங்கள் எழுதிய வகையில், எந்தமாதிரியான பொதுமைப்படுத்தல்கள் சாத்தியம் (அ) சாத்தியமில்லை என்பதை நீங்களே தீர்மானித்துக் கொள்ளுங்கள்.

எங்களது நோக்கம், சமூக ஊடகங்களை நேர்மறையாகவோ அல்லது எதிர்மறையாகவோ மதிப்பிடுவது அல்ல. மாறாக அந்த நோக்கம் கல்வி சார்ந்தது. ஒவ்வொரு இடங்களிலும், சமூக ஊடகங்கள் என்னவாக மாறியிருக்கின்றன, இவற்றின் உள்ளூர் விளைவுகள் மற்றும் மதிப்பீடுகள் என்னென்ன என்பதற்கான விரிவான ஆதாரங்களை சமர்ப்பிப்பது தான் எங்கள் நோக்கம்.

ஒவ்வொரு புத்தகமும், 15 மாத விரிவான ஆராய்ச்சியின் அடிப்படையிலானது. இந்த காலக்கட்டத்தில் அநேகமாக அனைத்து ஆராய்ச்சியாளர்களும் அவரவர் பணிபுரிந்த ஆய்வுக்களத்திலேயே தங்கி, வாழ்ந்து, உள்ளூர் மக்களுடன் அவர்களுடைய மொழியிலேயே உரையாடி இருக்கின்றனர். இருந்தும் அவர்கள், சமூக அறிவியல் புத்தகங்கள் எழுதும் ஓங்கிய பாரம்பரியத்தில் இருந்து மாறுபட்டிருக்கிறார்கள். முதலில் அவர்கள், சமூக ஊடகங்கள் பற்றிய ஏற்கனவே இருக்கும் இலக்கிய நூல்களுடன் தங்களை சம்பந்தப்படுத்திக் கொள்ளவில்லை. ஒன்பது புத்தகங்களிலும் அதே விவாதங்கள் இருப்பது மாபெரும் மறுநிலைப்படியாக இருக்கும். மாறாக இந்த இலக்கியநூல்களைப் பற்றிய விவாதங்களை இந்த ஒற்றை, ஒட்டுமொத்த ஒப்பீட்டுத் தொகுதியில் காணலாம். இரண்டாவதாக, இந்த தனிவரைவுகள் ஒப்பிடக்கூடியவை அல்ல. அது இந்தப்புத்தகத்தின் முதன்மையான வேலையாகும். மூன்றாவதாக, சமூக ஊடகங்களின் மீதான பொதுமக்களின் கணக்கற்ற ஆர்வத்தை கருத்தில் கொண்டு, இந்தப் புத்தகத்தை நாங்கள் அணுகக்கூடிய திறந்த பாணியில் எழுத முனைந்திருக்கிறோம். இதனால் விளங்குவது என்னவென்றால், தனிவரைவுகள், அனைத்து மேற்கோள்கள், பரவலான கல்வி விசாரங்களைப் 
பற்றிய கலந்தாய்வுகள் ஆகிய அனைத்தையும் முடிவுக்குறிப்புகளில் வைக்கும், மிகவும் பாரம்பரியமான ஒரு எழுதுமுறையை சுவீகரித்துக்கொண்டிருக்கிறது என்பது தான்.

நீங்கள் இந்த ஆராய்ச்சியின் விளைவுகளை ரசிப்பீர்கள் என்றும், இந்த ஒப்பீட்டு தொகுதியுடன் கூடுதலாக, தனிவரைவுகளில் ஒன்று அல்லது அனைத்தையுமே படிப்பீர்கள் என்று நாங்கள் நம்புகிறோம்.

\section{ஒப்புரை}

இந்தப்புத்தகம், லண்டனின் பல்கலைக்கழக கல்லூரியில், மானுடவியல் துறையில் இருந்தசமயம் எடுத்துக்கொண்ட முனைவர் பட்டத்திற்கான (2012-2016) ஆராய்ச்சியின் விளைவு. இந்த ஆய்வு, “உலகளாவிய சமூக ஊடக பாதிப்பு ஆய்வு" என்றும், "நாம் ஏன் பதிவிடுகிறோம்" என்று பிரபலமாக அறியப்பட்ட ஒரு பெரிய செயல்திட்டத்தின் பகுதியாகும். இது உலகெங்கிலும் ஒன்பது ஆய்வுக்களங்களில் எட்டு வெவ்வேறு நாடுகளில் நடத்தப்பட்டது. இது ஐரோப்பிய ஆராய்ச்சிக்கழகம் மற்றும் யயுிஎல்-ன் மானுடவியல் துறையின் தாராளமான நிதியுதவி (grant ERC-2011- AdG- 295486 Socnet) இல்லாமல் சாத்தியமாகியிருக்காது.

நான் குறிப்பாக எனது வழிகாட்டி மற்றும் கண்காணிப்பாளரான பேராசிரியர் டேனியல் மில்லர் மற்றும் எனது செயல்திட்ட குழு, எலிசபெட்டா கோஸ்டா, நெல் ஹெய்ன்ஸ், டாம் மெக்டொனால்ட், ரஸ்வான் நிகோலஸ்கு, ஜோலின்னா சினனன், ஜூலியானோ ஸ்பையர், க்சின்யுவான் வாங் மற்றும் இரண்டு அற்புதமான செயல்திட்ட நிர்வாகிகள் பாஸ்கல் செயர்லே மற்றும் லாரா ஹாபியோ-கிர்க் ஆகிய அனைவருக்கும் கடன்பட்டிருக்கிறேன். நாங்கள் அனைவரும் சகபணியாளர்களாக துவங்கி இந்த செயல்திட்டத்தை காலகட்டத்தில் நெருங்கிய நண்பர்களாக ஆகிவிட்டோம். நான் எனது இரண்டாவது கண்காணிப்பாளரான லூசியா மிச்சேலுட்டி, மானுடவியல் துறையின் ஆசான்கள், மற்றும் என்னுடன் பயின்ற மற்ற முனைவு மாணவர்கள் அனைவருக்கும் அவர்கள் இந்த ஆராய்ச்சியின் காலகட்டம் முழுமையிலும் அளித்த ஊக்கத்திற்கும் மதிப்புமிக்க ஆலோசனைகளுக்கும் நன்றிக்கடன்பட்டிருக்கிறேன்.

ஐஐஎம், கோழிக்கோடை சேர்ந்த எனது களப்பணி கண்காணிப்பாளர் திரு அனுபம் தாஸ் அவர்களுக்கு அவரது 
அனைத்து வழிகாட்டல்களுக்கும் குறிப்பாக சரியான சமயத்தில் இந்திய ஆராய்ச்சி நெறிமுறை குழு அமைப்பதில் உதவியதற்காகவும் மிகவும் நன்றிக்கடன் பட்டிருக்கிறேன். இந்தக்குழு இல்லாமல் எனது களப்பணி துவங்கியிருக்காது. எனது மதிப்புறு ஆராய்ச்சி கூட்டாளி, நிம்மி ரங்கஸ்வாமி அவர்களுக்கு, அவரது மதிப்புமிக்க ஆலோசனைகளுக்காக மட்டுமல்லாமல், எனது களப்பணியின் போதான அவருடைய சீரிய நுண்ணறிவுக்காகவும் எனது மனமார்ந்த நன்றிகளை தெரிவித்துக்கொள்ள விரும்புகிறேன். நான் குறிப்பாக கலாஸ்ரீன், சிசிஹெச்டி, சென்னை மதிப்புறு ஆராய்ச்சி கூட்டாளி மற்றும் தென்னிந்திய ஆராய்ச்சி காணொளிகள் படப்பதிவாளர் அவருக்கும் நன்றிக்கடன் பட்டிருக்கிறேன். அவருடைய உதவி இல்லாமல் எனது ஆராய்ச்சியின் காட்சியமைப்பு கூறுகள் முழுமையடைந்திருக்காது.

நான் எனது முன்னாள் வழிகாட்டி. பேராசிரியர் கோவிந்த ரெட்டி அவர்களுக்கும் அவரது நுண்ணறிவு மிக்க ஆலோசனைகள் மற்றும் இந்த செயல்திட்டம் முழுமையிலுமான அவரது ஊக்குவிப்பிற்காக எனது மனமார்ந்த நன்றியை தெரிவித்துக்கொள்ள விரும்புகிறேன். ஹரிப்ரியா நரசிம்மன், எஸ். வெங்கட்ராமன், அபர்ணா, என்.வெங்கட்ராமன், அர்ச்சனா, அனுஷா, சகோதரி.லூர்த்தி மேரி, மெர்லின், சித்ரா, ஷாலினி, ப்ரீத்தி, பத்மலதா, சீதாலட்சுமி, ஜனனி, பாண்டியராஜ், குணநிதி, பத்மாவதி சேதுராமன், ஞானி சங்கரன், எஸ்.சுமதி, எம்.பி.தாமோதரன், க்ராஸ், ஜெகன், ராய் பெனடிக்ட் நவீன், அஸ்மா, பிரியதர்ஷினி கிருஷ்ணமூர்த்தி, விஷ்ணுபிரசாத், ஜில் ரீஸ், முரளி, ஷண்முகவேலன் மற்றும் ஜி.பி. யோகேஸ்வரன் ஆகியோரின் உதவி மற்றும் ஆதரவிற்கு மிகவும் நன்றியுள்ளவனாக இருக்கிறேன்.

இந்த புத்தகத்தை கையெழுத்துப்பிரதியில் இ இருந்து அச்சுப்பிரதியாக எடுத்துச்செல்ல உதவிய யூசிஎல் பதிப்பகத்திற்கும் எனது நன்றியை தெரிவித்துக்கொள்கிறேன்.

இந்த ஆராய்ச்சி எனது பெயரில்லா தகவலாளர்கள் இல்லாமல் முடிந்தேயிருக்காது. அவர்களுடைய நம்பிக்கை, நேரம், பொறுமை மற்றும் அவர்களது இயல்புநிலை மற்றும் நிகழ்நிலை வாழ்க்கையை பற்றி என்னுடன் பகிர்ந்துகொள்வதில் உள்ள ஆர்வம் அனைத்திற்கும் மனமார்ந்த நன்றியை தெரிவித்துக்கொள்கிறேன். 


\section{குறிப்பு}

நான்கு வரைபடங்கள் (படம்: 1.1-1.4) கூகுளில் இருந்து எடுக்கப்பட்ட பிரதிகள் தான். அவை ஆய்வுக்களத்தை காட்டுவதற்கும் அதன் வளர்ச்சியை அளவை காட்டுவெற்குமாக எடுக்கப்பட்டவை. (Non commercial use of Google Earth - https://www.google. co.uk/permissions/geoguidelines.html)

இந்தக்களப்பணி ஏப்ரல் 2013 முதல் ஆகஸ்ட் 2014 வரையிலான காலகட்டத்தில் எடுக்கப்பட்டது. அந்தசமயம் தமிழ்நாட்டின் முதலமைச்சராக செல்வி ஜெ.ஜெயலலிதா தான் இருந்தார். இருப்பினும் 5 திசம்பர் 2016 அன்று செல்வி ஜெ. ஜெயலலிதா மற்றும் 7 ஆகஸ்டு 2018 அன்று திரு எம். கருணாநிதி ஆகியோர் மறைவுக்குப் பின் அரசியல் நிலையில் மாற்றம் ஏற்பட்டிருக்கிறது. 



\section{பொருளடக்கம்}

படங்களின் பட்டியல்

அட்டவணைப் பட்டியல்

1. பஞ்சக்கிராமியும் அதன் சிக்கற்பாடுகெளும்

2. சமூக ஊடக நிலப்பரப்பு: மக்கள், அவர்களின் புலனுணர்வு மற்றும் சமூக ஊடக இருப்பு

3. காட்சிக்குரிய பதிவுகள்: தொடரும் காட்சிக்குரிய இடப்பரப்பு

4. உறவுகள்: சமூக ஊடகத்தில் சுற்றம்

5. பணியில் வீட்டைக் கொணர்தல்: பணி மற்றும் பணியல்லாதவைகளின் எல்லைகளை தெளிவற்றதாகச் செய்தல்

6. பரந்த உலகம்: அறிவார்ந்த பொருளாதாரத்தில் சமூக ஊடகங்கள் மற்றும் கல்வி

7. முடிவுரை: சமூக ஊடகங்களும் அவற்றின் தொடரும் சிக்கற்பாடுகளும் 



\section{படங்களின் பட்டியல்}

படம்: 1.1 பஞ்சக்கிராமியின் வானலைக் காட்சி

(கூகுள் பூமி வரைபடம்)

படம்: 1.2 2002-ல் பஞ்சக்கிராமி

(கூகுள் பூமி வரைபடம்)

படம்: 1.3 2010-ல் பஞ்சக்கிராமி

(கூகுள் பூமி வரைபடம்)

படம்: 1.4 2014-ல் பஞ்சக்கிராமி (கூகுள் பூமி வரைபடம்) 19

படம்: 1.5 1980-களில் பஞ்சக்கிராமி 19

படம்: 1.6 2014-ல் பஞ்சக்கிராமி 20

படம்: 1.7 2014-ல் பஞ்சக்கிராமியில் இருளர்கள் குடியிருப்பு 25

படம் 2.1 சமூூக வலைத்தொடர்புத் தளங்கள் -

மத்தியதர வகுப்பு

படம் 2.2 சமூக வலைத்தொடர்புத் தளங்கள் -

கீழ்மட்ட சமூகப்பொருளாதார வகுப்பு 67

படம்: 3.1 வணிக வளாகத்தில் புகைப்பட சுற்றுலா 92

படம்: 3.2 முகூலில் பதிவேற்றப்பட்டுள்ள குடும்ப புகைப்படம் 94

படம்: 3.3 நடிகர் விஜய் அவரது உடன் நடிக்கும் நட்சத்திரத்துடன் 94

படம்: 3.4 அம்மன் - இந்துசமய தாய்க்கடவுள் 98

படம்: 3.5 தனிப்பட்ட சாதனையை வெளிப்படுத்தும் புகைப்படம் 101

படம்: 3.6 பொதுவிடத்தில், குடும்ப விழா அறிவிப்பு 102

படம்: 3.7 வீரம் திரைப்படத்தில் நடிகர் அஜித் 107

படம்: 3.8 புலி திரைப்படத்தில் நடிகர் விஜய் 108

படம்: 3.9 திரைப்படங்கள் - நடிகர்கள் அஜித் மற்றும் விஜய்யின் பல்வேறு முகங்கள் 
திரைப்படங்கள் - முன்னாள் நடிகர் எம்.ஜி.ஆர் அவர்களின் பல்வேறு முகங்கள் (எம்.ஜி. ராமச்சந்திரன்)

படம்: 3.11 திரைப்படங்கள் - நடிகைகள் நயன்தாரா, அனுஷ்கா மற்றும் நஸ்ரியா

படம்: 3.12 அரசியல் - முனைவர். கருணாநிதி மற்றும் திரு. ஸ்டாலின்

படம்: 3.13 அரசியல் - திரு. அம்பேத்கர் அவர்களின் உருவச்சிலைக்கு மாலையிடுதல்

படம்: 3.14 அரசியல் - தலித் தலைவர் திருமாவளவன்

அரசியல் - சமூக பிரச்சினைகளை பற்றிய பரிகாசமான மற்றும் சீர்திருத்த நோக்கங்கொண்ட நையாண்டி தாக்குதல் மீம்கள்

படம்: 3.16 அரசியல் - விஜயகாந்த் அவர்களை பழிப்பதன் ஒரு உதாரணம்

படம்: 3.17 தனிப்பட்டவை - குவியம் என்மேல்

படம்: 3.18 தனிப்பட்டவை - காட்சிமுறை படமும்

அதற்கான நட்பார்ந்த கிண்டலும்

படம்: 3.19 தனிப்பட்டவை - நீ என்ன செய்கிறாய் என்பதைப் பற்றியது

படம்: 3.20

தனிப்பட்டவை - அந்தஸ்த்தை

காண்பிக்கும் பின்னணி

படம்: 3.21 தனிப்பட்டவை - குழுவில் (நண்பர்கள்) தான் எனும் தன்மை

படம்: 3.22 தனிப்பட்டவை - குழுவில் (குடும்பம்) தான் எனும் தன்மை

படம்: 3.23

இடைப்பட்டவை - வாழ்த்துடன் கூடிய விநாயகரின் படம்

படம்: 3.24

இடைப்பட்டவை - அன்றாட வாழ்த்துக்களுடனான எழுத்துவடிவ மீம்கள்

படம்: 3.25 இடைப்பட்டவை - ஊக்கமளிக்கக்கூடிய அல்லது சமயம் சார்ந்த மேற்கோள்களுடன் கூடிய படங்கள்

படம்: 3.26

இடைப்பட்டவை - காட்சியமைப்புகளுடன் கூடிய அன்றாட வாழ்த்துக்கள்

படம்: 3.27

இடைப்பட்டவை - வித்யாஷங்கர் பதிவேற்றிய கடவுள் கிருஷ்ணரின் படம்

படம்: 3.28 இடைப்பட்டவை - வாட்சப் குழுவில் சுதாஸ்ரீயின் பிரார்த்தனை 
படம்: 3.29 கலவையானவை - சர்ச் வாட்சப் குழுவில்

இருந்து யேசுகிறிஸ்துவின் படம்

படம்: 3.30 கலவையானவை - குடியிருப்பு வளாக

வாட்சப் குழுவில் இருந்து ஊக்கமளிக்கும்

மீம்

படம்: 3.31 கலவையானவை - வாட்சப்பில் உள்ள

நண்பர்கள் குழுவில் பூண்டு புளிக்குழம்பு

படம்: 3.32 கலவையானவை - முகநூல் பக்கத்தில்

உள்ள இயற்கை காட்சியுடனான மீம்

படம்: 3.33 கலவையானவை - அபிமான

திரைநட்சத்திரம் தனுஷ் - ஒரு ரசிகரின்

முகநூல் பக்கத்திலிருந்து

படம்: 3.34 கலவையானவை - சகபணியாளர்களுக்கு முன்னனுப்பிய வேடிக்கை மீம்

படம்: 4.1 கீழ்மட்ட சமூகப்பொருளாதார குடும்பத்தில் போன் வைத்திருப்பு

படம்: 5.1 - பணியமைப்பு முரண்பாடு

படம்: 6.1 - அகஇணைய பள்ளி வலைத்தொடர்பு வலைதளத்தின் உள்நுழைவுப் பக்கம்

\section{அட்டவணைப் பட்டியல்}

அட்டவணை: 3.1 - பஞ்சக்கிராமியில் உள்ள

காட்சியமைப்புகள் பற்றிய முகநூல் அளவீடுகள். 



\section{1 \\ பஞ்சக்கிராமியும் அதன் சிக்கற்பாடுகளும்}

ஏப்ரல் மாதம் 2013-ஆம் வருடம், ஒரு சுட்டெரிக்கும் கோடைக்கால மதியம், தன் குடும்பத்தின் முதல் பட்டதாரியான 24 வயது செல்வா, சமூக ஊடகங்கள் பற்றிய தன்னுடைய அனுபவத்தை பகிர்ந்து கொள்வதற்காக என்னை ஒரு சாலையோர தேநீர் கடையில் சந்தித்தார். நான்கு ஆண்டுகளுக்கு முன் கல்லூரியில் இருந்தபோது முதன்முதலாக முகநூல் அவருக்கு பரிச்சயமானது. அதன் பிறகு அவர் வாட்சப்-ஐயும் உபயோகித்துப் பார்த்து விட்டார். அவர் டுவிட்டரையும் பரீட்சித்துப் பார்த்துவிட்டார். ஆனால் அதில் அவரது வாசம் சொற்பகாலமாகத்தான் இருந்தது. அந்தத் தளம் அவரை சற்று பயமுறுத்தி விட்டது. அவரது மொழியிலேயே சொல்லவேண்டும் என்றால், “டுவிட்டர்ல இருக்கறதுக்கு நாமெல்லாம் பீட்டரு லெவெலுக்கு இங்கிலிஷ் பேசணும்" அதாவது, டுவிட்டரில் பதிவிடுவதற்கும், பின்பற்றுபவர்களைப் பெறுவதற்கும் அதிகமான அளவு ஆங்கிலப் புலமை பெற்றிருக்க வேண்டும்.

செல்வா, தான் எந்தளவு முகநூலையும் வாட்சப்பையும் நேசிப்பதாக கூறினார். தனக்கு முகநூலில் ஏகப்பட்ட பெண் நண்பர்கள் இருப்பதாகவும், அவர்களில் ஒருசிலர் மிகவும் நெருக்கமானவர்களாக இருப்பதாகவும் அதனால் அப்படிப்பட்டவர்களுடனான அவரது உரையாடல், முகநூலில் இருந்து வாட்சப்பிற்கு மாறிவிட்டதாகவும் கூறினார். எங்களுடைய உரையாடல் முழுமையிலும் தனது தனிப்பட்ட வாழ்வில் சமூக ஊடகங்களின் நேர்மறையான தாக்கம் பற்றி புகழ்ந்து தள்ளிவிட்டார்.

இருப்பினும், இரண்டு மாதங்களுக்குப் பின், செல்வா தன் முகநூல் கணக்கை தொடர்பறுத்துவிட்டு வாட்சப் மூலமாக 
மட்டும் உரையாடிக்கொண்டிருந்தார். நாங்கள் மீண்டும் அதே தேநீர் கடையில் சந்தித்த போது, தன் வாழ்வையும், குடும்ப கௌரவத்தையும் கெடுத்துவிட்டதாக கூறி முகநூலை சபித்தார். சில வாரங்களுக்கு முன்பு, அவருடைய 17 வயதான பள்ளி செல்லும் தங்கை, இவர் படித்த பல்கலைக்கழகத்திலேயே படிக்கும் மற்றொரு மாணவனுடன் காதல் வயப்பட்டிருப்பதாக கண்டறிந்தார். அந்த மாணவன், வேறு குலத்தைச் சேர்ந்தவன். அவர்கள் இ இருவரும் செல்வாவின் முகநூல் பக்கத்தின் மூலம் ஒருவருக்கொருவர் அறிமுகமாகியிருக்கின்றனர். இதையறிந்த செல்வாவின் பெற்றோரும், குடும்பத்தைச் சேர்ந்த மற்றவர்களும், தங்கையை சமூக ஊடகங்களை உபயோகிக்க தூண்டியதாக, செல்வாவை பழித்தனர். அவருடைய குடும்பம் அவர் தங்கையின் காதலை, தங்கள் குடும்ப மற்றும் குல கௌரவத்திற்கு இழுக்காக கருதினர். எரிச்சலுற்ற செல்வா தன்பங்கிற்கு, தனது மற்றும் தன் தங்கையினது முகநூல் கணக்குகளை தொடர்பறுத்துவிட்டார்.

தங்கையை முகநூலுக்கும், அலைபேசிக்கும் அறிமுகப்படுத்த வேண்டாம் என்ற தனது கிராமத்தை சேர்ந்த உறவினர்கள் மற்றும் நண்பர்களின் அறிவுரையை தான் கேட்டிருக்க வேண்டும் என்று செல்வா புலம்பினார். இதுபோன்ற மென்மையான பெண்களை காதல் என்ற வலையில் வளைத்துப்போடவென்றே காத்திருக்கும் பிற குலத்தை சேர்ந்த இளம் ஆண்களிடம் இருந்து தங்கையை காப்பதே செல்வாவின் தலையாய கடமை என்று அவரது உறவினரும் நண்பர்களும் கூறியிருந்தனர். மேலும் அவர்கள், ஒரு ஒழுக்கமான, திருமணமாகாத இளம் தமிழ்ப் பெண், அலைபேசியுடனோ அல்லது இது போன்ற ஆபத்தான தளங்களிலோ காணப்படமாட்டாள் என்றும் கூறியிருந்தனர்.

ஒரு வாரத்திற்கு பின், இந்த தேநீர் கடையில் இருந்து சில நூறு அடிகள் தள்ளியிருந்த ஒரு உயர்மட்ட காஃபி ஷாப்பில், தன் மத்திய இருபதுகளில் இருக்கும் விஜயா என்ற மென்பொருள் பணியாளர், தன்னுடைய சமூக ஊடக பயணத்தை விவரித்தார். அப்போது அவருக்கு மணமாகி இரண்டு வயது பெண்குழந்தை இருந்தது. இரண்டாம் குழந்தை வயிற்றில் ஐந்து மாதக் கருவாக இருந்தது. அவர் பல்வேறு சமூக ஊடகத்தளங்களில் இருந்தார். முகநூலிலும் டிவிட்டரில் அதிக செயல்பாட்டில் இல்லாமலும், வாட்சப்பிலும், லிங்க்ட்-இன்னிலும் மிகவும் பரபரப்பான செயல்பாட்டிலும் இருந்தார். வாட்சப் அவருக்கு குடும்பத்துடன் சிறந்த தொடர்பில் இருக்கவும், லிங்க்ட்-இன் அவரது பணி தொடர்பான ஆர்வங்களை கவனி- 
த்துக் கொள்ளவும் உதவின. தன் சிறு குழந்தையை பணியிடத்திற்கு அருகில் இருக்கும் குழந்தைகள் பராமரிப்பகத்தில் விட்டுவிட்டு பணிக்கு செல்ல வேண்டியிருப்பது விஜயாவிற்கு குற்றக் குறுகுறுப்பு கொடுக்கும் விஷயம். அவருடைய புகுந்த வீட்டாரைப் பொறுத்தமட்டில் இவ்வாறு செய்வது ஒரு நல்ல தாய்க்கு அழகல்ல. தான் தன் குழந்தையை விடும், பெரும்பாலும் தகவல் தொழில்நுட்பத் துறை பணியாளர்களின் குழந்தைகளை கொண்டிருக்கும் அந்தப் பராமரிப்பகம், ஒரு கூடுதலான கட்டணத்திற்கு, ஒரு மணி நேரத்திற்கு ஒரு முறை, வாட்சப் மூலம், குழந்தைகளை பற்றிய தகவலை தெரிவிப்பதாக விஜயா அறிந்து கொண்டார். உடனே அவர் அந்த சேவைக்கு கட்டணம் செலுத்தி விட்டார். வாட்சப் மூலம், நாள் முழுவதும் தன் மகள் என்ன செய்தாள் என்று அறிந்து கொள்ள முடிவது அவருக்கு மன நிம்மதியையும் தன குற்றக் குறுகுறுப்பில் இருந்து விடுதலையையும் அவருக்கு பெற்றுத்தந்தது.

இந்தப்புத்தகம், தமிழ்நாட்டின் சென்னை நகர்புறத்தை ஒட்டிய ஒரு பகுதியில், 15 மாத மக்கள் இனஅமைப்பியலின் ${ }^{2}$, கூறுரை விளக்கம் ஆகும். இந்த நூற்றாண்டின் துவக்கத்தில் ஐந்து கிராமங்களுக்கு மத்தியில் உள்ள இந்தப் பகுதியை, அரசாங்கம், தொழில்நுட்ப நிறுவனங்களுக்கான சிறப்புப் பொருளாதார மண்டலமாக அறிவித்ததில் இருந்து, தென்னிந்தியாவின் இந்தப் பிராந்தியம், கிராமப்புறம் என்ற நிலையில் இருந்து நகர்புறமாக அதிவேக மாற்றம் அடைந்து வருகிறது. இந்தப்பகுதியை நாம் இப்போது முதல் பஞ்சக்கிராமி ${ }^{3}$ என்று குறிப்பிடுவோம். தொழில்நுட்பத் துறையின் வருகை, பஞ்சக்கிராமியை பழமை புதுமையை சந்திக்கும் இடமாகவும், உள்ளூர் உலகளாவலை சந்திக்கும் இடமாகவும் மாற்றிவிட்டது. சமூக ஊடகங்களைப் பற்றிய ஆய்வை, புதிய தொழில்நுட்ப மையம் என்னும், இந்தியாவில் புதுமைக்கான உதாரணச் சின்னத்தை உள்ளடக்கிய ஒரு அமைப்புடன் தொடர்புபடுத்துவது மிகவும் பொருத்தமானதாக தோன்றியது.

பஞ்சக்கிராமி சுமார் 30000 மக்கட்தொகையை கொண்டது. இது தலைமுறை தலைமுறையாக இங்கேயே வாழ்ந்து வரும் 14000 உள்ளூர் கிராமவாசிகளையும், 16000 புதிய குடியிருப்பு வாசிகளையும் ொண்டது. இந்தப் புதியவர்களில், தொழில்நுட்பத் துறை மற்றும் அதைச் சேர்ந்த சேவைத்துறையைச் சார்ந்த பணியாளர்கள், சிறுதொழில் வணிகர்கள், தொழில் முனைவோர்கள், கட்டுமானப்பணியாளர்கள் மற்றும் வேலைவாய்ப்பு தேடி வந்திருக்கும் திறமையற்ற தொழிலாளர்கள் 
ஆகியோர் அடக்கம். மேற்கூறிய நிரந்தர குடியிருப்பாளர்களுடன் சேர்ந்து, பஞ்சக்கிராமியில் சுமார் 200,000 மிதக்கும் மக்கட்தொகை, அதாவது, தொழில்நுட்ப நிறுவனங்கள் மற்றும் இதர சேவைத்துறையில் பணிக்கு வந்து செல்லும் மக்கள் தொகையும் உண்டு.

இந்தப்பகுதியை ஆய்வுக்களமாக தேர்ந்தெடுத்ததற்கான கற்பிதங்களில் ஒன்று என்னவென்றால், இந்தப் பகுதி, தொழில்நுட்பத்துறையை சார்ந்தவர்கள் மற்றும் உள்ளூர் கிராமவாசிகள் ஆகிய இரு வேறு மக்கட்தொகைகளுக்கிடையே சமூக ஊடக பயன்பாட்டில் உள்ள மாறுபாடுகளை புரிந்துகொள்ள எனக்கு உதவும் என்பது தான். இதில், தொழில்நுட்பத்துறையை சார்ந்தவர்கள், அநேகமாக, நகர்புறவாசிகளாகவும், வசதிப்படைத்தவர்களாகவும், சமூக ஊடக பயன்பாட்டில் தேர்ச்சிபெற்றவர்களாகவும் உள்ளனர். அதேசமயம், உள்ளூர் கிராமவாசிகள், அதிக வசதியற்றவர்களாகவும், புதிய தொழில்நுட்பங்களில் அனுபவமற்றவர்களாகவும் இரருக்கின்றனர். இந்த இரண்டு மக்கட்தொகையை சேர்ந்தவர்களின் சமூக ஊடக பயன்பாடும், வெறும் தொழில்நுட்பத்துறை பணியாளர்கள் மற்றும் கிராமவாசிகள் என்ற இரண்டு இ இணையெதிர்வுகள் மட்டுமல்லாமல், பாலினம், உறவுமுறை, வயது, குலம், வர்க்கம், மதம் போன்ற பல்வேறு சமூக வகைகளால் பாதிக்கப்பட்ட பாரம்பரியத்தின் ஆழமான அடுக்குகளால் ஆளப்படுகின்றன என்பது இனஅமைப்பியல் தொடங்கிய உடனேயே தெள்ளத்தெளிவாகிவிட்டது. இது போன்ற பாரம்பரியங்களும், அவற்றை நிலைநிறுத்தும் சமூக வகைகளும், பஞ்சக்கிராமியின் குடியிருப்பு வாசிகளின் வாழ்வில் இரண்டறக் கலந்திருக்கிறது. அதுவே அவர்களின் சமூக ஊடக பக்கங்களிலும் வெளிப்படுகிறது.

செல்வாவின் வழக்கு, அவர் எப்படி, குலம், குடும்ப கௌரவம், பெண்மையின் இலக்கணம் பற்றிய போதனை, அதீத-ஆண்மையின் ${ }^{5}$ இலக்கணம் பற்றிய சிந்தனை போன்ற கருத்துக்களை, தனது இயல்புநிலை உலகில் இருந்து நிகழ்நிலை சமூக ஊடக உலகிற்கு சுமந்துவந்தார் என்பதை எடுத்துக்காட்டுகிறது. அதே போல விஜயா அவர்களின் வழக்கில், அவர், ஒரு சிறந்த தாய் பற்றிய எதிர்பார்ப்புகளை அவர் தன் மனதில் சுமந்துகொண்டு, தன் தாய்மை உணர்வுகளை வாட்சப் மூலமாக வெளிப்படுத்தி அந்த எதிர்பார்ப்புகளை நிறைவேற்ற முயற்சிக்கிறார். இந்த இரண்டு வழக்குகளையும் பயன்படுத்தி, ஒரு தகவல் தொழில்நுட்பத்துறை பணியாளருக்கும் (விஜயா) ஒரு கிராமவாசிக்கும் (செல்வா) இடையே உள்ள வேறுபாட்டை எடுத்துரைக்க, இந்த 
ஆராய்ச்சியின் பின்னிருக்கும் உண்மையான நோக்கம் வழிவகுத்திருக்கக் கூடும். இருப்பினும், இந்த இரு வழக்குகளையும், ஒரு ஆழமான பொதுத்தன்மை இணைக்கிறது. மக்கள் தங்கள் இயல்புநிலை வாழ்வில் உள்ள, பாலினம், உறவுமுறை, வயது, குலம், மதம் போன்றவற்றை சேர்ந்த பாரம்பரியங்களை சமூக ஊடகங்களின் பயன்பாட்டில் எடுத்து வந்து புகுத்துகிறார்கள். இயல்புநிலையில் உள்ள சமூக வகைகளை, நிகழ்நிலையிலும் பிரதிபலிக்கும் வகையில், பாரம்பரியம், சமூக ஊடகங்களில் பெருமளவு ஒப்பிடப்பட்டு மீண்டும் மீண்டும் வலியுறுத்தப்படுகிறது. நிகழ்நிலை என்பது, தனிநபர்கள், சமூக ஊடகங்களில், தங்களது உறவினர்கள், நண்பர்கள் போன்ற சமூக குழுக்களையும் உட்கொண்டு வர முயலும் ஒரு இடமாகக் இருக்கிறது. இவ்வாறு செய்வதன் மூலம், அவர்கள் சமூக ஊடகங்களை குழு ஊடகங்களாக காட்சிமைப்படுத்தி, அதில், தாங்கள் தரநெறிசார் இந்திய பாரம்பரியங்களை நிலைநிறுத்துவதை பரவலான உலக அரங்கத்தினரின் பார்வைக்கு செயல்காட்சி நடத்துகிறார்கள்.

இயல்புநிலை மற்றும் நிகழ்நிலை இடங்களுக்கு இடையேயான, இது போன்ற தொடர்ச்சி பற்றிய எண்ணச்சார்பு, இந்தியச் சூழலில் ஒன்றும் இன்று நேற்று புதிதாக உருவானது அல்ல. சொல்லப்போனால், இது போன்ற எண்ணச்சார்பு பற்றிய கோரிக்கைகள், இந்திய அண்டவியல் எண்ணவோட்டத்தின் அடிப்படை அங்கமாக இருந்து வந்திருக்கிறது. இது பஞ்சக்கிராமியில் வன்பொருள் கடை வைத்திருக்கும் 56 வயதான நாகமணியின் வழக்கில் தெளிவாக எடுத்துக்காட்டப்படுகிறது. நாகமணி, ஆறு வருடங்களுக்கு முன்பு, தன்னுடைய மூன்றாவது மகனை புற்றுநோய்க்கு பலிகொடுத்தார். அதைக் குறிக்கும் ஆறாம் ஆண்டு திதி நாள் சம்பிரதாயங்களில், பிரிந்து போன ஆத்மாவிற்கு அளிக்கப்படும் உணவுப் படையலும் அடக்கம். படையல் இடப்பட்டிருந்த வாழையிலைக்கு அருகில், நவீன கைக்கடிகாரம், 'சிந்தால்' வாசனை சோப்பு, குளிர்கண்ணாடி, 'பார்க்கர்' பால்பாயிண்ட் பேனா, மற்றும் ‘ஆக்ஸ்'8 வாசனை திரவியம் போன்ற பொருட்களும் வைக்கப்பட்டிருந்தன. இவையனைத்தும் தனது மகனுக்கு மிகவும் விருப்பமான பொருட்கள் என்றும், அவருக்கு அது மறுபிறவியிலும் தேவைப்படும் என்பதால் வைத்திருப்பதாகவும் நாகமணி கூறினார். எண்ணச்சார்பு பற்றிய நம்பிக்கை இடம் மற்றும் நேரத்தையும் மிஞ்சும் ஆற்றல் கொண்டிருந்தால், - அது இந்தப்பிறவி மற்றும் மறுபிறவிகளுக்கிடையே ஆனாலும் சரி அல்லது கிராமப்புறம் மற்றும் நகர்ப்புறம் 
ஆகியவற்றுக்கிடையே ஆனாலும் சரி, - இயல்புநிலை மற்றும் நிகழ்நிலைகளுக்கு இடையே எண்ணச்சார்பு இருக்கக்கூடும் என்பதில் ஆச்சரியம் ஒன்றும் இல்லை.

பஞ்சகிராமியில், நிகழ்நிலை சமூக ஊடகங்களில், இயல்புநிலை பழக்கவழக்கங்கள் மற்றும் சமூக வகைப்பாடுகளின் எண்ணச்சார்பு, பல வடிவங்களை கொண்டுள்ளது. சமூக ஊடகங்களில் பொதுவாக கவனிக்கப்பட்ட ஒரு இயல்புநிலை பழக்கவழக்கம் என்னவென்றால், ஒத்தத்தன்மை ${ }^{10}$ தொடர்பு என்றழைக்கப்படும் ஒரேமாதிரியான பின்னணியில் இருப்பவர்களுடன் தொடர்பு கொள்வது தான். பஞ்சக்கிராமியில் இது போன்ற ஒத்தத்தன்மை தொடர்பு கொள்ளுதல் என்பது குறிப்பாக குலம் மற்றும் வர்க்கம் சார்ந்ததாக இருக்கிறது. இது போன்ற உட்குழு நடத்தை, நிகழ்நிலையில் மற்ற அனைவராலும் உருவகப்படுத்தப்படும் 'பிறத்தன்மை'க்கு வழிவகுக்கிறது. இதுபோன்றவர்களுடனான உரையாடல்கள், தேவைக்கானவையாக இருக்கிறதே தவிர, சமூக பழக்கத்திற்கானதாக இருப்பதில்லை.

இது போன்ற ஒத்தத்தன்மை தொடர்பு, டிஜிட்டல் சமத்துவமின்மைக்கும் வழிவகுக்கிறது. இந்த ஒ ஒட்டுமொத்த செயல்திட்டத்தின் ${ }^{11}$ முக்கிய கண்டுபிடிப்புகளில் ஒன்று என்னவென்றால், நிகழ்நிலை சமத்துவம் என்பது இயல்புநிலை சமத்துவத்தின் பிரதிநிதியாக இருக்கவேண்டுமென்ற அவசியமில்லை என்பது தான். இது பஞ்சக்கிராமி விஷயத்தில் நிதரிசனமான உண்மை.

ஒரு சந்தர்ப்பத்தில், ஸ்மார்ட்போன்கள் மற்றும் இணையதள தரவு திட்டங்கள் போன்ற தகவல் தொழில்நுட்பங்கள் கட்டுப்படியாகக் கூடிய விலையில் கிடைப்பது, வாய்ப்புகளில் சமத்துவத்தை ஏற்படுத்தியிருக்கிறது. இரருப்பினும், ஊடக சமத்துவத்திற்கான வாய்ப்பு, நிகழ்நிலை சமத்துவத்திற்கான வாய்ப்பாகாது. அதேபோல, ஒருவரால் பல்வேறு பின்னணியில் இருந்து வரும் நபர்களுடன் நட்புதொடர்பு ஏற்படுத்திக்கொள்ள முடிகிறது என்பதால், அனைவராலும் அது முடியும் என்பதாகாது. அதிலும் குறிப்பாக, அப்படிப்பட்டவர், கீழ்மட்ட சமூக-பொருளாதார பின்னணி12 உடையவராக இருக்கும் போது.

இது போன்ற பாரம்பரிய குழுக்களின் பேணுகை, காட்சிக்குரிய, எழுத்துவாயிலான அல்லது வேறு பல வகைகளிலான பதிவுகள் வாயிலான சமூக ஊடக உரையாடால்களின் மூலம், வெளிப்படுத்தப்படும் சமூக அனுசரித்தலை வலியுறுத்துகிறது. பலர், தங்களது தகவலை, தங்கள் குழுக்களின் எதி- 
ர்பார்ப்பிற்கு ஏற்ப மூலோபாய வகையில் வடிவமைப்பதின் மூலம் அனுசரிக்க முயலுகிறார்கள். இதுபோன்ற குழுக்களில், அதிருப்தியை தெரிவிப்பது என்பது, தனிப்பட்ட வகையிலோ அல்லது அமைதி மற்றும் உதாசீனத்தின் மூலமாகவோ நடைபெறுகிறது. தரநெறிசார் எதிர்பார்ப்புகளுக்கு அதிருப்தி தெரிவிப்பதற்காகவே சிலர் பல்வேறு பொய்யான சமூக ஊடக கணக்குகளை உருவாக்கிக் கொண்டனர். பின்வரும் அத்தியாயங்களில் நாம் பார்க்கப்போவது போல, ஒரு சிலருக்கு நம்பத்தக்கதான எனும் தன்மை என்பது, இப்போதெல்லாம், பல புனைபெயர் அடையாளத்தில் பல்வேறு தளங்களிலோ அல்லது ஒரே தளத்திலோ வெளிப்படுத்தப்படும் வெவ்வேறு வகையான பதிவுகள் தான்.

நிகழ்நிலை மற்றும் இயல்புநிலை இடங்களுக்கு இடையே, மதம், வர்க்கம் போன்ற பல்வேறு சமூக வகைகளின் செல்வாக்கினால் உருவான எண்ணச்சார்பு, சமுதாயத்தில் வெவ்வேறு வலையமைப்பிற்கு வழிவகுப்பது போல் தோன்றினாலும், அவர்களுடைய சமூக ஊடக நடவடிக்கைகள் மற்றும் பதில்களை பார்க்கும் போது, அவற்றுக்கிடையே வேறுபாடுகளை விட, ஆழமான தமிழ் கலாசார செல்வாக்கு கொண்ட பல ஒற்றுமைகள் புலப்படுகின்றன (உதாரணத்திற்கு, அவைகளின் காட்சிமை கலாச்சாரம் அல்லது வலையமைப்பு அனுசரித்தல் போன்றவை). இது, தகவல் தொழில்நுட்ப பணியாளர்களின் "மேதாவி குழுக்களுக்கும்", கிராமவாசிகளுக்கும் இடையேயான அதீத ஒற்றுமையையும் விளக்குகிறது. இந்த புத்தகத்தின் வெவ்வேறு அத்தியாயங்கள், மக்கள் இன அமைப்பியல் மூலம் கிடைத்த உதாரணங்களோடு இவை அனைத்தையும் விரிவாக விளக்குகின்றன.

இந்த எண்ணச்சார்பு பற்றிய கருத்தை, நிகழ்நிலை மற்றும் இயல்புநிலை இடங்களை தனித்தனியே தெளிவாக புரிந்து கொள்வதன் மூலம் சரியாக மதிப்பிடமுடியும். முதல் இரண்டு அத்தியாயங்களின் முக்கிய பணியே இதுதான். இந்த அத்தியாயம், பஞ்சக்கிராமியையும், அங்கு குடியிருப்போரையும், அவர்களின் அன்றாட வாழ்க்கை பற்றியும் அறிமுகப்படுத்தும் வகையில் அமைக்கப்பட்டிருக்கிறது. இது, தகவல் தொழில்நுட்பத்துறை மற்றும் விவசாய ஆதிக்கம் உள்ள பாரம்பரிய கிராமிய விஸ்தீரணம் ஆகியவற்றால் ஊக்கமூட்டப்பட்டு, அடிப்படையாக பக்க அணிமைநிலையில் அமைக்கப்பட்டிருக்கும் ஒரு மாபெரும் அறிவார்த்த பொருளாதாரத்தினால் உருவாகக்கூடிய சிக்கல்களையும் ஆராய்கிறது. 
இதை தொடர்ந்து, இரண்டாம் அத்தியாயத்தில்,சமூக ஊடக இடப்பரப்பு மற்றும் தகவல் பரிமாற்ற பழக்கவழக்கங்கள் பற்றிய ஆராய்ச்சி செய்யப்பட்டிருக்கிறது. இந்த அத்தியாயம், பஞ்சக்கிராமியின் தகவல் பரிமாற்ற வரலாற்றில் தொடங்கி பல்வேறு சமூக குழுக்களுக்கு இடையிலான பல்வேறு சமூக ஊடகத்தள பயன்பாட்டைப் பற்றிய விவரங்களுக்கு நகர்கிறது. இயல்புநிலை தகவல் பரிமாற்றத்தோடு தொடர்புடைய விதிமுறைகள் எவ்வாறு அவர்களின் சமூக ஊடக உரையாடல்களில் பிரதிபலிக்கிறது என்பதையும் இரண்டாம் அத்தியாயம் பரிசீலிக்கறது.

இந்தப்பகுதியின் இயல்புநிலை மற்றும் சமூக ஊடக இடப்பரப்பின் புரிதலோடு, பஞ்சக்கிராமியில் மிகவும் பொதுவான சமூக ஊடக தகவல் பரிமாற்ற வகையான காட்சிக்குரிய பதிவுகளை ${ }^{13}$ ஆராய்வதற்கு நாம் செல்லலாம். இந்த காட்சிக்குரிய பதிவுகளெல்லாம் பெரும்பாலும் இயல்புநிலையில் உள்ள காட்சிமைகளின் தொடர்ச்சி தான் என்பதை மூன்றாம் அத்தியாயம் எடுத்துக்காட்டுகிறது. சமூக ஊடக காண்புலன் விஷயங்களை பொதுவானவை, தனிப்பட்டவை, இடைப்பட்டவை ${ }^{14}$ (அதாவது பொதுவிற்கும், தனிப்பட்டவைக்கும் இடையிலானவை) என்று இயல்புநிலை இடப்பரப்பிற்கேற்ற வகையில் பிரிப்பதன் மூலம் இந்த எடுத்துக்காட்டுதல் செய்யப்படுகிறது. மக்கள் எவ்வாறு தங்கள் காட்சிக்குரிய கருத்துக்களை சமூக விதிமுறைகளுக்கு ஏற்றவகையிலும், தாங்கள் சார்ந்திருக்கும் வலையமைப்புகளோடு அனுசரித்துப் 15 போகும் வகையிலும் இருக்குமாறு கவனமாக வடிவமைக்கிறார்கள் என்பதையும் இந்த அத்தியாயம் ஆராய்கிறது.

தரநெறிசார் குழு நடத்தை மற்றும் அனுசரித்துப் போதல் பற்றிய கருத்து ஆகிய இரண்டிற்கும் மையமானது தான் உறவுமுறை ${ }^{16}$ நான்காம் அத்தியாயம், அன்றாட தகவல் பரிமாற்றத்தின் முதன்மை களமாக விளங்கும் குடும்பம், உறவுமுறைகள் போன்றவற்றின் உள்வட்டத்தில் கவனம் செலுத்துகிறது. ஆகவே, பெரும்பாலான உறவுமுறைகள் பற்றிய ஒரு விரிவான கலந்தாய்வு மிகவும் அவசியமாகிறது. உண்மையில், இந்தியாவில் காணப்படும் ஒரு பொதுவான சமூக வகை என்னவென்றால் உறவுமுறைகள் தான். குலம் என்பது அகமணத்தை தம் குலத்தை மீறி வெளியே எங்கும் திருமணம் செய்ய தடை) இதன் மூலம் குலம் என்பது நீட்டிக்கப்பட்ட உறவுமுறைகளே என்று ஆகிவிடுகிறது. இது, சமூக கட்டுப்பாடுகள், கண்காணிப்பு, பாலின இடப்பரப்பு|', அதிகாரம், குழு செயல்பாடுகள், 
அதிகார படிநிலை போன்ற பல பரிமாணங்களை தன்னுடன் கொண்டுவருகிறது. இவற்றில் சில, தன் தங்கையை சமூக ஊடகங்களின் பிடியில் இருந்தும், நிகழ்நிலையில் உலவும் பிற சமூக குழுக்களை சேர்ந்த ஆண்களிடம் இருந்தும் தான் காக்க வேண்டும் போன்ற செல்வாவின் எண்ணங்களில் வெளிப்படுகின்றன. அவர் வகையில், ஒரு இளம்பெண்ணை மற்ற ஆபத்து நிறைந்த ஆணின இடப்பரப்பில் இ19 இருந்து காப்பாற்றக்கூடிய சமூக கட்டுப்பாடு என்பது, ஒருவருக்காக விதிக்கப்படும் ஒட்டுமொத்த தடையில் இருந்து, அவரது வீட்டிலேயே அவருக்கு விதிக்கப்படும் சமூக ஊடகப் பயன்பாட்டுக்கான கட்டுப்பாடுகள் வரை, பல வகைப்படும். இதற்கு மாறாக, விஜயாவின் புகுந்த வீட்டினரின் விழுமிய தாய்மை பற்றிய எதிர்பார்ப்பு உண்டாக்கிய அழுத்தம், இந்த தொழில்நெறிப் பெண்ணை, வாட்சப்பை தாய்மைக்கான ஒரு பெண்ணின இடப்பரப்பாக தழுவிக்கொள்ளச் செய்தது.

குடும்ப வட்டங்களுக்குள்ளான அதிகாரம் மற்றும் அதிகாரப் படிநிலை, தலைமுறைகளுக்கு இடையேயான தகவல் பரிமாற்றத்தில் அதிலும் குறிப்பாக பெரியவர்களை உடைய குடும்பங்களில் மிகவும் தெளிவாகத் தெரிகிறது. பல பெரியவர்கள், தங்களோடு உரையாடுவெதற்கான சரியான தளம் எது என்பதை தாங்கள் தான் முடிவு செய்ய வேண்டும் என்ற அதிகார மனப்பான்மையோடு முயல்கிறார்கள். பல குடிம்பங்களில், குரல்வழியாக எந்தெந்த செய்திகள் தெரிவிக்கப்படவேண்டும், முகநூலில் போட முடியாத அளவுக்கான தனிப்பட்ட தகவல்கள் என்னென்ன, எவையெவை தனிப்பட்ட முறையில் வாட்சப் மூலம் தெரிவிக்கப்படவேண்டும் போன்ற விஷயங்கள் எல்லாம் பெரும்பாலும் வயதில் மூத்த குடும்பத்தினரால் முடிவு செய்யப்படுகிறது. பொதுவாக, தனிப்பட்ட குடிம்ப தகவல் பரிமாற்றங்கள் வாட்சப் மூலமாகவும், விழுமிய குடும்பம் என்ற தோற்றத்தை பரந்த உலகிற்கு வெளிப்படுத்தும் வகையிலான செயல்கள் முகநூல் மூலமாகவும் செயல்படுத்தப்படுகின்றன. சமூக ஊடகங்களில் உள்ள கற்பனையான உறவினர் குழுக்களில் வெளிப்படுத்தப்படும் நெருக்கங்களும் இந்த அத்தியாயத்தில் கலந்தாய்வு செய்யப்பட்டுள்ளன.

ஒரு நவீன பணியமைப்பில், பணி சார்ந்த மற்றும் பணிசாரா வட்டங்களுக்கிடையேயான எல்லைகளை சமூக ஊடகங்கள் எவ்வாறு கீழறுக்கக்கூடும் என்பதை ஐந்தாம் அத்தியாயம் கலந்தாய்வு செய்கிறது. இது மிகவும் முக்கியம் ஏனென்றால், தகவல் தொழில்நுட்பத்துறையும் இன்னபிற நவீன பணியமைப்புகளும் தான் முதன்முதலில், பஞ்சக்கி- 
ராமியின் சமூகப் பொருளாதார மாற்றத்திற்கான முக்கியக் காரணங்கள். மக்கள் எவ்வாறு பாரம்பரிய சமூக வகைகளின் அதிகாரத்திற்கு, அவற்றை நவீன பணியிடங்களின் அதிகாரத்துடன் இ இணக்குவதன் மூலம் அனுசரித்துப் போகிறார்கள் என்பதை இந்த அத்தியாயம் எடுத்துக்காட்டுகிறது. பத்தாண்டுகளுக்கு முன்பு வரை ஒரு விவசாய பொருளாதாரத்தின் அங்கமாக இருந்தமையால், மக்கள் இதுவரை பணியையும் பணியல்லாதவற்றையும் இருபிரிவுகளாகவோ அல்லது ஒன்றிணைந்ததாகவோ பார்த்ததில்லை. இரண்டும் ஒன்றோடொன்று இணைந்தே இருந்தது. அவற்றுக்கிடையேயான எல்லைகள் அநேக சமயங்களில் இளகியதாகவே இருந்தது. இது தென்னிந்திய பணி கலாச்சாரத்தில் ஓரளவு உண்மையும் தான். இங்கு பணிசாரா இடப்பரப்புடனான உரையாடல்கள், அன்றாட சமூகமயமாதலின் அங்கமாக கருதப்படுகிறது. இருப்பினும், தகவல்தொழில்நுட்பத்துறை மற்றும் அதன் நவீன பணியிட விதிமுறைகளின் வருகையால், பணி மற்றும் பணிசாராதவை பற்றிய கருத்துக்கள் மாறத்துவங்கின. பணியிடம் தாண்டியும் பணியினை செய்வது, நவீன பணியிட எதிர்பார்ப்புகளுடன் அனுசரித்துப் போவதாக கருதப்பட்டாலும், பணிசாரா விஷயங்களை பணியிடத்திற்கு கொண்டுவருவது, ஒத்துழையாமையாக கருதப்பட்டு, நிர்வாகத்தினரால் ${ }^{20}$ அதிருப்தியுடன் பார்க்கப்பட்டது. சமூக ஊடகங்கள், இது போன்ற கட்டுப்பாடுகளை சுற்றிச்செல்வதற்கும், இது போன்ற அமைப்புகளில் உள்ள பணி சார்ந்த மற்றும் பணிசாரா எல்லைகளை கீழறுப்பதற்கும் உதவியுள்ளன. குலம் மற்றும் வர்க்கம் போன்ற முந்தைய பழமையான அதிகார வகைகள் தான், உறவினர்-அடிப்படையிலான ${ }^{21}$ பணியமர்த்தம் மற்றும் சமூக ஊடகங்கள் வாயிலான குடும்பத் தகவல் பரிமாற்றம் ஆகிய வகைகளில் இப்போதெல்லாம் பணியிடங்களை ஊடுருவியுள்ளன.

ஆறாம் அத்தியாயம், சமூக ஊடகங்கள் மற்றும் கல்வியை ஆராய்கிறது. அது, வெவ்வேறு பங்குதாரர்களின், உதாரணத்திற்கு, ஆசிரியர்கள், மாணவர்கள், பெற்றோர்கள், கல்விநிறுவனம் போன்றவர்களின், சமூக ஊடகங்கள் மீதான வேறுபட்ட மனப்பாங்கு மற்றும் பதற்றம் பற்றி விரிவாக எடுத்துரைக்கிறது. இந்த அத்தியாயத்தில், நாங்கள் கல்வியின் உட்பரப்பில் சமூக ஊடகங்களின் தாக்கத்தை ஆராய்கிறோம். இந்த ஆய்வுக்களம், புதிய அறிவார்த்த பொருளாதாரம் ${ }^{22}$ என்ற கருத்தை எவ்வாறு எதிரொலிக்கிறது என்ற வகையில் இந்த தலைப்பு குறிப்பாக முக்கியத்துவம் பெறுகிறது. 
கோமதி என்ற 54 வயதான ஆசிரியை, ஒரு அருமையான வீட்டுத்தயாரிப்பான மதிய உணவுடன், சமூக ஊடகங்கள், மாணவர்களுக்கு எப்படிப்பட்ட கவனச்சிதறலாகவும், நேர விரயமாகவும் இருக்கிறது என்பதை விளக்கினார். அவற்றை பயன்படுத்துவதிலிருந்து ஏன் மாணவர்களை தடை செய்யவேண்டும் என்பது பற்றிய வலுவான கருத்துக்களை அவர் கொண்டிருந்தார். சமூக ஊடகங்களின் தீமைகளை விவரிக்கும் பிரபலமான ஊடகக் கட்டுரைகளையும் அவர் மேற்கோள் காட்டினார். அவர், ஆசிரியர்களும் மாணவர்களும் சமூக ஊடகங்களில் நட்புதொடர்பில் இருப்பதையும் எதிர்த்தார். இதன் மூலம், ஆசிரியர்களுக்கு வகுப்பறையில் உள்ள கட்டுப்படுத்தும் ஆற்றல், குறையக்கூடும் என்று அவர் கருதினார்.

கோமதி அவர்கள் வெளிப்படுத்திய கருத்துக்களை எடுத்துக்கொண்டு, பாரம்பரியமான ஆசிரியர்-மாணவர் அதிகாரப் படிநிலையை, சமூக ஊடகத் தோழமை என்ற புதிய உறவுமுறையுடன் எவ்வாறு வரிசைப்படுத்துவது என்பதான இயல்பான பதற்றத்திற்கு சமூக ஊடகங்கள் எந்தவகையில் பங்களிக்கின்றன என்பதை நாம் பார்க்கலாம். சமூக வர்க்கங்கள் மற்றும் பள்ளி அமைப்பு வகைகள், ஆசிரியர்கள் மற்றும் மாணவர்களுக்கிடையே சமூக ஊடகங்களில் இருக்கும் இந்த மிக மெல்லிய உறவுமுறைக்கு, கூடுதலான சிக்கல் தன்மையை அளிக்கிறது.

பல்வேறு அத்தியாயங்களின் தலைப்புகளை அறிமுகப்படுத்தி விட்டதால், நாம் இப்போது பஞ்சக்கிராமி, அதன் மக்கள் மற்றும் அவர்களின் வாழ்வு பற்றிய விரிவான விளக்கங்களுக்குச் செல்லலாம்.

\section{பஞ்சக்கிராமி எங்கிருக்கிறது?}

ஐந்து கிராமங்களின் குழுமத்திற்கான புனைபெயரான பஞ்சக்கிராமி, தென்னிந்தியாவின், தமிழ்நாடு என்ற மாநிலத்தில் உள்ள 375 வயதான ${ }^{23}$ சென்னை மாநகரத்தின் புறநகர்ப் பகுதியில் அமைந்திருக்கிறது. இது காஞ்சிபுரம் ${ }^{24}$ மாவட்டத்தை சேர்ந்ததாகும்.

சராசரியாக 14.25 சதுர கி.மீ பரப்பளவை ஆக்கிரமித்திருக்கும் இந்த ஐந்து கிராமங்களும் தனித்தனி அலகுகள் தான். இவை ஒன்றிணைந்த நிர்வாக அமைப்பு இல்லை. இந்த மக்கள் இன அமைப்பியல் குறிக்கோள்களுக்காக, இந்த இடத்தின் விரைவான மாறுநிலையை விளக்கும் வகையில் 
பஞ்சக்கிராமியின் எல்லைகள் செயற்கையாக வரையப்பட்டுள்ளன.(படம்: 1.1)

பஞ்சக்கிராமி என்பது ஒற்றை நிலப்பகுதி கிடையாது. சென்னை நகரத்தின் உள்ளில் இருந்து காஞ்சிபுரம் மாவட்டத்தின் பகுதிகளுக்கு ெெல்லும் நெடுஞ்சாலையின் (தகவல் தொழில்நுட்ப ெெடுஞ்சாலை என்றழைக்கப்படும்) இருமருங்கிலும் அமைந்திருக்கும் நிலப்பகுதியாகும். மேற்கூறிய தகவல்தொழில்நுட்ப நெடுஞ்சாலையின் ஒரு பகுதி மட்டுமே பஞ்சக்கிராமியின் வழியாகச் செல்கிறது. பஞ்சக்கிராமியின் எல்லை ஒருபுறம், பிரபலமான பக்கிங்காம் கால்வாயின் காயலால் அமையப்பெற்றிருக்கிறது. இ இது தமிழ்நாட்டு கடற்கரையான வங்காள விரிகுடாவில் இருந்து 2 கி. மீ (1.25 மைல்கள்) தொலைவில் அமைந்திருக்கிறது. சில வருடங்களுக்கு முன்பு வரை இந்தக் கால்வாய் முக்கியமான நீர்வழிப் பாதையாக செயல்பட்டு இந்தப் பகுதியின் வணிகத்தை விருத்தி செய்வதற்கு உதவிக்கொண்டிருந்தது. ஆனால் இந்த நீர்வழிப்பாதையின் பயன்பாடு, பல காரணங்களுக்காக ${ }^{25}$ ரத்து செய்யப்பட்டிருக்கிறது. இது நடந்து பத்தாண்டுகளுக்கு மேல் ஆகிவிட்டாலும் இநந்தப்பகுதியில் உள்ள நீண்டகால முதிர்ந்த குடியிருப்புவாசிகள், இந்தக் கால்வாயில் தாங்கள் பயணித்த நாட்களை வாஞ்சையுடன் நினைவுகூருகிறார்கள். இந்த அழகான நீர்வழிப்பாதை மூடப்பட்டதில் தம் வருத்தத்தை தெரிவிக்கிறார்கள்.

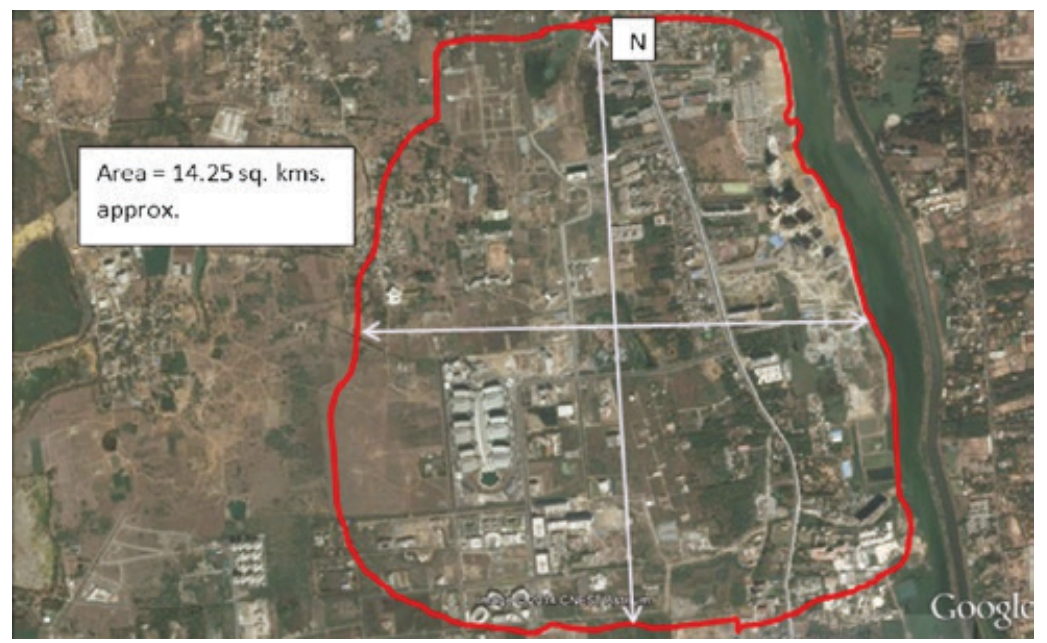

படம்: 1.1 பஞ்சக்கிராமியின் வானலைக் காட்சி (கூகுள் பூமி வரைபடம்) 
இன்றைய நிலையில் கடற்கரைக்கு செல்ல வேண்டும் என்றால், பஞ்சக்கிராமியில் இருந்து விலகி சில கிலோமீட்டர்கள் பயணித்து, மற்றொரு நெடுஞ்சாலையுடன் இணையும் ஒரு இணைப்புச்சாலைக்கு சென்று, அங்கிருந்து பிரிந்து கடற்கரைக்குச் செல்லும் சிலபல சிறிய சாலைகளின் மூலமாகத்தான் கடற்கரையை சென்றடைய முடியும். பஞ்சக்கிராமியின் மேற்கே, பல நெல்வயல்களும், வர்த்தகம், தகவல் தொழில்நுட்பத்துறை மற்றும் வீட்டு மனை விரிவாக்கங்களுக்காக பயன்படுத்தப் பட்டுக்கொண்டிருக்கும் பல காலி மனைகளும், அமைந்திருக்கின்றன. இந்த மேற்குப்பகுதி, தென்தமிழ்நாட்டை சென்னை மற்றும் இதர மாநிலங்களுடன் இணைக்கும் மற்றொரு தேசீய நெடுஞ்சாலையை ஒட்டி அமைந்திருக்கிறது.

பஞ்சக்கிராமியின் தெற்கே இன்னும் சில கிராமங்களின் தொடர்ச்சி அமைந்திருக்கிறது. இவற்றின் அமைப்புப்பாதை பத்தாம் நூற்றாண்டை சேர்ந்த இந்து சமய புனிதத்தலத்துடன் இணைகிறது. இந்தத் தலம், தமிழ்க் கடவுள் என்று அழைக்கப்படும் முருகக்கடவுளுக்கானது. இவர், மும்மூர்த்திகளில் ஒருவரான சிவபெருமானின் மகனாவார். இன்னும் தெற்கே போனால், சிற்பக்கலைக்கு பெயர்பெற்ற யுனெஸ்கோ ${ }^{26}$ உலகப் பாரம்பரிய ஸ்தலமான மாமல்லபுரம் என்றும் மகாபலிபுரம் ${ }^{27}$ என்றும் அழைக்கப்படும், ஏழாம் நூற்றாண்டில் பல்லவ சாம்ராஜ்யத்தின் ${ }^{28}$ துறைமுக நகரமாக விளங்கிய இடம் இருக்கிறது. பஞ்சக்கிராமியின் வடக்கே சென்னை மாநகரம் (முன்பு மெட்ராஸ், மதராசப்பட்டினம், சென்னைப்பட்டினம் என்றழைக்கப்பட்ட) $)^{29}$ அமைந்திருக்கிறது.

சென்னை நகரத்தின் மையத்தில் இருந்து பஞ்சக்கிராமி நோக்கிய நெடுஞ்சாலைப் பயணம் சுமார் ஒன்றரை மணிநேரம் எடுக்கிறது. இந்தத்தருணத்தில், நிலப்பரப்பு, நகர்ப்புறம் என்பதில் இருந்து மாறி, அரை-நகர்புறமாக, அதாவது நெடுஞ்சாலையின் இருமருங்கிலும் வானுயர்ந்து நிற்கும் நவீன வர்த்தக மற்றும் குடியிருப்பு கட்டிடங்களும் அவற்றுக்கருகில் விரிந்திருக்கும் விவசாய நிலங்களுமாக காட்சியளிக்கிறது. பஞ்சக்கிராமியை அமைத்திருக்கும் ஒரு கிராமத்தின் தனியான பெயர்ப்பலகை உங்களை பஞ்சக்கிராமிக்கு வரவேற்கும். இதைத் தாண்டிய பின், நீங்கள் வெகு சுலபமாக 20 பசுக்கள் சாலையில் நிதானமாக உலவுவதையோ, உட்கார்ந்திருப்பதையோ பார்க்கலாம். அதையொட்டியே தகவல்தொழில்நுட்பத்துறை பணியாளர்களின் கார்கள் அதிவேகமாக கடந்து செல்வதையம் பார்க்கலாம். இந்தக் கிராமங்களில் உள்ளவர்கள் மற்றும் அவர்களின் 
வயல்கள் ஆகியவற்றுக்கும் தகவல் தொழில்நுட்பத்துறை வளர்ச்சியால் ஊக்கமூட்டப்பட்டு செழித்தோங்கி வரும் நவீனத்திற்கும் இடையிலான திட்பமிக்க மாறுபாடு இதை ஒரு விசேஷமான காட்சியாக சித்தரிக்கிறது.

வழக்கமான தமிழ் கிராமங்களில் பார்க்க முடியாதவை என்றால், கே.எஃப்.சி எனப்படும் கென்டுக்கி ஃபிரைட் சிக்கன் மற்றும் டொமினோஸ் ீட்சா ஆகியவை தான். ஆனாலும், பஞ்சக்கிராமியில் நுழைந்தவுடன் சாலையின் ஒருபக்கம் அமைந்திருக்கும் பிரம்மாண்டமான பல்கூட்டு திரையரங்கு கட்டிடத்தின் தரைத்தள பகுதியில் இவை அமைந்திருப்பதை நீங்கள் காண முடியும். சாலையின் மறுபக்கம் பிரம்மாண்டமான பல அடுக்குமாடிகளை கொண்ட குடியிருப்பு வளாகத்தையும், அதைத்தொடர்ந்து ஒரு பெரிய பன்னாட்டு தகவல் தொழில்நுட்ப நிறுவனத்தின் அலுவலகங்கள் அமைந்திருப்பதையும் காணலாம். சாலையின் இருமருங்கிலும், பேக்கரி கடைகள், சிற்றுண்டி கடைகள், சிறு உணவகங்கள், முடித்திருத்தகங்கள், அழகுநிலையங்கள், பன்மாடி குடியிருப்புக்கட்டிடங்கள், பாரம்பரிய கிராமிய வீடுகள், சாலையோர தேநீர் கடைகள், உயர்மட்ட காபி ஷாப்கள், நட்சத்திர ஹோட்டல்கள், பல்பொருள் அங்காடிகள், வன்பொருள் மற்றும் செல்போன் உதிரி பாகங்கள் விற்கும் சிறிய கடைகள், கிராமச்சந்தை, தகவல் தொழில்நுட்பத்துறையை சார்ந்த மாபெரும் நிறுவனக்கட்டிடங்கள், சர்வதேசப் பள்ளிகள், கிராமப் பள்ளிக்கூடங்கள், மருத்துவமனைகள், சிறிய வைத்தியசாலைகள், மருந்தகங்கள், கல்லூரிகள், பஞ்சாயத்து அஅலுவலகங்கள் மற்றும் நெல்வயல்களுக்கு இட்டுச்செல்லும் பிரிவுச்சாலைகள் ஆகியவை அமைந்திருப்பதை பார்க்கலாம். ஒருவகையில் இந்தப் பகுதியின் போக்குவரத்து, வளர்ந்து வரும் பொருளாதார செழுமையையும் மாற்றங்களையும் பிரதிபலிக்கிறது. பேரூந்து போன்ற பொது போக்குவரத்து வாகனங்களோடு, சைக்கிள், ஸ்கூட்டர், மோட்டார்சைக்கிள், போன்ற தனிப்பட்ட இருசக்கர வாகனங்களும், சாதாரண கார் முதல் சொகுசுக் கார்கள் வரையிலான நான்கு சக்கர வாகனங்களையும் இங்கு பார்க்க முடியும். மக்களின் ஆடை வகைகளிலும் இந்த நிலப்பரப்பின் பன்முகத்தன்மை வெளிப்படு|கிறது. பாரம்பரியமான சேலை மற்றும் வேட்டிகள் முதல், சுரிதார், சல்வார்-கமீஸ், ஜீன்ஸ், டி-ஷர்டுகள், கோட்-சூட் போன்ற மேற்கத்திய ஆடைகள் வரை அனைத்து வகைகளையும் இங்கு பார்க்கலாம். 
பஞ்சக்கிராமி ஒரு சிறப்புப் பொருளாதார மண்டலமாகவும் விளங்குகிறது. இந்த இடம் பல்வேறு தகவல் தொழில்நுட்ப கூட்டுத்திரள்களுக்கும் இருப்பிடமாக இருப்பதோடு மேலும் விரிவடைந்து கொண்டே இருக்கிறது. இருப்பினும், பத்தாண்டுகளுக்கு முன்பிருந்த நிலப்பரப்பை நினைவூட்டும் வகையிலான, நவீன வளர்ச்சிக்கு உட்படுத்தப்படாத பல்வேறு காலி மனைகளையும் கொண்டுள்ளது. இந்தியாவின் தற்போதைய வளர்ச்சியை வளர்ந்து வரும் பொருளாதாரமாக விவாதிக்கும் பல்வேறு கோட்பாட்டளவான கணக்குகளும் பஞ்சக்கிராமியில் கண்கூடாக புலனாகும்.

\section{பஞ்சக்கிராமியின் சுருக்கமான வரலாறு}

பஞ்சக்கிராமியை அமைக்கும் கிராமங்கள், சுமார் 150-200 வருடங்களுக்கு முன்பு ஒரு குறிப்பிட்ட குலத்தை ${ }^{30}$ சேர்ந்த மக்கள் குடும்பங்களுடன் குழுக்களாக வந்து இங்கு குடியிருப்பு அமைத்தபோது உருவானவை.

இந்த கிராமங்களின் பண்டைய கோவில் நகரத்திற்கும் பண்டைய துறைமுக நகரத்திற்குமான அருகாமை, இந்தப்பகுதிக்கு மேற்கூறிய 150-200 வருடங்களுக்கு மேலான வரலாறு இருக்கக்கூடும் என்பதை உணர்த்துகிறது. பல்லவர்களின் நிர்வாகம் மற்றும் மாமல்லபுரம் ${ }^{31}$ ஆகியவற்றை பற்றிய இலக்கிய நூல்களும், இந்தப்பகுதியில் சேகரிக்கப்பட்ட வாய்வழி வரலாற்று தகவல்களும் சிலபல தொடர்புகளை சுட்டிக்காட்டுகின்றன. இன்றும் காணப்படும் வரலாற்றுச் சின்னங்களான சில கல்மண்டபங்களும், தற்சமயம் இந்தப் பகுதியில் மணல் அள்ளுதற்கு பயன்படும் நீரில்லா ஏரிகளும், குளங்களும் மேற்கூறிய தொடர்புகளின் சாத்தியக்கூறுகளை நிரூபிக்கின்றன. இதுபோன்ற கட்டிட அமைப்புகள், நீர்நிலைகள் அமைத்தல், பயணிகளுக்கான இளைப்பாறும் இடமாக கல்மண்டபங்கள் கட்டுதல் போன்ற பல்லவர்களின் கொள்கைகளுக்கு ஏற்றவாறு இருக்கின்றன. இந்தப் பகுதியைச் சுற்றி சிவபெருமானுக்கும் ம2 மஹாவிஷ்ணுவிற்கும் ${ }^{33}$ சிறிய கோவில்கள் அமைந்திருக்கின்றன. பஞ்சக்கிராமிக்கு வடக்கே சற்று தொலைவில், 1000 வருட புராதனமான மஹாவிஷ்ணு கோவில் ${ }^{34}$ ஒன்று இருக்கிறது. 


\section{0-க்குப் பிந்தைய அகக்கட்டமைப்பு வளர்ச்சிகள்}

இந்தியாவில் தகவல் தொழில்நுட்பத்துறை 1990களின் மத்தியில் பொருளாதார தாராளமயமாக்கலுக்கு ${ }^{35}$ பின் பெருவளர்ச்சி பெறத்துவங்கியது. பெங்களூரும் ஹைதராபாதும் தான் இந்தத் துறை சார்ந்த பெரு வளர்ச்சியையும், அகக்கட்டமைப்பு வளர்ச்சி வாய்ப்புகளையும் முதலில் பெற்ற மையங்கள். இந்த வரிசையில் அடுத்து இருந்தது சென்னை. 1990களின் இறுதியில் பஞ்சக்கிராமியில், மிகப்பிரபலமான தகவல் தொழில்நுட்பத்துறை நிறுவனத்தின் ஸ்தாபிதம் இந்தப் பகுதியின் அடுத்தடுத்த மற்றும் அதிதீவிர வளர்ச்சிக்கும் மாற்றத்திற்கும் அடித்தளமாக அமைந்தது. முதலில், சென்னையில் தகவல் தொழில்நுட்பத்துறையில் பிரம்மாண்ட நுழைவு மெதுவாகவும் அதேசமயம் சீரானதாகவும் இருந்தது. 2000-மாவது ஆண்டு, தமிழக அரசு, சென்னை நகரத்தினுள் டைடல் பார்க் என்ற தகவல் தொழில்நுட்பத்துறை நிறுவனங்களுக்கான சிறப்புப் பொருளாதார மண்டலத்தை திட்டமிட்டு நிறுவும் வரை, நகரத்தில் முதலில் ஸ்தாபிக்கப்பட்ட ஒருசில தகவல் தொழில்நுட்பத்துறை நிறுவனங்கள் இங்குமங்குமாக சிதறியிருந்தன. டைடல் பார்க்கின் ஸ்தாபிதம், பல தகவல் தொழில்நுட்பத்துறை நிறுவனங்களை சென்னை நோக்கி கவர்ந்திழுத்தது. 2000-ங்களின் துவக்கத்தில் இ இந்தியாவை சார்ந்த பன்னாட்டு நிறுவனங்களான டி.சி.எஸ் என்றழைக்கப்படும் டாடா கன்சல்டன்சி சர்விசஸ் (டாடா குழும நிறுவனங்களின் தகவல் தொழில்நுட்பத்துறை கிளையமைப்பு) இன்று தகவல் தொழில்நுட்பத்துறை நெடுஞ்சாலை என்றழைக்கப்படும் இந்தப் பகுதியின் நாடியைப்போன்ற சாலைகளை ஒட்டி பிரம்மாண்டமான அலுவலக வளாகத்தை அமைத்தது.

இதன் விளைவாக சென்னயன் புறநகர்ப்பகுதியாக மட்டுமே வளர்ந்திருக்கக்கூடிய நிலப்பரப்புகள், மாபெரும் தகவல் தொழில்நுட்ப வளாகங்களுக்காக ஒதுக்கி வைக்கப்பட்டன. சென்னையை ஒட்டி நகருக்கு வெளியே அமைந்திருப்பதால் பஞ்சக்கிராமி, சுலபமான உடமையாட்சிக்காக நிலங்களை மலிவு விலையில் கிடைக்கச்செய்தது. இதனாலேயே 2000மாவது வருடம், தகவல் தொழில்நுட்பத்துறைக்காக மட்டுமான ஒரு சிறப்பு பொருளாதார மண்டலத்தை தமிழ்நாடு அரசு திட்டமிட்டு பஞ்சகிராமியில் நிறுவியது. துறை-சார்ந்த சிறப்பு பொருளாதார மண்டலமாக, இந்த இடம், ஐ.டி/ஐ.டி.இ.எஸ் 
றைப்புகளும் அடிப்படை அகக்கட்டமைப்பு வசதிகளும் செய்துகொடுத்தது. இது எதிர்காலத்தில் இந்தப்பகுதி சென்னை நகர்புறப்பகுதியுடன் இ இணைக்கப்படக்கூடிய வாய்ப்புகளுக்கு வழிவகுத்துள்ளது.

பஞ்சக்கிராமியில் இதுபோன்ற முன்னணி இந்திய ஐ.டி/ஐ.டி.இ.எஸ் நிறுவனங்கள் மட்டுமல்லாமல், தங்கள் பணியிடங்களுக்கு அருகிலேயே குடியிருக்க விரும்பும் இங்கு பணிபுரியும் தகவல் தொழில்நுட்பத்துறை பணியாளர்களின் வீட்டு முதலீட்டு வாய்ப்புகளாக இருக்கக்கூடிய கட்டிமுடிக்கப்பட்ட மற்றும் கட்டப்பட்டுக்கொண்டிருக்கும் அடுக்குமாடி குடியிருப்பு வளாகங்களும் இருக்கின்றன. இதைத்தொடர்ந்து, இங்கு குடியிருப்பவர்களுக்கான குழந்தைகளுக்காகவென்று, சில தனியார் பள்ளிகள், குடியிருப்பு கட்டுமான நிறுவனங்களுடன் இணைந்து தனியார் பள்ளிகளை இங்கு அமைத்துக் கொண்டன. அதன்பின், இந்தப் பகுதியை சேர்ந்த சிறிய அளவிலான வணிகர்கள் மற்றும் இங்கு வந்து சேர்ந்த புதிய வணிகர்கள் ஆகியோர், இங்குள்ள மக்கட்தொகைக்கு என வணிகங்களையும், கடைகளையும் அமைத்தனர்.

[படம்: 1.2, 1.3 \& 1.4 - கடந்த பத்தாண்டுகளில் பஞ்சக்கிராமியில் நிகழ்ந்துள்ள அகக்கட்டமைப்பு மாறுதல்களை எடுத்துக்காட்டும் வானலை காட்சிப் புகைப்படங்கள்]

பஞ்சக்கிராமியின் நீண்டகால குடியிருப்புவாசிகள், இந்தப்பகுதியில் ஏற்பட்ட தகவல் தொழில்நுட்பத்துறை பொருளாதார வளர்ச்சியால் கிடைக்கப்பெற்ற கணிசமான பொருளாதார அனுகூலங்களை ஒப்புக்கொண்டாலும், அதேமூச்சில் தங்கள் ஆதங்கத்தையும் தெரிவிக்கின்றனர். தற்போது இருக்கும் ஆறு-தடமுள்ள நெடுஞ்சாலையைப் பார்க்கும் போதெல்லாம், முன்பிருந்த ஒரு-தட சாலையையும், அதன் இருமருங்கிலும் செழித்து அடர்ந்திருந்த மரங்களையும், சுட்டெரிக்கும் வெய்யிலில் இருந்து மனிதர்களுக்கும் மிருகங்களுக்கும் ஒரே மாதிரி அவை நிழல் அளித்ததையும் இந்த மக்கள் வாஞ்சையுடன் நினைவுகூருகின்றனர். உதாரணத்திற்கு, 43 வயதாகும் விஜயன் என்ற பஞ்சக்கிராமியின் நீண்டகால குடியிருப்பாளர், இங்கு தன்னுடைய இளம் பருவத்தை நினைவுகூருகிறார். குறைந்த மக்கட்தொகையுடனும், அதிலும் குறைந்த போக்குவரத்து நெரிசலுடனும் இருந்த வாத்சல்யமான சுற்றுச்சூழல் கொண்ட பகுதி என்று இந்தப் பகுதியை அவர் விவரித்தார். 1980-களில் இருந்த பஞ்சக்கிராமியின் கிராமங்களை நினைவூட்டும் வகையிலான தெருக்கள் மிகவும் குறைவாகவே உள்ளன. விஜயன் போன்ற 


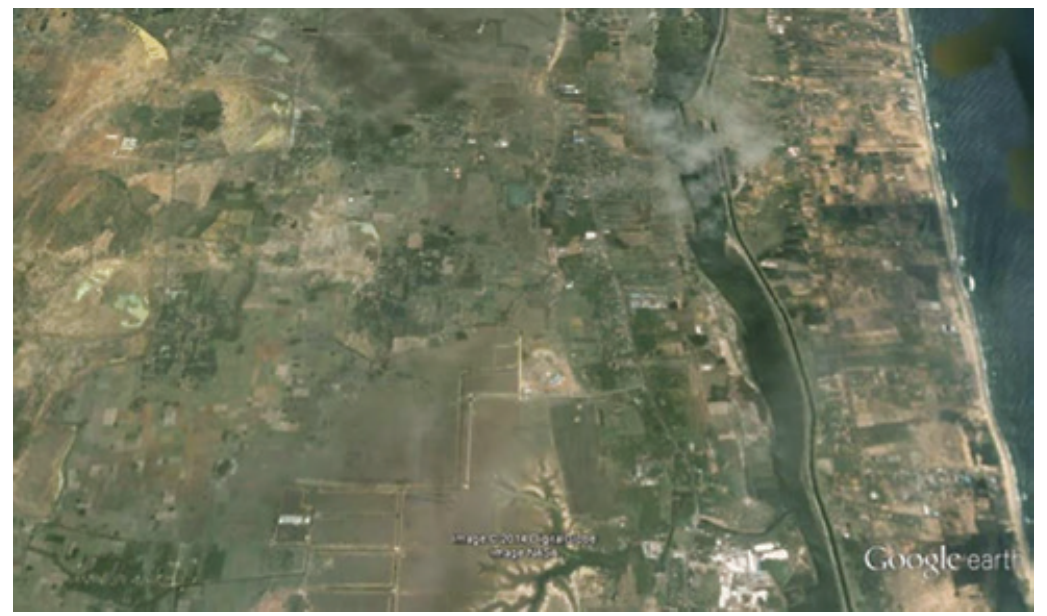

படம்: 1.2 2002-ல் பஞ்சக்கிராமி (கூகுள் பூமி வரைபடம்)

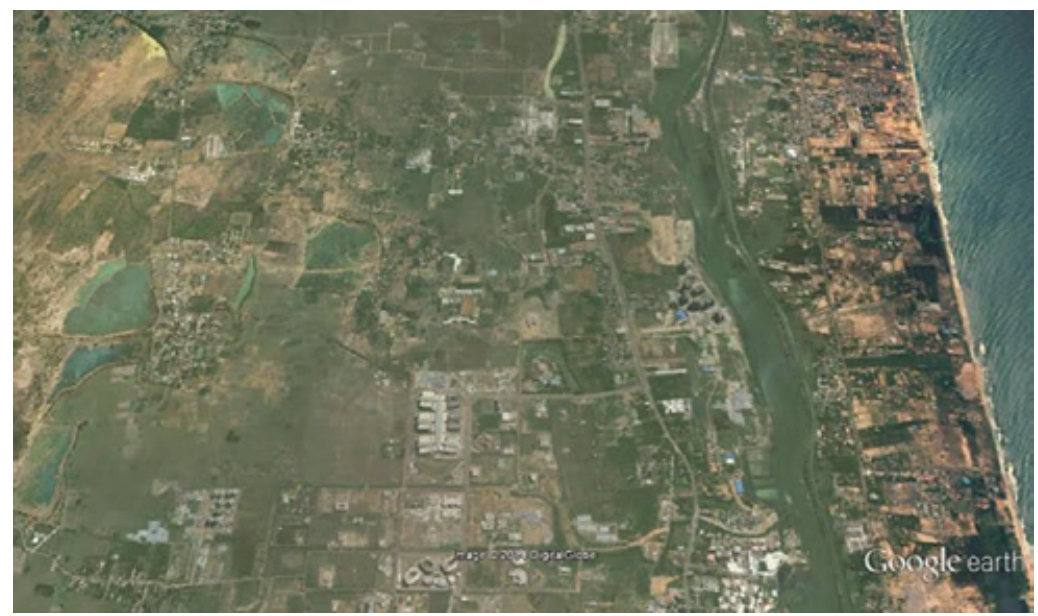

படம்: 1.3 2010-ல் பஞ்சக்கிராமி (கூகுள் பூமி வரைபடம்)

மற்ற நீண்டகால குடியிருப்பாளர்களிடம் இருந்து பெற்ற தகவல்களை வைத்து, இங்குள்ள ஒரு உள்ளூர் கலைஞர், அப்போதைய பஞ்சக்கிராமி எப்படியிருந்திருக்கும் என்று சித்தரிக்க உதவியிருக்கிறார். (படம்: 1.5)

இதற்கு மாறாக, அடுத்த படம், இப்போதுள்ள பஞ்சகிராமியை சித்தரிக்கிறது (படம்: 1.6) 


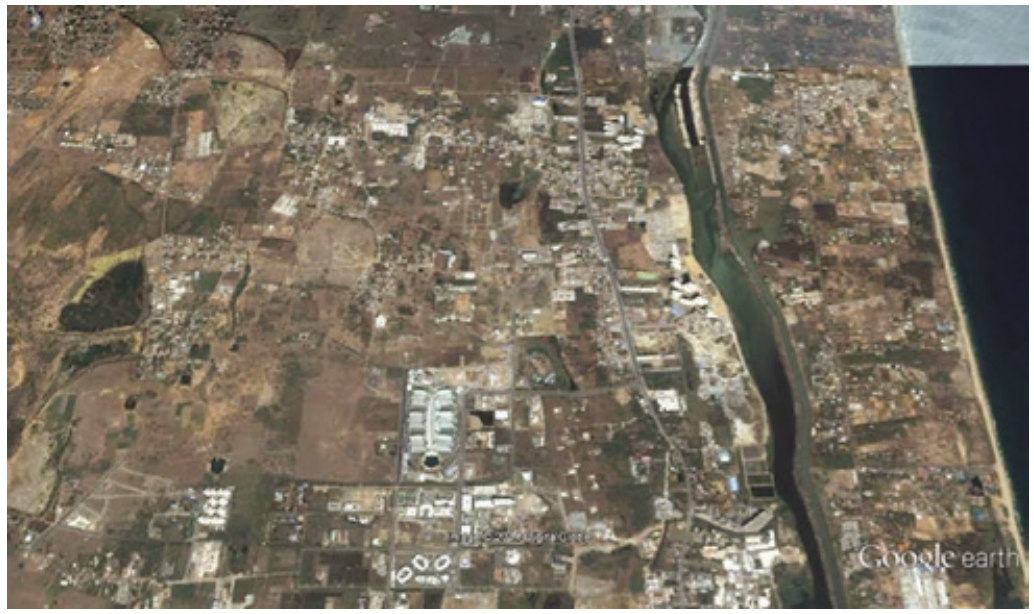

படம்: 1.4 2014-ல் பஞ்சக்கிராமி (கூகுள் பூமி வரைபடம்)

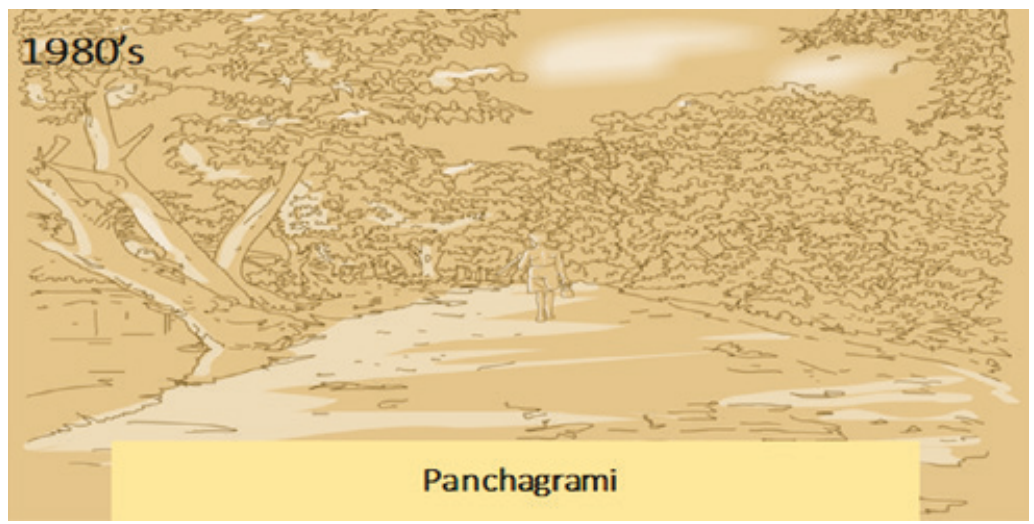

படம்: 1.5 1980-களில் பஞ்சக்கிராமி

இந்த அத்தியாயத்தின் கடைசி சில பகுதிகள், பஞ்சக்கிராமியின் வரலாற்றைப் பற்றியும், கடந்த பத்தாண்டுகளில் இந்தப் பகுதி அனுபவித்திருக்கும் அகக்கட்டமைப்பு மாற்றங்களையும் பற்றியும் மேலோட்டமான கருத்தை அளித்திருக்கிறது. அடுத்துள்ள சில பகுதிகள், இந்தப்பகுதியில் உள்ள மக்களைப்பற்றியும் சமூக கட்டமைப்பு பற்றியும் மேலோட்டமான கருத்துக்களை அளிக்கிறது. அதன்பின் பஞ்சக்கிராமி கட்டப்பட்ட சுற்றுச்சூழலைப் பற்றிய ஒரு சிறிய கலந்தாய்வுக்கு செல்லலாம். 


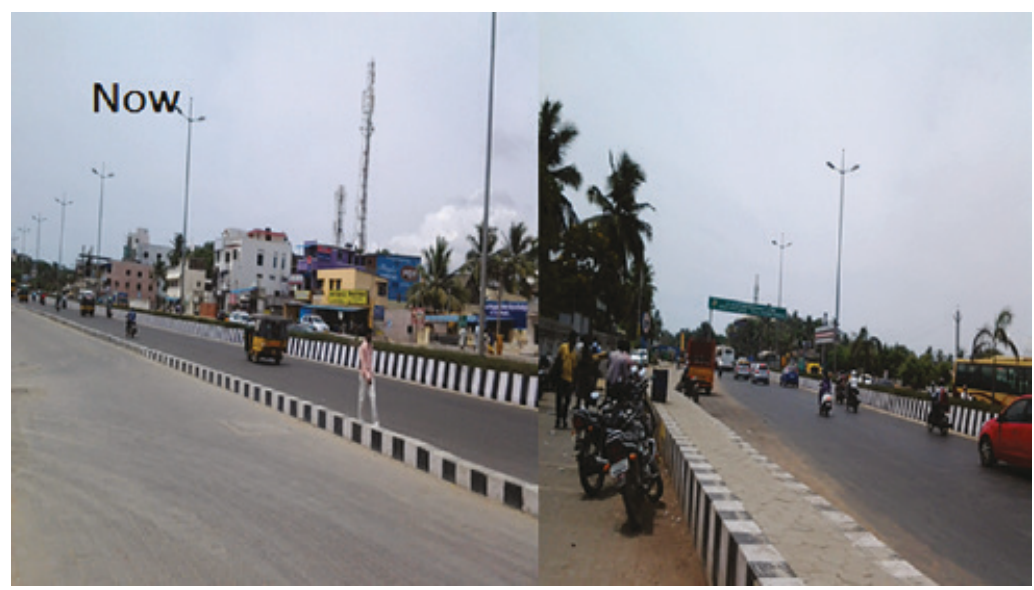

படம்: 1.6 2014-ல் பஞ்சக்கிராமி

\section{பஞ்சக்கிராமியின் மக்கள்:}

பஞ்சக்கிராமி, பெரும்பாலும் இந்துக்களைக் கொண்டிருந்தாலும், இங்கு கிறிஸ்தவர்களின் கணிசமான மக்கட்தொகையும் இருக்கிறது. இஸ்லாமியர்களும் சீக்கியர்களும் சிறிய அளவில் இங்கிருக்கிறார்கள். இவர்களை, பல்வேறு பொருளாதார, மொழிசார்ந்த மற்றும் குலம் சார்ந்த குழுக்களாக வகைப்படுத்த முடியும். பஞ்சக்கிராமியில் குறைந்தபட்சம், சிறிதில் இருந்து நடுத்தர அளவு வரையிலான 10 இந்துக்கோவில்கள் இருக்கின்றன. வருடாந்திர கோவில் திருவிழாக்கள் ஜூலை மற்றும் ஆகஸ்ட் மாதங்களில் (தமிழில் ஆடி மாதம்) நடைபெறுகின்றன. இவை பெரும்பாலும் இந்துக்களின் அன்னையாக கருதப்படும் அம்மன் கடவுளின் வழிபாடாக இருக்கின்றன.

இந்தப்பகுதி, ஒரு சில சிறிய பள்ளிவாசல்களையும் குறைந்தபட்சம் 5 சர்ச்சுகளையும் கொண்டிருக்கிறது. ஞாயிற்றுக்கிழமைகளில், நாள் முழுவதும் பெண்களும் குழந்தைகளும், குழுக்களாக இநந் சர்ச்சுகளுக்கு பிரார்த்தனைக்கு செல்வதை பார்க்க முடியும். உயர்மட்ட குடியிருப்பு வளாகங்களில் குடியிருக்கும் அநேக கிறிஸ்தவர்கள் உள்ளூர் சர்ச்சுகளுக்கு செல்வதில்லை. மாறாக, சென்னை நகரத்தில் அவர்கள் சார்ந்த பிரிவிற்கான சர்ச்சுகளுக்கு செல்கிறார்கள். பஞ்சக்கிராமியின் மற்றொரு பகுதி பள்ளிவாசல்களில் இருந்து வரும் 'அசன்' எனப்படும் அதிகாலை தொழுகை அழைப்பை கேட்டு தங்கள் நாட்பொழுதை துவங்குகின்றனர். இந்துக்கள் மற்றும் 
கிறிஸ்தவர்களின் அதிகமான மக்கட்தொகையால் பொங்கல், தீபாவளி, கிறிஸ்துமஸ் போன்ற சமய விழாக்கள் இந்தப்பகுதியில் மிகவும் சிறப்பாக கொண்டாடப்படுகின்றன.

இந்தப்பகுதியின் சிக்கலான தன்மை, இ இங்குள்ள உள்ளூர்வாசிகள் மற்றும் புலம்பெயர்ந்தவர்கள் ஆகியவர்களின் சமூக-பொருளாதார பின்னணிகளின் கலவையை நினைத்துப் பார்க்கும் போது தான் உறைக்கிறது. இவ்வளவு மாறுபட்ட தன்மையுடைய மக்கட்தொகை இருந்தாலும், இங்கு கவனிக்கவேண்டிய விஷயம் என்னவென்றால், இங்குள்ள அநேகமான உள்ளூர்வாசிகள் தமிழர்கள் ${ }^{37}$ என்பதால் இங்கு அதிகம் பேசப்படும் மொழியும் தமிழ் தான். இருப்பினும், புலப்பெயர்வின் விளைவாக, தெலுங்கு, மலையாளம், ஹிந்தி போன்ற பிற இந்திய மொழிகளை பேசுபவர்களின் எண்ணிக்கையும் அதிகரித்து வருகிறது. இவர்கள் பல்வேறு பொருளாதார வகைகளை சேர்ந்தவர்களாக இருக்கிறார்கள். பள்ளிகளிலும் ஐ.டி நிறுவனங்களிலும் முன்னுரிமைப்படுத்தப்படும் மொழி ஆங்கிலமாக இருந்தாலும், உள்ளூர்வாசிகளுடனான அன்றாட பரிமாற்றங்கள், தமிழ் மற்றும் ஆங்கிலக் கலவையில் நிகழ்கின்றன. மக்கள் ஓணம் தம் தம் மற்றும் தெலுங்கு வருடப்பிறப்பு 39 'கர்வா சவுத்"40 போன்ற பிராந்திய விழாக்களை கொண்டாடுவதற்காக தங்களுக்குள்ளேயே குழுக்களை அமைத்துக்கொள்கின்றனர்.

உள்ளூர் வாய்வழி வழக்குகளின் படி, தெலுங்கு பேசும் மக்கள், இங்கு சுமார் அரை நூற்றாண்டாக இருந்து வருகிறார்கள். இங்குள்ள உள்ளூர்வாசிகள், தமிழ் மற்றும் தெலுங்கு பேசும் மக்கட்தொகையினரிடையே பேதம் பாராட்டுவதில்லை. இதற்கு மாறாக, ஹிந்தி பேசுபவர்கள் மற்றும் இந்தியாவின் பிற மாநிலங்களில் இருந்து வந்தவர்களை இங்குள்ளவர்கள், கலாச்சார மற்றும் பாரம்பரிய வகையில் மாறுபட்டவர்களாக கருதுகிறார்கள்.

பஞ்சக்கிராமி, பெரும்பணக்காரர் முதல் பரமஏழை வரை பலதரப்பட்ட பொருளாதார வர்க்கங்களை உள்ளடக்கியதாக இருக்கிறது. இங்குள்ள உள்ளூர் வாசிகள் அநேகமாக, மத்திய வகுப்பை சேர்ந்தவர்களாகவோ அல்லது, கடந்த பத்தாண்டுகளில் தங்கள் நிலங்களை கட்டுமானப்பணிக்காகவோ அல்லது குடியிருப்பு வளாகங்களுக்கோ விற்றதில் பணக்காரர்களாக ஆனவர்களாகவோ இருக்கிறார்கள். சுலபமான புரிதலுக்காக சொல்லவேண்டுமென்றால், உள்ளூர் லட்சாதிபதிகள், ஐ.டி நிறுவனங்களின் முதுநிலை நிர்வாக ஆட்சியர்கள், இந்தப்பகுதியிலுள்ள சொத்துக்களில் 
முதலீடு செய்திருக்கும் வணிகர்கள் ஆகியோர் பணக்காரர்கள் மற்றும் உயர்மட்ட வர்கத்தை சார்ந்தவர்கள் என்ற வகைப்பிரிவில் இருக்கிறார்கள். மத்தியதர வர்கத்தை சேர்ந்தவர்களை, உயர்-மத்திய வர்க்கம், (அதாவது ஐ.டி நிறுவனங்களின் நடுநிலை மற்றும் முதுநிலை நிர்வாகிகள், தொழில்முனைவோர்கள்), மற்றும் ீீழ்-மத்திய வர்க்கம்,(அதாவது, விவசாயிகள், ஆரம்பநிலை ஐ.டி நிறுவனப் பணியாளர்கள், சிறுதொழில் வைத்திருப்பவர்கள்) என்று வகைப்படுத்தலாம். கீழ்த்தட்டு வர்க்கத்தினர், பெரும்பாலும் விவசாயத் தொழிலாளர்கள், சாலையோர விற்பனையாளர்கள் போன்றவர்களை உள்ளடக்கியதாக இருக்கிறது. பரம ஏழைகள் என்ற பிரிவு, திறன்சாரா எடிபிடி வேலை செய்பவர்கள், தெருவோர சோதிடர்கள், பஞ்சக்கிராமியின் வளர்ச்சியினால் சூடுபிடித்திருக்கும் கட்டிட வேலைகளுக்காக புலம்பெயர்ந்திருக்கும் கட்டுமான தொழிலாளர்கள் ஆகியவர்களை கொண்டதாக இருக்கிறது. இவர்கள், மிகச்சிறிய தற்காலிக வீடுகளில் குழுக்களாக தங்கியிருக்கிறார்கள். வெறும் 200 சதுர அடி கொண்ட ஒரு சிறு வீட்டில் குறைந்தது நான்கு பேராவது சேர்ந்து இருப்பது என்பது இங்கு மிகவும் சாதாரணமான விஷயம்.

இந்திய சமுதாயம், ஒரு தனிநபருக்கு பிறப்பிலிருந்தே ஒரு குறிப்பிட்ட அந்தஸ்தை அளிக்கும் குலங்கள் எனப்படும், சமூக அமைப்பை சுற்றி அமைக்கப்பட்டிருக்கிறது. அவனோ அவளோ, தங்கள் பிறவி முழுமைக்கும் சமூக அளவில் ஒரு தனிப்பட்ட அகமண உறவுடைய குழுவை சார்ந்தவர்கள் ஆகிவிடிகிறார்கள். இது 2000 வருடங்களாக மக்களை அவர்களின் தொழில் அடிப்படையில் வகைப்படுத்தும் பண்டைய இந்துசமய வர்ண அமைப்பின் வழித்தோன்றலாகும். கடந்த நூற்றாண்டுகளில் இந்த தொழில் அடிப்படையிலான, வகைப்படுத்தல் என்பது கொஞ்சம் கொஞ்சமாக வழக்கொழிந்து, ஒருவர் தன் வாழ்வில் பின்னாளில் எந்த தொழில் செய்கிறார் என்பது பற்றிய எண்ணமே இல்லாமல் ஒருவரின் பிறப்பிலேயே அவர் மீது இடப்படும் அடையாளம் ஆகிவிட்டது. இந்தியாவில் குல அமைப்பு என்பது அதிகார நிலைப்படி மற்றும் சமூக விதிமுறை என்ற வகையில் அமைக்கப்பட்டிருக்கிறது. ஆகவே, குலங்களுக்கு இடையேயான கலப்பு என்பது சாத்தியமில்லாததாக கருதப்படுகிறது. வர்ண அமைப்பு மக்களை நன்கு வகையாக மட்டுமே வகைப்படுத்தியது. ஆனால் இன்றுள்ள குல வகைகள், உட்பிரிவுகளையும் சேர்த்து எண்ணற்றவையாக ஆகிவிட்டன. 
குல அமைப்பின் கெடுதிகள், உயர்குலத்தவர் என்றழைக்கப்படுபவர்கள் காலம்காலமாக, தாழ்ந்த குலத்தவர் என்றழைக்கப்படுபெர்களின் மீீது காட்டிய பாகுபாட்டினால் நிலைநிறுத்தப்பட்டது. பி.ஆர். அம்பேத்கர் ${ }^{41}$ மற்றும் பெரியார் 12 போன்ற சமுதாய சிந்தனையாளர்கள் குல அமைப்பை வழக்கொழிக்க வேண்டும் என்று வலியுறுத்தியும், இந்த அமைப்பு இன்றளவும் இருந்து வருகிறது. மக்கள் மதம் மாறினாலும், தங்கள் குல அடையாளத்தை இருத்திக் கொள்கிறார்கள். குலங்கள் அவற்றின் உள்ளூர்ப் பெயரால் அடையாளம் காணப்பட்டாலும், (பஞ்சக்கிராமியில் செட்டியார்கள், பிராம்மணர்கள், முதலியார், வன்னியர், தலித் ${ }^{43}$ இருளர் குடியினர் போன்ற குலத்தை சேர்ந்தவர்கள் இருக்கிறார்கள்) நிர்வாக காரணங்களுக்காக இந்திய அரசாங்கம் 44 இவற்றை ஐந்து முக்கிய வகைகளாக பிரித்திருக்கிறது. பிற குலம், பின்தங்கிய குலம், மிகவும் பின்தங்கிய குலம், ஆதி திராவிட வகுப்பினர், ஆதி திராவிட பழங்குடியினர். அரசின் இந்த வகைப்படுத்துதல், உள்ளூர் சொல்லியல்களுக்கு ஈடான வகையில் முக்கியத்துவம் வாய்ந்ததாக, அதிலும் குறிப்பாக அரசியல் மற்றும் கொள்கை அதிகார எல்லைகளில் அதீத முக்கியத்துவம் வாய்ந்ததாக கருதப்படுகிறது.

பஞ்சக்கிராமியில், குலப்பெயர்கள், இந்தப்பகுதியில் நீண்டகாலமாக வாழ்ந்துவரும் உள்ளூர் வாசிகளால் மட்டுமே குறிப்பிடப்படுகிறது. பலரும், இந்த குல அமைப்பின் அதிகார நிலைப்படி அமைப்பை புரிந்துகொண்டிருக்கிறார்கள். சிலபல சமயங்களில், தங்களையும் மற்றவர்களையும், இந்தப் புரிதலின் அடிப்படையில் குறிப்பிடுகிறார்கள். குலம் பற்றிய பேச்சுக்கள் பொதுவான உரையாடல்களில் வெளிப்படாவிட்டாலும், இங்குள்ளவர்கள் தேவைப்படும் போது குலத்தைப் பற்றி குறிப்பிட சிறிதும் தயங்கமாட்டார்கள். ஒருசில உள்ளூர்வாசிகள் தங்கள் குலத்தை அரசாங்க வகைகளில் குறிப்பிட்டாலும், பெரும்பான்மையினர் பாரம்பரிய குலப்பெயர்களின் மூலமே தங்களை அடையாளப்படுத்திக் கொள்கின்றனர்.

பஞ்சக்கிராமியில் 150 வருடங்களுக்கு முன்பு குடியேறிய குடும்பங்களின் தலைவர்கள், "தலைக்கட்டு" என்று குறிப்பிடப்படுகின்றனர். வெகு விரைவிலேயே, இந்த ஆரம்பகாலக் குழு விரிவடைந்து, வணிகம் விவசாயம் மற்றும் பிற தொழில்களுக்காக மற்ற குலக்குழுக்கள் இவற்றுடன் இணைந்துகொண்டன. இந்தக் குழுக்கள் பிரிக்கப்பட்டு, அவர்களின் நிலங்கள் மற்றும் வாழும் பகுதிகள், ஆகியவை மறுசீரமைக்கப்பட்டன. கீழ்குலத்தை சேர்ந்தவர்கள், உயர்குலத்தை சேர்ந்த 
கிராமங்களின் அருகில் குடியேறுவதை தவிர்க்கும் வகையில் எல்லைகள் வரையப்பட்டன. தூய்மை மற்றும் தூய்மைக்கேடு ஆகிய கருத்துக்கள் உருவாகின. இதனால் உறைவிடங்கள் ஊர் என்றும்(உயர்குலத்தவர் என்றழைக்கப்படுபவர் வாழும் இடம்) காலனி என்றும்(கீழ்குலத்தவர் என்றழைக்கப்படுபவர் வாழும் இடம்) எல்லை (குறிக்கப்பட்டன. இதுபோன்ற குலம் அடிப்படையிலான எல்லைக்குறியீடுகள், தமிழ்நாடு| ${ }^{45}$ மற்றும் தென்னிந்தியாவில் நடத்தப்பட்ட மானுடவியல் ஆய்வுகளில் ஆவணப்படுத்தப்பட்டுள்ளபடி, சாதாரண நடைமுறை தான். இது இந்தப்பகுதிக்கு மட்டும் உரியதல்ல.

பண்டைய முறையில் பொருளாதார அமைப்பு எப்படி இருந்ததென்றால், உயர்குலத்தவர் என்றழைக்கப்படுபவர் நிலச்சுவான்தாரர்களாகவும் மற்றவர்கள் இவர்களின் நிலங்களில் தொழிலாளிகளாக பணிபுரிபவர்களாகவும் இருப்பார்கள். வேலைக்கான கூலி, பணமாகவும் பொருளாகவும் வழங்கப்படும். (பெரும்பாலும், விளைச்சலில் ஒரு பகுதியை பங்கிட்டு விநியோகம் செய்யும் சிக்கலான கூலி முறை பின்பற்ற பட்டது). 1970 மற்றும் 1980-களில், இந்தப்பகுதியில் பள்ளிகள், உள்ளூர் கிராம பஞ்சாயத்துக்களாலும் ${ }^{46}$, ஒரு சில கிறிஸ்தவ பிரசாரகர்களாலும் நடத்தப்பட்டன. 1990கள் வரை இந்தப்பகுதியில் பல்கலைக்கழக அளவிலான வசதிகள் எதுவும் இருக்கவில்லை. ஆகவே, உயர் கல்விக்காகவும், பொருளாதார இயங்குதிறனுக்காகவும், உயர்குலத்தவர் என்றழைக்கப்படும் பலர், சென்னை நகர்புறத்திற்கு குடிபெயர்ந்தனர். இவர்களில் சிலர், தங்கள் நிலங்களை தங்களிடம் பணிபுரிந்தவர்களிடமே (ஆதி திராவிட வகுப்பினர் மற்றும் தலித்துகள்) விற்றுவிட்டு சென்றனர். அவர்கள், தாங்கள் வாங்கிய நிலங்களை 2000ங்களில் ஐ.டி நிறுவனங்களுக்கு மறுவிற்பனை செய்து விட்டனர்.

ஆதி திராவிட வகுப்பினர் மற்றும் தலித்துகள், முன்பெல்லாம், இந்தப்பகுதியில் உள்ள உயர்குலத்தவர் என்றழைக்கப்படுபவர்களால் பாகுபாடாக நடத்தப்பட்டனர். ஆனால் காலப்போக்கில் அவர்களில் பலர், சமூக மற்றும் பொருளாதார இயங்குதிறனை சாதித்தனர். வேறு சில மற்றவர்களால் அது முடியவில்லை. அவர்களுடைய பொருளாதார செழிப்பினால், ஒரு சில தலித்துகள் உயர்-மத்திய வர்க்கத்தினராகவோ அல்லது பணக்காரர்களாகவோ கூட கருதப்படலாம். இருப்பினும், இங்குள்ள பெரும்பான்மையான தலித்துகள் கீழ்-மத்தியதர வர்க்கம் மற்றும் கீழ்த்தர வர்க்கம் ஆகிய வகைகளில் தான் பொருந்துகின்றனர். 
இங்குள்ள நீண்டகால குடியிருப்பாளர்களில், மிகவும் ஏழ்மை நிலையில் இ இருப்பவர்கள், இருளர்கள் ${ }^{47}$ என்றழைக்கப்படும் ஆதி திராவிட பழங்குடியினர் தான். அவர்களை உள்ளூரில் பாம்புப் பிடாரர்கள் அல்லது பாம்பு பிடிப்பவர்கள் என்று குறிப்பிடிகிறார்கள். அது தான் அவர்களின் பாரம்பரிய தொழில். அவர்கள் இந்தப் பகுதியில் உள்ள பழங்குடியினர் குடியிருப்புகளில் வாழ்கிறார்கள். (படம்: 1.7). இந்தக் குடியிருப்பில் உள்ள இருளர்கள், பெண்களை விட ஆண்களை படிக்க வைப்பதில் தான் அதிக அக்கறை செலுத்துகிறார்கள். ஆனால் பல ஆண்கள் படிப்பறிவை பெறுவதில் தோற்றுவிடுகிறார்கள். அவர்கள், பள்ளியில் இருந்து வெளியேறி, குடும்ப வருமானத்தை உயர்த்துவதற்காக ஏதாவதொரு வேலை செய்ய முற்படுகிறார்கள். அவர்களுடைய சமூகத்தில் இருக்கும் பொதுவான ஏழ்மை நிலையை பார்க்கும் போது, இதில் ஆச்சிரியம் ஒன்றும் இல்லை. படிப்பறிவில்லாத பல இளம் இருளர் பெண்கள், இந்தப்பகுதியில் உள்ள வீடுகளுக்கு வீட்டு வேலைக்காக அனுப்பப்படுகிறார்கள். இவர்கள் தான் பஞ்சக்கிராமியில் மிகவும் மலிவான சம்பளத்தில் கிடைக்கும் வீட்டு வேலைக்காரர்கள். இளம் இருளர் ஆண்கள் குறைந்த நிலையில் உள்ள வேலைகளுக்கு போனாலும், இவர்களில் சிலர் தங்கள் பாரம்பரிய தொழிலான பாம்பு பிடிப்பதையும்

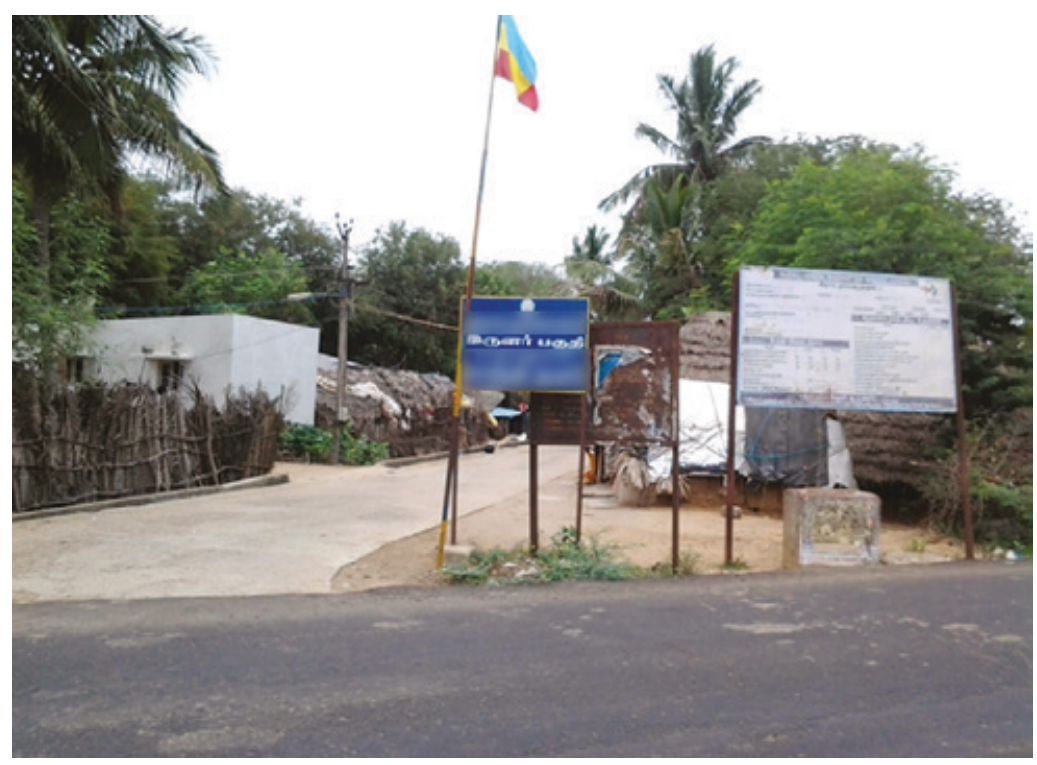

படம்: 1.7 2014-ல் பஞ்சக்கிராமியில் இருளர்கள் குடியிருப்பு 
செய்து கொண்டு தான் இ இருக்கிறார்கள். இ இங்குள்ள மற்ற உள்ளூர்வாசிகள், இருளர்களை, அவர்களுடைய கருத்த நிறத்தையும், ஏழ்மை தோற்றத்தையும் குறிப்பிட்டு, அவர்களின் தோற்றத்தை வைத்து அடையாளம் காண்கிறார்கள். மற்ற குலத்தவர்களுடன் ஒப்பிட்டுப் பார்க்கும்போது, மிகவும் குறைந்த அளவிலான இருளர்கள் மட்டுமே ஐ.டி துறையின் மிகவும் அடிமட்ட வேலைகளுக்கு போகிறார்கள். இருப்பினும் இந்த நிலைமை, ஒருசில இளம் இருளர்கள் இந்தப் பாரம்பரிய கட்டுக்களில் இருந்து விடுபட விழைந்து செயல்படுவதால், கொஞ்சம் கொஞ்சமாக மாறிவருகிறது.

இந்தப்பகுதியில் உள்ள மற்ற குலக்குழுக்களை அரசாங்கத்தின் பரந்த வகைகளான மிகவும் பின்தங்கிய வர்க்கம், பின்தங்கிய வர்க்கம் மற்றும் இதர வர்க்கங்கள் ஆகியவற்றில் வகைப்படுத்திவிட முடியும். இவற்றில் அநேகமானோர், மத்தியதர வகுப்பினர் என்ற பரந்த வகையில் தான் வருவார்கள். இதில் சிலர் முன்னாள் நிலச்சுவான்தாரர்களாக இருந்து நிறுவனங்களுக்கும், கட்டுமானக்காரர்களுக்கும் நிலங்களை விற்றதன் மூலம் பணக்காரர்கள் ஆனவர்கள். அவர்களுக்கு இந்தப்பகுதியில் செல்வாக்கு அதிகம். பலர் இந்தப்பகுதியில் கட்டிடங்களை கட்டி வாடகைக்கு விட்டிருக்கின்றனர். இவர்களில் பலர் நன்கு படித்திருக்கிறார்கள். மேலும் அநேக இளைஞர்கள் குறைந்த பட்சம் ஒரு பட்டமேனும் பெற்றிருக்கின்றனர். ஒருசிலர் சுயதொழிலில் ஈடுபட்டிருக்கிறார்கள். இன்னும் பலர், ஐ.டி துறையில் பணியில் இ இருக்கின்றனர். இநந்தப்பகுதியில் ஆண் பெண் பாலின விகிதம் 1.1க்கு 1.0. பஞ்சக்கிராமியின் அதிகாரபூர்வ எழுத்தறிவு சதவிகிதம், 76ஆக இருந்தாலும், இது இன்னும் சில காலத்தில் புதிதாக குடியேறியுள்ள திறன்வாய்ந்த ஐ.டி பணியாளர்களால் சில சதவிகிதம் உயர்ந்தாலும், இன்றும் கீழ்க்குலத்தை சேர்ந்த பல ஆண்களும் பெண்களும் அடிப்படை கல்வியறிவு கூட இல்லாமல் இருக்கிறார்கள். இந்தப் பகுதியில் உள்ள எழுத்தறிவு சதவிகிதம், இந்தியாவின் எழுத்தறிவு சராசரியை விட அதிகம். இது தமிழ்நாட்டின் சராசரி எழுத்தறிவு சதவவிகிதத்திற்கு ${ }^{48}$ இணையானதாக கூட இருக்கலாம்.

இந்த இடத்தின் பொருளாதார வளர்ச்சியினால், பஞ்சக்கிராமி, மாறுபாடான சமூக-பொருளாதார பின்னணியில் இருந்து வரும் பல்வேறு புலப்பெயர்வாளர்களை கவர்ந்திழுத்திருக்கிறது. ஏழ்மையான புலப்பெயர்வாளர்கள், கட்டுமானப் பணியாளர்களாகவோ அல்லது பல்வேறு கீழ்நிலை சேவகப் பணியாளர்களாகவோ (உதாரணத்திற்கு, ஹோட்டல்- 
களிலும் ஐ.டி நிறுவனங்களிலும் துப்புரவுப் பணியில் ஈடுபடுபெவர்கள்). கட்டுமானப் பணியாளர்கள் (பெரும்பாலும் பரம ஏழைகள்) பொதுவாக மிகவும் குறைவான கல்வியறிவு பெற்றவர்களாகவும் பீகார், ஆந்திரப்பிரதேசம், மேற்கு வங்காளம் மற்றும் ஒதிஷா போன்ற இடங்களில் இருந்து வந்தவர்களாகவும் இருப்பார்கள். இதுவே, ஹோட்டல்களிலும் ஐ.டி நிறுவனங்களிலும் துப்புரவுப் பணியில் ஈடுபடுபவர்கள், மணிப்பூர், மிசோரம், நேபாள் போன்ற இடங்களில் இருந்து வந்தவர்களாகவும், பொதுவாக, ஓரளவு கல்வியறிவு பெற்றவர்களாகவும் இருப்பார்கள்.

கட்டுமானப் பணியாளர்கள் பொதுவாக, குழுக்களாக வருவார்கள். நாடோடிகளைப் போன்ற வாழ்க்கைமுறையில் அவர்கள் ஒரு கட்டுமானக் களத்தில் இருந்து மற்றதிற்கு ${ }^{49}$ மாறிப் போய்க்கொண்டே இருப்பார்கள். அவர்கள் ஒவ்வொரு காலத்திலும் கூலியாளர்களின் தேவை இருப்பதற்கேற்ப இருந்துவிட்டு, பின்னர் ஆறு மாதத்தில் இருந்து ஒரு வருடத்திற்குள், மறு இடத்திற்கு மாறிப்போய்விடுவார்கள். இவற்றின் புலப்பெயர்வு வாழ்க்கை முறையினால் இது போன்ற குழுக்கள் பொதுவாக, அரசாங்கத்தின் மக்கட்தொகை கணக்கெடுப்பில் வராதவையாக இருக்கும். மக்கள் சாலையோரங்களில் தற்காலிக வீடுகள் அல்லது கொட்டாரங்கள் அமைத்துக்கொண்டோ, அல்லது உள்ளூர்வாசிகளிடம் தற்காலிக வாடகையிடம் பேசி அமைத்துக்கொண்டோ வாழ்வார்கள். பஞ்சக்கிராமியில் உள்ள குப்பை கோலம் திரட்டுபவர்களும், சோதிடர்களும் கூட இவ்வாறு வாழ்பவர்கள் தான். இவர்கள் தமிழ்நாட்டின் தென் பகுதியில் இருந்து வந்து நாடோடிகள் போல வாழ்பவர்கள்.

இந்தப்பகுதியில் உள்ள ஐ.டி நிறுவனங்களில் பணிபுரிய வரும் ஆரம்ப நிலை ஐ.டி பணியாளர்கள் அதிகபட்சம் இரண்டிலிருந்து மூன்றாண்டுகள் வரை இங்கு இருக்கிறார்கள். அவர்கள் அநேகமாக ஒற்றை ஆட்களாக வந்திருந்து, தங்கும் விடுதிகள் அல்லது தங்களைப் போன்ற மற்றவர்களுடன் பகிர்ந்து கொள்ளும் வாடகை இடங்களில் தங்கிக்கொள்கிறார்கள். பலசமயங்களில் இவர்கள் தாங்களாகவே இடம் மாறிச்சென்றுவிடுகிறார்கள். ஆன்-சைட் வேலைகளுக்காக (வெளிநாடுகளில் முனைப்பான தகவல் தொழில்நுட்ப செயல்திட்டங்களில் பணிபுரிவது) ஒன்றிரண்டு வருடங்களிலேயே இடம் பெயர்வது தான் இவர்களிடையே அதிகம். இது போன்ற நிலையற்ற பணியாளர்கள் தான் பலசமயங்களில் உள்ளூர்வாசிகளின் பணவீக்கம் பற்றிய குற்றச்சாட்டுகளு- 
க்கு ஆளாகிறார்கள். இருப்பினும், நடுநிலையில் இருக்கும் பணிநிலைகளில் உள்ளவர்கள், திருமணமாகி, குழந்தைகள் இருப்பவர்கள் போன்ற பணியாளர்கள், நீண்ட காலம் இங்கு நிலைத்திருக்க முற்படுகிறார்கள். அவர்களில் சிலர் அடுக்குமாடி வீடுகள், வீட்டுமனைகள் போன்றவற்றில் முதலீடு கூட செய்கிறார்கள்.

பஞ்சக்கிராமியை சுற்றி, இரண்டு பல்கலைக்கழகங்களும், 10 கல்லூரிகளும் இ இருப்பதால், இங்கு புலப்பெயர்வு மாணவர்களின் மக்கட்தொகையும் காணப்படுகிறது. அவர்கள் இங்கு பொறியியல் துறை படங்களுக்காக நான்கு ஆண்டுகளும், பிற படங்களுக்காக இரண்டு அல்லது மூன்றாண்டுகளும் இங்கு தங்குகிறார்கள். பொறியியல் துறை மாணவர்கள், இந்தியாவின் பல பகுதிகளில் இருந்தும் இங்கு கல்வி பயில வருகிறார்கள். குறிப்பாக, ஆந்திரப்பிரதேசம், அசாம், மிசோரம், மணிப்பூர், ஒதிஷா, மேற்கு வங்காளம், மஹாராஷ்டிரா, கர்நாடகா, கேரளா, பீகார் போன்ற இடங்களில் இருந்து நிறைய மாணவர்கள் வருகிறார்கள். இந்தப் பகுதியில் ஏகப்பட்ட சிறிய உணவகங்களும், சிற்றுண்டிச்சாலைகளும் (பணியிடங்கள் மற்றும் கல்விநிலையங்கள் ஆகியவற்றை சுற்றி) இருக்கின்றன. இவை, இது போன்ற மாணவக்குழுக்களின் உணவுத்தேவைகளை பூர்த்தி செய்கின்றன.

குறுகிய கால வணிகர்கள், பல்வேறு அளவிலும், பல்வேறு இடங்களிலும் இருந்து புலம் பெயர்ந்து வந்து, உணவுத்தொழிலில் கவனம் செலுத்த முனைகிறார்கள். இவர்கள் தேநீர் கடைகள், சிற்றுண்டிச்சாலைகள் போன்றவற்றை வைத்து நடத்துகிறார்கள். குறிப்பிட்ட புலப்பெயர்வு இனத்தின் உணவுத்தேவைகளை பூர்த்தி செய்யும் வகையிலான உணவகங்களும் ஏராளமாக உண்டு. உதாரணத்திற்கு, ராஜஸ்தானி தாபா, கேரளா மெஸ், ஆந்திரா மெஸ் போன்றவை. சிகரெட்டு, சூயிங்கம், சாக்லேட், மிட்டாய், வாசனை மிட்டாய்கள் போன்றவற்றை விற்கும் சாலையோர வியாபாரிகள், ஐ.டி நிறுவனங்களின் முன் தற்காலிக கடை போன்ற அமைப்பை வைக்கிறார்கள். இதுபோன்றவை, ஒரு சிகரெட்டு அல்லது சூயிங்கமிற்காக தொலைவாக நடந்து போக வேண்டியிருப்பதை தேவையற்றதாக ஆக்கிவிடிகின்றன என்பதால் இவை ஐ.டி பணியாளர்களுக்கு விருப்பமானதாக இருக்கிறது. இதுபோன்ற நடைமுறைகள், பல குறுகிய கால வியாபாரிகளை, ஐ.டி குழுக்களுக்காகவே, இந்தப்பகுதிக்கு புலம்பெயரச்செய்கிறது. டீக்கடைகள் என்றழைக்கப்படும் தேநீர் கடைகளும் இதுபோன்றவையே. பல புலப்பெயர்வாள- 
ர்கள், ஐ.டி நிறுவனங்களின் முன் தற்காலிக தேநீர் கடைகளை துவங்கி, ஐ.டி பணியாளர்கள் மற்றும் அங்குள்ள கட்டுமானப் பணியாளர்களை தங்கள் வாடிக்கையாளர்களாக ஆக்கிக்கொண்டுள்ளார்கள். ஒரு சாதாரண தேநீர் கடையில், ஆண்களும் பெண்களுமாய், பணியாளர்களின் குழுக்களை வெகு சாதாரணமாகப் பார்க்கலாம். இதுவே, தற்காலிக சிகரெட்டு கடைகளில் ஆண் பணியாளர்களின் குழுக்களை மட்டுமே பார்க்க முடியும்.

மத்தியதர மற்றும் உயர் மத்தியதர வகுப்பினருக்கான குடியிருப்பு அடுக்குமாடிக்கட்டிட வளாகங்களின் கட்டுமானத்துறையில் ஏற்பட்ட திடீர் வளர்ச்சியும் கூட, புலப்பெயர்வாளர்களுக்கு வழிவகுத்திருக்கிறது. சென்னையின் புறநகர்ப்பகுதிகளில் வாழ விரும்பும் வயது முதிர்ந்த பணிஓய்வு பெற்றவர்களும் இந்தப் பகுதியில் குடியிருப்பு வளாகங்களில் முதலீடு செய்கிறார்கள். இந்தப்பகுதியில் குடியேறும் அநேகம் பணிஓய்வு பெற்ற வயது முதிர்ந்தவர்கள் பிள்ளைகள், வெளிநாட்டிலோ அல்லது இங்குள்ள ஐ.டி துறை நிறுவனங்களிலோ பணிபுரிகிறார்கள். கூடுதலாக, நடுநிலையில் இருக்கும் பணிநிலைகளில் உள்ளவர்கள், திருமணமாகி, குழந்தைகள் இருப்பவர்கள் போன்ற பணியாளர்களும், இங்குள்ள அடுக்குமாடி வீடுகள் மற்றும் வீட்டுமைகள் போன்றவற்றில் முதலீடு செய்கிறார்கள். அதேபோல சென்னையில் உள்ள வளமான வணிகர்களும் தங்கள் குடிம்பங்களுடன் விஸ்தாரமான குடியிருப்புகளில் குடியேற, இந்தப் பகுதியில் முதலீடு செய்கிறார்கள். இங்குள்ள அடுக்குமாடி குடியிருப்பு வளாகங்களில் உள்ள ஐ.டி அல்லாதவர்களின் மக்கட்தொகை சுமார் முப்பது சதவிகிதம் இருக்கும்.

\section{இடப்பரப்பில் மாற்றம்.}

இந்தப்பகுதியின், கிராமப்புறம் என்பதில் இருந்து நகர்ப்புறம் என்பதான சமூகப்பொருளாதார மாற்றம், இங்குள்ள சமூக இடப்பரப்பிலும் கணிசமான மாற்றத்தைக் கொண்டுவந்திருக்கிறது. இந்த மாற்றம், தகவல் தொழில்நுட்பத்துறையால் ஊக்கமூட்டப்பட்டாலும், இது, கட்டுமானத்தொழில் மற்றும் இந்தப்பகுதியின் புலப்பெயர்வு மக்கள் ஆகியவற்றுடன் தொடர்புடையதாக இருக்கிறது. இந்த அத்தியாயத்தில் முன்னமே குறிப்பிட்டது போல, இந்தப்பகுதி, பணக்காரர்களில் இருந்து பரம ஏழைகள் வரையிலான மாபெரும் வர்க்க வகைகளைக் 
கொண்டுள்ளது. இருப்பினும், கடந்த பத்தாண்டுகளில் நிஜமாகவே மாறியிருப்பது என்னவென்றால், சில வருடங்களுக்கு முன்பு, வருமானங்களில் உள்ள வேறுபாடுகள், குலங்களின் அதிகார நிலைப்படியை ஒத்ததாக இருந்திருக்கும். ஆனால் இன்றைய நிலையில், பணக்காரராக இருப்பதற்கு ஒருவர் உயர்குலத்தவர் என்றழைக்கப்படுபவராகத்தான் இருக்கவேண்டும் என்ற அவசியமோ, அல்லது பரம ஏழையாக இருப்பவர், கீழ்குலத்தவர் என்றழைக்கப்படுபவராகவோ தான் இருக்கவேண்டும் என்ற அவசியமோ இல்லை.

எனினும், முன்பு குறிப்பிட்டது போல, சமீப காலம் வரை, குலம் தான், பண்டைய முறையில், அதாவது, கிராமம் (அ) ஊர்(பின்தங்கிய வகுப்பினர், மிகவும் பின்தங்கிய வகுப்பினர் மற்றும் இதர வகுப்பினர் குடியிருக்கும் இடம்) மற்றும் காலனி (ஆதி திராவிட வகுப்பினர் குடியிருக்கும் இடம்) மற்றும் பழங்குடியினர் குடியிருப்பு என்ற மூன்று கூறுகளாகப் பிரிக்கப்பட்ட இந்தக் கிராமங்களின் ${ }^{50}$ இடப்பரப்பு சீரமைப்பை முடிவு செய்தது.

குறிப்பாக, பஞ்சக்கிராமியின் கிராமங்களில் ஒன்றாக அமைந்திருக்கும் ஒரு கிராமத்தில், நெடுஞ்சாலையின் ஒருபுறம் கிராமமும் (பின்தங்கிய வகுப்பினர், மிகவும் பின்தங்கிய வகுப்பினர் மற்றும் இதர வகுப்பினர் குடியிருக்கும் இடம்) மறுபுறத்தில் காலனியும் (ஆதி திராவிட வகுப்பினர் குடியிருக்கும் இடம்) அவற்றின் சொந்த மயானத்துடன் அமைந்திருக்கின்றன. இது இந்தியக் கிராமங்களில் மக்கள் தூய்மை மற்றும் தூய்மைக்கேடு எல்லைகளை தாண்டாத பொதுவான ஒரு அமைப்பாகும். இதுபோன்ற நடைமுறைகள், சென்ற நூற்றாண்டு வரை நடைமுறையில் இருந்திருந்தாலும், தகவல் தொழில்நுட்பத்துறை மட்டும் கட்டுமானத்துறையின் வளர்ச்சியால், இவை இப்போது அநேகமாக மறைந்தே போய்விட்டது என்று கூறலாம். கடந்த ஐந்து முதல் எட்டாண்டுகளாக, காலனி என்று கூறப்பட்ட இடத்தில், பல்வேறு புதிய வர்த்தக மையங்களும், குடியிருப்பு வளாகங்களும் உருவாக்கப்பட்டு வருகின்றன. காலனியை ஒட்டித்தான் விவசாய நிலங்களும், மாந்தோப்புகளும் அமைந்திருந்தன. அந்த இடம் பெறப்பட்டு, பெரியளவிலான அடுக்குமாடி குடியிருப்பு வளாகங்களுக்காக மறுசீரமைக்கப்படுகின்றன. காலனியின் முன்னாள் குடியிருப்பாளர்கள், தங்கள் வீடுகளை, சிறிய நிலவுடைமைகளுடன் கட்டுமான நிறுவனங்களுக்கும், சமீபத்தில் அங்கு குடியேறியவர்களுக்கும் விற்றுவிட்டனர். 
நெடுஞ்சாலையின் இருமருங்கிலும், குறைந்த பட்சம், 17 குடியிருப்பு வளாகங்களும் (ஒவ்வொன்றும் 170 முதல் 800 வரையான குடியிருப்பு தளங்களை கொண்டவை) கட்டுமானத்தில் இருக்கின்றன. மேலும் 25 பிரம்மாண்டமான அடுக்குமாடி குடியிருப்பு வளாகங்கள் பஞ்சக்கிராமியில் ஏற்கனவே இருக்கின்றன. இவற்றை கட்டமைப்பவர்களில் இந்தியாவின் முன்னணி கட்டிடநிபுணர்களும் அடக்கம். இரண்டு படுக்கையறைகளை கொண்ட குடியிருப்புத்தளம், குறைந்தது இந்தியச் செலாவணி ${ }^{11} 35$ லட்சத்தில் இருந்து 90 லட்சம் வரை விலையிருக்கக்கூடும். முன்னணி கட்டுமான நிறுவனத்தின் மூலம் பெறக்கூடிய, சிறந்த ஓவியக்காட்சி போன்ற தோற்றம் கொண்ட ஐந்து படுக்கையறை குடியிருப்புத்தளம், சுமார் 50 லட்சத்திற்கு மேல் இருக்கக்கூடும். மாளிகை போன்ற தனிவீடுகள், சுலபமாக 65 லட்சம் முதல் 7 கோடி வரை விலையிருக்ககூடும்.

கிராமம் இருந்த பக்கம் வளர்ச்சி இருந்தாலும், கால்வாயின் உப்பங்கழியை ஒட்டி அமைந்திருந்த காலனி, அதிக பொருளாதார மதிப்பை உடையதாக இருந்தது. ஏனெனில், அது உயர்ந்த அடுக்குமாடி குடியிருப்பாளர்களுக்கு, ஓவியக்காட்சி போன்ற தோற்றத்திற்கான உத்திரவாதத்தை அளித்தது. காலனியின் முந்தைய குடியிருப்பாளர்கள், தங்களுக்கு புதிதாக கிடைத்த நிதிவளத்தை வைத்துக்கொண்டு, சாலைக்கு அந்தப்புறமாகவோ, அல்லது ரோட்டின் மற்ற பகுதிகளுக்கோ புலம் பெயர்வதற்கு பயன்படுத்திக்கொண்டனர். முன்பு சிதறியிருந்த குலங்கள் எல்லாம், ஒரே இடத்தில் குடியேறி விட்டன. முன்பு இருந்த இடப்பரப்பின் எல்லைக்குறியீடுகளும் மறைந்துவிட்டன. காலனி என்பதே வழக்கொழிந்து போய்விட்டது. முன்பு தூய்மைக்கேடானவர்களாக கருதப்பட்டு, கீழ்குலத்தவர் என்றழைக்கப்பட்டு ஒதுக்கி வைக்கப்பட்டவர்கள், இன்று உயர்குலத்தவர் என்றழைக்கப்படுபவர்களுடன் மிக அண்மையில் குடியிருக்கிறார்கள்.

பஞ்சக்கிராமியிலுள்ள ஒரு விஸ்தாரமான அடுக்குமாடி குடியிருப்பின் மத்தியில் உள்ள இடுகாடு, இந்தப் பண்டைய மற்றும் நவீன இடப்பரப்பின் இணைதலை, தெளிவாக்குகிறது. இந்தப் பல்மாடி குடியிருப்பு வளாகங்களில் உள்ள குடியிருப்புத் தளங்களின் விலைகள், 1.7 கோடியில் இருந்து 5 கோடி வரை துவங்குகின்றன. இது உப்பங்கழிக்கு அருகிலும், ஓவியக்காட்சி போன்ற தோற்றத்துடனும் சில ஆண்டுகளுக்கு முன்னர் தான் கட்டப்பட்டது. ஆனால், இது ஆதி திராவிட வகுப்பினரின் நிலப்பரப்பில் கட்டப்பட்டதால், 
இதில் அவர்களுக்கான ஒரு மயானமும் இருக்கிறது. இது இந்தக் குடியிருப்பின் பல்வேறு கட்டிடங்களின் ஒன்றின் முன் அமைந்திருக்கிறது. இந்தக் கட்டுமான நிறுவனத்தின் இதை அப்புறப்படுத்துவதற்கான அனைத்து முயற்சிகளும் வீணாகத்தான் போயின.

இந்தக் கட்டிடத்தில் இருக்கும் குடியிருப்புவாசிகள், இப்போது இந்த மயானப் பரப்பை வெறுக்கிறார்கள். அது இந்த நிலப்பரப்பின் காட்சிமையை கெடுப்பதாகவும், தங்கள் உணர்ச்சிக்கூறுகளை பாதிப்பதாகவும் அவர்கள் கருதுகிறார்கள். தன்னுடைய பிந்தைய அறுபதுகளில் இருக்கும் ஒரு குடியிருப்பாளர், தன் சொந்த சாக்காட்டை, இது நினைவுபடுத்துவதாக கூறுகிறார். இருப்பினும், இந்தப் பகுதி, இந்தக் காரணத்திற்காக இங்கு நீண்டகாலமாக வாழ்ந்துவரும் உள்ளூர்வாசிகளால் பயன்படுத்தப்பட்டு வருகிறது. பணத்தின் ஆதிக்கம் மற்றும் குடியிருப்பு தளங்களின் சொந்தக்காரர்கள் மற்றும் குடியிருப்பாளர்கள் (பொதுவாக உயர்குலம் என்றழைக்கப்படும் வகையைச் சேர்ந்தவர்கள்) கொடுக்கும் நெருக்கடி ஆகியவற்றை வைத்துப் பார்க்கும் போது, ஒரு தீர்வு வெகு அருகில் இருப்பது போல் தான் தெரிகிறது. மயானம், சாலையின் மறுபுறத்தில் மாற்றியமைக்கப்படக்கூடும். மொத்தத்தில் ஒரு சமயம் குடியிருப்புகளின் அமைப்பு பற்றி குலம் சார்ந்த போராட்டமாக இருந்த ஒன்று, இப்போது வர்க்கம் மற்றும் குலம் சார்ந்த சச்சரவாக மாறிவிட்டது.

இதுபோன்ற இடப்பரப்பின் இணைதலுக்கு விதிவிலக்காக இருப்பவை, இருளர்களின் குடியிருப்பு தான். அவர்கள் இன்னும் அவர்களுக்கென்று ஒதுக்கப்பட்ட குடியிருப்புகளில் தான் வாழ்கிறார்கள். அவர்களின் நிலையில் பெரிய மாற்றம் எதுவும் ஏற்பட்டுவிடவில்லை.

இந்தத்தருணத்தில், குலத்தின் அடிப்படையிலான பிரிவினைகளை தாண்டி, அந்தஸ்து வர்க்கம் பிரதானமாகி வருகிறது என்று அறிவிக்க ஆவலாகத் தான் இருக்கிறது. ஆனால், உள்ளூர் அரசியல் பிரிவுகளை பார்க்கும் போது, குலம் இன்னும் முக்கியமானதாகத்தான் கருதப்படுகிறதது என்பது புலனாகிறது.

\section{அரசியலும் ஆட்சிமுறையும்}

தமிழ்நாட்டில், இரண்டு மாபெரும் அரசியல் கட்சிகள், திமுக (திராவிடர் முன்னேற்றக் கழகம்) 52 மற்றும் அதிமுக (அண்ணா 
திராவிடர் முன்னேற்றக் கழகம்) 53 இந்தக் கட்சிகளின் ஆதிக்கம், மாநிலம் முழுமையிலும் விரவியிருக்கிறது, பஞ்சக்கிராமி உட்பட. இவ்விரண்டு கட்சிகளும் மாநில அளவில் ஒன்றுக்கொன்று ஜென்ம விரோதிகளாக இருக்கின்றன. அவை தங்கள் அதிகாரத்தைப் பற்றிய தற்பெருமையை மார்தட்டிக்கொள்வதோடு, குலம் மற்றும் பாலின வகைகளில் மிகவும் வேறுபட்டவையாக இருக்கின்றன. இந்தக் கட்சிகளை தவிர, மதிமுக (மறுமலர்ச்சி திராவிட முன்னேற்றக் கழகம்) 54 , தேமுதிக (தேசிய முற்போக்கு திராவிட கழகம்) ${ }^{55}$, விசிக (விடுதலை சிறுத்தைகள்

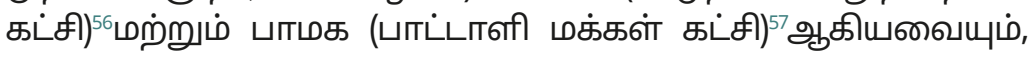
கணிசமான பின்தொடர்வாளர்களை கொண்டிருக்கின்றன. இதில் கவனிக்க வேண்டிய விஷயம் என்னவென்றால், விசிக (விடுதலை சிறுத்தைகள் கட்சி) மற்றும் பாமக (பாட்டாளி மக்கள் கட்சி) ஆகியவை, குலம் அடிப்படையில் அமைந்த கட்சிகள். விசிக, தலித் (ஆதி திராவிடர் வகுப்பு) சார்ந்த அரசியல் கட்சி, பாமக, வன்னியர் (மிகவும் பின்தங்கிய வகுப்பு) சார்ந்த அரசியல் கட்சி, இந்தக் கிராமங்களில் ஒருசிலவற்றில், கணிசமான தலித் மக்கட்தொகையும், மற்றவற்றில், கணிசமான மிகவும் பின்தங்கிய வகுப்பைச்சார்ந்த மக்கட்தொகையும் உள்ளவை. இந்தப் பகுதி மாறிக்கொண்டே இருக்கும் இந்நிலையில், இந்தப்பகுதியில் பன்முகத்தன்மை அதிகரித்திருக்கிறது என்பதை ஒத்துக்கொள்ளத்தான் வேண்டும். இருப்பினும் குலம் அடிப்படையிலான அரசியல்கட்சிகளுக்கான ஆதரவு இங்கு இருக்கத்தான் செய்கிறது. இங்குள்ள மக்கள் தாங்கள் சார்ந்திருக்கும் கட்சிகளுக்கு விசுவாசமாக இருந்தாலும், மாநில மற்றும் தேசிய அளவிலான அரசியல் போராட்டத்தில் நடக்கும் வன்முறைகள் இந்தப்பகுதியை பாதிப்பதாக தெரியவில்லை. அதேபோல, பஞ்சக்கிராமியில் பலதரப்பட்ட மதங்களை சேர்ந்த மக்கட்தொகையை கொண்டிருந்தாலும், மதம் சார்ந்த விழாக்களின் போது, மற்ற மதங்களில் இருந்து வரக்கூடிய எதிர்ப்பு இங்கு காணப்படுவெதில்லை. அதற்கான ஒரு காரணம், மதம் அடிப்படையிலான அரசியல் கட்சிகள் இங்கு இல்லாமல் இருப்பதால் கூட இருக்கலாம்.

பஞ்சக்கிராமியை உருவமைக்கும் ஒரு சில கிராமங்கள், தமிழ்நாடு அரசின், ஒப்புதல் செயல்திட்டக் கொள்கைகளின் ${ }^{58}$ கீழ் வருகிறது. இதன்படி, பஞ்சாயத்து தலைவருக்கான பதவி ஆதி திராவிட வகுப்பினர் அல்லது ஆதி திராவிட பழங்குடியினர் ஆகிய வகைகளை சேர்ந்தவர்களால் மட்டுமே நிரப்பப்பட முடியும். இதன் விளைவாக இங்குள்ள மக்கள் சிலசமயங்களில், அவர்கள் ஏதாவது அரசியல் கட்சியை 
வெளிப்படையாக ஆதரித்தாலும், தேர்தலில் சுயேச்சை வேட்பாளர்களாக போட்டியிடவே விரும்புகின்றனர். உதாரணத்திற்கு, இங்குள்ள ஒரு பஞ்சாயத்தில், தலைவராக இருப்பவர் அதிமுக வை சேர்ந்த ஆதிதிராவிட வகுப்பினர். அவர் இந்தப் பதவிக்கான ஒதுக்கீட்டிற்காக சுயேச்சை வேட்பாளராக போட்டியிட்டு வென்றவர்.

பஞ்சக்கிராமியையும் அதன் மக்களையும் அறிமுகப்படுத்திவிட்டபடியால், இப்போது நாம் அடுத்த பகுதிக்கு செல்லலாம். இதில் இந்த செயல்திட்டத்தில் பயன்படுத்தப்பட்டுள்ள ஆராய்ச்சி கட்டமைப்புகளையும் வழிமுறைகளையும் பற்றிய விரிவான விவரங்களை பார்க்கலாம்.

\section{செயல்திட்ட கட்டமைப்பு}

இந்தப்புத்தகம், பஞ்சக்கிராமியில் 15 மாத இயல்புநிலை மற்றும் நிகழ்நிலை மக்கள் இன அமைப்பியல் ஆய்வின் விளைவாகும். மக்கள் இன அமைப்பியல் 59 ஒரு ஆராய்ச்சி வழிமுறையாக, பங்கேற்பாளர்களை கூர்நோக்குதல், பேட்டிகள், கணக்கெடுப்புகள் போன்றவற்றை உள்ளடக்கியது. இவையனைத்தும், நிகழ்நிலை ${ }^{60}$ மற்றும் இயல்புநிலை இரண்டிலுமே நடத்தப்பட்டன. (நிகழ்நிலை செயல்பாடுகள், முகநூலில் மக்களுடன் நட்புதொடர்பு ஏற்படுத்திக்கொள்வது, பல்வேறு வாட்சப் குழுக்களில் உறுப்பினராவது, டுவிட்டரில் பலரைப் பின்தொடர்வது, லிங்க்ட்-இன் -ல் பல தொழில்நெறிஞர்களுடன் தொடர்பு ஏற்படுத்திக்கொள்வது, மற்றும் அவர்களின் நிகழ்நிலை நடவடிக்கைகளை இந்த சந்தர்ப்பத்தில் கூர்நோக்குவது போன்ற அனைத்தையும் உள்ளடக்கியதாகும்). இந்த வழிமுறை, பஞ்சக்கிராமியில் தங்கியிருந்து, அங்குள்ளவர்களுடன் வாழ்ந்து, 15 மாதங்களுக்கு அவர்களை ஒவ்வொரு நாளும் சந்தித்து, அவர்களின் அன்றாட வாழ்வில் பங்கெடுத்துக்கொள்வது அனைத்தையும் உள்ளடக்கியதாகும். மக்களை பலமுறை சந்தித்து அவர்களின் சம்பிரதாய பேட்டி எடுப்பது மற்றும் சம்பிரதாயமற்ற உரையாடல்கள் நடத்துவது ஆகிய அனைத்தும் இந்த செயல்பாட்டின் ஒரு அங்கம் தான். மக்களையும் அவர்களின் சமூக ஊடகப் பயன்பாட்டையும் ஆழமாக புரிந்துகொள்வதற்காக, நூறுக்கும் மேற்பட்ட சம்பிரதாய பேட்டிகளும், எண்ணற்ற சாதாரண உரையாடல்களும் நடத்தப்பட்டன. 
பஞ்சக்கிராமியில் மக்கள் இன அமைப்பியல் ஆய்வு நடத்துவது அவ்வளவு சுலபமானதல்ல. இதில் உள்ள முக்கிய சவால், மக்களின் நம்பிக்கையையும் அவர்களின் உள் வட்டங்களுக்கான அணுகலையும் பெறுவது தான். அப்போது தான் அவர்களின் தனிப்பட்ட சமூக ஊடக உரையாடல்களை அணுக முடியும். இதற்கு பரிசோதித்துப் பார்க்கும் திறன், கற்பனாத்திறன் மற்றும் மட்டற்ற பொறுமை ஆகியவை மிகவும் அவசியம். பஞ்சக்கிராமியின் ஒரு பகுதி மிகவும் பழமை வாய்ந்ததாகையால், முதல் மூன்று மாதங்களுக்கு, எந்தவொரு இளம்பெண்ணையும் ஆய்வுப் பங்கேற்பாளராக நியமிப்பது சவாலுக்குரியதாக இருந்தது. என்னுடைய உடை, என்னை நான் வெளிப்படுத்திக்கொள்ளும் விதம் ஆகியவை மிகவும் முக்கியத்துவம் வாய்ந்தவையாக இருந்தது. ஒருபக்கம் ஜீன்சும் டி-ஷர்ட்டும் அணிந்து செல்வது இந்த ஆய்வின் உண்மைத்தன்மைப் பற்றிய சந்தேகங்களை எழுப்பியது. மறுபுறம், சம்பிரதாயமான மேற்கத்திய பாங்கிலான உடைகள் என்னை ஒரு விற்பனையாளராக சித்தரித்தது. இறுதியில், "இந்திய அறிவாளியாக" என்னைக் காட்டக்கூடிய குர்தா போன்ற உடைகளை அணிந்தபின் தான் ஒரு கல்வியாளராக என்னை நிலைப்படுத்திக்கொள்ள முடிந்தது. அதன் பின் தான் பெண்கள் என்னுடன் உரையாடுவவை பாதுகாப்பானதாக கருதினர்.

15 மாத ஆராய்ச்சி காலக்கட்டத்தில், ஆராய்ச்சி காரணங்களுக்காக, முகநூலில் நட்புதொடர்புக்கு ஒப்புக்கொண்டவர்களின் எண்ணிக்கை 172. இதில் 132 பேர், இந்த ஆராய்ச்சிக்காகவென்றே உருவாக்கப்பட்ட முகநூல் கணக்கில் நட்பானார்கள் என்றால், மீதமுள்ள 40 பேர், எனது சொந்த முகநூல் கணக்கில் நட்பானார்கள். ஏனெனில் அவர்கள் ஆராய்ச்சிக்கான முகநூல் கணக்கின் நட்பாக விரும்பவில்லை. மேலும், 53 தனிப்பட்ட தொடர்புகள் வாட்சப்பில் நிறுவப்பட்டன. களப்பணி காலகட்டத்திலும் அதற்கு பிறகும், இந்த எண்ணிக்கை உயர்ந்து கொண்டே போய் 210 என்ற எண்ணிக்கையை எட்டியது. வாட்சப் குழுக்களுக்கும் இதே நிலை தான். டிவிட்டரில் 41 தகவலாளர்கள் பின்தொடரப்பட்டனர். தனியாக இதற்கென்றே உருவாக்கப்பட்ட லிங்க்ட்இன் பக்கத்தில் 67 தொடர்புகள் நிறுவப்பட்டன. இந்த எண்ணிக்கையும், களப்பணி காலகட்டத்திலும் அதற்கு பிறகும் அதிகரித்துக் கொண்டே போயின.

இரண்டு கணக்கெடுப்பு வினாத்தாள்கள் ம மேற்கொள்ளப்பட்டன ${ }^{61}$ வினாத்தாள் 1 (Q1) மற்றும் வினாத்தாள் 2 
(Q2). இ இவ்விரண்டும் சமூூக ஊடகங்களில் இருப்பவர்களுக்கு நிர்வகிக்கப்பட்டன. Q1, களப்பணியின் துவக்கத்தில் 130 பேருக்கும், Q2 களப்பணியில் இறுதியில் 150 பேருக்கும் நிர்வகிக்கப்பட்டது. இந்த செயல்திட்டம் ${ }^{62}$ நடத்தப்பட்ட ஒன்பது ஆய்வுக்களங்களிலும் ஒரே சீராக நிர்வகிக்கப்பட்டு இந்த இரண்டு வினாத்தாள்களை தவிர, பஞ்சக்கிராமியில் மூன்று சிறிய கணக்கெடுப்புகளும் நடத்தப்பட்டன. இவை, அந்த சமுதாயத்தின் சில நோக்குகளை இன்னும் விரிவாக புரிந்து கொள்வதற்காக நடத்தப்பட்டன. களப்பணி, சமூக ஊடக உரையாடல்களையும் பயன்படுத்திக்கொண்டது. (முகநூல் மற்றும் வாட்சப் ஆகியவற்றில் இருந்து பெறப்பட்ட தரவுகள் மூலம்) தகவல் பரிமாற்ற குறிப்பேடுகள், தகவல் பரிமாற்ற வரைபடங்கள், உறவுமுறை வட்டங்கள், இணையத்தில் உலாவிய தளங்களை பற்றிய இணையதள வரலாற்று பதிவுகள், மற்றும் ஆய்வுக்காப்பக பதிவேடுகள் ஆகியவை, இந்தப்பகுதியில் சமூக ஊடகங்களின் தாக்கத்தை தெளிவாக புரிந்து கொள்வதற்காக பயன்படுத்தப்பட்டன.

அனாமதேயத்திற்கான உத்திரவாதமளிக்கும், கையொப்பமிடப்பட்ட நெறிமுறை மற்றும் ஒப்புதல் படிவங்கள், ஆராய்ச்சி தகவலாளர்களாக பேட்டி எடுக்கப்பட ஒப்புக்கொண்ட ஒவ்வொருவரிடமிருந்தும் பெறப்பட்டது.

\section{முடிவுரை}

இந்திய சமூகத்தைப் பற்றிய ஆய்வைப் பற்றி பரிச்சயமில்லாத எவருக்கும், இந்த அத்தியாயத்தில் கொடுக்கப்பட்டுள்ள சில விவரங்கள் சற்று அச்சுறுத்துவதாக இருக்கக்கூடும். குலம் என்றால் என்ன? அது எப்படி அந்தஸ்துடன் தொடர்புடையதாகிறது? மக்களைப் பற்றிய அரசாங்கத்தின் வகைப்படுத்தல் எவ்வாறு அவர்கள் தங்களை வகைப்படுத்திக்கொள்ள உபயோகித்தவையுடன் ஒத்துப்போகிறது? இவற்றுக்கான தெளிவான புரிதலை, அசைவற்ற நிலையில் இருந்து அடைவது கடினமாக இருக்கும். ஆனால் இந்த மக்கள் இன அமைப்பியலின் சூழல் அசைவற்றது தவிர மற்ற அனைத்துமாக இருந்தது. நிலத்தின் மதிப்பில் உண்டாகும் மாற்றத்தால் மட்டுமே, முன்புபோலவே இருந்துவிட்ட சில உள்ளூர் கிராமவாசிகள் தங்களை, இந்த பணவீக்கத்துக்கு காரணமாக இருந்த தகவல் தொழில்நுட்பத்துறை பணியாளர்களுக்கு ஈடான வருமானமும் சொத்துக்களும் உடையவ- 
ர்களாக பார்த்தால் என்ன நடக்கும்? இதனால் பயனடையாத மற்ற குடியிருப்பாளர்களுடன் அவர்கள் எவ்வாறு தங்களை தொடர்பு படுத்திக்கொள்கிறார்கள்? கடைசிக்கு முந்தைய அத்தியாயம், இந்த விஷயத்திற்கு ஈடான விசாரங்களைப் பற்றி அலசுகிறது. அனைவராலும், அறிவார்த்த பொருளாதாரத்தின் தூண்டுகோலாக பார்க்கப்படும் கல்விஅமைப்பு, கிராமக்குழந்தைகளையும், ஐ.டி பணியாளர்களின் குழந்தைகளையும் ஒருங்கே அரவணைத்தால், அந்த கல்வியமைப்புக்கு என்ன நேரும்?

அதிர்ஷ்டவசமாக, இந்தச் சூழலை நாம் ஒட்டுமொத்தமாக பார்க்கும்போது, இந்த மாற்றங்களின் ஒன்றுக்கொன்று உதவும் தன்மையை நம்மால் பார்க்க முடிகிறது. முழுவதுமாக கணக்கில் எடுத்துக்கொள்ளப்படவேண்டிய அத்தனை வகையான வித்தியாசங்களும், பிரிவுகளும் இ இருப்பது தெள்ளத்தெளிவாக தெரிகிறது. இவற்றில் மிகவும் முக்கியமான ஒன்று அனைத்திலும் ஊடுருவியுள்ள பாலினம். மற்றது, குடும்பங்களில் உள்ள உட்சுற்று இயக்கவியல் மற்றும் அவற்றின் வெளிப்புற தோற்றத்திற்கிடையேயான வித்தியாசங்கள். ஆனால் இங்குள்ள முக்கிய விஷயம் என்னவென்றால், இந்த வித்தியாசங்கள் அனைத்தும், ஆயிரம் வருடங்கள் பின்னோக்கி செல்லும் குலம் போன்ற வகைக்கூறுகளின் பாரம்பரிய பிரிவுகள் தான். ஒருவகையில், இது இந்தக் குழுக்களின் பொதுத்தன்மையை நமக்கு எடுத்துரைக்கிறது. இவையனைத்துமே, குல மற்றும் பாலின வித்தியாசங்கள் மற்றும் குடும்ப வாழ்வின் விதிமுறைகளால் நெறிப்படுத்தப்படுகின்றன என்பது தான் அது. இந்த அத்தியாயத்தின் துவக்கத்தில் குறிப்பிட்டது போல, இந்தப் புத்தகத்தின் உண்மையான கதையே அது தான். வேறுபாடுகளால் குறியிடப்பட்டதாக காணப்படும் ஒரு சமூகம், உண்மையில் அதன் பின்னிருக்கும் நீண்டகால பாரம்பரியங்களுடனான எண்ணச்சார்பை வெளிக்காட்டுகிறது.

இந்தக்குறிப்புகளை நினைவில் இ இருத்திக்கொள்வதன் மூலம் மட்டுமே, சமூக ஊடகங்களின் மூலமாகவும் வழியாகவும் சொல்லப்பட்டிருக்கும் இந்தக் கூறுரையின் வழியில் நாம் பயணிக்கமுடியும். ஏனெனில் இந்தப் புத்தகம், இந்த வரிசையில் உள்ள மற்றவற்றைப் போலவே, சமூக ஊடகங்கள் எவ்வாறு சமுதாயத்தை மாற்றியிருக்கின்றன என்ற ஆதாரங்களால் ஆதிக்கம் செலுத்தப்படவில்லை. அதற்கு நேர்மாறாக, எவ்வாறு சமூக ஊடகங்களே இந்தச் சூழலை தழுவிக்கொள்வதன் மூலம் மாற்றப்பட்டிருக்கின்றன என்பது 
தான். மிகவும் பழமையான பரந்துவிரிந்த தமிழ்ச் சமுதாயத்தில், வெகு குறுகிய காலகட்டத்திலேயே சமூக ஊடகங்கள் எவ்வாறு மிகவும் சக்திவாய்ந்த கருத்து தெரிவிப்பு ஊடகமாக மாறியிருக்கிறது என்பதை நாம் அங்கீகரித்தால் தான் தென்னிந்தியாவில் சமூக ஊடகங்கள் என்னவாக இருக்கிறது என்பதை நம்மால் பாராட்ட முடியும். 


\section{சமூக ஊடக நிலப்பரப்பு: மக்கள், அவர்களின்}

\section{புலனுணர்வு மற்றும் சமூக ஊடக இருப்பு}

“சென்னை சமூக ஊடகங்களின் மாபெரும் உபயோகிப்பாளர் தளங்களில் ஒன்றாக விளங்குகிறது.....புதிய தொழில்நுட்பங்களை சுவீகரித்துக் கொள்வதில் முதன்மையாக விளங்கும் நாடுகளில் ஒன்றாக இந்தியா இருப்பதில் நமது பங்களிப்பும் இருக்கிறது." - வேணுகோபால், டிஜிட்டல் ஊடக பகுப்பாய்வாளர்

பஞ்சக்கிராமியின் செழித்த தகவல் தொழில்நுட்பத்துறை வளர்ச்சியையும், அதன் அருகாமை நகரமான சென்னை, இந்திய நகரங்களின் முகநூல் உபயோகிப்பாளர்கள் ${ }^{1}$ எண்ணிக்கையில் ஐந்தாவது இடத்தில் இருப்பதையும் பார்க்கும் போது, பஞ்சக்கிராமி, தகவல் தொழில்நுட்ப வளர்ச்சியில் முன்னணியில் இருப்பதில் எந்த ஆச்சரியமும் இல்லை. பஞ்சக்கிராமியில், தகவல் தொழில்நுட்பங்கள், படிப்படியான வளர்ச்சியின் காரணமாக ஒவ்வொன்றாக வந்தது என்று சொல்வதை விட, தொடர்ச்சியான வளர்ச்சிப்பாய்ச்சலாக ${ }^{2}$ வந்தது என்று தான் சொல்லவேண்டும். இதன் சமீபத்திய நிகழ்வு தான் சமூக ஊடகங்களின் ${ }^{3}$ வருகை. பஞ்சக்கிராமியின் சமூக ஊடக நிலப்பரப்பை தெளிவாக புரிந்துகொள்ள வேண்டுமென்றால், இந்தப்பகுதியில், தகவல் பரிமாற்ற வரலாற்றைப் பற்றிய சிறிய கலந்தாய்வில் துவங்குவது தான் சிறந்தது.

பஞ்சக்கிராமியில் பாரம்பரிய தகவல் பரிமாற்றம், எப்போதுமே மக்கள் நேருக்கு நேர் ச சந்தித்துக் கொள்வதுடன் 
சம்பந்தப்பட்டது. அது மக்களது அன்றாட நிகழ்வுகளை பற்றி உரையாடுவதற்காக இருந்தாலும் சரி அல்லது பிறப்பு, திருமணம், இறப்பு போன்ற மிகவும் முக்கியமான விஷயங்கள் பற்றிய கலந்தாய்வாக இருந்தாலும் சரி. அந்தத்தகவலின் இயல்பும், அதன் பெறுநரின் சமூக அந்தஸ்தும், அந்தத்தகவலை அறிவிப்பவர் ${ }^{5}$ யார் என்பதையும் அது போன்ற தகவல்களை எந்தளவிலான சம்பிரதாயத்துடன் தெரிவிக்கப்படவேண்டும் என்பதையும் தீர்மானித்தன. உதாரணத்திற்கு, ஒரு குடிம்பத்தலைவி, உணவு தயாரித்துக்கொண்டிருக்கும் சமயம், அதற்கு தேவையான எதாவது ஒரு முக்கிய செய்பொருள் தீர்ந்து விட்டதென்றால், அவர், தன் குழந்தைகளில் ஒருவரை பக்கத்துக்கு வீட்டிற்கு அனுப்பி அந்த செய்பொருளை அப்போதைக்கு கடனாக பெற்றுக்கொள்வது ஒத்துக்கொள்ளப்பட்ட ஒன்று. ஆனால் அதுவே, ஒரு குடும்பம், தங்கள் இல்லத்தில் எந்த ஒரு நிகழ்வுக்காவது தங்கள் அண்டை அயலாரை அழைக்க விரும்பினால், மரபுப்படி, இல்லத்தில் உள்ள பெரியவர்கள், அண்டை வீட்டுக்குச் சென்று அழைப்பு விடுக்க வேண்டும் என்று கூறப்படுகிறது. கூடிதலாக, மரணம் தொடர்பான செய்திகள், கோவில் திருவிழாக்கள் பற்றிய செய்திகள், உள்ளூரில் நடைமுறையில் இருக்கும் கிராம பஞ்சாயத்தின் அறிவிப்புகள், அல்லது சமூகம் தொடர்பான கொள்கைகள் போன்றவை அந்தந்த குறிப்பிட்ட செயல்களுக்காக ${ }^{6}$ நியமிக்கப்பட்டிருக்கும் ஆண் செய்தியறிவிப்பாளர் மூலம் மட்டுமே அறிவிக்கப்பட்டன.

இது போன்ற தகவல் பரிமாற்ற வழிமுறைகளுடன் சேர்த்து, கிசுகிசு போன்ற வீணுரைகளும், இந்த நெருக்கமாக பிணைந்திருக்கும் கிராமச்சமுதாயத்தில் சமூகச் செய்திகளை அதிதீவிரமாக பரப்ப உதவின. குடிம்பத்தில் உள்ள உட்பூசல்கள், காதல் விவகாரங்கள், மணமகன் வீட்டார் கேட்ட வரதட்சிணை விவரங்கள் போன்ற விசாரங்கள், கிசுகிசுக்கள் மூலம் பரப்பப்பட்டன. இது போன்ற தகவல்கள் எப்போதுமே வாய்வழிச்செய்தியாகவே பரப்பப்பட்டன. பலதரப்பட்ட இந்தச் செய்திகளை போலவே, இதை பரப்பியவர்களும் பலதரப்பட்டவர்கள். மூர்த்தி என்ற 27 வயதான தொழில்முனைவாளர், சுமார் 15 வருடங்கள் முன்பு நடந்த ஒரு சம்பவத்தை நினைவுகூர்கிறார். பக்கத்து கிராமத்தில் வாழ்ந்து கொண்டிருந்த அவரது தூரத்து உறவான அத்தைப்பாட்டி ஒருவர், அவரது சித்தப்பாவின் திருமண நிச்சயம் பற்றிய செய்தியை கேள்விப்பட்டு, அதைப்பற்றி நேருக்கு நேர் கேட்பதற்காக அவர்கள் வீட்டுக்கு வந்திருந்தார். இந்த விஷயத்தை தனக்கு ஏன் நேரடியாக 
தெரிவிக்கவில்லை என்று அவர் கோபமாக கேட்பதற்காக வந்திருந்தார். இது மூர்த்தியின் குடும்பத்தினருக்கு மிகவும் ஆச்சரியமாக இருந்தது. ஏனென்றால், முந்தைய மாலையில் தான் அவர்கள், அவரின் சித்தப்பாவிற்கு ஏற்ற மணப்பெண்ணை தேடுவது பற்றி தற்செயலான உரையாடலாக பேசிக்கொண்டிருந்தனர். அவர்கள் வீட்டில் வேலை செய்யும் பெண்மணி, அவரது அத்தைப்பாட்டியின் கிராமத்தில் வாழும் தனக்கு தெரிந்த ஒருவரிடம் இந்த கலந்துரையாடலைப் பற்றி குறிப்பிட்டிருக்கிறார். ஆனால் எந்தவொரு திருமண நிச்சயத்தைப் பற்றியும் அவர் குறிப்பிட்டிருக்கவில்லை. ஆனால் இந்தச் செய்தி, அத்தைப்பாட்டியின் காதுகளை, கூடுதல் அலங்காரங்களுடன் சேர்த்து சென்றடைந்திருக்கிறது. அது அவரை தான் அலட்சியப்படுத்தப்பட்டதாக எண்ண வைத்திருக்கிறது.

இது போன்ற பல சம்பவக்கதைகளை இந்தப் பகுதியில் கேட்க முடியும். ஆண்கள், பெண்களை கிசுகிசுக்களை பரப்புவதாக குற்றம் சாட்டினாலும், இருபாலாரும் இந்த நடைமுறையில் சம-அளவில் ஈடுபட்டிருந்தனர். தங்கள் வீடுகளுக்கு அருகே இருக்கும் தெருக்களில் பெண்கள் ஒருவரையொருவர் சந்தித்து குடும்பம் மற்றும் தனிமனிதர்கள் சம்பந்தப்பட்ட செய்திகளை பரிமாறிக்கொண்டார்கள் என்றால், ஆண்கள் தேநீர் கடைகள் மற்றும் இதர பொது இடங்களில் சந்தித்துக்கொண்டனர். இங்கு அவர்கள், இந்தப் பகுதியில் உள்ள பிற ஆண்கள் பெற்ற சமீபத்திய வியாபார ஒப்பந்தங்களை பற்றியும், உள்ளூர் மற்றும் மாநில அளவிலான அரசியல் நிலவரங்களை பற்றியும் அரட்டையடித்தனர். இளம் ஆண்கள், அந்தப்பகுதியின் இளம் பெண்கள் மற்றும் தங்கள் அபிமான நட்சத்திரங்களின் சமீபத்திய திரைப்படங்கள் பற்றியும், இளம் பெண்கள், வீட்டுப்பிரச்சினைகள், திரைப்படங்கள், நாகரீகம் மற்றும் அந்தப்பகுதியின் இளம் ஆண்கள் ஆகியவற்றைப் பற்றியும் கிசுகிசுத்தனர். வீட்டுப்பெண்கள் தங்கள் வீட்டு ஆண்களிடம், தனிமனித மற்றும் குடும்பங்கள் பற்றிய அரட்டை தகவல்களை தெரிவித்தாலும், இதன் நேர்மாறு பொதுவாக நடப்பதில்லை.

பஞ்சக்கிராமியில் இருந்து புலம் பெயர்ந்த மக்கள் தங்கள் உறவினர்களுடன் தபால்தலையிட்ட தபால் அட்டைகள் மூலமாகவோ அல்லது இன்லாண்ட் கடிதங்கள் மூலமாகவோ கடிதத்தொடர்பில் இருந்தனர். இந்தப்பகுதியில் உள்ள தபால் நிலையம், கடிதப் போக்குவரத்துக்கான இடமாக மட்டுமல்லாமல், உள்ளூர்வாசிகள் சந்திக்கும் 8 சமூகக் கூடமாகவும் 
இருந்து வந்தது. தந்திகள் ${ }^{9}$ அவசர செய்திகளை தெரிவிப்பதற்காக பயன்படுத்தப்பட்டன. இரருப்பினும், மக்கள் தந்தி வந்தாலே ஒருவித பதட்டம் அடைந்தனர். தாங்கள் அறிந்த எவரது மரணச்செய்தியோ தந்தியில் இ இருக்கக்கூடும் என்று அவர்கள் அச்சத்துடன் அனுமானித்துக்கொண்டனர். இவ்வாறு, விரைவிலேயே தந்திச் சேவை, அமங்கல செய்தியுடன் தொடர்புடையதாக கருதப்பட்டது. தந்திகளைப் பற்றிய இவ்வாறான எண்ணவோட்டம், பஞ்சக்கிராமிக்கு மட்டும் உரித்தானதல்ல. இந்தியாவின் பல கிராமங்களிலும் இது தான் நடப்பதாக இருந்தது. 2013ஆம் வருடம் ஜூலை மாதம், 162 வருட சேவைக்கு ந்தரமாக நிறுத்தப்பட்டது. அதன் மூலம் இந்த வகையிலான தகவல் பரிமாற்றம் ஒரு முடிவுக்கு வந்தது. உள்ளூர், தேசீய மற்றும் சர்வதேச கூரியர் சேவை, இருபத்தி ஒன்றாம் நூற்றாண்டின் துவக்கத்தில் தான் நடைமுறைக்கு வந்தது. அவை இப்போது இ இங்குள்ள உள்ளூர் மக்கட்தொகைக்கும், தகவல் தொழில்நுட்ப நிறுவனங்களுக்கும் அவசர செய்திகள் மற்றும் தகவல்களை அனுப்பதல் மற்றும் பெறுதல் பணியை செய்துகொண்டிருக்கின்றன.

வானொலி, தொலைக்காட்சி, செய்தித்தாள்கள் மற்றும் பத்திரிகைகள் ${ }^{11}$, காலங்காலமாக சர்வதேச, தேசீய மற்றும் பிராந்திய செய்திகளையும், அவற்றோடு சேர்த்து திரை நட்சத்திரங்கள், அரசியில்வாதிகள், விளையாட்டு வீரர்கள் மற்றும் இன்ன பிற பிரபலன்களைப் பற்றிய குறுஞ்செய்திகளையும் கிசுகிசுக்களையும் தெரிவித்து வருகின்றன. அரசாங்கத்தின் "ஆகாச வாணி" மட்டுமே 1990-களின் இறுதி வரை ஒற்றை வானொலி அலைவரிசையாக இருந்து வந்தது. 2000ங்களில் இருந்து தனியார் வானொலி அலைவரிசைகளான சூரியன் எஃப்.எம், ரேடியோ மிர்ச்சி, பிக் எஃப்.எம் ${ }^{12}$ போன்றவை பஞ்சக்கிராமியில் பிரபலமடையத் துவங்கின. பல்வேறு அளவிலான வானொலி சாதனங்களை வைத்திருப்பதும், மொபைல் போன்களின் மூலமாக வானொலி அலைவரிசைகளை கேட்பதும், உள்ளூர் செய்திகளை கேட்பது மற்றும் பொழுதுபோக்கு காரணங்களுக்காக இந்த தனியார் அலைவரிசைகளின் பிரபல்யத்தை அதிகரித்துள்ளன.

1980களிலும் 1990களின் துவக்கத்திலும், பஞ்சக்கிராமியில் பல குடும்பங்கள் ஒரு தொலைக்காட்சி சாதனத்தை பெற்றிருக்கவில்லை. அவர்கள் அநேகமாக எப்போதும், செய்திகளின் ஒளிபரப்பை பார்ப்பதற்கும், வெள்ளிக்கிழமை இரவுகளில் ஒலியும் ஒளியும் என்ற திரைப்பட பாடல்கள் நிக- 
ழ்ச்சியை பார்ப்பதற்கும் பஞ்சாயத்து அலுவலகத்தில் ஒன்று கூடினர். 1990களின் இறுதியிலும் 2000-ங்களின் துவக்கத்திலும், அதிகமான குடும்பங்கள் தொலைக்காட்சிப் பெட்டி வாங்கினர். அத்தோடு சேர்த்து, தமிழ்நாட்டில் உள்ள இரண்டு முன்னணி அரசியல் கட்சிகளும் ஒன்றோடொன்று போட்டி போட்டுக்கொண்டு, ஓட்டு பெறுவதற்காக கீழ்த்தட்டு சமூக பொருளாதார நிலையில் இருக்கும் மக்களுக்கு கொடுக்கும் இலவச பொருட்களில் ${ }^{13}$ தொலைக்காட்சிப் பெட்டியும் ஒன்று. இன்று பஞ்சக்கிராமியில் அநேகமாக அனைத்து இல்லங்களிலும் தொலைக்காட்சிப் பேட்டிகள் உள்ளன. 1990களின் இறுதியிலும், 2000-ங்களின் துவக்கத்திலும், தொலைகாட்சி அலைவரிசைகளை அதிகரித்துள்ளன. 1980களிலும் 1990களின் துவக்கத்திலும் அரசாங்கத்தால் நடத்தப்பட்ட தூரதர்ஷன் என்ற ஒற்றை அலைவரிசை மட்டும் இருந்தது போய், இப்போது கேபிள் மற்றும் தனியார் வலைத்தொடர்புகள் நடத்தும் அலைவரிசைகளான சன் டி.வி, விஜய் டி.வி, ஜெயா டி.வி14 போன்றவை மற்றும் இதர பல தமிழ் அலைவரிசைகளும், ஆங்கிலம் மற்றும் பிற இந்திய மொழி அலைவரிசைகளும் நடைமுறையில் இருக்கின்றன. அநேகம் மக்கள், தங்கள் கேபிள் தொடர்புக்கு தாங்களே கட்டணம் செலுத்தினாலும், ஒருசிலர், ஒரே தொடர்பில் இருந்து சட்டத்திற்குபுறம்பாக, கொத்தாக பல தொடர்புகள் எடுத்துக்கொள்கின்றனர். 1 மற்றும் 3 ஆம் அத்தியாயத்தில் குறிப்பிட்டுள்ளது போல, தமிழ்நாட்டின் மற்ற பகுதிகளைப் போலவே இந்தப்பகுதியிலும் திரைப்படங்கள் மிகவும் பிரபலம். மேற்கூறிய அனைத்து தொலைகாட்சி அலைவரிசைகளும் திரைத்தொடர்பான செய்திகளைக் கொண்ட நிகழ்ச்சிகளையே அதிகம் அலைபரப்புகின்றன.

தினத்தந்தி மற்றும் தினமலர் ${ }^{15}$ போன்ற தமிழ் செய்தித் தாள்கள் தேநீர்க் கடைகள் போன்ற இடங்களில் பொதுவாக காணப்பட்டாலும், “தி ஹிந்து", “தி நியூ இந்தியன் எக்ஸ்பிரஸ்",:டெக்கான் கிரோனிக்கல்", “தி டைம்ஸ் ஆப் இந்தியா" மற்றும் “தி எகனாமிக் டைம்ஸ்"116 போன்ற ஆங்கில தினசரிகளும் மத்தியதர மற்றும் உயர் மத்தியதர வாசகர்களிடையே தங்கள் இடத்தை பிடித்துள்ளன. இவை அச்சுப் பதிவுகளாகவும், நிகழ்நிலை பதிவுகளாகவும் கிடைக்கின்றன. அதேபோல, தமிழ் வாராந்திர பத்திரிகைகளான குமுதம், ஆனந்த விகடன்17, போன்றவை, மத்தியதர மற்றும் கீழ்மட்ட சமூகப் பொருளாதார வகுப்பினரிடையே பொதுவாகக் காணப்படும். அதேசமயம், இந்தியா டுடே, பிசினெஸ் டுடே, ஃபெமினா, 
வோக் 18 போன்ற ஆங்கில பத்திரிகைகள் உயர் மத்தியதர வகுப்பினரின் இல்லங்களில் காணப்பட்டன. டேட்டாக்வஸ்ட் மற்றும் டிஜிட் 19 போன்ற தொழில்நுட்ப பத்திரிகைகள் மத்தியதர வகுப்பை சேர்ந்த ஐ.டி பணியாளர்களின் இல்லங்களில் காணப்பட்டன. தெலுங்கு, ஹிந்தி மற்றும் மலையாளம் மொழிகளைச் சேர்ந்த செய்தித்தாள்களும், பத்திரிகைகளும், ஐ.டி பணியாளர்கள் மற்றும் அவர்களது குடும்பத்தினராலும் மற்றும் பிற மாநிலங்களில் இருந்து வந்து குறைந்த கூலிக்கு பணிபுரியும் புலம்பெயர் பணியாளர்களாலும் வாசிக்கப்படுகின்றன.

\section{மொபைல் போன் (அலைபேசி), இணையதளம் மற்றும் ஆர்குட்}

தந்தி மற்றும் தபால் சேவைகளை தொடர்ந்து, அந்த வரிசையில், தனிப்பட்ட செய்திகளையும், தகவல்களையும் பரிமாறிக்கொள்ள வந்தது தான் லேண்ட்லைன் எனப்படும் தொலைபேசி. 1970களில், உள்ளூர் நிர்வாகக்குழு ஒரு தொலைபேசித் தொடர்பு வைத்திருந்தாலும், இவைகளின் அதிகமான தேவை மற்றும் அளிப்பு விகிதாச்சாரம் மற்றும் இதன் விளைவாக, அவற்றின் ஒதுக்கீட்டில் 20 நிகழ்ந்த அதிகாரவர்க்க செயல்பாடுகள் ஆகியவை சேர்ந்து, 1990களில் இது போன்ற தொலைபேசிகளின் தனிப்பட்ட உடைமை, பஞ்சக்கிராமியின் ஒருசில செல்வாக்கான குடும்பங்களுக்கிடையே மட்டுமே மையமிட்டிருந்தன. இந்தப் போன்களின் உபயோகம், அண்டைஅயலார், உறவினர் மற்றும் தங்கள் குல உறுப்பினரிடையே ${ }^{21}$ பகிர்ந்து கொள்ளப்பட்டது. தொலைபேசி எண்கள், தொலைதூரத்தில் வாழும் உறவினர் மற்றும் இங்கிருந்து புலம்பெயர்ந்து சென்ற உள்ளூர்வாசிகள் ஆகியோரிடையே பகிர்ந்து கொள்ளப்பட்டன. இதன் மூலம் எந்தவொரு அவசரத்தேவைக்கும் அவர்களால் சுலபமாக தொடர்பு கொள்ள முடிந்தது.

இதைத் தொடர்ந்து எஸ்.டி.டி/ஐ.எஸ்.டி பூத் எனப்படும் பொது தொலைபேசி அமைப்புகள் உருவாகின. இவை மக்களுக்கு உள்ளூர், வெளியூர் மற்றும் வெளிநாட்டு தொலைபேசி அழைப்புகளை ஒரு குறிப்பிட்ட கட்டணத்திற்கு 22 செய்வதற்கு உதவின. மொபைல் போன்கள் இந்தியாவில் 1995ஆம் ${ }^{23}$ வருடம் அறிமுகமாகி இருந்தாலும், 2000-மாவது வருடத்திற்கு பிறகு தான் இந்தப்பகுதி மக்கள் அவற்றை 
சொந்தமாக உபயோகிக்க துவங்கினர். போன் உற்பத்தியாளர்களின் எண்ணிக்கையில் அதிகரிப்பு, அதிக அளவிலான தொலைத்தொடர்பு அளிக்கும் நிறுவனங்களின் தெரிவு, மற்றும் அதிவேக வளர்ச்சியினால் குறைந்த விலையில் கிடைக்கும் மொபைல் போன்கள் போன்ற பலதரப்பட்ட காரணிகள் ஸ்மார்ட்போன் அல்லாத ${ }^{24}$ சாதாரண மொபைல் போன்களின் அதிவேக பரவுதலுக்கு வழிவகுத்தன. இந்த மொபைல் போன்கள் விரைவில் கட்டுப்படியாகக்கூடிய ஒரு பொருளாக ஆகிவிட்டது. இதனால் இந்தப்பகுதியில் தகவல் பரிமாற்ற வழிமுறைகளும் அலைவரிசைகளும் அதிதீவிர மாற்றமடைந்தன.

இந்தப்பகுதியில் ொபைல் தொலைத்தொடர்பு வளர்ச்சிக்கு இணையாக, பஞ்சக்கிராமியில் தகவல் தொழில்நுட்ப நிறுவனங்களின் வருகை, கணினியை வாங்குவதிலும் பொதுவான ஆர்வத்தைத் தூண்டியது. இந்தப்பகுதியில், நிலம் மனைகளின் லாபகரமான விற்பனை மூலம் தமது வளத்தை அதிகரித்துக்கொண்ட ஒருபகுதி மக்கட்தொகையின் பொருட்ச்செழுமையுடன், இந்த ஆர்வம் பொருந்திப் போயிற்று. இவர்கள் பெற்ற புதிய வளங்களின் ஒரு பொதுவான பயன்பாடு, குடும்பத்தில் உள்ள இளைய தலைமுறையினருக்காக கணினிகள் ${ }^{25}$ வாங்குவதாக இருந்தது. குடும்பத்தில் உள்ள ஒரு இளைய உறுப்பினர் கணினி வைத்திருப்பது என்பது, உள்ளூர்வாசிகளால் ஒரு அளவில் தலைமுறைகளுக்கு இடையேயான பேறாக ${ }^{26}$ கருதப்பட்டது. இருப்பினும், நடைமுறையில், இந்தக் கணினிகள், இங்குள்ள நீண்ட கால குடியிருப்பாளர்கள் குழந்தைகளால், சம்பிரதாயமான கல்வியை விடவும் அதிக அளவில், விளையாட்டுகளுக்கும், திரைப்படங்கள் பார்ப்பதற்கும், இசை கேட்பதற்கும் பயன்படுத்தப்பட்டது.

இந்த சமயங்களில், அதிக திறமை வாய்ந்த தகவல் தொழில்நுட்ப பணியாளர்கள், சென்னை நகரத்தில் இருந்து, தங்களது தனிப்பட்ட (அ) நிறுவனத்திற்கு சொந்தமான (அ) பொது போக்குவரத்து ஊர்திகளின் மூலம் இங்குள்ள தங்கள் அலுவலகங்களுக்கு வந்து போய்க்கொண்டிருந்தனர். அவர்களது அலுவல் இடத்திற்கு அருகாமையிலான பன்மாடிக் குடியிருப்பு வளாகங்கள் அப்போது கட்டுமான நிலையில் தான் இருந்தன. ஆகவே, ஐ.டி பணியாளர்கள், சொந்தமாக கணினிகள் - பெரும்பாலும் மேசைக் கணினிகள் வைத்திருந்தாலும், இந்த நிலையில் அவர்களில் அநேகர், சென்னை குடியிருப்புவாசிகளாகத் தான் 
இருந்தனர். பஞ்சக்கிராமியில் இல்லை. இருப்பினும், 2000-ங்களின் இறுதியிலும், 2010-க்களின் துவக்கத்திலும், மேசைக் கணினி மற்றும் மடிக்கணினி வைத்திருப்பவர்களின் எண்ணிக்கை இந்தப் பகுதியில் பெருமளவில் அதிகரித்தது. அது பஞ்சக்கிராமியில் பணிநிமித்தம் ${ }^{27}$ குடும்பத்தோடு புலம்பெயரும் திறமை வாய்ந்த ஐ.டி பணியாளர்களின் எண்ணிக்கையோடு ஒத்துப்போயிற்று. இந்த சமயத்தில் தான் கணினிகள் (மேசைக் கணினி மற்றும் மடிக்கணினி) கட்டுப்படியாகக் கூடிய விலையில் கிடைக்கத்துவங்கின. இதே சமயத்தில் தான் அரசாங்கம், ஒரு மாணவருக்கு ஒரு மடிக்கணினி என்ற திட்டத்தையும் செயல் படுத்தியது. ஆகையால் உயர்நிலைப்பள்ளி செல்லும் குழந்தைகள் உள்ள குடும்பங்களில் கணினியின் வருகை துரிதமானது. ஒரு சில வழக்குகளில், இவ்வாறு அரசாங்கத்தால் வழங்கப்பட்ட மடிக்கணினிகள், தங்கள் தூரத்து உறவினருக்கோ அல்லது வேறு யாருக்காவதோ குறைந்த விலையில் விற்கப்பட்ட சம்பவங்களும் நடந்திருக்கிறது. எது எப்படியிருப்பினும், கீழ்த்தட்டு சமூக பொருளாதார நிலையில் இருக்கும், கணினி வாங்கும் திறன் இல்லாத மற்றும் உயர்நிலைப்பள்ளியில் படிக்கும் குழந்தைகள் இல்லாதவர்களுக்கு, கணினி என்பது ஒரு நெடுந்தூர கனவாகவே இருக்கிறது.

2000-ங்களின் துவக்கத்தில், இணையத்தளத்திற்கான அணுகல் வாய்ப்பு சமமற்றதாக இருந்தது. ஏனெனில், அப்போது, டயல்-அப் தொடர்பு என்றழைக்கப்படும் அணைப்பு வழி இணைப்பு மூலமாக தான் இணைய தொடர்பு கொள்ள முடியும். இதற்கு தரைவழி தொலைபேசி இணைப்பு அவசியமாக இருந்தது. பஞ்சக்கிராமியின் குடியிருப்பாளர்களில் பெரும்பாலானோர் தொலைபேசி இணைப்பு பெற்றிருக்காத காரணத்தால், அவர்களுடைய கணினிகள் (யாரெல்லாம் வைத்திருந்தார்களோ அவர்களது மட்டும்) இணையத்தொடர்பில் இருக்கவில்லை. இந்தப்பகுதியில் இணையத்துக்கான அணுகல் வாய்ப்பு, பிரௌசிங் மையம் அல்லது சைபர் கஃபே 28 என்றழைக்கப்படும் இணையதள மையங்கள் மூலமாக மட்டுமே சாத்தியமாக இருந்தது. இவை பெரும்பாலான சமயம் கல்லூரி செல்லும் ஆண் மாணவர்கள் அல்லது ஐ.டி பணியாளர்கள் கும்பலால் சூழப்பட்டிருந்தது. பொதுவான இணையதள உலாவலைத் தவிர, இந்த இணையதள மையங்களின் மற்ற பிரபலமான இணையதள பயன்பாடுகள் என்னவென்றால், நிகழ்நிலை விளையாட்டு- 
க்கள், மின்னஞ்சல் அனுப்புதல் மற்றும் பெறுதல், பாலியல் காணொளிகள்/புகைப்படங்கள் பார்த்தல், யாஹூ மற்றும் ஐ.சி.க்யூ மூலமாக அளவளாவுதல் ஆகியவை தான்.

கூகிள்-க்கு சொந்தமான ஆர்குட் தான் இந்தியாவில் 29 புழக்கத்தில் வந்த முதல் சமூக ஊடகத்தளம். இப்போது அந்தத் தளம் நிறுத்தப்பட்டு விட்டாலும், உலகம் முழுவதும் அதே போன்ற சமூக ஊடகத்தளங்களான முகநூல் போன்றவற்றிற்கு இடம் பெயர்ந்து விட்டாலும், இந்தியாவில், நிகழ்நிலை நட்புதொடர்புகளுக்கு ஆர்குட் ஒரு பிரபலமான பாதையாக இருந்தது. 2000-ங்களின் மத்தியில், ஆர்குட், அளவில் பெருகிய போது, அந்த தளத்தின் இணையதள போக்குவரத்தில், பெரும்பான்மை இந்தியாவில் ${ }^{30}$ இருந்து தான் வந்தது. மக்கள் யாஹூ குழுக்களையும், மின்னஞ்சல் குழுக்களையும், இதற்கு முன்னோடியாகப் பார்த்தாலும், ஆர்குட் ஏற்படுத்திய தாக்கத்தை, அவை ஏற்படுத்த தவறிவிட்டன.

தகவல் தொழில்நுட்ப பன்னாட்டு நிறுவனம் ஒன்றில், அது பஞ்சக்கிராமியில் 2002ல் நிறுவப்பட்ட போதிலிருந்தே பணிபுரியும் விக்ரம் என்ற 34 வயதான மூத்த ஆலோசகர் கூர்நோக்கியது:

“2005 இறுதியில், நாங்கள் அனைவரும் ஆர்குட்-ல் இருந்தோம். அது மிகவும் வேடிக்கையாக இருந்தது. நாங்கள் இதைப் பற்றி அலுவலகத்தில் எந்நேரமும் பேசிக்கொண்டிருப்போம். எனக்கு அப்போது திருமணமாகியிருக்கவில்லை. என்னுடைய ஐ.டி நண்பர்கள் சிலருடன் சேர்ந்து தங்கிக்கொண்டிருந்தேன். இந்தப் பகுதி எப்படியிருந்தது என்று உங்களுக்கு தெரியுமல்லவா....மோசமான சாலைகள்....சென்னையை அடைவதற்கு ஏறத்தாழ ஒரு மணிநேரம் ஆகும். சென்னையை அடைந்த பின், ஏதாவது ஒரு இணையதள மையத்திற்கு சென்று ஆர்குட்-ல் அமர்ந்தால், மாலை வெகு நேரம் வரை அது தான், அது மட்டும் தான். சனிக்கிழமைகள், ஞாயிற்றுக்கிழமைகள் எல்லாம் ஆர்குட் தான் எங்கள் உலகமாக இருந்தது."

பஞ்சக்கிராமியில் வாழும் சுஜாதா என்கிற 29 வயதான குடும்பத்தலைவி குறிப்பிடுகிறார்:

“எங்கள் கல்லூரியில் அந்த சமயம் ஆர்குட் தான் மிகவும் பிரபலமான ஒன்று. ஒருவர் ஆர்குட்-ல் இருக்கிறார் 
என்றால் அதன் மதிப்பே தனி. அதற்காக, ஆர்குட்ல் இல்லையென்றால், நண்பர் குழாமில் இருக்க முடியாது என்பதெல்லாம் கிடையாது. எனது நண்பர்களில் பலர் அப்போது ஆர்குட்-ல் இல்லை. அவர்கள், சென்னைக்கு வெளியேயுள்ள கிராமங்களில் இருந்து வந்தவர்கள்.... ஆர்குட்-ல் எப்போதும் ஒரு எட்டிப்பார்க்கும் மனப்பான்மை இருந்தது.....இப்போதும் மக்கள் முகநூலில் அதுபோல் செய்கிறார்கள். ஆனால் ஆர்குட்-ல் உங்களது பக்கத்தை யார்யாரெல்லாம் பார்த்தார்கள் என்பதை கண்டுகொள்ள முடியும்.....அது துவக்கத்தில் மிகவும் வேடிக்கையாக இருந்தது. ஆனால் போகப்போக எரிச்சலான ஒரு விஷயமாக ஆகிவிட்டது."

அர்ஜுன் என்ற 31 வயதான குடியுரிமை அல்லாத இந்தியர் ${ }^{31}$ பஞ்சக்கிராமியில் ஒரு அடுக்குமாடிகுடியிருப்பில் சொந்தமாக வீடு வைத்திருக்கிறார். அவர், சென்னையில் கணினியில் இளங்கலை பொறியியல் பட்டம் முடித்துவிட்டு முதுகலை பட்டத்திற்காக அமெரிக்கா சென்றுவிட்டார். அவர் கூறுகிறார்:

“முன்பெல்லாம், ஆர்குட் மூலமாக மட்டும் தான் நான் என்னுடைய பல இந்திய நண்பர்களுடன் தொடர்பில் இருக்க முடியும். இப்போதெல்லாம் பலப்பல வழிகள் இருக்கின்றன. ஆனால் 2005-ல் மின்னஞ்சலுக்கு பிறகு, ஆர்குட் மட்டும் தான். மின்னஞ்சலை விட, ஆர்குட் கண்டிப்பாக அதிக வேடிக்கையான விஷயமாக இருந்தது."

இருப்பினும், அவர்களின் ஆர்குட் பயன்பாடு 2010-களின் 32 துவக்கத்தில் தேய்வுற தொடங்கியது. மேலே குறிப்பிட்டிள்ள எவருமே, தங்களது ஆர்குட் கணக்கை ரத்து செய்யவில்லை. முகநூல், வாட்சப் போன்ற மற்ற ஊடகங்களுக்கு மாறிய பின், அவர்கள் தங்கள் ஆர்குட் கணக்கின் விவரங்களை அப்படியே விட்டுவிட்டார்கள். அவ்வளவுதான். ஆர்குட் பற்றிய நினைவுகள், சமீபத்தில் நகரத்தை விட்டு பஞ்சக்கிராமியில் குடியேறியவர்களிடையே பொதுவான ஒன்றாகும்.

ஆர்குட்-ஐ பற்றி வாஞ்சையுடன் நினைவுகூரும் மற்ற நகரங்களில் இருந்து பஞ்சக்கிராமிக்கு புலம்பெயர்ந்த மத்தியதர மற்றும் உயர்மத்தியதர வகுப்பை சேர்ந்தவர்களைப் போலல்லாமல், பஞ்சக்கிராமியின் உள்ளூர்வாசிகளிடையே இந்த சேவை அப்படி ஒன்றும் பிரபலமானது அல்ல. பஞ்சக்கிராமியின் வெகுசில நீண்டகால குடியிருப்பாளர்கள் மட்டுமே 
ஆர்குட் தளத்தை பயன்படுத்தியிருக்கிறார்கள். போக்குவரத்து வணிகத்தில் ஈடுபட்டிருக்கும் சிவா என்கிற 37 வயதான தொழில் முனைவர் கூறுவது போல:

“அந்தசமயத்தில் தான் இந்தப்பகுதியில் வளர்ச்சி முழுவீச்சில் நடந்துகொண்டிருந்தது. எங்களது தலைமுறையில், நன்கு படித்தவர்கள் அதிகம் கிடையாது. பலருக்கும் இணையதள தொடர்பும் கிடையாது. எனது தலைமுறையில் பலரும் ஆர்குட்-ஐ தவறவிட்டுவிட்டார்கள் என்று நினைக்கிறேன். ஆனால் முகநூலை கண்டுகொண்டார்கள். இணையதள மையங்கள் மிகவும் பிரபலமாக இருந்தன. ஆனால் அவையனைத்தும் சென்னையில் இருந்தன. அப்போது இங்கு ஒரே ஒரு இணையதள மையம் மட்டுமே இருந்தது. உங்களுக்கு தெரியுமா, பிற நகரங்களில் இருந்து இங்குள்ள பொறியியல் கல்லூரியில் படிக்க வந்திருந்த மாணவர்கள் அநேகமாக அனைவரும், எப்போதுமே இந்த மையங்களில் தான் இருப்பார்கள். அவர்கள் தான் ஆர்குட்-ஐ பயன்படுத்தியவர்கள், நாங்கள் அல்ல. நான் அதில் இருந்த காரணம், சென்னையில் இருந்த எனது நண்பர் ஒருவர் அதைப் பற்றிக் கூறியதால் மட்டும் தான்.

பஞ்சக்கிராமியின்

குடியிருப்பாளரான

சுந்தர் என்கிற

32 வயதானவர் விளக்கியதாவது:

“நான் ஆர்குட்-ல் இ இருந்தேன். கல்லூரியில் படிக்கும் போது நண்பர் ஒருவர் எனக்கு அதை அறிமுகப்படுத்தி வைத்தார். இது நடந்தது நகரத்தில்....இங்கு அல்ல...எனது பகுதியில் இருந்த நண்பர்கள் யாரும் அதில் இருக்கவில்லை...நான் அவர்களுக்கு அதைப் பற்றி கூறினேன்... அதில் முதலில் ஆர்வம் காட்டிய அவர்கள், நாள் செல்லச்செல்ல ஆர்வத்தை இழந்துவிட்டனர். ஏனெனில் அவர்கள் யாரிடமும் ஆர்குட்ஐ பயன்படுத்துவதற்கு கணினி இருக்கவில்லை. எனது ஆர்குட் நண்பர்கள் அனைவருமே, என்னுடைய நகரத்து நண்பர்கள் தான். இப்போது முகநூலில் எல்லாம் வித்தியாசமாக இருக்கிறது. முகநூலை, மொபைல் போன்களின் மூலம் அணுக முடியும். பத்தாண்டுகளுக்கு முன்பு, இது சாத்தியமில்லை. இணையதள மையங்களுக்கு போனால் மட்டுமே ஆர்குட்ஐ அணுக முடியும். என்னுடைய நண்பர் 
ஒருவர், சிலகாலம் அதில் இருந்தார்...பின்னர் ஆர்வமிழந்துவிட்டார். இப்போது அவர் முகநூலில் இருக்கிறார். இங்குள்ள பலர் மின்னஞ்சல் கணக்கு கூட வைத்திராத போது, ஆர்குட்-ஐ பற்றி ஏன் பேச வேண்டும்ி"

தனிப்பட்ட சாதனங்களின் மூலமான சமூக ஊடக அணுகல், இணையதள வசதிகள் முழு வளர்ச்சியடைந்து, பிராட்பேண்ட் பிரபலமடைந்து, கட்டுப்படியாகக்கூடிய நிலை வரும்வரை காத்திருக்க வேண்டியிருந்தது. பிராட்பேண்ட் மற்றும் வைஃபை இணைப்புகளை துவக்கத்திலேயே தத்தெடுத்துக் கொண்டவர்கள் பஞ்சக்கிராமியில் உள்ள மத்தியதர மற்றும் உயர்-மத்தியதர வகுப்பை சேர்ந்தவர்கள் தான். மடிக்கணினியின் வருகையுடன், யு.எஸ்.பி. டாங்கிள் எனும் சிறு சாதனத்தின் மூலமான இணையதள தொடர்பும் பிரபலமடையத் துவங்கியது. இதுபோன்ற டாங்கிள்-களின் பிரயோகம், ஐ.டி பணியாளர்கள், விடிதியில் வாழும் கல்லூரி மாணவர்கள், மற்றும் வீட்டில் பிராட்பேண்ட் தொடர்பு வாங்குவதற்கு வசதியில்லாத கீழ்-மத்தியதர வகுப்பைச் சேர்ந்த உள்ளூர்வாசிகள் ஆகியோரிடையே பிரபலமாக இருந்தது.

பிராட்பேண்ட் மற்றும் யு.எஸ்.பி. டாங்கிள் மூலமான இணையதள தொடர்பு இவற்றுக்கு இணையாக, இந்திய சந்தைக்கென்றே ${ }^{33}$ தயாரிக்கப்பட்ட மலிவான, சீன தயாரிப்பு மொபைல் போன்கள், தரமுத்திரையுள்ளவை (சாம்சங் போன்றவை) மற்றும் தரமுத்திரை அல்லாதவை போன்றவற்றின் வருகை இருந்தது. இதையொட்டி, கட்டுப்படியாகக் கூடிய கட்டங்களில் தரவுத் திட்டங்கள் அறிமுகமாயின. இதனால், குறைந்த வருமானமுள்ளவர்கள் கூட இணையத்தொடர்பு ${ }^{34}$ பெறமுடிந்தது.இந்த மலிவான போன்கள், சராசரி ஸ்மார்ட்போன்களின் குறைக்கப்பட வடிவங்களாகும், அதாவது, குறைவான தொழில்நுட்ப செயல்திறன் கொண்டவை. இது போன்ற போன்கள் இந்தியாவின் தகவல் பரிமாற்ற அமைப்பை ${ }^{35}$ முற்றிலும் மாற்றியமைத்தன. இவை பஞ்சக்கிராமியின் மீதும் குறிப்பிடத்தக்க தாக்கத்தை ஏற்படுத்தின. ஸ்மார்ட்போன்களின் ${ }^{36}$ தேசீய ஊடுருவல் 2014-ல் சராசரியாக 21 சதவிகிதம் ${ }^{37}$ இருந்தது. ஆனால் பஞ்சக்கிராமியிலோ, ஒரு கூர்நோக்கு ஆய்வு மற்றும் மாதிரி கணக்கெடுப்பின்படி, குடியிருப்பாளர்களிடையேயான ${ }^{38}$ ஊடுருவல், 48 சதவிகிதம் இருந்தது. திறன் வாய்ந்த ஐ.டி பணியாளர்களின் ${ }^{39}$ மிதக்கும் மக்கட்தொகையின் மத்தியிலான இந்த சராசரி, 70 சதவிகிதம் அளவு உயர்ந்ததாக இருக்கக்கூ- 
டும். இருப்பினும், மக்கள் வைத்திருந்த ஸ்மார்ட்போன்கள், அவற்றின் தர அடையாளம், தொழில்நுட்ப திறன் மற்றும் விலைகள் ஆகிய வகைகளில் வித்தியாசப் பட்டன. உயர்வகை ஸ்மார்ட்போன்கள், பெரும்பாலும், உயர்-மத்தியதர வகுப்பை சேர்ந்த ஐ.டி பணியாளர்கள் மற்றும் அவர்களின் குடிம்பத்தினர் அல்லது இந்தப் பகுதியின் வளமான நீண்டகால குடியிருப்பாளர்கள் ஆகியோரால் பயன்படுத்தப்பட்டன. இருப்பினும், ஒரு சில சமயங்களில், கீழ்த்தட்டு சமூகப்பொருளாதார வகுப்புகளில் இருந்து வந்த இருபதுகளின் ஆரம்பத்தில் இருக்கும் இளம் ஆண்களும் இது போன்ற விலையுயர்ந்த ஸ்மார்ட்போன்கள் வைத்திருந்தனர். அவர்கள், தங்களது சமூக வட்டங்களில் இருந்து கடன் வாங்கியோ அல்லது இதுபோன்ற போன் விற்பனையாளர்கள் ${ }^{40}$ வழங்கும் தவணைமுறை திட்டங்கள் மூலமாகவோ இந்தப் போன்களை வாங்கியிருந்தனர். இதுபோன்ற வாடிக்கையாளர்களுக்கு, போன்களின் அளவும், செயல்பாடுகளும் தம்மையொத்தவர்களிடையே ஒரு அந்தஸ்துச் சின்னமாக இருந்தது.

பஞ்சக்கிராமியில் உள்ள மொபைல் போன் கடைகளில் எடுக்கப்பட்ட பேட்டிகள் வெளிப்படுத்திய தகவல் என்னவென்றால், ரூ. 3000/- முதல் ரூ. 5000/-41 வரையிலான விலையில் இருக்கும் ஆரம்ப நிலை போன்கள் தான் மிகவும் பிரபலமான ஸ்மார்ட்போன்கள் என்பது தான். சாம்சங் நிறுவனத்தின், இந்திய சந்தைக்கான மொபைல் போன் வடிவங்கள் தான், இந்த விலை அளவில் போன்களை தேர்வு செய்யும் வாடிக்கையாளர்களிடையே மிகவும் பிரபலமாக இருந்தவை. பஞ்சக்கிராமியில், ஐ-போன்கள், ஹெச்.டி.சி, சோனி, மற்றும் விலையுயர்ந்த சாம்சங் ஸ்மார்ட்போன்கள் போன்ற உயர் செயல்திறன் கொண்ட மொபைல் போன்களை வாங்க விரும்பும் வாடிக்கையாளர்கள், அவற்றை, பஞ்சக்கிராமியில் உள்ள கடைகளில் வாங்குவதை விட, சென்னையில் உள்ள தர அடையாளக்கடைகளில் வாங்குவதையே விரும்பினர். இதற்கான முக்கிய காரணம், அது போன்ற பெரிய கடைகளில் கிடைக்கும் அதிகமான விருப்பத்தேர்வுகள், தவணை முறைகள் மற்றும் விற்பனைக்கு பின்னான சேவை ஆகியவை தான்.

மற்ற இடங்களைப் போலவே, இங்கும் ஸ்மார்ட்போன்கள், அழைத்தல், குறுஞ்செய்தி அனுப்புதல், விளையாடுதல், மின்னஞ்சல் அனுப்புதல்/பெறுதல், முகநூல், டுவிட்டர், லிங்க்ட்-இன், வாட்சப் போன்ற சமூக ஊடகங்களை அணுகுதல், புகைப்படங்களை எடுத்து சமூக ஊடகங்களில் பதிவிடல், திரைப்படங்கள் மற்றும் காணொளிகள் பார்த்தல் போன்ற பலவற்றை செய்வ- 
தற்கு பயன்படுத்தப்பட்டன. ஸ்மார்ட்போன்களில் விளையாட்டுக்கள் விளையாடுவது தங்கள் இருபது மற்றும் முப்பதுகளில் இருப்பவர்களுக்கு ஒரு பொழுதுபோக்காக ${ }^{42}$ மட்டும் இருந்தாலும், பல பதின்பருவத்தினருக்கு அது அவர்களின் வாழ்வின் ஒரு முக்கிய அங்கமாகும். உண்மையில், பஞ்சக்கிராமியில் உள்ள பல பதின்பருவத்தினர், முகநூல் உறுப்பினர்களானதே இதுபோன்ற விளையாட்டுக்கள் விளையாடுவெதற்காகத் தான். அவர்கள் ஸ்மார்ட்போன்கள், கணினிகள், டாப்லேட்-கள் ${ }^{33}$ ஆகியவற்றை இந்தக் காரணங்களுக்காக ${ }^{44}$ பயன்படுத்துகிறார்கள்.

குறைந்த வருமானமுள்ள குழுக்களில் மடிக்கணினி வைத்திருந்த (தமிழக அரசால், 'ஒரு குழந்தைக்கு ஒரு மடிக்கணினி' என்ற திட்டத்தின் மூலம் பெறப்பட்டது| ${ }^{45}$ ஒரு சிலர், அவற்றை, மலிவான ஸ்மார்ட்போன்களின் மூலம் பெறப்பட்ட முன்கட்டணம் ெலுத்திய இணையதள தொடர்போடு இணைத்து ${ }^{46}$ பயன்படுத்தினர். இது, யூட்யூபில் புதிய தொலைக்காட்சி நிகழ்ச்சிகளைப் பார்ப்பதற்கும், புதிய தமிழ் திரைப்படங்களின் திருட்டு வடிவங்களைப் ஒரு வழிவகையாக அமைந்தது. சுருங்கச்சொன்னால், அது அவர்களின் பிரத்யேக தொலைக்காட்சி மற்றும் திரையரங்காகவும், கட்டுப்படியாகக்கூடிய பொழுதுபோக்கை வழங்கக்கூடியதாகவும் இருந்தது. மத்தியதர வகுப்பினரிடையேயும் இதே தான் நடந்தது. என்னவொன்று, அவர்கள் இவையனைத்தையும் செய்வதற்கு, தங்கள் தர அடையாளமுள்ள மடிக்கணினி அல்லது ஒருங்கிணைத்த மேசைக் கணினியில் 48 பிராட்பேண்ட் அல்லது யு.எஸ்.பி. டாங்கிள் மூலமான இணையதள தொடர்பை பயன்படுத்தினர். தற்போதுள்ள ஆளுங்கட்சி, மொபைல் போன்கள், மடிக்கணினிகள், இலவச வைஃபை தொடர்பு ${ }^{49}$ போன்ற இலவசங்களை அறிவித்துள்ளதில் இருந்து, எதிர்காலத்தில், இணையதள மற்றும் சமூக ஊடக உபயோகத்தில் கூரான உயர்வு இருக்கும் என்று எதிர்பார்க்கப்படுகிறறது.

\section{குரல்வழி தகவல் மற்றும் சமூக ஊடகங்களுக்கு இடையேயான விருப்பத்தேர்வு.}

ஸ்மார்ட்போன்கள் வைத்திருப்பதால் ம மட்டுமே அனைத்து தகவல் பரிமாற்றங்களும், சமூக ஊடகங்கள் வாயிலாக மட்டுமே நடப்பதாகிவிடாது. உண்மையில், பல்வேறு சமூக காரணிகள், பஞ்சக்கிராமியில் தகவல் பரிமாற்ற முறைகளை நிர்ணயம் செய்கின்றன. உதாரணத்திற்கு, குடும்பத்தின் மூத்த 
உறுப்பினர்களுடனான தகவல் பரிமாற்றம் பெரும்பாலும் குரல்வழியாகத்தான் நிகழும். இதற்கு, பழமையான ஊடக முறையின் பரிச்சயமான சௌகரியம், பெற்றோர்களின் கட்டுப்பாடு மற்றும் இளம் உறுப்பினர்களுக்கு, மற்ற ஊடகங்களை உபயோகிக்க அனுமதி மறுப்பு போன்ற பல காரணங்கள் இருக்கலாம். சிலசமயங்களில், கல்வியறிவு, ஆங்கிலம் போன்ற குறிப்பிட்ட மொழிகளில் தேர்ச்சியின்மை, புதிய திறன்களை கற்றுக்கொள்வதில் ஆர்வமோ, நம்பிக்கையோ இல்லாமல் இருப்பது போன்றவை கூட குரல்வழி தகவல் பரிமாற்றத்திற்கு வழிவகுக்கும் காரணிகளாக இருக்கக்கூடிம்.

இது ரவியின் அன்னையின் விஷயத்தில் தெளிவாகத் தெரிகிறது. அவரது குறைபாடு, அன்றாட வழமையான தகவல் பரிமாற்றத்திற்கு ${ }^{50}$ கூட ரவியை, அவருக்கு குரல்வழி தொலைபேசி அழைப்பு விடுப்பதற்கு வற்புறுத்துகிறது.

கீழ்-மத்தியதர வகுப்புக் குடும்பத்தை சேர்ந்த 25 வயதாகும் ரவி, பஞ்சக்கிராமியில் உள்ள ஒரு மருத்துவத் தகவல் வகைப்பாட்டு நிறுவனத்தில் தரவு உள்ளீட்டாளராக பணிபுரிகிறார். அவரது தங்கை, திருமணமாகி சென்னையில் வாழ்கிறார். விவசாயியான அவரது தந்தை, கூடுதல் வருமானத்திற்காக குழாய்ப் பணியாளராகவும் பணிபுரிகிறார். அவரது தாய் ஒரு இல்லத்தரசி. அனைத்து குடும்ப உறுப்பினர்களும் தற்சமயம் தனித்தனியே மொபைல் போன் வைத்திருந்தாலும், அவரது தங்கை, தனக்கான தனிப்பட்ட மொபைல் போனை, திருமணத்திற்கு பிறகு தான் பெற்றார். அதற்கு முன், அவர் தனது தாயினது போனை தான் உபயோகித்துக் கொண்டிருந்தார். ரவியினது பெற்றோர்கள், ஸ்மார்ட்போன் அல்லாத சாதாரண மைக்ரோமேக்ஸ் போன் வைத்திருந்தார்கள். ரவி சாம்சங்கின் காலக்சி கோர் ஸ்மார்ட்போன் வைத்திருக்கிறார்.

பணிநாட்களில், சாதாரணமாக, ரவி, மதிய உணவை வீட்டிலிருந்தே எடுத்துச் சென்றுவிடுவார். உணவு தயாராவதற்கு தாமதமாகும் நாட்களில், அவர் பின்காலைப் பொழுதில், தனது அன்னைக்கு மொபைல் போனில் அழைப்பு விடுத்து, மதிய உணவுக்கு வீட்டுக்கு வரலாமா என்று கேட்டுக்கொள்வார். இந்த மொத்த உரையாடலும் சுமார் இரண்டு நிமிடங்கள் தான் எடுக்கும். அதோடு சேர்த்து தனது அன்னையிடம், ஏதாவது அவசர வேலையை வரும் வழியில் முடிக்க வேண்டுமா என்றும் ரவி கேட்டுக்கொள்வார். அவர்களுக்கிடையேயான புரிதல் என்னவென்றால், ரவி தன் தாய்க்கு அழைப்பு விடுக்கவில்லை என்றால், அவரது தாய் அவருக்காக மதிய உணவு தயாரித்து வைத்திருக்க மாட்டார் என்பது 
தான். ஒருசமயம், ரவிக்கு அலுவலகத்தில் இருந்த அவசர வேலையினால், அவரால் தனது அன்னையை அழைக்க முடியவில்லை. பதிலாக அவர், தன் அன்னைக்கு தான் மதிய உணவுக்கு வருவதாக ஒரு குறுஞ்செய்தியை ${ }^{51}$ அனுப்பினார். அவர் வீட்டிற்கு சென்ற பொழுது, அவரது அன்னை உணவு எதுவும் தயாரித்திருக்காதது கண்டு ஆச்சரியப்பட்டுப் போனார். கூடுதலாக, அவரது அன்னையும், ரவி உணவுக்கு வருவதாக தகவல் தெரிவிக்கவில்லை என்று கூறி மேலும் அதிர்ச்சியூட்டினார். அப்படியில்லை என்று நிரூபிப்பதற்காக, ரவி, தனது அன்னையின் மொபைல் போனை வாங்கி, தான் அனுப்பிய குறுஞ்செய்தியை அவருக்கு காட்டினார். அப்போது தான் அவருக்கு, தன் தாய் எந்தவொரு குறுஞ்செய்தியையும் படிப்பதில்லை என்பது தெரிய வந்தது. குறுஞ்செய்திகளை எப்படிப் படிப்பது என்பது, குறிப்பாக ஆங்கிலத்தில் உள்ள செய்திகளை எப்படிப் படிப்பது என்பது அவருக்கு தெரிந்திருக்கவில்லை. இதைக்கேட்டு ரவிக்கு மிகவும் ஆச்சரியமாகிப் போயிற்று. ஏனெனில், முன்பொருமுறை, அவர் இதுபோல் அனுப்பிய குறுஞ்செய்தி ஒன்றிற்கு, அவருக்கு தாயின் மொபைல் மூலம் பதில் வந்திருக்கிறது. அந்த பதில், அவரது தங்கை அனுப்பியது என்பதை அவர் பின்னர் தான் அறிந்து கொண்டார்.

இப்போதெல்லாம், ரவி தவறாமல் தனது தாயை அழைத்துப் பேசிவிடுகிறார். தன் தாயுடன் உரையாடுவெதற்கு, பேசுவதே சிறந்த வழி என்பதை அவர் கண்டுகொண்டார். அவரின் கூற்றுப்படி, அவரது தாய்க்கு, தமிழில் குறுஞ்செய்தி அனுப்புவது எப்படி என்றறிந்து கொள்ளும் ஆர்வம் கூட இருக்கவில்லை. இப்போது, இருவரும் சேர்ந்து, ரவி, தன் தாயுடன் பேச முடியாத அளவிற்கு பணியில் மும்முரமாக இருக்கும் சமயத்திலும், தகவல் பரிமாறிக்கொள்ள ஒரு வழியை கண்டுபிடித்து விட்டனர். அவர், தன் தாய்க்கு ஒரு தவறிய அழைப்பு அதாவது மிஸ்டு கால் 52 கொடுப்பார். அன்னையின் போன் இரண்டு முறை ஒலித்து அடங்கினால், ரவி மதிய உணவிற்கு வருவார் என்பது செய்தி. அவர் அழைக்கவில்லை என்றால், வரமாட்டார் என்பது செய்தி. ரவியைப் பொறுத்தவரை அவரது தாய், தரைவழி தொலைபேசியை மொபைல் போன் ஈடுகட்டியிருக்கிறது அவ்வளவே. ஏனெனில், அவரது தாய், தனது மொபைல் போனை, அழைப்பதற்கன்றி வேறு எதற்கும் பயன்படுத்துவது இல்லை.

ரவி, தனது தந்தையை வெகு அபூர்வமாகத் தான் மொபைல் போனில் அழைக்கிறார். அதுவும் பணிநேரங்களில் 
அழைப்பதே இல்லை. ஏனெனில் அவரது தந்தை, வயலிலோ அல்லது குழாய்ப் பணியிலோ மும்முரமாக இருக்கக்கூடும். எனினும், அவரது தந்தைக்கு குறுஞ்செய்திகளை படிக்கத்தெரியும். ஆகவே, ரவி, தனது தந்தைக்கு, தமிழிலேயோ அல்லது ஆங்கிலத்தில் எழுதப்பட்ட தமிழ் வார்த்தைகள் வடிவிலோ குறுஞ்செய்தி அனுப்பி விடுவொர். அவருடைய செய்திகளெல்லாம் மிகவும் குறுகியவை. சொல்லவேண்டிய தகவலை சுருக்கமாகச் சொல்பவை. உதாரணத்திற்கு, அவர் தந்தைக்கு அவர் அனுப்பிய ஒரு செய்தி, "அரிசி வாங்கியாச்சு" என்பதாகும். தன் தந்தையும் அரிசி வாங்கிவிடக்கூடாதே என்பதற்காக அனுப்பிய குறுஞ்செய்தி தான் அது. அவரது தந்தை அபூர்வமாகத் தான் பதில் அனுப்புவார். ஆனாலும், ரவிக்கு தன தந்தை செய்தியை பிடித்துவிட்டார் என்பது தெரியும். யாருக்காவது தெரிவிக்கவேண்டிய ஏதாவது செய்தியை தன் தாயிடம் கூறினால், அது ஒட்டுமொத்த குடும்பத்திற்கும் தெரிவிக்கப்பட்டுவிடும் என்பதும் அவருக்குத் தெரியும். அவர் விளையாட்டாக, தன் தாயை, தொலைபேசி இணைப்பகம் என்று குறிப்பிடுவார்.

ரவி, தான் பணியில் இருக்கும் நேரங்களில், தன் தங்கையுடனான உரையாடல்களை கட்டுப்படுத்திக்கொண்டாலும், (வேலை நேரத்திற்கு பிறகோ அல்லது வார இறுதியிலோ தான் அவருக்கு மொபைல் போனில் அழைப்பு விடுப்பார்) அவருக்கு எப்போதாவது குறுஞ்செய்திகள் அனுப்புவார். அவர் தன் தங்கைக்கு அனுப்பும் அநேகமான குறுஞ்செய்திகள், வேடிக்கைகளாக இருக்கும் (ஆனாலும் விரசமானவற்றை தவிர்த்துவிடுவார்). அவரது தங்கை அவர்களின் தாயை, ஒரு நாளில் பலமுறை வெறும் அரட்டையடிப்பதற்காக போனில் அழைப்பார். அவர், அவர்களது தந்தையுடனும் நாளில் ஒருமுறையேனும் பேசிவிடுவார். பொதுவாக, மொத்த குடும்பத்துடனான அளவளாவல்கள், வார இறுதிகளில் மட்டுமே நடைபெறும். தன் பெற்றோர், தன் தங்கையின் குரலை நாளில் ஒருமுறையேனும் கேட்டுவிடவேண்டும் என்று நினைப்பர் என்று ரவி குறிப்பிடுகிறார்.

ரவியின் தாயின் வழக்கு, கல்வியறிவு மற்றும் தொழில்நுட்ப அறிவு சம்பந்தப்பட்ட விசாரங்களால், எழுத்துவழியை விட, குரல்வழி தகவல் பரிமாற்றம் எவ்வாறு விருப்பத்தேர்வாக இருக்கிறது என்பதை எடுத்துக்காட்டுகிறது. பெற்றோருக்கும் பெண்களுக்கும் இடையே உணர்வுபூர்வ விசாரங்களும் எழக்கூடும் என்பதையும் இது எடுத்துக்காட்டுகிறது.

இப்போது, தனது படிப்பின், இறுதியாண்டில் இருக்கும் ஷோபனா என்ற 22 வயதாகும் கல்லூரி மாணவியின் 
வழக்கைப் பார்ப்போம். அவரிடம், அவரது சித்தப்பா பரிசளித்த நோக்கியா ஸ்மார்ட்போன் ஒ ஒன்றும், நோக்கியாவின் சாதாரண (ஸ்மார்ட்போன் அல்லாத) போன் ஒன்றும் இருக்கிறது. ஷோபனா, தனது ஸ்மார்ட்போனை, தன் நண்பர்களுடன் அளவளாவுவதற்கும், சமூக ஊடக நடவடிக்கைகளுக்கும் பயன்படுத்துகிறார். தனது மற்ற போனை, தன் பெற்றோர் மற்றும் குடும்பத்தினருடன் உரையாடுவதற்கும் அவர் பயன்படுத்துகிறார். இந்த இரண்டாவது எண்ணை, தனது நண்பர்கள் யாருக்கும் அவர் கொடுப்பதில்லை. இதுபோன்ற, தகவல் பரிமாற்ற வழிகளின் தனிப்பிரிவினை, அவர் ஒருமுறை கல்லூரியில் இருந்து தாமதமாக வந்தபோது நடந்த, அவரது பெற்றோர் சம்பந்தப்பட்ட ஒரு நிகழ்ச்சிக்கு பிறகு அமலுக்கு வந்தது. ஷோபனா, தான் தாமதமாக வருவது பற்றி, தன் தாய்க்கு செய்தி அனுப்பியதாகவும், உடனே அவரது தாய் அவரை போனில் இரண்டு மூன்று முறை அழைத்ததாகவும், பதில் எதுவும் வராமல் போகவே, அவர் எரிச்சலும், கவலையும் அடைந்ததாகவும் கூறுகிறார். அவரது தாயின் கண்ணோட்டம் என்னவென்றால், அவருக்கு வேண்டியதெல்லாம், ஷோபனா, பாதுகாப்பாக இ இருக்கிறார் என்று அறறந்துகொள்ளவேண்டியது மட்டுமே, ஆதலால், இதுபோன்ற சமயங்களில், அவரது போன் அழைப்பிற்கு பதில் அளித்தல் மிகவும் அவசியம் என்பது தான்.

ஒற்றைக் குழந்தையான ஷோபனா, தன் பாதுகாப்பு, தனது குடும்பத்தாருக்கு எவ்வளவு முக்கியம் என்பதை நன்கு அறிவார். தனது தாய், நாட்டில் நடக்கும் பல்வேறு பாலியல் பலாத்காரம் ${ }^{33}$ மற்றும் கொலை பற்றிய செய்திகளை பார்த்தும் கேட்டும், அதிக பதட்டமடைகிறார் என்று அவர் கருதுகிறார். அவரது தாயும், ஓரளவிற்கு அவரது தந்தையும், தமது அழைப்புகள் உடனே பதிலளிக்கப்படவில்லை என்றால், மிகவும் பதட்டமும் எரிச்சலும் அடைகின்றனர். ஷோபனாவின் அன்னை, அவர் நண்பர்களுடன் மணிக்கணக்கில் பேசுவதாகவும், அதனாலேயே, அவரது அழைப்புகள் பதிலளிக்கப்படவில்லை என்றும் குற்றம்சாட்டுகிறார். அதனாலேயே, ஷோபனா, தனது குடும்பத்திற்கும், நண்பர்களுக்கும் ஒரே எண்ணை பயன்படுத்துவது இல்லை. தனது பெற்றோருக்காக, தனிப்பட ஒரு போன் எண்ணை வைத்திருக்கிறார். பெற்றோருக்காக வைத்திருக்கும் எண்ணுள்ள போனில், இணைய தரவுத் திட்டம் எதுவும் கிடையாது. ஆனால் அவரது ஸ்மார்ட்போனில், 3ஜி இணைய தரவு இணைப்பு வைத்திருக்கிறார். அவரது 
பெற்றோர் சாதாரணமாக குறுஞ்செய்திகள் மூலம் தகவல் பரிமாறிக்கொள்வார்கள். ஆனால் ஷோபனாவின் விஷயத்தில், அவருடன் குரல்வழி தகவல் பரிமாற்றம் தான் வேண்டும் என்று குறிப்பாக இருக்கிறார்கள்.

சமூக ஊடக அறிவுத்திறன் கொண்ட பெரியவர்கள் உள்ள உயர்-மத்தியதர வகுப்பைச் சேர்ந்த குடும்பங்களிலும் கூட, அவர்களின் குடும்ப வட்டத்தினுள்ளான அதிகாரநிலைப்படி மற்றும் மரியாதை குறித்த எதிர்பார்ப்புகள், தலைமுறைகளுக்கு இடையேயான தகவல் பரிமாற்ற தளத்தின் தேர்வில் ஆதிக்கம் செலுத்துகின்றன. ஒருசில வகையான தகவல் பரிமாற்றத்திற்கு புது ஊடகங்களை பயன்படுத்துவது, ஆட்சார்பற்றதாகவும், மரியாதைக்குறைவானதாகவும் அவர்கள் கருதுகின்றனர். குடும்ப மூத்தவர்களிடமிருந்து இந்த வகையிலான எதிர்பார்ப்பு, ஒரு பெரிய மருத்துவ நிறுவனத்தின் பயிற்சி மற்றும் வளர்ச்சித் துறையின் தலைவராக இருந்து பணி ஓய்வு பெற்ற 65 வயதான ராகவனின் வழக்கில் தெளிவாகத் தெரிகிறது. சமூக ஊடகங்களின் வாயிலாக தகவல் அனுப்புவதைவிட, மக்கள் தன்னை அழைத்து தங்கள் வாழ்க்கையின் நிகழ்வுகளைப் பற்றி தெரிவிப்பதையே அவர் விரும்புகிறார். ஒருசில வாழ்க்கை நிகழ்வுகளைப் பற்றி மின்னஞ்சல் அனுப்புவதை கூட ஆட்சார்பற்றதாகவும் பெரியவர்களிடத்து மரியாதை குறைவான செயலாகவும் அவர் கருதுகிறார்.

இங்கு கால இசைவற்ற தகவல் பரிமாற்றத்தை ${ }^{54}$ விட, குரல்வழி மற்றும் ஒத்தியங்கும் தகவல் பரிமாற்றத்திற்கான விருப்பத்தேர்வை நாம் பார்க்கிறோம். ஆகவே, வாட்சப் மூலமாக குரல்வழி தகவல் அனுப்புவதென்பது அவர்களுக்கு போன் உரையாடல்களைப் போலவோ அல்லது வெகு தூரத்தில் இருப்பவர்களுக்கு ஸ்கைப்/கூகுள் ஹாங்அவுட் உரையாடல்களைப் போலவோ திருப்தி அளிக்காது. இருப்பினும், இந்தக் கண்ணோட்டம், பணி அல்லது கல்வி நிமித்தம் வெளிநாடுகளுக்கு புலம்பெயர்ந்த குடும்ப உறுப்பினர்கள் விஷயத்தில் சற்றே மாறுபடுகிறது. குடும்ப உறவுமுறைகளை ஆராயும் அத்தியாயம் 4, இந்த விஷயத்தை இன்னும் விரிவாக கலந்தாய்வு செய்வதோடு, அதை மற்ற வகையான உறவுமுறைகளுக்கும் நீட்டிக்கிறது.

என்றாலும், மூத்தவர்கள் மற்றும் பெற்றோர்களின் தகவல் பரிமாற்ற தளங்களின் பயன்பாட்டிலிருந்து நாம் நகர்ந்தோமானால், சமூக ஊடகங்களால் நிறைந்திருக்கும் ஒரு பரந்த உலகத்தை நாம் சந்திக்கலாம். முதல் சமூக ஊடக கணக்கெ- 
டுப்பான Q1, அத்தியாயம் 1-ல் குறிப்பிட்டுள்ளபடி, 2013ஆம் ஆண்டு களப்பணி துவங்கியபோது, சமூக ஊடகங்களை 55 பயன்படுத்தும் 130 பதிலளிப்பாளர்களின் மாதிரியின் மீது நடத்தப்பட்டது. இதன் முடிவுகள், சமூக ஊடக பயன்பாட்டாளர்களின் இந்த மாதிரிக்கு முகநூல் மிகவும் பிரபலமான சமூக ஊடகத் தலமாகவும் (84 சதவிகிதம்), அடுத்த இடத்தில் வாட்சப் (62 சதவிகிதம்) மற்றும் டுவிட்டர் (34 சதவிகிதம்) இருப்பதாக தெரிவித்தன. லிங்க்ட்-இன்(31 சதவிகிதம்) போன்ற தளங்கள் இந்த கணக்கெடுப்பில் காணப்பட்டாலும், அவை ஐ.டி மக்கட்தொகை மற்றும் இதர பெருநிறுவனத்தில் பணிபுரியும் மக்கள் சார்ந்ததாக மட்டுமே இருந்தது. களப்பணியில் காலப்போக்கில், சமூக ஊடகங்களை பயன்படுத்தும் பஞ்சக்கிராமியின் குடியிருப்பாளர்களிடையே இந்த தளப் பிராபல்யம் பற்றிய பொதுவான போக்கிற்கான தொடர்ச்சியான ஆதாரங்கள் கிடைத்துக்கொண்டே இருந்தன. வாட்சப் மட்டும் இதற்கான விதிவிலக்காக இருக்கக்கூடிய வாய்ப்பிருக்கிறது. ஏனென்றால், களப்பணியின் காலத்தில் அதன் பிராபல்யம் முகநூலை ${ }^{56}$ மிஞ்சிவிட்டது.

மிகக்குறைவானவர்கள் மட்டுமே இ இ இ ம்்டாகிராமில் கணக்குகள் வைத்திருந்தனர். இன்றும் ப்ளாக்பெர்ரி போன்கள் வைத்திருக்கும் உயர்-மத்தியதர வகுப்பைச்சேர்ந்த பெருநிறுவன பணியாளர்களிடையே பி.பி.எம் இன்னும் பிரபலமாக இருந்தாலும், ஸ்னாப்ச்சாட்டோ அல்லது இன்ஸ்டாகிராமோ குறிப்பிடக்கூடிய அளவிற்கு ${ }^{57}$ காணப்படவில்லை. மக்கள் பல்வேறு தளங்களில் ஒரே சமயத்தில் உறுப்பினர்களாக இருந்தனர். உதாரணத்திற்கு, முகநூலில் உறுப்பினராக இருக்கும் மக்கள் வாட்சப் மற்றும் டுவிட்டரில் உறுப்பினர்களாக இருந்தனர். இதற்கு நேர்மாறும் காணப்பட்டது.

ஸ்மார்ட்போன் வகைகள் மற்றும் அவற்றிற்கான தரவுத்திட்டங்கள் முன்னமே கூறியபடி பரவலாக வேறுபட்டாலும், Q1 மூலம் கணக்கெடுக்கப்பட்ட சமூக ஊடக பயன்பாட்டாளர்களில் 83 சதவிகிதத்தினர் ${ }^{58}$, தாங்கள் ஸ்மார்ட்போன்கள் மூலமாக சமூக ஊடகத்தளங்களை பயன்படுத்தியதாக கூறியுள்ளனர்.சமூக ஊடகங்களை கையாளுதற்கு பயன்படுத்தப்பட்ட மற்ற பிரபலமான வழிகள், மடிக்கணினிகள் (61 சதவிகிதம்) மற்றும் மேசைக் கணினிகள் (47 சதவிகிதம்). பலர் பிராட்பேண்ட் மற்றும் யு.எஸ்.பி. டாங்கிள் ஆகியவற்றை இணையதள தொடர்புக்கு பயன்படுத்தினாலும், இதற்காக தங்கள் போன்களை இணைத்தவர்களும் உண்டு. இந்த அத்தியாயத்தின் பின்வரும் பகுதிகள், மூன்று முக்கிய சமூக 
ஊடகத்தளங்களான முகநூல், வாட்சப் மற்றும் ஆகியவை பஞ்சக்கிராமியில் தோன்றியபடியான அறிமுகத்தை அளிக்கிறது. லிங்க்ட்-இன் ஐ.டி துறையினரால் தகவல் ஞானம் பெறுவதற்கான வழிமுறையாகவும், தொழில்நெறிஞர் தொடர்புக்கான சாதனமாகவும் பயன்படுத்தப்படுகிறது. லிங்க்ட்-இன் குழுக்கள், உபயோகிப்பாளர்களுக்கு தொழில் துறை தொடர்புகள் ஏற்படுத்திக்கொள்ளவும், வேலைவாய்ப்புகளை மேம்படுத்திக்கொள்ளவும், அதேசமயம், அவரவர் தொழிநுட்ப களங்களில் ஏற்பட்டுள்ள புதிய முன்னேற்றங்களைப் பற்றிய செய்திகளை அறிந்துகொள்வதற்கும், தலைமை, தனிப்பட்ட மற்றும் தொழில்நெறி வளர்ச்சி பற்றிய கட்டுரைகளை பெறுவதற்கும் பெரிதும் உதவுகின்றன. ஐ.டி துறையினுள், லிங்க்ட்-இன் அறிவார்ந்த வலை தொடர்பாகவும், தொழில்நெறி சார் வாழ்வை மேம்படுத்திக்கொள்ள உதவும் தொடர்பாகவும் பார்க்கப்படுகிறது.

\section{முகநூல் - வர்க்கம், குலம் மற்றும் பாலினம்}

முகநூலின் இருப்பு, தங்கள் குடும்பங்களுடன் பஞ்சக்கிராமியில் குடியேறியுள்ள ஐ.டி பணியாளர்கள் மற்றும் இந்தப்பகுதியின் நீண்டகால குடியிருப்பாளர்கள் ஆகிய இருசாரார் இடையேயும் காணப்பட்டாலும், வர்க்கம், குலம், வயது, பாலினம் போன்ற சமூகக் காரணிகளின் தாக்கம் இந்தக் குழுக்களின் முகநூல் பயன்பாட்டின் மீது இருக்கத்தான் செய்கிறது. இந்தக் காரணிகளின் செல்வாக்கை ஆராய்வது, பஞ்சக்கிராமியில் முகநூல் எப்படி பொருந்துகிறது என்பதை பொதுவாக புரிந்துகொள்வதற்கு உதவியாக இருக்கும்.

பஞ்சக்கிராமியில் உள்ள கீழ்-மத்தியதர வகுப்பினர் மற்றும் குறைந்த வருமானமுள்ள குழுக்களிடையே இளைஞர்கள் தான் முக்கியமாக முகநூலில் இருப்பவர்கள். இந்தக் குழு பெரும்பாலும் படித்த, வாகன ஓட்டுநர், வீட்டுப் பொறுப்பாளர், நுழைவு-நிலை ஐ.டி ஆதரவுப் பணியாளர் போன்ற ஏதாவதொரு பணியில் இருப்பவர்களை உள்ளடக்கியதாகும். இவர்கள் பொதுவாக, முகநூலை தங்களது ஸ்மார்ட்போன்களில் முன்கட்டணம் செலுத்திய இணையதள இணைப்பின் மூலம் உபயோகிக்கிறார்கள். அவர்களுடைய நண்பர்கள் பட்டியலில் இருக்கும் பெண் நண்பர்களில் பலர், மற்ற பகுதிகளையோ அல்லது மற்ற இந்திய மாநிலங்களையோ சேர்ந்தவர்களாக இருப்பார்கள். ஏனெனில், இந்தப்பகுதியில், 
இவர்களின் சமுதாயப் படிநிலையை சேர்ந்த மணமாகாத இளம்பெண்கள், போன்கள் மற்றும் முகநூல் உபயோகிப்பதில் இருந்து தடுக்கப்படுகிறார்கள். இந்த சமுதாய படிநிலைகளில் உள்ள சில இனக்குழுக்களை சேர்ந்த ஆண்கள், முகநூலை, தங்களை குடும்பங்களில் உள்ள பெண்களின் மீது ஆபத்தான செல்வாக்கு உடைய ஒன்றாக பார்க்கிறார்கள். முகநூல் பற்றிய இதுபோன்ற கண்ணோட்டம், அவர்கள் அந்தத் தளத்தை, சரசப்பேச்சிற்கான ஒரு தளமாக உபயோகிப்பதாலும், குலத்தினிடை காதல்கள் பற்றிய பயத்தாலும், ஒருபெண் இதுபோன்ற நட்புறவுக்கெல்லாம் ${ }^{59}$ ஆட்படுத்தப்படுகிறாள் என்று அவர்கள் சமூகத்தில் உள்ள மற்றவர்கள் அறிய நேர்ந்தால், தங்களுடைய குடும்ப கௌரவத்தை இழக்க நேரிடுமோ என்ற பயத்தாலும், வருவது தான். இந்தப்பகுதியில் உள்ள தத்தம் சமூகத்தை சேர்ந்த பல பெண்கள், முகநூலில் இ இருக்கக்கூடும் என்று இந்த ஆண்கள் ஒத்துக்கொள்கிறார்கள். ஆனால் அவ்வாறு அவர்கள் இருப்பது தெரியவந்தால் அவர்களை இதை விடுக்குமாறு கூறுவார்கள் என்றும் அவர்கள் கூறுகின்றனர்.

ஆகவே, முகநூலில் குலம் சம்பந்தமான முக்கிய தாக்கம், பாலின விசாரங்களின் வடிவில் இருக்கிறது. உதாரணத்திற்கு, குலத்தின் அடிப்படையிலான ஒரு அரசியல் குழுவின் தலைமை (இந்தக் குலம், மற்ற ஆதி திராவிட வகுப்பினரை விட, சமூக படிநிலையில் தாங்கள் உயர்ந்த நிலையில் இருப்பதாக கூறிக்கொள்கிறது), தங்கள் குலப்பெண்கள், ஆதிதிராவிட வகுப்பைச் சேர்ந்த இளம் ஆண்களால், குறிவைத்து கவரப்படுகிறார்கள் என்றும், இது குலத்தினிடை திருமணங்களுக்கு வழிவகுப்பதால், உடனே நிறுத்தப்படவேண்டும் என்றும் அறிவித்துள்ளது. பெரும்பாலும் கீழ்-மத்தியதர வகுப்புப் பின்னணி கொண்ட இந்தக் குலக்குழுவில் உள்ள இளம் ஆண்கள், தங்கள் தலைமையுடன் ஒத்துப்போகக் கூடியதான கருத்துக்களை வெளியிட்டனர். இதனால், இந்தக் குடும்பங்களில் ஏற்கனவே முகநூல் மற்றும் மொபைல் போன்களை உபயோகிப்பதில் இருந்து தடை செய்யப்பட்ட பெண்களின் மீதான கட்டுப்பாடுகள் இன்னும் இறுக்கப்பட்டது. இது அனைத்தும், தங்கள் குலப்பெண்கள், தாழ்ந்த குலத்தைச் சேர்ந்த இளம் ஆண்களிடம் இருந்து காப்பாற்றப்பட வேண்டும் என்ற ஒரு கூற்றால் நியாயப்படுத்தப் படுகிறது. இதுபோன்ற கட்டுப்பாடுகௌால், இவர்கள் குலத்தைச் சேர்ந்த இளம் பெண்கள், தாழ்ந்த குலத்தைச் சேர்ந்த ஆண்களால் கவரப்பட்டு, தூய்மைக்கேடாக ${ }^{60}$ அவர்கள் நினைக்கும் குலத்தினிடை மணம் 
புரிந்துகொள்ளும் அபாயம் தவிர்க்கப்படும் என்று அவர்கள் நம்புகிறார்கள்.

இந்த வர்க்கம் மற்றும் குலத்தைச் சேர்ந்த இளம்பெண்களிடம் நடத்தப்பட்ட பேட்டிகளின் மூலம், அவர்களில் பெரும்பாலானோர், முகநூல் கணக்குகள் வைத்திருப்பது தெரியவந்தது. நகரத்தில் இருக்கும் தங்களையொத்தவர்கள் அனுபவிக்கும் சமூக வலைதொடர்பில் இருந்து விடுபடப்படுவதை அவர்கள் விரும்பவில்லை. இருப்பினும், இந்தக் கணக்குகளின் பயன்பாடு, அவர்கள் விரும்பும் அளவிற்கு தொடர்ச்சியாகவோ முனைப்பாகவோ இருக்கவில்லை. இவர்கள் தங்கள் முகநூல் கணக்குகளை, கல்லூரியில் சென்னையில் இருந்தோ அல்லது கட்டுப்பாடு குறைவான பின்னணியில் இருந்தோ வரும் தங்கள் நண்பர்கள் கொண்டுவரும் ஸ்மார்ட்போன்கள் மூலம் உபயோகிக்கிறார்கள் ${ }^{61}$. இது, பெண்கள் சமூக ஊடகங்களை பயன்படுத்துவதில் ${ }^{62}$, கடுமையான கட்டுப்பாடுகெளை விதிக்கும், இதே பொருளாதார படிநிலையில் உள்ள மற்ற குலக்குழுக்களுக்கும் பொருந்தும். இதுபோன்ற கட்டுப்பாடுகள், சமூக ஊடகங்களை பயன்படுத்துவத்திலான ஒட்டுமொத்த தடையில் இருந்து, அதுபோன்ற பயன்பாட்டிற்கான நேர மற்றும் இடக்கட்டுப்பாடு வரை பலவகைப்படும். உதாரணத்திற்கு, மஞ்சுளா என்பவர் 20 வயதான, கீழ்-மத்தியதர வகுப்பைச்சேர்ந்த, ஒப்பீட்டளவில் குறைந்த கட்டுப்பாடுகெள விதிக்கும் குடிம்பத்தைச் சேர்ந்த கல்லூரி மாணவி. இவருக்கு சமூக ஊடகங்களை தங்கள் குடும்ப மேசைக் கணினி மூலம் பயன்படுத்துவதற்கு அன்றாடம், இரவு எட்டுமணி வரை மட்டுமே அனுமதி உண்டு.

மஞ்சுளாவிற்கு விதிக்கப்பட்ட கட்டுப்பாடுகள் பயன்பாட்டின் நேரம் அடிப்படையிலானதாக இருப்பது போல, பயன்பாட்டின் இடம் அடிப்படையிலான கட்டுப்பாடுகளும் இருக்கிறது. உதாரணத்திற்கு, 20 வயதாகும் அர்ச்சனா, பஞ்சக்கிராமியில் உள்ள ஒரு கல்லூரியில் பயிலுகிறார். அவரிடம் ஒரு ஸ்மார்ட்போன் இருக்கிறது. அவருக்கு அதை வீட்டிற்கு வெளியே பயன்படுத்த தடை விதிக்கப்பட்டிருக்கிறது. இது, போன்கள் மற்றும் சமூக ஊடகங்களை வீடு மற்றும் வீட்டிற்கு வெளியே பயன்படுத்துவது பற்றிய மற்றொரு கருத்தாகும் - பெரும்பாலான குடும்பங்கள், தங்கள் இளம் பெண் உறுப்பினர்களை, போன்கள் மற்றும் சமூக ஊடகங்களை, வீட்டிலிருந்து உபயோகிக்க அனுமதிக்கிறார்கள். ஏனெனில், அது அவர்களுக்கான பாதுகாப்பான சூழலாக இருப்பதோடல்லாமல், ஊடகத்தொடர்பு, குடும்பத்தினரின், கவனமான கண்பார்வையில் நடக்கும் 
என்பதும் தான் இதற்கான காரணம். வீட்டுக்குள்ளிருக்கும் குடும்பச்சூழல் பெண்களுக்கு பாதுகாப்பானது என்றும், வீட்டிற்கு வெளியில் இருக்கும் சூழல், ஆபத்தானது மற்றும் ஆணுக்குரியது ${ }^{63}$ என்பதான கருத்து, இந்த சமூகத்து மக்களுடனான உரையாடலில் பலமுறை வெளிப்பட்டது. அர்ச்சனா, தன்னுடைய நண்பரின் மொபைல் போனை பயன்படுத்தி, (அவரது நண்பர், அதே குலத்தைச் சேர்ந்தவர் ஆனால் கட்டுப்பாடு குறைவான குடும்பத்தில் இ இருந்து வந்தவர், சென்னையில் வாழ்பவர்) கல்லூரியில் இருக்கும் சமயம் முகநூலை உபயோகிக்கிறார். வீட்டிற்கு திரும்பியபின், தன்னுடைய மொபைல் போனை பயன்படுத்தி முகநூல் உபயோகத்தை தொடர்கிறார்.

இந்த இளம்பெண்களுக்கான கட்டுப்பாடுகள், அவர்களது தாய்மாரால் விதிக்கப்பட்டவை அல்ல. அவர்களை விட சுமார் மூன்றே வயது வித்தியாசத்தில் இருக்கும் அவர்களது சகோதரர்களால் விதிக்கப்பட்டவை. அவர்கள், தங்கள் சகோதரிகளை, அவர்கள் முகநூலில் இருக்கிறார்களா, அப்படி இருந்தால் யார்யாருடன் தொடர்பில் இருக்கிறார்கள் என்றெல்லாம் தொடர்ந்து கண்காணிப்பதன் மூலம், அவர்கள் மீது கடுமையான கட்டுப்பாட்டை செலுத்தினர். பொதுவாக ஒரு பெண்ணின் கல்லூரி நண்பர்களோ அல்லது நகரத்தில் உள்ள ஒன்றுவிட்ட சகோதர/சகோதரிகளோ தான் அவர்களுக்கு முகநூல் கணக்கை துவக்க உதவியாக இருந்தனர். இந்த இளம் பெண்களின் முகநூல் சுயவிவரப்பக்கங்கள், இவர்கள் மீது விதிக்கப்பட்ட கட்டுப்பாடுகளை ஒருவகையில் பிரதிபலித்தன. உதாரணத்திற்கு, அவர்கள் ஒருபோதும் தங்களது புகைப்படங்களை தங்கள் பக்கங்களில் பதிவிடமாட்டார்கள். அவர்களது சுயவிவர புகைப்படம் அநேகமாக ஏதாவது ஒரு நடிகையினதோ அல்லது இயற்கை காட்சியாகவோ அல்லது குழந்தையின் புகைப்படமாகவோ இருக்கும். கூடுதலாக, இவர்களுடைய நட்புதொடர்புகளின் எண்ணிக்கை 60க்கும் குறைவானதாகவே இருக்கும். அதிலும் பெரும்பாலானோர், அவர்களது நீட்டிக்கப்பட்ட உறவினர்களாகவோ அல்லது அவர்களது கல்லூரி நண்பர்களாகவோ இருப்பார்கள்.

ஷில்பா என்கிற 21 வயது கல்லூரி மாணவி, ஒரு முகநூல் கணக்கு வைத்திருக்கிறார். அதில் அவருடைய குடும்ப உறுப்பினர்கள் (சகோதரர்கள், ஒன்றுவிட்ட சகோதர-சகோதரிகள், மாமா, சித்தப்பா, அத்தை, சித்தி போன்றவர்கள்) மட்டுமே அவரது நண்பர்களாக இருக்கிறார்கள். அவருடைய பல ஆண் வகுப்புத்தோழர்களிடமிருந்து வந்த நட்புக் கோரிக்கையை அவர் மறுத்துவிட்டார். ஏனெனில், முகநூலில் உள்ள அவரது குடும்பத்தாருக்கு அவரது 
முகநூல் கணக்கில் ஆண் நண்பர்கள் இருப்பது விருப்பமில்லை என்பதே காரணம். அவருக்கு அளிக்கப்பட அதிகபட்ச சலுகை, அவருடைய வகுப்புத்தோழியும், அண்டை வீட்டுக்காரருமான அவருடைய நெருங்கிய தோழியான 21 வயது வசுதாவுடன் முகநூலில் நட்புதொடர்பு கொள்ள அனுமதித்தது தான். அவரும் அதேபோன்ற நிலையில் இருப்பவர் தான். 20 வயது கம்ப்யூட்டர் சயின்ஸ் மாணவியான கே. ப்ரீத்தி-யின் வழக்கும் இதே போன்றது தான். அவருக்கு முகநூலில் மொத்தம் 5 நண்பர்கள் தான். அதில் இருவர் அவரது மாமாவும் சித்தப்பாவும், மற்றவர் அவரது சகோதரர். இந்த இளம்பெண்கள் யாரும் தங்களுக்கு முகநூல் கணக்குகள் இருப்பதாக முதல் பேட்டியில் குறிப்பிடவில்லை. அவர்களது குடிம்ப ஆண்கள் அருகில் இருந்ததே அதற்கு காரணம். ஆண்கள் யாரும் அருகில் இல்லாத அடுத்தடுத்த பேட்டிகளில் தான் அவர்கள் தங்களது முகநூல் இருப்பைப்பற்றி ${ }^{64}$ மனம்திறந்து பேசினர்.

எனினும், ஆண் சகோதரர்கள் இல்லாத பெண்களின் கதை முற்றிலும் வேறு மாதிரியாக இருந்தது. அவர்களில் பலர், தங்களது ஆண் வகுப்புத்தோழர்களில் பலருடன் முகநூலில் நட்புதொடர்பில் இருந்தனர். நிறைய ஒன்றுவிட்ட சகோதரர்களை (சராசரியாக அவர்களுக்கு சகோதரர்கள் இருந்தால் அவர்களின் வயதிலேயே இருக்கக்கூடியவர்கள்) உடைய பெரிய குடும்பங்களில் வளரும் பெண்களுக்கு, கட்டுப்பாடுகள் நிறைந்த சூழலே மீண்டும் இருந்தது. தங்கள் சகோதரர்களுக்கும் தனக்கும் இடையே நீண்ட வருட வயது வித்தியாசம் இருந்த பெண்களுக்கு மிகவும் தளர்வான கட்டுப்பாடுகளே இருந்தன. முற்றிலும் சகோதரிகளை மட்டுமே கொண்ட குடும்பங்களுக்கும் இது பொருந்தும். இருப்பினும், கல்யாணமோ அல்லது வேலைவாய்ப்போ, ஒரு பெண்ணின் வாழ்வில் எது முதலில் வந்தாலும், அது அவர் ஸ்மார்ட்போன் வைத்துக்கொள்வதற்கும், சமூக ஊடகங்களை பயன்படுத்துவதற்கும் அவருக்கு உரிமையளிக்கும் ஒன்றாக இருந்தது. திருமணம் என்பது ஒரு பெண்ணின் குடும்பத்தினருக்காக பெரும் பொறுப்பின் முடிவாக கருதப்பட்டது. வேலைவாய்ப்புடன் இணைந்து வரும் வருவாய் வசதிகள், பெண்களுக்கு முன்னெப்போதும் இல்லாத ஒருவித அந்தஸ்தை அளித்தன. இது போன்ற நிலைகளில், முகநூல் உபயோகத்தில் முன்போன்ற தடைகளும் கட்டுப்பாடுகளும் இல்லாவிட்டாலும், அவரது குடிம்பத்தை சேர்ந்த ஆண் உறவினர்களும், அவர்களது சமூகத்தை சேர்ந்த மணமான பெண்களும் அவர்களுடன் இப்போது நட்புதொடர்பு கொள்ள துவங்கி விடுவார்களாதலால் 
ஓரளவு மென்மையான கட்டுப்பாடு அந்த இடத்தை நிரப்புகிறது. ஆகவே இந்தப் பெண்கள், தங்களது முகநூல் பதிவுகள், தங்களது சமூக உறுப்பினர்கள் முன் தங்களை சங்கடப்படுத்திவிடாதபடி கவனமாக இருக்க வேண்டும். இயற்கையாகவே, இதுபோன்ற குழுவில் உள்ள மணமான பெண்கள், சமூக அளவில் பொருத்தமான பதிவுகளான சமய சம்பந்தமான விஷயங்களையோ, தங்கள் குடும்ப புகைப்படங்களையோ தான் பதிவிடுவார்கள். இது போன்ற பதிவுகள் தரநெறிசார்புள்ளவையாகவும், ஆதர்ச தமிழ் பெண்ணைப்பற்றிய குழுவின் எதிர்பார்ப்புக்கு ஏற்றதாகவும் கருதப்படுகிறது.

எனினும், சமீபத்தில் பஞ்சக்கிராமிக்கு புலம்பெயர்ந்த உயர்-மத்தியதர வகுப்பைச் சேர்ந்த மக்களைப் 65 பார்க்கும்போது இதுபோன்ற கட்டுப்பாடுகளோ தடைகளோ எதுவும் புலப்படவில்லை. இந்த வகுப்பைச் சேர்ந்த பெரும்பாலான பெண்கள், முகநூலிலோ அல்லது வேறு பல சமூக ஊடகங்களிலோ இருந்தார்கள். அவர்கள் அப்படி இதுபோன்ற தளங்களில் இல்லாவிட்டால், அது அவர்களின் சுய விருப்பத்தால் தானே தவிர, எந்தவித கட்டுப்பாட்டினாலும் இல்லை. அவர்களின் சமூக ஊடக இருப்பு, அவர்களின் இயல்புநிலை சமூக தொடர்புகளின் செல்வாக்கு உடையதாக இருந்தது. இந்தப் பெண்களின் நண்பர்கள் அனைவரும் ஏதாவது ஒரு சமூக ஊடகத்தளத்தில் இருக்கிறார்கள். ஆதலால், அவற்றின் உறுப்பினர் நிலை தானாகவே ஏற்பட்டுவிடுகிறது. நிகழ்நிலையில் புகைப்படங்களை பதிவேற்றுவதோ அல்லது பரிச்சயமற்றவர்களுடன் நட்புதொடர்பு கொள்வது பற்றியோ உள்ள ஆபத்துகளைப் பற்றி தங்கள் மகள்களை எச்சரித்திருப்பதாக பல பெற்றோர்கள் சொன்னாலும், இதனால் அவர்களின் மகள்களின் ஊடகப் பிரயோகம் இடம் நேரம் போன்ற எந்தவகையிலும் கட்டுப்படுத்தப்படவில்லை.

இருப்பினும், பல இளம் பெண்கள், தங்களது பெற்றோர் அல்லது பிற குடும்ப உறுப்பினர்களை, முகநூலில் நண்பர்களாக கொண்டிருந்தனர். இதன் மூலம் தங்களின் பதிவுகளின் மீது தாங்களே ஒருவித மென்மையான கட்டுப்பாட்டை கொணர்ந்தனர். உதாரணத்திற்கு, நூற்றுக்கும் மேலான கல்லூரி நண்பர்களுடன் தனது பெற்றோர் மற்றும் இதர குடும்ப உறுப்பினர்களுடன் முகநூலில் நட்புதொடர்பில் இருக்கும் கீர்த்தி என்கிற 19 வயது கல்லூரி மாணவி, தனது குடும்பத்தை சங்கடப்படுத்தாத விஷயங்களை மட்டுமே தான் முகநூலில் பதிவு செய்வதாக குறிப்பிட்டார். இந்தப் போக்கு, இதுபோன்ற 
குழுக்களை சேர்ந்த மணமான பெண்களிடமும் காணப்பட்டது. தங்களுடைய குடும்ப உறுப்பினர்களை உள்ளடக்கிய தங்களது சமூக தொடர்புகளுக்கு சங்கடம் ஏற்படுத்தாத விஷயங்களை மட்டுமே பதிவிடுவதில் அவர்கள் உறுதியாக இருந்தனர். சமூக ஊடகங்கள் மற்றும் போன்களை பயன்படுத்துவதில் விதிக்கப்படும் பாலின கட்டுப்பாடுகள் பல காரணிகளின் சிக்கலான கலவையே என்பது தெளிவாக விளங்குகிறது. அவற்றில், குலம், வர்க்கம், பதியாண்மை, உணர்வுகள் போன்றவை குறிப்பிடத்தக்க சிலவாகும். ஒரே குலத்தில் இருக்கும் வெவ்வேறு வர்க்கத்தைச் சேர்ந்த மக்கள், சமூக ஊடகங்களை வெவ்வேறுவிதமாக கருதிய வழக்குகளும் இருக்கின்றன. இதுபோன்ற வழக்குகளில், ஒரு குறிப்பிட்ட காரணியை மட்டும் சுட்டிக்காட்ட முயல்வது என்பதே கட்டுப்படுத்தக்கூடிய ஒன்றாக இருக்கக்கூடிம்.

அதேசமயம், பஞ்சக்கிராமியில் உள்ள ஆண்கள், அவர்களது சமூகப்பொருளாதார அந்தஸ்த்தைப் பொருட்படுத்தாமல், மிகக்குறைந்த வயதிலேயே முகநூலில் உறுப்பினர்கள் ஆகிவிட்டனர். மத்தியதர வகுப்பைச் சேர்ந்த பல இளம் ஆண்கள், ஏழு முதல் பத்து வயதிற்குள்ளாக இந்தத்தளத்தை முதலில் உபயோகித்திருக்கிறார்கள். இதுவே, கீழ்மட்ட சமூகப்பொருளாதார வகுப்பைச்சேர்ந்தவர்களின் முதல் உபயோகம், 12 அல்லது 13 வயதாக இருந்திருக்கிறது. இந்த வயது வரம்பைச் சேர்ந்த பெரும்பாலான இளம் ஆண்கள், முகநூலை விளையாட்டுக்கள் விளையாடுவெதற்காகத் தான் உபயோகிக்கின்றனர். அவர்களைப்பொறுத்தவரை, வலைத்தொடர்பு என்பது விளையாட்டின் மூலம் ஏற்படும் ஒன்று. அவர்களுடைய உரையாடல்கள் அனைத்தும் வேறெதையும் விட விளையாட்டுக்களையே மையமாகக் கொண்டவை. மத்தியதர வகுப்பைச்சேர்ந்த 14-15 வயதுக்குட்பட்ட இளம் ஆண்கள், தங்களது ஒன்றுவிட்ட சகோதர-சகோதரிகளை (பெரும்பாலும் வெளிநாட்டிலோ அல்லது மற்ற இந்திய மாநிலங்களிலோ வாழ்பவர்கள்) தங்கள் முகநூல் நண்பர்களாகக் கொண்டிருக்கின்றனர். மத்தியதர வகுப்பைச்சேர்ந்த பதின்பருவத்தினர் பலர் தங்களது பிளேஸ்டேஷன், வீ, மற்றும் முகநூலுக்கிடையே மாற்றி மாற்றி விளையாடினாலும், கீழ்மட்ட சமூகப்பொருளாதார பின்னணியில் உள்ள மற்றவர்களுக்கு, முகநூல் அவர்களது விளையாட்டு உலகின் முதன்மை தோற்றுவாயாக இருக்கிறது. படங்கள் 2.1 மற்றும் 2.2-ல் இ இருந்து மதத்தியதர வகுப்பு மற்றும் பணக்கார வகுப்பைச்சேர்ந்த பையன்களும், கீழ்மட்ட சமூகப்பொருளாதார பின்னணியைச் சேர்ந்த 


\begin{tabular}{|l|l|l|}
\hline & Age & Use \\
\hline Age of Access & $7-10$ years & Games \\
\hline $12-13$ years & $\begin{array}{l}\text { Friending, } \\
\text { Access of } \\
\text { other aspects } \\
\text { on FB }\end{array}$ \\
\hline
\end{tabular}

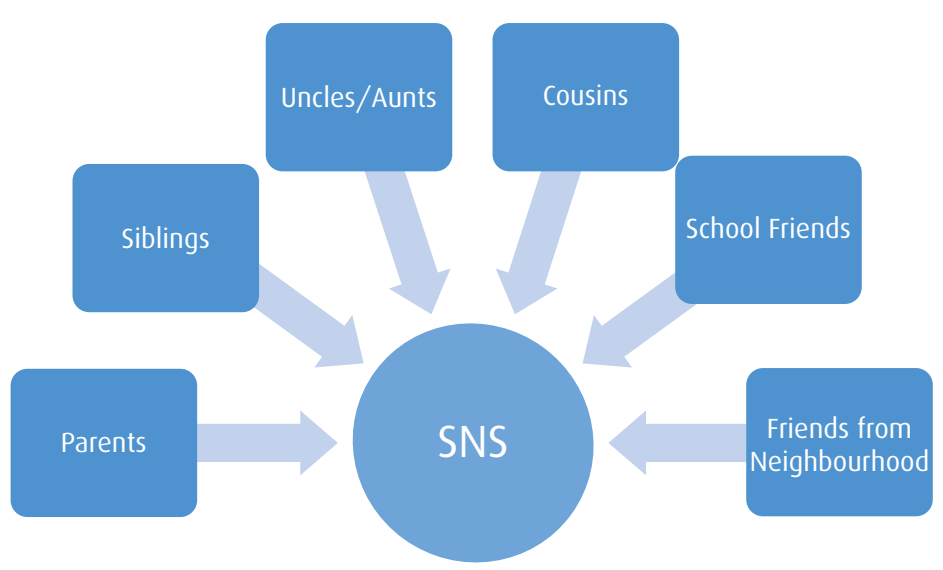

படம்: 2.1 சமூக வலைத்தொடர்புத் தளங்கள் - மத்தியதர வகுப்பு

பையன்களும் எந்த வயதில் முகநூலுக்கு அறிமுகமாகிறார்கள் என்ற விவரத்தையும், அவர்களை இந்தத் தளத்திற்கு அறிமுகப்படுத்திய மாபெரும் செல்வாக்குக்குரியவர்களின் பட்டியலையும் நாம் பார்க்கலாம். (படம்: 2.1 \& 2.2)

16-17 வயதில், சினிமா, விளையாட்டுக்கள் மற்றும் எதிர்பாலினத்தின் மீதான ஆர்வம் ஆகியவை முகநூலில் உள்ள விளையாட்டுக்களோடு சேர்ந்து முதன்மை பெறுகின்றன. இருப்பினும், கீழ்மட்ட சமூகப்பொருளாதார வகுப்பைச் சேர்ந்த பெண்களில் பெரும்பாலானோர் இந்த வயதில் முகநூலில் இருப்பதில்லையாதலால் இந்தப் படிநிலையில் உள்ள ஆண்களுக்கு நிகழ்நிலை மாற்றுப்-பாலின நட்பு வரையறுக்கப்படுத்தப்பட்டதாகவே இ இருக்கிறது. இருப்பினும், மத்தியதர வகுப்பைச்சேர்ந்த 16-17 வயதுள்ள இளம் ஆண்கள், தங்கள் பள்ளியில் இருந்தோ, அண்டை-அயலில் இருந்தோ தங்களுக்கு பரிச்சயமான பெண்களுடன் முகநூலில் நட்புதொடர்பில் இருக்கிறார்கள். 


\begin{tabular}{|l|l|l|}
\hline & Age & Use \\
\hline Age of Access & $12-13$ years & Games \\
\hline $14-15$ years & $\begin{array}{l}\text { Friending, } \\
\text { Access of } \\
\text { other aspects } \\
\text { on FB }\end{array}$ \\
\hline
\end{tabular}

on FB

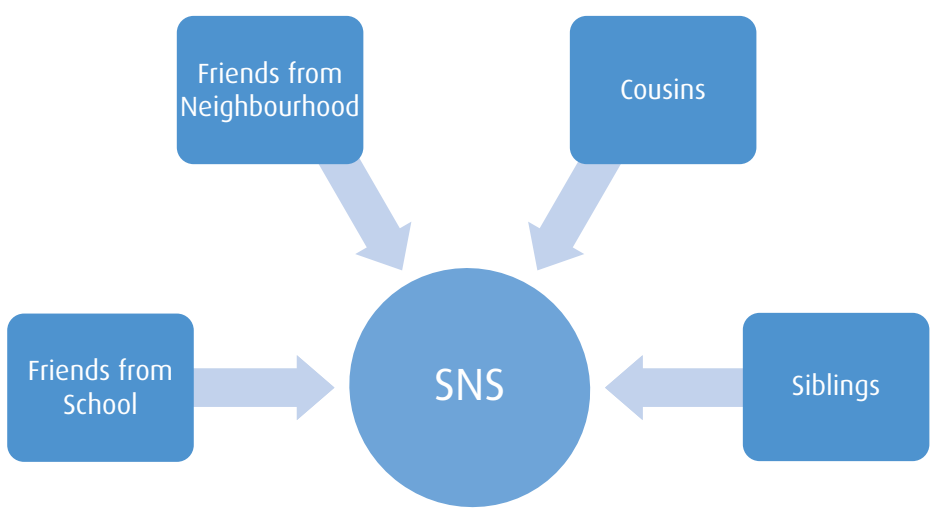

படம்: 2.2 சமூக வலைத்தொடர்புத் தளங்கள் - கீழ்மட்ட சமூகப்பொருளாதார வகுப்பு

கல்லூரி மாணவர்களை கூர்நோக்கும்போது, விஷயங்களில் மாறுதல்கள் புலப்படுகின்றன. இன்னும் கல்லூரியில் படித்துக்கொண்டிருக்கும் 22 வயதுக்கு கீழுள்ள ஆண்கள், பெண்களை கவர்வதற்கும், அவர்களுடன் சரசப்பேச்சு ${ }^{66}$ வைத்துக்கொள்வதற்கு, இயன்றால் அவர்களுடன் நேருக்கு நேர் சந்திப்பதற்கும் உதவும் சாதனமாகத்தான் முகநூலை பார்க்கிறார்கள். இந்த வயது வரம்பைச்சேர்ந்த முகநூலில் இ இருக்கும் கல்லூரி மாணவிகள் (பெண்கள்), தங்கள் வகுப்புத்தோழர்களை (ஆண்கள்) அன்றாடம் சந்திப்பதாலும், அவர்களைப்பற்றி அறிந்ததாலும் முகநூலில் நண்பர்களாக்கிக்கொண்டதாக குறிப்பிடுகிறார்கள்.

மற்றொரு பொதுவான பயன்பாடு என்னவென்றால், தமிழ்த் திரைப்பட நகைச்சுவை காட்சிகளின் காணொளிகள், திரைப்பாடல்கள், திரைச்செய்திகள் மற்றும் அரசியல் செய்திகள் ${ }^{77}$ ஆகியவற்றை பகிர்ந்து கொள்ளுதல் தான். ஒருசிலர், முகநூலை, நாகரீகம், இசை, உடற்பயிற்சி, 
சைவஉணவு முறை போன்ற குறிப்பிட்ட ஆர்வங்களை வளர்ப்பதற்கு பயன்படுத்தினர்.

பெரும்பாலும் முகநூல், வேடிக்கை மட்டும் சமூகமாகப் பழகுதல் ஆகியவற்றுக்கான இடமாகத் தான் கருதப்பட்டது. இதில் சமூகமாகப் பழகுதல் என்பது அத்தியாயம் 3-ல் குறிப்பிட்டுள்ளபடி, அன்றாடம் காலை மற்றும் மாலை வணக்கங்களை தெரிவிக்கும் வாழ்த்துச்செய்திகளையும் குறிக்கும்.

இளம் ஆண்களிடையே, தங்கள் பதிவுகளுக்கு குறிப்பாக புகைப்படங்களுக்கு அதிகபட்ச விருப்புக்களையும், கருத்துகளையும் பெறுவதில் போட்டி இருப்பது தெளிவாகத் தெரிகிறது. ஏனெனில் அது அவர்களையொத்தவர்களிடையே அவர்களது அந்தஸ்தை உயர்த்துகிறது. வழக்கமாக அவர்கள் ஏதாவது ஒரு பதிவிட்டபிறகோ அல்லது புகைப்படத்தை பதிவேற்றியபிறகோ, தங்கள் நண்பர்களை அழைத்து அதற்கு விருப்புத்தெரிவிக்க கோருவார்கள். அவர்கள், தங்கள் நண்பர்களை நேரில் சந்திக்கும் போதும், முகநூலில் அவர்களுடன் நடப்பில் அளவளாவும் போதும், தங்களது பதிவிற்கு விருப்பு தெரிவிக்க கோரி பிரச்சாரம் செய்வார்கள். சிலசமயங்களில், விருப்புத்தெரிவிக்கக் கோரும் தனிப்பட்ட குறுஞ்செய்திகளும் அனுப்பப்படும். இதுபோன்ற போக்கு, இந்த வயதையொத்த பெண் கல்லூரி மாணவிகளிடமும் காணப்படுகிறது.

பஞ்சக்கிராமியில், 22 முதல் 25 வயதுக்குட்பட்ட முகநூல் உபயோகிப்பாளர்களில் பெரும்பாலானோர் புதுப் பட்டதாரிகள் (அவர்களில் சிலர் வேலை தேடிக்கொண்டிருப்பவர்கள்), முதுகலை பட்டதாரிகள் மற்றும் நிறுவனங்களில் புதிதாக வேலைக்குச் சேர்ந்திருப்பவர்கள். பாலினம் மற்றும் வர்க்கம் ஆகியவை பற்றிய பொருட்டில்லாமல் இந்த புதிய பட்டதாரிகள் முகநூலை, முக்கிய காரணங்களுக்காக பயன்படுத்தினர். ஒருசிலர் வேலைவாய்ப்பு, வேலை-தொடர்பான திறமைகள் மற்றும் நேர்காணலுக்கு தயாராவதற்கான உத்திகள் ஆகியவற்றை பதிவிடிம் பக்கங்களை பின்தொடர்ந்தனர். மற்றவர்கள், சமூக விசாரங்கள் மற்றும் சார்புநலம் போன்றவற்றிற்கு ஆதரவு தெரிவிக்கக்கூடிய குழுக்களில் இணைந்தனர். இந்த வயதினருக்கு, முகநூல் என்பது கூட்டு அறிவு மற்றும் தகவல் பகிர்தலுக்கான ஒரு களமாக இருந்தது.

இந்தப்பகுதியில் உள்ள 25 முதல் 40 வயதுக்குட்பட்ட உபயோகிப்பாளர்கள், அவர்களது இளம்வயது எதிரிணையாளர்களைப் போல அவ்வளவு சுறுசுறுப்பாக சமூக ஊடகங்களில் இருப்பது இல்லை. எனினும், அவர்கள் அவ்வப்போது முகநூலை பயன்படுத்தி, தங்கள் தொடர்புகளை பேணுகிறா- 
ர்கள். பலர் தாங்களாக எந்தப்பதிவுகளும் இடாமல், தங்கள் நண்பர்களின் பதிவுகளுக்கு விருப்பமும் கருத்தும் மட்டும் தெரிவிக்கும் அடக்க-உபயோகிப்பாளர்களாக ஆகிவிடுகிறார்கள். அவர்கள் அப்படியே பதிவிட்டாலும் அது அநேகமாக முன்னனுப்பிய செய்தியாகவோ அல்லது செய்திகள் மற்றும் கட்டுரைகளுக்கான இ இணையத்தொடர்பாகவோ தான் இருக்கும். இந்த வயது வரம்பினர், பாலினம் மற்றும் வர்க்கம் பற்றிய எந்த பேதமும் இல்லாமல் நெருக்கமான சமூகத்தொடர்புள்ள வாட்சப் குழுக்களில் மிகவும் சுறுசுறுப்பாக செயல்படுகிறார்கள். இருப்பினும், மத்தியதர வகுப்பைச் சேர்ந்த ஐ.டி பணியாளர்கள் மட்டுமே பணிநிமித்த வலைத்தொடர்புகளான லிங்க்ட்-இன் போன்றவற்றில், அவ்வளவு ஏன், டுவிட்டரிலும் முனைப்புடன் ஈடுபடுகெறார்கள். இவர்கள் முகநூலில், தங்களது சுயவிவர பக்கம் எப்படி இருக்கவேண்டும் என்பதில் குறிப்பாக இருந்தாலும், அதன் அந்தரங்க அமைப்பு பற்றி பொதுவாக கவலைப்பட்டுக்கொள்வதில்லை. இந்த வயது வரம்பில் உள்ள மணமான இளம் தம்பதியினர், தங்கள் குழந்தைகளின் புகைப்படங்களை (குறிப்பாக இளம் குழந்தைகள்) பொதுப்பார்வைக்கு ெரியும்படி பதிவேற்றுகிறார்கள். இவர்களும் அந்தரங்க அமைப்பைப் பற்றி எந்தக் கவலையும் கொள்வதில்லை. இந்த வயது வரம்பில் உள்ள உயர் மத்தியதர வகுப்பைச்சேர்ந்த பெண்கள், தங்கள் எதிரிணையாளர்களை விட முகநூலில் அதிக சுறுசுறுப்பான செயல்பாட்டில் இருக்கிறார்கள்.

40 முதல் 60 வயதுக்குட்பட்ட உபயோகிப்பாளர்கள், லிங்க்ட்-இன்னை தொழில்சார் உபயோகத்துக்கென்று ஒதுக்கிவிட்டு, முகநூல் என்பது தனிப்பட்ட உபயோகித்திற்கு மட்டுமே என்பதில் மிகவும் தெளிவாக இருக்கிறார்கள். ஊடகங்களின் பாகுபாடு இவர்கள் தங்கள் வாழ்க்கையை எப்படி பிரித்துப்பார்க்கிறார்கள் என்பதன் அடிப்படையில் இருக்கிறது. அவர்கள் பொதுவாக மத்தியதர அல்லது உயர்-மத்தியதர வகுப்பைச்சேர்ந்தவர்களாகவும், தொழில்முனைவோர் அல்லது திறன் வாய்ந்த ஐ.டி பணியாளர்கள் அல்லது அவர்கள் குடும்பத்தை சேர்ந்தவர்களாக இருக்கிறார்கள். அவர்கள், தங்களது பழைய பள்ளி அல்லது கல்லூரி நண்பர்களுடன் மீண்டிம் தொடர்பு கொள்வதில் முனைப்பாக செயல்படுகிறார்கள். அத்தோடு, தங்கள் நண்பர்கள் பட்டியலைப் பற்றி கவனமாகவும் தெரிவுடனும் இருக்கிறார்கள். அவர்கள் தங்கள் முகநூல் பக்கத்தில் உள்ள அந்தரங்க அமைப்பைப் பற்றி மிகவும் விசாரப்படுபவர்க- 
ளாக இருக்கிறார்கள் ஏனெனில் அவர்கள் இதை மிகவும் தனிப்பட்ட உபயோகத்திற்கானதாக நினைக்கிறார்கள். அவர்கள் ஏதேனும் குழுக்களை அமைத்தாலும், அவற்றை பல்வேறு காரணங்களுக்காக மூடிய குழுக்களாக அமைக்கிறார்கள். தங்கள் குழந்தைகளின் புகைப்படங்களை பதிவேற்றும் தாய்மார்கள், அந்தரங்க அமைப்பைப் பற்றி மிகவும் கவனமாக இருக்கிறார்கள். இருப்பினும், இந்தக்குழுவிலும், தனக்கு நட்புதொடர்பு கோரிக்கை அனுப்பும் எவரையும் நண்பர்களாக்கிக் கொள்பவர்களும் இருக்கிறார்கள். அவர்கள் தங்கள் தொடர்பில் உள்ளவர்களை, அவர்கள் தங்களால் ஒ ஒப்புக்கொள்ள இயலாத நடவடிக்கை ஏதேனும் செய்தால் மட்டுமே, நட்புநீக்கம் செய்கிறார்கள். உதாரணத்திற்கு பாலியல் புகைப்படங்கள், பெண்ணின வெறுப்புப் பதிவுகள் போன்றவை.பொதுப்பார்வையில் (அவர்களுடைய முகநூல் நட்பு வலைத்தொடர்பு) அவர்களுடைய மதிப்பிற்கு பங்கம் விளைவிக்கக்கூடிய எதையும் அவர்கள் மிகவும் தீவிரமாகப் பார்க்கிறார்கள்.

60 வயதுக்கு மேற்பட்ட உபயோகிப்பாளர்கள் பலரும் பணிஓய்வு பெற்றவர்கள். அவர்கள் பொதுவாக உயர்-மத்தியதர வகுப்பையோ அல்லது பணக்கார வகுப்பையோ சேர்ந்தவர்களாக இ இருக்கிறார்கள். 60 வயதுக்கு மேற்பட்ட பெரியவர்களுடனான பேட்டிகள், முகநூல் பற்றிய அவர்களின் கண்ணோட்டம், அத்துடன் இணைந்த பலவகையான உணர்வுகளைப் பொறுத்து வித்தியாசப்படுகிறது என்பதை வெளிக்காட்டியது. ஒருசிலர் முகநூலை முயன்று பார்த்து, அது சிறுபிள்ளைத்தனமாக இருப்பதாக கருதி, அதுபிடிக்காமல் விட்டுவிட்டனர். வேறுசிலர் இந்தத்தளத்தை மிகவும் பிடித்துப்போய் அதை முயன்றுபார்ப்பதில் மகிழ்ச்சியடைகின்றனர். ஒருசில மூத்த பெண் உபயோகிப்பாளர்கள், மணமாகி வெளிநாட்டில் ${ }^{68}$ வாழும் தங்கள் மகள்களுக்காக சமையல் குறிப்பு காணொளிகளை பதிவேற்றுகிறார்கள். அவர்கள், தங்கள் பேரக்குழந்தைகள் மற்றும் வெளிநாட்டில் வாழும் உறவினரின் புகைப்படங்களை பகிர்ந்துகொள்ளவும், அவற்றில் தங்கள் கருத்துக்களை பதிவு செய்யவும் முகநூலை பயன்படுத்துகின்றனர். இ இருப்பினும், பலர் வெளிப்படுத்திய அபிப்ராயம் என்னவென்றால், இந்தியக் கலாச்சாரம் ${ }^{69}$ முன்பு உத்திரவாதமளித்த தனிப்பட்ட தகவல் பரிமாற்றத்தை, முகநூல் அகற்றிவிட்டது என்பது தான்.

இந்த அத்தியாயத்தில் நாம் முன்பு சந்தித்த திரு. ராகவன் அவர்களின் வழக்கு, இந்த உணர்வுகளையும், தகவல் பரிமா- 
ற்றத்தில் உள்ள அதிகார படிநிலை எதிர்பார்ப்புகளையும் எடுத்துக்காட்ட உதவுகிறது.

65 வயதாகும் திரு. ராகவன், சமீப காலம் வரை முகநூலில் இருந்தார். இந்தியாவில், முகநூலின் வருகையின் விளைவால், இளம்தலைமுறை உபயோகிப்பாளர்கள், பெரியவர்களை மதிப்பதில்லை என்ற காரணத்தால், அவர் முகநூலில் உள்ள தன் கணக்கை ரத்து செய்துவிட்டார். குறிப்பிட்ட ஒருசில சம்பவங்கள் அவரை எரிச்சலூட்டி விட்டதாகத் தெரிகிறது. முதலாவது, அவருடைய உடன்பிறந்தாரின் மகள், தன்னுடைய மகனின் முதல் பிறந்தநாள் விழாவிற்கு, அவரை நேரிலோ, தொலைபேசியிலோ அழைக்காமல், அவருக்கு முகநூல் மூலம் அழைப்பு விடித்தது. இரண்டாவது, அவரது உடன்பிறந்தாரின் மகன், தனக்கு ஒரு மகன் பிறந்ததை, அவருக்கு நேரிடையாகத் தெரிவிக்காமல், முகநூலில் குழந்தையின் புகைப்படத்தை பதிவேற்றியதன் மூலம் அறிவித்தது. இந்த சம்பவங்களை எங்களுடன் பகிர்ந்து கொள்ளும் போது, அவர், மக்கள் முக்கியமான வாழ்க்கை சம்பவங்களை பெரியவர்களுக்கு தெரிவிக்க வேண்டிய சரியான முறைகளை தெரிந்து வைத்திருக்க வேண்டும் என்பதை வலியுறுத்தினார். முகநூல், தனிப்பட்ட தகவல் பரிமாற்ற தளமாக இருப்பதைவிட, வெகுஜன ஊடகமாக ஆகிவிட்டது என்பது அவரது கருத்து. இதற்கு மாறாக, அவரது மனைவி, தன் கணவரின் எதிர்பார்ப்புகள் யதார்த்தமற்றவை என்றும் தனிப்பட்ட தகவல் பரிமாற்றம் (குறிப்பாக குரல்வழிச் செய்திகள்), ஒருவரது உடனடி குடிம்பத்தாரிடம் இருந்து மட்டுமே எதிர்பார்க்க முடியும் என்று கருதினார்.

மறுபக்கம் பார்த்தால், 66 வயதான கரண், முகநூல் உபயோகிப்பதில் தனக்கு எந்தவொரு வெறுப்பும் இல்லையென்றும், வெளிநாடுகளில் வாழும் தன் உடன்பிறந்தாரின் மகன் மற்றும் மகள்களுடன் தொடர்பில் இருப்பதற்கு அது உதவுகிறது என்றும் குறிப்பிட்டார். அவர், தான் வாழும் குடியிருப்பு சமூகத்திற்காகவும், தன் குடியிருப்பின் அருகில் இருக்கும் ஒரு ஏரியை பாதுகாப்பது குறித்த சமூகக் காரணத்திற்காகவும் தனிப்பட்ட முகநூல் பக்கங்களை உருவாக்கியிருக்கிறார்.

இதுபோல, முகநூல் சில வயதில் மூத்த உபயோகிப்பாளர்களுக்கு, சமூகசேவை செய்வதில் உதவியாகவும், வெளிநாட்டில் வாழும் தங்கள் குழந்தைகள் மற்றும் இதர உறவினர்களுடன் தொடர்பில் இருப்பதற்கு உதவியாகவும் இருக்கிறது. அதுவே, வேறு சிலருக்கு அவர்கள் தங்கள் 
குடும்ப உறுப்பினர்களிடம் இருந்து எதிர்பார்க்கும் தனிப்பட்ட தகவல் பரிமாற்றங்களை அகற்றிவிடுகிறது.

பஞ்சக்கிராமியில், பொய்யான மற்றும் பன்மடங்காக முகநூல் கணக்குகளும் இ இருக்கின்றன. தங்களது முந்தைய கணக்கின் விவரங்களை மறந்து போவதில் இருந்து தனது பல்வேறு சமூகத் தொடர்புகளை மூலோபாய முறையில் பிரித்து வைப்பதற்காக, பாலியல் காணொளிகளை காண்பதற்காக, பெண்களை கவர்வதற்காக, சமூகக் கட்டுப்பாடுகளில் இருந்து தப்பிப்பதற்காக என்பது வரை, உபயோகிப்பாளர்கள், பல்வேறு காரணங்களுக்காக பொய்யான முகநூல் கணக்குகளை வைத்திருக்கிறார்கள். குடியுரிமை அல்லாத இந்தியர்கள், வெளிநாட்டில் ஒ ஒன்றும் இந்்தியாவில் ஒன்றுமாக ${ }^{70}$ உறவுமுறைகளை வைத்திருப்பதற்காக இருவேறு முகநூல் கணக்குகளை வைத்திருந்த வழக்குகளெல்லாம் இருக்கின்றன. பஞ்சக்கிராமியில், சமூக அனுசரித்தலுக்காகவும், ஒதுக்கி வைக்கப்படாமல் இருப்பதற்காகவும் பெயரிடப்படாமல் இருக்கும் ஒருசில ஓரினச்சேர்க்கையாளர்களின் கணக்குகளும் முகநூலில் காணப்பட்டன. முகநூல் நிறுவனம், இதுபோன்ற பல கணக்குகள் வைத்திருப்பதை, சிதறியிருக்கும் செயலாகவும், நிகழ்நிலையில் ஒற்றை அடையாளம் இருக்க வேண்டும் என்ற தங்கள் கொள்கைகளுடன் ஒத்துப்போகாத செயலாகவும் கருதினாலும், பஞ்சக்கிராமியில் இருக்கும் சில மக்களுக்கு, பல்வேறு கணக்குகளின் வாயிலாக தங்கள் தனித்தன்மையை பிரித்து வைத்திருப்பது என்பது, தங்களது அடையாளத்தை ${ }^{71}$ பேணுவதற்கான அவசியமான மற்றும் நம்பத்தகுந்த வழிமுறையாகும். பஞ்சக்கிராமியில் முகநூலின் இருப்பைப்பற்றிய இந்த பொதுவான கருத்தை நிறுவிக்கொண்டபிறகு, இப்போது நாம், பஞ்சக்கிராமியில், மொபைல் போன்கள் மற்றும் கட்டுப்படியாகக்கூடிய தரவுத்திட்டங்களின் வருகையால் பிராபல்யம் பெற்றுவரும் ஊடகத்தளமான வாட்சப் பற்றிய கலந்தாய்வுக்கு செல்லலாம்.

\section{வாட்சப்}

2013களின் துவக்கத்தில், பஞ்சக்கிராமியில், வாட்சப் 72 முகநூலை ${ }^{73}$ விட, மிகக்குறைவான பிராபல்யம் கொண்டிருந்த ஊடகத்தளம். உண்மையில், மக்கள் இதை குறுஞ்செய்தி அனுப்புதலின் நீட்டிப்பாகத்தான் கருதினர் (தனிநபர்களுக்கு அல்லது குழுக்களுக்கு) ${ }^{74}$. வாட்சப்பின் இருப்பு முகநூலின் 
அடிப்படையிலான நிபந்தனை இருப்பு என்பது தெளிவாகிறது. அதாவது, தாங்கள் வாட்சப் கணக்கு வைத்திருப்பதாக சொன்ன மக்கள் அனைவரும் அனேகமாக எல்லா நேரங்களும் முகநூலிலும் இருந்துகொண்டே தான் இருந்தார்கள். வாட்சப்பில் இருக்கும் ஒருசிலர் மட்டுமே, முகநூலில் இல்லாமல், வாட்சப்பில் மட்டுமே இருந்தனர். குறிப்பிட்ட சில வகையான தகவல் பரிமாற்றத்திற்கு ${ }^{75}$ வாட்சப் ஏற்றது தானா என்று மக்கள் வாட்சப்பை சோதித்துக் கொண்டிருந்த சமயம் தான் அது.

இருப்பினும், 2013ஆம் வருடத்தின் இறுதியில், வாட்சப்-ன் பிராபல்யம் உயர்ந்து கொண்டே போகத்துவங்கியது. பலசமயங்களில் முகநூலில் இருந்து விடுபட்டும் கூட. அதாவது, வாட்சப்பில் இருந்த மக்கள் எல்லோருமே முகநூலில் 76 இருக்கவில்லை. கட்டுப்படியாகக்கூடிய கட்டணத்தில் கிடைத்த மொபைல் இணையத்தொடர்பு தான் இந்தப்பகுதியில் வாட்சப் பிராபல்யத்திற்கு முக்கிய காரணமாக இருந்தது. 2013களின் இறுதிக்கு முன்பு, முன்கட்டணம் செலுத்தப்பட்ட மொபைல் இணையத்தொடர்பு அட்டைகளை வாங்கும் பாணி, 200எம்.பி இணையதள தரவுக்கு, ரூ. 25/ஆக இருந்தது. அதுவே, குறுஞ்செய்தி அனுப்புவதற்கான செயலூக்கி தொகுப்பு, ரூ. 15/- திற்கு கிடைத்தது. (இதன்மூலம் 3000 குறுஞ்செய்திகள் வரை இலவசமாக அனுப்பலாம்). கீழ்த்தட்டு பொருளாதார படிநிலையில் உள்ளவர்கள், ஒரே ஊடகத்தளத்தில் அவர்கள் தொடர்பில் இருக்கக்கூடிய குறைவான சமூக வட்டத்தைக் கருத்தில் கொண்டு, வாட்சப்பை விட குறுஞ்செய்தி தொகுப்புகளை விரும்பிப்பெற்றனர். மேலும், எவரேனும், நிகழ்நிலை காணொளிகளை நடப்புநிலை பாய்வோட்டத்தில் பார்த்தால், உதாரணத்திற்கு யூட்யூப் காணொளிகள் பார்த்தல், அவரது தரவு இருப்பு, அதிவேகமாக குறைந்து போனது. இந்த நுகர்வு போக்கு, இணையத்தொகுப்பின் விலை மாற்றங்களால் மாறத்துவங்கியது. இவை கட்டுப்படியாகக் கூடிய கட்டணங்களில் கிடைக்கதுவங்கின. அதாவது, உபயோகிப்பாளர்கள், ஒரே முறை கட்டணமாக வெறும் ரூ.20/- செலுத்தி 500எம்.பி வரையிலான தரவுத்தொகுப்பைப் பெற்று, மாதம் முழுவதும் நிகழ்நிலையில் உலவமுடிந்தது. இந்த மொபைல் கடைகளுக்கு ஓருவர் தினமும் வருகை தரவேண்டும். ஏனெனில், இதுபோன்ற சலுகைகள், நாளுக்கு நாள் மாறுபட்டுக் கொண்டேயிருக்கும். ஒருநாள் விட்டாலும், ஏதேனும் ஒரு சிறந்த சலுகையை நழுவவிட்டுவிடக்கூடும். இது மிகவும் முக்கியமானது ஏனெனில், 
இணையத்தளத்தில் உள்ள திட்டங்கள் கூட மற்ற முறைகளைவிட, வாய்வழிச்செய்தி மூலம் தான் அதிகம் பகிரப்படுகின்றன. தொலைத்தொடர்பு நிறுவனங்கள் மட்டுமே தங்களது சமீபத்திய சலுகைகளை பற்றிய குறுஞ்செய்திகளை தங்கள் வாடிக்கையாளருக்கு மொபைல் போனில் அனுப்புகின்றனர்.

கட்டுப்படியாகக்கூடிய இணையத்தரவு தொகுப்புகளின் அதிகரிப்பால், மத்தியதர மற்றும் உயர்-மத்தியதர வகுப்பைச்சேர்ந்த ஐ.டி யில் பணிபுரியும் மக்கள் தான் முதன்முதலில் 3ஜி தொடர்புடன் வாட்சப்-ஐ பயன்படுத்தத் துவங்கியவர்கள். ஆகவே, கட்டுப்படியாகும் தன்மையுடன் கூடிய, எழுத்து, காட்சி மற்றும் குரல்வழி ஆகிய மூன்றும் ஒன்றிணைந்த தகவல் பரிமாற்ற செயல்திறன் மற்றும், தகவல் பரிமாற்றங்களை, தனிநபரில் இருந்து குழுக்களுக்கு77 அனுப்பக்கூடிய பெருக்க அளவீடு ஆகிய அனைத்தும் சேர்ந்து, வாட்சப்-ஐ முகநூலுக்கு அடுத்தபடியான பிரபலமான ஊடகத்தளமாக ஆக்கிவிட்டது. இது நீண்டகால அளவில் ஒருவரின் சமூக வட்டத்துள் தகவல் பரிமாற்ற செலவைக் குறைத்து, பொருளாதார வகையில், கட்டுப்படியாகக்கூடிய ஒன்றாக தோன்றியதும் இதற்கான காரணம்.

இந்த வளர்ச்சி, தனிப்பட்டதல்ல. இநந்தியாவில், ஸ்மார்ட்போன் வணிகம், நல்ல வடிவம் பெற்றுக்கொண்டிருக்கும் சமயத்தில் தான் இந்த வளர்ச்சியும் நடைபெற்றது. ஸ்மார்ட்போன் வணிகத்தில் இப்படிப்பட்ட ஒரு விரிவாக்கம், மலிவான மற்றும் கட்டுப்படியாகக்கூடிய ஸ்மார்ட்போன்கள் கிடைப்பதற்கு வழிவகுத்தது. அத்தியாயம் 1-ல் பார்த்தது போல, வாட்சப்பின் மாபெரும் பயன்பாட்டாளர் அடித்தளமாக ${ }^{78}$ இந்தியாவின் வளர்ச்சி, கண்டிப்பாக அதே சமயத்தில் நடைபெற்ற ஸ்மார்ட்போன் வணிகத்தின் வளர்ச்சியுடன் தொடர்புடையது.

பஞ்சக்கிராமியின் குடியிருப்பாளர்களுக்கு, வாட்சப் என்பது முகநூலுக்கும், போனில் குறுஞ்செய்தி அனுப்புவதற்கும் இடைப்பட்ட ஒன்று. அவர்களைப்பொறுத்தவரை, இந்தத் தளம், தனிப்பட்ட ஒருவருக்கொருவரான அந்தரங்க தகவல் பரிமாற்றத்திற்கு ஏதுவாக இருப்பதோடு, ஒத்த ஆர்வமுள்ள ${ }^{79}$ குழுக்களுக்கிடையேயான தகவல் பரிமாற்றத்திற்கும் வழி செய்தது. குழுத்தகவல் பரிமாற்றத்தை பொறுத்தவரை, ஒரு பெரிய ஒத்த ஆர்வக்குழுவின் அங்கமாக உபயோகிப்பாளர்கள் இ இருக்கும்பட்சத்தில், அவர்கள் அந்தக்குழுவில் உள்ள மற்றவர்களை, பரிச்சயமுள்ளவர்களாக தான் பார்த்தார்களே தவிர, அந்நியர்களாக அல்ல. ஆனால், எந்தவிதமான ஒத்த ஆர்வமும் இல்லாத முற்றிலும் அந்நியமானவர்களுடன் 
நட்புதொடர்பு ஏற்படுத்திக்கொள்ளக்கூடிய வாய்ப்பளிக்கும் முகநூல், வாட்சப் அளவிற்கு அந்தரங்கமானதாக கருதப்படவில்லை. டுவிட்டர், அந்தரங்கத்துடன் தொடர்புடையதாகவே கருதப்படவில்லை. அது, முகமற்ற பரந்த உலகத்துடன் தகவல் பரிமாறிக்கொள்ளும் ஒரு பொதுப்பார்வைக்குரிய ஊடகத்தளமாகத்தான் கருதப்படுகிறது. மேலும், பெரும்பாலான உபயோகிப்பாளர்கள், வாட்சப், முகநூலை விட அடிமைப்படுத்தும் தன்மையுடையது என்று அபிப்ராயப்பட்டாலும், யாரும் அதைவிட்டு விலகிச்செல்ல நினைக்கவில்லை ${ }^{80}$.

எனினும், வாட்சப், போன்களில் மட்டுமே உபயோகப்படுத்தப்பட்டுக் கொண்டிருந்தது. நாம் ஏற்கனவே பார்த்தது போல, குலத்தினிடை காதல் மற்றும் குலம்சார்ந்த வரம்புமீறுதல் பற்றிய பயங்களால், இளம்பெண்களுக்கு மொபைல் போன்கள் பயன்படுத்துவதில் ஏகப்பட்ட தடைகள் விதிக்கப்பட்டிருந்தன. ஆகவே, அவர்களுடைய வாட்சப் பயன்பாடும், கட்டுப்படுத்தப்பட்டிருந்தது. முகநூலுக்கு தடையாக இருந்த குலம், வர்க்கம், பாலினப் பாணிகள் ஆகியவை வாட்சப்பிற்கும் அதேபோல் வந்து நின்றன.

பொதுவான தகவல்களான, வேடிக்கைகள், நல்வாழ்த்துக்கள், பிரார்த்தனைகள், ஆகியவற்றை பகிர்தல், ஒரு பொதுவான நடவடிக்கையாக கருதப்பட்டது. அன்றாட காலை மாலை வணக்கங்களை குறிக்கும் "நன்னாள்" மற்றும் “நல்லிரவு" போன்ற வாழ்த்துச்செய்திகள் அதைவிட பொதுவானதாக கருதப்பட்டன. இந்த அனைத்து நடவடிக்கைகளும் அத்தியாயம் 3 மற்றும் 4-ல் விரிவாக அலசப்பட்டிருக்கின்றன.

புகைப்படங்களை பகிர்ந்து கொள்வது, அது, கலாரசனைக்குரியதாகவோ, சுயபுகைப்படமாகவோ, குழுப்புகைப்படமாகவோ, இயற்கைக்காட்சியாகவோ அல்லது உபயோகிப்பாளர்களின் குழந்தைகளின் புகைப்படமாகவோ81இருக்கலாம், காணொளிகள் பகிர்வதைவிட பொதுவான ஒன்றாக இருந்தது. வாட்சப்பில் உள்ள தனிப்பட்ட காணொளித்துண்டுகள் பொதுவாக குடும்ப நிகழ்ச்சி அல்லது விழாக்கள் தொடர்புடையதாக இருந்தது. திரைப்பட நகைச்சுவைக்காட்சிகள், திரைப்பாடல்கள் ஆகியவற்றைப் பற்றிய காணொளித்துண்டுகள், சமூகத்திற்கு பொருத்தமான தகவல்கள், அரசியல்வாதிகளைப் பற்றிய எள்ளல் துண்டுகள், ஆகியவை பொதுவானதாக இருந்தாலும், நிலையான காட்சிக்குரிய படங்களை விட, குறைந்த அளவே பிரபலமாக இருந்தன. இருப்பினும், வாட்சப்பில் காணொளிகளை பகிரும் அடுக்குநிகழ்வு, அதிகரிக்கும், கட்டுப்படியாகக்கூடிய அதிவேக இணையத்தொ- 
டர்புடன் இ இணைந்து அதிகரித்தது. இந்தக் காணொளிகள், இணையத்தில் இருந்து பதிவிறக்கியதாகவோ, அல்லது உபயோகிப்பாளர்களுக்கு முன்னனுப்பப்பட்டதாகவோ இருக்கலாம். முன்பு பல போன்களில் இருந்த ப்ளூடூத் செயல்பாட்டின் மூலம் நடத்தப்பட்ட பாடல் காணொளிகள் மற்றும் கேட்பொலிக் கோப்புகளின் பகிர்தல் இப்போது வாட்சப் மூலம் நடத்தப்படுகிறது.

பஞ்சக்கிராமியின் குடியிருப்பாளர்களுக்கு, வாட்சப், அதன் குரல்வழி செய்தி அனுப்பக்கூடிய செயல்திறனால், கூடுதல் மதிப்புடையதாக இருக்கிறது. உதாரணத்திற்கு, 33 வயதான லட்சுமி, இப்போது, பிரபலமான ஐ.டி நிறுவனம் ஒன்றில், ஒரு வணிக ஆய்வுக்குழுவின் நிர்வாகியாக தனது மூன்றாவது பணியில் இருக்கிறார். அவருக்கு பஞ்சக்கிராமியில் உள்ள பள்ளி ஒன்றில் பயிலும் 8 வயது மககனும், மூன்று வயது மகளும் இருக்கிறார்கள். அவருடைய கணவர், சென்னையில் உள்ள மற்றொரு ஐ.டி நிறுவனத்தில் பணியில் இருக்கிறார். இவர், வாட்சப்பை அடிக்கடி பயன்படுத்துகிறார். தன்னுடைய பணியில் தீவிரமான காலக்கெடு உள்ள சமயங்களில், வாட்சப் தான் தன்னை புத்தி தெளிவுடன் வைத்திருக்க உதவுகிறது என்று அவர் குறிப்பிடுகிறார்.

“நான் சந்தோஷமாக வேலையை விட்டுவிட்டு, என் குழந்தைகளுடன் நேரம் செலவிட விரும்புகிறேன். என் மகளுக்கு மூன்று வயது தான் ஆகிறது. நான் அவளை என் தாயாரிடமோ அல்லது என் மாமியாரிடமோ விட்டுவிட்டு தான் பணிக்கு வருகிறேன். எனினும், அது ஆவலுடன் நானே இருப்பது போலாகாது"

வாட்சப்பின் மூலம் அவர் தன் தாயின் மொபைல் போனுக்கு ${ }^{82}$ குரல்வழிச்செய்தி அனுப்புகிறார். இவை பெரும்பாலும் அவரது மக்களுக்கான செய்திகள். அவருடைய செய்திகள், அன்பும் மென்மையும் வாத்சல்யமும் நிறைந்த குரலில் இருக்கின்றன. இந்தச் செய்திகளில், லட்சுமி தன் மகளை தான் அவள் நேரிலேயே இருப்பது போல விளிக்கிறார். அவருடைய தாய், இந்தச்செய்திகளை தன் பேத்திக்கு போட்டுக்காட்டியபின், அவளிடமிருந்து செய்திகளை பதிவு செய்து, லட்சுமிக்கு வாட்சப் மூலம் அனுப்புவார். லட்சுமி இதேபோல் தன் மகனுக்கும், அவரது வீட்டுப்பாடத்தைப்பற்றியும், அன்றைய தினத்தைப் பற்றியும் கேட்பதற்கு செய்திகள் அனுப்புகிறார். இவை தான் தன்னை செயல்படவைப்பவை என்று கூறும் அவர், தொலைபேசி அழைப்புக்கு அடுத்தபடியாக இது தான் சிறந்த வழி என்று குறிப்பிடுகிறார். அலுவலகத்தில் 
அவருடைய மும்முரமான பணி அட்டவணையின் காரணத்தால், அவரால் ஒத்தகால இயல்புள்ள தொலைபேசி அழைப்புகளில் ஈடுபட முடிவதில்லை. எனினும் ஒரு விரைவான வாட்சப் குரல்வழி தகவல், அவருக்கு, தன குழந்தைகளுடன் தொடர்பில் இருப்பது போல உணர்வதற்கு உதவுகிறது. இது தன்னை பெருமளவு குற்றவுணர்ச்சியில் இருந்து காப்பதாகவும், பணியில் இருந்துகொண்டே தன குழந்தைகளுக்கு தாயாக செயல்பட உதவுவதாகவும் அவர் ஒத்துக்கொள்கிறார். அதேபோல, பஞ்சக்கிராமியில், ஐ.டி பணியாளர்கள் மற்றும் தொழில்முனைவோர்களின் மனைவிகளான, மத்தியதர வகுப்பைச்சேர்ந்த இல்லத்தரசிகளுக்கு, வாட்சப், தனிப்பட்ட தகவல் பரிமாற்றத்திற்கான நிகழ்நிலை காட்சி சாதனமாக விளங்குகிறது. வேடிக்கைகள் மற்றும் கைவேலைப்பாடுகளை பகிர்ந்துகொள்வது என்பது, அவர்களது அன்றாட சுறுசுறுப்பான வாட்சப் பங்கேற்பின், முக்கிய அங்கமாகும். கைவேலைப்பாடுகள் மற்றும் புகைப்படங்களை, சுற்றோட்டம் விடுவதும், சேகரிப்பதும், ஒரு வகையான மின்னணு பொழுதுபோக்காக இருக்கிறது. இதற்கு மின்னணு இடம் தான் தேவையே தவிர, இயல்புலகில் இடம் தேவையில்லை. வாட்சப் குழுக்களில் உள்ள தயார் அரங்கத்தினரால், கைவேலைப்பாடுகளின் புதுமை பற்றிய பாராட்டுதல், ஒரு புதிய வடிவமெடுக்கிறது. மேலும், இதுபோன்ற சுற்றோட்டங்கள், ஒரு வேடிக்கை அல்லது புகைப்படத்தின் சமூக வாழ்வோடு ${ }^{83}$ உரையாடுகிறது. வேடிக்கைகளுக்கு பதிலாக அளிக்கப்படும் வேடிக்கைகள், உண்மையான நகைச்சுவை கருத்தை மீறிய வாழ்வோட்டத்தை பெறுகின்றன.

பஞ்சக்கிராமியில் உள்ள தம்பதிகளுக்கு, வாட்சப் ஒரு தனித்தன்மையான முடிவெடுக்கும் திறனை அளிக்கிறது. வீட்டிற்கு தேவையான பொருட்களை வாங்குவது பற்றிய முடிவுகள், ஒன்றுகூடியவையாகவும், பலசமயங்களில் வாட்சப் மூலம் எடுக்கப்பட்டவையாகவும் இருக்கின்றன. கணவர், அலுவலகத்தில் இருந்து கொண்டே, பல்பொருள் அங்காடியில் இருக்கும் மனைவிக்கு, ஒரு பொருளை வாங்குவது பற்றிய ஆலோசனையை, அதன் படத்தை, வாட்சப் மூலம் பார்த்தே தரமுடியும். இது ஒருவர் நேரடியாக இருக்க முடியாததன் தடைகளை கடப்பதற்கும், அதேசமயம் நெருக்கத்தை அதிகரிக்கவும் உதவுகிறது.

அதேபோல, பஞ்சக்கிராமியில் உள்ள மத்தியதர வகுப்பை சேர்ந்த இல்லத்தரசிகள், வாட்சப்பை குறிப்பாக, அது அளிக்கும் மனத்தால் உணரக்கூடிய ${ }^{84}$ இயங்குதிறனுக்காகவே 
நேசித்தனர். இல்லத்தரசிகள், வாட்சப்புடன் தாங்கள் பலபணிகளை செய்வதைப் பற்றியும், சமைத்துக்கொண்டிருக்கும் போதோ அல்லது மற்ற முக்கிய பணிகளை செய்துக்கொண்டிருக்கும் போதோ தகவல் அனுப்புவது பற்றியும், அடிக்கடி விவரித்தனர்.

வாட்சப் போன்ற ஒரு சமூக ஊடகத்தளம், ஒரு குறிப்பிட்ட மக்கள் குழுவினிடையே (இந்த வழக்கில் இல்லத்தரசிகள்) பிற தளங்களை விட அதிகமாக விரும்பப்பட்டது ஏன் என்பதை புரிந்து கொள்வதில் பல்வேறு காரணிகள் பங்களித்துள்ளன. பதில் அளிப்பதில்/பெறுவதில் உள்ள வேகம் (இது கால இசைவற்றதாக இருந்தாலும், கால இசைவுள்ளது போன்ற தோற்றமளிக்கிறது), ஊடகத்தளத்தின் சுலபத்தன்மையான அணுகல் (மொபைல் போன்களின் மூலம்) மற்றும் இந்த தளத்தை பயன்படுத்துவதில் உள்ள பொருளாதாரம் போன்றவை, இந்த விருப்பத்தேர்விற்கான சில முக்கிய காரணிகள். வாட்சப் இந்த அனைத்துக் காரணிகளையும் உள்ளடக்கியதாக இருந்ததால், குறிப்பாக ஒரேசமயத்தில் கால இசைவுள்ளதாகவும், கால இசைவற்றதாகவும் இருத்தல் (ஒருவர் எப்படி எடுத்துக்கொள்கிறார் என்பதைப் பொறுத்து) அது ஒரு விருப்பத்தேர்வு ஊடகமாக இருக்கிறது. மேலும், பல இல்லத்தரசிகள் முகநூலில் இருந்தாலும், அவர்களது சமூக ஊடகத்தள நடவடிக்கை வட்டம், அவர்கள் முகநூலை ${ }^{85}$ விட வாட்சப்பில் அதிக சுறுசுறுப்புடன் இயங்குவதாக சான்றளிக்கிறது.

கால இசைவற்ற, குரல்வழி தகவல் பரிமாற்றம் என்ற உத்தி, முதன்மையானது. லட்சுமி போன்ற அனைத்து பணிபுரியும் அன்னைகளுக்கோ அல்லது மேற்கூறிய இல்லத்தரசிகளுக்கோ மட்டுமல்லாமல், தொலைதூர காதலை பேணுவதற்கு பாடுபடும் ஆனந்த் போன்ற இளம் ஆண்களுக்கும் தான். ஆனந்த் என்கிற 26 வயது இளைஞர், தனது முந்தைய பணியிடத்திலுள்ள ஒரு பெண்ணுடன் நிலையான உறவுமுறையில் இருக்கிறார். அவரது காதலி தமிழ்நாட்டின் தென்மேற்கில் உள்ள நகரமான கோயம்பத்தூருக்கு பணிமாற்றம் செய்யப்பட்டுவிட்டார். இந்த தம்பதி கடந்த ஆறு மாதங்களாக பிரிந்திருக்கின்றனர். அவர் பல்வேறு அமைப்புகளில் எடுக்கப்பட்ட தன்னுடைய படங்களை (சுய-புகைப்படம் மற்றும் காட்சிகொடுத்து எடுக்கப்பட்ட புகைப்படங்கள்) தன் காதலை வெளிப்படுத்தும் ஒரு குரல்வழிச்செய்தியுடன் சேர்த்து தன் காதலிக்கு அனுப்புவார். அவர்கள் பிரிந்திருந்த காலத்தின் துவக்கத்தில், ஆனந்த் தன் காதலியையோ அல்லது அவர் ஆனந்தையோ அழைத்துப் பேசுவதற்கு அடிக்கடி முயன்றனர். 
இருப்பினும், இதற்கு அவர்கள் அதிக அளவு தங்களின் ஓய்வு நேரங்களையும் திட்டமிட்டு செயல்பட வேண்டியதாக இருந்தது. எனினும், வாட்சப் மூலமான தனது தகவல்கள் மிகவும் தன்னிச்சையானதாக இருப்பதால் அது தன் காதலியுடனான உறவுமுறையை அடுத்த நிலைக்கு எடுத்துச்சென்றுவிட்டதாக ஆனந்த் கூறுகிறார். இந்த தம்பதியினர் ஒரு நாள்பொழுதில், எண்ணற்ற தூதுரைகளை பரிமாறிக்கொள்கின்றனர் ${ }^{86}$.

வாட்சப்பில் பெண் உபயோகிப்பாளர்கள் சந்தித்த மாபெரும் பிரச்சினை என்னவென்றால், தங்கள் மொபைல் எண்ணை பகிர்ந்து கொள்ள நேரும் விசாரம் தான். ஒத்த ஆர்வக் குழுவின் உறுப்பினர்களை பரிச்சயமானவர்களாகப் பார்த்தாலும், ஒரு சில பெண்கள், தங்களுக்கு முழுமையாக பரிச்சயமற்றவர்களுடன் தங்களது மொபைல் எண்களை பகிர்ந்து கொள்வதில் அசௌகர்யமடைகிறார்கள். இருப்பினும், வாட்சப்பில் ஒத்த ஆர்வக்குழுவில் உறுப்பினராக இருப்பதற்கு அவ்வாறு செய்து தான் தீரவேண்டும். மற்றவர்களின் விசாரம், தங்கள் சமூக வட்டங்களை பிரித்து வைப்பது தான். இயல்புநிலையில் அவர்கள் எப்போதோ ஒருமுறை சந்தித்து தங்களின் மொபைல் எண்ணை பகிர்ந்து கொண்ட யாரோ ஒருவர் கூட இவர்கள் வாட்சப்பில் இருந்தால் அவருக்கு தகவல் அனுப்பக்கூடும். இது அவர்களின் தனிப்பட்ட நேரம் மற்றும் இடத்தில் குறுக்கிடுவெதாகக் கருதப்படுகிறது. உதாரணத்திற்கு, 45 வயது இல்லத்தரசியான சிந்து, அவரது வீட்டில் முன்பு பணிபுரிந்த ஆண் பணியாளரிடமிருந்து வாட்சப் மூலம் வாழ்த்துச் செய்திகளைப் பெற்றார். 30 வயது இ இல்லத்தரசியான சரோஜாவின் வழக்கும் அதேபோன்றது தான். அவரது முந்தைய இல்லத்தை வடிவமைபதில் உதவிய ஆண் உட்புற வடிவமைப்பாளரிடமிருந்து அவருக்கு வாழ்த்துச் செய்திகள் அனுப்பப்பட்டது. இரண்டு பெண்களும், இதுபோன்ற செய்திகளை வரவேற்கவில்லை. அவர்களைப் பொறுத்தவரை, இது அவர்களின் தனிப்பட்ட நேரம் மற்றும் இடத்தில் ஏற்பட்ட குறுக்கீடு தான்.

இருப்பினும், அந்தரங்கம் தொடர்பான இந்த விசாரங்கள், பெண்களை வாட்சப் பயன்படுத்துவதில் இருந்து தடுக்கவில்லை. உண்மையில், பஞ்சக்கிராமியில், உயர்-மத்தியதர வகுப்பைச் சேர்ந்த இல்லத்தரசிகளிடையே வாட்சப் மிகவும் பிரபலமான ஒரு சாதனமாக இருக்கிறது. அவர்கள் வாட்சப்பை குடியிருப்பு சமூக விழாக்கள், உள்ளூர் சர்ச் நிகழ்ச்சிகள் மற்றும் சீட்டாட்ட கேளிக்கை விருந்துகள் ${ }^{87}$ போன்றவற்றை ஏற்பாடு செய்ய பயன்படுத்துகிறார்கள். உதாரணத்திற்கு, 
இந்த அத்தியாயத்தின் துவக்கத்தில், பழங்காலத்து இல்லத்தரசிகள் எவ்வாறு தங்கள் குழந்தைகளை, அண்டைவீட்டில், தங்களுக்கு அவசரமாக தேவைப்படும் ஏதாவதொரு சமையல் உட்பொருள் கடன் வாங்குவதற்காக அனுப்புவார்கள் என்பதை பார்த்தோம். இப்போதெல்லாம் இல்லத்தரசிகள், இதுபோன்ற விஷயங்களுக்கு தங்கள் வாட்சப் குழுவில் முதலில் தகவல் அனுப்புகிறார்கள். அதற்கான பதில் பெற்ற பிறகு, தங்கள் குழந்தைகளை அந்தப் பொருளை பெறுவதற்கு அனுப்புகிறார்கள். கீழ்மட்ட சமூக பொருளாதார வகுப்பைச் சேர்ந்தவர்களின் விஷயத்தில், இது போன்ற கடன்வாங்குதல் என்பது, இன்றும், குழந்தைகளை அனுப்புவதாகத்தான் இருக்கிறது. வாட்சப் தகவலுக்கான தேவையே இல்லாமல்.

தம்பதிகள் ஒருவருக்கொருவர் பொருட்கள் வாங்குவதில் வாட்சப் மூலம் உதவுவது பற்றி முன்பு குறிப்பிப்டது போல, மத்தியதர வகுப்பைச்சேர்ந்த கணவர்கள், வீட்டிற்கான மளிகை சாமான்கள் வாங்கும் பொறுப்பை ஏற்றுக்கொண்டு, சரியான பொருள் தான் வாங்குகிறோமா என்று மனைவியுடன் சரிபார்த்துக்கொள்வதற்காக, அவற்றை புகைப்படம் எடுத்து, மனைவிக்கு வாட்சப் மூலம் அனுப்பி சரிபாத்துக்கொள்வது போன்ற பலசம்பவங்களும் நடந்ததுண்டு. சில இல்லத்தரசிகள், வாட்சப்பை, தங்களது விற்பனை வழியாக பயன்படுத்தி, தங்கள் நண்பர்களையும், அண்டை வீட்டாரையும் ${ }^{88}$ தங்களது நுகர்வோராக கருதி தங்களது பொருட்களை அவர்களுக்கு விற்பனை செய்து, தொழில் முனைவர்களாகவும் மாறியிருக்கிறார்கள்.

ஒரு சில வழக்குகளில் வாட்சப் மூலம் அனுப்பப்பட்ட தனிப்பட்ட புகைப்படங்கள் முகநூல் மூலம் அனுப்பியவற்றைக் காட்டிலும் அதிகமானவை. ரங்கன் என்ற 32 வயதானவர், சென்னையின் பிரபலமான வாடகைக்கார் நிறுவனத்தில் வாகன ஓட்டெராக பணிபுரிகிறார். இவர் பஞ்சக்கிராமிக்கு பணிநிமித்தம் அடிக்கடி பயணம் மேற்கொள்வார். அப்படி போகும்போதெல்லாம், ஹோட்டல்களில் இ இருந்து அங்குள்ள அலுவலகங்களுக்கு அவர் அழைத்துச் செல்லும் வெளிநாட்டவருடன் சுயபுகைப்படம் எடுத்துக்கொள்வார். இவர் அந்த புகைப்படங்களை தனது வாட்சப் நண்பர்களுக்கு அனுப்பி, தனக்கு சர்வதேச தொடர்புகள் இருப்பது போன்ற தோற்றத்தை ஏற்படுத்தி அவர்களிடையே தனது அந்தஸ்தை உயர்த்திக்கொள்ள முனைவார். பணியில் இருக்கும்போது எடுத்துக்கொள்ளப்பட்ட மற்ற சுயபுகைப்படங்களை, புதிதாக திருமணமான அவர், தனது மனைவிக்கு வாட்சப் மூலம் அனுப்பிவைப்பார். பஞ்சக்கிராமியில், புகைப்படங்கள், பொதுவாக முகநூலை- 
க்காட்டிலும், வாட்சப் மூலமாகவே பகிரப்படுகின்றன. இதன் மூலம் அவற்றின் அந்தரங்கம் காக்கப்பட்டு, அவை அனுப்பப்படவேண்டிய நபர்களை உபயோகிப்பாளர்களால் கட்டுப்படுத்தமுடியும் (குறைந்தபட்சம் கட்டுப்படுத்துவாக கருத முடியும்) என்பதால் தான் இப்படி. குடும்பம் மற்றும் உறவுமுறைகளைப் பற்றி கலந்தாய்வு செய்யும் அத்தியாயம் 4-ல், புதிதாகப் பிறந்த குழந்தைகளின் புகைப்படங்களை, நெருங்கிய குடும்ப உறுப்பினர்கள் மத்தியில் சுற்றோட்டம் விடுவதைப் பற்றிய வழக்குகளையும் நாம் அலசப்போகிறோம். இதுபோன்ற புகைப்படங்கள், கண்ணேறை ${ }^{89}$ தவிர்ப்பதற்காக, முகநூலை விட வாட்சப் மூலமாகவே பகிரப்படுகின்றன.

வாட்சப் முகநூலை விட சம்பிரதாயமானது என்ற கருத்து, சில அலுவலகப் பணியாளர்கள், தங்களது நிர்வாகிகளுக்கு, வாட்சப் ${ }^{90}$ மூலம் தகவல் அனுப்பியதால் உருவானது. வாட்சப் இலவசமானது என்பது ஒரு கூடுதெல் கவர்ச்சி. 2013-ல் வாட்சப், ஓராண்டு உபயோகத்திற்கு ${ }^{91}$ பிறகு, கட்டணச்சேவை உடையதாக ஆகிவிடும் என்று கூறப்பட்டது. பஞ்சக்கிராமியில் உள்ள பல உள்ளூர்வாசிகள், இதுகுறித்த தங்களது அறியாமையை ஒத்துக்கொண்டனர். இது அறிந்த ஒருசிலர், அந்த ஒரு வருடத்தின் இறுதியில், வாட்சப்பை அகற்றிவிட்டு, மீண்டும் தங்களது போன்களில் நிறுவிக்கொண்டனர். இது ஜனவரி 2016-ல் வாட்சப் வாழ்நாள் இலவச சேவையைய12 அறிவித்தபோது மாறியது. எப்படியிருப்பினும், பஞ்சக்கிராமியின் குடியிருப்பாளர்களுக்கு இந்த மாற்றம் பற்றி தெரிந்தாவது இருக்குமா என்பது சந்தேகம் தான். அவர்களைப்பொறுத்தவரை, வாட்சப் எப்போதுமே இலவசமானது தான்.

குலம், வர்க்கம், பாலினம் போன்ற காரணிகளின் தாக்கம், வாட்சப் பயன்பாட்டின் மீதும் இருந்தாலும், அதைப்பற்றி முகநூலைப்பற்றியதை போன்ற விரிவான கலந்தாய்வுகள் எதுவும் இந்தப்பகுதியில் நடத்தப்படவில்லை. இதற்கான பகுதிக்காரணம், முன்பே குறிப்பிட்டது போல, இந்தக்காரணிகளால் உருவாக்கப்பட்டு முகநூலில் காணப்பட்ட பல போக்குகள், வாட்சப்பிலும் மீண்டும் காணப்பட்டன என்ற குறிப்பிடல் தான். முக்கியமாக இந்தக் காரணிகள், தனிநபர்களின் பொது விவரங்களைக் கொண்டிருக்கும், பொதுநோக்கிய சமூக ஊடகங்களான முகநூல் போன்றவற்றில் தான் குவியம் கொண்டுள்ளன. முகநூலை விட பெருமளவில் தனிப்பட்ட தளமான வாட்சப், இதுபோன்ற காரணிகளை, பின்னணிக்கு தள்ளிவிட்டது. இதன் விளைவாக, பஞ்சக்கிராமியில் வாட்சப் பற்றிய இந்தப் பகுதி, இந்தத்தளத்தையும் அதன் உபயோ- 
கங்களையும் பற்றிய பொதுவான கலந்தாய்வில் மட்டும் கவனம் செலுத்தியுள்ளது.

\section{டுவிட்டர்}

பஞ்சக்கிராமியில், டுவிட்டர், வாட்சப் அல்லது முகநூலைப்போல அதிக பிராபல்யமோ, அல்லது பரவலான உபயோகமோ கொண்டிருக்கவில்லை. கணக்கெடிப்பின் முடிவுகளும், பேட்டிகளும் தெளிவாக எடுத்துரைத்தது என்னவென்றால், முகநூல், வாட்சப்பும் அனைவருக்கும் பொதுவான ஊடகத்தளங்களாகவும், டுவிட்டர், படித்தவர்கள் மற்றும் பணக்காரர்களுக்கான ${ }^{93}$ ஊடகத்தளமாக கருதப்பட்டது என்பது தான். இதற்கான காரணம், ஏதோவொரு வகையில், டிவிட்டர் ஆங்கிலப்புலமையுடன் இணைத்துப் பார்க்கப்பட்டதும், பின்தொடர்வாளர்களை சேகரிப்பதற்கு அதிக மெனக்கடவேண்டிய தளமாகவும் பார்க்கப்பட்டது தான். மேலும், டுவிட்டரின் மூலமான தகவல் பரிமாற்றம், திசையற்றது என்பதும், அதனால் ஆயத்த அரங்கத்தினரை கொண்டிராததும் தான். கூடுதலாக, மற்றவரது டுவீட்களை பார்ப்பதற்கு, அனைத்து டுவீட்களும் பொதுப்பார்வைக்கானவை, ஒருவருக்கு டிவிட்டர் கணக்கு தேவைப்படாவிட்டாலும், தங்களது அரங்கத்தினரை சேகரிப்பதற்கு, ஒருவர், டுவிட்டரில் மும்முரமான பங்கேற்பு கொண்டிருக்கவேண்டும்.

இதுபோன்ற பலகாரணங்கள் குறிப்பிடப்பட்டன. பஞ்சக்கிராமியின் சில குடியிருப்பாளர்கள், ுவெட்டர் கணக்குகளை உருவாக்கிக்கொண்டாலும், அதை அவர்கள், அந்த ஊடகத்தைப் பற்றி அறிந்துகொள்ளும் ஆர்வத்தால் செய்தார்களே தவிர, உண்மையில் டுவிட்டரை பயன்படுத்தவேண்டும் என்ற எண்ணத்தால் அல்ல என்பதை ஒத்துக்கொண்டார்கள். ஒருசிலர் இதை உபயோகிக்க முயற்சித்து தோற்றுவிட்டார்கள். வினோத் என்ற 23 வயது பட்டதாரியைப் போல. அவர், நான்கு முறை டுவிட்டரில் சேர்ந்து, ஒவ்வொருமுறையும், அதைப் பயன்படுத்தாமல் விட்டுவிட்டார். டுவிட்டரில் தகவல் பரிமாற்றம் அவருக்கு மிகவும் கடினமானதாக இருந்தது. இருப்பினும் அவர் தனது அபிமான திரைநட்சத்திரங்கள் அனைவரின் டுவீட்களையும் படித்தார். கோலிவுட் ${ }^{94}$ என்றழைக்கப்படும் தமிழ்த் திரையுலகத்தில் உள்ள பல பிரபலங்கள் தங்கள் கருத்துக்களை, தங்களது டுவிட்டர் கணக்கின் மூலம் வெளிப்படுத்துகின்றனர். அவர்களுக்கு மாபெரும் பின்தொடர்வாளர் 95 கூட்டம் இருக்கிறது. சரத் என்ற 22 வயதான கல்லூரி மாணவர், 
தனது அபிமான திரை நட்சத்திரமான தனுஷ் என்பவரை பின்தொடர்வதற்காக மட்டுமே, தனக்கென்று ஒரு டுவிட்டர் கணக்கை ஏற்படுத்திக்கொண்டிருக்கிறார். அவர், டுவீட்கள் அல்லது மறு-டுவீட்கள் இடுவதோ, இந்தத்தளத்தில் ஏதேனும் பகிர்ந்து கொள்வதோ இல்லை. அவரது டுவிட்டர் பயணத்தின் துவக்கத்தில், சரத், தனது அபிமான நட்சத்திரத்தின் புகைப்படங்களை தனது பக்கத்தில் பகிர்ந்து கொண்டார். அவை அவருக்கு பெரிய பின்தொடர்வாளர் எவரையும் பெற்றுத்தரவில்லை.

வினோத்தை போலவே சரத்திற்கும், அவரது தோல்வியின் விளைவாக பின்தொடர்வாளர்களை கவருவதற்காக பல டுவிட்டர் கணக்குகள் உண்டு. உத்தேசமாக 22 முதல் 25 கணக்குகளை அவர் வைத்திருக்கிறார். அவ்வப்போது, தனது நண்பர்கள் சிலரையும், டிவிட்டர் கணக்கு துவங்கி தனது அபிமான நட்சித்திரத்தை பின்தொடருமாறு அரித்தெடுத்துக்கொண்டிருக்கிறார். இதற்கான காரணம் மிகவும் எளிமையானது. சரத், டிவிட்டரில் தனது அபிமான நட்சத்திரத்திற்கான பின்தொடர்வாளர்களின் எண்ணிக்கையை அதிகரிக்க விழைகிறார். அதேபோல பிரியா என்கிற 22 வயதான கல்லூரி மாணவி, தனது தோல்வியை ஒத்துக்கொள்ளுமுன், அவரது அபிமான திரை நட்சத்திரங்களின் டுவீட்களை மறு-டுவீட் செய்வதற்கு பலமுறை முயன்றார். அவர், இதை விட்டதன் காரணமும், பின்தொடர்வாளர்கள் இல்லாதது தான். இயல்புநிலை வாழ்வில் பிரபலமாக இருந்தால் தான் டுவிட்டரில் பின்தொடர்வாளர்களை சேகரிப்பது சுலபம், இல்லையேல் அது அதிக மெனக்கெட வேண்டிய ஒரு செயல் என்று அவர் கருதினார்.

பல திறன்-வாய்ந்த ஐ.டி பணியாளர்கள், டுவிட்டரில் ஓரளவிற்கு சுறுசுறுப்பான செயல்பாட்டுடன் இருந்தார்கள் அல்லது குறைந்தபட்சம், முடக்க நிலையிலேனும் அதில் ஈடுபட்டிருந்தனர். அவர்களைப்பொறுத்தவரை, சிலிக்கான் பள்ளத்தாக்கின் எண்ணத்தலைவர்களான, கய் கவாஸாகி அல்லது ராபர்ட் ஸ்காப்ளே போன்ற, புகழ்பெற்ற பிரபலங்கள் எவரையேனும் பின்தொடர்ந்தால், டுவிட்டர் என்பது ஒரு சிறந்த அறிவார்த்த தளமாக உதவக்கூடும் என்று கருதினர். பலர், இந்திய பிரதமர் திரு. நரேந்திர மோடியை டுவிட்டரில் பின்தொடர்ந்து கொண்டிருந்தனர். இதில் பெரும்பாலானோர், செய்திகளையும் தகவல்களையும் மறு-டுவீட்கள் மூலம் பகிர்ந்துகொண்டிருந்தாலும், அவர்கள், முதலில் தங்களுக்கென பின்தொடர்வாளர்களை பெறுவதற்கு முய- 
ற்சிகள் செய்ய வேண்டும் என்பதை ஒத்துக்கொண்டனர். ஒரு பெரிய ஐ.டி நிறுவனத்தில் பணிபுரியும் சுடேன் என்கிற 30 வயதான வணிக ஆய்வாளர், தான் தன் நண்பர்களில் பலரையும் டுவிட்டரில் பின்தொடர்ந்ததாகவும், அவர்களும் பதிலுக்கு இவரை பின்தொடர்ந்ததாகவும் கூறினார். இந்தவகையில், இவர்கள் ஒருவருக்கொருவர், பின்தொடர்வாளர்களின் எண்ணிக்கையை அதிகப்படுத்திக்கொண்டனர்.

பஞ்சக்கிராமியில் ஒரு பெண் ஐ.டி பணியாளர் கொலை செய்யப்பட்ட பிறகு, டுவிட்டர் கருத்துக்களை வெளுப்படுத்துவதற்கான மாபெரும் சாதனமாக ஆகிவிட்டது. ஐ.டி தொழில்நெறிஞர்கள் பலரும் ஆண் பெண் என்ற பேதமின்றி, டுவிட்டர் மூலமாக, தங்கள் ஆதரவையும், இந்தத்துறையில் பணிபுரியும் பெண்களின் பாதுகாப்பு பற்றிய தங்களது விசாரத்தையும் வெளிப்படுத்தினர். தேவி என்கிற 28 வயதான ஐ.டி தொழில்நெறிஞர் விளக்கியதாவது:

“இந்தச்சம்பவத்திற்கு பிறகு, பெண்களுக்கு பாதுகாப்பு வேண்டும் என்று நான் ஒரு டுவீட் பதிவு செய்தேன். திடீரென்று, எனது பதிவு பலராலும் மறு-டுவீட் செய்யப்பட்டது. இந்த சம்பவத்தின் அலை ஓய்ந்தபிறகு, எனது எந்தவொரு பதிவும் மறு-டுவீட் செய்யப்படவில்லை.... என்னைப்பின்தொடரும் எனது நண்பர்கள் சிலர், தகவல் பகிர்வதற்காக சிலசமயம் மறு-டுவீட் செய்வார்கள். இப்போது நான் முன்பின் அறியாதவர்கள் எல்லாம் எனக்கு பின்தொடர்வாளர்களாக இ இருக்கிறார்கள்.... எனது பின்தொடர்வாளர்கள் மீதான கட்டுப்பாட்டை நான் இழந்துவிடுவேன் போலிருக்கிறது....அவர்களில் பலர் யாரென்று எனக்கு நிஜமாகவே தெரியாது"

ஒருசில தொழில் முனைவாளர்கள், தங்கள் தர அடையாளங்களை பற்றிய விழிப்புணர்வை மேம்படுத்துவதற்கு டுவிட்டரை பயன்படுத்தினர். பல தொழில்முனைவாளர்கள், தங்களை டுவெட்டரில் "எண்ணத்தலைவர்களாக" காட்டிக்கொள்வதை விரும்பினர். அவர்களில் ஒருவர் ஆனந்தி, 44 வயதான, நுண்திறன் மற்றும் தகவல்துறை வல்லுநர். இவர் தனது சுயவிவரப்பக்கத்தை, தகவல் துறையில் எண்ணத்தலைவராக காட்டிக்கொள்ளும் வகையில் டுவீட்களை பதிவு செய்தார். அவர், டுவிட்டரில் தனது தர அடையாளம் அமைக்கும் இருப்பு அவருக்கு வணிகத்தை பெற்றுத்தந்தது என்று கூறினார். 40 வயதான தொழில்நுட்ப தொழில்முனை- 
வரும் எழுத்தாளருமான ரிஷியின் வழக்கும் இதுபோன்றது தான். அவர் டுவிட்டரில் இருப்பதற்கான ஒரே காரணம், தனக்கான தனிப்பட்ட தர அடையாளத்தை அமைத்துக்கொள்வது தான். பஞ்சக்கிராமியில் உள்ள பல சிறுதொழில்கள், டுவிட்டரில் இருந்தன. அனால் அவற்றில் குறைந்தது 70 சதவிகிதம் தற்போது செயலற்றதாக இருக்கின்றன ${ }^{96}$. பஞ்சக்கிராமியில் இருக்கும் பன்னாட்டு நிறுவனங்கள் பல டுவிட்டரில் இருந்தன. ஆனால் அவர்களின் டுவீட்கள் பெரும்பாலும் உலகளாவியவையாக இருந்தன. அவர்களின் சமூக ஊடகக்குழு, பஞ்சக்கிராமியில் அமைந்திருப்பதாக தெரியவில்லை.

பஞ்சக்கிராமியில், டுவிட்டரை உபயோகிப்பதில் வேறும் அன்றாட தகவல் பரிமாற்ற கருவியாக எண்ணாமல், ஒரு திடமான நோக்க உணர்வு இருந்தது. முகநூல், வாட்சப் போன்ற பிற சமூக ஊடகத்தளங்களுடன் டுவிட்டரை ஒப்பிட்டுப் பார்க்கும்போது, டுவிட்டரில் பரிமாறிக்கொள்ளப்பட்ட தகவல்கள், முகநூலில் பரிமாறிக்கொள்ளப்பட்ட தகவல்களில் இருந்து மிகவும் வித்தியாசமாக இருந்தது தெளிவாக விளங்கியது. ஒருவர் தரநெறிசார்புடையவராகவும், அரசியல் ரீதியாக சரியானவராகவும் இருக்கவேண்டிய தளமாக டுவிட்டர் கருதப்பட்டது. டுவிட்டரில் முட்டாள்தனமாக ஏதாவது செய்துவிட்டால், இணையதள சமூகம் அவரை பழித்துத்தள்ளிவிடும் என்ற அச்சம் நிலவுகிறது. ஆதலால் இங்கு பதிவிடப்படும் தகவல்கள், கவனமானதாகவும், நடுநிலையானதாகவும், தரநெறிசார்புள்ளதாகவும் இருக்க வேண்டியிருக்கிறது. இதற்கு மாறாக, முகநூலிலோ அதை விட அதிகமாக வாட்சப்பிலோ, ஒருவர், தங்களது சுய இயல்புடன் இருப்பது மிகவும் சுலபம்.

\section{முடிவுரை}

இந்த அத்தியாயத்தின் நோக்கம், பாரம்பரியமான தகவல் பரிமாற்ற வகைகளில் துவங்கி, இந்தப்பகுதியில் பல்வேறு சமூக ஊடககதளங்களை ஆராய்வது நோக்கி நகர்வதன் மூலம் பஞ்சக்கிராமியிலுள்ள சமூக ஊடகங்களையும், தகவல் பரிமாற்ற நிலப்பரப்பையும் அறிமுகப்படுத்துவது தான். இவ்வாறு செய்வதன் மூலம், இந்த அத்தியாயம், பஞ்சக்கிராமியில் சமூக ஊடக பயன்பாட்டை நிர்வகிக்கும் சிக்கலான அடுக்குகளையும் அளித்திருக்கிறது. தெளிவாக தெரிந்த இன்னொரு விஷயம் என்னவென்றால், குடும்பத்தைப் 
பற்றிய அக்கறை, விசாரம் போன்ற உணர்வுபூர்வ காரணிகள் மற்றும், வயது, அதிகாரப்படிநிலை, சமூக அந்தஸ்து, கல்வியறிவு, குலம், வர்க்கம், பாலினம் போன்ற சமூகக் காரணிகள் ஆகியவை ஒன்றுக்கொன்று ஆதிக்கம் செலுத்தி பாரம்பரிய இயல்புநிலை தகவல் பரிமாற்றத்தை பாதிப்பதோடல்லாமல், சமூக ஊடகங்களின் மீதும் பாதிப்பை ஏற்படுத்துகின்றன என்பது தான்.

குடும்பங்களுக்குள்ளான போன் மூலமான குரல்வழி தகவல் பரிமாற்றம் கூட வயது, அதிகாரப்படிநிலை, கல்வியறிவு, உறவினர்களுக்குள்ளான அந்தஸ்து போன்ற காரணிகளால் பாதிக்கப்படுகின்றன என்பது திரு. ராகவன் மற்றும் ரவியின் தாயார் ஆகியோரின் வழக்குகளின் மூலம் தெளிவாகிறது. இவை, குழந்தைகளின் நலம் பற்றிய உணர்வுபூ ர்வ விசாரங்களால் மேலும் சிக்கலாக்கப் படுகிறது என்பது, ஷோபனா மற்றும் லட்சுமி ஆகியோரின் உதாரணங்கள் மூலம் தெளிவாகிறது. ேோபனாவின் வழக்கில் இதற்கான தீர்வு கால இசைவுள்ள தொலைபேசி அழைப்பாக இருந்தாலும், லக்ஷ்மியின் வழக்கில், இதற்கான தீர்வு, கால இசைவற்ற வாட்சப் குரல்வழி தகவலாக இருந்தது.

இதுபோன்ற உணர்வுபூர்வ விசாரங்கள், வர்க்கம், குலம் மற்றும் குடும்ப கௌரவம் போன்ற காரணிகளால் பாதிக்கப்படும்போது, சமூகக் கட்டுப்பாடு மற்றும் சமூக ஊடகங்களின் கண்காணிப்பு என்ற வடிவத்தை எடுக்கிறது. இதுபோன்ற விசாரங்களின் பாதிப்பு குறிப்பாக பாலின விஷயங்களில் உணரப்படுகிறது. சமூக ஊடக பயன்பாட்டை பாதித்த சமூக கட்டுப்பாடு மற்றும் பெண்களின் மீதான கண்காணிப்பு ஆகியவற்றை கடிமையான தடைகள் மற்றும் மென்மையான கட்டுப்பாடுகள் என இருவகைப்படுத்தலாம். கீழ்மட்ட சமூகப்பொருளாதார வகுப்புகள் மற்றும் கீழ்-மத்தியதர வகுப்புகள் ஆகியவற்றில் கடிமையான தடைகள் நான்கு தனிப்பட்ட வகைகளில் அமல்படுத்தப்பட்டன:

1. ஒட்டுமொத்த தடை: இது, தங்கள் குடும்பங்கள் மற்றும் குலத்தில் உள்ள மணமாகாத இளம் பெண்களின் மீது ஆண் உறுப்பினர்கள் ஒரு கண்காணிக்கும் பார்வை வைத்துக்கொள்ள முற்படும் வழக்குகளில் நடக்கிறது. இந்த ஆண்கள், இந்தப் பெண்களை, தங்கள் குடும்பத்தையும் தங்களையும் தங்கள் சமூகத்தில் அவமானப்படுத்தக்கூடிய குலத்தினிடை காதல் உறவுகள் பற்றிய 
பயத்தால் சமூக ஊடகங்களை பயன்படுத்துவதற்கு தடை விதிக்கின்றனர்.

2. நேரக்கட்டுப்பாடுகள்: இந்தவகையான கட்டுப்பாடு, சமூக ஊடகப்பயன்பாட்டை அனுமதிப்பதால் தங்களை குறைந்த அளவு பழமைவாதிகளாக ${ }^{97}$ கருதும் குடும்பங்களில் விதிக்கப்படுகிறது. பல்வேறு சாதனங்களை உபயோகிக்கக்கூடிய நேரங்களின் மீது கட்டுப்பாடிகள் விதிக்கப்பட்டு அவை கடுமையாக கடைபிடிக்கப்படுகிறது.

3. இடக்கட்டுப்பாடுகள்: இது மீண்டும், தங்களை குறைந்த அளவு பழமைவாதிகளாக கருதும் குடும்பங்களில் விதிக்கப்படுகிறது. வீட்டின் வெளியே உள்ள இடப்பரப்பு, ஆணுக்குரியதாகவும், ஆபத்து நிறைந்ததாகவும், பெண்களுக்கு கேடுவிளைவிப்பதாகவும் கருதப்படுகிறது. ஆகவே, அவர்களுக்கு வீட்டினுள் மட்டுமே குடும்பத்தாரின் கவனமான பார்வையின் கீழ் சமூக ஊடகங்களை பயன்படுத்துவதற்கான அனுமதி வழங்கப்படுகிறது. பாதுகாப்பான இடப்பரப்பு மற்றும் மற்றவகையான இடப்பரப்பு போன்ற கருத்துக்கள், இதுபோன்ற குடிம்பங்களில் அடிக்கடி உச்சரிக்கப்படுகின்றன.

4. உள்நோக்கத்துடனான கண்காணிப்பு: இது, தங்கள் குடும்பத்தின் பெண் உறுப்பினர்களை, சமூக ஊடகத்தை பயன்படுத்துவதற்கு அனுமதித்துவிட்டு, அவர்களின், நட்புதொடர்புகளை, குடும்ப உறுப்பினர் அல்லது இயல்புநிலை வாழ்வில் அவர்கள் அறிந்தவர்கள் ஆகியோருக்கு மட்டுமே கட்டுப்படுத்தும் குடும்பங்களில் தெளிவாகத் தெரிகிறது. இளம்பெண்களின் சமூக ஊடகப் பக்கங்களின் மீதும் அவர்களின் சமூக ஊடகப் பதிவுகளின் மீதும் கடுமையான கண்காணிப்பு, குடும்பத்தாரால் வைக்கப்படுகிறது. இதுபோன்ற உள்நோக்கத்துடனான கண்காணிப்பு, நேரம் மற்றும் இடக்கட்டுப்பாடுகள் விதிக்கும் குடும்பங்களிலும் காணப்படுகெிறது.

பொதுவில் உள்நோக்கம் இல்லாத மென்மையான கட்டுப்பாடுகள், மற்றவர்களுடன் சேர்த்து குடும்ப உறுப்பினர்களும் நிகழ்நிலையில் நட்புதொடர்பு கொள்ளும் குடும்பங்களில் காணப்படுகிறது. சமூக ஊடக நடவடிக்கைகளில் எந்த குறிப்பிட்ட கட்டுப்பாடுகள் விதிக்கப்படாவிட்டாலும், நண்பர்களாக இருக்கும் குடும்ப நண்பர்களின் இருப்பே, இந்தப் பெண்கள் 
சுய தணிக்கை செய்யப்பட்ட பதிவுகள் இடிவதை உறுதிப்படுத்திவிடுகிறது. இதுபோன்ற கட்டுப்பாடு, பலவகையான வகுப்புகளை சேர்ந்த இளம் ஆண்கள் மீதும் விதிக்கப்படுகிறது. சமூக ஊடகங்களில் ஒருவரையொருவர் நட்புதொடர்பு கொண்ட ஆசிரியர்களும் மாணவர்களும், இது போன்ற நட்புதொடர்பு, மென்மையான கட்டுப்பாடுகளுக்கு வழிவகுக்கிறது என்று உணர்ந்தனர். இந்த நிலவரம் அத்தியாயம் 6-ல் விரிவாக ஆராயப்பட்டிருக்கிறது.

கடினமோ, மென்மையோ, இதுபோன்ற கண்காணிப்புகளில் இருந்து தப்புவதற்காக, பொய்யான மற்றும் பல சமூக ஊடக கணக்குகள், துவங்கப்படுகின்றன. பஞ்சக்கிராமியின் பல சமூக ஊடக உபயோகிப்பாளர்களுக்கு, இது போன்று பல ஊடகக் கணக்குகள் மூலம் தங்களை வெளிப்படுத்திக்கொள்வது என்பது தங்கள் அடையாளங்களை முழுமையடைய செய்கிறது.

இந்தப்பிரச்சினைகள், இநந்த அத்தியாயத்தின் முகநூல் பகுதியில் விரிவாக கலந்தாய்வு செய்யப்பட்டிருக்கிறது. இருப்பினும், இந்தப்பிரச்சினையின் பல பரிமாணங்கள், மற்ற ஊடகத்தள உபயோகத்திலும் புலப்பட்டன.

வாட்சப்பின் கட்டுப்படியாகும் தன்மையும், அதன் செயல்திறனும், அதை, பஞ்சக்கிராமியின் குடியிருப்பாளர்களிடையே பிரபலமான விருப்பத்தேர்வாக ஆக்கினாலும், அது பலராலும் குறுஞ்செய்தி அனுப்புதலின் நீட்டிப்பாகத்தான் பார்க்கப்பட்டது. இதன் செயல்பாட்டுத்திறன், உபயோகிப்பாளர்களை தங்கள் தகவல் பரிமாற்றத்தை பெருக்க அளவீடு செய்ய அனுமதிப்பதன் மூலம், பொது மற்றும் தனிப்பட்ட வட்டங்களுக்கிடையே ${ }^{98}$ தடையற்ற இயக்கத்திற்கு வழிவகுத்தது. உணர்வுபூர்வ விசாரங்கள், வர்க்கம், பாலினம் போன்ற காரணிகள், வாட்சப்பின் பயன்பாட்டுப் பாணிகளையும் பாதித்தன.

அதேபோல, டுவிட்டர், வர்க்கம் மற்றும் ஆங்கில கல்வியறிவு ஆகியவற்றுடன் நெருங்கிய தொடர்புடையதாக கருதப்பட்டது. பஞ்சக்கிராமியில் வாழும் பலருக்கு, இநந்தத்தளம், பின்தொடர்வாளர்களை பெறுவதற்கு, முகநூல் மற்றும் வாட்சப்பை விட அதிக அளவிலான மெனக்கெடலுடன் தொடர்புடையதாக இருந்தது. ஒருரிலர், டுவிட்டரில் பிராபல்யம் மற்றும் அடையாளம்-அமைத்தலை, இயல்புநிலை பிராபல்யத்தோடு ஒப்பிட்டாலும், மற்றவர்கள், “எண்ணத்தலைவர்களாக" தங்களது அடையாளத்தை அமைத்துக்கொள்ள அதைப் பயன்படுத்திக்கொண்டனர். டுவிட்டரில் பழித்துப் பேசப்படுவெது 
பற்றிய பயம், உபயோகிப்பாளர்களின், தரநெறி சார்புள்ள, சர்ச்சைக்குட்படாத பதிவுகளுக்கு வழிவகுத்தது.

இருப்பினும், ஒரே ஒரு சமூக ஊடகத்தளத்தில் இருப்பதும், அதன் பிரத்யேகமான உபயோகமும், மிகவும் அபூர்வம். பஞ்சக்கிராமியில், பன்னூடகங்கள் ${ }^{99}$ என்ற வலுவான வழக்கு நிலுவையில் இருந்தது. வலைத்தொடர்புகளில் தங்களது பங்களிப்பை இயக்கமுள்ளதாக காட்டுவதற்காக, ஒரே விஷயத்தை பல்வேறு தளங்களில் (உதாரணத்திற்கு, ஒரே செய்தியைப் பற்றிய தொடர்பை, ஒரேசமயத்தில் முகநூல், வாட்சப், டுவிட்டர் லிங்க்ட்-இன் ஆகிய அனைத்திலும் பகிர்தல்) பதிவிடுவோர் இருந்தாலும், தனிநபர்களுடன் தகவல் பரிமாறிக்கொள்வது என்று வரும்போது, உரையாடல்கள் மிகவும் அந்தரங்கமானதாகவும், சூழ்நிலைக்கேற்றதாகவும் ஆகிவிடுகிறது. இது அளவீட்டுச் சமுதாயத்திற்கான ${ }^{100}$ ஒரு வலுவான நிலைப்பாடாகவும் இருக்கிறது.

சமூக ஊடகங்களை பற்றிய விசாரமே இல்லாமல், முகநூலில் குடிம்பங்களால் விதிக்கப்படும் சமூகக் கட்டுப்பாடு, அல்லது வாட்சப் குழுக்களில் உள்ள குழு விதிமுறைகள் அல்லது டுவிட்டரில் உள்ள பழிச்சொற்கள் பற்றிய பயம் - ஆகிய அனைத்தையும் விட வெளிப்படும் மற்றொரு வலுவான கருத்து, சமூக அனுசரித்தலைப் பற்றிய எதிர்பார்ப்புகள் தான். இது வலுவான இயல்புநிலை எண்ணச்சார்பை கொண்டது. நிகழ்நிலை மற்றும் இயல்புநிலை இடங்களுக்கு இடையேயான இந்த எண்ணச்சார்பு, எவ்வாறு சமூக அனுசரித்தலை பாதிக்கிறது மற்றும் இதுபோன்ற அனுசரிப்பு குறித்த அழுத்தங்கள் எவ்வாறு, ஒருவரின் வலைதொடர்பில், தன்னின் மூலோபாய முன்னிலைப்படுத்தலுக்கு வழிவகுக்கிறது என்பதை அத்தியாயம் 3 ஆராய்கிறது. 


\section{காட்சிக்குரிய பதிவுகள்: தொடரும் காட்சிக்குரிய இடப்பரப்பு}

\section{அறிமுகவுரை}

திரு என்கிற 21 வயதான தானியங்கி வாகன பொறிமுறையாளரும், அவரது நண்பர்களான 23 வயது வடிவேலு மற்றும் 19 வயது லக்ஷ்மணசுவாமி ஆகியோரும், ஒன்றுவிட்ட ஞாயிற்றுக்கிழமைகளில் சென்னையை சுற்றியுள்ள ஷாப்பிங் வளாகங்கள், ரசனையான காட்சிக்குரிய சுற்றுலா தலங்கள் போன்ற இடங்களுக்கு சென்று தங்களை அந்த இடங்களில் பல புகைப்படங்கள் எடுத்துக்கொள்வர். மூவரும் இதுபோன்ற பயணங்களை, "புகைப்பட சுற்றுலாக்கள்" என்று குறிப்பிடுகின்றனர். இவற்றில் எடுக்கப்படும் புகைப்படங்கள் குறிப்பாக, அவர்களது சமூக ஊடகத்தள பக்கங்களில் பதிவேற்றுவதற்காகவே எடுக்கப்படுகின்றன. வேறு சில நண்பர்களும் எப்போதாவது அவர்களுடன் சேர்ந்துகொள்ள அழைக்கப்படுகிறார்கள். ஆனால் பெரும்பாலும், இந்த மூவர் மட்டுமே இதுபோன்ற புகைப்பட சுற்றுலாக்களுக்கு திருவின் வாடிக்கையாளர்களின் மோட்டார்சைக்கிள்களில் கிளம்பிவிடிகின்றனர். இம்மூவரின், முகநூல் பக்கங்களில் உள்ள புகைப்படங்களில் 60 சதவிகிதம், இதுபோன்ற புகைப்பட சுற்றுலாக்களில் எடுக்கப்பட்டவை தான்.

இந்தக்குழுவால் 2013-ல் எடுக்கப்பட்ட பெரும்பாலான புகைப்படங்கள், கேமரா முன் காட்சி கொடுக்கும் வகையில் நின்று எடுக்கப்பட்டவையாக இருந்தாலும், 2014-ல் இருந்து எடுக்கப்பட்டவை சுய-புகைப்படங்கள் ${ }^{1}$ என்ற வகையை 
நோக்கி நகர்ந்து விட்டன. இதில் மாறாமல் இருந்த ஒரு விஷயம் என்னவென்றால், இம்மூவரும், ஒரு குழுவாக நின்று புகைப்படங்கள் எடுத்துக்கொண்டது தான். அவரவர் ஊடக பக்கங்களில் இவர்களின் தனிப்பட்ட புகைப்படங்கள் காணப்பட்டாலும், இவர்களுக்கு மிகவும் முக்கியமாக இருப்பது இவர்கள் மூவரும் சேர்ந்திருக்கும் புகைப்படங்கள் தான். இந்தக் குழு புகைப்படங்கள் இந்த இளைஞர்களின் நட்பை மட்டுமல்லாமல் அவர்களின் வாழ்க்கை முறை மற்றும் அவர்கள் தங்கள் நட்பின் ${ }^{2}$ மூலம் தங்கள் வாழ்க்கையை அனுபவிக்கும் விதம் ஆகிய அனைத்தையும் காட்சிப்படுத்துகின்றன. இந்த புகைப்படங்களை, தங்களது சமூக வலைத்தொடர்புகள் ${ }^{3}$ கூடும் சமூக ஊடகத்தளங்களில் பதிவேற்றுவது இந்த மூவருக்கும் மிகவும் முக்கியமானதாக இருந்தது. ஏனெனில் இது, இம்மூவரின் வாழ்நாள் முழுமைக்கான நட்பை, இவர்களுக்கு வேண்டியவர்களுக்கு எடுத்துக்காட்டுகிறது.

நட்புறவு என்பது தமிழ்நாட்டில் ${ }^{4}$ கொண்டாடப்படும் ஒரு சீர்மை. ஒருவரின் சிறந்த நண்பர், அவரது குடும்பத்தார் மற்றும் உறவினர்களை விட உயர்ந்த அந்தஸ்தில் கருதப்படுகிறார். இதுபோன்ற நட்புறவுகள், மிகவும் புனிதமான உறவுமுறைகளாக கருதப்படுகின்றன. இந்தசீர்மை, தமிழ்த் திரைப்படங்கள் போன்ற பிரபலமான காட்சிக்குரிய ஊடகங்களின் வாயிலான பொதுப்பிரசங்கங்களின் மூலம் மீீ்டிம் மீண்டும் வலியுறுத்தப்படுகிறது. இளைஞர்களின் கருத்தியலில் இதுபோன்ற கருத்துக்கள் ஆழமாகப் பதிந்திருப்பதால், இவற்றைப் பின்பற்றுவதும், அதைவிட முக்கியமாக இவற்றை பரவலான அரங்கத்தினருக்கு காட்சிப்படுத்துவதும், மிகவும் முக்கியத்துவம் பெற்றதாகிவிடுகிறது. இந்த மூவரும், தங்களின் முகநூல் புகைப்படங்களின் மூலம், அதையேதான் செய்கிறார்கள். (படம்: 3.1)

வீணா என்பவர் 42 வயதான இல்லத்தரசி. பள்ளி செல்லும் இரண்டு குழந்தைகளின் தாயும் ஆன அவர், அடுக்குமாடி குடியிருப்பு ஒன்றில் உள்ள நளினமும் அழகும் கொண்ட 3 படுக்கையறை வீட்டில் குடியிருக்கிறார். அவர், கடற்கரையை சுத்தப்படுத்துவது, தரும காரியத்திற்காக நெடுந்தொலைவு ஓட்டம் அல்லது அறக்கட்டளைகளுக்காக சமைப்பது போன்ற தரும காரியங்கள் மற்றும் பொதுநல நிகழ்ச்சிகளில் தனது நண்பர்களான 37 வயது அனுஷ்யா மற்றும் 45 வயது கௌரி ஆகியோருடன் பங்கேற்றுக்கொள்வார். அவருக்கு, இந்த நிகழ்ச்சிகள் எதுவுமே, சுயபுகைப்படமோ அல்லது காட்சி கொடுத்து எடுக்கப்படும் புகைப்படமோ இல்லாமல் நிறைவுறாது. இந்த 


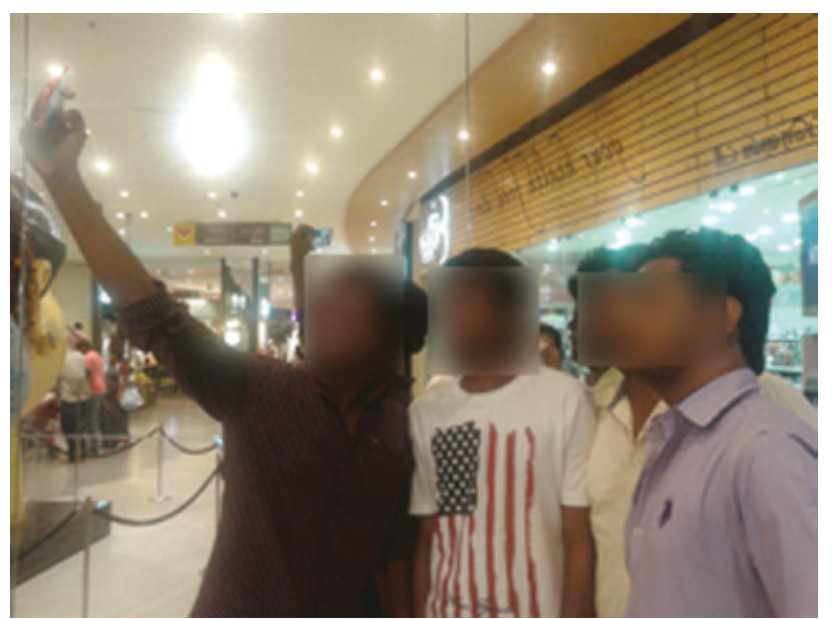

படம்: 3.1 வணிக வளாகத்தில் புகைப்பட சுற்றுலா

புகைப்படங்கள் உடனே, குடும்பம் மற்றும் நண்பர்கள் இருக்கும் வாட்சப் குழுக்களில் பகிரப்படும். இதுபோன்ற புகைப்படங்கள், பொது நிகழ்ச்சிகளில் தனிப்பட்ட தருணங்களை பிரதிபலிக்கின்றன என்பது வீணாவின் எண்ணம். அதனால், இவை அவருக்கு நன்கறிந்தவர்கள் இ இருக்கும் குழுக்களில் மட்டுமே பகிரப்படும் என்று அவர் கருதுகிறார். வீணாவிற்கு முகநூல் கணக்கு கிடையாது. தர்மகாரியங்களில் பங்கேற்பது குறித்த புகைப்படங்களை பொதுப்பார்வைக்கு காட்சிப்படுத்துவது பகட்டுக் காட்சிமைத்தனமாகும் என்பது இவரது கருத்து. இருப்பினும், இதுபோன்ற புகைப்படங்கள், இவ்வாறு பொதுநலக் காரியங்களில் பங்கேற்பது எவ்வளவு மகிழ்ச்சிகரமானது என்பதை அவரது வலைதொடர்பில் உள்ள நண்பர்களுக்கும் குடும்ப உறுப்பினர்களுக்கும் எடுத்துக்காட்டி அவர்களையும் இதுபோன்ற பொதுநல காரியங்களில் பங்கெடுத்துக்கொள்ள ஊக்குவிக்கக் கூடும் கொடைக் குணமுள்ள செய்கைகள், பொதுநல நிகழ்ச்சிகளுக்கு தன்னார்வல ஊழியராக இருத்தல், சமுதாயத்திற்கு தன்னால் முடிந்ததை திருப்பிச்செய்வது போன்றவை, பெண்களுக்கான முக்கியமான சீர்மைகளாக இந்தியாவில் 6 பொதுவாக கருதப்படுவதோடு, குறிப்பிட்ட மத்தியதர வகுப்பு கருத்தியலின் ${ }^{7}$ முக்கியமான பண்புக்கூறை வடிவமைக்கிறது. வீணாவுக்கு இதுபோன்ற நிகழ்ச்சிகள், பொதுநல நோக்கோடு சேர்ந்து, நண்பர்களோடு சேர்ந்திருக்கும் தருணங்களைப் பற்றி, பின்னர் நினைத்துப் பார்த்து மகிழக்கூ- 
டிய வாஞ்சையான நினைவுகளை உருவாக்கிக்கொள்ள கிடைக்கும் ஒரு வாய்ப்பாகவும் இருக்கிறது ${ }^{8}$.

ரத்தினவேலு என்கிற 42 வயதான தொழில் முனைவர், கட்டுமான நிறுவனங்களுக்கு வன்பொருட்களை விநியோகம் செய்வதில் ஈடுபட்டிருக்கிறார். ரத்தினவேலுவும், 38 வயது இல்லத்தரசியான அவரது மனைவியும், முகநூல் கணக்குகள் வைத்திருக்கிறார்கள். அதில் அவர்கள், தங்களது புகைப்படங்களை மட்டுமல்லாமல், தங்களது உடனடி குடும்பத்து உறுப்பினர்களின் புகைப்படங்களையும் பதிவேற்றுகின்றனர் (பள்ளிசெல்லும் தங்களது இரண்டு குழந்தைகள் மற்றும் தங்களது நீட்டித்த குடும்பங்கள் மற்றும் அவர்கள் உறவினர்கள் ${ }^{9}$ போல் கருதும் நண்பர்கள் ஆகியோர் பங்கேற்கும் குடும்ப விழாக்கள் மற்றும் நிகழ்ச்சிகளின் புகைப்படங்கள் ஆகியவையும் இதில் அடங்கும்). அவர்களின் வலைத்தொடர்புகளுடனான வாட்சப் தகவல்கள் கூட, குடும்பத்துடனான உல்லாசப்பயணம், ஒன்று கூடிய நிகழ்ச்சிகள் போன்றவற்றின் புகைப்படங்களாக இருக்கும். இவற்றைத்தவிர, அவர்கள் ஒழுக்கம் சார்ந்த மீம்களையும் தாயத்திற்கு பொருத்தமான கருத்துக்கள், வேடிக்கைகளை, இந்து சமயக் கடவுள்களின் படங்கள் ஆகியவற்றையும் முன்னனுப்புகின்றனர். அவரது சமூக ஊடகத்தள பக்கங்களில் உள்ள புகைப்பட தொகுப்புகள், உடனடி மற்றும் நீட்டித்த குடும்பங்களின் புகைப்படங்களால் நிரம்பியுள்ளன (படம்: 3.2). ரத்தினவேலுவும் அலமேலுவும், தங்களது குடும்பப்பிணைப்பை, தங்கள் சமூக வலைதொடர்பில் எடுத்துக்காட்டுவதை மிகவும் அவசியமாக் கருதுகின்றனர். ஏனெனில், உறுதியான குடும்ப மற்றும் உறவுப் பிணைப்புகள், தமிழ்க் கலாச்சாரத்தின் ${ }^{11}$ சீர்மைகளாக கருதப்படுகின்றன. அவர்களது முகநூல் பக்கத்தில் உள்ள இதுபோன்ற படங்கள், அவர்களின் சமூக வலைதொடர்பில் ${ }^{12}$ இருப்போரிடமிருந்து, தொடர்ச்சியான விருப்புகளையும், கருத்துக்களையும், தனிப்பட்ட தகவல்களையும் பெற்றவண்ணம் இருக்கின்றன.

குருநாத் என்கிற 20 வயதான உயிர்வேதியியல் மாணவர், தனது அபிமான தமிழ்த்திரை நட்சத்திரமான விஜய் அவர்களின் புகைப்படங்களையும், அவருடன் இணைந்து நடிக்கும் நடிகைகளின் புகைப்படங்களையும், திரை நட்சத்திரத்தின் வெளியான, வரப்போகிற திரைப்படங்களின் சுவரிதழ்களையும் தொடர்ந்து பதிவேற்றிக்கொண்டே இருப்பார் (படம்: 3.3). இவையெல்லாம், குருநாத்தின் சமூக ஊடகப்பக்கங்களில் உள்ள ரசிகர்களின் வலைதொடர்பில் உள்ளவர்களுக்காகவும், 


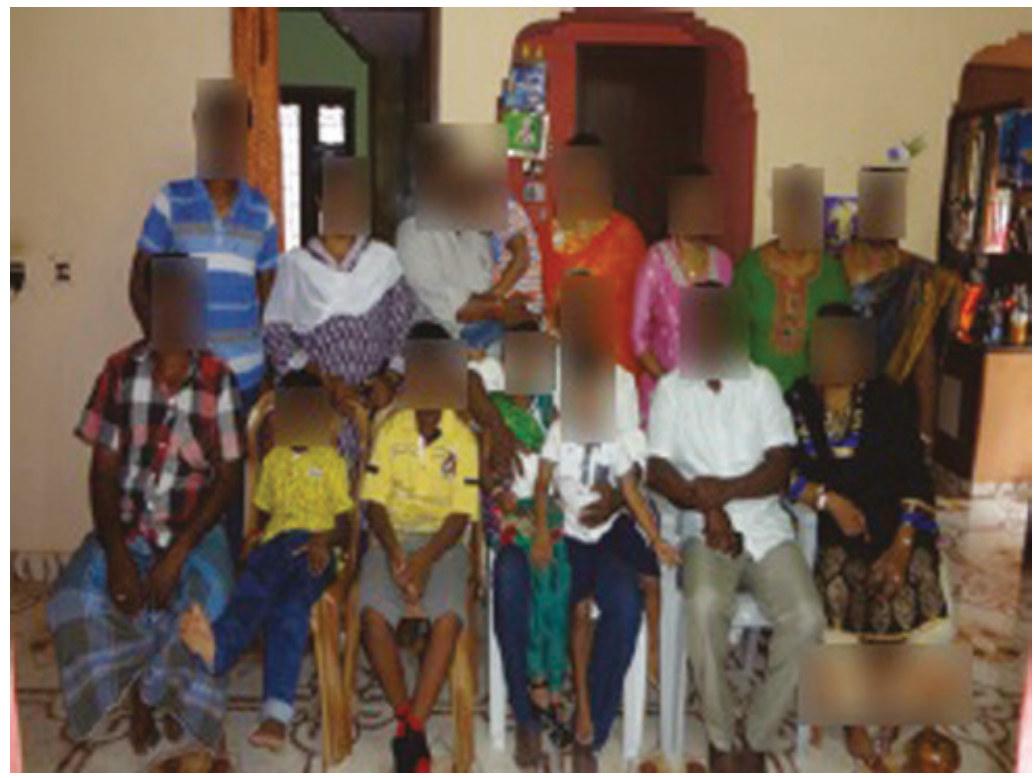

படம்: 3.2 முகநூலில் பதிவேற்றப்பட்டுள்ள குடும்ப புகைப்படம்

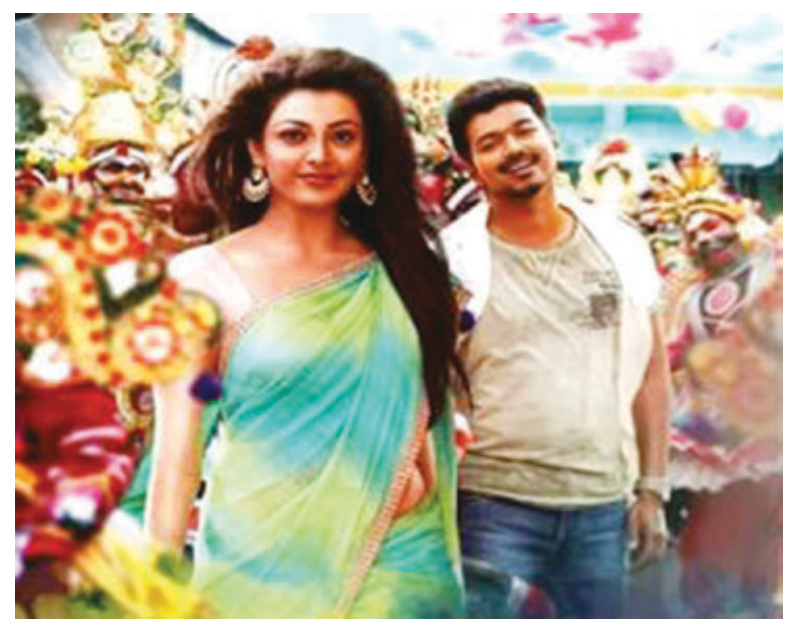

படம்: 3.3 நடிகர் விஜய் அவரது உடன் நடிக்கும் நட்சத்திரத்துடன் 
முகநூலில் உள்ள அந்த திரை நட்சத்திரத்தின் ரசிகர் மன்றப் பக்கத்திற்காகவும் பதிவேற்றப்படுபெவ. பிரபலமான ஆண் திரை நட்சத்திரங்களுக்கு ரசிகர் மன்றங்கள் இருப்பது தமிழ்த் திரைக்கலாச்சாரத்தின் முக்கியமான கூறாகும். இது தமிழ்நாட்டில் அரை நூற்றாண்டுக்கும் 13 மேல் வழக்கத்தில் உள்ள ஒரு அம்சமாகும். இந்த மன்றங்கள் பெரும்பாலும் ஆண்களைக் கொண்டதாகவே இருக்கும். இதுபோன்ற மன்றங்களில் உறுப்பினராக உள்ள ரசிகர்கள், தங்களின் விசுவாசத்தையும், உடைமை உணர்வையும், காட்சிப்படுத்த வேண்டும் என்று எதிர்பார்க்கப்படுகிறார்கள். குருநாத், இதை, முகநூலில், நடிகர் விஜய்யின் ரசிகர்கள் உள்ள அவரது வலைத்தொடர்பு பக்கத்தில் செய்கிறார். அவர் பெரும் விருப்புகளும், கருத்துக்களும் பெரும்பாலும் நடிகர் விஜய்யின் ரசிகர்களிடம் இருந்துதான்.

மேற்கூறிய ஒவ்வொரு வழக்குகளும், பஞ்சக்கிராமியில் வாழும் பலவித மக்கள் குழுக்களில், ஒன்றில் உள்ள குறிப்பிட்ட வகையான காட்சிப்பதிவுகள் தான். இந்த வழக்குகள் ஒவ்வொன்றிலும் குறிப்பிடப்படவேண்டிய விஷயம் என்னவென்றால், இதுபோன்று நிகழ்நிலைத் தளங்களை, தங்கள் கலாச்சார சீர்மைகள், விதிமுறைகள், அன்றாடப் பண்புகள் போன்றவற்றை காட்சிப்படுத்துவதற்கு என ஒதுக்கிவைப்பது என்பது, கூட்டு நெறிமுறைகளின் சில பெரிய மற்றும் ஏற்கனவே நிறுவப்பட்ட கூறுகளை, அலைபரப்பும் வழிவகையாகும். உதாரணத்திற்கு, நட்புறவுகளை மதித்தல், தர்மகாரியங்கள், குடும்ப பிணைப்பு, உபயோகிப்பாளர்கள் தங்களை அடையாளம் கண்டுகொள்ளும் மற்றும் தாங்கள் முக்கியமானதாகக் கருதும் சமூக வலைத்தொடர்பு அல்லது குழுவின் மீதான விசுவாசத்தை வெளிக்காட்டுவது போன்றவை.

காட்சிக்குரியவை பற்றிய இந்த அத்தியாயம், பஞ்சக்கிராமியில் உள்ள மக்களுக்கு, நிகழ்நிலை சமூக ஊடகங்களில் உள்ள காட்சிக்குரிய இடப்பரப்புகள் என்பவை, இந்தப் பகுதியின் இயல்புநிலை காட்சிக் கலாச்சாரத்தில் ஏற்கனவே இடம்பெற்றுள்ள பெரிய மற்றும் ஏற்கனவே நிறுவப்பட்ட கூட்டுச் சீர்மைகளை காட்சிப்படுத்தும் இடமாக இருக்கிறது என்பது பற்றி விவாதிக்கிறது.

இது, பஞ்சக்கிராமியின் இயல்புநிலை காட்சிகாலச்சாரத்தைப் பற்றிய சுருக்கமான பின்னணித் தகவலை, பொதுநோக்கிய காட்சிக் கலாச்சாரம், வீட்டிலுள்ள காட்சிக்குரியவை மற்றும் இந்த இரண்டுக்கும் இடையே உள்ளவை ஆகியவற்றை ஆராய்வதன் மூலம் அளிக்கிறது. இது பின்னர், இவற்றின் பிரதிகள் மற்றும் சமூக ஊடகத் தொடர்ச்சி ஆகியவற்றை, 
சினிமா மற்றும் அரசியல் ஆகியவற்றைப் பற்றிய பொதுநோக்கிய காட்சிப் பதிவுகள் மற்றும் தனிப்பட்ட அகநிலைப் பண்புகளை முன்னிலைப்படுத்தும் காட்சிகள் ஆகியவற்றை கூர்நோக்குவதன் மூலம் ஆராய்கிறது. அன்றாட வாழ்த்துச்செய்திகள், அவற்றைத் தொடர்ந்த நிகழ்நிலை சமூகத்துவத்தை அமைக்க உதவும் மீம்கள், போன்றவையும், பொதுநோக்கிய மற்றும் தனிப்பட்ட ஆகியவற்றுக்கிடையே விழும் மூன்றாவது வகையான காட்சிகளாக ஆராயப்படுகின்றன. மேற்கூறியவற்றைக் கருத்தில் கொண்டு, இந்த அத்தியாயம், நிகழ்நிலை காட்சிக்குரிய இடப்பரப்பில் உள்ள பதிவுகள், முகநூல் மற்றும் வாட்சப் போன்ற சமூக ஊடகத்தளங்களில், மக்கள் தங்களுக்காக அமைக்கும் சமூக வலைப்பரப்பின் எதிர்பார்ப்புகளுக்கு, அனுசரணையாக இருக்கிறது என்பதை எடுத்துக்காட்டுகிறது.

\section{பஞ்சக்கிராமியில் உள்ள இயல்புநிலை காட்சிக்கலாச்சாரத்தைப் பற்றிய மேலோட்டக்கருத்து}

ஒன்பது ஆய்வுக்களங்களிலும் நடத்தப்பட்ட இந்த மக்கள் இன அமைப்பியல் ஆராய்ச்சியின் முக்கியமான கண்டுபிடிப்பு என்னவென்றால், சமூக ஊடகங்கள் நமது தகவல் பரிமாற்றத்தை, எழுத்து வடிவமாக ${ }^{14}$ இருப்பதைவிட அதிக அளவு காட்சி வடிவமானதாக ஆக்கிவிட்டது என்பது தான். இது ஒரு முக்கிய மாற்றத்தைக் குறித்தாலும், ஒவ்வொரு குறிப்பிட்ட சந்தர்ப்பத்திலும், இந்த மாற்றம் மற்றும் காட்சிக்குரிய உள்ளடக்கம் ஆகியவை, முந்தைய வகைகள் மற்றும் மரபுகளின் வழிச்சார்பாக இருக்கக்கூடிய சாத்தியக்கூறுகள் இருக்கின்றன.

தமிழ்நாடு இந்தியாவின் மற்ற பகுதிகளைப் போலவே ${ }^{15}$ பொதுநோக்கிய மற்றும் தனிப்பட்ட வட்டங்களில், காட்சிக்குரிய சமூகமாக இருந்திருக்கிறது. கடவுள்கள், அரசியல்வாதிகள், திரைநட்சத்திரங்கள், விளம்பரங்கள் ${ }^{16}$, கேலிச்சித்திரங்கள் ${ }^{17}$ ஆகியவற்றின் அசையா புகைப்படங்களாகவும், சுவரொட்டிகள், விளம்பரப்பதாகைகளாகவும், கட்-அவுட் என்று உள்ளூரில் குறிப்பிடப்படும் விளம்பரப்பலகைகளாகவும், பொதுவிடத்தில், சினிமா போன்ற அசையும் படங்களாகவும் ${ }^{18}$ அல்லது சமயம் சார்ந்த படங்களாகவும் ${ }^{19}$ இல்லங்களில், நாட்காட்டிகள், சட்டமிடப்பட்ட புகைப்பட- 
ங்கள் ${ }^{20}$ மற்றும் தனிப்பட்ட காணொளிகளாகவும் ${ }^{21}$ படங்கள் வழிவழியாக தோன்றிக்கொண்டு தான் இருக்கின்றன.

\section{பொதுநோக்கிய காட்சிக் கலாச்சாரம்:}

தமிழ்நாட்டில், சினிமா, கிரிக்கெட், சமயம், அரசியல், பிறந்தநாள் விழாக்கள், பெண்கள் பூப்பெய்துவது குறித்த விழாக்கள், திருமண விழாக்கள், மரணம் அல்லது எந்தவொரு வாழ்க்கை சுழற்சி தொடர்பான விழாக்கள் போன்ற பல நிகழ்ச்சிகள் பொதுநோக்கியவையாகவும் காட்சிக்குரியவையாகவும் கொண்டாடப்படும் ஒரு இடமாகும். இவற்றில், கண்ணைக் கவருபவை, சமயம்சார்ந்த விழாக்களும், சினிமா மற்றும் அரசியல் சார்ந்த விழாக்களும் தான். பருவக்காலத்திற்கு ஏற்றவாறு, சமயம்சார்ந்த விழாக்கள், தமிழ்நாட்டின் பொதுநோக்கிய காட்சிக் கலாச்சாரத்தின் மீது தமது ஆதிக்கத்தை செலுத்துகின்றன. உதாரணத்திற்கு, ஆடி மாதத்தில் 22 (ஜூலை-ஆகஸ்ட்)பொதுவாக, அம்மன் ${ }^{23}$ என்றழைக்கப்படும், இந்துசமய தாய்க் கடவுளுக்கு, விழாக்கள் நடைபெறுகின்றன. இதைத்தொடர்ந்து, இநந்துசமயக் கடவுள்களான கிருஷ்ணர் மற்றும் கணேசருக்கும் ${ }^{24}$ விழாக்கள் நடத்தப்படுகின்றன. இவையனைத்துமே, பொதுப்பார்வைக்கு வைக்கப்பட்ட, கோவில்களின் விவரங்கள் மற்றும் விழாக்கொண்டாட்டங்களின் விவரங்கள் போன்றவற்றைக் குறிக்கும், சுவரொட்டிகள், பதாகைகள் போன்றவற்றுடன் கொண்டாடப்படுகின்றன. அருகில் உள்ள சர்ச் மற்றும் மசூதிகளில் நடக்கும் கிறிஸ்துமஸ் மற்றும் ரமதான் போன்ற விழாகொண்டாடட்டங்களைப் பற்றி அறிவிக்கும் சுவரொட்டிகளும் பதாகைகளும் கூட பல இடங்களில் காணப்படுகின்றன. கிராமத்திருவிழாக்களும், இதைப்பின்பற்றி, பக்தியை 25 பொதுப்பார்வைக்கு காட்சிப்படுத்துகின்றன.

தமிழ்நாட்டை ஆளும் மற்றொரு கவர்ச்சிக்குரிய விஷயம் சினிமா ${ }^{26}$. பல ஆராய்ச்சியாளர்கள், உள்ளூர் சினிமாவின் (கோலிவுட் என்று பிரபலமாக அறியப்படுகிறது) சக்திக்கும் தமிழ்நாட்டு அரசியல் வெற்றியுடன் ${ }^{28}$ அதற்கு இருக்கும் நெருக்கமான உறவுக்கும் ஆதரவுசான்றளிப்பார்கள். விவாதிக்கக்கூடிய வகையில், ஒட்டுமொத்த இந்தியாவிலும் தமிழ்நாடு மட்டுமே, கடந்த அரைநூற்றாண்டில், சினிமாவுடன் தொடர்புடைய ஐந்து முதன்மந்திரிகளை (மாநில முதல்வர்கள்) கொண்டிருப்பதாக பெருமையுடன் பறைசாற்றிக்கொள்ள முடியும். உண்மையில், தமிழ்நாட்டின் தற்போதைய முதல்வ- 
ரும், அதன் மாபெரும் எதிர்க்கட்சியின் தலைவரும், திரைத்துறைப் 29 பின்னணியில் இருந்து வந்தவர்கள் தான்.

காட்சிக்குரிய கலாச்சாரத்தை வெளிப்படுத்துவதில், பஞ்சக்கிராமி எந்தவகையிலும் வித்தியாசமானது அல்ல. அதன் பிரதான சாலைகளின் இருமருங்கிலும் உள்ள பளீரென்ற வண்ணமயமான சுவரொட்டிகள் இதற்கு சான்றளிக்கின்றன. உதாரணத்திற்கு, இங்குள்ள கிராமத்தினர், ஆடி மாத (இந்துசமயத் தாய்க்கடவுளுக்கான காணிக்கைகளுடன், கிராமத்தில் திருவிழாக்கள் நடக்கும் ஒரு தமிழ் மாதம்) கொண்டாட்டங்களுக்கு தயாராகிக்கொண்டிருக்கும் இந்த சமயத்தில், அதன் ஒரு பகுதியாக இங்குள்ள சாலைகளும், தெருக்களும், இந்துசமய கடவுள்களின் உருவங்களை கொண்ட சுவரொட்டிகள் மற்றும் பெரிய பதாகைகளால் அலங்கரிக்கப்பட்டிருக்கின்றன (படம்: 3.4). தீபாவளி போன்ற சமயம் சார்ந்த பண்டிகை, தெய்வங்களின் ${ }^{30}$ உருவமுள்ள சுவரொட்டிகள் அல்லது பதாகைகள், புதுத் திரைப்பட வெளியீடுகள், திரை நட்சத்திரங்களின் படங்கள் மற்றும் தள்ளுபடி விலையில் பொருட்களின் விற்பனை பற்றிய விளம்பரங்கள் ஆகியவற்றின் கலவையால் முன்னறிவிக்கப்படுகிறது. பல சமயங்களில், இந்துசமய கடவுள்கள், பதாகை மற்றும் சுவரொட்டிகளின் இடப்பரப்பை, உள்ளூர் அரசியல்வாதிகள், இனக்குழுத் தலைவர்கள் மற்றும் அன்னதான உபயதாரர்களான உள்ளூர் வணிகர்கள் அல்லது கலைநிகழ்ச்சி பற்றிய விவரங்கள் ஆகியவற்றுடன் பகிர்ந்து கொள்வதை பார்க்கலாம். மேற்கூறிய கலைநிகழ்ச்சி, பெரும்பாலும், பஞ்சக்கிராமியில் உள்ள மக்களுக்கான மெல்லிசை நிகழ்ச்சியாகவோ (தமிழ்திரைப் பாடல்களை மேடைகளில் பாடுவது) அல்லது தெருக்கூத்துக்களாகவோ இருக்கக்கூடும்.

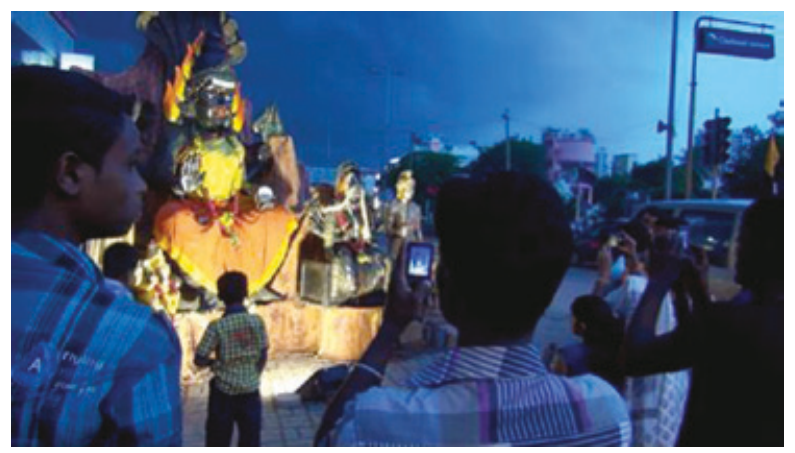

படம்: 3.4 அம்மன் - இந்துசமய தாய்க்கடவுள் 
திரை நட்சத்திரங்கள் மற்றும் திரைப்படங்களின் சுவரொட்டிகள், பொதுச்சுவர்களை அலங்கரிக்கின்றன. அவை புதுத் திரைப்பட வெளியீடுகள் ${ }^{31}$, அல்லது திரை நட்சத்திரங்களின் பிராபல்யம் போன்றவற்றை பிரதிபலிக்கும் வகையில் மாற்றப்படுகின்றன. கட்-அவுட் வகையிலான காட்சிப்படுத்தல் திரையரங்குகளை சுற்றி மட்டுமே காணப்பட்டாலும், தனிப்பட்ட திரைப்படங்களின் சுவரொட்டிகள் இந்தப்பகுதி முழுவதும் காணப்படுகின்றன. தேர்தல் அல்லது வேறு அரசியல் நிகழ்ச்சிகள் நடக்கும் சமயங்களில், அந்த நிகழ்ச்சிக்கேற்றவாறான அரசியல் தலைவர்களின் ஆளுயர பதாகைகள் பஞ்சக்கிராமியின் பிரதான சாலைகளை சுற்றி முளைத்திருக்கும். பல சமயங்களில் அவை, அரசியல் தலைவர்களை புகழும் வகையிலான பசப்புரை வார்த்தைகளை உள்ளடக்கியதாக இருக்கும். உதாரணத்திற்கு, அவர்களின் கௌரவத்தை அதிகரிக்கும் வகையில் "சிங்கமே", சீர்மையான தமிழன் என்பதை குறிக்கும் வகையில் “தமிழனே" அல்லது “தமிழ்த்தாயே" போன்ற புகழுரைகளை இந்தப் பதாகைகள் கொண்டிருக்கும். இது போன்ற அடையாளச்சொற்கள், தமிழ்நாட்டில் பொதுவாக பயன்படுத்தப்படும் பசப்புரைகளை பிரதிபலிக்கின்றன ${ }^{32}$.

பஞ்சக்கிராமியில் உள்ள பெரும்பாலான பொதுப்பார்வைக்குரிய காட்சிப் பொருட்கள், தற்காலிகமானவையே. இவை காலத்திற்கும் வருடத்தின் ${ }^{33}$ போக்கைக் குறிக்கும் நிகழ்ச்சிகளுக்கும் ஏற்றவகையில் மாறிக்கொண்டேயிருக்கும். அவை, தகவலுக்காகவும் அவை ஏற்படுத்தக்கூடிய தாக்கத்திற்காகவுமே வடிவமைக்கப்படுகின்றன. நினைவில் இருப்பதற்காக அல்ல. தேர்தல்கள், அரசியல் நிகழ்ச்சிகள், புதிய திரைப்பட வெளியீடுகள் மற்றும் விழாக்கள் ஆகியவை தான் இவற்றின் அடுத்தடுத்த மாற்றத்திற்கான வினையூக்கிகளாக இருக்கின்றன. இருப்பினும், இல்லங்களில் காட்சிக்குரிய பொருட்கள் என்பவை முற்றிலும் வேறுவகையானவை. தனிப்பட்ட புகைப்படங்கள் நீண்டகாலத்திற்கு காட்சிப்படுத்தும் வகையில் வைக்கப்படுகின்றன. இவை நினைவலைகளை தூண்டும் கலைப்பொருட்களாகவும் இருக்கின்றன.

\section{இல்லங்களில் காட்சிப்படுத்தும் கலாச்சாரம்.}

உள்ளூர்வாசிகளின் இல்லங்களில், அவர்களின் சொந்த புகைப்படங்களோடு சேர்த்து, கடவுள்களின் புகைப்படங்கள், 
தேசீயத் தலைவர்கள் மற்றும் குடும்ப உறுப்பினர்களின் புகைப்படங்கள், இறந்து போன உறவினர்களின் புகைப்படங்கள் போன்றவை, வரவேற்பறை சுவர்கள் மற்றும் புகைப்பட தொகுப்புகளின் பக்கங்கள் ஆகியவற்றை அலங்கரிக்கின்றன. பிறந்தநாள் விழாக்கள், பூப்புநீராட்டு விழாக்கள், திருமணங்கள், குலதெய்வக்கோவிலில் நடந்த விழாக்கள், புதுமனைபுகுவிழாக்கள் போன்று குடும்பத்தில் கொண்டாடப்பட்ட அனைத்து விழாக்களின் காணொளிப்பதிவுகளும், டிவிடி வடிவத்தில், அவற்றின் நினைவாக இல்லங்களில் குடியிருக்கும். மேற்கூறிய புகைப்பட மற்றும் காணொளிப் பதிவுகளில் பெரும்பாலானவை, தொழில்முறை புகைப்பட நிலையங்களிலோ அல்லது தொழில்முறை ஒளிப்பதிவாளர்களாலோ ${ }^{34}$ தான் பதியப்படுகின்றன.

இந்துக்கள் மற்றும் கிறிஸ்தவர்களின் இல்லங்களில் கடவுள்கள் மற்றும் சாதுக்களின் படங்கள், அவர்களின் சிறிய அளவிலான உருவ மூர்த்திகளுடன், பூஜை மாடங்களில் அலங்காரமாக வைக்கப்பட்டிருக்கும்.இந்துக்களின் இல்லங்களில், கடவுளின் உருவப்படங்கள் கொண்ட நாள்காட்டிகள் இரண்டு வகைப்படும். ஒன்று, சாதாரண மாதாந்திர நாட்காட்டிகள், மற்றது பஞ்சாங்கத்துடன் இணைந்த விரிவான நாட்காட்டிகள். கிறிஸ்தவர்களின் இ இல்லங்களில் மாதாந்திர நாட்காட்டிகள் மட்டுமே இருக்கும்.

புகைப்படத்தொகுப்புகளில் இருந்து, வரவேற்பறை சுவர்களிலோ அல்லது அலமாரிகளிலோ, விருந்தினர் பார்க்கும் வகையில் அலங்காரமாக வைப்பதற்காக எடுக்கப்படும் புகைப்படங்கள், ஒருவகையான தணிக்கைமுறைக்கு உள்ளாகும். காட்சி கொடுக்கப்பட்டு எடுக்கப்பட்ட புகைப்படங்களும், அந்தக் குடும்பம் வெளிப்படுத்த வேண்டும் என்று விரும்பும் ஒரு சில கூறுகளை வெளிக்காட்டும் புகைப்படங்களும் மட்டுமே இவ்வாறு பொதுப்பார்வைக்கு வைக்கப்படும் (படம்: 3.5) வரவேற்பறையை அலங்கரிக்கும் படங்கள் எல்லாம், ஏதோவொரு சாதனையை எடுத்துக்காட்டும் படங்கள் (பட்டமளிப்பு விழாக்கள், பரிசு வெல்லும் விழாக்கள் போன்றவை), குடும்பத்தின் ஒற்றுமையை பறைசாற்றும் விதமான படங்கள், அல்லது, வம்சாவளியை ${ }^{36}$ நினைவுபடுத்தும் விதமாகவும் அவர்கள் மிகவும் நேசித்ததை நினைவுகூரும் விதமாகவும் வைக்கப்பட்டிருக்கும் இறந்து போன மூதாதையர்களின் படங்கள் போன்றவை தான். மற்ற புகைப்படங்களில் எல்லாம், நண்பர்களும் ெருங்கிய குடும்பத்தினரும் வருகை தரும் சமயத்தில், அவ்வப்போது ஞாபகங்களை புதுப்பித்துக்கொ- 


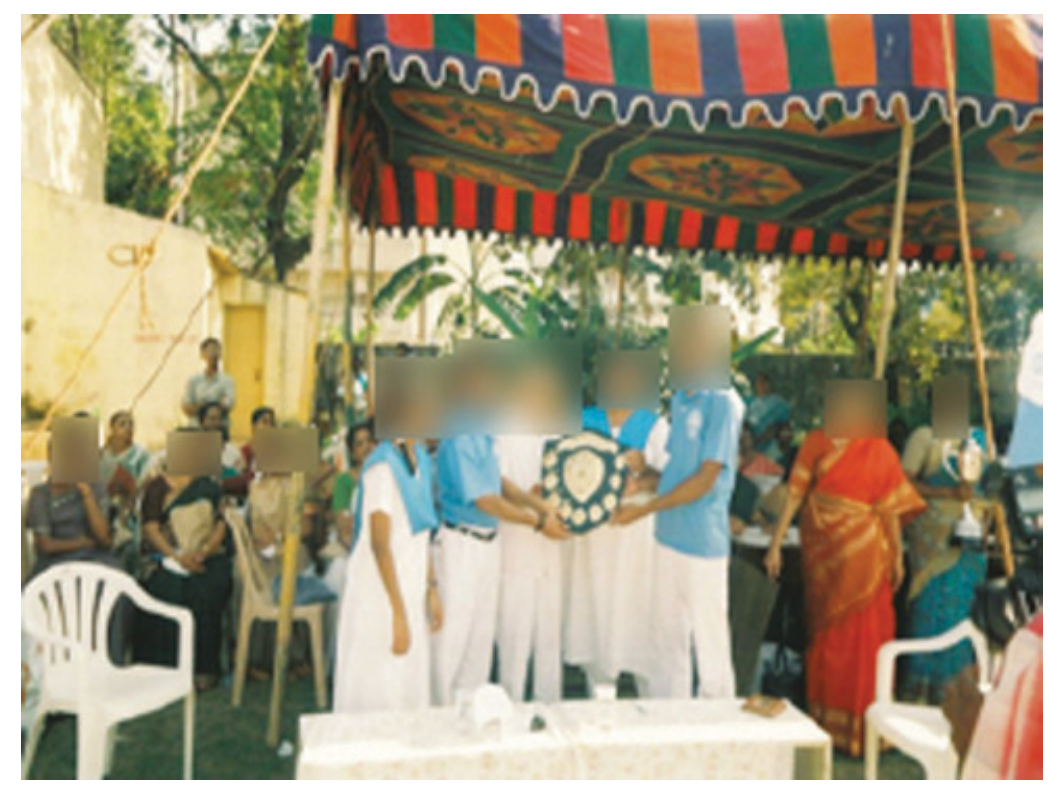

படம்: 3.5 தனிப்பட்ட சாதனையை வெளிப்படுத்தும் புகைப்படம்

ள்வதற்கு ஏற்றவாறு, புகைப்பட தொகுப்புகளில் வைக்கப்பட்டிருக்கும். சங்கடப்படுத்தக்கூடிய படங்கள் எதுவுமே வரவேற்பறைக்கு வராது. அவை எப்போதுமே புகைப்படத்தொகுப்புகளில் மறைத்து வைக்கப்பட்டிருக்கும்.

இல்லங்களில் காட்சிக்கு வைக்கப்பட்டிருக்கும் படங்கள், அடிக்கடி பாதுகாத்து வைக்கப்படிகின்றன. ஆனால் எப்போதுமே அழிக்கப்படுவதில்லை. இது பஞ்சக்கிராமியில் காணப்படும் பொதுக்காட்சி கலாச்சாரத்திற்கு முற்றிலும் மாறுபட்டதாகும். இவை, பல சமயங்களில், விருந்தினர்களுக்கு குறிப்பான தகவல்களை (சாதனைகள் அல்லது குடும்ப ஒற்றுமை) தெரிவிப்பதற்கோ, அல்லது குடும்பத்தினுள்ளேயே நினைவுச்சின்னமாக கருதப்படுவதற்கோ ஆன கலைப்பொருட்களாக பயன்படுகின்றன.

பஞ்சக்கிராமியில் வாழும் மக்கள், ஆளுயர சுவரொட்டிகளை பொதுவாக தங்கள் இல்லங்களுக்கும் கொண்டு வருவது இல்லை. அது போன்ற காட்சிப்படுத்தலுக்கான எல்லைகளை அவர்கள் தெளிவாக வரையறுத்து வைத்திருப்பார்கள். ஆனால், முற்றிலும் பொதுவானதாகவும் அல்லாமல், முற்றிலும் தனிப்பட்டதும் அல்லாமல், இரண்டிற்கும் இடைப்ப- 
ட்டதாகக் கருதப்படும் ஒரு சில காட்சிப்படுத்தல்கள் மட்டும் இதற்கு விதிவிலக்கானவை.

\section{இடைப்பட்டவைகள்.}

ஒரு சில படங்கள், பொதுக்காட்சிப்படுத்தலுக்கும், இல்லங்களில் காட்சிப்படுத்தலுக்கும் இடைப்பட்டவையாக இருக்கும். இவை வழக்கமாக, திருமணம், பூப்புநீராட்டு விழா போன்றவற்றை அறிவிக்கும் பெரிய அளவிலான பதாகைகளாக இருக்கும். இவற்றை, பெரும்பாலும் பஞ்சக்கிராமியின் பிரதான சாலைகளின் பிரிவுகளில் பார்க்கலாம் (படம்: 3.6) இது போன்ற பதாகைகள், மணப்பெண் மற்றும் மணமகன் ஆகியோரின் படங்களையோ அல்லது பூப்பெய்திய பெண்ணின் படங்களையோ அல்லது பிறந்தநாள் விழாக்காணும் குழந்தைகளின் படங்களையோ மட்டுமல்லாமல், இந்த விழாக்கள் பெருவெற்றியடைய வாழ்த்தும் நெருங்கிய நண்பர்கள் மற்றும் உறவினர்களின் படங்களையும் கொண்டிருக்கும். இந்தப் பதாகைகள் மற்றும் படங்களெல்லாம், அவர்களின் சமூக வலைத்தொடர்புகளுக்கு, (குலக்குழு உறுப்பினர்கள்,

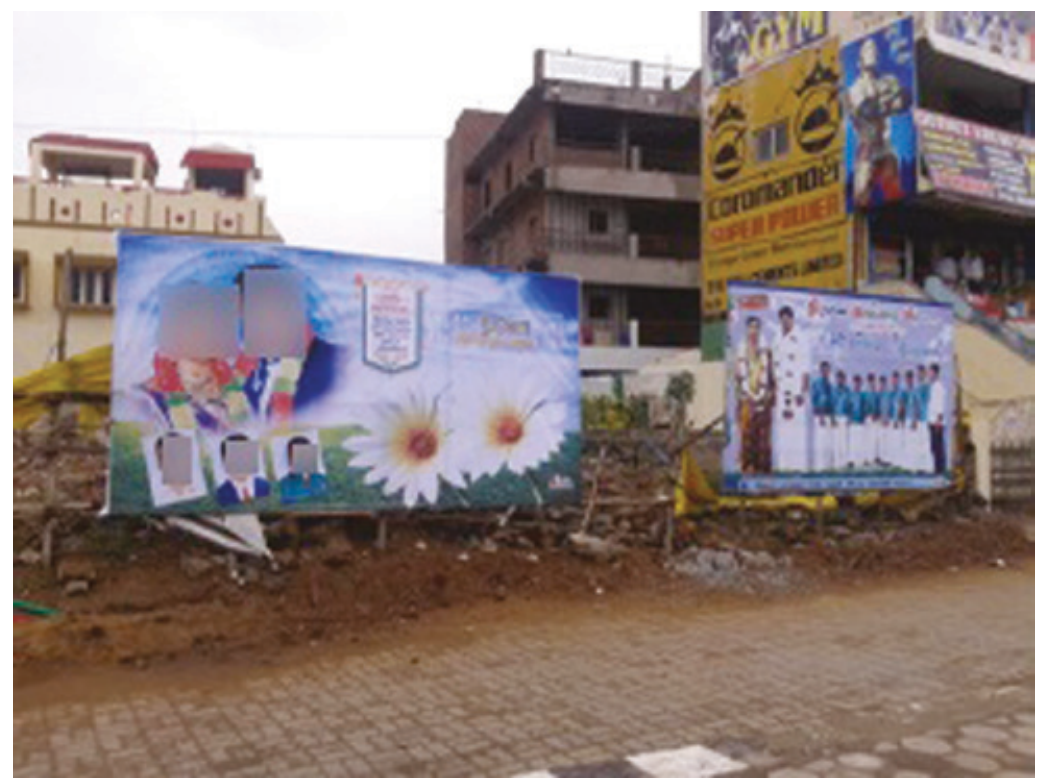

படம்: 3.6 பொதுவிடத்தில், குடும்ப விழா அறிவிப்பு 
அண்டை கிராமத்தை சேர்ந்த மக்கள், தூரத்து உறவினர்கள் போன்றவை) தங்கள் குடிம்ப விழாவைப் பற்றி தெரிவிப்பதாகவும், யாருக்காக விழா நடத்தப்படுகிறதோ, அவர்களுடனான தங்களின் நெருங்கிய பிணைப்பை தெரியப்படுத்தும் விதமாகவும், அமைக்கப்படுகின்றன. இந்தப் பதாகைகள் மற்றும் படங்களில் பெரும்பாலானவை, தற்காலிகமானவையே. விதிவிலக்காக, ஒரு சில மட்டும், உணர்ச்சிபூர்வ காரணங்களுக்காக பாதுகாத்து வைக்கப்படுகின்றன. மற்றவையெல்லாம் திரைப்பட மற்றும் அரசியல் சுவரொட்டிகள்/பதாகைகள் போலவே அழிக்கப்பட்டுவெடுகின்றன.

இங்கும், குடிம்ப நிகழ்ச்சி சம்பந்தமான வீட்டிற்குரிய படங்கள், பொது இடத்திற்கு நகரக்கூடும். அவற்றில் பெரும்பாலானவை வீடு திரும்புவதில்லை. அவை குறிப்பிட்ட சமூக வலைத்தொடர்பு நோக்கிய குறிப்பிட்ட செய்திகளுக்காக அமைக்கப்பட்டவை. அத்தோடு அவற்றின் பணி முடிந்து விடுகிறது.

\section{சமூக ஊடகங்கள், இந்தக் காட்சிப்படுத்தல் கலாச்சாரத்தை எவ்வாறு பிரதிபலிக்கின்றன.}

காட்சிப்படுத்தும் பொருட்கள் மற்றும் காட்சிப்படுத்துவது தொடர்பான செயல்பாடுகள், ஆகியவை, பஞ்சக்கிராமியில் அன்றாட சமூக ஊடக நடவடிக்கைகளின் பெரும்பகுதியை ஆக்ரமித்துக்கொள்கின்றன. இவை, படங்களை பதிவேற்றுவதில் இருந்து, பகிர்வது, விருப்பு தெரிவிப்பது, கருத்துத் தெரிவிப்பது வரை பலவகைப்படும். பஞ்சக்கிராமியில், காட்சிப்படுத்துதல் தொடர்பான செயல்பாடுககள், சமூக ஊடகப்பயன்பாட்டின் மையமாக இருப்பதில், கட்டுப்படியாகும் தொழில்நுட்பங்களான காமெராவுடனான (முன்-பின் இருபுறங்களிலும் இருக்கக்கூடியவை) ஸ்மார்ட்போன்கள் மற்றும் முன்கட்டணம் செலுத்தப்பட்ட மொபைல் இணையதள தொடர்புகள் போன்றவை முக்கிய பங்கு வகிக்கின்றன.

பஞ்சக்கிராமியில் உள்ள மக்கள், தங்கள் சமூக ஊடகப்பக்கங்களில் (முகநூல் மற்றும் வாட்சப் போன்றவை) பதிவேற்றும் படங்கள், இயல்புநிலையில் பார்ப்பவையிலிருந்து, அதிகம் மாறுபட்டவை அல்ல. அவை, பொதுவாக மேற்கூறிய வகைகளில், அதாவது, பொதுவானவை, தனிப்பட்டவை அல்லது இடைப்பட்டவை, ஆகியவற்றில் எதோ ஒன்றிலோ 
அல்லது ஒன்றுக்கும் மேற்பட்டதிலோ உரியதாக இருக்கின்றன. பஞ்சக்கிராமியின் பொதுவான காட்சிப்படுத்துதல் கலாச்சாரத்தை சேர்ந்ததாக கருதப்படும் படங்கள், அதாவது, சமய விழாக்கள், தமிழ்த்திரை நட்சத்திரங்கள், அரசியல்வாதிகள் மற்றும் அரசியல் நிகழ்ச்சிகள்/[பிரச்சினைகள், போன்றவற்றின் புகைப்படங்கள் அந்த நிகழ்ச்சியோ அல்லது காலமோ முடிந்தபின் நீக்கப்பட்டுவிடுகின்றன (அவற்றின் இயல்புநிலை சமனானவற்றில் நடப்பது போல). இல்லாவிட்டால், முகநூலில் படத்தொகுப்பு இடப்பரப்பில், கீழ்நிலைக்கு (காலவரிசைப்படியான) தள்ளப்பட்டுவிடுகின்றன. புதியப்படங்கள் முன்னிலையில் வைக்கப்படுகின்றன. இதுபோன்ற படங்கள் முகநூலில் பகிரவோ அல்லது பதிவேற்றவோ படுகின்றன. இதுபோன்ற படங்களை தங்கள் மொபைல் போன்களில் இருந்து முகநூலில் பதிவேற்றும் மக்களில் பலர், தங்களின் மொபைல் போனின் சேமிப்பு இடப்பரப்பை மிச்சப்படுத்துவதற்காக, முகநூலை, படங்களை சேமித்து வைக்கப்படுவெதற்கான மேகக்கலமாக உபயோகிக்கின்றனர்.

முகநூல் பக்கங்களில் பதிவேற்றப்பட்ட தனிப்பட்ட புகைப்படங்கள், புதிய படங்கள் பதிவேற்றப்பட்டாலும், நீக்கப்படுிெதில்லை. அவை படத்தொகுப்பில் சேமித்து வைக்கப்படுகின்றன. பெரும்பாலும் உயர் மத்தியதர வகுப்பை சேர்ந்த பெண்கள், தங்கள் வாழ்வின் நிகழ்ச்சிகளுக்கு ஏற்றவாறான சமீபத்திய புகைப்படங்களுடன் ${ }^{38}$, தங்கள் சுயவிவரப்பக்கங்களை மேம்படுத்திக்கொள்கின்றனர் என்பதை கூர்ந்து நோக்கினால் கவனிக்க முடியும். இந்த தனிப்பட்ட புகைப்படங்கள், பெரும்பாலும் அவர்களின் மொபைல் போன்கள் மற்றும் முகநூல் பக்கங்கள் ஆகிய இரண்டிலும் சேமித்துவைக்கப்படுகின்றன. திருமண அழைப்புகள், விழாக்கள் அல்லது குடும்ப நிகழ்ச்சிகளுக்கான வாழ்த்தட்டைகள், சமூக வலைத்தொடர்பில் உள்ள நண்பர்கள் மற்றும் உறவினர்களை குறிவைத்து அனுப்பப்படும் வார்த்தை மீம்களைக் கொண்ட அன்றாட வாழ்த்துச் செய்திகள் ("இடைப்பட்டவை" போன்றவை) மொபைல் போனில் மட்டும் சேமித்து வைக்கப்பட்டு, முகநூலில் பதிவேற்றப்படுகின்றன. எனினும், இவற்றில் ஒருசில மட்டுமே, அவர்களின் போனிலோ அல்லது முகநூல் தொகுப்பிலோ அந்த நிகழ்ச்சியோ அல்லது காலமோ முடிந்தபின்னும் சேமித்துவைக்கப்படுகின்றன. பெரும்பாலானவை புதியவைகளுக்கு இடமளிப்பதற்காக நீக்கப்பட்டுவிடுகின்றன. பலசமயங்களில், முகநூலில் உள்ள படங்களின் தற்காலிக தன்மை, இவற்றின் இயல்புநிலைத் தன்மையை பிரதி- 
பலிக்கின்றன. இது மேற்கூறிய வழக்குகளான படங்களை ஞாபகார்த்தத்திற்காக சேர்த்துவைப்பது என்பதிலும், அவற்றை, தம் சமூகவலைதொடர்பிற்கு குறிப்பிட்ட தகவலை தெரிவிப்பதற்காக பயன்படுத்துவது என்பதிலும் காணப்படுகிறது.

\section{தொடரும் காட்சிப்படுத்தும் கலாச்சாரம்}

இயல்புநிலை காட்சிப்படுத்தும் கலாச்சாரம், சமூக ஊடகங்களுக்கும் தொடர்கிறது என்பதை காட்டுவதற்காக, இந்தப்பகுதியும், மேலே கூறியுள்ளபடி, பொதுப்பார்வைக்கானவை, இல்லம்சார்ந்தவை மற்றும் இடைப்பட்டவை ஆகிய மூன்று வகைகளாக பிரிக்கப்பட்டிருக்கிறது. முதல் பகுதி, பொதுப்பார்வைக்கானவை என்ற வகையைச் சாரும், திரைப்படம் மற்றும் அரசியல் படங்களின் நிகழ்நிலை காட்சிப்பதிவுகளை கையாள்கிறது. இதைத் தொடர்வது, உபயோகிப்பாளர்களின் சொந்த காட்சிப்படுத்தல்கள். இவை, இல்லம்சார்ந்த காட்சிப்படுத்தல்களின் வகையை சேரும். இறுதியாக தமிழ் கலாச்சாரத்தில் பிணைந்துள்ள அன்றாட வாழ்த்துக்களை, மக்கள் சமூக ஊடகங்களுக்கு எப்படி கொண்டுவெருகிறார்கள் என்றும் இந்துசமய இறையியலின் அண்டவியல் பற்றிய புரிதலுடன் எவ்வாறு தொடர்புபடுத்துகிறார்கள் என்றும் கண்டறிவதன் மூலம் நாம் அலசுவோம். இந்த அத்தியாயத்தின் இறுதிப்பகுதி சமயத்தைப் பற்றி கலந்தாய்வு செய்வதால், பொதுப்பார்வைக்கான காட்சிப்படுத்துதல் பற்றிய பகுதி, திரைப்படம் மற்றும் அரசியல் ஆகியவற்றை மட்டுமே கலந்தாய்வு செய்யும். இந்த வகைகளை விரிவாகக் கருதுவதற்கு முன், பஞ்சக்கிராமியில் சமூக ஊடகங்களில் உள்ள காட்சிப்படுத்தும் பதிவுகளின் அளவை புரிந்துகொள்ளுதல் அவசியம்.

இந்த அத்தியாயத்தில் கலந்தாய்வு செய்யப்படவுள்ள காட்சிக்குரிய பதிவுகள் குறித்த ஒருசில விஷயங்கள் கவனிக்கப்படவேண்டும். முதலில், திரைப்படம் மற்றும் அரசியல் தொடர்பான காணொளிகள் மக்களின் சமூக ஊடக பக்கங்களில் காணப்பட்டாலும், இந்த அத்தியாயம் அசையா படங்களை மட்டுமே கையாளும், ஏனெனில், இவை அசையும் படங்களை விட அதிக அளவில் காணப்படுகின்றன. கூடுதலாக, பஞ்சக்கிராமியில் உள்ள காட்சியமைப்புகள், சொந்தக் காட்சிகள் மற்றும் முன்னனுப்பப்பட்ட காட்சிகள் ஆகியவற்றின் கலவையாக இருக்கின்றன. ஆகவே, பஞ்சக்கிராமியில் உள்ள ஒவ்வொருவரும், தங்களது சொந்த காட்சியமைப்புகளை 
சமூக ஊடகங்களில் உருவாக்கவில்லையென்றாலும், பலர், தாங்கள் முகநூலிலோ அல்லது வாட்சப்பிலோ பார்த்த காட்சியமைப்புகளை பகிர்வதிலும் முன்னனுப்புவதிலும் பெரும் ஆர்வம் காட்டினர்.

\section{பஞ்சக்கிராமியில் உள்ள காட்சியமைப்புகளின் புகைப்பட நொடிப்பு}

காட்சிக்குரிய படங்களின் அளவை பற்றிய புகைப்பட நொடிப்பை அளிப்பதற்காக, பஞ்சக்கிராமியிலுள்ள 20 முகநூல் நண்பர்களின் முகநூல் ${ }^{39}$ பக்கங்கள் மாதிரிகளாக பயன்படுத்தப்பட்டு, பின்வரும் செயல்முறை நடத்தப்பட்டது (அட்டவணை: 3.1). மாதிரிக்குழு, 15 முதல் 67 என்ற பரந்த வயது வரம்பில் உள்ள 10 ஆண்கள் மற்றும் 10 பெண்களைக் கொண்டதாக இருந்தது. இங்குள்ள படங்கள், அவர்களின் முகநூல் பக்கங்களில் காணப்பட்ட அனைத்து காட்சியமைப்புகளையும், அதாவது, அவர்களாலேயே பதிவேற்றப்பட்டவை மற்றும் அவர்களுடன் இணைக்கப்பட்டவை, உள்ளடக்கியது. பின்வரும் புள்ளிவிவரம், சொந்த மற்றும் முன்னனுப்பப்பட்ட பொருளடக்கங்களை குறிக்கிறது. உதாரணத்திற்கு, பொதுவாக, மீம்கள் முன்னனுப்பப்பட்ட அல்லது பகிரப்பட்ட பொருளடக்கங்களாக இருக்கும்.

\section{பகுதி 1: பொதுப்பார்வைக்கானவை}

திரைப்படங்கள்

முன்பே கூறியபடி, பஞ்சக்கிராமியில், திரைப்படங்கள், மிகவும் பிரபலமான ${ }^{40}$ பொழுதுபோக்காக கருதப்படுகிறது. பலசமயங்களில், இளம் கல்லூரி மாணவர்களின் முகநூல் சுயவிவர படங்கள், திரைநட்சத்திரங்களின் படங்களால் நிரம்பியிருப்பதை நாம் பார்க்கமுடியும். (படம்: 3.7 \& 3.8). இந்தப்பகுதியில் மிகவும் பிரபலமானவர்களாக இரண்டு நடிகர்கள் இருக்கிறார்கள். ஒருவர் அஜித் (தலை என்று குறிப்பிடப்படுபவர்) மற்றவர் விஜய் (தளபதி என்று குறிப்பிடப்படுபவர்). ரசிகர்கள் தாங்கள் மிகவும் விரும்பும் ஒரு குறிப்பிட்ட நடிகரை ஆதரிக்கும் வலைத்தொடர்புகளில் சேர்ந்து கொண்டு, பின் அந்த நடிகருடைய புகைப்படங்களையும், 
அட்டவணை: 3.1 - பஞ்சக்கிராமியில் உள்ள காட்சியமைப்புகள் பற்றிய முகநூல் அளவீடுகள்.

\begin{tabular}{ll}
\hline காட்சியமைப்புகளின் மொத்த எண்ணிக்கை & 1367 \\
காட்சியமைப்புகளின் குறைந்த பட்ச எண்ணிக்கை & 18 \\
காட்சியமைப்புகளின் அதிகபட்ச எண்ணிக்கை & 102 \\
காட்சியமைப்புகளின் சராசரி எண்ணிக்கை & 68 \\
தகவலாளர்களையும் சேர்த்து படங்களின் & \\
சராசரி சதவிகிதம் & $43 \%$ \\
தகவலாளர்களை மட்டுமே காண்பிக்கும் & \\
படங்களின் சராசரி சதவிகிதம் & $20 \%$ \\
குழுக்களுக்குள் உள்ள தகவலாளர்களை & \\
காண்பிக்கும் படங்களின் & $23 \%$ \\
சராசரி சதவிகிதம் மீம்ஸ்கள் இடம் பெறும் & \\
படங்களின் சராசரி சதவிகிதம் & $38 \%$
\end{tabular}

புகைப்படத்தொகுப்புகளையும் பதிவேற்றுகின்றனர். இது போன்ற நடவடிக்கைகளின் உச்சத்தை, அந்த நடிகரின் புதிய திரைப்படம் வெளியாகும் சமயத்திலும் அந்த நடிகரின் பிறந்தநாள் சமயத்திலும் பார்க்கலாம். வெளியிடவிருக்கும் அந்தத் திரைப்படத்தின் விளம்பரப்படங்கள் இது போன்ற வலைத்தொடர்புகளின் வழியாக, சமூக ஊடகங்களில் பகிர்ந்துகொள்ளப்படும்.

இதுபோன்ற பகிர்வுகள் பெரும்பாலும், திரைப்படவெளியீட்டின் சமயம், இந்தப்பகுதி முழுவதும் சுவரொட்டிகள் மற்றும் பதாகைகள் ஆகியவற்றில் இருக்கும் படங்கள் தான்.

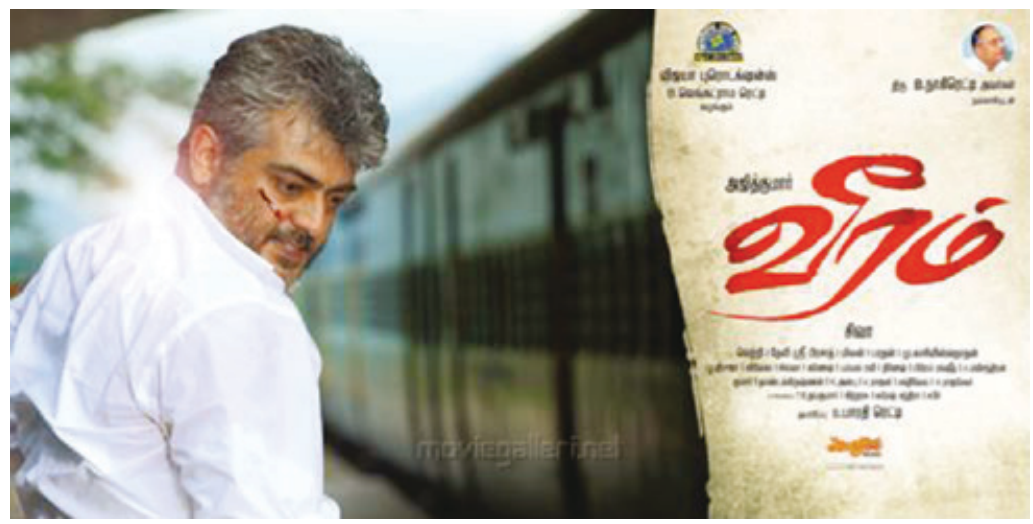

படம்: 3.7 வீரம் திரைப்படத்தில் நடிகர் அஜித் 


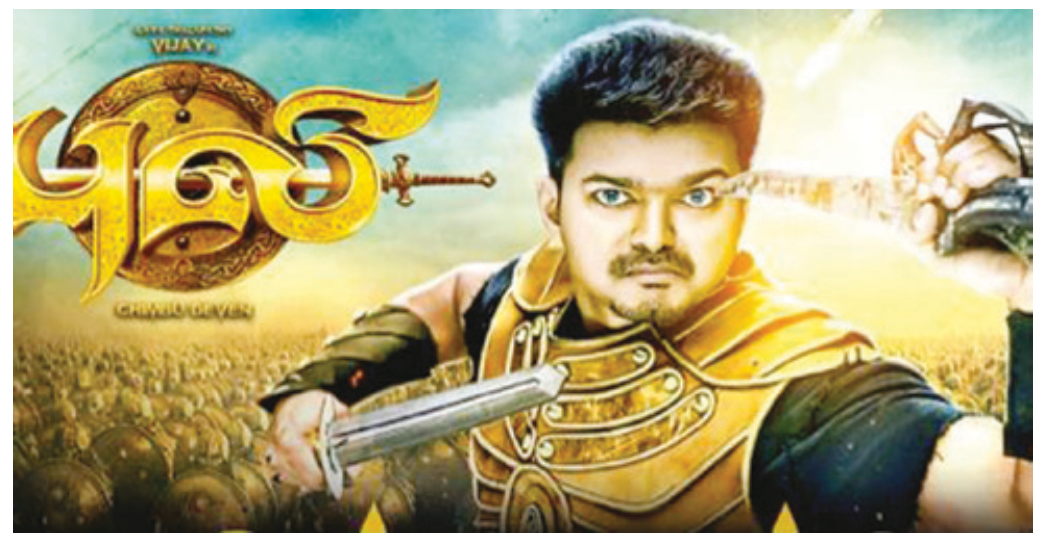

படம்: 3.8 புலி திரைப்படத்தில் நடிகர் விஜய்

மக்கள் தங்கள் அபிமான நட்சத்திரங்கள், தங்கள் குடும்பத்துடன் இருக்கும் படங்களையும் (சிறந்த குடும்பத்திற்கான லட்சிய நோக்கை குறிக்கும் வகையில்), தங்கள் அபிமான நட்சத்திரத்தின் சமூகத்தன்மையை எடுத்துக்காட்டும் விதத்தில் இருக்கும், சக நட்சத்திரங்களுடன் ஒரு பொது நிகழ்ச்சியில் மகிழ்வுடன் இருக்கும் தருணங்களை குறிக்கும் படங்களையும், சமூக ஊடகங்களில் பதிவேற்றுகின்றனர் (படம்: 3.9)

காலங்காலமாக தமிழ்நாட்டில், இந்த வகையிலான படங்கள் காணப்பட்டுக்கொண்டு தான் இருக்கின்றன. மீகப்பிரபலமான திரைநட்சத்திரமாகவும் இருந்த, முன்னாள் முதலமைச்சர், காலஞ்சென்ற திரு. எம்.ஜி. ராமச்சந்திரன் ${ }^{41}$ (எம்.ஜி.ஆர் என்று பிரபலமாக அறியப்பட்டவர்) அவர்களின் படங்கள் 40 ஆண்டுகளுக்கு முன் தமிழ்நாடு முழுவதும் சுவரொட்டிகளில் காணப்பட்டன (படம் 3.10). இது போன்ற செயல்கள், இன்று அவர்கள் முயற்சியாலேயே42 பிரபலமாக இருக்கும் திரை நட்சத்திரங்களுக்கும் நடத்தப்படுகின்றன.

இந்த அத்தியாயத்தின் துவக்கத்தில் குறிப்பிடப்பட்ட குருநாத்தின் வழக்கில் காணப்பட்டது போல், நடிகர்களின் ரசிகர்களால் பதிவிடப்பட்ட இது போன்ற படங்கள், அவர்களுக்கு அந்த நடிகரின்பால் இருக்கும் விசுவாசத்தை எடுத்துக்காட்டுகின்றன. ஆதலால் அவர்கள், அந்த வலைதொடர்பில் இருக்கும் மற்ற ரசிகர்களிடம் இருந்து விருப்புகளையும் கருத்துகளையும் பதில்களாக பெறுகின்றனர். இருப்பினும், இது போன்று ஒவ்வொரு ரசிகரும் பதிவிடும் படங்களுக்கு கிடைக்கும் பதில்களின் அளவு, இயல்புநிலையை ${ }^{43}$ விட, 

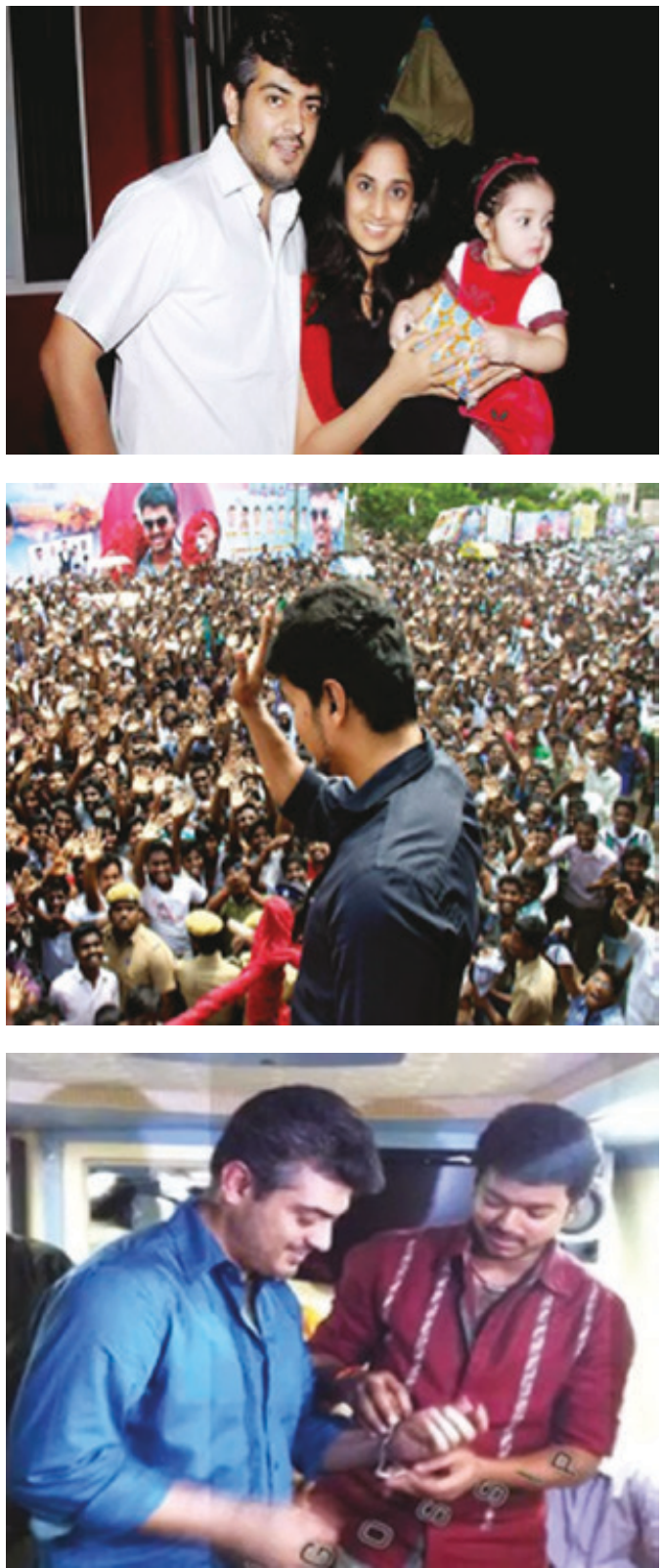

படம்: 3.9 திரைப்படங்கள் - நடிகர்கள் அஜித் மற்றும் விஜய்யின் பல்வேறு முகங்கள் 

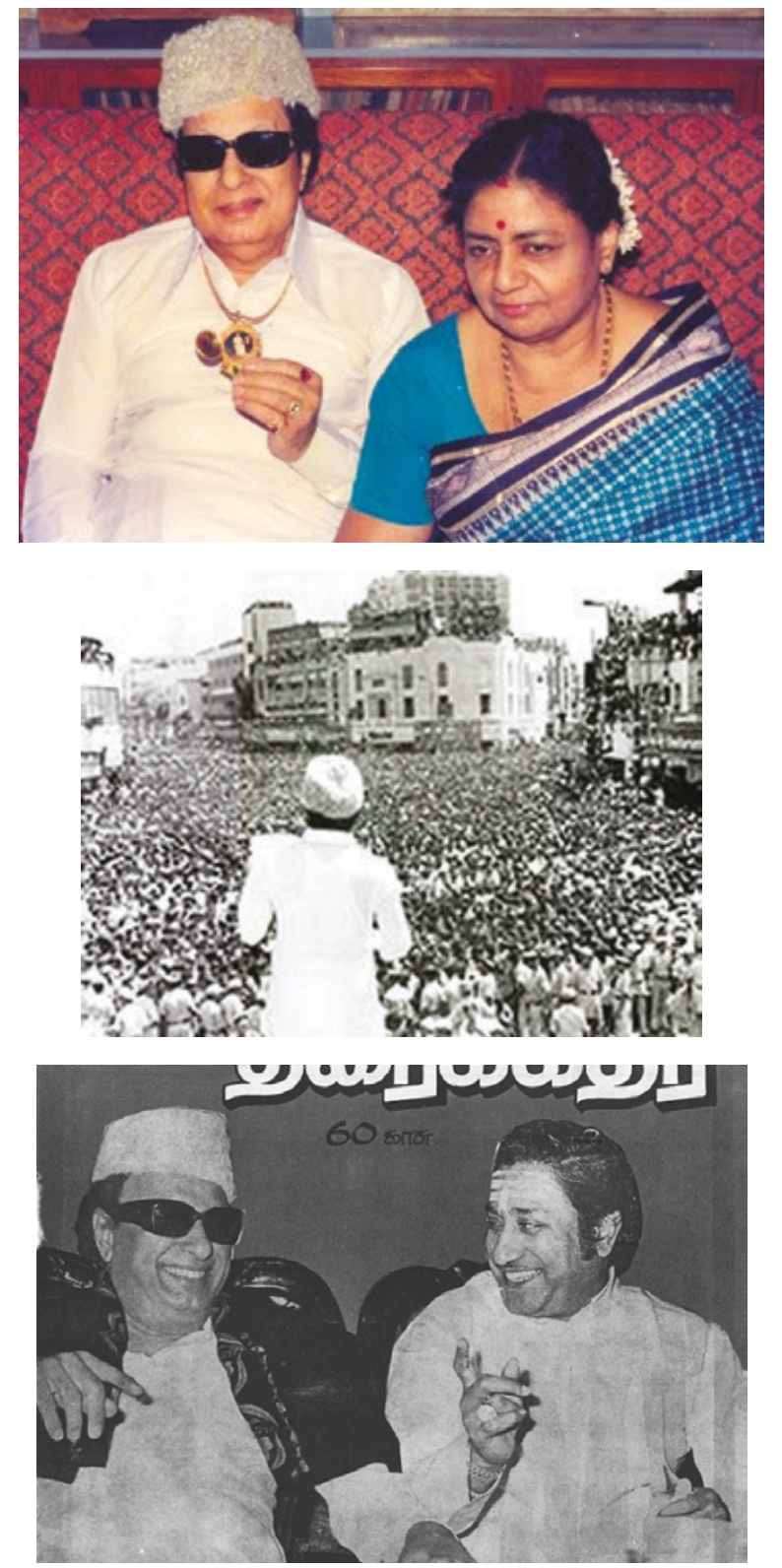

படம்: 3.10 திரைப்படங்கள் - முன்னாள் நடிகர் எம்.ஜி.ஆர் அவர்களின் பல்வேறு முகங்கள் (எம்.ஜி. ராமச்சந்திரன்) 
சமூக ஊடகங்களில் செய்யப்படும்போது, தெளிவாகத்தெரிவதாகவும் அதிகமாக மிகுதிபடுத்தப்பட்டதாகவும் தெரிகிறது. தமிழ்த்திரைப்பட நடிகைகளின் படங்களும், சமூக ஊடகங்களில் சுற்றனுப்பப்படுகின்றன (படம்: 3.11). திரைப்பட நடிகர்களின் படங்களை பதிவிடெது இளம் ஆண்களாக இருந்தாலும், இளம் பெண்களும், சற்றே வயதில் மூத்த ஆண்களும், திரைப்பட நடிகைகளின் படங்களை, தங்கள் சமூக ஊடக சுயவிவர பக்கங்களில் பதிவிடுகிறார்கள். பிரபல நடிகர்களின் படங்கள் பெரும்பாலும் அப்படியே தான் இருக்கின்றன. ஆனால் தமிழ் நடிகைகளின் படங்கள், (குறிப்பாக கதாநாயகியாக நடிப்பவர்கள்) அதிக அளவு மாறிக்கொண்டேயிருக்கின்றன. இயல்புநிலையில் திரைப்பட சுவரொட்டிகளில் மாறும் அளவிற்கு சமூக ஊடகங்களிலும் அவை மாறிக்கொண்டேயிருக்கின்றன. இது வரலாற்று முன்னுதாரணத்தைக் 44 கொண்டதாகவும், இந்திய திரைப்படங்களில், நடிகைகளின் மாறிக்கொண்டேயிருக்கும் நிலையை பிரதிபலிப்பதாகவும் இருக்கிறது.

இளம் ஆண்களும் பெண்களும், நடிகர் மற்றும் நடிகைகளின் படங்களை தங்கள் சுயவிவர பக்க புகைப்படமாக உபயோகிக்க தலைப்படுகின்றனர். இதுபோன்ற படங்களை அவர்கள் உருவாக்குவதில்லை. மாறாக இணையத்திலிருந்தோ, திரைப்படத்தின் வலைத்தளத்தில் இருந்தோ அல்லது முன்னனுப்பப்பட்ட படங்களையோ அவர்கள் பயன்படுத்துகின்றனர்.

\section{அரசியல்}

பஞ்சக்கிராமியில், கட்-அவுட்டுகள் மற்றும் பதாகைகள் என்றழைக்கப்படும் அரசியல் சம்பந்தப்பட்ட இயல்புநிலை காட்சியமைப்புகள் நான்கு வகைப்படும்: உள்ளூர் அளவிலானவை, பிராந்திய அளவிலானவை, தேசீய அளவிலானவை மற்றும் சர்வதேச அளவிலானவை. உள்ளூர் அளவிலானவை பொதுவாக மாநில, சிலசமயங்களில் தேசீய அளவிலான பெரிய அரசியல் கட்சிகளின் ${ }^{45}$ உள்ளூர் பிரதிநிதித்துவத்தை கொண்டதாக இருக்கும். உள்ளூர் அளவில் அலுவலர்கள், கிராம மற்றும் நகர மன்றங்களை நிர்வகிப்பதில் பெரும்பங்கு வகிக்கிறார்கள். இயல்புநிலை உள்ள கட்-அவுட்டுகளும், பதாகைகளும், கட்சி தலைவரை சித்தரிக்கின்றன. அதன் ஓரத்தில் சிறிதாக, உள்ளூர் பிரதிநிதியின் படமும் இடம்பெற்றிருக்கும். இங்கு பாலினத்தின் முக்கியத்துவத்தையும் நீங்கள் தெரிந்து 

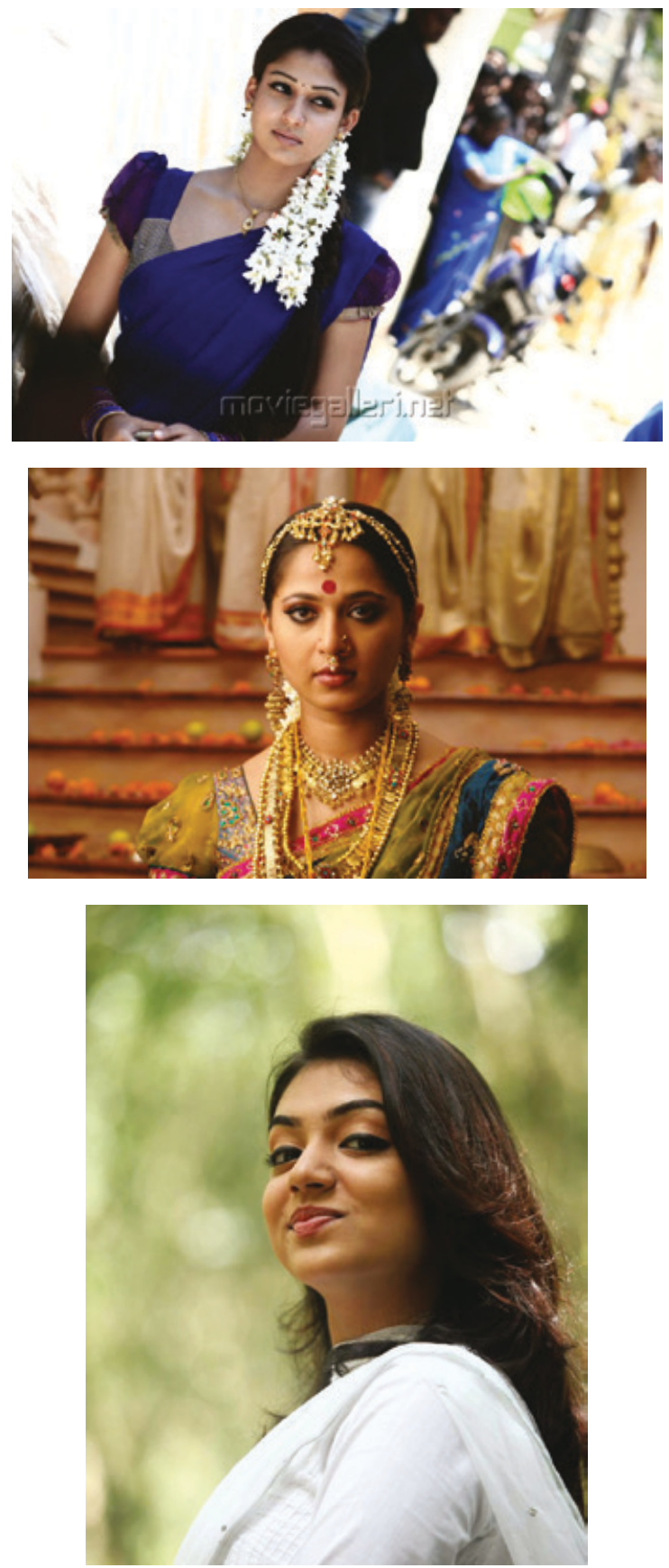

படம்: 3.11 திரைப்படங்கள் - நடிகைகள் நயன்தாரா, அனுஷ்கா மற்றும் நஸ்ரியா 
கொள்ள வேண்டும். பொதுவாக இங்கு ஆண்கள் தான் இயல்புநிலை காட்சியமைப்புகளின் மூலம் அரசியல் அதிகாரத்தை வெளிப்படுத்துவதில் ஈடுபட்டிருப்பார்கள். இ இந்தப்பகுதியில் பெண்கள் தங்கள் படங்களை, அவர்களுக்கு அரசியல் ஆர்வம் ${ }^{46}$ இருந்தாலும் கூட, பொதுப்பார்வைக்கான பதாகைகளில் வெளியிடிவது, பொதுவாக முகச்சுளிப்புடன் பார்க்கப்படுகிறது. இது போன்ற வெளிப்பாடுகள், சமூக அளவில் பிறழ்ந்த நடத்தையாக அவர்களின் சமூகத்தொடர்புகளால் பார்க்கப்படுகிறது. இதன் விளைவாக பெரும்பாலான பேர்கள் இதைத் தவிர்க்க விரும்புகிறார்கள். உள்ளூர் கட்சி அரசியலில் பெண் பிரதிநிதிகள் இருந்தாலும் கூட, உள்ளூர் பெண்களின், குறிப்பாக மணமாகாத பெண்களின் படங்களை கட்சி சுவரொட்டிகளில் வெளியிடும் நடைமுறை, வெகு அபூர்வமாக, அவர்கள் மிகவும் அறியப்பட்டவராக ${ }^{47}$ இருந்தால் மட்டுமே நடக்கிறது.

சுவரொட்டிகள் பெரும்பாலும், கட்சித்தலைவர்களை புகழும் வகையில் அமைக்கப்பட்டிருக்கும். அவை, அந்தக் குறிப்பிட்ட கட்சியை சேர்ந்த தொண்டர்களால், பொதுச்சுவர்களில், ஒட்டப்பட்டிருக்கும். இதுபோன்ற நடவடிக்கைகள், நீண்டகாலமாக, தமிழக அரசியலின் அங்கமாக இருந்து வருகிறது. இப்போது இவை பஞ்சக்கிராமியில் உள்ள கட்சித் தொண்டர்களின் சமூக ஊடக காட்சியமைப்புகளிலும் பிரதிபலிக்கின்றன.

பின்வரும் உதாரணங்கள் எல்லாமே, மாநில அளவிலான அரசியல் தலைவருக்கு காட்டப்படும் ஆதரவை வெளிப்படுத்துகின்றன. உதாரணத்திற்கு திமுக-வின் ${ }^{48}$ தலைவர், முனைவர்.மு. கருணாநிதி மற்றும் அவரது மகன் (கட்சியின் எதிர்காலத் தலைவர்) திரு. ஸ்டாலின் அவர்களின் படங்கள், இந்தக் கட்சியின் உறுப்பினர்கள் அல்லது தேர்தல் சமயத்து கூட்டணி கட்சியின் உறுப்பினர்கள் ஆகியோரால் மட்டுமே பதிவேற்றப்படுகின்றன (படம்: 3.12) திரு. ஸ்டாலின் போன்ற ஒருசில கட்சித்தலைவர்கள் சமூக ஊடகங்களில் ${ }^{49}$ இருப்பதால், அவர்கள், தங்களது அதிகாரபூர்வ சமூக ஊடகத்தள பக்கங்களில் பதிவேற்றும் கட்சிக்கூட்டங்களிலான அவர்களது படங்களும், கட்சித்தொண்டர்களால், மறு-பதிவிடலும் பகிர்தலும் செய்யப்படுகின்றன.

அதேபோல, அஇஅதிமுக50 தலைவர், செல்வி. ஜெ. ஜெயலலிதா அவர்களின் படங்களும், அந்தக்கட்சியின் உறுப்பினர்களின் சுயவிவர பக்கங்களில் காணப்படுகின்றன.

இந்தப்பாணியில் இருந்து காணப்படும் ஒரு விலகல், இனம் சம்பந்தப்பட்ட விசாரங்களில் இருந்து வரக்கூடும். உதா- 

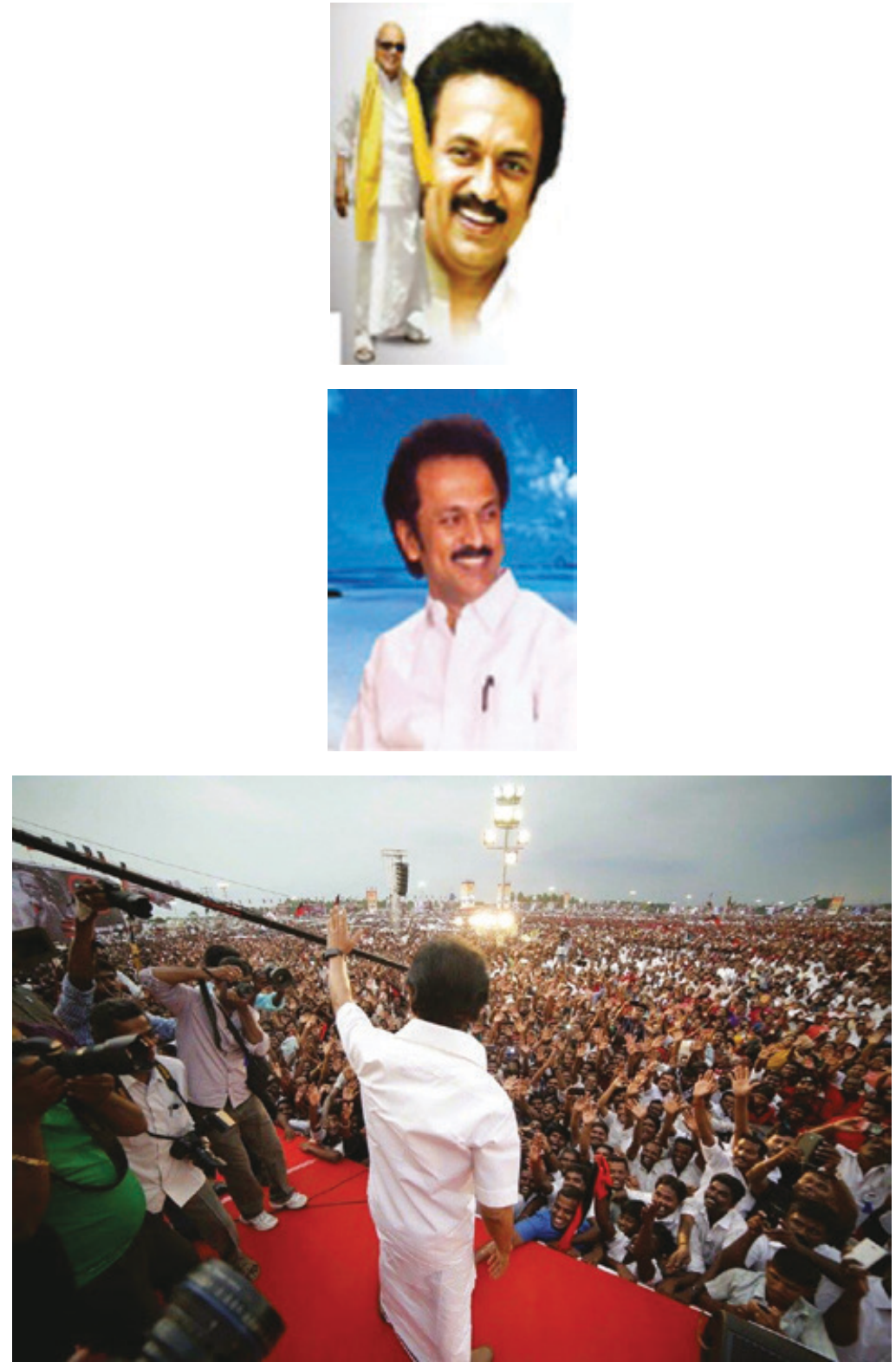

படம்: 3.12 அரசியல் - முனைவர். கருணாநிதி மற்றும் திரு. ஸ்டாலின் 
ரணத்திற்கு, பஞ்சக்கிராமியில் குறிப்பிடத்தக்க அளவிலான தலித்-களின் (ஆதிதிராவிட வகுப்பினர்) மக்கட்தொகை இருக்கிறது. சமூக நீதிக்கும் சமத்துவத்திற்குமான போராட்டத்தில் தலித்துக்களின் பிரதிநிதியாக இருந்த தேசீயத் தலைவர், இந்திய அரசியல் அமைப்புச்சட்டத்தின் தந்தை, காலஞ்சென்ற திரு. அம்பேத்கர் அவர்களின் படங்கள், ஆதிதிராவிட வகுப்பு மக்களின் கட்சியாக தன்னை காட்டிக்கொள்ளும் விடுதலை சிறுத்தைகள் கட்சியின் (வி.சி.க) உறுப்பினர்களின் முகநூல் பக்கங்களில் அடிக்கடி காணப்படும். உள்ளூர் கட்சிப் பிரமுகர்கள், சமூக நீதிக்கும் சமத்துவத்திற்குமான தங்களின் ஆதரவை பறைசாற்றும் விதமாக, அடிக்கடி இவ்வாறு செய்ய முற்படுவார்கள் (படம்:3.13). அரசியல் உத்தியாக, இங்கு
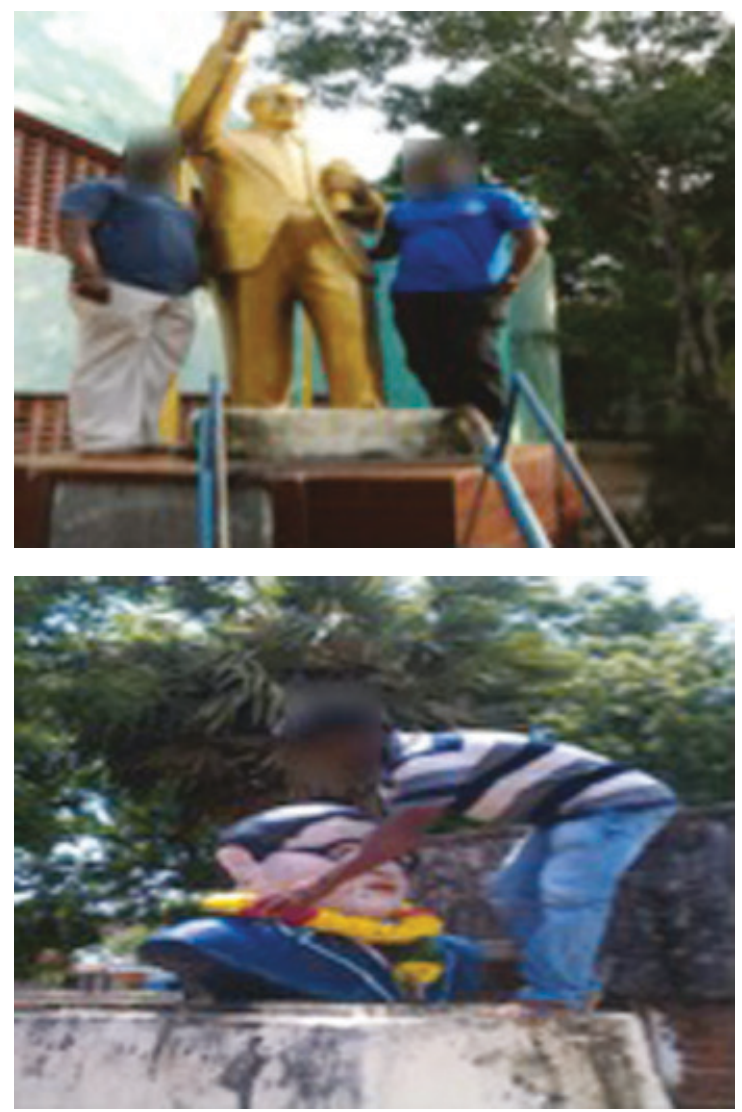

படம்: 3.13 அரசியல் - திரு. அம்பேத்கர் அவர்களின் உருவச்சிலைக்கு மாலையிடுதல் 
இருக்கும் பல்வேறு அரசியல் கட்சிகளின் பிரமுகர்களும், இதே போல் திரு. அம்பேத்கர் அவர்களின் படங்களை, போட்டிபோட்டுக்கொண்டு சமூக ஊடக பக்கங்களில் பதிவேற்றி வருகின்றனர்.

முதலாம் அத்தியாயத்தில் குறிப்பிட்டது போல, பஞ்சக்கிராமியின் ஒரு பகுதி, கிராம மன்றத் தலைவர் பதவிகளுக்காக, ஆதி திராவிட வகுப்பு மற்றும் பழங்குடியினர் இனத்தைச் சேர்ந்தவர்களுக்காக, தேர்தலில் ஒதுக்கீடு செய்யப்பட தொகுதியாகும். இங்குள்ள பலர் வி.சி.க கட்சியை சேர்ந்தவர்கள். திரு. திருமாவளவன் என்ற தலித் தலைவர், இந்த கட்சியின் மாநில அளவிலான தலைவராக இருக்கிறார். இவரின் படங்கள், ஒரு சில தகவலாளர்களின் சுயவிவர பக்கங்களில் ஏராளமாகக் காணப்படுகின்றன. இவர்கள் இந்தக் கட்சியை சேர்ந்தவர்களாகவோ அல்லது இதன் கொள்கைகளுக்கு ஆதாரவானவர்களாகவோ இ இருக்கிறார்கள் (படம்: 3.14). கட்சித்தலைவருடன் எடுத்துக்கொள்ளப்பட்ட படங்களும் மிகவும் பொதுவான காட்சிகளாக இருக்கின்றன. இவை, இதை பதிவேற்றுபவருக்குள்ள ஆதரவு, அந்தஸ்து, அதிகாரம்,செல்வாக்கு ஆகியவற்றை வெளிக்காட்டுவதாக இருக்கின்றன. முன்பே குறிப்பிட்டது போல், இந்தப் பகுதியில், வலுவான, நீண்டகாலமாக நிறுவப்பட்ட காட்சிப்படுத்தும் கலாச்சாரம் இருக்கிறது. சிலசமயங்களில், இந்த காட்சியமைப்புகளில், மிகவும் வலுவான வடிவங்கள் தான் - அதாவது, இயல்புநிலையில் காணப்படும் தலித் தலைவர்களின் கட்-அவுட், சுவரொட்டிகள் போன்றவை - முகநூல் ${ }^{51}$ அல்லது வாட்சப்பில் மறு-பதிவேற்றப்படுகின்றன.

இருப்பினும், இது மக்களை, ஊழல் மற்றும் மதுவுக்கு அடிமையாதல் போன்ற சமூக நோக்கங்கொண்ட சுவரொட்டிகளை ஒட்டுவதிலிருந்து தடுக்கவில்லை. இது போன்ற சுவரொட்டிகள் பெரும்பாலும் விசாரத்தை வெளிப்படுத்துவனவாகவோ அல்லது சீர்திருத்த நோக்கங்கொண்ட நையாண்டி தாக்குதல்களாகவோ இருக்கின்றன. இவை மாநில அல்லது தேசீய அளவில் உள்ள தலைமையை மட்டும் தான் தாக்குகின்றன. இவை சில சமயத்தில், மாநிலம் அல்லது தேசத்தை தாக்கும் சர்வதேச பிரச்சினைகள் குறித்ததாகவும் இருக்கின்றன. பெரும்பாலும், உள்ளூர் பிரமுகர்கள் விமர்சிக்கப்படுவெதே இல்லை. இதேபாணி, ஏறத்தாழ அப்படியே சமூக ஊடகங்களிலும் பின்பற்றப்படுகிறது. இது கண்டிப்பாக பாதுகாப்பான உத்தி தான். ஏனெனில், உள்ளூரில் வெவ்வேறு கட்சிகளுக்கு ஆதாரவானவர்களான முகநூல் மாற்று இதர சமூக ஊடகங்க- 

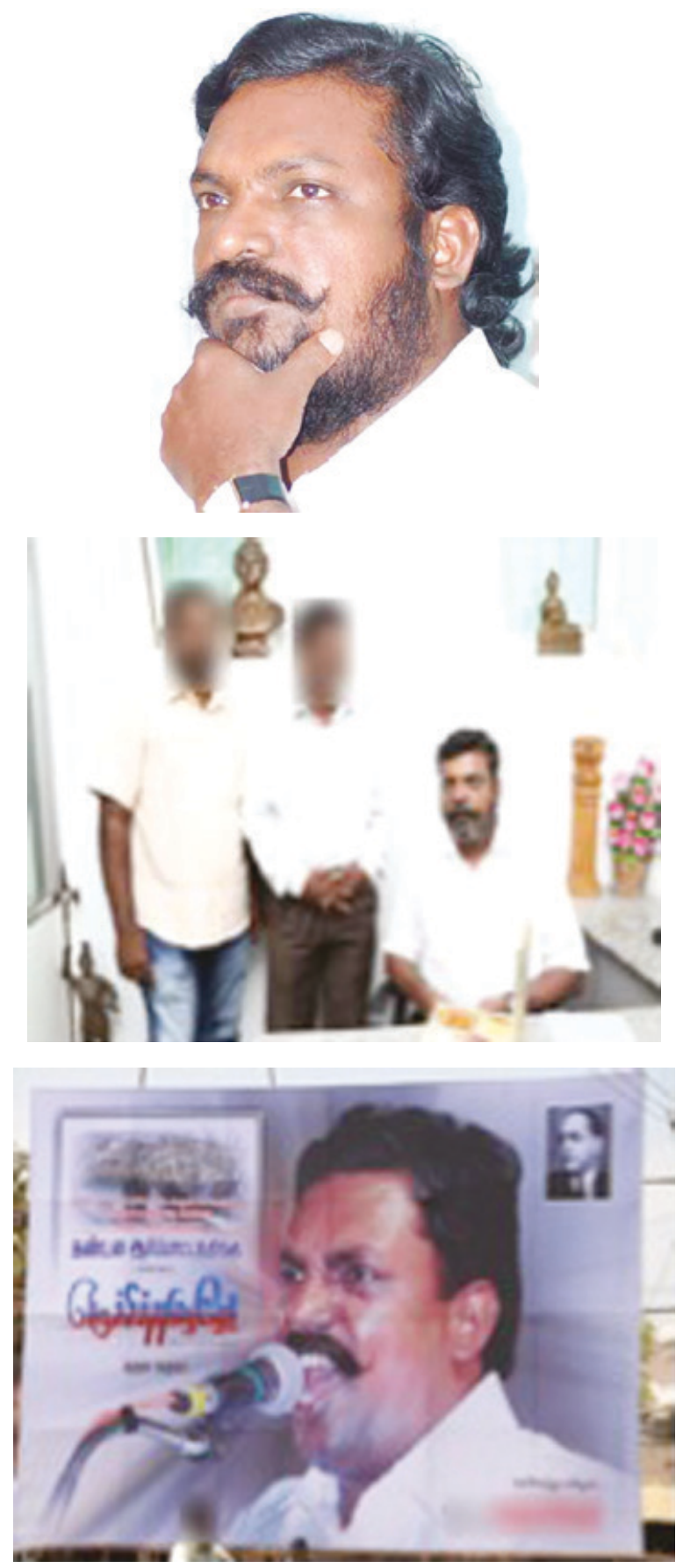

படம்: 3.14 அரசியல் - தலித் தலைவர் திருமாவளவன் 
ளில் இருக்கும் நண்பர்கள் மற்றும் உடன் பணியாற்றுபவர்கள் கோபத்திற்கு ஆளாக நேரிடாதல்லவா! இது போன்ற பிரச்சினைகளும், கட்சித்தலைவர்களும் பலசமயங்களில் கேலிச்சித்திரங்களாக ${ }^{52}$ வரையப்பட்டு அந்த காட்சியமைப்புகள் சமூக ஊடகப் பக்கங்களில் பகிரப்படுகின்றன (படம்: 3.15).

வழக்கமாக, கீழ்மட்ட சமூகப் பொருளாதார வகுப்புகளில் இருந்து வரும் நீண்டகால குடியிருப்பாளர்கள் (பொதுவாக அரசியலில் ஏற்கனவே ஈடுபட்டிருக்கும் ஆண்கள்) தான்
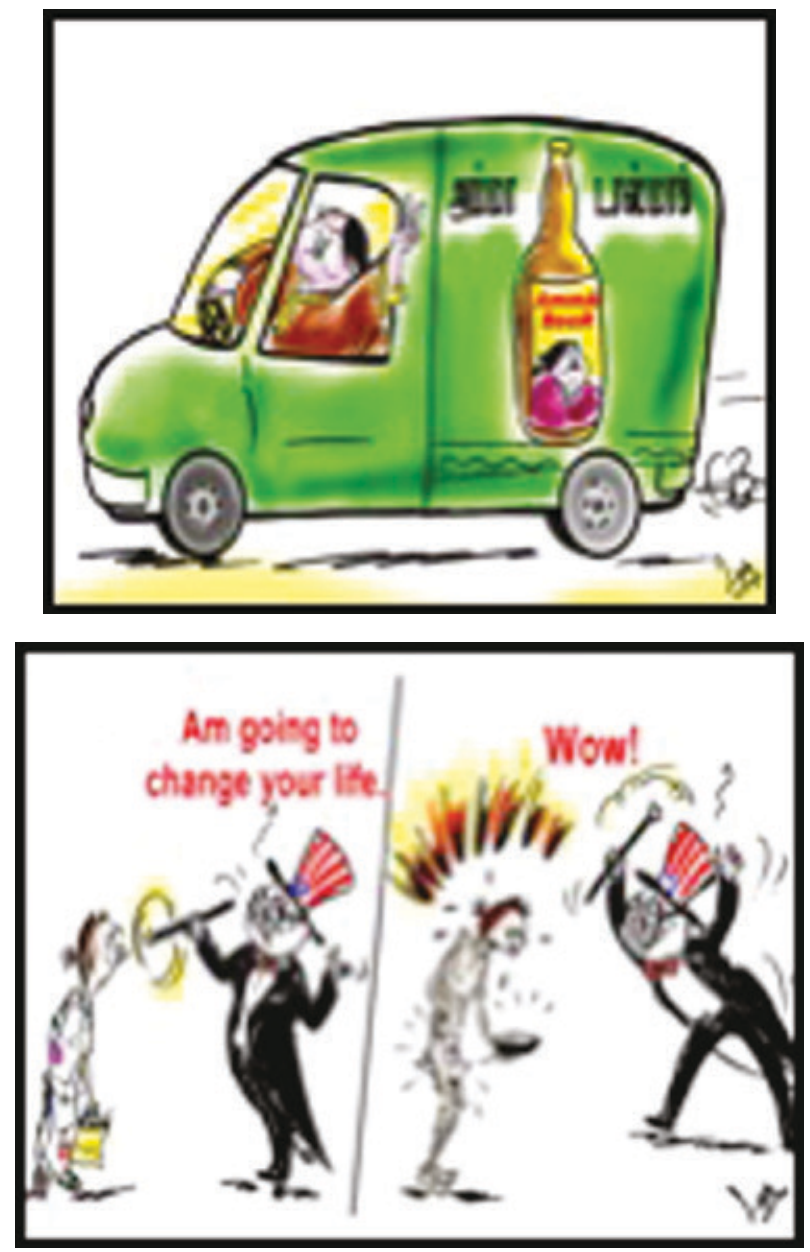

படம்: 3.15 அரசியல் - சமூக பிரச்சினைகளை பற்றிய பரிகாசமான மற்றும் சீர்திருத்த நோக்கங்கொண்ட நையாண்டி தாக்குதல் மீம்கள் 
பஞ்சக்கிராமியில் இ இயல்புநிலை காட்சியமைப்புகளான பதாகைகள் போன்றவற்றை அமைப்பவர்கள். சமூக ஊடகங்களில் பதிவிடப்படும் அரசியல் காட்சியமைப்புகள், வர்க்க எல்லைகளை தாண்டியதாக இருக்கின்றன. கீழ்மட்ட சமூகப் பொருளாதார வகுப்புகளை சேர்ந்தவர்களின் பதிவுகள், பெரும்பாலும் ஒரு கட்சியை புகழும் விதமாகவும் மற்றவற்றை இகழும் விதமாகவும் இருக்கும். ஆனால் மத்தியதர வகுப்பைச்சேர்ந்தவர்களின் பதிவுகள், மேலே கொடுக்கப்பட்டுள்ளதைப்போல ${ }^{53}$ மாநில, தேசீய மற்றும் சர்வதேச பிரச்சினைகளைப் பற்றிய சீர்திருத்த நோக்கங்கொண்ட நையாண்டி மீம்களாக இருக்கின்றன.

இது போன்ற இழிவு படுத்துதல்கள், இன்றளவில், மக்கள் அதிகம் ஆட்சேபிக்காத பொதுவான சமூக வலைத்தொடர்பு நடவடிக்கையாக ஆகிவிட்ட பழிக்கும் மீம்களின் வடிவத்தை எடுக்கின்றன. ஆட்சேபணைக்கு பதிலாக, மக்கள் இதை ஒருவகையான வேடிக்கையாக எடுத்துக்கொள்கின்றனர் (வம்பிழுப்பது அல்லது தமிழில் கலாய்க்கிறது என்றழைக்கப்படும் வகையான சமூக வேடிக்கை முறை). இது நெறிசார்ந்ததாகவும், எதிர்பார்க்கப்படும் வகையான நடத்தையை ${ }^{54}$ அனுசரிப்பதாகவும் கருதப்படுகிறது. அரசியல்வாதிகளை கேலிச்சித்திரங்கள் மூலம் கிண்டல் செய்வது, இந்தியக் கலாச்சாரத்தின் ${ }^{55}$ அங்கமாகவே இருந்துவிட்ட போதிலும், சமூக ஊடகங்களில் மின்னணு பழித்தல் மீம்களை பகிர்வது இன்னும் அதிகமான ஜனநாயக செயலாக ஆகிவிட்டது. உதாரணத்திற்கு, நடிகராக இருந்து அரசியல் தலைவராக மாறிய விஜயகாந்த் அவர்களை பழிப்பது, முகநூல் மற்றும் வாட்சப் ஆகிய இரண்டிலும் நடைபெறுகிறது (படம்: 3.16). இளைஞர் குழுக்கள், இவற்றை முகநூலில் பகிர்ந்து கொண்டாலும், நடுத்தர வயதை சேர்ந்தவர்கள், இவற்றை வாட்சப் குழுக்களில் பகிர்ந்து கொள்கின்றனர்.

அடுத்த பகுதி, இல்லங்களில் காட்சிப்படுத்தும் கலாச்சாரத்துடன் ஒப்பிடக்கூடிய, சமூக ஊடகங்களில் உள்ள மக்களின் காட்சிக்குரிய பதிவுகளை கையாளும்.

\section{பகுதி 2: தனிப்பட்டவை மற்றும் இல்லம் சார்ந்தவை}

பஞ்சக்கிராமியில், சமூக ஊடகங்களில் காணப்படும் ஒருவரின் படங்கள், அவரின் வரவேற்பறையில் காணப்படும் 


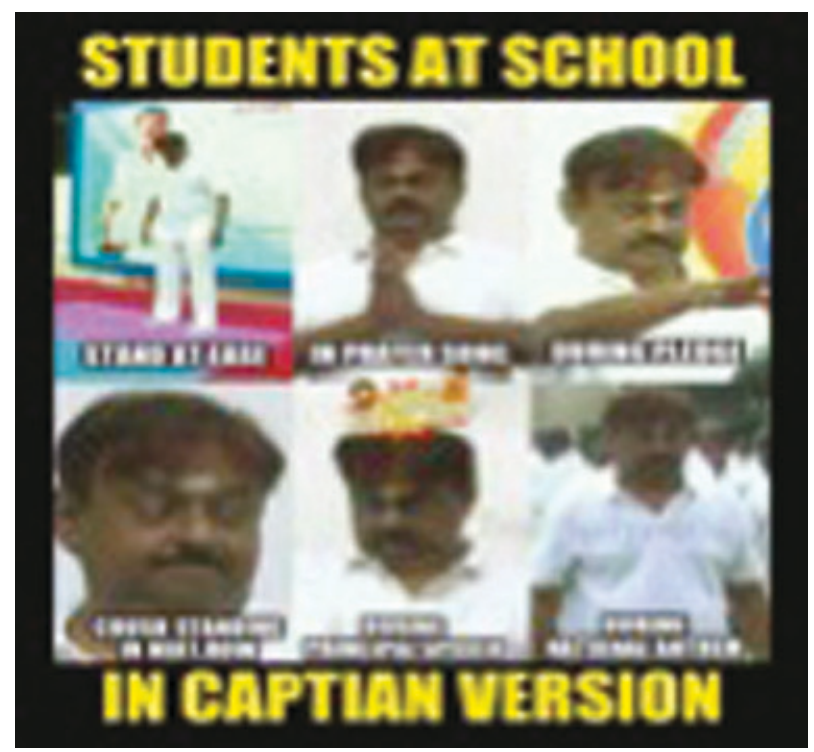

படம்: 3.16 அரசியல் - விஜயகாந்த் அவர்களை பழிப்பதன் ஒரு உதாரணம்

படங்களுடன் ஒப்பிடக்கூடியவையாக இருக்கும். வரவேற்பறையில் இருக்கும் புகைப்படங்கள் விருந்தினரின் பார்வைக்காக வைக்கப்பட்டிருப்பதால், அவை, பெரும்பாலும், ஒழுங்குமுறைக்கான விதிமுறைகள் மற்றும் பாலின எதிர்பார்ப்புகள் ஆகியவற்றுடன் ஒத்துப்போகும் வகையில் அமைந்திருக்கும். சமூக ஊடகங்களில் இருக்கும் ஒருவரின் புகைப்படங்கள், இதே விதிமுறைகள் மற்றும் எதிர்பார்ப்புகளுடன் ஒத்துப்போவதாக இருக்கின்றன. பொதுவாக, இது போன்ற படங்கள், அவர்களின் வலைதொடர்பில் இருந்து, முடிந்த அளவிலான நேர்மறையான கருத்துகளையும் விருப்புகளையும் பெரும் வகையில் வடிவமைக்கின்றன.

இந்தப்பகுதி ஒருவரின் மிகவும் சாதாரணமான மற்றும் அதீதமான முன்னிலைப்படுதலுடன் துவங்குகிறது. அங்கிருந்து நகர்ந்து, மக்கள் தங்களை எவ்வாறு மற்றவர்களுடன் தொடர்புபடுத்தி முன்னிலைப்படுத்துகிறார்கள் என்றும், எந்தெந்த சூழல்களில் தங்களை இருத்திக்கொள்கிறார்கள் என்றும் எடுத்துக்காட்டுகிறது. ஆகவே, இந்தப்பகுதி, சுலபமான புரிதலுக்காக உபபகுதிகள் மற்றும் வகைகளாக பிரிக்கப்பட்டிருக்கிறது. இது முகநூலில் இருந்து படங்களையும் கொண்டிருக்கிறது. 
வகை 1: குவியம் என்மேல்.

அதீதமாக தன்னை முன்னிறுத்திக்கொள்ளும் உதாரணத்துடனான துவக்கம். பல படங்கள், சமூக ஊடகங்களில் பதிவிடப்படுவதத்கான ஒரே நோக்கத்துடன் எடுக்கப்படுகின்றன (படம்:3.17).

இங்குகொடுக்கப்பட்டுள்ள

புகைப்படங்களில் தெளிவாகத் தெரிவது போல், இவற்றின் பின்னணியில் ஏதாவது முக்கியத்துவமோ அல்லது, இதில் உள்ளவரின் இருப்பிடம் அல்லது மற்ற விவரங்களைப் பற்றிய தகவலோ காணப்படவில்லை. இது போன்ற படங்களின் முக்கிய நோக்கமே, இதில் உள்ளவர்களின் மீது ஒட்டுமொத்த குவியமும் இருப்பது தான். அவற்றின் பின்னணியில் அல்ல.

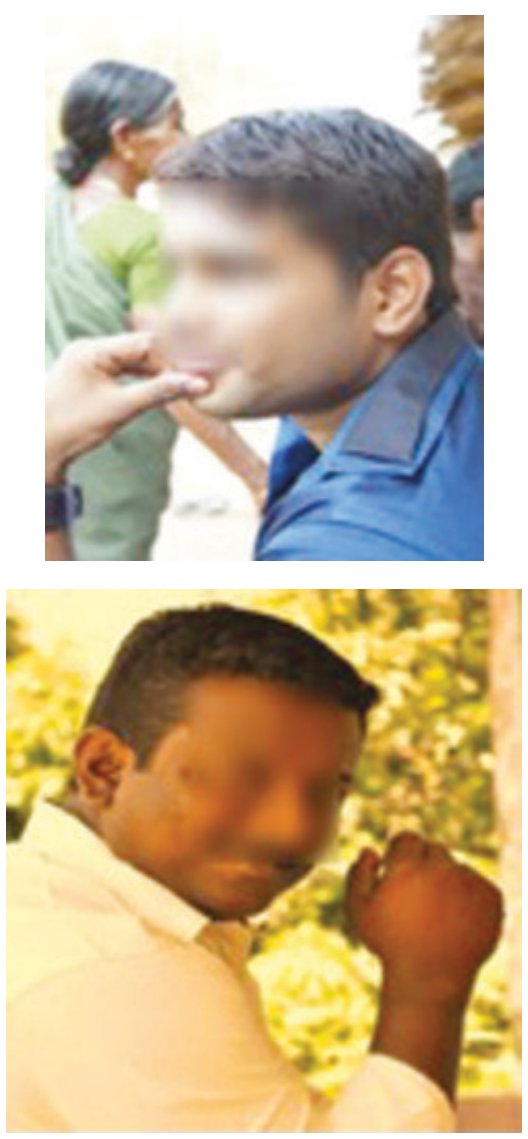

படம்: 3.17 தனிப்பட்டவை - குவியம் என்மேல் 
பெரும்பாலான சமயங்களில், இது போன்ற படங்களில் இருக்கும் நபருக்கு, தாம் புகைப்படம் எடுக்கப்படுகிறோம் என்பது தெரியும். அதற்கேற்ப, அவர்கள் காட்சிகொடுக்கிறார்கள். இதுபோன்ற வடிவமைக்கப்பட்ட படங்கள், எங்களது வேறு சில ஆய்வுக்களங்களில் காணப்படும், சாதாரணத்தன்மை மற்றும் நம்பகத்தன்மை பற்றிய விழுமங்களில் இருந்து, இந்தப்படங்கள் முற்றிலும் வேறானதாக இருக்கின்றன. மேலும், இது போன்ற படங்களின் முக்கிய கூறே, காட்சிகொடுப்பது தான் ஆகையால், இவை சுய-புகைப்படங்களாக இருக்க முடியாது. வேறு ஒருவரால் எடுக்கப்பட்டவையாக தான் இருக்க முடியும்.

அநேகமாக, இது போன்ற அனைத்துப் படங்களும், முகநூலில் வெளியிடப்படுவெத்கு முன், அந்தந்த தனிநபர்களின் ஒப்புதலை பெற்றுவிடுகின்றன. பெரும்பாலும், இதில் உள்ளவர்களே இவற்றை சமூக ஊடகங்களில் பதிவேற்றுகிறார்கள். இதுபோன்ற படங்களெல்லாம் புகைப்படநிலையத்தில் எடுக்கப்பட்டவையும் அல்ல, ஸ்கேன் செய்து சமூக ஊடகங்களில் பதிவேற்றப்பட்டவையும் அல்ல. அவை, டிஜிட்டல் காமெரா அல்லது ஸ்மார்ட்போன்கள் உபயோகித்து, டிஜிட்டல் வடிவத்தில் எடுக்கப்பட்டவை. அனைத்து சமூக வகுப்புகளை சேர்ந்த ஆண்களும் இது போன்ற படங்களை பதிவேற்றினாலும், உயர் மத்தியதர பெண்கள் மட்டுமே இதுபோன்ற படங்களை பதிவிடுகிறார்கள். ஆண்கள் பெண்கள் இருசாராரும், இதுபோன்ற படங்களில், கண்ணியம் மற்றும் சுயமரியாதையை வெளிப்படுத்தும் விதமாக கௌரவமான உடையணிந்து காணப்படுகின்றனர்.

இதுபோன்ற படங்கள் சாதாரணமாக, நேர்மறையான கருத்துக்கள் மற்றும் அதிக அளவிலான விருப்புகளைப்பெற்று காட்சிமுறை படங்களாகவோ அல்லது சுயவிவரப்பக்க படங்களாகவோ ஆனாலும், ஒரு சில வழக்குகளில் இவை, கீழுள்ள உதாரணத்தில் இருப்பது போல, நட்பார்ந்த பழிப்புக்களையும், கிண்டல்களையும் பெறக்கூடிம் (படம்: 3.18).

இது போன்ற பழித்தல்களும் கிண்டல்களும், அந்த தகவலாளர் இயல்புநிலை வாழ்க்கையில், நன்கறிந்த, இதே போன்ற ஒருவருக்கொருவர் வம்பிழுத்துக்கொள்ளும்படியான நட்புறவு கொண்ட நண்பரிடம் இருந்து வந்தால் மட்டுமே ஒத்துக்கொள்ளப்படும். இதுபோன்ற வேடிக்கையான கிண்டல்கள் (தமிழில் கலாய்க்கிறது என்றழைக்கப்படுவெது), ஒரு உறுதியான நட்புறவுத்தொடர்பின் எதிர்பார்ப்புகளுக்கு ஒத்ததாக இருக்கிறது. இது போன்ற படங்களாலேயே பதில- 


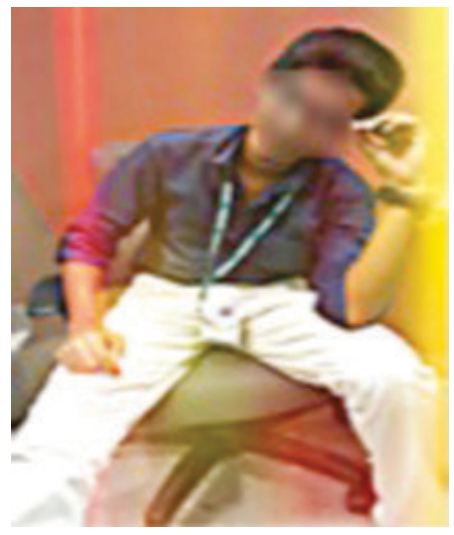

Profile Picture

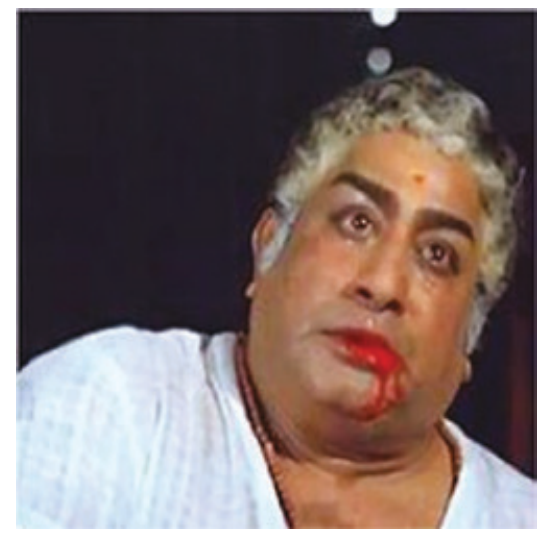

Comment

Ayoooooo! (meaning OMG!)

படம்: 3.18 தனிப்பட்டவை - காட்சிமுறை படமும் அதற்கான நட்பார்ந்த கிண்டலும்

ளித்தல் என்பது இங்கு குறிப்பிடத்தக்கதாக இருக்கிறது. அதிகபட்சம் இரண்டு மூன்று பரிமாற்றங்களுக்கு பிறகு அவை நிறுத்தப்பட்டு விடுகின்றன.

\section{வகை 2: நீ என்ன செய்கிறாய் என்பதைப் பற்றியது.}

ஒரு சாதாரண தனிப்பட்ட புகைப்படம் போதுமானதாக இருக்கக்கூடிய இடத்தில், முதல் வெளிப்படையான விரிவாக்கம், ஒரு நபர் அவர் செய்துகொண்டிருக்கும் செயல்/பணி உடன் தொடர்புடைய காட்சிப்படுத்தல். இது அவருடைய தொழில் அல்லது பணியாக இருக்கலாம். அல்லது அவர் தன்னை தீவிரமாக தொடர்புபடுத்திக்கொள்ளும் ஆர்வ ஈடுபாட்டுத்துறையாக இருக்கலாம் (படம்:3.19).

இது போன்ற காட்சியமைப்புகள் ஒரு தனிநபரின் சாதாரண சித்தரிப்பு அல்ல. இது போன்ற படங்கள், பல சமயங்களில் ஒரு நபரின் அடையாளத்தை வடிவமைக்கும் காரியத்தின் உள்ளார்ந்த அங்கமாகும். அவை சாதாரணமாக, தனிநபர்களின் நிகழ்நிலை புகைப்பட தொகுப்புகளில் காணப்படும். இவற்றில் சில வாட்சப் குழுக்களிலும் பகிரப்படும். இவை அடையாளம் குறிக்கப்பட்டவையோ அல்லது படவிளக்க வரி அளிக்கப்பட்டவையோ அல்ல. இவை ஒரு குறிப்பிட்ட விஷயத்தை புரியவைப்பதற்காக உத்தேசிக்கப்பட்டவை. பெருவாரியான சமயங்களில், இந்தப்படங்களில் 
உள்ள நபருடன் இணைத்து காட்டப்பட்டுள்ள பொருள் தான், அந்த நபர் உருவாக்க விரும்பும் அடையாளத்தை நோக்கிய கவனத்தை ஈர்க்கிறது. இது ஒரு நுண்-ஒலிவாங்கியாகவோ, கணினியாகவோ இருக்கக்கூடிம். படம்: 3.19-ல் உள்ள படங்களில் காட்டப்பட்டுள்ளபடி, இவை ஏதேனும் குறியீட்டு அர்த்தத்துடன் தொடர்புடையவையாக இருக்கலாம். இது போன்ற படங்களில் அவற்றின் பின்னணியும் மிகவும் முக்கியம் வாய்ந்ததாக இருக்கிறது.

இவை, பஞ்சக்கிராமியில் உள்ள வீடுகளின் வரவேற்பறைகளில் காணக்கூடிய படங்களுக்கு மிகவும் ஒத்ததாக இருக்கிறது. இதுபோன்ற குடும்பத்திற்குரிய இடங்களில், ஒரு நபரின் அடையாளம் விளக்கக்கூடிய இடப்பரப்பில் அல்லது அடையாளம் விளக்கக்கூடிய பொருட்களுடனான சித்தரிப்பு உள்ள படங்கள் வைக்கப்படுகின்றன. உதாரணத்திற்கு, பஞ்சக்கிராமியில் உள்ள வழக்கறிஞர்கள், அடிக்கடி, கருப்புக்கோட்டுடனான தங்களின் படங்களை, தங்கள் வரவேற்பறைகளின் பிரதான இடங்களில் வைத்துக்கொள்வார்கள்.

இந்த அடையாளம் வடிவமைக்கும் படங்கள், அவற்றின் குறியீட்டுப் பொருட்கள் மற்றும் அவற்றின் பின்னணி ஆகிய இரண்டின் மீதும் கவனத்தை ஈர்த்து, பாலினப்பணிகளை விளக்க உதவுகின்றன. உதாரணத்திற்கு, பஞ்சக்கிராமியில், பாடுவது என்பது பல சமயங்களில், பெண்களுக்கான செயலாக கருதப்படுகிறது. இது பெண்மை பற்றிய கருத்தமைவையும் உயர்த்துகிறது. இந்தப்படத்தில் உள்ள ஒலிவாங்கி, இந்தத்தொடர்பை, பூர்த்தி செய்கிறது. ஆண்களுக்கு, உடற்பயிற்சி நிலையங்கள், கட்டுமமஸ்தான உடற்கட்டு போன்றவை ஆண்மையின் அடையாளமாக கருதப்படுகிறது. இது போன்ற தொடர்புகள், படத்தில் உள்ள நபரின் அடையாளத்தை மேம்படுத்துவதற்காக கவனமான முறையில் வடிவமைக்கப்படுகிறது.

\section{வகை 3: அந்தஸ்த்தை காண்பிக்கும் பின்னணி}

மூன்றாவது வகையானது, ஒரு தனிநபரின் அந்தஸ்தை, பொருட்கள் அல்லது பின்னணிகளுடன் தொடர்புபடுத்துவதன் மூலம் உயர்த்தும் படங்கள் இருக்கும் வகையாகும். இது சமூக அமைப்பின் மூலம் சாத்தியமாகக்கூடும். உதாரணத்திற்கு, வெளிநாட்டு பயணங்களைக் காட்டுவது, பொருட்களுடனான நெருக்கத்தன்மையை வெளிப்படுத்துவது அல்லது சாதிக்கும் ஆர்வத்தை எடுத்துக்காட்டுவது போன்றவை (படம்: 3.20). 

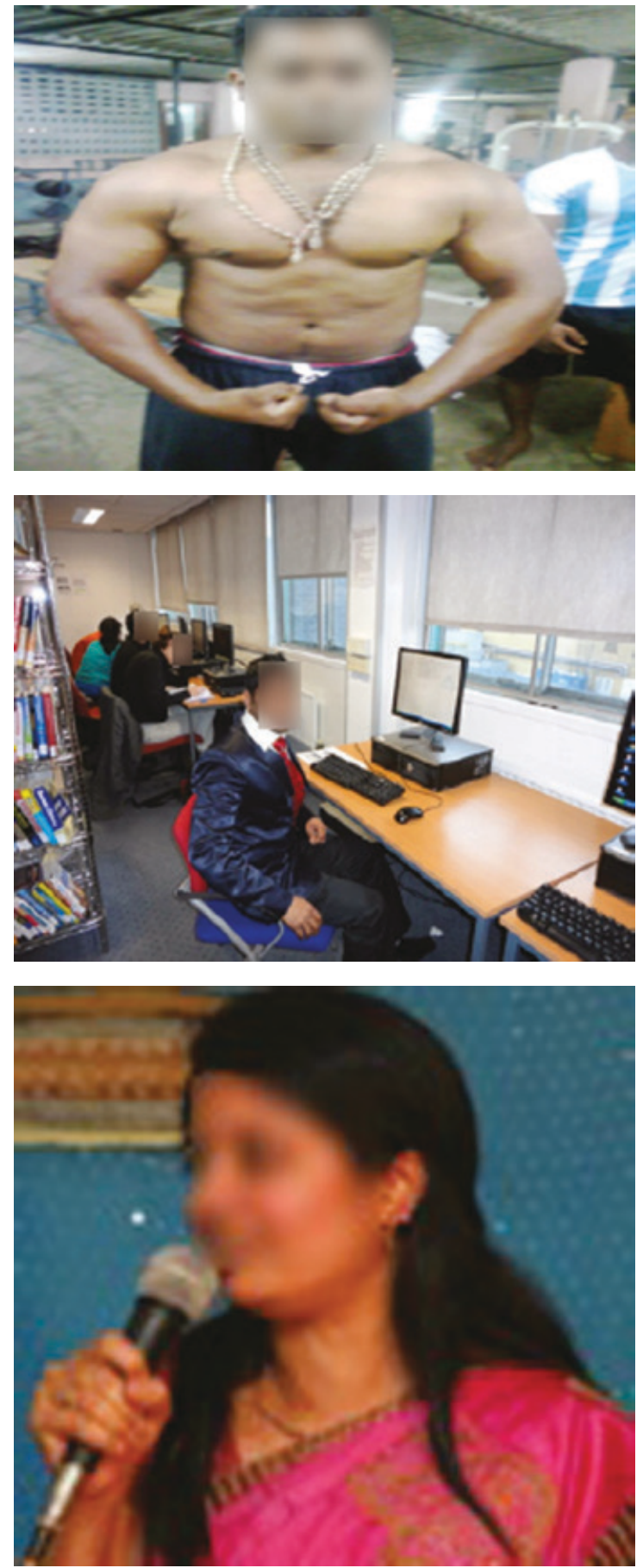

படம்: 3.19 தனிப்பட்டவை - நீ என்ன செய்கிறாய் என்பதைப் பற்றியது 

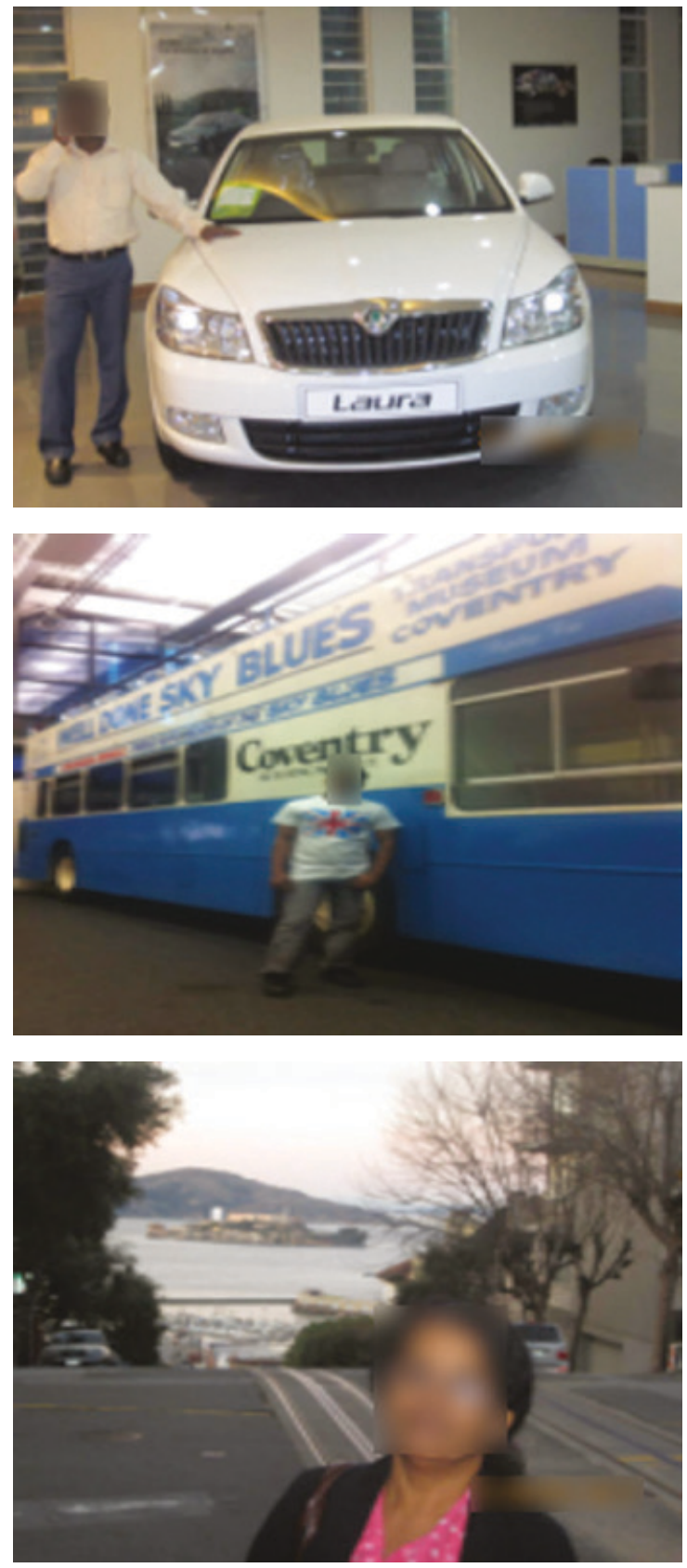

படம்: 3.20 தனிப்பட்டவை - அந்தஸ்த்தை காண்பிக்கும் பின்னணி 
இந்தப்படங்கள், ஒரு நபரின் சமூக அந்தஸ்தை பிரகடனப்படுத்துவதன் மூலமும், ஒரு குறிப்பிட்ட சாதனையை குறித்துக்காட்டுவதன் மூலமும், வகை 2-ல் உள்ளவற்றில் இருந்து வித்தியாசப்படுகின்றன. இந்தப்படங்கள், இருபத்தியோராம் நூற்றாண்டு சமூகத்தின் எதிர்பார்ப்புகளையும் பேரார்வங்களையும் சித்தரிக்க முற்படுகின்றன. ஒரு அறிவார்ந்த பொருளாதாரமாக பஞ்சக்கிராமி, தனிநபர்களின் மீது வைக்கும் சமூக எதிர்பார்ப்புகளுடன் இவை ஒத்துப்போகின்றன. உதாரணத்திற்கு சொகுசுக்கார், அடுக்குமாடிக்குடியிருப்பு, வெளிநாட்டு பயண அனுபவம் போன்றவை.

\section{வகை 4: குழுவில் (நண்பர்கள்) தான் எனும் தன்மை}

இளைஞர்கள், பெரும்பாலும் தனியாக புகைப்படமெடுக்கப்படுவதை விட, நண்பர்களுடன் எடுக்கப்படுவதையே விரும்புகின்றனர். இதன் கடைக்கோடி நுனியில் இரருப்பவர்கள், கீழ்-மத்தியதர வகுப்பு அல்லது கீழ்மட்ட சமூகப்பொருளாதார வகுப்பைச் சேர்ந்த திருமணமாகாத இளம் பெண் மாணவியர் தான். இவர்களிடம், முகநூலில், தங்களின் தனிப்பட்ட புகைப்படம் ஒன்று கூட இருக்காது. அதுவே நண்பர்களுடனான படங்கள் அவர்களிடம் அவசியம் இருக்கும் (படம்: 3.21). இதற்கான மிக எளிய காரணம், இந்தப்படங்கள் எல்லாம், இவர்களால் பதிவேற்றப்படாமல், இவர்கள் பெயர் இணைக்கப்பட்டிருப்பதாலேயே இவர்களின் சுய-விவர பக்கங்களில் தோன்றுவனவாக இருக்கலாம்.

சிலசமயங்களில், மணமாகாத இளம் பெண்களின் ஊடகப்பக்கங்களில், அவர்கள் இயல்புநிலையில் அறிந்த இளம் ஆண்களின் குழுப்புகைப்படங்கள், அங்குள்ள படத்தொகுப்புகளில் காணப்படலாம். இவை அந்த இளம்பெண்களாலேயே பதிவேற்றப்பட்டதாகவும் இருக்கலாம் அல்லது மேற்கூறியது போல இவர்கள் பெயர் இவற்றுடன் இணைக்கப்பட்டதாகவும் இருக்கலாம். இருப்பினும், திருமணமாகாத இளம்பெண், திருமணமாகாத இளம் ஆணுடன் இணைந்து நிற்பது போன்ற புகைப்படத்தை பார்க்கவே முடியாது. அப்படியே ஏதாவது ஒரு படம் இருந்தாலும் அது திருமணம் போன்ற ஏதாவது சமூக அமைப்பில் எடுக்கப்பட்டதாகவோ, அல்லது நெருங்கிய ஆண் உறவினருடன் எடுக்கப்பட்டதாகவோ இருக்கும். வழக்கமாக, இளம் பெண்கள் ஒன்றாக காட்சிகொடுக்கும் குழுப் படங்களையோ அல்லது இளம் ஆண்கள் மற்ற இளம் ஆண்களுடன் காட்சி கொடுக்கும் புகைப்படங்களையோ தான் பார்க்க முடியும். 

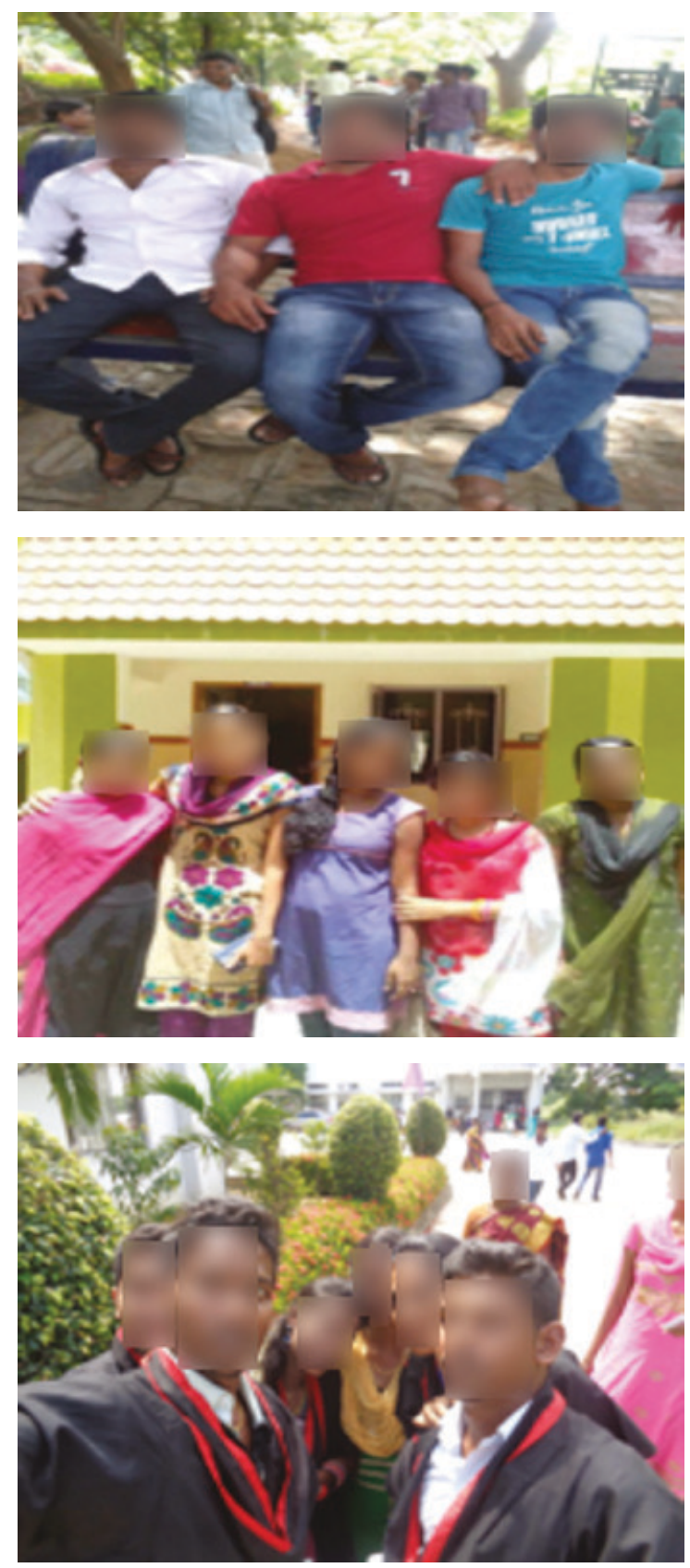

படம்: 3.21 தனிப்பட்டவை - குழுவில் (நண்பர்கள்) தான் எனும் தன்மை 
பொதுவாக, மணமாகாத, இரு பாலாரும் சேர்ந்த படங்கள், அது சுய-புகைப்படமாகவோ அல்லது காட்சி கொடுத்து எடுக்கப்பட்ட புகைப்படமாகவோ இருக்கலாம், - நிகழ்நிலையில் தோன்றினால், அது அநேகமாக குழுப்படமாக தான் இருக்கும். தனிஇருவர் மட்டும் இருக்கும் படமாக இராது. இதற்கான விதிவிலக்கு, மணமாகாத, உயர் மத்தியதர வகுப்பைச்சேர்ந்த இளம் பெண்கள் சம்பந்தப்படும் சமயங்கள் மட்டுமே. இந்தக்குழுவிற்கு, மணமாகாத இளம் ஆணுடன் புகைப்படத்திற்கு காட்சி கொடுப்பது என்பது, சமூக அனுசரிப்பு இல்லாமை என்று அர்த்தமாகாது.

\section{வகை 5: குழுவில் (குடும்பம்) தான் எனும் தன்மை}

திருமணத்திற்கு பிறகு, பொதுப்பார்வைக்கான முன்னிலைப்படுத்தல் என்பது தனிநபர் மீது மட்டும் குவியம் கொண்டதாக இல்லாமல், அந்தத்தம்பதிக்ளின் “குடிம்பத்தின் அங்கம்" என்ற புதிய தகுதி நிலையின் மீீது குவியம் கொள்ளவேண்டும் என்பதே பொருத்தமானது என்று பொதுவாக கருதப்படுகிறது. இது மணமான பெண்களின் வழக்குகளில் மிகவும் உண்மையான ஒன்று (படம்:3.22)

பஞ்சக்கிராமியில் உள்ள அன்னையர்கள் சமூக ஊடக பக்கங்களில், சீர்மையான தாய்மையையும், குடும்ப வாழ்வையும் எடுத்துக்காட்டும் விதமான குழந்தைகளின் படங்கள் மற்றும் உடனடி குடும்பத்தின் உறுப்பினர்களின் படங்கள் ஆகியவை அடிக்கடி காணப்பட்டுக்கொண்டே இருக்கும். சிலசமயங்களில், இந்தப் படங்களின் பின்னணிகள் முக்கியத்துவம் பெற்றாலும், இங்கு குடும்பம் தான் அதிக முக்கியத்துவம் உடையதாக இருக்கிறது. குடும்பத்திலுள்ள நெருக்கத்தை, சமூக ஊடகங்களில் உள்ள படங்கள் வெளிக்காட்ட முற்படுகின்றன. இந்தப்படங்கள், இவற்றில் உள்ளவர்கள் தங்களை அடையாளப்படுத்திக் கொள்ளக்கூடிய பின்னணிகளைவிட, சிறுகுடும்பம் என்ற அமைப்பின் மீது தான் அதிக குவியத்தை ஈர்க்கின்றன. இந்தப்படங்கள், வீட்டிற்கு உள்ளேயும் வெளியேயும் சமமாக எடுக்கப்பட்டவையாக இருக்கின்றன. இந்தப்படங்கள் ஒருவித உடைமை உணர்வை வெளிப்படுத்துவனவாகவும், சிலசமயங்களில், இயல்புநிலையில் உள்ள பழைய புகைப்பட தொகுப்புகளில் இருந்து ஸ்கேன் பண்ணப்பட்டவையாகவும் இருக்கின்றன. இவை, குடும்ப நெருக்கம் மற்றும் நினைவுகள் ஆகியவற்றை காட்சிப்படுத்தும் நோக்கம் கொண்டவையாக இருக்கின்றன. 

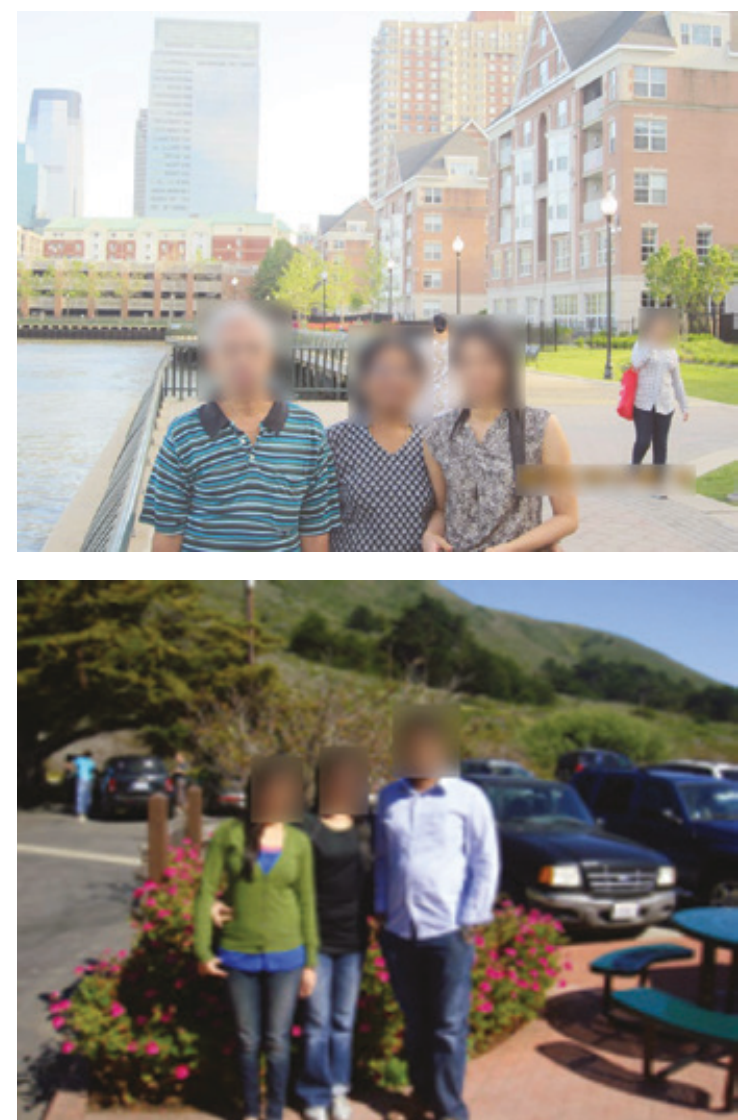

படம்: 3.22 தனிப்பட்டவை - குழுவில் (குடும்பம்) தான் எனும் தன்மை

முடிவாக, இந்தப்படங்கள், உபயோகிப்பாளர்கள், சித்தரிக்க விரும்பும் தான் எனும் தன்மையின் வெவ்வேறு கூறுகளை வெளிப்படுத்தினாலும், அவை பெரும்பாலும் அவர்கள் சார்ந்திருக்கும் சமூகம் மற்றும் சமூக வலைத்தொடர்பு ஆகியவற்றின் எதிர்பார்ப்புகளையும் சமூக விதிமுறைகளையும் அனுசரிப்பவையாகவே இருக்கின்றன. பேரார்வங்கள் குறித்த படங்கள் கூட, சமூகம் தனக்கென கொண்டிருக்கும் கூட்டு பேரார்வங்களை எடுத்துக்காட்டுவதாக இருக்கிறதே தவிர, இவற்றிலிருந்து விலகியதாக எதுவும் காட்டப்படுவதில்லை. ஒருவித சமூக ஒப்பந்தம் போல, இதுபோன்ற படங்களுக்கு கிடைக்கும் பதில்கள் அனைத்துமே நேர்மறையானதாகவே இருக்கிறது. இருக்கும் ஒருசில பரிகாசங்களும், மென்மை- 
யானவையாகவும், நட்பார்ந்தவையாகவும், இயல்புநிலையில் உள்ள நெருக்கமான பிணைப்பை வலுயுறுத்துவனவாகவும் இருக்கின்றன.

மேலே விவாதிக்கப்பட்டுள்ள ஐந்து வகைகளும், தான் எனும் தன்மையின் பல்வேறுவிதமான முன்னிலைப்படுதலை எடுத்துக்காட்டுகின்றன. இப்போது நாம் பஞ்சக்கிராமியின் காட்சிக்குரிய பதிவுகளில் மற்றொரு வகையான அன்றாட வாழ்த்துக்கள் என்பதை கருதுவதை நோக்கி நகரலாம். இந்தவகை, பொது மற்றும் தனிப்பட்ட வகைகளுக்கு இடையேயுள்ள கூறுகளான "இடைப்பட்டவை" என்பதோடு ஒத்ததாக இருப்பவை. அன்றாட வாழ்த்துக்கள் பெரும்பாலும், ஒருவகையான மரபு கண்காணிப்பாளர்களான ${ }^{56}$ மீம்களால் தொடரப்படுகிறது. பஞ்சக்கிராமியில் மீம்கள் மிகவும் பிரபலமானவை மற்றும் பலதரப்பட்டவை. இந்த அனைத்துவகை மீம்களையும் இந்த அத்தியாயத்தில் அலசுவது என்பது இயலாததாகையால், அனைத்து வகுப்பைச்சேர்ந்த மக்களாலும் அடிக்கடி (ஒருநாளில் சுமார் ஐந்து முறை) பதிவிடப்படும் வகையான அன்றாட வாழ்த்துச்செய்திகளுடன் தொடர்புடைய மீம்கள் மட்டும் அடுத்தப்பகுதியில் அலசப்பட்டிருக்கின்றன. தனிப்பட்ட புகைப்படங்கள், குடும்பம் அல்லது நண்பர்கள் என்கிற உடனடி வட்டத்துடனான உறவுமுறையை சமூக ஊடகங்களில் உள்ள பெரிய வலைத்தொடர்புக்கு எடுத்துக்காட்ட உதவுகின்றன. அன்றாட வாழ்த்துச்செய்திகள், இந்த பெரிய வலைத்தொடர்பை, அன்றாட அடிப்படையில் அங்கீகரிக்கவும் நிர்வகிக்கவும் உதவுகின்றன. இவை பஞ்சக்கிராமியின் அன்றாட இயல்புநிலை கலாச்சாரத்தின் அங்கமான சமூகத்தன்மையை, பேணுவதற்கும் வலியுறுத்துவதற்குமான ஒரு வழியாகவும் செயல்படுகின்றன.

\section{பகுதி 3: "இடைப்பட்டவை"}

\section{அன்றாட வாழ்த்துச்செய்திகள்}

ராஜப்பா என்கிற 67 வயதான ஓய்வு பெற்ற அஞ்சல் நிலைய அதிகாரி, அவருடைய குடியிருப்பு வளாகத்தில் செயல்படும் மூத்த குடிமக்கள் கழகத்தின் மூலம் வாட்சப்பிற்கு அறிமுகப்படுத்தப்பட்டார். இந்தக் குழு (60 வயதுக்கு மேற்பட்டவர்களை கொண்டது) 45 உறுப்பினர்களை உடையது. இவர்களில் பெரும்பாலானோருக்கு குறைந்தபட்சம் அவர்களின் ஒரு பிள்ளை- 
யேனும் வெளிநாட்டில் பணிபுரிபவர்களாக இ இருக்கிறார்கள். ராஜப்பாவின் வாட்சப் குழுவில் உள்ள தகவல்களின் அடித்தளத்தில், பல கருத்துக்கள் மீண்டும் மீண்டும் தோன்றுவனவாக இருக்கும். ப்ரார்தனைகளுடன் கூடிய அன்றாட வாழ்த்துச்செய்திகள், ஆரோக்கியம், உடற்பயிற்சி, உணவுப்பழக்கம் ஆகியவை பற்றிய முன்னனுப்பப்பட்ட காட்சிதகவல்கள், முன்னனுப்பப்பட்ட படப்புதிர்கள் மற்றும் இயற்கை பற்றிய காட்சிகள். இந்தக்குழுவின் அன்றாடத் தகவல்கள் காலை 4:30 மணியளவில், காலை வணக்கத்துடனும், அதைத்தொடர்ந்து பிரார்த்தனையுடன் கூடிய மீம்களுடனும் துவங்குகின்றன. காலை 6:00 அல்லது 6:30 மணியளவில், அநேகமாக அனைத்து உறுப்பினர்களும் ஒருவருக்கொருவர், காலை வணக்கச்செய்தியையோ அல்லது காட்சியுடன் கூடிய வாழ்த்தையோ தெரிவித்து முடித்திருப்பார்கள். பெரும்பாலான பிரார்த்தனைகள் பொதுவானவை. அவை இந்துசமயக் கடவுளின் படத்தைக் கொண்டிருந்தால், அது அநேகமாக விநாயகர் அல்லது ஷீர்டி சாய்பாபா ${ }^{57}$ ஆகியோருடையதாக இருக்கும். இவற்றில் பல, முன்னனுப்பப்பட்டதாகவோ அல்லது கூகுள் படங்களின் பிரதியாகவோ இருக்கும்.

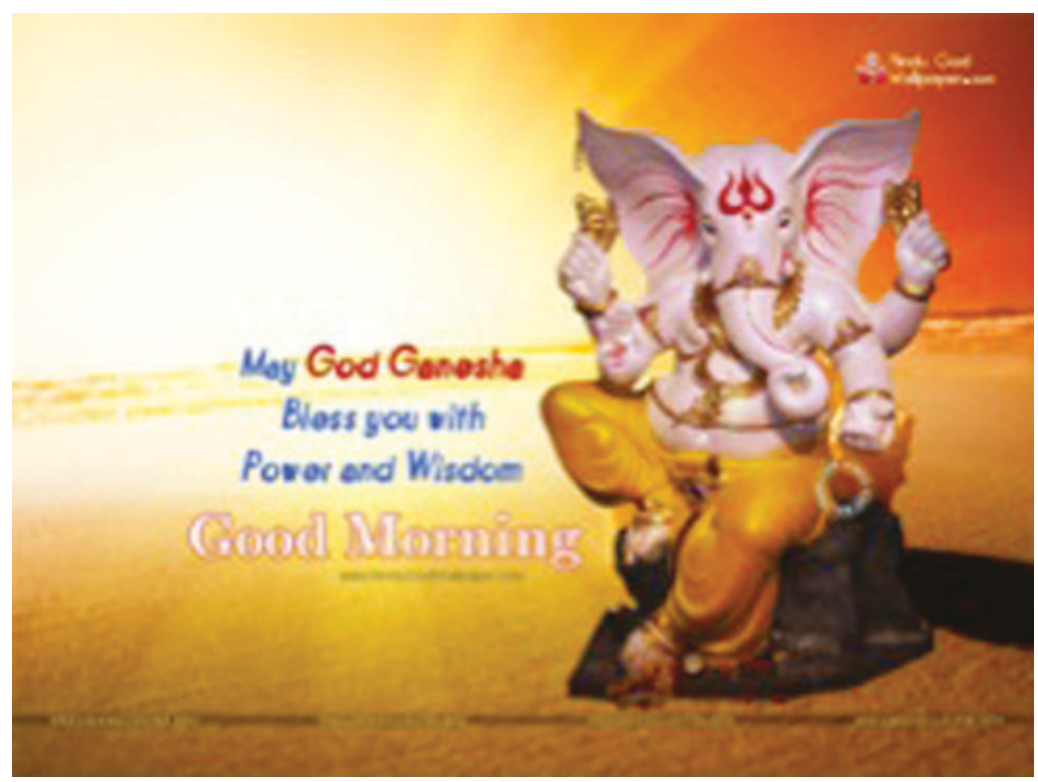

படம்: 3.23 இடைப்பட்டவை - வாழ்த்துடன் கூடிய விநாயகரின் படம் 
ராஜப்பா, தான் காலையில் வாட்சப்பில் என்ன படிக்கிறோம் என்பது பற்றி சற்றே உணர்ச்சிவயப்படுபவர். நல்லதான எதையாவது படித்தால் தான் அன்றைய பொழுது நல்லதாக இருக்கும் என்பது அவரது கருத்து. ராஜப்பாவுக்கும் அவரது மூத்த குடிமக்கள் கழகத்தில் உள்ள பலருக்கும், சமூகத்தன்மையின் முக்கியமான கூறே, ஒவ்வொருவருக்கும் புன்னகையுடன் வாழ்த்து தெரிவிப்பது தான். அவர்களைப்பொறுத்தவரை, இது ஒரு நாளின் துவக்கத்திற்கான நேர்மறை அறிகுறி. இந்தக் குழு வாட்சப்பில் உள்ள ஸ்மைலி சின்னத்தை நிஜப்புன்னகைக்கு ஈடானதாக கருதாததால், ஒரு ப்ரார்தனையோ அல்லது நல்லதொரு தகவலோ, அதற்கு சிறந்தவொரு ஈடாக கருதப்படுகிறது.

பஞ்சக்கிராமியில் அன்றாட வாழ்த்துக்கள், சமூகத்தன்மையின் ஒரு முக்கியமான வகையாகும். இதை, மத்தியதர வகுப்பினரால் விரும்பப்படும் காலை நடைப்பயிற்சியில் போதான “காலை வணக்கங்களிலும்", பால்காரர்களின் நட்பார்ந்த வணக்கங்களிலும் ${ }^{58}$ மற்றும் மாணவர்களின் பணிவான வணக்கங்களில் காணமுடியும். இது போன்ற வாழ்த்துக்கள், சமூகத்தன்மையை வடிவமைப்பனவாக மட்டுமல்லாமல், ஒரு நாளின் துவக்கத்திற்கான சிறந்த வழியாகவும் கருதப்படுகிறது. இது பிரபலங்களின் ஊக்கமளிக்கும் சொற்களோடு இணைந்து, பள்ளிகள், பஞ்சாயத்து அலுவலகங்கள் மற்றும் இதர பொது இடங்களில் எழுதிவைக்கப்பட்டிருக்கும்போது, முற்றிலும் மாறுபட்ட பரிமாணத்தைப் பெற்றுவிடுகிறது. இதுபோன்ற வாழ்த்துக்கள் பலசமயங்களில் சமூக ஊடகத்தளங்களுக்கும் எடுத்துச்செல்லப்படுகின்றன. அங்கு இ இவை ஊக்கமளிக்கும் தகவல்களுடன் சேர்ந்து, கண்ணுக்கு குளிர்ச்சியான காட்சியமைப்புகளுடன் இணைந்த அன்றாட வாழ்த்துக்களாக வடிவமெடுக்கின்றன.

ஊக்கமளிக்கக்கூடிய, தத்துவார்த்த அல்லது சமயம்சார்ந்த தகவல்களை சித்தரிக்கும் படங்கள், மற்றவர்களுடன் பகிரப்படுவெதற்காகவே வடிவமைக்கப்படுகின்றன. இந்தகாட்சியமைப்புகளுடன் பலசமயங்களில் வார்த்தைகள் இணைந்திருக்கக்கூடும். வேறுசிலசமயங்களில் இவை, மீம்கள் என்று வகைப்படுத்தக்கூடிய வகையில், படவிளக்கச்சொற்களுடன் காணப்படக்கூடும். இந்த மீம்கள், நேர்மறையானதாகவும், நல்ல உணர்வை கொடுக்கக்கூடியதாகவும் இருக்கும். இது போன்ற தகவல்கள், பெரும்பாலும் ஒரு நாளின் நேரத்துடன் தொடர்பான வாழ்த்துடன் இணைந்ததாக இருக்கும் (காலை/ மாலை வணக்கங்கள்; இந்நாள் நன்னாள் போன்றவை). இந்த 
வாழ்த்துக்கள், ஒருவர் சமூக ஊடகத்தில் அமைத்துக்கொண்ட சமூக வலைத்தொடர்புகளுடன் தொடர்பில் இருக்க உதவும் (நடப்பில் இருக்கும் உரையாடல் மூலம்) சிறந்த வழியாகவும் பார்க்கப்படுகின்றன.

இந்த காட்சியமைப்புகளை ஒரு சில உப-வகைகளாக பிரிக்கலாம். உதாரணத்திற்கு, ஊக்கமளிக்கும் மேற்கோள்கள், சமயம்சார்ந்த மேற்கோள்கள் போன்றவை. பஞ்சக்கிராமியில், வார்த்தைகளுடனான இந்தப் படங்கள், பொதுவாக அன்றாட எழுத்துவடிவ வணக்கத்தால் தொடரப்படுகின்றன (படம்: 3.24).

இந்தப்படங்களில் சில, வெறும் வார்த்தைகளை மட்டுமே கொண்டதாகவும், பல்வேறு வண்ணங்களையும், எழுத்துவடிவங்களையும் கொண்டு அழகுற வடிவமைக்கப்பட்டதாகவும் இருக்கும். இவை வழக்கமாக பிரபலமான ஒருவரின் மேற்கோள்களை கொண்டதாகவோ அல்லது நிகழ்நிலையில் இருந்து எடுக்கப்பட்ட படமாகவோ இருக்கும். ஒருசிலர், தத்துவார்த்த அல்லது ஊக்கமளிக்கும் சொற்களுடன் கூடிய படங்களை இணையத்தில் கூகுளின் படத்தேடல் மூலம் தேடி குறிப்பாக அவற்றை பதிவிறக்கி, அவற்றை, தங்கள் சமூக ஊடக தொடர்புகளுக்கு முன்னனுப்புகின்றனர்.

ஒருசிலர் இதுபோன்ற படங்களை, தங்களின் முகநூல் மற்றும் வாட்சப் தொடர்புகளுக்கு ஒவ்வொரு காலையிலும் அனுப்புவதை தமது முதன்மையான கடமையாக நினைக்கின்றனர். அவர்கள், இது போன்ற நேர்மறையான கருத்துக்கள் கொண்ட படங்களை பகிர்வதை, பரந்த சமூகத்திற்கான பரிசாகவும், நம்மைச்சுற்றியிருக்கும் எதிர்மறை எண்ணங்களை சமன் செய்ய உதவும் ஒன்றாகவும் கருதுகின்றனர். இல்லத்தரசிகள், இதை வாட்சப் மூலமாகவும், கல்லூரி மாணவர்கள், இதை வாட்சப் மற்றும் முகநூல் மூலமாகவும் பரிமாறிக்கொ-

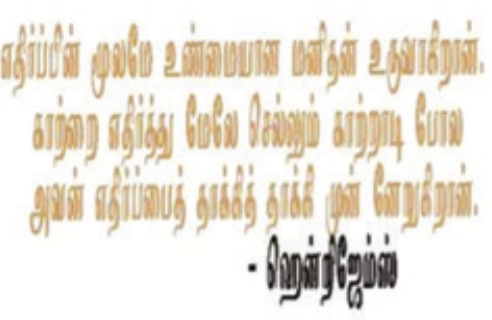

Gud mrng frnds (:):-:)

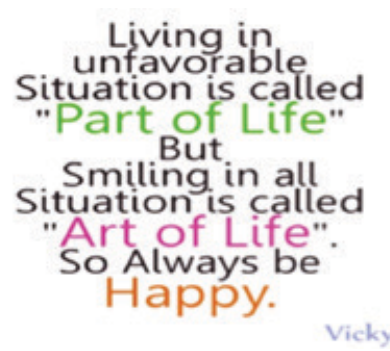

Gud mrg guys... Wake up...

படம்: 3.24 இடைப்பட்டவை - அன்றாட வாழ்த்துக்களுடனான எழுத்துவடிவ மீம்கள் 
ள்கிறார்கள். உதாரணத்திற்கு, கண்ணா என்கிற 21 வயதான, வணிக நிர்வாகம் பயிலும், கீழ்த்தட்டு சமூகப்பொருளாதார பின்னணியில் இருந்து வந்த மாணவர், இறுதியாண்டு மாணவராக, இரண்டாண்டுகளுக்கு முன்பு, இதை செய்யத்துவங்கினார். அவர் இதை, தன் வகுப்புத்தோழர்களுக்கும் நண்பர்களுக்கும் உதவும் வகையில் (குறிப்பாக கீழ்த்தட்டு சமூகப்பொருளாதார பின்னணியில் இருந்து வந்து, தங்கள் குடும்பங்களுக்கு பொருளாதார வகையில் உதவுவதற்காக படித்துக்கொண்டே பணிபுரிபவர்கள்) செய்யத்துவங்கினார். அவர், இதை தொடர்ந்து செய்து, தனது கல்லூரி வட்டத்திற்கும் இதை விரிவாக்கி விட்டார். கண்ணனது உடன் பயிலும் மாணவர்கள் இதை ஆதரிக்கின்றனர். ஒவ்வொரு காலையிலும், தனது முகநூல் பக்கத்தில் எந்த மேற்கோளை அவர் பதிவிடுவார் என்று பார்ப்பதற்காக காத்திருப்பதாக அவர்கள் கூறினர். இந்தப்பழக்கம், அவரது வலைதொடர்பிற்குள்ளாக சமூகத்தன்மையை கட்டமைக்கும் விதமாக நடத்தப்படுகிறதது. பல்வேறு உபயோகிப்பாளர்களும், அன்றாட ஊக்கமளிக்கும் அல்லது சமயம் சார்ந்த மேற்கோள்களை பகிர்ந்து கொண்டனர் (இவையும் ஊக்கமளிப்பவையாக இருக்கும்). இவை வழக்கமாக, அவை உருவாகும் சமயத்தைக்குறிக்கும் சமயச்சின்னத்தை கொண்டிருக்கும் (படம்: 3.25). எனினும், அனைத்துச் செய்திகளும், அன்றாட வணக்கம் தெரிவித்தலான “காலை/ மாலை வணக்கங்கள்" “இந்நாள் நன்னாள்" போன்றவற்றை பின்பற்றுகின்றன.

இதுபோன்ற சில படங்கள், எந்தப்பின்னணியும் இல்லாமல் வெறும் வார்த்தைகளை மட்டுமே கொண்டவை (படம்: 3.26). மற்ற அன்றாட வணக்கம் தெரிவித்தலுக்கு, வார்த்தைகளுடன் சேர்ந்து பொருத்தமான காட்சி ஒன்றும் சேர்ந்திருக்கும்.

கண்ணைக்கவரும் இயற்கை காட்சிகள், அன்றாட வணக்கம் தெரிவித்தலுக்கு பயன்படுத்தப்படுவெது, வழக்கத்திற்கு மாறானது அல்ல. இவை, பலசமயங்களில் சமயம்சார்ந்த அல்லது ஊக்கமளிக்கும் மேற்கோள்களில் பயன்படுத்தப்படுகின்றன. இவை இயற்கையின் அழகை காட்டும் நோக்கத்துடன் மட்டுமல்லாமல், இந்த உலகம் என்பது மிகப்பெரிய இடப்பரப்பு என்றும், இதை உருவாக்கியவர், இந்தத்தகவலைப் படிப்பவரையும் பார்த்துக்கொள்வார் என்பதையும் குறிப்பிட்டுக்காட்டும் விதமாக அமைக்கப்படுகிறது.

ஆகவே, அன்றாட வாழ்த்துத்தெரிவித்தல், ஏற்கனவே நிறுவப்பட்டு நண்பர் குழுவுடன் தொடர்பில் இருக்க உதவும் 


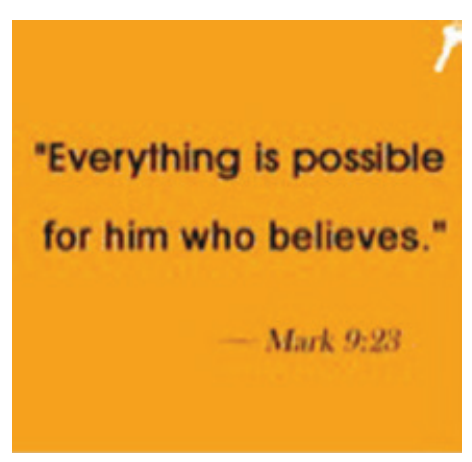

GOOD MORNING !

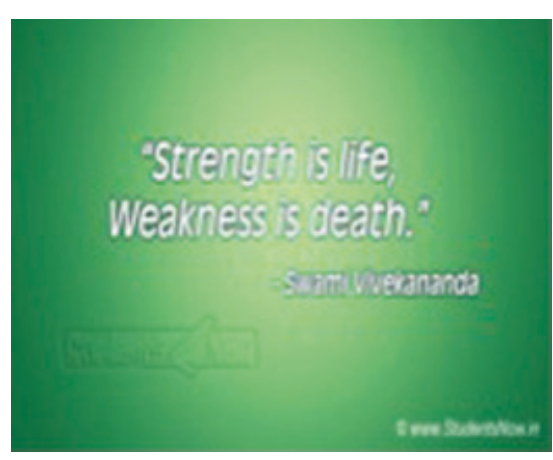

Have a grt...dy

படம்: 3.25 இடைப்பட்டவை - ஊக்கமளிக்கக்கூடிய அல்லது சமயம் சார்ந்த மேற்கோள்களுடன் கூடிய படங்கள்

விதமாக பயன்படுத்தப்படுகின்றன. தகவலாளர்களை பேட்டியெடுத்ததில் தெரியவந்தது என்னவென்றால், ஒருமுறை அவர்கள் முகநூல் அல்லது வாட்சப்பில் நண்பர்கள் குழுவை அமைத்துவிட்டார்கள் என்றால், அவர்கள் ஒவ்வொருவருடனும் தொடர்பில் இருப்பது மிகவும் முக்கியமானதாக கருதப்படுகிறது என்பது தான். இல்லையென்றால் சமூக ஊடகங்களில் இவ்வளவு நண்பர்களை சேர்த்து என்ன பயன் என்ற கேள்வி எழுகிறது. இதுபோன்ற விசாரங்களை தவிர்ப்பதற்கு, அன்றாட வணக்கம் தெரிவித்தால் ஒரு சிறந்த வழியாக 59 ஆகிவிட்டது. இதன்மூலம் தகவலாளர்களின் நண்பர்கள் பட்டியலில் உள்ள ஒவ்வொருவருடனும் மோதலற்ற வகையில் ஈடுபட்டதாகிவிடுகிறது. ஒரு சில உபயோகிப்பாளர்கள் கூறியது என்னவென்றால், தங்கள் தொடர்புகளில் அதிகம் பேசாமல் இருக்கும் மக்கள் கூட, இது போன்ற நடைமுறை நிறுவப்பட்டபின், அதிகம் பங்கேற்பவர்களாக ஆகிவிடிகிறார்கள் என்பது தான்.

இருப்பினும், இது போன்ற தகவல்கள், சமூகத்தன்மையை வடிவமைப்பவை ஆகவும் நண்பர்குழுவுடன் தொடர்புகொள்ள பயன்படுபவை ஆகவும் மட்டுமல்லாமல், ஒரு குறிப்பிட்ட சமயம் சார்ந்த நேர்மறையான கர்மாபுள்ளிகளை சேகரிப்பதற்காகவும் பயன்படுகின்றன. பஞ்சக்கிராமியில் இருந்து வரும் பல நடுத்தர வயதை சேர்ந்த தகவலாளர்கள், முகநூலில் ஏதோவொரு சமயம் சார்ந்த நடவடிக்கைகளில் பங்கெடுத்துக்கொண்டார்கள். அவர்கள் அதை சமயம் சார்ந்ததாக வகைப்படுத்தவில்லை என்றாலும், அவர்களை பொறுத்தவரை அது நல்ல கர்மாவை ${ }^{60}$ வடிவமைப்பதுடன் தொடர்புடையதானதாகும். இது “நாம் பிறருக்கு என்ன செய்கிறோமோ அது தான் 


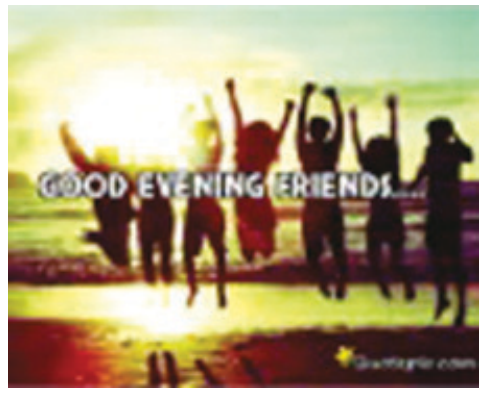

Hope u hd a grt day Good Evening...

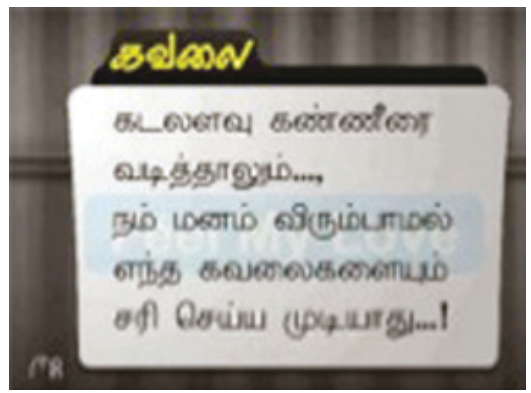

Good Evening (:-):-
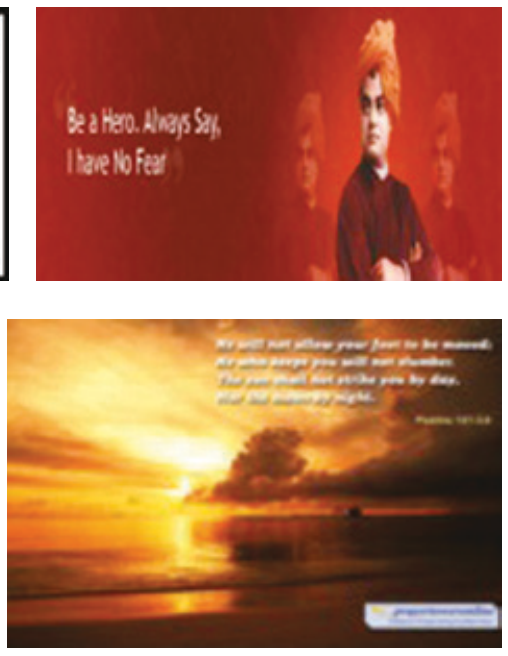

Good Evening Friends

Have a good Day...

படம்: 3.26 இடைப்பட்டவை - காட்சியமைப்புகளுடன் கூடிய அன்றாட வாழ்த்துக்கள்

நமக்கு வந்து சேரும்' என்று கூறப்படும் நல்ல செயல்கள் நல்ல விளைவுகளுக்கு வழிவகுக்கும் என்ற சமய நம்பிக்கையில் இருந்து விளைந்ததாகும். இது போன்ற பங்கெடுப்பு, நேர்மறையான எண்ணங்களை தரக்கூடிய சமயச்செய்திகளை பகிர்வது, கடவுள்களின் உருவப்படங்களை பகிர்வது போன்றவை முதல் பரந்த சமூகத்தில் நேர்மறையான எண்ணங்களை வலியுறுத்தக்கூடியதான ஊக்கமளிக்கும் கவிதைகள், கதைகள் மற்றும் கட்டுரைகளை பகிர்வது வரை பல வகைப்படும். இதனால் இவற்றை பகிர்பவர்களுக்கு நல்ல கர்மா விளையும் என்று நம்பப்படுகிறது. வித்யாஷங்கரை போலவே, சிலர் இதை அன்றாட நடைமுறை ஒழுங்காக பின்பற்றினார்கள். 
வித்யாஷங்கர் என்ற, 47 வயதான கட்டக்கலைஞர், முகநூலில் இதுபோன்ற நல்ல கர்மாக்களை அன்றாடம் வடிவமைப்பவர். அவருடைய பெரும்பாலான சமூக வட்டம் முகநூலில் இருப்பதால், தனக்கான நல்ல கர்மாவை வடிவமைத்துக்கொள்வதற்கான செயல்களை பெறக்கூடிய அங்கத்தினராக தன்னுடைய வலைதொடர்பில் இருப்பவர்களை பயன்படுத்திக்கொள்ளலாம் என்பது அவரது கருத்து. ஷங்கரின் அன்றாட நடைமுறை ஒழுங்கு, ஏதாவது ஒரு இந்துசமயக் கடவுளின் உருவப்படத்தை (வழக்கமாக கிருஷ்ணர் அல்லது விநாயகர்) முகநூல் பக்கத்தில் காலை ஆறு மணிக்குள் பதிவிடுவதாகும் (படம்: 3.27).

ஷங்கர் இந்த நடைமுறையை மிகவும் கண்டிப்புடன் கடைபிடிக்கிறார். ஏனென்றால், முகநூலில் உள்ள நடுத்தர வயதைச் சேர்ந்த அவர் நண்பர்களில் பலர், காலையில் முதல் காரியமாக முகநூலைப் பார்ப்பதை வழக்கமாக கொண்டிருக்கிறார்கள் என்று அவருக்கு தெரியும். அவர்கள், தினமும் காலையில் ஒரு மங்களகரமான சின்னத்தில் விழிக்கவேண்டும் என்பதற்காக, அவர் கடவுள்களின் படங்களை காலை ஆறு மணிக்கு சற்று முன்பே தனது முகநூல் பக்கத்தில் பதிவிடுகிறார்.

ஒரு பேட்டியில் ஷங்கர் குறிப்பிட்டதாவது:

“அவர்கள் இதை பார்த்துவிட்டார்கள் என்று எனக்குத் தெரியும். நான் பதிவிட்டவுடனே வரும் விருப்புகளில்

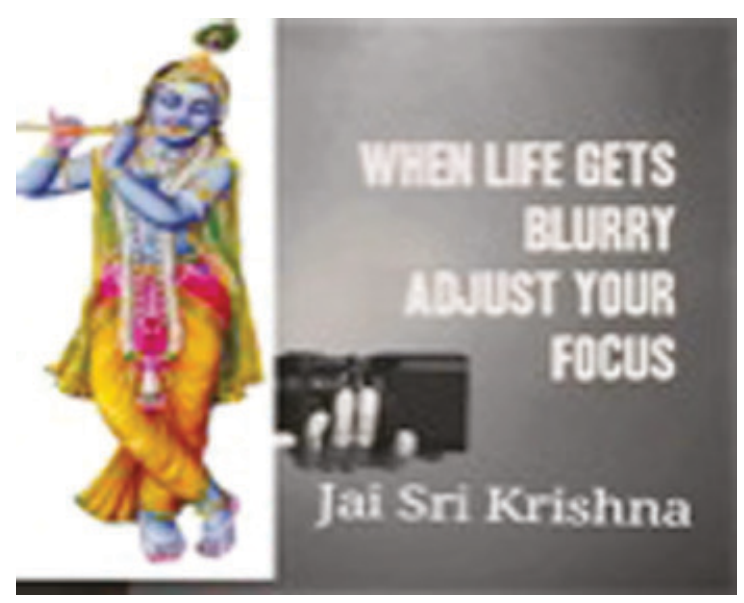

படம்: 3.27 இடைப்பட்டவை - வித்யாஷங்கர் பதிவேற்றிய கடவுள் கிருஷ்ணரின் படம் 
இருந்து, நான் இதை அறிந்து கொள்கிறேன். பெரும்பாலும் இது வழக்கமான அதே 40-45 நண்பர்கள் தான், ஆனால் உடனடி பதிலைப் பெறுவது மிகவும் பயனுள்ளது. ஏனெனில், அன்றைக்கான எனது நல்ல கர்மாவை நான் அமைத்துக்கொண்டுவெட்டேன் என்று எனக்குத் தெரியும். மேலும், அவர்கள் இதை பகிரும்போது, நான் அவர்களதை வடிவமைக்க உதவியதால் இது அவர்களுடைய நல்ல கர்மாவை மட்டுமல்லாமல் என்னுடையதையும் வடிவமைக்கும்."

சுதாஸ்ரீ என்கிற 37 வயதான இ இல்லத்தரசி, தன்னுடைய கர்மாவை, தினமும் காலையில் நேர்மறையான எண்ணங்களை தூண்டும் தகவல்களை 35 உறுப்பினர்களை கொண்ட வாட்சப் குழுவில் பகிர்ந்துகொள்வதன் மூலம் வடிவமைத்து கொள்கிறார். இவர், ஏதாவது ஒரு சமயப்புத்தகத்தில் இருந்து எடுக்கப்பட்ட நேர்மறையான செய்தியை, காலை வணக்க வாழ்த்துடன் (படம்: 3.28) இந்தக்குழுவில் பகிர்ந்து கொள்கிறார். சுதாஸ்ரீ தன்னுடைய நம்பிக்கையை பின்வருமாறு விவரித்தார்:

“என்னுடைய தகவல்கள், மக்கள் தங்கள் நாட்பொழுதை நேர்மறையான எண்ணங்களுடன் துவங்க உதவுகிறது. அன்றாடம் காலை கண் விழிப்பதே ஒரு அதிசயமாதலால், மக்கள் கடவுளால் வழங்கப்பட்ட நாட்பொழுதை வீணடி-

\section{Serenity Prayer}

God...

Grant me the serenity to accept

the things I cannot change...

The courage to change

the things $I$ can...

And the urisdom to know

the difference.

படம்: 3.28 இடைப்பட்டவை - வாட்சப் குழுவில் சுதாஸ்ரீயின் பிரார்த்தனை 
ப்பதை நான் விரும்பவில்லை.....நல்லதொரு துவக்கம் மகிழ்ச்சியான நாளை கொடுக்கக்கூடும்.....இதன்மூலம் இந்தநாட்பொழுதில் நான் ஒரு நல்லசெயலை செய்திருக்கிறேன்"

வித்யாஷங்கரும் சுதாஸ்ரீயும் தனியாக இல்லை. சமூகத்திற்கு (அவர்களுடைய உடனடி சமூக வட்டம்) நன்மையை அளிக்கும் செயல்களில் பங்கெடுத்துக்கொள்வது அவர்களுக்கு நல்ல கர்மாவை அளிக்கும் என்று பல தகவலாளர்கள் நம்புகின்றனர்.

இதுபோன்ற காட்சியமைப்புகளில் பெரும்பாலானவை உருவாக்கப்பட்டவை (ஆக்கபூர்வமாக படைக்கப்பட்டவை) அல்ல. இவை முகநூல் அல்லது வாட்சப் மூலம் பகிரப்பட்டவை. சந்தீப் என்கிற 31 வயதான மென்பொருள் பொறியாளரை பொறுத்தவரை, இதுபோன்ற படங்களைப் பெறுபவர்கள், உண்மையில் நல்ல கர்மாவை அமைப்பதற்காக கடவுள் கொடுக்கும் சந்தர்ப்பங்களை தங்கள் மடியில் நேரடியாக விழப்பெற்றவர்கள். அவர்கள் செய்யவேண்டியதெல்லாம், இதை மற்றவர்களுடன் பகிரவேண்டியது தான். இதுபோன்ற பகிர்தல்களின் மூலம், இந்தப்படங்கள், ஊடகங்களிடையே மாறி மாறி, மின்னஞ்சலில் இருந்து முகநூலிற்கு அங்கிருந்து வாட்சப்பிற்கு என்று பலசுற்றுக்கள் பல்வேறு தளங்களிடையே செல்வதன் மூலம் தனக்கென ஒரு சமூக வாழ்வை ${ }^{61}$ பெறுகின்றன.

\section{கலவையான வகைகள்}

மேலே விளக்கப்பட்டுள்ள காட்சிக்குரிய பதிவுகளின் வகைகள் பிரத்யேகமானவை அல்ல. ஜோத்ஸ்னா மற்றும் சகாயம் ஆகியோரின் வழக்காய்வுகள், பஞ்சக்கிராமியில் உள்ள மக்கள் எவ்வாறு, தாங்கள் தொடர்பில் இருக்கும் பல்வேறு வலைத்தொடர்புகளின் எதிர்பார்ப்புகளுடன் அனுசரிப்பதாக காட்டிக்கொள்ளும் விதமாக, வகைகளின் கலவையை பயன்படுத்தி சாமர்த்தியமாக தங்களுக்கென ஒரு தோற்றத்தை வடிவமைத்து முன்னிலைப்படுத்திக்கொள்கிறார்கள் என்பதை நமக்கு எடுத்துக்காட்டுகிறது.

ஜோத்ஸ்னா என்பவர் 33 வயதான இல்லத்தரசி. அவர் 39 வயதான தன் கணவர் சாமுவேல் மற்றும் அவர்களின் இரண்டு குழந்தைகளுடன், பஞ்சக்கிராமியில் உள்ள உயர்தர குடியிருப்பு வளாகத்தில் வசிக்கிறார். அவர், தமிழ்நாட்டின் ஒரு 
சிறுநகரத்தில் வளர்ந்து, 10 வருடங்களுக்கு முன், சாமுவேலுடனான அவரது திருமணத்திற்கு பிறகு தான் சென்னைக்கு இடம்பெயர்ந்திருக்கிறார். சாமுவேல் பஞ்சக்கிராமியில் உள்ள பெரிய ஐ.டி நிறுவனம் ஒன்றில் அமைப்புக் கட்டட வடிவமைப்பாளராக பணிபுரிவதாலும், அவர் அந்தப்பகுதியில் 2011-ஆம் வருடம் இரண்டு படுக்கையறைகள் கொண்ட அடுக்குமாடி குடியிருப்பு தளத்தை விலைக்கு வாங்கியிருந்ததாலும், இந்தத் தம்பதியினர், பஞ்சக்கிராமியில் குடியேற முடிவு செய்தனர்.

ஆதர்ச கிறிஸ்தவரான ஜோத்ஸ்னா, பஞ்சக்கிராமிக்கு அருகில் உள்ள சர்ச்சில் பிரார்த்தனைக் கூட்டங்களுக்கு செல்வார். சர்ச்சின் ஒழுங்கான உறுப்பினர்களுடன் தொடர்பில் இருப்பதற்காகவும் நிகழ்ச்சிகளை திட்டமிடுவதற்காகவும், ஒரு வாட்சப் குழு நிறுவப்பட்டது. ஜோத்ஸ்னா, விரைவில் இதில் ஒரு நிரந்தர பங்களிப்பாளராக ஆனார். இந்தக்குழுவில் அவரது பகிர்வுகள் அனைத்தும், ஏசு கிறிஸ்துவின் படங்கள், பிரார்த்தனை மீம்கள் போன்ற காட்சிக்குரிய பதிவுகள் தான் (படம்: 3.29). ஜோத்ஸ்னாவின் படங்களில் பெரும்பாலானவை, அவரது மற்ற தொடர்புகளால் முன்னனுப்பப்பட்டவை அல்லது கூகுள் படத்தொகுப்பில் இருந்து பதிவிறக்கம் செய்யப்பட்டவை.

ஜோத்ஸ்னாவின் குடியிருப்பு வளாகம், அவ்விடத்தின் முறையான பராமரிப்பு விசாரங்களை விவாதிப்பதற்காக ஒரு முகநூல் குழுவையும், குடியிருப்பாளர்களிடையே நட்புதொடர்பை ஊக்குவிப்பதற்காக ஒரு வாட்சப் குழுவையும் ஏற்ப-

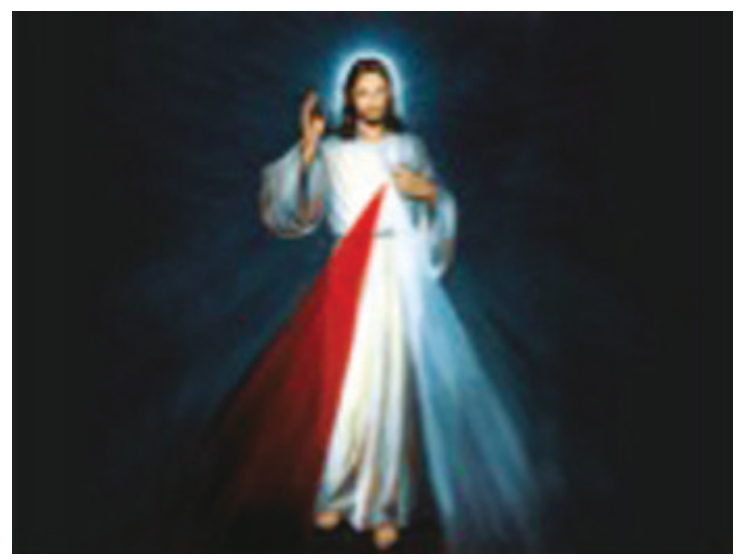

படம்: 3.29 கலவையானவை - சர்ச் வாட்சப் குழுவில் இருந்து யேசுகிறிஸ்துவின் படம் 
டுத்தியிருக்கிறது. இஇந்த வாட்சப் குழுவில் ேோத்ஸ்னாவின் பங்களிப்பு, அன்றாடம் காலையில் ஒரு நேர்மறை தகவலை முன்னனுப்புவதாகும். இந்தத்தகவல்கள் எதுவுமே இயல்பில், சமயம் சார்ந்தவை அல்ல. மாறான அவை, ஊக்கமளிக்கக்கூடியவையாக வகைப்படுத்தக் கூடிய நேர்மறைக் கருத்துக்கள் தாம் (படம்: 3.30). இது போன்ற மீம்களுக்கு குழு உறுப்பினர்களிடம் இருந்து பெறப்படும் பதில்கள் எப்போதும் ஊக்கமளிப்பதாகவே இருந்திருக்கிறது.

ஜோத்ஸ்னா, நான்கு நெருங்கிய நண்பர்கள் மட்டும் இருக்கும் மற்றொரு வாட்சப் குழுவிலும் உறுப்பினராக இருக்கிறார். 38 வயதான பிரியா மற்றும் 37 வயதான தேவி ஆகிய இருவரும் இல்லத்தரசிகள். 33 வயதான வசந்தி என்பவர் உள்ளூர் கல்லூரி ஒன்றில் தமிழ் விரிவுரையாளர். இந்தக்குழுவில், ஜோத்ஸ்னா வேடிக்கையான மீம்களையும், தங்கள் குடும்ப புகைப்படங்களையும், தங்கள் குழந்தைகளின் பள்ளிவிழா புகைப்படங்களையும், தான் வீட்டில் சமைக்கும் பதார்த்தங்களின் புகைப்படங்களையும் (படம்: 3.31) தொடர்ந்து பகிர்ந்து கொள்கிறார். இதுபோன்ற பகிர்வுகள், மற்ற உறுப்பினர்களிடம் இருந்தும் இதுபோன்ற காட்சிக்குரிய பதிவுகளை வெளியிடச் செய்கின்றன.

சகாயம் என்கிற 21 வயதான உள்ளூர் கலைக்கல்லூரியில் பயிலும் வணிகவியல் மாணவர், அவரது முகநூல் கணக்கில் 110 நண்பர்களை கொண்டிருக்கிறார். இந்த நண்பர்கள்

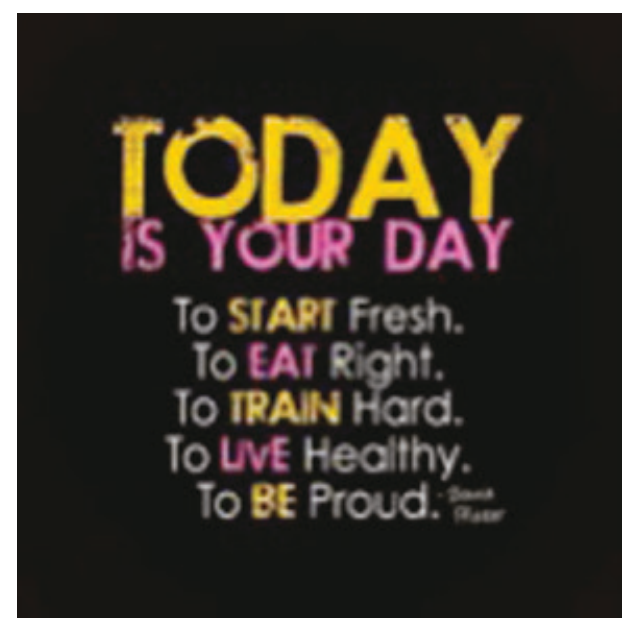

படம்: 3.30 கலவையானவை - குடியிருப்பு வளாக வாட்சப் குழுவில் இருந்து ஊக்கமளிக்கும் மீம் 
குழாம், கல்லூரி நண்பர்கள் மற்றும் அவர் முகநூலில் மட்டுமே சந்தித்த பெண்கள் ஆகியோரின் கலவை. சகாயத்தின் முகநூல் பக்கம், அழகான இயற்கை காட்சிகளின் படங்களையோ (கூகுள் படத்தொகுப்பிலிருந்து அவரால் பதிவிறக்கப்பட்டு, தனது முகநூல் பக்கத்தில் பகிரப்பட்டது) அல்லது குழந்தைகளின் படங்களையோ மட்டுமே கொண்டதாக இருக்கிறது (படம்: 3.32). இந்தப்படங்கள் பெரும்பாலும் காலை

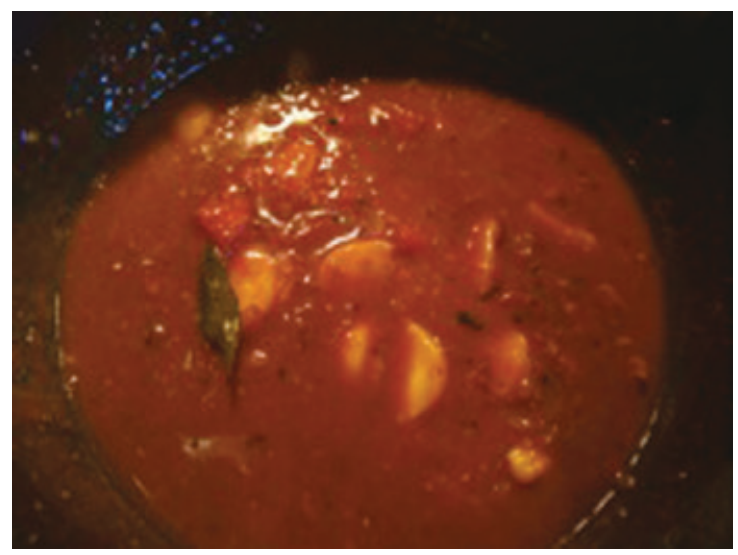

படம்: 3.31 கலவையானவை - வாட்சப்பில் உள்ள நண்பர்கள் குழுவில் பூண்டு புளிக்குழம்பு

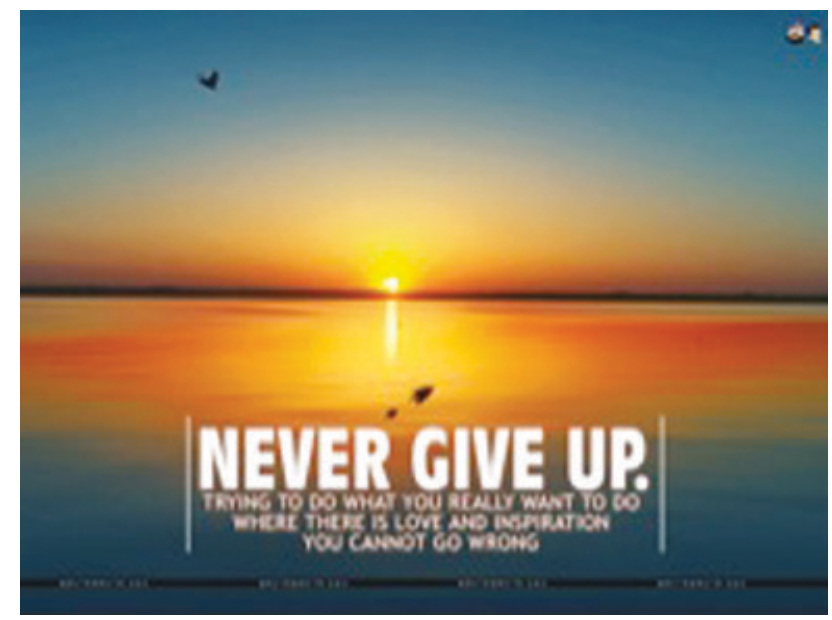

படம்: 3.32 கலவையானவை - முகநூல் பக்கத்தில் உள்ள இயற்கை காட்சியுடனான மீம் 
அல்லது மாலை வணக்கத்துடன் இணைந்ததாக இருக்கிறது. இவை அவரது நண்பர்களிடம் இருந்து (குறிப்பாக பெண் நண்பர்களிடமிருந்து) அதிக அளவிலான பதில்களை பெறுகின்றன. வழமையான பதில்கள் எல்லாம் விருப்பு தெரிவிப்புடன் சேர்ந்த காலை அல்லது மாலை வணக்கங்கள் தான். சகாயத்தின் படத்தொகுப்பில் அவர் காமெராவுக்கு காட்சிகொடுக்கும் வகையிலான படங்கள், ஒருசில சுய-புகைப்படங்கள், நண்பர்களுடனான பல புகைப்படங்கள் ஆகியவை காணப்படுகின்றன. இந்த இயற்கை காட்சிகள் மற்றும் குழந்தை படங்களுக்கு மாறாக, அவரது தனிப்பட்ட படங்கள், அவரது கல்லூரித்தோழர்களாலும் அவற்றில் இருக்கும் வேறுசில நண்பர்களாலும் மட்டுமே விருப்பு தெரிவிக்கப் பட்டுள்ளது. சகாயம், அவரது அபிமான திரைநட்சத்திரம் தனுஷிற்காக அர்ப்பணிக்கப்பட்ட ஒரு முகநூல் குழுவிலும் உறுப்பினராக இருக்கிறார். இந்த நடிகரைப் பற்றிய சமீபத்திய செய்திகளுடனான புகைப்படங்களை, கூகுள் தொகுப்பிலிருந்தோ அல்லது மற்ற திரைதளங்களில் இருந்தோ பதிவிறக்கி அவர் தொடர்ந்து இந்தக் குழு பக்கங்களில் பகிர்ந்து கொண்டிருக்கிறார் (படம்: 3.33). மற்ற குழு உறுப்பினர்களிடம் இருந்து பெறப்படும் பதில்கள் எப்போதுமே நடிகரை மட்டுமல்லாமல், சகாயத்தின் நடவடிக்கைகளையும் வியந்து பாராட்டுவதாகவும், இந்தக் குழுவிற்கான அவரது முயற்சிகளை அங்கீகரிப்பதாகவும் இருக்கிறது.

சகாயம் ஒரு உற்சாகமான வாட்சப் உபயோகிப்பாளரும் கூட. அவர் பஞ்சக்கிராமியில் உள்ள பல்பொருள் அங்காடியில் பகுதி நேர விற்பனை உதவியாளராகவும் இருக்கிறார். இங்குள்ள அவரது சகபணியாளர்கள் மற்றும் மேலாளர்களுடன் பணிநேரம் தாண்டிய சமயங்களில் வாட்சப் மூலம் தொடர்பில் இருக்கிறார். சகாயம் இவர்களை வாட்சப் குழுவினர் என்று டிஜிட்டல் முறையில் வகைப்படுத்தாவிட்டாலும் வேடிக்கையான மற்றும் சமூகத்திற்கு பொருத்தமான மீம்களை தன்னுடைய தேர்ந்தெடுத்த சகபணியாளர்கள் வலைதொடர்பிற்கு தொடர்ந்து முன்னனுப்புகிறார் (படம்: 3.34).

ஜோத்ஸ்னா மற்றும் சகாயம் ஆகியோரின் சுயவிவரங்கள் அவர்களுடைய வலைத்தொடர்புகள் உடனான 70 சதவிகிதத்திற்கும் மேற்பட்ட தகவல் பரிமாற்றங்கள் காட்சியமைப்புகளாக இருப்பதை வெளிப்படுத்துகின்றன. இதில் முக்கியமான விஷயம் என்னவென்றால், அவர்களது காட்சியமைப்பு தகவல் பரிமாற்றத்தின் அடித்தளத்தில் காணப்படும் பாணி தான். இவர்களின் காட்சியமைப்பு தகவல் பரிமாற்றங்களின் வகை- 


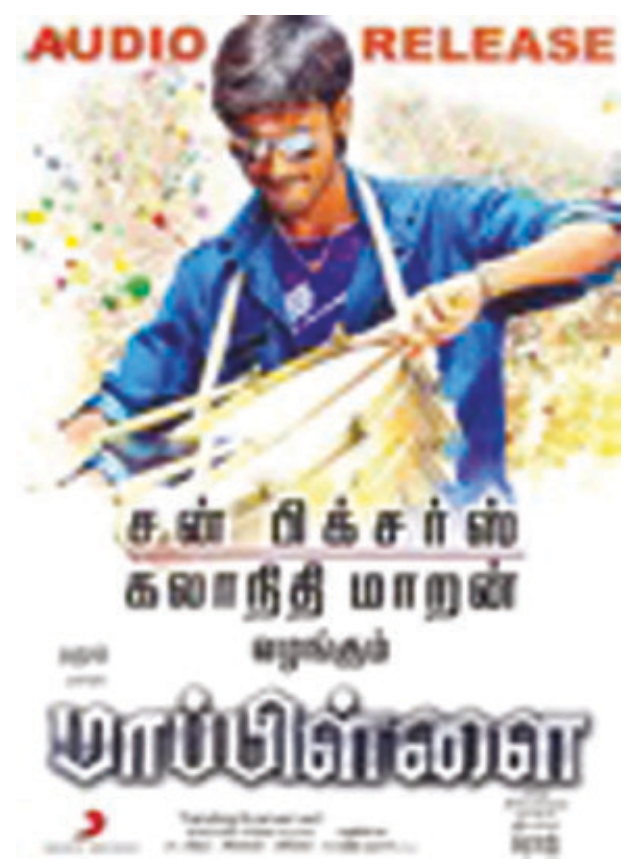

படம்: 3.33 கலவையானவை - அபிமான திரைநட்சத்திரம் தனுஷ் - ஒரு ரசிகரின் முகநூல் பக்கத்திலிருந்து

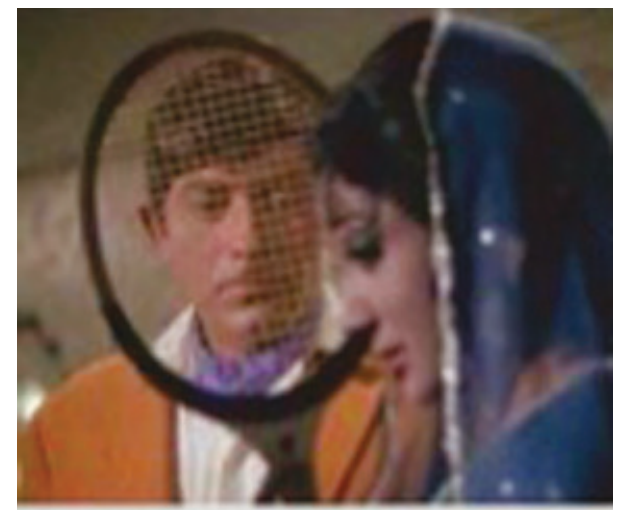

Net chatting in 1970

படம்: 3.34 கலவையானவை - சகபணியாளர்களுக்கு முன்னனுப்பிய வேடிக்கை மீம் 
யிலக்கணம் அவர்கள் தகவல் பரிமாறிக்கொள்ளும் வலைதொடர்பிற்கு ஏற்றவகையில் மாறுகிறது. ேோத்ஸ்னாவின் சர்ச் குழுவிற்கான தகவல்கள் அவர் குடியிருப்புவாசிகளுடனும் நெருங்கிய நண்பர்களுடனும் பரிமாறிக்கொள்ளும் தகவல்களில் இருந்து முற்றிலும் வித்தியாசப்படுகிறது. அவர், யேசுகிறிஸ்துவின் படங்களை, குடியிருப்புவாசிகள் குழுவிற்கு அனுப்புவதில்லை என்பதில் தெளிவாக இருந்தார். ஏனெனில், அந்தக்குழுவில் மற்ற சமயங்களை சேர்ந்தவர்களும் உறுப்பினர்களாக இருந்தனர். அவருடைய நெருங்கிய நண்பர்கள் குழுவிலும் இதே கதை தான். அதிலுள்ளவர்களில் இருவர் இந்துசமயத்தை சேர்ந்தவர்கள். ஆகவே, இந்தக் குழுக்களில் சமயம் சார்ந்த பதிவுகள் சேர்க்கப்படாமல் அவர் கவனமாக இருந்தார். அவருடைய நண்பர்கள் குழுவிற்கு நேர்மறையான தகவல்களை அனுப்புவது சரிதான். ஆனால் குடியிருப்புவாசிகள் குழுவிற்கும் சர்ச் குழுவிற்கும் அவரது சமையல்பதார்த்தங்களின் படங்களை அனுப்புவது சரியானதல்ல. அவர் அவ்வாறு செய்யவும் மாட்டார். ஏனெனில் இது அந்தக் குழுக்களின் எதிர்பார்ப்பு கிடையாது. அவருடைய நெருங்கிய நண்பர்களின் குழுவில், உறுப்பினர்கள் குடும்ப புகைப்படங்களை பகிர்ந்து கொள்ளவேண்டும் என்று எதிர்பார்க்கப்படுகிறது. ஆகையால் அவர் அதற்கேற்ப படங்களை பகிர்ந்து கொள்கிறார்.

சகாயத்தின் வழக்கும் இது போன்றது தான். இயற்கை காட்சிகள் மற்றும் குழந்தைகளின் படங்களை பகிர்வது பரவலான விருப்புக்களையும் கருத்துகளையும் பெறும் என்று அவர் உணர்ந்தார். இந்தத் தகவல்கள் உரையாடலையும் ஊக்குவித்து இவரது சுயவிவர பக்கத்தை சுறுசுறுப்பாக வைத்திருந்தது. அவருடைய குறிப்பிட்ட நண்பர்கள் குழுவில் அவர் நடிகர் தனுஷின் படங்களை பகிர்ந்திருந்தால் இதுபோல் நடந்திருக்காது. அதேபோல நடிகர் தனுஷிற்கான ரசிகர்கள் பக்கத்தில், இயற்கை காட்சிகளையும் குழந்தைகள் படங்களையும் பகிர்ந்திருந்தால் இணக்கமான பதில்கள் கிடைத்திருக்காது. அதேபோல, வேடிக்கையான மற்றும் சமூகத்திற்கு பொருத்தமான மீம்களை தனது சகபணியாளர்களுக்கு அனுப்புவது நேர்மறையான பதில்கள் வாட்சப்பிலும் அவர்களை நேரில் சந்திக்கும் சமயங்களிலும் கிடைக்கும் என்று சகாயம் எண்ணினார்.

ஜோத்ஸ்னா மற்றும் சகாயம் ஆகியோரின் வழக்கில் முக்கியமான விஷயம் என்னவென்றால், அவர்களது காட்சியமைப்பு தகவல் பரிமாற்றத்தின் வகையிலக்கணங்கள் 
வெவ்வேறு வ வலைத்தொடர்புகளுக்கு வெவ்வேறுமாதிரியாக இருந்தன. ஒவ்வொன்றும் அந்தந்த வலைத்தொடர்பின் எதிர்பார்ப்பிற்கு ஏற்றவகையில் கவனமாக வடிவமைக்கப்பட்டிருந்தன. இதுபோன்ற மாற்றங்கள், அவர்கள் தகவல் பரிமாறிக்கொள்ளும் வலைத்தொடர்புகளில், அதன் அளவைப் பற்றிய பொருட்டில்லாமல் தாக்கத்தை ஏற்படுத்தும் வகையில் சாமர்த்தியமாக செய்யப்படுகின்றன.

ஜோத்ஸ்னாவின் வழக்கில், இல்லம்சார்ந்தவை மற்றும் இடைப்பட்டவை ஆகிய காட்சியமைப்புகளின் ஒன்றன்மேல் ஒன்றான கவிந்திருக்கை காணப்பட்டது. சகாயத்தின் வழக்கில் இதுபோன்ற கவிந்திருக்கை, பொதுப்பார்வைக்குரியவை மற்றும் இடைப்பட்டவை ஆகிய காட்சியமைப்புகளில் காணப்பட்டது. அவர்கள் இருவரின் ஊடகப்பக்கங்களும், பஞ்சக்கிராமியில் உள்ள சமூக ஊடக பக்கங்கள், எவ்வாறு படங்களின் வகைகளை ஒன்றிணைத்து, இந்த மூன்று வகையிலக்கணங்களின் கலவையை உருவாக்குகின்றன என்பதற்கான இயல்பான எடுத்துக்காட்டாக இருந்தன.

\section{முடிவுரை:}

இந்த அத்தியாயத்தின் நோக்கமே, நிகழ்நிலை மற்றும் இயல்புநிலை இடப்பரப்புகளுக்கு இடையே, ஒரு தொடர்ச்சிநிலை இருக்கிறது என்பதையும், ஒன்று மற்றொன்றை பல்வேறு வகைகளில் பாதிக்கிறது என்பதையும், இரண்டில் எதையும் மக்கள் வாழ்வில் தனித்தனி கூறுகளாக ஆய்வு செய்ய முடியாது என்பதையும் எடுத்துக்காட்டுவது தான். பிரபலமான தமிழ்நாட்டுக் கலாச்சாரத்தில், காட்சிக்குரிய பொருட்கள் எப்போதுமே முக்கியப்பங்கு வகித்திருக்கின்றன. இந்த பழக்கவழக்கங்கள், மக்களின் சமூக ஊடக தகவல் பரிமாற்றத்தில் ${ }^{33}$ பெரும் தாக்கத்தை ஏற்படுத்தியிருக்கின்றன. இது இந்த அத்தியாயத்தில் எண்ணச்சார்பு பற்றிய வலியுறுத்தலுக்கு வழிவகுத்திருக்கிறது.

இந்த அத்தியாயம், பஞ்சக்கிராமியில் அடிக்கடி நிகழும் காட்சியமைப்புகள் வகையிலக்கணங்களை அலசியிருக்கிறது. அவையாவன, திரைப்படம், அரசியல், தான் எனும் தன்மை மற்றும் அன்றாட வாழ்த்துச்செய்திகள் பற்றிய காட்சிப் பதிவுகள். இவை, பொதுவட்டங்கள், இல்லங்கள் மற்றும் இடைப்பட்டவை ஆகியவற்றுடன் இணைந்துள்ள காட்சியமைப்பு கலாச்சாரத்துடன் ஒ ஒப்பிடப்பட்டுள்ளன. சமூக 
ஊடக காட்சியமைப்பு இடப்பரப்பு இயல்புநிலை கலாசார சீர்மைகளை வெளிப்படுத்தவும் ஒ ஒதுக்கீடு செய்யப்பட்டுள்ளதையும் நாம் கண்டோம்.

எடுத்த எடுப்பில், நாம் மக்களின் தனிப்பட்ட தன்மைகளை அல்லாமல், சமூக ஊடகங்களில் காட்டப்பட்டுள்ள வகையிலான மக்களின் பிம்பங்களை மட்டுமே கூர்நோக்குவதைப் போல் தோன்றக்கூடும். எனினும், பஞ்சக்கிராமி போன்ற ஒரு சமூகத்தில், அடிப்படை அளவில், இந்த சமூக ஊடக உபயோகிப்பாளர்களுக்கு பொதுவானது எது, தனிப்பட்டது எது என்ற கேள்வி எழுகிறது. நாம் மேற்கண்ட பெரும்பாலான நிகழ்நிலை மற்றும் இயல்புநிலை தகவல் பரிமாற்றங்கள் எல்லாமே, உபயோகிப்பாளர்கள், பல்வேறு அளவு மற்றும் வகையிலான தங்கள் வலைத்தொடர்புகளுடன் பரிமாறிக்கொண்டவை தான். இது போன்ற தகவல் பரிமாற்றங்களின் நோக்கமே, அவர்கள் சார்ந்துள்ள வலைத்தொடர்புகளின் எதிர்பார்ப்புகளுடன் ஒ ஒத்துப்போவது தான். அந்தரங்கம் என்பது சார்புடையது. முழுமையான அந்தரங்கம் ஒரு தனிநபருக்கும் அவரது எண்ணங்களுக்கும் இடையே மட்டுமே இருக்கக்கூடியது. இருப்பினும், மிகவும் நெருங்கிய உறவினர் மற்றும் நண்பர்கள் வட்டத்திற்கான தகவல் பரிமாற்றங்களுக்கும் மற்ற வட்டங்களுக்கான தகவல் பரிமாற்றத்திற்கும் இடையே முக்கிய வித்தியாசங்கள் இ இருக்கின்றன. இவை அளவீட்டு சமுதாயம் ${ }^{64,65}$ பற்றிய கதிர்நிரலை கலந்து பேசி முடிவு செய்கின்றன. உறுப்பினர்களுக்குள் வலுவான நம்பிக்கை இருக்கக்கூடிய நெருக்கமான குழுக்களில், மிகக்குறைந்த அளவிலான சுய தணிக்கையே தேவைப்படு|கிறது. பரந்த வட்டங்களில் மக்கள் அனுசரித்துப் 66 போக வேண்டியிருக்கிறது. இல்லையேல் எதிர்ப்பு தெரிவிப்பதற்காக பொய்யான கணக்குகள் உருவாக்குதல் போன்ற மாற்று உத்திகளை யோசிக்க வேண்டியிருக்கிறது.

உதாரணத்திற்கு, யாராவது, தங்களின் ஊடகப்பக்கங்களில், தங்களது சுய-புகைப்படங்களையோ அல்லது தங்களது வேறுபடங்களையோ பதிவிட்டால், இந்தப்படங்கள் அதீத தற்காதல் தன்மையுள்ளவை என்பது போன்ற நேரடியான விமர்சனங்களை கருத்துப் பகுதியில் பார்ப்பது மிகவும் அபூர்வம். இதுபோன்ற வழக்குகளில் எதிர்ப்பு தெரிவிப்பது என்பது எந்த கருத்தோ/விருப்போ தெரிவிக்காமல் அமைதியாக இருப்பதன் மூலம் வெளிப்படுத்தப்படுகிறது. நெருக்கமான நண்பர்கள், தமது அபிப்ராயத்தை வேறுவகையான தகவல் பரிமாற்ற வழிகள் மூலம் தெரிவிக்கக்- 
கூடும். ஆனால் இதுபோன்ற கருத்துக்கள் தெரிவிப்பதற்கு முன், இயல்புநிலையில் உள்ள அவர்களது உறவுமுறையின் தன்மை மிகவும் முக்கியமானது.

இது பலசமயங்களில், திரைப்படம் மற்றும் அரசியல் தொடர்பான பொதுப்பார்வைக்குரிய பதிவுகளிலும் காணப்படுகிறது. மீண்டும், இவையெல்லாம், உபயோகிப்பாளர்கள் அவர்களின் சமூக ஊடகத்தளங்களில் பேணும் வலைத்தொடர்புகள் சம்பந்தப்பட்டவை. ஒருவர் அவரது அபிமான திரைநட்சத்திரத்தின் படத்தை பதிவிட்டால், அவரின் வலைதொடர்பில் இருக்கும் வேறு நடிகரை விரும்புபவர்கள் கூட அதை நேரடியாக எதிர்க்க மாட்டார்கள். இதனால், அவரின் வலைதொடர்பில் இருப்பவர்கள் எதிர்மறையான கருத்துக்களே தெரிவிக்க மாட்டார்கள் என்பது இல்லை. அதுபோன்ற கருத்துக்கள் தெரிவிக்கப்படும் போது மிகவும் பாதுகாப்பான வட்டத்துள் இருந்துகொண்டு தெரிவிக்கப்படுகின்றன.

அதேபோல, பாலியல் படங்கள்/காணொளிகள் போன்ற சர்ச்சைக்குரிய காட்சிப்பொருட்களை யாரும் பதிவிடும் சமயங்களில், அவருடைய தொடர்புகள், வெளிப்படையாக எதிர்ப்பு தெரிவிப்பதை விட, அவர்களுடனான உறவை, அவர்களை நட்பு ரத்து செய்வதன் மூலம் துண்டித்துக்கொண்டு விடுகிறார்கள். இருப்பினும், முகநூலைவிட வாட்சப்பில், நண்பர்களின் அந்தரங்க வட்டத்துள், பாலியல் படங்கள்/காணொளிகள் பகிர்ந்துகொள்ளப்பட்ட வழக்குகளும் இருக்கின்றன. மீண்டும், இந்த உதாரணங்கள் அனைத்துமே, தனிநபர்கள் சார்ந்துள்ள வலைத்தொடர்புக்கு ஒத்ததாக இருப்பவையே.

எனினும், இந்த வெளிப்பாடுகள், நிகழ்நிலையால் மட்டுமே காணப்படுபவை அல்ல. இவை, இந்த அத்தியாயத்தில் முன்பே குறிப்பிட்டுள்ளபடி, இயல்புநிலையில் சமூகத்தொடர்புகளுக்கிடையே சர்ச்சைகளை தவிர்ப்பதற்காக அனுசரித்தலை ஊக்குவிக்கும் பழக்கவழக்கங்களின் முந்தைய பாணிகளுடன் வலுவான தொடர்புடையவை.

ஆகவே, பொது மற்றும் தனிப்பட்டவை பற்றிய பொருட்டோ அல்லது வலைத்தொடர்புகளின் அளவு பற்றிய பொருட்டோ இல்லாமல், வலைத்தொடர்புகளின் ஆதிக்கம் நிறைந்த எதிர்பார்ப்புகள், பஞ்சக்கிராமியின் காட்சியமைப்பு தகவல் பரிமாற்றத்தை பாதிக்கின்றன. பலசமயங்களில், மக்கள் இந்த எதிர்பார்ப்புகளுக்கு அனுசரித்துப்போவது போல் காணப்படுவைதே விரும்புகிறார்கள். மீம் வடிவம் எடுக்கும் அன்றாட வாழ்த்துக்கள் போன்ற இடைப்பட்ட காட்சியமைப்புகள் கூட, பரந்த வலைதொடர்பின் தரநெறிசார் 
எதிர்பார்ப்புகளுக்கு அனுசரித்துப்போக தலைப்படுகின்றன. நிகழ்நிலை சமூகத்தன்மையை பேணுவதில் அவர்கள், பஞ்சக்கிராமியின் அன்றாட இயல்புநிலை கலாச்சாரத்துடன் தொடர்புடைய சமூகத்தன்மையை மறு-வலியுறுத்துவதோடு, மறைமுக மரபு கண்காணிப்பாளர்களாகவும் செயல்படுகிறார்கள்.

ஒருவகையில், இயல்புநிலை இடப்பரப்பில் உள்ள பாரம்பரியமான காட்சியமைப்பு வெளிப்பாடுகளுக்கும், நிகழ்நிலையில் உள்ளவற்றிற்கும் இடையே உள்ள கணிசமான எண்ணச்சார்பை, இந்த அதத்தியாயம் எடுத்துக்காட்டியுள்ளது. இருப்பினும், நிகழ்நிலை காட்சிப்பதிவுகளுக்கு, அவை உத்தேசிக்கப்பட்ட அங்கத்தினர், மற்றும் அவற்றிற்கு தேவையான அந்தரங்கம் போன்ற கூடுதல் விசாரங்கள் இருக்கின்றன. இது, அடுத்துவரும் அத்தியாயங்களில் கலந்தாய்வு செய்யப்படும் சில விசாரங்களுக்கு வழிவகுத்துக்கொடுக்கிறது. அத்தியாயம் 4, குடும்பம் மற்றும் நெருங்கிய நட்புறவுகளின் தனிப்பட்ட களத்தைப் பற்றியதாக இருக்கிறது. பின்வரும் மற்ற அத்தியாயங்கள் மேலும் பரந்த மற்றும் பொதுவான விஷயங்களை கருதுகின்றன, குறிப்பாக கல்வி மற்றும் பணி ஆகியவை. 


\section{4 \\ உறவுகள்: சமூக ஊடகத்தில் சுற்றம்}

டிசம்பர் 2013 ல் ஒரு அமைதியான மதியப் பொழுதில், கோவிந்தன், ஒரு 33 வயது ஹார்ட்வேர் பொருட்கள் வியாபாரி, பஞ்சகிராமியில் உள்ள அவரது 200 சதுர அடி அளவுள்ள இட நெருக்கடி மிகுந்த கடையில் தேநீர் அருந்திக் கொண்டே தனது ஃபேஸ்புக் புரொஃபைலைப் பற்றி விவாதிக்க என்னை அழைத்திருந்தார். விரைவிலேயே பேச்சு ஃபேஸ்புக் 'நண்பர்களைப்' பற்றி திரும்பியது ((அந்த நேர்காணலின் போதே ஏறக்குறைய 120 பேர் இருந்தனர்). அவர் சீக்கிரமே தனது ஃபேஸ்புக் 'நண்பர்களைப்' பற்றி 'மாமா', 'அண்ணன்', 'மச்சான்', ‘சிஸ்டர்', 'ப்ரோ' சொந்தங்கள் பற்றிய வார்த்தைகளில் அல்லது 'சார்' அல்லது 'மேடம்' போன்ற பணிவிணக்கம் குறித்த வார்த்தைகளில் குறிப்பிட ஆரம்பித்தார்; ஆனால் பின்னால் உள்ள சொற்கள் குறைவாகவே உபயோகப்படுத்தப்பட்டன. முதல் பார்வையில், கோவிந்தனின் குடும்பத்தில் பெரும்பான்மையினர் (அவரது விரிநிலைக் குடும்பமும் சேர்ந்து) ஆன்லைனில் இருப்பது போல் தெரிந்தது. ஆனால், விரைவிலேயே, அவர் சொந்தம் கொண்டாடிக் கொண்டிருக்கும் குழு, அவரது விரிநிலை குடும்பம் மட்டுமில்லாமல், அவரால்கற்பனைச் சொந்தம் சொல்லி அழைக்கப்படும் அவரது பல நண்பர்களின் சேர்க்கையும்தான் என்பது விரைவிலேயேத் தெளிவாயிற்று.

அவர் குறைந்த பட்சம் தனது 17 நண்பர்களையாவது 'மச்சான்' என்று அழைத்தாலும், கோவிந்தனுக்கு ஒரு உண்மையான மச்சான்தான் இருந்தார், ஆனால் அவர் ஃபேஸ்புக்கில் இல்லை. அவர் ஏறக்குறைய தனது ஒன்பது நண்பர்களை 'பங்காளி' என்று அழைத்தாலும், அவருக்கு ஒரு பங்காளி கூட இல்லை. இவ்வாறுதான் அவரது 'சகோத- 
ரிகளை'2 வகைப்படுத்துவதும், (சொந்த சகோதரிகள், இவர்களுடன் ஒன்று விட்ட சகோதரிகள் ${ }^{3}$, மற்றும் அவரது பெண் நண்பர்கள்) இன்னொரு பயிற்சியாக இருந்தது. ஆனால் அவர் தன்னுடன் தொழில்முறை சம்பந்தம் உள்ளவர்களையும், தன்னை விட உயர்ந்த சமூகப் பொருளாதார வகுப்பில் இருப்பவர்களை மட்டுமே 'சார்' அல்லது 'மேடம்' என்பது விரைவிலேயேத் தெளிவானது; மற்றவர்கள் அனைவரும் அவருடன் ஏதோ ஒரு வழியில் சொந்தமானவர் போன்றே அழைக்கப்பட்டார்கள். ${ }^{4}$

கோவிந்தனின் நடத்தை அந்த, இடத்தில் இருந்த, தொடர்புகளை உறவுமுறை ${ }^{5}$ சொல்லி அழைக்கும் பலரை ஒத்தே அமைந்திருந்தது. கோவிந்தனைப் போன்றவர்கள் தங்கள் தொடர்புகளைக் குறிப்பிடும் முறையை அறிந்திருந்தாலும், ஒரு வெளியாளிற்கு இது முதலில் அச்சுறுத்தலாகவும், குழப்பமானதாகவும் இருக்கக் கூடும். ${ }^{6}$

பஞ்சகிராமியின் சமூகக் கட்டமைப்புகளை அறிந்து கொள்ள, நமக்குப் பல அடுக்கு மக்களுக்குள்ளும், இடையேயும் உள்ள தொடர்புகளின் இயல்பையும், வடிவங்களையும் அறிந்து கொள்ளுதல் அவசியம். பலதரப்பட்ட தொடர்புகள் பஞ்சகிராமியில் ஆட்சி செய்கின்றன, அவை குடிம்பத்திற்கு உள்ளேயாகட்டும், விரிநிலைக் குடும்பங்களுக்கு இடையேயாகட்டும் அல்லது சாதிக்குழுகளுக்கு உள்ளேயாகட்டும். இவ்வாறே, இந்தத் தொடர்புகள் சமூக ஊடகங்களுள் குடிபெயர்கின்றன என்பது இந்தமண்டலத்தில் சமூக ஊடகத்தின் பயன்களையும், விளைவுகளையும் புரிந்து கொள்ள நடிநாயகமானது.

பஞ்சகிராமியில் அடிப்படை சமூகக் குழு ஒரு குடும்பமாகவோ அல்லது சாதியாகவோ, ஒரு அமைப்பகவோ அல்லது நிறுவனமாகவோ, ஒரு சுற்றுப்புறமாகவோ அல்லது குடியிருப்பு வளாகமாகவோ இருக்கலாம், இந்த ஒவ்வொரு சமூகக் குழுவும், தனக்குச் சொந்தமான தொடர்பு வடிவங்களைக் கொணர்கின்றன (உறவு, பணியளிப்பவர்-பணிபுரிபவர், இணைநிலையினர், நட்பு, காதல் தொடர்புகள் போன்றவை.), சில படிநிலை மற்றும் அதிகாரத்தால் ஆதிக்கம் செலுத்தப் படுகின்றன, மற்றும் சில சமநோக்கு இயல்பை உடையவனவாக இருக்கின்றன. நாம் இவ்வாறு பிணைந்திருக்கும் தொடர்புகளின் சிக்கலான பாங்குகளைப் புரிந்து கொள்ள வேண்டிம் என்றால், மேற்கூறிய உதாரணத்தைப் பின்பற்றுதலே மேன்மையானது. இது பெரும்பான்மையானத் தொடர்புகளின் மீது ஆதிக்கம் செலுத்தும் மரபுச் சொற்கள் மிகவும் 
அடிப்படை நிலையிலேயே, அதாவது உறவுமுறையிலேயே, எவ்வாறு ஆரம்பிக்கின்றன என்பதைக் காட்டுகிறது.

ஆகையால், இந்த அத்தியாயம், உறவு என்று பரந்த அளவில் வகைப் படுத்தக்கூடிய தொடர்புகளைப் பற்றிப் பேசுகிறது. அத்தியாயம் 5 அலுவலக இடங்களில், மேலும் அத்தியாயம் 6,கல்வி நிறுவனங்களிலும், குறிப்பாகப் பள்ளிகளில் உள்ளத் தொடர்புகளைப் பற்றி விவாதிக்கிறது. ஆனால், நாம் கோவிந்தனின் உதாரணத்தில் பார்த்ததைப் போலவே பஞ்சகிராமியில் அது கற்பனை உறவு வடிவத்தில் இணைத்துக் கொள்ளப்படுவெதால், இந்த அத்தியாயத்தில் நட்பையும் உள்சேர்த்துக் கொள்ள வேண்டும். உறவு வட்டங்களுக்குள் சமூக ஊடகங்களின் பயன்பாடு இவ்வாறு இந்த அத்தியாயத்தின் மையப் பொருளாக இருக்கிறது.

\section{பஞ்சகிராமி உறவுமுறைகளின் ஒரு அறிமுகம்}

நாம் 1ம் அத்தியாயத்தில் பார்த்ததைப் போலவே, பஞ்சகிராமியின் உறவு முறை, ஆணாதிக்க ${ }^{8}$ வகையைச் சேர்ந்தது; அது தனி அல்லது விரிவுநிலைக் குடும்பம் என்று வகைப்படுத்தப் படலாம். பஞ்சகிராமியின் ஒரு வழக்கமான தனிக் குடும்பத்தில் நான்கு அல்லது ஐந்து உறுப்பினர்கள் இருக்கிறார்கள், கணவன், மனைவி, இரண்டு அல்லது மூன்று குழந்தைகள். ஆனால் இந்த மூல அலகு பல காரணிகளைப் பொறுத்து மாறுகிறது, உதாரணத்திற்கு, குழந்தைகளின் திருமண நிலை அல்லது கணவரின் நெருங்கிய உறவுகள் (விதவைச் சகோதரி அல்லது தாய் போன்றவர்) குடும்பத்துடன் வசித்தல் ஆகியவை. பெரும்பாலும், ஒருதனிக் குடும்ப அமைப்பு ஒரு விரிவுநிலைக் குடும்பத்தின் அடிப்படையே, பின்னவற்றில், தாத்தா, பாட்டி, சித்தப்பா மற்றும் சித்தி போன்றவர்கள் ஒரே வீட்டில் ஒன்றாகவே வாழ்வார்கள். ${ }^{10}$

தெற்காசிய உறவுகளின் பாரம்பரிய முறையைப் பாதுகாத்து வரும் பஞ்சகிராமியின் நீண்ட நாள் வாசிகளுக்கு நடிவில் இந்த மாதிரியான அமைப்பு வெளிப்படையாக அமைந்துள்ளது. ${ }^{11}$ கணேஷின் உதாரணத்தை எடுத்துக் கொள்ளலாம், இவர் 23 வயதான பஞ்சகிராமி வாசி, ஒரு கல்லூரி மாணவர், அவர் தன் பெற்றோர், தந்தை வழி விதவை சித்தி பாட்டி, இரண்டு மணமாகாத தங்கைகள், மற்றும் மூன்று சகோதரர்கள் (அனைவரும் மூத்தவர்கள் மற்றும் திருமணமானவர்கள்), மேலும் அவர்களது குடிம்பங்கள் ஆகியவர்களுடன் ஒரு தனி 
வீட்டில் (அபார்ட்மென்ட் அல்ல) வசித்து வருகிறார். இந்த உயர் மத்தியவகுப்பு என வகை செய்யப்படக் கூடிய15 உறுப்பினர்களை உடைய குடும்பம் ஒரே கூரையின் கீழ் வசிக்கிறது, ஒரு பெரிய சமையலறையில் உணவு தயாரிக்கிறது.

இன்னொரு உதாரணம், சங்கீதா, இவர் ஒரு மகனுக்கும், மகளுக்கும் தாயாயுள்ள 33 வயது இ இல்லத்தரசி. இவர் தன் கணவர் கங்காதரன் மற்றும் புகுந்த வீட்டாருடன் பஞ்சக்கிராமியில் வசிக்கிறார். இந்தக் குடும்பம் எளிமையான ஒரு படுக்கையறை உள்ள வீட்டில் வசிக்கிறது, இந்தக் குடும்பத்தை கீழ்நிலை சமூகப் பொருளாதார வகுப்பைச் சேர்ந்தது என வகைப்படுத்த முடியும்.

இதனால் தாய், தந்தை மற்றும் குழந்தைகளை உடைய 'வழக்கமான' தனிக் குடும்பங்கள் பஞ்சக்கிராமியில் இல்லை என்று சொல்லி விட முடியாது. மாத்யூ, ஒரு 30 வயது திருமணமான பிளம்பர், தன்னுடைய மனைவி மற்றும் ஒரு வயது மகளுடன் ஒரு வாடகை வீட்டில், தன் சகோதரர் வீட்டிற்கு அருகிலேயே, வசிக்கிறார். மாத்யூ இரண்டு வருடங்களுக்கு முன், தனது திருமணமான பொழுது, இந்த இடத்திற்குக் குடி புகுந்தார். இரு சகோதரர்களும் அவர்களின் குடும்பங்களும் தினமும் ஒருவரையொருவர் சந்தித்தாலும், அவரவர் குடிம்பங்களிலேயே சமையல் தனியாக நடக்கிறது.

நீண்ட நாள் வாசிகள் (நடுத்தர மற்றும் கீழ்நிலை சமூக மற்றும் பொருளாதார வகுப்பைச் சேர்ந்தவர்கள்), கூட்டுக் குடிம்ப அமைப்பில் வாழ்ந்தாலும், அல்லது அவர்களது உறவினர்கள் அருகில் வசித்தாலும், கீழ் நிலை சமூகப் பொருளாதார வகுப்பைச் சேர்ந்த புலம் பெயர்ந்தோர் மத்தியில் உறவுகள் அருகில் இல்லாத மாதிரி தனிக்குடும்பங்கள் காணப்படுகின்றன.

மத்திய வகுப்புக் குடும்பங்களைக் காணும் பொழுது, ஐடி துறையில் உள்ள ஒரு சராசரி தொழிற்பண்பட்டவர், மற்ற தொழில் துறைகளைச் சார்ந்த அவரது எதிரிணையாளர்களை விட அதிகம் சம்பாதிக்கிறார் என இருக்கும் பட்சத்தில், இந்த பல அடுக்கு குடியிருப்புக் கட்டிடங்களில் வசிப்பவர்கள்தான், பஞ்சகிராமியின் புதிய நடுத்தர வர்க்கத்தினர் ${ }^{33}$ ஆவார்கள். பல அ அபார்ட்மென்ட் வளாகக் குடியிருப்புவாசிகளின் குடும்ப முறையை தனிக்குடும்பம் என பலர் வரையறுக்க முற்பட்டாலும், இவர்களின் குடும்ப முறை ${ }^{14}$ முழுமையாகத் தனியாகவோ அல்லது பூரணமான கூட்டாகவோ இல்லாத ஒரு தனிப் பாங்கிற்கு மாறிக் கொண்டிருக்கிறது என்பது தெளிவாகத் தெரிகிறது. இந்த அபார்ட்மென்ட் வளாகத்தில் வசிக்கும் 
பெரும்பான்மையான மத்திய வகுப்புக் குடும்பங்கள், தனி அலகுகளாகப் பார்க்கப்படும் போது தனிக் குடும்பங்களாகத்தான் தெரிகின்றன. ஆனால் பெற்றோரும், உடன் பிறந்தோரும் ஒரே வளாகத்திலுள்ள அபார்ட்மென்டுகளில் 15 முதலீடு செய்யும் போக்கு இப்பொழுது பரவலாகக் காணப்படுகிறது. உதாரணத்திற்கு, ஒரு திருமணமான மகளோ அல்லது மகனோ ஒரு குடியிருப்பு வளாகத்தில், ஒரு அபார்ட்மென்ட்டில் முதலீடு செய்தால், பெற்றோர்களோ அல்லது உடன் பிறந்தோர்களோ அதே வளாகத்தில் பக்கத்து பிளாக்கில் முதலீடு செய்யலாம். அவர்கள் அருகருகே உள்ள அபார்ட்மென்ட்டில் இருந்தால் ஒரே குடிம்பமாக சமையல் செய்ய முற்படலாம், அல்லது தேவைப்படும் பொழுது உண்வைப் பரிமாற்றம் செய்துக் கொள்ளலாம். ஒரு சில குடும்பங்களின் ஆணாதிக்க முறைகளைப் பொறுத்து மூத்த உறவினர்கள் தங்கள் மகன்களுடன் தங்கும் முறையும் காணப்படுகிறது. கூட்டுக் குடும்ப முறையின் இன்னொரு ஆர்வமூட்டும் பரிமாணம் பேரக் குழந்தைகளினால் ஏற்படுகின்றன, அவர்களுக்கு அம்மாதிரியான குடும்பங்களில் அதிக முன்னுரிமை அளிக்கப் படுகிறது. ஐடி தொழில் பண்பட்டவராகவோ, தொழில் முனைவராகவோ பெற்றோர்கள் வேலையில் இருக்கும் பொழுது, தாத்தா பாட்டிகள் தங்கள் பேரக் குழந்தைகளைப் பார்த்துக் கொள்கிறார்கள். இந்த ஏற்பாட்டிற்கு பாரம்பரிய கிராம வாழ்க்கையில் முன்னுதாரணங்கள் இல்லாமல் இல்லை. அங்கு ஒரு விரிவு நிலைக் குடும்ப உறுப்பினர்கள், வெவ்வேறு வீடுகளில் வசிப்பது போல் இருப்பார்கள், ஆனால் உண்மையில், ஒரு குறிப்பிட்ட வீட்டின் அடுப்பங்கரையில் உணவு தயாரிக்க அவர்கள் ஒன்றாகக் கூடுவர்.

சாந்தியின் உதாரணத்தைப் பாருங்கள். இவர் ஒரு 28 வயது, திருமணமான, ஐடி தொழிலாளி, இவரது கணவர் சுந்தரமும் ஐடி துறையில் பணி புரிபவர். இவர்கள் தனது ஐந்து வயது மகனுடன் பஞ்சக்கிராமியின் ஒரு பல மாடி அபார்ட்மென்ட் வளாகத்தின் பனிரெண்டாவது மாடியில் வசிக்கின்றனர். சாந்தி ஒரே மகள் என்பதால், அவரது பெற்றோரும் அதே அபார்ட்மென்ட் வளாகத்திற்கு குடி வந்து விட்டனர்; அவர்கள் அதே கட்டிடத்தின் எட்டாவது மாடியில் உள்ள ஒரு இரட்டைப் படுக்கையறை அபார்ட்மென்ட்டில் வசிக்கிறார்கள். இது அவர்களுக்கு, சாந்தியின் குடும்ப அந்தரங்கத்தில் தலையிடாமலேயே, தன் மகளுக்கு அருகில் இருக்கவும், தன் பேரனைப் பார்த்துக் கொள்ளவும் வழி வகுக்கிறது.

புவனா, 36 வயது ஐடி தொழிலாளியின் விஷயமும் இது மாதிரியானதுதான்; இவர் விஷயத்தில் இவரது புகுந்த 
வீட்டினர் இவரது அபார்ட்மென்ட்டிற்கு அருகிலுள்ள அபார்ட்மென்ட்டில் குடியேறி இருக்கின்றனர். சமையலை புவனாவின் மாமனார்-மாமியார் செய்கிறார்கள், உணவு புவனா வீட்டிற்கு அனுப்பப் படுகிறது அல்லது இரர்டு வீடுகளில் ஏதாவது ஒன்றில் ஒன்று சேர்ந்து உண்ணப் படுகிறது. பஞ்சகிராமியில் பல வீடுகள் இதே பாங்கில் அமைந்திருக்கின்றன; சமையல் ஒரு வீட்டில் செய்யப் படுகிறது (வழக்கமாகப் பெரியவர்கள் வாழும் வீட்டில்), இளைய தலைமுறை அவர்களுடன் உணவருந்துகிறது, அல்லது உணவுகட்டப்பட்டு அவர்களது வீட்டிற்கு உண்பதற்காக அனுப்பப் படுகிறது. ${ }^{16}$ அடுப்படிதான் ஒரு குடும்பத்தை வரையறுப்பது என்றால், மேலே விவரிக்கப் பட்டவை ஆர்வத்தைத் தூண்டுபவையாக உள்ளன. ஆரம்பத்தில் அவை இரு தனி வீடுகளில் வசிக்கும் இரண்டு தனிக்குடித்தனங்களாகத் தெரிந்தாலும், உண்மையில் அவை சுதந்திரமாக வாழும் ஒரு கூட்டுக் குடித்தனம்தான்.

ஆனால், தன்னுடன் எந்த உறவினரும் வசிக்காத, அல்லது உறவினர்கள் அருகில் வசிக்காத தனிக் குடித்தனங்கள் பஞ்சக்கிராமியில் இல்லவே இல்லை என்பதைச் சொல்வதற்காக இவை சொல்லப்படவில்லை என மீண்டும் ஒரு முறை சொல்லலாம். இதற்கு எதிராக, இந்த இடத்தில் ஐடி புலம் பெயர்ந்தோர் மற்றும் பிற முதலீட்டாளர்களின் அதிக வருகையால் அவைகள் மிகுந்துக் காணப்படுகின்றன. ரவீந்திரன், ஒரு 44 வயது ஐடி தொழில் முனைவோர், முழுமையான தனிக் குடும்பங்களின் வளர்ந்து வரும் படிமத்தின் பிரதிநிதியாக உள்ளார்.17 அவர் பஞ்சகிராமியில் ஒரு பெரிய அபார்ட்மென்ட் வளாகத்தில் தன் மனைவி மற்றும் பள்ளி செல்லும் மூன்று மகள்களுடன் ஒரு மூன்று படுக்கையறை அபார்ட்மென்ட்டில் வசிக்கிறார். அவரது சகோதரர் கோல்கத்தாவில் (இது இந்தியாவின் வடகிழக்கில் உள்ள ஒரு நகரம், முன்னர் கல்கட்டா என அழைக்கப்பட்டது.) வசிக்கிறார், இவரது சகோதரி, தன் கணவரின் இந்திய ராணுவ வேலை காரணமாக பஞ்சாபில் (இந்தியாவின் வடமேற்கில்) வசிக்கிறார்.

மதன் மற்றும் அவரது மனைவி பூர்வி, தனது பிந்தைய நாற்பதுகளில் இருக்கும் ஒரு நடுத்தர வயது தம்பதியினர், இதே மாதிரி நிலையில் உள்ளனர். இருவரும் தமக்கு சொந்தமான் ஒரு குடும்பத் தொழிலில் பணியாற்றுகிறார்கள், பூர்வியின் சகோதரி யூஎஸ்ஏவில் வசிக்கிறார், மதனின் சகோதரி ஆஸ்திரேலியாவில். இந்த தம்பதி தனது கோல்டன் ரிட்ரீவருடன் பஞ்சகிராமியில் ஒரு மூன்று படுக்கையறை அபார்ட்மென்ட்டில் வசிக்கின்றனர். 
மேற்கண்டவாறு, விரிவு நிலைக் குடும்பங்கள், ஒரு அபார்ட்மென்ட் வளாகத்திலோ அல்லது கிராமத்தில் தனி வீடுகளிலோ, அருகிலேயே வசிப்பதுதான் பஞ்சகிராமியில் காணப்பட்ட ஒரு முக்கியமான விசேஷ குணம், ஆனால், விரிவு நிலைக் குடும்பங்கள், திருமணம் அல்லது பிறப்பினால் பெற்ற அதிகக் குடும்ப உறுப்பினர்களுடன், ஒரு குறிப்பிட்ட இடத்தில் தனி வீடுகளில் வாழும் போது, அந்த இடம் குறிப்பிட்ட இடையுறவுள்ள சாதி சமூகத்தால் ஆதிக்கம் செலுத்தப்படுகிறது. ${ }^{18}$ உதாரணத்திற்கு பஞ்சகிராமியை உருவாக்கும் கிராமங்களில் ஒன்றில் நீண்டகால அசல் குடியிருப்போர் அனைவரும் உறவினர்களாக இருக்கின்றனர். அவர்கள் அனைவரும் ஒரே சாதிக் குழுவைச் சேர்ந்தவர்கள், தனது தொடக்கத்தை ஒரே சாதிக் குழுவைச் சேர்ந்த இந்தப் பகுதியின் 12 ஆரம்பக்கால குடியேற்றத்தார்களிடமிருந்தே அடையாளம் காண்கின்றனர். இந்த 12 குடியேற்றத்தார் ஒ ஒவ்வொருவரும் 'தலைக்கட்டு' அல்லது குடும்பத்தின் ஆண் தலைவர் என அழைக்கப்பட்டனர், இதைப்பற்றி முகவுரையிலேயேப் பார்க்கப்பட்டது. வாய்மொழி வரலாறு இந்தக் குழுவை அகமண உறவுடையக் குழு என ஆவணப்படுத்தினாலும், மற்றக் கிராமங்களில் இருந்து திருமண சம்பந்தங்கள் ஏற்பட்டே இருந்தன (சாதி அடிப்படை அகமண உறவுக்குட்பட்டே). ஆகையால் இப்பொழுது, 120 வருடங்களுக்குப் பிறகு, இந்த கிராமம் முழுவதிலும் உள்ள ஆதிகுடியினர் ஒருவரை ஒருவர் உறவுமுறை 19 இட்டே அழைக்கின்றனர், ஆனால் அனைவருக்கும் அவர்களின் உண்மை உறவுமுறை தெரியாத பொழுதும் கூட. உண்மையான உறவு முறைகளுக்குப் பதில் கற்பனை உறவு முறைச் சொற்களை பயன்படுத்துதல் இந்த இடத்தில் மிகுந்து காணப்படுகிறது.

இந்த இடத்தில் குடும்ப அமைப்புகளைப் புரிந்து கொள்ளுதல் நமக்கு பதிலுக்கு எவ்வாறு நிறுவனக் கோட்பாடுகளைப் புரிந்து கொள்ள உதவுகிறது என்பதைப் பார்க்கலாம். சாதிகளும், துணை சாதிகளும் குடும்பத்துடன் நெருங்கிய சொந்தத்தில் உள்ளன, அவைகள் உண்மையில் ஒரு விரிவு நிலை குடும்பம் போலக் கூட காட்சியளிக்கக் கூடும், ஏனென்றால் அதிக திருமணங்கள் அகமண உறவுடையவனவாகவே இருக்கின்றன. இந்த இடத்தின் இது போன்ற அகமண உறவு முறைகள் முதலில் ஒருவரின், உறவு மூலமாக பிணைக்கப்பட்ட குடும்பங்கள் உள்ள துணை சாதிக் குழுக்குள்ளேயே திருமணம் நடைபெறுவதை உறுதி செய்கின்றன. இது மேலும் ஒருவரின் பிறப்பிலிருந்தே அவ்வாறான அமைப்புடன் தொடர்பிலிருப்பது மூலம் தனது அடையாளத்தைப் 
பெறுவதின் மூலம் வலியுறுத்தப் படுகிறது. ஆகையால் ஒருவர் தனது கண்டுபிடிக்கக்கூடிய உறவுகளின் நெட்வொர்க்கை தன்னுடைய வம்சாவளி மூலமாக மட்டும் அல்லாமல், ஆனால் இரண்டு அல்லது மூன்று முறை நீக்கப்பட்ட அதிக தூரத்து சொந்தங்களிடமிருந்தும் ஒ ஒப்பிடலாம். அவ்வாறான அகமண உறவுடைய துணை சாதிக் குழுக்கள் இந்த இடத்தில் ஒரு சாதி சமூகத்தை உண்டாக்க முற்படுகின்றன. ${ }^{20}$

பஞ்சக்கிராமியில் தொடர்புகளின் பாங்கு மற்றும் சமூக ஊடகங்களைப் புரிந்து கொள்ள, ஒருவர் குடும்பத்துடன், வகுப்பு, சாதி, பாலினம் மற்றும் வயது ஆகியவற்றின் அயன் மகரந்தச் சேர்க்கையைக் கருத்தில் கொண்டு வர வேண்டும். ${ }^{21}$ குடும்பத்தை ஒரு பிரதானமான வகையாக உபயோகப்படுத்தும் பொழுது, நாம் தலைமுறைகளுக்கு இடையேயான தொடர்பில் கவனத்தை செலுத்தலாம் (பெற்றோர்-பிள்ளைகள்) மற்றும் தாத்தா, பாட்டி-பேரப் பிள்ளைகள்), ஜோடிகளுக்கு இடையேயான தொடர்பு, உடன்பிறந்தோருக்கு இடையேயான தொடர்பு, விரிவு நிலைக் குடும்பங்களுக்கு இடையேயானது மற்றும் கற்பனைச் சொந்தங்களாக நட்புகளுக்கிடையே உள்ள தொடர்பு ஆகியவை.

இந்த அத்தியாயத்தின் ஒரு முக்கிய பாகம், இந்த வெவ்வேறு தொடர்புகள் எவ்வாறு வேறு வேறு சமூக ஊடக தளங்களின் மேல் ஒப்பிடப்படுகின்றன என்பதைப் புரிந்து கொள்வதும், அவற்றுக்குள் உண்டாகும் சாத்தியக்கூறுகளை ஆராய்வதும்தான். ஒரு நெருங்கிய குடும்பத்திற்கு அல்லது ஒரு குறிப்பிட்ட சமூக நெட்வொர்க்கிற்குஉள்ளேயான தொடர்பு (விரிவு நிலைக் குடும்பம் அல்லது கற்பனை உறவு எதுவாக இருந்தாலும்), வழக்கமாகத் தனிமையை அளிக்கக்கூடிய அலைவரிசை மூலமாகவே ஏற்படுகிறது; இந்த மாதிரியான சந்தர்ப்பங்களில் ஃபேஸ்புக் ஒரு விரும்பக்கூடிய தளமே அல்ல. ஆனால், வாட்ஸ் அப் இவ்வாறான குடும்ப அடிப்படையான தனிப்பட்ட தொடர்புகளுக்கு இணங்கக்கூடிய தளமாகப் பார்க்கப்படுகிறது. ஃபேஸ்புக் ஏறக்குறைய ஒரு ஆர்ப்பாட்டமான தளமாகப் பார்க்கப் படுகிறது; பல்வேறு குடும்ப உறுப்பினர்களால் ஒரு குறிப்பிட்ட அளவு நிகழ்வு அங்கு நடத்தப் படுகிறது, ஆனால் இது எப்பொழுதும் வெளியுலகத்திற்காகவே நடத்தப் படுகிறது. இது பெற்றோர்-பிள்ளை அல்லது தாத்தா, பாட்டி-பேரப்பிள்ளை உறவுகள் விஷயத்திலேயே நடைபெறுகின்றது, மேலும் குறிப்பிட்ட முறையில் உயர் மத்திய வகுப்புக் குடும்பங்களிலேயே ஏற்படுகின்றது. இந்த வகுப்பு குடும்ப உறுப்பினர்கள் ஃபேஸ்புக்கில் இல்லாத காரணத்தி- 
னால், இது கீழ் மத்திய வகுப்பு மற்றும் குறைந்த வருமானச் சூழல்கள் விஷயத்தில் இவை உண்மையாக இல்லை.

\section{தலைமுறைகளுக்கு இடையேயான தொடர்பு}

பஞ்சகிராமியின் வாரிசுரிமையுள்ள உறவுகளைப் புரிந்து கொள்வதில் சமூக ஊடகத்தின் பங்கு மேலும் தொடர்பு முறைகளை மாற்றுவதில் அதன் செல்வாக்கு நமக்கு உறவுகளைப் பற்றிய ஒரு சிறந்தப் புரிதலை அளிக்கிறது. பஞ்சகிராமியின் ஒரு குடும்பத்தின் உள்ளே உள்ள ஒரு கருத்தைக் கவரும் பண்பு மொபைல் ஃபோனின் முக்கியமான பங்காற்றல் ஆகும். இந்த அத்தியாயம் தலைமுறைகளுக்கு இடையேயான தொடர்பின் கட்டமைப்பின் மீது சமூக ஊடகத்தின் பங்கை ஆராயும் முன் ஒரு குடும்பச் சூழலின் உள்ளே ஒரு மொபைல் ஃபோன் பற்றிய சுருக்கமான கருத்தை வழங்குகிறது.

குடும்பத்திற்கு உள்ளேயானத் தொடர்புக்கு மொபைல் ஃபோன் ${ }^{22}$ ஒரு பெரிய நெட்வொர்க்கிங் உபகரணம் ஆகும். இது பாரம்பரிய லேண்ட்லைன் தொலைபேசியை மிஞ்சியும், சில இடங்களில் அதன் இடத்தைப் பெற்றும் இருக்கிறது. மொபைல் ஃபோன்கள் குரல் மற்றும் எழுத்துத் தொடர்புகளை வழங்கினாலும், பஞ்சகிராமியின் பெரும்பாலான குடிம்பங்களின் பெரியவர்கள் தொலைபேசி பற்றிய தங்கள் கருத்தான பேசுவதற்கு மட்டுமான சாதனம் என்பதை தொடர்ந்து வைத்திருக்கிறார்கள். மேலும், இன்டர்நெட் மற்றும் நிறைந்த சமூக நெட்வொர்க்கிங் தளங்கள் போன்ற பல தொடர்பு மீடியாக்களின் இடையிலும் ஒரு குடும்பத்திற்கு உள்ளேயான தொடர்பு பெரும்பாலும் குரல் மூலமாகவே நடை பெறுகிறது. பெற்றோர்கள், முக்கியமாக தாய்மார்கள், தம் பிள்ளைகளிடமிருந்து எழுத்துச் செய்திகளைப் பெறுவதை விட அவர்கள் குரலைக் கேட்பதையே அதிகம் விரும்புகிறார்கள். குடும்ப உறுப்பினர்கள் ஒருவர் இன்னொருவருடன் செய்தி அனுப்புவதைக் காட்டிலும் அழைப்பதன் மூலமே ஒருவர் இன்னொருவருடன் தொடர்பு கொள்கிறார்கள். பிள்ளைகள் தங்களுடன் தொடர்பு கொள்ளும் பொழுது பிள்ளைகள் பின்பற்ற வேண்டிய தொடர்பு முறைகளைப் பற்றி வலியுறுத்தும் விதம் உபயோகப்படுத்த வேண்டிய ஊடகத்தை முடிவு செய்வதில் வயது எவ்வாறு படிநிலைக் கோட்பாடாக இயங்குகிறது என்பதற்கு செயல்முறை விளக்கமளிக்கிறது. கீழ் சமூக பொருளாதார வகுப்புகளில் கல்வித்தரம் மற்றும் எழுத்தறிவு திறமை மற்ற- 
வற்றைக் காட்டிலும் ஒரு குறிப்பிட்ட ஊடகத்தைத் தேர்வு செய்ய தாக்கத்தை ஏற்படுத்துகிறது. ஆயினும் குடும்பத் தொடர்புகளை முழுமையாகக் குரலுலகிலேயே வைத்திருத்தல் குடிம்பம் சார்ந்திருக்கும் சமூக பொருளாதார வகுப்பைப் பொறுத்து அமையவில்லை.

பஞ்சகிராமியில் ஒரு கீழ் சமூக பொருளாதார பின்புலத்தைச் சேர்ந்த ஒரு தனிக் குடும்பம், வழக்கமாக, மூன்று மொபைல் ஃபோன்களைக் கொண்டுள்ளது - பெற்றோர் இருவருக்கும் ஒவ்வொன்று, மற்றும் மகனுக்கு ஒன்று. மகள்களைப் பொதுவாக மொபைல் ஃபோனை உபயோகிக்க அனுமதிப்பதில்லை.

தாயால் உபயோகிக்கப்படிம் மொபைல் ஃபோன் பொதுவாக ஒரு லேண்ட்லைனைப் போல் உபயோகிக்கப் படுகிறது, அது மொத்தமாக குடும்பத்தினர் அனைவருக்கும் சொந்தமானது; அது ஒரு பகிரப்படும் பொருளாக மாறி விடுகிறது. தாயார் பொதுவாக இல்லத்தரசியாக ${ }^{23}$ இருக்கிறார் (சில நேரங்களில் அவர் தன் கணவருக்கு வருடங்களில் சில மாதங்கள் விவசாய வேலைகளில் உதவி செய்யலாம்). மகளுக்கு கல்லூரி செல்லும் வரையிலுமோ அல்லது வேலைக்குச் செல்லும் வரையிலுமோ மொபைல் ஃபோன் கொடுக்கப் படெவில்லை - அல்லது சில நேரங்களில் அவளுக்குத் திருமணம் ஆகும் வரையில். ${ }^{24}$ பஞ்சகிராமியில் திருமணம் அல்லது வேலை மட்டுமே மகள்களுக்கு மொபைல் ஃபோன் வைத்திருக்கத் தகுந்த அந்தஸ்தைக் கொடுக்கும் பல நிகழ்வுகள் நடந்துள்ளன. இந்தக் குடும்பத்து இளைஞர்கள் மேல் இம்மாதிரியான தடைகள் விதிக்கப் படிவதில்லை. ஒரு மாதிரிக் குடும்பத்தில், தொடர்பு தந்தை, தாய் மற்றும் மகனுக்கிடையில் மொபைல் ஃபோன் வாயிலாக நடக்கிறது; மகள் மற்றக் குடும்ப உறுப்பினர்களுடன் தன் தாயின் மொபைல் ஃபோன் வாயிலாகவே தொடர்பு கொள்கிறார், அத்தியாயம் 2ல் விவாதிக்கப்பட்ட ரவியின் குடிம்பத்தைப் போல. இவ்வாறு மொபைல் ஃபோன் குடும்பத்தின் பெண் உறுப்பினர்கள் மத்தியில் ஒரு பகிரப்பட்ட உபகரணமாகிறது. ஒரு மகள் தனது இருபது வயதுகளின் தொடக்கத்தில் கூட மொபைல் ஃபோனை உபயோகிப்பதில் தடையைச் சந்திக்க முடியும், ஆனால் மகன் தனது 15வது வயதிலேயே சொந்தமாக ஒரு ஃபோனைப் பெற முடியும். பாலின அடிப்படையில் இவ்வாறான தொடர்புச் சாதனங்களைப் பெறுவதில் வயது ஏ ஏற்றத்தாழ்வு பஞ்சகிராமியின் கீழ் மத்திய வகுப்பு மற்றும் கீழ் சமூகப் பொருளாதார வகுப்பில் சமூக ஊடகங்களின் பயன்பாட்டைப் பாதிக்கிறது. 
கீழே கொடுக்கப்பட்டுள்ள உறவுப் படம் பஞ்சகிராமியின் கீழ் சமூகப் பொருளாதார வகுப்பைச் சேர்ந்த மாதிரித் தனிக் குடும்பங்களில் மொபைல் ஃபோன் உபயோகத்தின் போக்கைக் குறிக்கிறது (படம் 4.1). இந்த விளக்கப்படத்தில் நீல முக்கோணம் ஆண்களைக் குறிக்கிறது, வெளிர் சிவப்பு வட்டம் பெண்களைக் குறிக்கிறது. 'சமம்'குறி திருமணத்தைக் குறிக்கிறது மற்றும் குறுக்கு, நெடுக்கு கோடுகள் குழந்தைகளைக் குறிக்கின்றன.

மீண்டும் அத்தியாயம் 2 ல், ரவியின் தாயின் விஷயம் மொபைல் ஃபோன் மற்றும் லேண்ட்லைன் இரண்டும் குரலுக்கு மட்டுமேயான ஊடகமாகக் கருதப்படும் பாங்கின் தொடர்ச்சியைக் குறிக்கிறது. பாலினத்தை அடிப்படையாகக் கொண்ட அவ்வித வேறுபாடுகள் (திருமணமாகாத இளம் பெண்களுக்கு மொபைல் ஃபோன் வைத்திருக்க அனுமதி இல்லாமல் இருக்குமிடத்தில்) சில நேரங்களில் கிராமங்களில் வசிக்கும் மத்தியவகுப்புக் குடும்பங்களிலும் (கீழ் மத்திய வகுப்புக் குடும்பங்களில் அதிகமாக) காணப்படுகின்றன. இங்கு சாதி அடிப்படையிலான மரபு குடும்பத்தை அந்த மாதிரியான முடிவுகளை எடுக்கக் கட்டுப் படுத்துகிறது.

கீழ் சமூகப் பொருளாதார வகுப்பு மற்றும் கீழ் மத்திய வகுப்பு குடும்பங்களில் மொபைல் ஃபோன் உபயோகத்தில் பாலின ஏற்றத்தாழ்வு இருந்தாலும், இம்மாதிரியான பாலின அடிப்படை வேறுபாடுகள், பெரிய அபார்ட்மென்ட் வளாக-

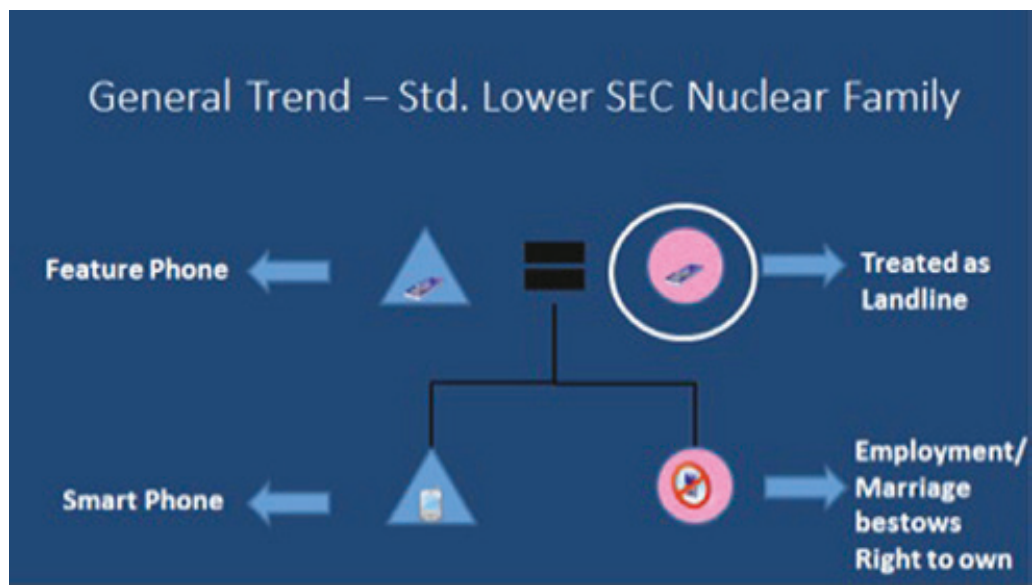

படம்: 4.1 கீழ்மட்ட சமூகப்பொருளாதார குடும்பத்தில் போன் வைத்திருப்பு 
ங்களில் குடியிருக்கும் மேல் மத்திய வகுப்புக் குடும்பங்களில் வெளித் தெரிவதில்லை.

பஞ்சகிராமியில் வாரிசுரிமை உள்ள உறவுகள் உணர்ச்சிப் பிணைப்புகளின் அழுத்தத்துடனேயே வருகின்றன. தம் குழந்தைகளின் நன்மையை உறுதி செய்யும் தொடர் தேவை முன்னுரிமைப் பெறுகிறது மேலும் முன்னெச்சரிக்கை மற்ற அனைத்து காரணிகளையும் விட முன்னிடம் பெறுகிறது - குறிப்பாக மகள்கள் விஷயத்தில், அத்தியாயம் 2ல் பார்த்த ஷோபனாவின் பெற்றோரைப் போல. ஷோபனா கிராமத்தின் ஒரு மத்திய வகுப்புக் குடிம்பத்தைச் சேர்ந்தவளாக இருந்தாலும், இதே விஷயம் பள்ளி செல்லும் வயதில் பிள்ளைகள் உள்ள மேல் வகுப்புக் குடும்பங்களுக்கும் இது பொருந்துகிறது.

உதாரணத்திற்கு, சுகீர்த்தி பஞ்சகிராமியின் ஒரு வளமான பன்னாட்டுப் பள்ளியில் ஒன்பதாவது வகுப்புப் படிக்கும் 14 வயது பெண். அவளுடைய பள்ளிக்குப்பின் செயல்களில் நீச்சல், சங்கீதம், கராத்தே டென்னிஸ் மற்றும் தனி அகாடமிக் ட்யூஷன் ஆகியவை அடங்கும். பெற்றோர் இருவரும் வேலைக்குச் செல்வதனால், இவள் ஒரு ஹோண்டா சிடி செடான் கார் மூலம் தமது ஓட்டுநரால் ஒரு ஆயாவுடன் கூட முன் மாலையில் இந்த வகுப்புகளுக்குக் கூட்டிச் செல்லப் படுகிறாள். அவளுடைய நிலையை எப்பொழுதும் அவளது பெற்றோருக்குத் தெரிவிப்பதற்காக, அவள் தான் இருக்குமிடம் பற்றி தன் பெற்றோருக்குத் தெரிவிப்பேன் என்று உறுதி அளித்ததின் பேரில், அவளுக்கு ஒரு சாம்சங் மொபைல் ஃபோன் பரிசளிக்கப் பட்டது. சென்ற வருடம் சுகீர்த்தியின் ஆயாவிற்கு அவளது பெற்றோர் தன் மகளின் அட்டவணையை அறிவதற்காக ஒரு நோக்கியா ஃஃசீச்சர் ஃபோனைப் பரிசளித்தனர். சுகீர்த்தி, இதனால் வெட்கமடைந்து, தம் அட்டவணையை தாமே கவனித்துக் கொள்வதாய் கூறினாள். அவளுடைய இயக்கத்தைக் கண்காணிப்பதற்காக, அவளுடைய பெற்றோர் சுகீர்த்திக்கு ஒரு ஸ்மார்ட்ஃபோனைப் பரிசளித்தனர், ஆனால் ஒரு நிபந்தனையின் பேரில், அதற்கு இன்டர்நெட் கனெக்ஷன் இருக்காது (3ஜி அல்லது 4ஜி). அந்த ஸ்மார்ட்ஃபோனின் முக்கிய நோக்கம் அவளுடையப் பெற்றோர்களை அழைப்பதுதான்; அவர்களால் பதிலளிக்க முடியவில்லை என்றால், அவள் பள்ளிக்குப் பிறகு எங்கிருக்கிறாள் என்பதை அறிவிக்க அவள் செய்தி அனுப்ப வேண்டும்.

குழந்தைகளின் நலனை உறுதிப்படுத்தும் வகையில் அவர்கள் குரலைக் கேட்பதில் கவனம் செலுத்தும் உணர்ச்சிப் 
பிணைப்பு பாலினத்தைப் பொறுத்து மாறுவது இல்லை; இது சிறிய பையனின் பெற்றோர்களிடையேவும் நடக்கிறது. ராகுலின் விஷயம் ஒரு மாதிரி உதாரணமாக விளங்குகிறது.

15 வயதான ராகுலும் ஒரு ஒன்பதாம் வகுப்பு மாணவன்; அவனும் பஞ்சக்கிராமிக்கு மிகவும் அருகில் அமைந்துள்ள இன்னொரு வளமான பன்னாட்டுப் பள்ளிக்குச் செல்கிறான். அவன் ஆய்வுக்களத்தில் அமைந்துள்ள ஒரு பலமாடி அபார்ட்மென்ட் வளாகத்தில் வசிக்கிறான். ராகுலின் பெற்றோர் இருவரும் ஐடி கம்பெனிகளில் பணி புரிகிறார்கள், அவர்கள் பொதுவாக மாலை 7 மணி சுமாருக்கோ அல்லது சில நேரங்களில் அதற்குப் பிறகோ வீடு திரும்புவார்கள். அதனால் ராகுல் மாலை சுமார் 4 மணிக்கு தான் பள்ளியிலிருந்து வீடு திரும்பிய பின்னர் தன் பெற்றோர் வரும் வரை தன் தாய்வழி தாத்தா-பாட்டியுடன் இருக்கிறான் (அவர்களும் அதே அபார்ட்மென்ட் வளாகத்தில் தமக்கு ஒரு வீடு வாங்கியுள்ளனர்). வலியுறுத்தப்படும் முறை என்னவென்றால் ராகுல் தான் தாத்தா பாட்டி வீட்டை அடைந்ததைத் தெரியப் படுத்த தம் பெற்றோர் யாராவது ஒருவரை அழைக்க வேண்டும். பிறகு அவன் பெற்றோர் 7 மணி சுமாருக்கு தாம் வீடு வந்து விட்டதைத் தெரியப்படுத்த அவனுடைய மொபைல் ஃபோனில் (ஒரு சாம்சங் ஸ்மார்ட் ஃபோன்) அழைப்பார்கள். அவர்கள் அவனுடைய தாத்தா பாட்டியையும் தம் வருகையைத் தெரிவிக்க அழைப்பார்கள்.

மேற்கூறிய அனைத்து உதாரணங்களும் ஒரு பதின்ம வயதினரையோ அல்லது திருமணமாகாத பிள்ளையையோ உள்ளடக்கிய பெற்றோர்- பிள்ளை தொடர்பைக் குறிக்கிறது. இந்த தருணத்தில், அத்தியாயம் 2ல் கூறப்பட்ட திரு.ராகவன் அவர்களை அறிமுகப்படுத்துவது உசிதமாகத் தெரிகிறது. அவர் தம் மகன்களுடன் தொடர்பு கொள்ளும் விஷயத்தில் ஒரு ஊக்கமுள்ள திட்டமிடுபெர். அவருடைய விஷயத்தில் திருமண நிலையோ அல்லது தொலைவோ ஒரு பொருட்டு இல்லை - வெவ்வேறு நகரங்களில் வாழும் திருமணமான மகன்கள் கூட அவருடன் மரபு சார்ந்த குரல் தொடர்பில் இருக்கிறார்கள்.

65 வயதான ராகவன், ஒரு உயர் மத்திய வகுப்பைச் சேர்ந்த பிராமணர். ${ }^{25}$ பணி ஓய்வுக்கு முன் அவர் ஒரு பிரசித்தி பெற்ற மருந்துக் கம்பெனி ஒன்றின் பயிற்சி மற்றும் வளர்ச்சித் துறைத் தலைவராக இருந்தார். அவர் தம் பணி ஓய்வுப் பெற்ற உடனேயே பஞ்சகிராமியில் ஒரு நல்ல இரண்டு படுக்கையறை அபார்ட்மென்ட்டில் முதலீடு செய்தார், இங்கு அவர் 
மழலையர் பள்ளி ஆசிரியை பயிற்சி பெற்றிருக்கும் தம் மனைவியுடன் வசித்து வருகிறார்; அவர் மனைவி பாரம்பரிய இந்திய நுண்கலையான தஞ்சாவூர் ஓவியத்திலும் ${ }^{26}$ வல்லுநர். இந்த தம்பதியின் ஒரு மகன் பெங்களூரில் வசிக்கிறார், ஒரு ஐடி கம்பெனியில் பணி புரிகிறார், மற்றொருவர் கனெக்டிகட், யூஎஸ்ஏவில் பணி புரிகிறார். ராகவன் மற்றும் அவரது மனைவியிடம் ஒரு பிளாக்பெர்ரி (திரு ராகவனுக்காக) மற்றும் சாம்சங் (திருமதி ராகவனுக்காக) ஃபோன்களைத் தவிர, ஐபாட்களும் சாம்சங் டாப்லட்களும் இருக்கின்றன. அவர்களிடம் இரண்டு லேண்ட்லைன் ஃபோனும் இருக்கின்றன. மேலும் அவர்களிடம் ஒரு ஏர்டெல் 27 (இந்தியாவின் மிகப்பெரிய மொபைல் டெலிஃபோன் சேவை வழங்குபவர்) போஸ்ட்-பெயிட் இணைப்பும் இருக்கிறது, அவர்களிடம் அதன் மூலம் ஒரு சியூஜி (Closed User Group) இருக்கிறது. இந்தக் குழுவிற்குள் பேசப்படும் அழைப்புகள் எல்லாமே இலவசம், பெங்களூரில் இருக்கும் ராகவனின் மகனும், மருமகளும் இந்தக் குழுவில் இருக்கிறார்கள். அவர்கள் வழக்கமாக வார நாட்களில் தம் மகன் மற்றும் மருமகளுடன் ஃபோனில் உரையாடுகிறார்கள், மற்றும் வாரக்கடைசியில் தன்னுடைய டாப்லெட்டெக் மூலம் ஸ்கைப்பில் தொடர்பு கொள்கிறார்கள், அவர்களுக்கு இதனால் தம் பேத்தியையும் பார்க்க முடிகிறது. இதைத் தவிர, திரு ராகவனிடம் மாஜிக் ஜாக் என அழைக்கப்படும் ஒரு விஓஐபி ${ }^{28}$ தொலைத் தொடர்பு சேவையும் இருக்கிறது. யூஎஸ்ஏவிற்குப் பேசப்படும் அழைப்புகளை இவற்றில் இலவசமாகப் பேசலாம், இதற்காக சந்தாதாரருக்கு இந்தியாவில் ஒரு யூஎஸ் எண் வழங்கப்படுகிறது. மாதம் ஒரு முறை குடும்பம் முழுவதும் (யூஎஸ், பெங்களூர் மற்றும் சென்னையிலிருந்து) திரு மற்றும் திருமதி ராகவன் தம் பேரக்குழந்தைகளை பார்க்க முடியும் வகையிலும், மற்றும் சிறுவர்கள் தம் சகோதர சகோதரிகளைப் பார்க்க முடியும் வகையிலும், குறைந்த பட்சம் ஒரு மணி நேரம் ஒன்று சேர முயற்சிக்கிறார்கள். வார இறுதியில் ஸ்கைப்பில் செல்வதற்கு முன் திரு.ராகவன் ஒரு சிறிய முறையைப் பின்பற்றுகிறார்.

அடி 1: இரண்டு மகன்களையும் அழைத்து², அவர்கள் குறிப்பிட்ட வார இறுதியில் ஓய்வாக இருப்பார்களா எனக் கேட்டல்

அடி 2: அழைப்புக்கு முன் இரு மகன்களையும் தனித்தனியாக அழைத்து அவர்கள் இருப்பதை உறுதிப்படுத்திக் கொள்ளுதல் 
அடி 3: மகன்கள் குறிப்பிட்ட நேரத்திற்குள் வரத் தவறினால் ஒவ்வொருவரையும் தனியாக மீண்டும் அழைத்தல்

அடி 4: ஸ்கைப்பில் நிதி சம்பந்தப்பட்ட விஷயங்கள் விவாதிக்கப்படுமேயானால், அந்த விவாதத்தின் பேரில் தொடர் கண்காணிப்புக் குறிப்பு அனுப்புதல்.

அவருடைய மனைவி அவர் இந்த விவாதங்களை முறைப்படுத்த முனைவதாகவும் இந்த விதமான அழைப்புகளை அமைத்தலை விரும்புவதாகவும் கூறுகிறார்.

திரு. ராகவன் தம் பேரப்பிள்ளைகள் 9 மற்றும் 10 வயதிலேயே மொபைல் ஃபோன் உபயோகிப்பதைக் காண தாம் ஏமாற்றம் அடைவதாகக் கூறினார். ஆகையால், அவர் அவர்களை அழைக்கும்போதெல்லாம் அவர்கள் வீட்டு லேண்ட்லைனுக்கு அழைக்கிறார், மொபைல் ஃபோனுக்கு அழைப்பதில்லை. அவரது மனைவி மேலும் பேரப்பிள்ளைகள் தம் தாத்தாவின் விருப்பத்தை அறிந்திருப்பதாகவும் அவர்கள் அவருடன் லேண்ட்லைன் அல்லது ஸ்கைப்பிலேயே பேச வேண்டும் என்பதைத் தெரிந்து வைத்திருப்பதாகவும் கூறினார்.

திரு. ராகவன், தெளிவான முறையில், தம் வயதையும், உறவு முறையையும்,தம் பேத்திகளுடனான தொடர்பு அலைவரிசையை நிர்ணயிக்க படிநிலைக் கோட்பாடாகவும் அதிகாரமாகவும் பயன் படுத்துகிறார். அவர் தன் குடும்பத்தினருடன் செய்திகளை அனுப்புவதுமில்லை, பெறுவதுமில்லை. அவர் ஸ்கைப் அழைப்பை நிறுவுவதற்கு முன் தம் மருமகளின் பெற்றோர் அவர்களுடன் பெங்களூரில் இருக்கிறார்களா என்பதைத் தெரிந்து கொள்கிறார். அவர்கள் அங்கு இருந்தால் திரு.ராகவன் தம் பேரப்பிள்ளைகளை தொலைபேசியில் மட்டுமே அழைக்கிறார்; அவர் அவர்களுடன் பேசி விட்டு தம் உரையாடலை விரைவிலேயே முடித்துக் கொள்கிறார். தன்னுடைய குடும்பத்தாருடன் ஊடகப் பயன்பாட்டைப் பற்றி அவரது வார்த்தைகளிலேயே இவ்வாறு சுருக்கிக் கூறலாம்:'ஒருவர் உயிரோடு இருக்கும் வரை, அவரது மௌனத்தை விட அவருடன் பேசுவதே சிறந்தது.' திரு.ராகவன் தம் பேரப்பிள்ளைகள் மேல் கொண்டுள்ள பாசம் ஸ்கைப் மற்றும் லேண்ட்லைன் மூலமான தொடர்பை தூண்டிவதால், இது ஒரு ஊடக பன்முகமாக்கல் ${ }^{30}$ விஷயமாகத் தென்பட்டாலும், நாம் கருத்தில் கொள்ள வேண்டியது என்னவென்றால் அவரது தொடர்பு அலைவரிசைகள் யாவும் குரலையே பிரதானமான விஷயமாகக் கொண்டுள்ளன. இந்தக் கொள்கை வலுவான 
பிணைப்புகள் தொடர்புக்கான அதிக அலைவரிசைகளை உபயோகிக்கின்றன என்பதை அறிவுறுத்துகிறது, ஆனால் இங்கு கவனிக்க வேண்டிய விஷயம், இது நேரானது அல்ல; இது, திரு.ராகவனின் விஷயத்தைப் போல, பண்பாட்டுச் சூழலால் தாக்கம் அடைந்துள்ளது,

இந்த விஷயம் நம்மை தலைமுறைகளின் இடையிலான தொடர்பின் இன்னொரு தோற்றத்திற்கு எடுத்துச் செல்கிறது, அதாவது தாத்தா-பாட்டி மற்றும் பேரப்பிள்ளைகளின் இடையே நடப்பதற்கு. ஐடி துறையில் தொழிற்பண்பட்டவராகப் பணி புரியும் பெற்றோர், ஆன்சைட் செயல் திட்டங்களுக்காக ${ }^{31}$ எப்பொழுதும் அதிகரித்துக் கொண்டிருக்கும் பயணத் தேவையை எதிர் கொள்ளலாம (இதில் ெெளிநாட்டு) நியமனங்களும் இருக்கலாம்), சில நேரங்களில் நீண்ட காலத்திற்கு கூட இருக்கலாம், இது குறிப்பிட்ட செயல் திட்டத்தையோ அல்லது அவர்கள் வேலை செய்யும் வெளிநாட்டு வாடிக்கையாளரையோப் பொறுத்தது. பல தடவை ஐடி பணியாளர்கள், ஒரு வீட்டில் முதலீடு செய்து, அங்கு சில ஆண்டுகள் வாழ்ந்தபின் ஒரு குறிப்பிட்ட செயல் திட்டத்திற்காக, யூஎஸ்ஏ, யூகே அல்லது வேறு ஒரு நாட்டுக்குச் செல்லப் பணிக்கப் படுகிறார்கள். தம் குடும்பத்துடன் வெளிநாட்டு செயல்திட்டக் களத்திற்கு செல்பவர்களுக்கு (மனைவி/கணவன், மற்றும் குழந்தைகள்), குடும்பம் திரும்பி வரும் வரை, இந்த வீட்டை காலியாக பூட்டி வைக்காமல், கணவர் வீட்டார் இந்த வீட்டில் வசிக்கத் திட்டமிடலாம். ${ }^{32}$

மற்றொருக் காட்சியில், பல வயதான தம்பதியினர், பணி ஓய்வுக்குப் பின் பஞ்சக்கிராமியில் வீடுகளில் முதலீடு செய்கின்றனர், அவர்கள் நிர்மாணிக்கப்பட்ட சமூகங்களில் வாழவும், தன் மாதிரியே இருக்கும் தம்பதியினரோடு பழகவும் முனைகிறார்கள். அவர்கள் வழக்கமாக இந்தியாவில் ஆறுமாதத்திற்கு வசிக்கிறார்கள், அதன் பின் யூஎஸ் அல்லது யூகேவில் வாழும் தம் மகன்/மகளோடு ஏறக்குறைய இன்னொரு ஆறு மாதங்களுக்கு தங்க செல்கிறார்கள் (தன் மகன் யூஎஸ்ஸில் இருக்கும் திரு. ராகவனைப் போல). இந்த மாதிரியான விஷயங்களில் வெளிநாட்டில் வசிக்கும் திருமணமான மகன் அல்லது மகள் மற்றும் பஞ்சக்கிராமியில் இருக்கும் பெற்றோர் ஆகியோருக்கு இடையில் தொடர்பு ஏற்படும் பொழுது, தம் பேரக்குழந்தைகளுடன் உரையாடும் ஆவல் குறிப்பாக அதிகமாகக் காணப்படுகிறது. அவர்களுடைய பேரப்பிள்ளைகளின் படங்களை அடிக்கடி ஃபேஸ்புக்கில் பார்த்தாலும், அல்லது அவை தாத்தா-பாட்டிக்கு வாட்ஸ் அப் மூலம் அனுப்பப் பட்டாலும், மாதமிருமுறை ஸ்கைப் 
அல்லது கூகுள் ஹாங்க் அவுட் மூலமான அழைப்பு ஒரு முறையாகி விட்டது. பல இடங்களில், இந்தியாவிலிருக்கும் வயதான பெற்றோருக்கும், வெளிநாட்டில் இருக்கும் மகன் அல்லது மகளுக்கும் இடையேயான வாராந்திரப் பொதுத் தொடர்பு ஃபோன் மூலமாக இருந்தாலும், பேரப்பிள்ளைகளுடனானத் தொடர்பிற்கு குரலுடன் காட்சியும் இணைந்தவை (ஸ்கைப் அல்லது கூகுள் ஹாங் அவுட் போன்றவை) முதலிடம் பெறுகின்றன. ஒரு வேளை காட்சி தொடர்பு சாத்தியமில்லை என்றால், தாத்தா-பாட்டி பேரப்பிள்ளைகளின் குரலைக் கேட்பதிலேயே திருப்தி அடைகிறார்கள். இநந்தப் பாங்கு 10 வயதிற்குக் கீழ் உள்ள பேரப் பிள்ளைகளுடனான தொலைவில் வசிக்கும் குடும்பங்களின் இடையில் காணப்படுகிறது.

உதாரணத்திற்கு வருண் மற்றும் வரரண்யா, 10 வயது இரட்டையர் திருமதி சாரதா மற்றும் திரு நமச்சிவாயம் தம்பதிகளின் பேரப்பிள்ளைகள், ஒவ்வொரு வாரமும் ஸ்கைப்பில் தம் தாத்தா பாட்டியுடன் உரையாடுகிறார்கள். சாரதாவிற்கு அவரது சென்ற யூஎஸ் பயணத்தின் போது கிறிஸ்துமஸ் பரிசாக ஒரு ஐபாட் கொடுக்கப்பட்டது, அது அவருக்கு அவருடையப் பேரப் பிள்ளைகளால் கொடுக்கப் பட்டது (அவர்களது பெற்றோர் அதை வாங்கியிருந்தாலும்). சாரதா தமக்கு ஃபேஸ்டைம் மற்றும் ஸ்கைப்பை உபயோகிக்க தம் பேரப்பிள்ளைகள் கற்றுக் கொடுத்ததாகக் கூறுகிறார். இப்பொழுது ஒவ்வொரு வாரமும், சுருக்கமாக இருந்தாலும் கூட அவர்கள் உரையாட இந்த தளங்களுக்குச் செல்கிறார்கள். சாரதா சில சமயம் தான் தன் மகளுடன் (இரட்டையர்களின் தாய்) உரையாடும் நேரத்தை விட தன் பேரப் பிள்ளைகளுடன் அதிக நேரம் உரையாட விரும்புவதை உணர்ந்து உள்ளார். அவரிடம் தன் பேரப் பிள்ளைகளின் பெயர்களை உடைய ஒரு வாட்ஸ் அப் குழுவும் இருக்கிறது, இதில் உறுப்பினர்களாக, அவரது திருமணமாகாத மகன், மகள், மாப்பிள்ளை, மகளின் புகுந்த வீட்டினர் (பெற்றோர், சகோதரி, போன்றோர்) ஆகியவர்கள் உறுப்பினர்களாக இருக்கின்றார்கள். அவர்கள் இந்த சமூகத்தில் எப்பொழுதும் செய்திகளையும், படங்களையும் பரிமாறிக் கொள்கிறார்கள்.

திரு. ராகவன் மற்றும் திருமதி சாரதா இருவரின் விஷயத்திலும், குரல் ஒரு முக்கியக் காரணியாக இருந்தாலும், தொடர்பு கொள்ள ஒன்றுக்கு அதிகமான ஊடகங்களை உபயோகிப்பது தெளிவாக உள்ளது. இரண்டு விஷயங்களுக்கும் உள்ள ஒரே வேற்றுமை, சாரதாவின் விஷயத்தில் காட்சி உடன்போதலின் அதிகபட்ச நிகழ்வு (ஸ்கைப்/ஃபேஸ்டைம் 
மூலமாக). மேற்கூறிய இரு உதாரணங்களும் ஊடகத்தின் பன்முகத்தன்மை மற்றும் பாலிமீடியாவின் கூறுகளை விளக்குகின்றன. ${ }^{33}$

திருமதி கீதா தியாகராஜன், இவர் தனது மகளுக்கு வாட்ஸ் அப் மூலம் பிறந்த குழந்தை பராமரிப்பு பற்றி அறிவுரை வழங்குகிறார், இன்னொரு இதே மாதிரியான உதாரணம். அவருடைய தனது மகளுடனான முந்தைய உரையாடல்கள் ஸ்கைப் மூலமாக இருந்தன, அதில் அவர்கள் குடும்ப வதந்திகளையும், மற்றும் அவரது மகள் யூஎஸ்ஸில் செய்வதற்காக இந்திய உணவுத் தயாரிப்பு முறைகளைப் பற்றியும் உரையாடுவார்கள். திரு தியாகராஜன் அவர்கள் தனது மகள் திருமணமாகி யூஎஸ்ஸிற்கு சென்ற பின் தமது மனைவி தன்னுடன் நேராக உரையாடுவதை ${ }^{34}$ விட ஸ்கைப்பில் அதிக நேரம் செலவிடிவதாக ஹாஸ்யமாகக் கூறுவார். அவர்களது முதல் பேரக்குழந்தை பிறந்த பிறகு, அவர்கள் பெரும்பான்மையான உரையாடல்கள் குழந்தைப் பேணுதலைப் பற்றியே இருக்கின்றன. மகளுடைய புகுந்த வீட்டார் யூஎஸ்ஸிற்கு சென்ற பொழுது, இந்த உரையாடல் டெலிஃபோனிற்கும் வாட்ஸ் அப்பிற்கும் நகர்ந்து விட்டது. தம் மகள் தம் புகுந்த வீட்டாருடன் நேரத்தை செலவழிக்க வேண்டும் என்பதை மதித்து, அவளுடைய புகுந்த வீட்டார் வந்திருக்கும் நேரத்தில் அவளை அதிக நேரம் ஸ்கைப்பில் இருத்தி வைத்திருப்பது சரியாகப் படவில்லை. (கர்ப்பத்தினால் ஏற்படும் குமட்டலும், அசதியும் ஸ்கைப்பில் அதிக நேரம் செலவிடாததற்கான காரணங்களின் ஒரு பகுதியாகும்). அவர்களது மகள் ஃபேஸ்புக்கில் இருந்தாலும், கீதா, மற்றும் அவளது புகுந்த வீட்டினர் கண் திருஷ்டி ${ }^{35}$ காரணமாக குழந்தையின் புகைப்படத்ட்தை அப்லோடு செய்ய வேண்டாம் என்று கூறியிருக்கிறார்கள். ஆகையால் படங்கள் இ-மெயில் அல்லது வாட்ஸ் அப் மூலமே பரிமாறிக் கொள்ளப் படுகின்றன. அவரது மருமகனால், குழந்தைப் பிறந்தவுடனேயே அனைவருக்கும் நற்செய்தியைத் தெரிவிப்பதற்காக, அப்லோட் செய்யப்பட்ட படம் கூட கீதாவின் அறிவுரையால் உடனடியாக விலக்கப்பட்டது.

பல தாத்தா பாட்டிகள் புதிதாகப் பிறந்த குழந்தையின் படத்தை ஃபேஸ்புக்கில் அப்லோடு செய்வது கண்திருஷ்டிக்குக் காரணமாகும் என நம்புகிறார்கள். ஆனால், அவர்கள் குழந்தையின் படம் சில மாதங்களுக்குப் பிறகு ஃபேஸ்புக்கில் தென்பட்டால் மகிழ்ச்சி அடைகிறார்கள். புதிதாகப் பிறந்தக் குழந்தையின் படத்திற்கு இடப்பட்டஃபேஸ்புக் 'லைக்குகள்' கூட 
கண் திருஷ்டியின் அறிகுறியாகவேக் கருதப்படுகின்றன. இது வெளியாட்களுக்கு மூட நம்பிக்கையாகத் தெரியும் என்பதை அவர்கள் ஒப்புக் கொள்கிறார்கள், ஆனாலும் அவர்களது அனுபவத்தின் படி கண் திருஷ்டி என்பது உள்ளது என்பதை வலியுறுத்திக் கூறுகிறார்கள், மேலும் ஃபேஸ்புக் போன்ற ஊடகங்கள் இதை ஏற்படுத்தக்கூடிய சாதனம் என்றும் கூறுகிறார்கள். இந்த மாதிரியான கருத்துக்கள் 68 வயதான, யூஎஸ்ஸில் ஒரு புதிய பேரனை அடைந்திருக்கும் திருமதி. கல்யாணி அவர்களின் உரை மூலம் சுருக்கமாக நன்கு விளக்கப்படுகின்றன.

உங்களுக்குத் தெரிந்தவர்களேதான் ஃபேஸ்புக்கிலும் இருக்கிறார்கள், ஆகையால் யாருடைய இயல்பு என்ன என்பது உங்களுக்கு நன்றாகத் தெரியும், ஆகையால் உங்களுக்கு கெடெல் செய்ய அவர்களுக்கு ஏன் ஒரு வாய்ப்பைக் கொடுக்க வேண்டும் (கண் திருஷ்டி இடிவதின் மூலம்)?

ஆனாலும், வாட்ஸ் அப் மூலம் நெருங்கிய உறவினர்களுக்கு (குறிப்பாக குழந்தை பிறப்பின் போது வர முடியாத தாத்தா பாட்டிக்கு) பிறந்த குழந்தையின் படத்தை அப்லோடு செய்தலும், அனுப்புதலும் முகஞ்சுளிக்கச் செய்வதில்லை. இந்தப் படங்கள் மிகவும் முக்கியமானவர்களுக்கும், இதைப் பற்றி தெரிவிக்காமலோ அல்லது இந்தப் படத்தைக் காட்டாமலோ இருந்தால் மிகவும் வருந்தக் கூடியவர்களுக்கும் அனுப்பப் படுகின்றன, ஆகையால் இந்த குடும்ப நெட்வொர்க்கின் எதிர்பார்ப்புகளுக்கு அனுசரித்து இருப்பதற்காக இந்தப் படங்கள் வாட்ஸ் அப்பின் மூலம் மட்டுமே அனுப்பப் படுகின்றன, ஃபேஸ்புக் ஒரு பொதுத் தளமாகப் பார்க்கப் படுகிறது (ஒரு பெரிய நெட்வொர்க்கிற்கு சேவை செய்யக்கூடிய தளம்), வாட்ஸ் அப் தனிப்பட்ட தளமாக பார்க்கப் படுகிறது (ஒரு உறுதியான பந்தத்தின் நெட்வொர்க்கை வளர்க்கக் கூடிய தளம்). மத்திய வகுப்பு மற்றும் உயர் மத்திய வகுப்பு மூத்தவர்கள் ஊடகங்கள் 'தனிப்பட்ட மற்றும் ‘பொது' என இரு விதமாகப் பிரித்து வகைப்படுத்துகிறார்கள் மேலும் இதன் மூலம் எது எங்கே இருக்க வேண்டும் என்பதையும் முடிவு செய்கிறார்கள். தலைமுறைகளுக்கு இடையேயான அந்தரங்கமான குடும்ப விவகாரங்களைப் பற்றி தொடர்பு கொள்ள மிகச் சிறந்த வழியை முடிவு செய்ய தளங்களின் பயன்பாடு பற்றி அவர்களது சொல்லே பின்பற்றப் படுகிறது. 
வயதானவர்கள் புதிய தொடர்பு ஊடகத்தைப் பற்றி தெரிவிக்கும் இன்னொரு கவலை, விரிவு நிலைக் குடும்ப உறுப்பினர்களின் முக்கிய வாழ்க்கை நிகழ்வுகள் அவர்களுக்கு எவ்வாறுத் தெரிவிக்கப் படுகிறது என்பதுதான். திரு. ராகவன் மற்றும் திரு. கரண் (இவரைப் பற்றி அத்தியாயம் 2ல் விவாதிக்கப்பட்டது), போன்ற பெரியவர்கள், சமூக ஊடகங்கள் வயதையோ அல்லது படிநிலையையோ கருத்தில் கொள்வதில்லை, ஆனால் குரல் மற்றும் தனிப்பட்ட அழைப்புகள் அவற்றை கருத்தில் கொள்கின்றன என உறுதி செய்கிறார்கள்.

வயதான உறவினர்களுக்கு, பெற்றோர் அல்லது தாத்தா பாட்டியுடன் விளக்கமான அல்லது முக்கியமான தொடர்புகளைப் பொறுத்தவரை அவை செய்தியை விட குரல் மூலமாகவே இருக்க வேண்டும். சமூக நெட்வொர்க்கிங் தளங்கள் மூலமாக சிறிதளவு செய்திகளே பரிமாறப்படுகின்றன அல்லது அவை மூலம் தொடர்பு ஏற்படுவதே இல்லை, மேலும் ஒன்றாக வாழும் பிள்ளைகள், பெற்றோர் மற்றும் தாத்தா பாட்டி இடையே ஒரு தொடர்பு ஊடகத்தை வளப்படுத்துவதில் இவைகளுக்கு பங்கேதும் இல்லை. கல்வி, வேலை போன்ற காரணங்களுக்காக இந்தியாவிற்குள்ளேயோ அல்லது வெளி நாடுகளுக்கோ புலம் பெயர்ந்து இருக்கும் நெருங்கிய குடும்ப உறுப்பினர்களுடன் கொள்ளும் தொடர்பில் மட்டுமே சமூக நெட்வொர்க்கிங் தளங்கள் பங்களிக்கின்றன. ஒரு நிலையற்றதாக இருக்கும் உடல் சார்ந்த தொலைவு, தொடர்புகளில் காட்சிகளின் பயன்பாடு மற்றும் இருதிசைப் பயன் விளைவு ஆகியவற்றை நிர்ணயிக்கிறது. தொடர்பு கொள்பவர்களுக்கு இடையே உள்ள தொலைவு கணிசமாக இருக்கும் பட்சத்திலேயே, ஸ்கைப் மற்றும் கூகுள் ஹேங்க் அவுட் போன்றவை பங்காற்ற வருகின்றன.

குடும்பத்தினுள் தொடர்புக்கான ஊடகம் தெளிவாக வரையறுக்கப் பட்டிருந்தாலும், நாம் இப்பொழுது குடும்பங்கள் எவ்வாறு சமூக ஊடகத்தைப் (முக்கியமாக ஃபேஸ்புக்) பயன் படுத்துகின்றன,, குறிப்பாக பொதுத் ${ }^{36}$ தொடர்புகளை வளப்படுத்துவதில் ஒரு முக்கிய மற்றும் திறமையான பங்காற்றுகின்றன, என்பதைப் பரீட்சிப்போம்.

நெருக்கமான குடும்ப உறுப்பினர்களுக்கு இடையே தொடர்பு கொள்ள ஃபேஸ்புக் விரும்பத்தக்க தளமாக இல்லாவிட்டாலும், இது குடும்ப நெருக்கத்தை நோக்கமுள்ள சமூக வட்டத்தின் முன் நிகழ்த்துவதற்கும் காட்டுவதற்கும் விரும்பித் தேர்வு செய்யும் ஊடகம் ஆகும். சுருக்கமாக, அன்பு மற்றும் 
நெருக்கம் இரண்டு சுழற்சிகளுக்கு இடையே ஏற்படுகிறது - ஒன்று தனிப்பட்ட கோளத்தில் இயங்குவது, அதாவது ஒரு பலத்த பந்த நெட்வொர்க்கின் உள்ளே, மற்றது பொதுக் கோளத்தில் ஒரு பெரிய நெட்வொர்க்கின் பார்வையில் இயங்குகிறது (முக்கியமாக ஃபேஸ்புக்).

எடுத்துக்காட்டாக, திருமதி மைதிலி விஜயன் மற்றும் திரு விஜயன் தம்பதிக்கு தம் பக்கத்து வீட்டில் வசிக்கக் கூடிய ஒரு ஏழு வயது பேத்தி இருக்கிறாள் (இது மேலே விவாதிக்கப்பட்ட அருகருகே இருக்கும் வீடுகளில் வசிக்கும் விரிவு நிலைக் குடும்பத்தின் ஒரு மாதிரி உதாரணம் ஆகும்). மைதிலியின் மகன் சங்கர் தனது மகளின் மேற்கத்திய சங்கீதம் முதல் வகுப்புக்குப் பின் எடுத்த படத்தை ஃபேஸ்புக்கில் போஸ்ட் செய்திருந்தார். இந்தப் படம் சில வாரங்களுக்கு வாட்ஸ் அப் மூலம் நெருங்கிய குடிம்பத்திற்கு நடுவில் சுற்றி வந்திருந்தது, இதில் சிலர் பெண் மிகவும் நன்றாக இருப்பதாக குறிப்பு எழுதியிருந்தார்கள்; இந்தப் பாராட்டு சில தினங்களிலேயே முடிந்திருந்தது. ஆனால் இந்தப்படம் ஃபேஸ்புக்கில் போஸ்ட் செய்யப்பட்ட பொழுது இரு தாத்தா பாட்டிகளும் அவர்களுடைய 'குட்டி தேவதை' மிக நன்றாக இருப்பதாகக் கூறியிருந்தார்கள், அவர்களது மருமகளின் குறிப்பு இதைத் தொடர்ந்திருந்தது. இதனை வெளிநாட்டில் வசிக்கும் சங்கரின் சகோதரியின் பலக் குறிப்புகள் பின் தொடர்ந்திருந்தன. அவர் ஒருவாரத்திற்கு முன்பே இந்தப் படம் தனக்கு வாட்ஸ் அப் மூலம் அனுப்பப் பட்ட போது இதைப் பார்த்திருந்தார், மேலும் இதைப் பற்றி குறிப்பும் எழுதியிருந்தார்.

இதே மாதிரி, திருமதி உமா பிரகாஷ், ஒரு 35 வயது இல்லத்தரசி, தனது 8 வயது மகன் வித்யுத் விளையாடுவது, பள்ளிக்குப் போவது மற்றும் வீட்டுப்பாடங்களைச் செய்வது போன்ற படங்களை அப்லோட் செய்கிறார். இந்த ஒவ்வொரு படங்களும் அவரது புகுந்த வீட்டார்களிடமிருந்தும் (கணவரின் சகோதரி, சகோதரி போன்றவர்கள்) மற்றும்பக்கத்து வீட்டில் வசித்துக் கொண்டு வித்யுத்தை தினசரி பார்க்கும் தனது பெற்றோரிடமிருந்தும் பதிலைக் குறிப்புகள் மூலமாகப் பெறுகிறது.

அரசுத் தொலைத் தொடர்புத் துறையிலிருந்து ஓய்வு பெற்ற, 67 வயதான கிருஷ்ணனின் விஷயத்தில் அவரும் அவரது மனைவியும் தமது மகன் மற்றும் அவர் குடும்பத்தாரிடமிருந்து (யூஎஸ்ஸில் வசிக்கும்) பெறும் வாராந்திர ஸ்கைப் அழைப்புகளின் நேரங்கள் தாம் தம் குடும்பச் செய்திகளைப் பகிர்ந்து கொள்ளும் நேரமாகும். உரையாடல்கள் பெரும்பாலும் பள்ளியில் ஐந்தாவது மற்றும் நான்காவது வகுப்பில் படிக்கும் 
அவர்களது பேரப்பிள்ளைகள் வர்ஷா மற்றும் ஷைலாவையே சுற்றி அமைக்கப் பெறுகின்றன, இந்த தம்பதியின் மருமகளான, சுமி, வர்ஷா மற்றும் ஷைலாவின் புகைப்படங்களை ஃபேஸ்புக்கில் அப்லோட் செய்கிறார், கிருஷ்ணன் மற்றும் சுமியின் பெற்றோர்கள் இதைப் பற்றி குறிப்பு வரைகிறார்கள் - இந்தப் படங்கள் ஏற்கனவே அவர்களுடன் தனிப்பட்ட முறையில் பகிரப்பட்டு அதற்கு இவர்கள் பாராட்டுக் குறிப்புகளை அனுப்பியிருந்தாலும் கூட.

பஞ்சகிராமி போன்ற சமுதாயத்தில் ஒரு நல்ல மற்றும் மாதிரிக் குடும்பத்தின் கொள்கைகள் சம்பந்தமான பல ஒரு குடிம்பத்தினுள் தரநெறிசார் உரைகள் இருக்கின்றன. தரநெறிசார் கொள்கைகளின் அடிப்படைகள் அந்தக் குடும்பத்தின் உள்ளேயே புதைந்து போவதைக் காட்டிலும் வெளிப்படையாக அதை வெளிப்படுத்துதலே அவசியமாகிறது. குடும்பங்கள் வெளி உலகத்திற்கு தன் குடும்பத்தின் நெருக்கத்தையும், மற்றும் குடும்பம் எப்படி விழுமியதாக இருக்கிறது என்பதையும் வெளிக்காட்டும் ஆஃப்லைன் உலகத்தைப் போலவே, ஃபேஸ்புக்கும் குடும்பங்கள் தன் பாசம், அன்பு மற்றும் பந்தத்தை உலகின் கண்களுக்கு - அதாவது அவர்களது பெரிய நெட்வொர்க்கிற்கு வெளிப்படையாகக் காட்டும் ஒரு ஆன்லைன் மக்கள் ஊடகமாகும் - இது அளவிடக்கூடிய சமூகத்தன்மையின் இ இன்னொரு மாதிரி உதாரணமாகும்; குடும்பங்கள் தம் குடும்பம் சம்பந்தப்பட்ட எதையும் போஸ்ட் செய்யும் முன்னர் குழுவின் அளவையும் மற்றும் இயல்பையும் உத்திப் பூர்வமாகத் தேர்ந்தெடுக்கிறார்கள்.

மூத்த மற்றும் இளைய தலைமுறை இருவரும் இந்த நிகழ்வில் பங்கேற்றாலும், மூத்தவர்கள் மட்டுமே இதை வெளிப்படையாகச் செய்கிறார்கள். இது கிராமங்களில் வசிக்கும் கீழ் சமூகப் பொருளாதார வகுப்பிலோ, கீழ் மத்திய வகுப்பிலோ தென்படுவ்து இல்லை, ஏனென்றால், இங்கு பெற்றோர்/ தாத்தா பாட்டி ஆகியோரின் தொழில்நுட்ப உபயோகம் மிகவும் குறைவாகவே உள்ளது. ஆனால் அவர்கள் சில சமயங்களில் தமது பிள்ளைகள் தம் செல்ஃபோனிலிருந்து காட்டும் படங்களைப் பார்த்து தம் பாராட்டை வெளிப்படுத்தலாம். ஆனால் இதனை அவர்கள் ஃபேஸ்புக் அல்லது வாட்ஸ் அப்பில் அல்லது வேறு எந்த ஒரு தளத்திலும் உள்ள படம் என்பதை விட மொபைல் ஃபோனில் உள்ள படம் என்கிற விதத்திலேயேக் காண்கிறார்கள். ஹார்ட்வேர் உபகரணம் (அதாவது மொபைல் ஃபோன் அல்லது கம்ப்யூட்டர்) மட்டும்தான் அவர்களுக்கு உணரத் தக்கதாகும். 
முடிவில், ஊடகத்தின் வாயிலான (ஃபோன் அல்லது சமூக ஊடகம்) தலைமுறைகளுக்கு இடையேயானத் தொடர்பு, பல தட்டுக்களை உடையது மேலும் அதிகார அமைப்பினால் தாக்கத்திற்குள்ளாவது; இது வயதுக்கு மரியாதை என்றக் கொள்கை மீது அமைந்துள்ள படிநிலை மீதுள்ள மிகுந்த மதிப்புடன் கூடிய ஒரு பண்பாட்டுச் சூழலிலிருந்து உருவாவது. இதன் அர்த்தம் ஊடகப் பயன்பாட்டை நிர்ணயிப்பதின் கட்டுப்பாடு நெருங்கிய குடும்ப உறுப்பினர்கள் மீது அக்கறை, பாதுகாப்பு, கவலை ஆகியவற்றால் அல்ல என்பது இல்லை. காட்சி மற்றும் தரநெறிசார் கோட்பாடுகள் பற்றிய விஷயங்கள், நெட்வொர்க்கின் எதிர்பார்ப்பை அனுசரித்தல் மற்றும் திறன்களும் மற்றவற்றை விட்டு ஒரு தொடர்பு ஊடகத்தைத் தேர்ந்தெடுத்தலின் மேல் தாக்கத்தை விளைவிக்கின்றன, பஞ்சக்கிராமியில் தலைமுறைகளுக்கு இடையேயான தொடர்புகளில் பாலிமீடியாவின் நம்ப வைக்கும் காட்சி அமைந்துள்ளது.

\section{விவாகமான் ஜோடிகளும் பாலிமீடியாவும்}

தலைமுறைகளுக்கிடையேயானத் தொடர்பு போல் அல்லாமல், விவாகமான ஜோடிகள் செய்திப் பரிமாற்றத்தை ஏற்றுக் கொள்ள மிகவும் தயாராயிருந்தார்கள், செய்தி மற்றும் குரல் தொடர்புகளுக்கு இடையே ஒரு சமநிலை ஏற்பட்டிருந்தது. ஆனாலும், அவர்களுடையத் தொடர்பு ஊடகம் எப்பொழுதுமே தனிப்பட்டதாகவே இருந்தது, அது வெளி உலகத்திற்குத் தெரியக்கூடியதாகவே இல்லை. ஒன்றாக வாழும் ஜோடிகள் தங்களுக்குள் தொடர்பு கொள்ள ஃபேஸ்புக்கை குறைவாகவேப் பயன் படுத்தினார்கள். அவர்கள் எப்பொழுதும் குரல் அழைப்பையோ, எழுத்துச் செய்திகளையோ அல்லது மற்ற சமூக ஊடகங்களை விட அவர்கள் அதிகம் விரும்பும் வாட்ஸ் அப்பையோ உபயோகித்தார்கள். அவர்கள் தொடர்பு கொள்ள தேர்ந்தெடுக்கும் ஊடகம் அந்தரங்கம், பாதுகாப்பு மற்றும் நெருக்கம் போன்ற இயல்புகளைக் கொண்டதாக இருக்கும். கீழே கொடுக்கப்பட்டுள்ள உதாரணங்கள் பெரும்பாலும் கீழ் சமூகப் பொருளாதார வகுப்புகளைக் காட்டிலும்மத்திய வகுப்பை (ஸ்ரீ லக்ஷ்மி மற்றும் கருப்பையாவைத் தவிர) சேர்ந்ததாகவே இருக்கின்றன, ஏனென்றால் முன் சொல்லப்பட்ட பின்புலத்தைச் சேர்ந்த ேோடிகளின் இடையேயானத் தொடர்பு வழக்கமாக குரல் அல்லது எழுத்து மூலமாகவே 
இருக்கிறது (இருவரும் படித்தவர்களாக இருந்தால், அல்லது எப்பொழுதும் குரல் மூலமாக மட்டும்தான்). கல்வி மற்றும் சில தொழில்நுட்பங்களை உபயோகப்படுத்துவதற்கான செலவு இரண்டும் கீழ் சமூகப் பொருளாதார வகுப்பினரது தொடர்பு ஊடகத்தை நிர்ணயிப்பதில் முக்கிய பங்கு அளிக்கின்றன.

\section{சந்திரலேகா மற்றும் ரங்கா}

சந்திரலேகா (சந்திரா) மற்றும் ரங்கா இருவரும் ஒரே ஐடிஈஎஸ் ${ }^{37}$ கம்பெனியில் பணி புரிகிறார்கள், மேலும் அவர்கள் இருவருக்கும் ஒரு நிச்சயிக்கப்பட்ட திருமணம் நடந்திருந்தது (அவர்கள் இரு குடும்பத்தாராலும் ஏற்பாடு செய்யப்பட்டது). திருமணமானவுடனேயே ரங்கா சந்திராவின் கிளைக்கே மாற்றல் வாங்கிக் கொண்டு விட்டார். கம்பெனி திருமணமான ஜோடிகளை ஒரே குழுவில் வேலை செய்ய அனுமதிப்பதில்லை என்பதால், ரங்காவும் சந்திராவும் வெவ்வேறு தளங்களில் அமைந்துள்ள இரண்டு தனித் தனிக் குழுவில் பணி புரிகிறார்கள். ஆனால், அவர்கள் இருவரும் மதிய உணவுக்கு தினமும் சந்திக்கிறார்கள், மேலும் தனது மதிய உணவு வேளையை இதற்கு ஏற்றவாறு அமைத்துக் கொள்கிறார்கள். இருவருக்கும் தங்கள் வேலை அட்டவணை மாற்ற முடியாதது அல்ல என்பது தெரிந்திருந்ததால், அவர்கள் மற்றவர் மதிய உணவுக்குத் தயாராக உள்ளாரா என்பதைத் தெரிந்து கொள்ள ஒரருவருக்கொருவர் செய்தி அனுப்பிக் கொள்கிறார்கள். ஆனால், அவர்கள் இ இந்தத் தொடர்புக்குத் தமது ஃபோனையோ அல்லது வாட்ஸ் அப் மற்றும் ஃபேஸ்புக் போன்றவற்றைக் கூட உபயோகப் படுத்துவதில்லை. இந்தத் தொடர்பு நிறுவனம் முழுமைக்கான உடனடி தூது சேவை மூலமாக செய்யப் படுகிறது, இதன் மூலமாக ஒரு பணிபுரிபவர் மற்றொருவருக்கு ஃபோனை உபயோகிக்காமல் எதைப் பற்றியும் கேட்க தனிப்பட்ட செய்திகளை அனுப்பலாம். ரங்காவும் சந்திராவும் ஒருவருடன் ஒருவர் தொடர்பு கொள்ள இந்த வசதியைப் பயன் படுத்துகிறார்கள். ஏனென்றால் இது அவர்களது மேலதிகாரிகளுக்கு அவர்கள் வேலை நேரத்தில் தனிப்பட்ட செய்திகளைப் பரிமாறிக் கொண்டிருக்கிறார்கள் என்ற எண்ணத்தை ஏற்படுத்துவதில்லை (சந்திராவின் மேலதிகாரி முக்கியமாக இதில் கவனம் உள்ளவராக இருக்கிறார்), இது ஒரு எளிமையான, மற்றும் ரகசியமான தொடர்பு வழியாகும், இவற்றை இந்த ஜோடிகள் பிறருடைய 
கவனத்தை ஈர்க்காமல் செய்ய முடியும். இதை அவர்கள் மதிய உணவு நேரத்தை நிர்ணயிக்க மட்டுமல்லாமல், நாளுக்கான வேலையை முடிக்கும் நேரத்தைப் பற்றிமுடிவு செய்யவும், சில நேரங்களில் வீட்டு சம்பந்தமான விஷயங்களைப் பற்றி விவாதிக்கவும் உபயோகிக்கிறார்கள்.

\section{ஆர்த்தி மற்றும் அகிலன்}

அகிலன் (வயது 31) மற்றும் ஆர்த்தி (வயது 30), தனது ஐந்து வயது மகனுடன் பஞ்சகிராமியில் வசிக்கும் இளைய வயது தம்பதியினர். அவர்கள் ஏறக்குறைய எட்டு வருடங்களுக்கு முன் தம் பெற்றோர்களின் விருப்பத்திற்கு எதிராக கலப்பு மணம் புரிந்தவர்கள். அப்பொழுதிலிருந்தே அவர்கள் தம் பெற்றோரிடமிருந்து விலக்கி வைக்கப் பட்டுள்ளனர். காலம் எந்தப் புண்ணையும் ஆற்ற வல்லது என்றாலும், அது இவர்கள் விஷயத்தில் நடப்பதாய் இல்லை. அவர்கள் தனிக் குடித்தனமாக வாழ்கிறார்கள், ஆர்த்தியின் தாய் ${ }^{38}$ மற்றும் சகோதரி மட்டும் நேரம் கிடைக்கும் பொழுது வந்து செல்கிறார்கள். பிற உறவினர்களும் அவர்களது திருமணத்திற்கு அனுகூலமாக இல்லை என அவர்கள் நம்புவதால், அவர்கள் இருவரும் ஐடி கம்பெனியில் பணி புரிந்தாலும், இருவரும் ஃபேஸ்புக்கில் இல்லை. ஃபேஸ்புக்கில் உறுப்பினர் ஆகுதல் இவர்களை இந்த உறவினர்களுடன் தொடர்பு படுத்தும் என்பதாலும், இதனால் இக்கட்டானக் கேள்விகளுக்கு ஆளாகக் கூடும் என்பதாலும், அவர்கள் இந்த தளத்தைத் தவிர்க்கிறார்கள். ஆனால் அவர்கள் லிங்க்ட் இன், மற்றும் பிற மன்றங்கள், குழுக்கள் (கம்ப்யூட்டர் மற்றும் பணி சம்பந்தப்பட்டவை) தொழில் நுட்ப சமூக நெட்வொர்க்கிங் தளங்களில் உறுப்பினர்களாக உள்ளார்கள். அவ்ர்கள் இருவரிடமும் ஒரு சாம்சங் ஃபோனும் ஒரு போஸ்ட் பெயிட் சியூஜி (க்ளோஸ்ட் யூஸர் குரூப் - இதில் இவர்கள் இருவர் மட்டுமே குரூப்பாக இருக்கிறார்கள்) கனெக்ஷனும் (வோடாஃபோன்) இருக்கின்றன.

பணியின் போது ேோடிகள் இ இருவரும் தங்களுக்குள் அழைக்குமுன் எழுத்துச் செய்திகளைப் பரிமாறிக் கொள்கிறார்கள். 2013 நவம்பர் 22, வெள்ளிக்கிழமையன்று நடந்த ஒரு மாதிரி தொடர்பு இவ்வாறு இருந்தது:

ஆர்த்தி: சாப்பிட்டாச்சா?

அகிலன்: ஹ்ம்ம்ம் நீ? 
ஆர்த்தி: இன்னும் இல்ல, நான் இப்போ உன்னைக் கூப்பிடலாமா?

அகிலன்: இன்னும் 10 நிமிஷம் கழிச்சுப் பண்ணட்டுமா?

ஆர்த்தி: சரி

அகிலன்: ஏதாவது அவசரமா?

ஆர்த்தி: இல்ல, தயிர் கொஞ்சம் புளிக்குது - (இந்தியாவில் தயிர் சாதம் வழக்கமான மதிய உணவு ஆகும்)

அகிலன்: தெரியும், கண்டுக்கல, அவசரமா சாப்பிட்டேன்

அகிலன்: சரி, 10 நிமிஷத்துல கால் பண்றேன்

ஆர்த்தி: சரி

இருவரும் ஐடி கம்பெனியில் பணி புரிவதால், அவர்கள் உடனடியாக அழைத்து விட முடியாது என்பதைத் தெரிந்து வைத்திருக்கிறார்கள், ஏனென்றால் ஒருவர் ஓய்வில்லாமல் இருக்கலாம், ஆகையால் இருவரும் மற்றொருவருக்கு அழைக்கும் முன் செய்தி அனுப்புகின்றனர். அவர்களுடைய செய்திகள் இப்பொழுது வாட்ஸ் அப்பில் குடியேறி விட்டன.

\section{தீபா மற்றும் வாசு}

வாசு (37 வயது) மற்றும் தீபா (35 வயது), இவர்களும் கலப்பு மணம் புரிந்தவர்கள், ஜோடிகளின் விஷயமும் இது போன்றதுதான். வாசு, இவரை நாம் 2ம் அத்தியாயத்தில் சந்தித்திருக்கிறோம், ஒரு சொந்தத் தொழில் செய்பவர், தீபா ஒரு இல்லத்தரசி. அகிலன் மற்றும் ஆர்த்தியைப் போல், இவர்கள் இருவர் குடிம்பங்களும் இவர்களது திருமணத்தை எதிர்த்தது. ஆயினும், இந்த ஜோடிக்கு திருமணமாகி 10 வருடங்கள் ஆகின்றன, மேலும் அவர்களுக்கு ஆரம்பப் பள்ளிக்குச் செல்லும் இரண்டு குழந்தைகள் உள்ளனர். வாசு, தீபா இருவரும் ஃபேஸ்புக் மற்றும் வாட்ஸ் அப்பில் இருக்கின்றனர். இருவரிடமும் சாம்சங் ஸ்மார்ட் ஃபோன் இருக்கிறது, இதைத் தவிர வாசு ஒரு ஐஃபோனையும் உபயோகிக்கிறார் (தொழில் விஷயமாக துபாய்க்கு சென்ற போது வாங்கப் பட்டது).

ஆயினும், தினசரி தொடர்புகளில் இருவரும் ஃபேஸ்புக்கை உபயோகிப்பதைக் காட்டிலும், ஒருவரை ஒருவர் அழைக்கவோ அல்லது வாட்ஸ் அப்பில் விரைவு செய்திகள் அனுப்பவோ விரும்புகிறார்கள். தீபா வாசுவை மதியம் 1லிருந்து 1.30க்குள்ளாகவே அவர் மதிய உணவு உண்ணும் நேரத்தில் அழைக்கிறார் (அவர் தான் உணவு அருந்தும் 
நேரம் பற்றி குறிப்பாக இருக்கிறார், ஏனென்றால் அவருக்கு வயிற்றில் அல்சர் நோய் ஆரம்பக் கட்டத்தில் இருக்கிறது). ஆகையால் வாசுவை 1லிருந்து 1.30க்குள் தொடர்பு கொள்ளமுடியும் என்பது தீபாவிற்குத் தெரிந்திருக்கிறது; அல்லது தீபா செய்தியை அனுப்பி விட்டு வழக்கமாக அவருடைய அழைப்பிற்குக் காத்திருக்கிறார். அவர்களுடைய உரையாடல் வழக்கமாக தினசரி வேலைகளைப் பற்றியே அமைந்திருக்கிறது. தீபா மற்றும் வாசுவிற்கு இடையே வாட்ஸ் அப் செய்திகள் மூலம் நடந்த ஒரு உரையாடல் பின் வருமாறு:

தீபா: வரும்போது மில்க் வாங்கணும் வாசு: சரி.

அவர்களது சுருக்கமான வாட்ஸ் அப் உரையாடலின் இன்னொரு உதாரணம் பின் வருமாறு:

தீபா: மதியம் சொன்னதை மறக்காதீங்க. வாசு: மறக்கல

\section{ஸ்ரீ லக்ஷ்மி மற்றும் கருப்பையா}

ஸ்ரீ லக்ஷ்மி ஒரு பட்டதாரி, இவர் ஒரு கால் சென்டரில் பணி புரிகிறார். அவரது 25 வயது கணவர், கருப்பையா, உள்ளூர் ஐடி கம்பெனிகளுக்கு சிறப்பு சேவை புரியும் டாக்சி கம்பெனியில் டிரைவராக இருக்கிறார். அவர் ஸ்கூல் படிப்பை முடிக்காவிட்டாலும், ஆங்கிலத்தில் நன்கு பேசுகிறார் - அது அவர் தனது வாடிக்கையாளார்களுடன் (வெளிநாட்டவர் மற்றும் இந்தியர் இருவரும்) தொடர்பு கொள்வதற்காக கற்றுக்கொண்ட திறன். கருப்பையா மற்றும் ஸ்ரீ லக்ஷ்மி இருவரிடமும் சாம்சங் ஸ்மார்ட்ஃபோன் இருக்கிறது மேலும் அவர்கள் வாட்ஸ் அப்பின் ஆர்வமுடைய உபயோகிப்பாளர்கள். கருப்பையாவிற்கு தாம் நாள் முழுவதும் செல்லும் இடங்களில் தம்முடைய ஃபோட்டோவை எடுத்துக் கொள்வது பொழுதுபோக்காக இருக்கிறது; அவர் இந்த உருவங்களை ஸ்ரீ லக்ஷ்மிக்கும் மற்றும் சில நண்பர்களுக்கும் அனுப்புகிறார். அவர் வாட்ஸ் அப்பில் அவர்கள் ஒவ்வொருவருடனும் தொடர்பு கொள்ள தனித்தனி சானல்களை வைத்திருக்கிறார் (ஸ்ரீ லக்ஷ்மிக்கு என்று தனியாக ஒன்று, மற்ற நண்பர்களுக்கு என்று ஒரு குழுப் பேச்சு). கருப்பையாவுடைய வாட்ஸ் அப் படம் அவர் 
ஐடி கம்பெனிகள், ஐந்து நட்சத்திர ஹோட்டல்கள், பல்கலைக்கழகக் கட்டிடங்கள் போன்றவை முன் நிற்பனவையாக இருக்கின்றன. ஸ்ரீ லக்ஷ்மி தன்னுடைய பங்கிற்கு அவர் வேலை நேரத்தில் ஓய்வாக இருந்தால் குரல் செய்திகள் மூலம் இந்தப் படங்களுக்கு குறிப்பு அளிக்கிறார், அல்லது அவர் வேலையாக இருந்தால் 'ஸ்மைலி' அல்லது 'தம்ஸ் அப்' சின்னங்கள் மூலமாகவோ பதிலளிக்கிறார்.

ஸ்ரீலக்ஷ்மி கருப்பையாவிற்கு ஏதும் வீட்டு வேலைகள் கொடுக்க விரும்பினால், அவர் வழக்கமாக அவரைக் கூப்பிடுவொர். அவர் பதிலளிக்கவில்லை என்றால், அவர் காரோட்டிக் கொண்டிருக்கிறார் என்பதைப் புரிந்து கொண்டு, திரும்பக் கூப்பிடச் சொல்லியோ அல்லது ஒரு குறிப்பிட்ட வேலையை செய்யச் சொல்லியோ அவருக்கு வாட்ஸ் அப் குரல் செய்தி அனுப்புகிறார். அவர்கள் விஷயத்தில் எழுத்து செய்திகள் அனுப்புவது நடப்பதில்லை; அவர்கள் குரல் செய்திகளையோ அல்லது காட்சி சின்னங்களையோ உபயோகிக்கிறார்கள்.

\section{இந்திரா மற்றும் அரவிந்த்}

43 வயதான இந்திரா ஒரு இல்லத்தரசி மற்றும் இரண்டு குழந்தைகளின் தாயார். அவருடைய கணவர், 48 வயதான ஒரு தொழில் முனைவோர், அவருக்குச் சொந்தமாக ஒரு சைக்கிள் ஷோரூம் இருக்கிறது. இந்திரா வீட்டு வேலை அனைத்தையும் பார்த்துக் கொண்டாலும், அரவிந்த்தும் சில நேரங்களில் இதில் பங்கெடுத்துக் கொள்கிறார், குறிப்பாக அவர் வேலையிலிருந்து வீடு திரும்பும் நேரங்களில். அவர் தனது காரியாலயத்திற்கு அருகில் நடைபாதை வியாபாரிகள் புதியப் பழங்களையோ அல்லது காய்கறிகளையோ விற்பதைக் கண்டார் என்றால், அவர் அதை வாங்குவதற்கு முன் இந்திராவின் ஒப்புதலுக்காக, அவைகளை படம் எடுத்து வாட்ஸ் அப் மூலம் இந்திராவிற்கு அனுப்புகிறார்.

வார இறுதி நாட்களில் இந்திரா சமையல் செய்வதில் மும்முரமாக இருக்கும் பொழுது, தம் கணவரிடம் ஏதாவது மசாலாப் பொருட்கள் வாங்கி வரச் சொன்னாலும் இதுவே நடக்கிறது. ஒரு வேளை அரவிந்த் வாங்க வேண்டியதை மறந்து விட்டாலோ அல்லது அதைப் பற்றி ஏதும் குழப்பம் ஏற்பட்டாலோ, அவர் அந்த மசாலா சாமானின் படத்தை எடுத்து அதை இந்திராவிற்கு வாட்ஸ் அப்பில் அவர் சம்மதத்திற்காக அனுப்புகிறார். இந்திராவும் தங்கள் பரஸ்பர 
நண்பர்களுக்கு பரிசு வாங்கும் பொழுது (அவர்களது, பிறந்த தினம் அல்லது திருமண தினம் போன்றவைகளுக்கு) அரவிந்த்திற்கு அவரது யோசனை பிடித்திருக்கிறதா மற்றும் விலைக்கு ஒத்துக் கொள்கிறாரா என்பது தெரிய இது போன்றே செய்கிறார். ஆனால் ஒப்புதலுக்காக படங்கள் அனுப்புவதைத் தவிர மற்றத் தொடர்புகள் எல்லாம், மற்ற ஊடகங்களைக் காட்டிலும், ஃபோன் மற்றும் குரல் மூலமாகவே நடைபெறுகின்றன.

\section{வசுதா மற்றும் மகேஷ்}

58 வயதான வசுதா தனது மகளின் இரண்டாவது பிரசவத்திற்கு உதவி செய்ய இப்பொழுது யூஎஸ்ஸில்39 இருக்கிறார். 62 வயதான இவர் கணவர் மகேஷ், ஒரு மேலாண்மை நிறுவனத்தில் தம் தொழிற்பண்பட்டவர் பணியை முன்னிட்டு இந்தியாவில் தங்க நேர்ந்தது. இந்த தம்பதி தினமும் ஃபோனிலும், இரண்டு அல்லது மூன்று நாட்களுக்கு ஒரு முறை ஸ்கைப்பிலும் தொடர்பு கொள்கிறார்கள். இந்திய நேரப்படி காலை 7 அல்லது மாலை 9 மணிக்கு ஃபோன் அடித்தால், வசுதாதான் அழைப்பது என்பது மகேஷுக்குத் தெரியும்.

வசுதா இந்தியாவை அழைக்க யூஎஸ்ஸில் ஒரு விஓஐபி ஃபோனை உபயோகித்து வந்தார். இதே போல் யூஎஸ்ஸில் அவரிடம் ஸ்மார்ட் ஃபோன் இல்லாதக் காரணத்தால், மகேஷுக்கு ஸ்கைப் செய்வதற்கான சரியான நேரத்தைத் தெரியப் படுத்த தன் ஐபாட் மூலம் மெயில்களை அனுப்பிக் கொண்டிருந்தார். ஆரம்பத்தில் அவர்கள் ஃபோன் அழைப்புக்குச் செய்தது போல, ஸ்கைப் செய்ய சமய நிர்ணயம் எதுவும் செய்யவில்லை. காலப் போக்கில் இதற்கான அட்டவணையும் ஏற்பட்டது, வசுதாவின் ஐபாட் மகேஷுடன் ஸ்கைப் செய்ய அவருக்கு மிகவும் உதவியாக இருந்தது. மகேஷும் தனது ஆசிரியப்பணியிலிருந்து சிறிது ஓய்வாக இருப்பதால், அழைப்புகள் பெரும்பாலும் புதன்கிழமை, வெள்ளிக்கிழமை மற்றும் ஞாயிற்றுக்கிழமைகளிலேயே விடுக்கப்பட்டன. சில தனிப்பட்ட உரையாடல்களைத் தவிர, அவர்களது பெரும்பான்மையான உரையாடல்கள், ஒரு குறிப்பிட்ட உண்வை எப்படித் தயாரிப்பது என்பதைப் பற்றியே இருந்தன. வசுதா மகேஷ் நன்றாகச் சாப்பிட வேண்டும் என்பதிலும், மேலும் அவர் என்னென்ன உணவுகள் சமைத்தார் என்பதைத் தெரிந்து கொள்வதிலுமேக் குறியாக இருந்தார். 


\section{ராதிகா மற்றும் சந்தானம்}

33 வயதான ராதிகாவும், 36 வயதான சந்தானமும், தம்பதிகள் மட்டுமல்ல, ஒரு தொழில் முனைவில் சம பங்குதாரர்கள் கூட. அவர்கள் ஒரு ஃபேஷன் உடைகள் ஷோரூமும் ஒரு முடி திருத்தகமும் நடத்துகிறார்கள். அவர்களுடைய மேலாண்மை மாதிரி பிரிக்கப்பட்டதாக இல்லை, அதாவது இருவரும் இரு தொழில்களையும் பார்த்துக் கொண்டார்கள். சந்தானம் நிதி, போக்குவரத்து மற்றும் செயல்பாடு ஆகியவற்றில் சம்பந்தப்பட்டிருந்தார், ராதிகா அதிகம் சந்தைப் படுத்துதல், விற்பனை மற்றும் மனித வளம் போன்றவற்றில் இ இணைந்திருந்தார். ஆயினும், வேலைகளின் இந்தப் பாகுபாடு கண்டிப்பானதாக இல்லை, இருவரும் கடைசியில் அனைத்தையும் கவனித்துக் கொண்டனர்.

இத்தம்பதிக்கு தனிப்பட்ட ஒரு ஃபேஸ்புக் பக்கம் இருந்தது, ஆனால் இதை அவர்கள் பரஸ்பரத் தொடர்பை விட தங்கள் நண்பர்களுடன் தொடர்பு கொள்ளவே உபயோகித்து வந்தார்கள். அவர்களுடைய பெரும்பாலான தொடர்பு குரல் செய்திகளாகவோ அல்லது எழுத்துச் செய்திகளாகவோதான் இருந்தன; ராதிகா தனிப்பட்ட செய்திகள் தொழில் சம்பந்தப்பட்ட செய்திகளுடன் சேர்ந்து விடுவதால், இது ஒரு குழப்பமாக இருப்பதாகக் கூறுகிறார். ஆயினும், அவர்கள் இப்பொழுது வாட்ஸ் அப்பில் ஒரு தொழில் குழுவை அமைத்து விட்டனர். தனிப்பட்ட செய்திகளையும் ஒருவருக்கொருவர் வாட்ஸ் அப் மூலமாகவே அனுப்பிக் கொள்கிறார்கள், ஆனால் தொழில் முறை செய்திகள் அவர்களது கம்பெனி பெயரை உடையக் குழுப் பெயரை உபயோகப்படுத்தி அனுப்பப் படுகின்றன, இதனால் குழப்பங்கள் தவிர்க்கப்படுகின்றன.

இந்த நிகழ்வு ஆய்வுகளில் பார்க்கப்பட்டது போல, தம்பதிகளுக்கிடையேயான தொடர்பு நெருக்கம், அன்பு மற்றும் அக்கறை ஆகியவற்றின் பாங்கைக் காட்ட முயன்றிருக்கிறது வெளி உலகத்திற்கு வீணானதாகத் தோன்றும் உரையாடல்களில் கூட. இதில் வெளிப்படும் இன்னொரு நுட்பமான பாங்கு தொடர்பு அலைவரிசைகளோடு செய்யும் பேரங்கள், அவைகள் சம்பந்தப்பட்ட இரு பாலருக்கும் எது பொருந்தும் மற்றும் எது வசதியாக இருக்கும் என்பதைப் பொறுத்தே இவைத் தேர்ந்தெ-

டுக்கப் படுகின்றன. தலைமுறைகளுக்கிடையேயானத் தொடர்பு ஒரு கட்டுப்பாட்டு பாங்கினை வெளிக்காண்பிக்கும் நேரத்தில், ஜோடிகளின் விஷயத்தில் ஊடகத்தின் தேர்வுக்கு முன் ஒரு விதமான ஒப்புக் கொள்ளப்பட்ட காரண விளக்கம் 
தென்படுகிறது. மேலேக் கூறப்பட்ட நிகழ்வுகளில் கூறப்பட்டது போல பயன் படுத்த வேண்டிய தளங்களின் தேர்வும் உரையாடலை ஆரம்பிப்பவரைப் பொறுத்து இல்லாமல், ஒவ்வொரு பங்குதாரர்களின் காலத்தையும், இடத்தையும் புரிந்து கொள்வதின் மூலமாகவே நிர்ணயிக்கப் படுகிறது.

இங்கு வெளிப்படும் இன்னொரு நுட்பமா பாங்கு என்னவென்றால் தொடர்பு கொள்ளும் பொழுது ஆண்களை விடப் பெண்களுக்கே ஏற்படும் கவனம். முன்னரே கூறியது போல, நெருக்கம் மற்றும் பாதுகாப்பு இவற்றைக் கொண்ட அலைவரிசைகளை உடைய தளங்களே மற்றவற்றை விட அதிகமாக விரும்பப் பட்டாலும், இது பயனர்களை, கீழ்க்கண்ட நிகழ்வுகளில் காட்டுவது போல், ஒரு குறிப்பிட்ட தளத்தைப் ஃபேஸ்புக் - பொறுத்த வரை மக்களின் ஒரு பெரிய நெட்வொர்க்கின் முன்னோ அல்லது பொதுவிலோ தமது நெருக்கத்தை வெளிக்காட்டுவதிலிருந்து விலக்குவதில்லை.

\section{தம்பதிகளுக்கான ஒரு நிகழ்த்துத்திறத் தளமாக ஃபேஸ்புக்}

ஃபேஸ்புக் வாரிசுரிமை உள்ள உறவுகளைப் போலவே, ஜோடிகளை ஒரு பரந்த நெட்வொர்க்கின் முன்னிலையில் தம் நெருக்கத்தை வெளிக்காட்ட அனுமதிக்கும் ஒரு நிகழ்த்துத் திறத் தளமாகவே இயங்குகிறது. தம்பதிகள் இணைந்து வாழும் போதும், அவர்களுடைய பரஸ்பர அன்பு மற்றும் அர்ப்பணிப்பின் செயலாக்கம் ஃபேஸ்புக்கில் வெளிக் காட்டப்பட்டது என்பதை பின் வரும் நிகழ்வுகள் காட்டும். ஆயினும், இங்கு இது உலகத்தின் பார்வைக்கான செயலாக்கமாகவே இருந்தது, அவர்களுக்குள்ளானத் தனிப்பட்ட தொடர்பாக இல்லை.

ஒரு விழுமியக் குடும்பத்தின் தரநெறிசார் உரைகளைப் போல ஒரு விழுமிய தம்பதி அல்லது விழுமிய திருமணம் பற்றிய உரைகள், தீவிரமாக எடுத்துக் கொள்ளப் படுகின்றன. ஃபேஸ்புக்கின் மூலமாக இந்த விழுமிய கூறுகள், குடும்ப அளவிலேயே புதைந்து போவதற்கு பதிலாக, உலகத்தின் பார்வைக்காக வெளிப்படுத்தப் படுகின்றன. ${ }^{40}$ வாரிசுரிமை உறவு நிகழ்வுகளைப் போல, ஃபேஸ்புக் அப்படிப்பட்ட 'உலகளாவிய' வெளிப்பாடுகள் ஒரு தாக்கத்தை ஏற்படுத்தக்கூடிய ஒரு தளமாகிறது. பின்வரும் மூன்று நிகழ்வு ஆய்வுகளும் செயலாக்கத்தின் உதாரணங்களைக் கொடுக்கின்றன. 


\section{சரண்யா மற்றும் ஸ்ரீஜித்}

24 வயதான சரண்யா மற்றும் 25 வயதான ஸ்ரீஜித் ஒரு நிதி நிறுவனத்தில் பணி புரிந்த போது காதல் வயப்பட்டார்கள். அவர்கள் தத்தம் பெற்றோர்களின் ஒப்புதலுடன், அவர்கள் ஒருவரை ஒருவர் சந்தித்த ஒரு வருடத்திற்குள்ளாகவே திருமணம் புரிந்தனர். ஃபேஸ்புக்கில் அதிக செயல்பாட்டுடன் இருந்த சரண்யா, தானும் ஸ்ரீஜித்தும் தாம் பழக ஆரம்பித்தக் காலத்திலிருந்தே சேர்ந்திருந்த படங்களை போஸ்ட் செய்தார். அவர்களுடைய திருமணம் முடிந்த உடனேயே, அவரது ஃபேஸ்புக் ப்ரொஃபைல் அந்த நிகழ்ச்சியின் படங்களால் நிறைந்தது. ஒரு மாதத்திற்குப் பிறகு அது தம்பதிகள் சேர்ந்து வெளியே செல்லும் படமாக மாறியது. இதுவரை ஸ்ரீஜித் சரண்யாவின் ப்ரொஃபைலைப் பற்றி குறிப்பு ஒன்றும் எழுதியதில்லை, அவர் ஃபேஸ்புக்கில் அதிக செயல்பாட்டுடனும் இல்லை. ஆனால் திருமணத்திற்கு சில மாதங்களுக்குப் பிறகு அவர் தாம் வேலை நேரத்தில் தம் மனைவி இல்லாக் குறையை எவ்வளவு தூரம் உணர்ந்திருக்கிறோம் என்பதைப் பற்றி ஃபேஸ்புக்கில் செய்திகள் போஸ்ட் செய்ய ஆரம்பித்தார் - வாஸ்தவத்தில் அவருடைய காரியாலயம் சரண்யாவின் காரியாலயத்திலிருந்து ஒ ஒரு மாடிக் கீழேயே இருந்தது. இருவரும் தாம் முதல் நாள் மாலை ஒரு உணவு விடுதியில் இரவு உணவை எவ்வளவு மகிழ்ச்சியுடன் உண்டோம் மற்றும் இரவில் வீட்டிற்குக் காரில் பயணம் ஆகியவற்றைக் கூறும் செய்திகளை ஃபேஸ்புக்கில் வெளியிட்டனர்.

\section{சாயா மற்றும் வருண்}

சரண்யா மற்றும் ்ரீஜித்தின் நிலையைப் போலவே சாயா மற்றும் வருணின் நிலையும் (இவர்கள் இருவரும் இருபதுகளின் நடுவில் இருப்பவர்கள்தான்). அவர்கள் சேர்ந்து எடுத்துக் கொண்ட விடுமுறைப் படங்களைத் தவிர, அவர்கள் ஒரு குறிப்பிட்ட இடத்தை எவ்வாறு ஒன்றாக மகிழ்ச்சியுடன் அனுபவித்தார்கள் அல்லது சேர்ந்து ஒரு ஐஸ்கிரீமை உண்டார்கள் என்பதும் கூட அவர்களது ஃபேஸ்புக் ப்ரொஃபைலில் போய் விடும். இருவரும் அந்த போஸ்ட்டுகள் ஞாபகங்களாக வேண்டும் எனக் கூறினார்கள், ஆனால் இவை எப்பொழுதும் ஃபேஸ்புக்கில் மற்றவர்கள் குறிப்பெழுதுவதற்கான ஒரு 
இடமாகக் கருதுவதற்கு பதிலாக, தம்பதிகளின் ஒரு உரையாடலினால் தொடரப்பட்டிருக்கும்.

\section{சந்தியா மற்றும் கோபால்}

23 வயதான சந்தியா கூறுகிறார் அவரது கணவர் 28 வயது கோபால் எப்பொழுதும் காதல் நிறைந்தவராகவும், காதலைப் பொதுவில் வெளிப்படுத்த விருப்பம் உள்ளவராகவும் இருந்தார். சந்தியா தாம் முன்பு சங்கடப்பட்டதாகவும், ஆனால் கோபாலுடன் வாழும் பொழுது அதற்கு பழக்கப்பட்டு விட்டதாகவும் தெரிவிக்கிறார். ஒரு மனித வள அதிகாரியான சந்தியா தாம் பணி புரியும் ஐடி கம்பெனிக்காக ஆட்களைப் பணியிலமர்த்த நாடெங்கிலும் பயணம் செய்கிறார். ஒரு சாஃப்ட்வேர் ப்ரோக்கிராமர் தொழிலில் இருக்கும் கோபால் அதிகம் பயணம் செய்வதில்லை, சந்தியாவின் பயணங்களின் போது அவர் வீட்டிலேயே இருக்கிறார். அவரது ஒவ்வொரு பிரயாணத்தின் போதும், கோபால் தம் மனைவி இல்லாக் குறையை உணர்த்த யூ-ட்யூப்பிலிருந்து ஒரு தமிழ் (கோலிவுட்) ${ }^{41}$ அல்லது ஹிந்தி (பாலிவுட்) ${ }^{42}$ காதல் பாட்டைபோடு|ெதை வழக்கமாகக் கொண்டிருந்தார். இந்த போஸ்ட்கள் சந்தியாவை சங்கடப்படுத்தின, ஆனாலும் அவர் தம் கணவர் தம்மை மிகவும் நேசிக்கிறார் என்ற உண்மையைச் சாதகமாகப் புரிந்து கொண்டு இதற்குத் தம்மை இணங்க வைத்துக் கொண்டார்.

இந்த அத்தியாயத்தில் முன்னர் நாம் தம்பதிகளின் தினசரி நெருக்கமான உரையாடல்களைப் பார்த்தோம். இந்தப் பகுதியில் நாம் இந்த உரையாடல்களின் ஒரு அதிகரிக்கப்பட்ட தேர்வு ஒரு பெரிய நெட்வொர்க்கின் முன் எவ்வாறு வெளிக்காட்டப்படுகிறது என்பதைப் பார்த்தோம், ஆனால் சாயா கூறியவாறு, இவ்விதமான போஸ்ட்கள் அவர்கள் சேர்ந்திருக்கும் நேரங்களின் நினைவுகளாகவும் இ இருக்கின்றன. ஆகையால் இந்த போஸ்ட்களை வெளி உலகத்துடனான ஒருத் தொடர்பாக மட்டும் பார்க்க முடியாது, ஆனால் ஒருவருக்கொருவர் பரஸ்பர தொடர்பாகவும் மற்றும் எதிர்காலத்தில் குறிப்பிட நினைவுகளின் ஒருங்கிணைப்பாகவும் பார்க்க வேண்டும். ${ }^{33}$ இதில் சம்பந்தப்பட்டுள்ள ஒருவரால் மட்டுமே இந்த விதமான போஸ்ட்களை ஆரம்பிக்க முடியும் என்றாலும், வெகு விரைவிலேயே, அவர்கள் ஆரம்பத்தில் சங்கடப்பட்டால் கூட, தமது கூட்டாளியின் சம்மதத்தையும் பங்கேற்பையும் பெறுகிறார்கள்,ஆனால், இதன் அர்த்தம் இவ்விதமாக போஸ்ட் 
செய்த அனைத்து தம்பதிகளுமே இந்த மாதிரியான சுழற்சிக்கு உள்ளானார்கள் என்பது அல்ல. ஒரு கூட்டாளி இந்த விதமான போஸ்ட்களை தடை செய்த நிகழ்வுகளும் உண்டு. அவர்கள் தம் நெருக்கமான தருணங்களை தமக்காகவே வைத்திருந்தார்கள் அல்லது குறிப்பிட்ட வலுவான பந்தமுள்ள நெட்வொர்க்கிடம் மட்டும் பகிர்ந்து கொண்டார்கள்.

\section{சகோதர உறவுகளுக்குள் தொடர்பு}

தம்பதிகளுக்குள்ளான அ அல்லது தலைமுறைகளுக்கு இடையேயான தொடர்புகளிலிருந்தூ சகோதர உறவுகளுக்கான தொடர்புகள் வேறுபட்டப் பாணிகளைக் கொண்டிருந்தன. பஞ்சகிராமியில் சேர்ந்து வாழும் சகோதர உறவுகளுக்கு இடையேயான தொடர்புகள் முக்கியமாக நேரடியானதாகவோ அல்லது ஃபோன் மூலமாகவோ இருந்தது. அவர்களில் ஒருவர் திருமணமாகி பிரிந்து சென்றவுடன் இது பேஸ்புக்கிற்கோ அல்லது வாட்ஸ் அப்புக்கோ மாறி விடுகிறது, முக்கியமாக ஒரு அல்லது இரு உடன் பிறப்புகளும் பெண்ணாக இருந்தால். ஒருவரை ஒருவரோ, அல்லது ஒருவர் மற்றவரின் குழந்தைகளையோ ஃபேஸ்புக்கில் ஆதரிப்பது ஒரு சாதாரணக் காட்சி ஆகும், குறிப்பாக ஒருவர் பெண்ணாக இருக்கும் பட்சத்தில். மற்றொரு விதத்தில் உடன்பிறப்புக்களுக்கிடையில் ஒரு தொடர்புத்தளம் என்ற வடிவில் ஃபேஸ்புக் மற்றும் வாட்ஸப்பின் பயன்பாடு சகோதரர்களை விட சகோதரிகளுக்கிடையேயோ அல்லது சகோதர சகோதரிகளுக்கிடையேயோ அதிகம் நடந்தது.

சகோதரர்களுக்கு இடையேயான வயது வித்தியாசம் நான்கிற்கு அதிகமாக இருந்தால், அவர்களுக்கு தனி நண்பர்கள் குழு இருக்கும் மற்றும் இருவரும் ஃபேஸ்புக்கில் இருந்தாலும், அவர்கள் வாட்ஸ் அப்பில் தனிப்பட்ட முறையில் தொடர்பு கொண்டாலும், அவர்கள் ஒருவருடனொருவர் நண்பர்களாவதில்லை என்பதைப் பற்றிய ஒரு தெளிவான போக்கு வெளியாயிற்று. இது குறிப்பாக திருமணமாகாத இளவயது சகோதரர்கள் நடுவே தெளிவாகக் காணப்பட்டது. ஆனால், திருமணமாகி குழந்தைகளுடன் வாழும் சகோதரர்கள் நடுவே இந்த நிலை மாறி விட்டது; அவர்கள் இப்பொழுதும் ஃபேஸ்புக்கில் இருந்தால் ஒருவருடனொருவர் நண்பர்களாகி விட்டனர். இது முக்கியமாக கீழ் மத்திய வகுப்பைச் சார்ந்தவர்களிடம் காணப்பட்டது. ஆனால், மேல் மத்திய வகுப்புகளில், குடும்பம் 
முழுவதும் (சகோதரர்களின் மனைவிகளும் கூட) ஃபேஸ்புக் நண்பர்களாக இருப்பர். வழக்கமாக கீழ் மத்திய வகுப்புக் குடிம்பங்களின் சகோதரர்களால், அவர்கள் மனைவிகள் அதில் இல்லாத காரணத்தால், வாட்ஸ் அப் குழுக்கள் அமைக்கப் படுவதில்லை. ஆனால் ஒரு மேல் மத்திய வகுப்புக் குடும்பத்தில் சகோதரர்களின் குடும்பங்களுக்கு நடுவில் ஒரு வாட்ஸ் அப் குழு என்பது ஒரு வழக்கமான நிகழ்வாகவே இருக்கிறது, இவை எல்லாவற்றுக்கும் மேலாக இந்தக் குழு சகோதரர் ஒருவரின் மனைவியால் தம் புகுந்த வீட்டார் துணையுடன் அமைக்கப் படுகிறது.

ஆனால், சகோதர-சகோதரி உறவில், விஷயங்கள் வேறுபட்டவனவாக இல்லை. இந்த ஆண்கள் பொதுவாக ஃபேஸ்புக்கை குடும்பப் பெண் உறுப்பினர்களைப் பொறுத்த வரையில் ஒரு சாத்தியமான ஆபத்து உபகரணமாக உணர்ந்திருந்தாலும், அவர்கள் தன்னுடைய அண்மை அல்லது விரிவு நிலைக் குடும்பத்திற்கு வெளியே உள்ள பெண்களின் விஷயத்தில் தம் நிலைப்பாட்டிலிருந்து மாறு பட்டார்கள். அவர்கள் குடும்பத்துப் பெண்கள் சமூக ஊடகங்களில் அடைந்துள்ள புலப்படும்தன்மை ஒரு நல்ல பெண்ணியல்பின் உதாரணமாக ஏற்றுக் கொள்ளப்படவில்லை; ஒரு நல்ல தமிழ், ஏன், இந்தியப் பெண்ணிற்கான தரநெறி சார் கொள்கையாகக் கருதப்படுவதில்லை. ${ }^{44}$ பாரதப் பண்பாட்டின் சூழலுக்கேற்ற பெண்ணியல்பைப் பற்றிய உரைகள், குடும்ப பெண் உறுப்பினர்களுக்காக ஆண்கள் இதை எப்பொழுதும் உறுதி செய்ய முற்படும் விதங்கள் ஆகியவை அரிதானதாக இல்லை. இவ்வாறான உணர்வுகள் பெரும்பாலும் பாரம்பரிய சமூகக் கோட்பாடுகளையும், ஆண்கள் சார்ந்திருக்கின்ற சாதி அடிப்படையிலான சில அரசியல் கட்சிகளின் கொள்கைகளையும் பிரதிபலிக்கின்றன.

ஆயினும், (இரண்டாம் அத்தியாயத்தில் விவாதிக்கப்பட்ட), ரவியின் நிகழ்வைப் போல, சகோதரிகள் திருமணமாகிச் சென்ற பின் நிலைமை மாறி விடுகிறது, அங்கு அவரது சகோதரி திருமணத்திற்குப் பின்னும் ஒவ்வொரு வாரமும், தம் குடும்பத்துடனான அழைப்பை ஆரம்பித்து வைக்கிறார். இதில் இன்னொரு உதாரணம், ரஞ்சித் மற்றும் ஸ்ரீலதாவின் நிகழ்வு, ஸ்ரீலதா திருமணமாகி பஹ்ரைனில் குடியேறிய பிறகு அவர் ஃபேஸ்புக்கை உபயோகிக்க ஆரம்பித்து தம் சகோதரரின் தோழியானார். அவர் ரஞ்சித்தை தம் பெற்றோரின் படங்களை ஃபேஸ்புக்கில் போடச் சொல்லி வலியுறுத்தினார் மேலும் அவற்றை ஒவ்வொரு வாரமும் மாற்றவும் சொன்னார், 
அதனால் அவர் ஒவ்வொரு முறையும் தம் பெற்றோர் எப்படி இருக்கிறார்கள் என்பதைப் பார்க்கலாம்.

ஸ்ரீலதா தம்முடைய பெற்றோருடன் ஸ்கைப் செய்யும் பொழுது, அவர்கள் டெஸ்க்டாப் கம்ப்யூட்டரை உபயோகிப்பததையும் அதில் ஸ்கைப் செய்வதையும் சௌகரியமாக உணராததால், ரஞ்சித் அங்கிருக்க வேண்டும். ஆகையால் ஸ்கைப் அழைப்புகள் மாதத்தில் ஒரு தடவையே நடக்கின்றன, ஸ்ரீலதா இடையில் தம் பெற்றோர்களை அவர்களைக் காண முடியாமல் இருந்தாலும் ஃபோனில் அழைக்கிறார். இதன் விளைவாக, ஸ்ரீலதா ரஞ்சித்தைத் தொடர்பு கொண்டு தம் பெற்றோர் மற்றும் உறவினர்களின் படங்களைப் பெறுகிறார்.

ஸ்ரீலதாவின் திருமணத்திற்கு முன் ரஞ்சித் தம் சகோதரியைக் கட்டுப்படுத்தியதை ஒப்புக் கொள்கிறார்; அவர் தம் சகோதரியை ஃபேஸ்புக்கைக் கூட உபயோகப்படுத்த அனுமதிக்காத அளவுக்குக் கடுமையாக இருந்தார். அவர் அது தன்னுடைய பங்கில் முட்டாள்தனமானது என்பதை இப்பொழுது ஒப்புக் கொள்கிறார். மேலும் அவர் ஸ்ரீலதா பஹ்ரைன் சென்ற பொழுதுதான் அவருடைய மதிப்பும், தான் அவர் மேல் கொண்டிருந்த அன்பும் தமக்கு விளங்கியது என்று கூறுகிறார். ஸ்ரீலதா தான் தமது வெகு நாட்கள் தொடர்பில்லாமல் இருந்த உறவினருடன் நட்புக் கொண்டது என்றும், இழந்த உறவை திரும்பவும் ஒருங்கிணைத்தது என்றும் ரஞ்சித் கூறுகிறார். அவர் தம் விரிவு நிலைக் குடும்பத்தைத் தொடர்பு கொள்ள தாம் என்றும் முயற்சித்ததில்லை, ஆனால் ஸ்ரீலதா தான் ஃபேஸ்புக்கில் சேர்ந்த உடனேயே அதைச் செய்தார் என ஒப்புக் கொள்கிறார், ரஞ்சித் தாம் காதலித்த பெண்ணுடன் முதலில் நட்பானது ஸ்ரீலதாதான் எனக் கூறுகிறார். இந்த உறவைப் பற்றி அவருக்கு தம் பெற்றோருடன் பிரச்சினை ஏற்பட்ட பொழுது ஸ்ரீலதா உடனான ஃபேஸ்புக் உரையாடல்களே அவரைச் சமாதானப் படுத்தியதாகவும் கூறுகிறார். ரஞ்சித் விரைவிலேயே இந்தப் பெண்ணை மணக்க இருக்கிறார், நன்றி ஸ்ரீலதாவிற்குத்தான், அவர்தான் பெற்றோரை சம்மதிக்கச் செய்துள்ளார். தன் சகோதரி இன்னும் பக்குவமடையவில்லை என தாம் முட்டாள்தனமாக நினைத்ததாகவும், ஆனால் அவர் தன்னைவிட மூன்று வயது சிறியவராக இருந்தாலும் அதிகம் பக்குவப்பட்டவர் என்றும் ரஞ்சித் கூறுகிறார். ஆனால் விநோதமாக சகோதரர் சகோதரி இருவரும், அவர்களிடம் ஸ்மார்ட்ஃபோன் இருந்தாலும், வாட்ஸ் அப் மூலம் தொடர்பு கொள்ளவில்லை. ரஞ்சித்துடன் ஒரு நேர்காணலில் இந்தக் 
கேள்வி எழுந்த போது, அவர் பொருள் பொதிந்த புன்னகையுடன் அதையும் விரைவிலேயே செய்யப் போவதாகக் கூறினார்.

சகோதரிகளுக்கிடையேயான தொடர்பு முற்றிலும் வேறு உலகில் இருந்தது. அவர்கள் வயது வித்தியாசம் அதிகம் இருந்தாலும் ஃபேஸ்புக்கில் தோழிகளாக இருப்பதைப் பற்றி கவலைப்படாமல் இருந்தார்கள், மேலும் பொதுவாக மற்றவரது ப்ரொஃஃைல் அல்லது போஸ்ட்டிற்கு லைக்கும் குறிப்பும் போட்டார்கள். குறிப்பிட்ட தனி விவாதங்கள் குரல் அடிப்படையான அழைப்புகளிலோ அல்லது எழுத்துப் பூர்வ செய்திகளிலோ அல்லது வாட்ஸ் அப்பில் கூட நடந்தாலும், ஃபேஸ்புக் தூதும் பல நிகழ்வுகளில் காணப்பட்டது.

கீழ் சமூகப் பொருளாதார வகுப்புகளில் பல திருமணமாகாத இளம்பெண்களுக்கு தொடர்புத் தொழில்நுட்பங்களின் உபயோகத்திற்கான அனுமதி மறுக்கப்பட்டு இருந்தது. ஆயினும், பணி கிடைத்தபின் அல்லது மணமான பின் (படித்திருந்தால்), குடும்ப உறுப்பினர்களுக்கிடையே தொடர்பை இடைவிடாதிருக்கச் செய்வதற்காக அவர்கள் முக்கியமான சமூக ஊடகப் பயனாளிகளாகி விடுகிறார்கள். இதன் அர்த்தம் இவர்கள் சமூக நெட்வொர்க்கிங் உபகரணங்கள்/தளங்களை உபயோகிப்பார்கள் என்பது அல்ல, ஏனென்றால் இவற்றின் உபயோகம் இந்தப் பெண்களின் சமூக வட்டத்திற்குள் யார் இவற்றை உபயோகிக்கிறார்கள் என்பதைப் பொறுத்தே அமைகிறது. ஆயினும் குரல் மற்றும் எழுத்துச் செய்திகள் மூலம் நெட்வொர்க்கிங் நிச்சயம் நடைபெறுகிறது.

ஒரு நெருங்கிய குடும்ப வட்டத்திற்குள் தொடர்பின் பாங்கைப் பற்றி ஆய்வு செய்த பின், நாம் இப்பொழுது பஞ்சக்கிராமியில் விரிவு நிலைக் குடிம்ப உறுப்பினர்களுக்கு மத்தியில் தொடர்பைப் பற்றி ஆய்வு செய்வோம்.

\section{விரிவுநிலைக் குடும்பத்துடனானத் தொடர்பு}

விரிவுநிலைக் குடும்ப உறுப்பினர்களுடனானத் தொடர்பு அவர்களுடைய ஆㅇபைன் தொடர்பைப் பொறுத்தே இருந்தது, ஏனென்றால் ஆஃப்லைன் பந்தங்களின் இயல்புகளே ${ }^{45}$ (பலமான அல்லது பலவீனமான) தொடர்புகளின் அடுக்கு நிகழ்வை நிர்ணயிக்கின்றன. ஆனால் நீண்ட நாட்களாகப் பிரிந்து இருந்த உறவுகள் ஃபேஸ்புக் மூலம் இணைந்த நிகழ்வுகளும் உண்டு. பஞ்சகிராமியில் இது சில உயர் மத்திய வகுப்பு வயதானவர்களுக்கு ஒரு பொழுது போக்காகவே 
இருந்தது. இது ஆண்களோ அல்லது பெண்களோ பெரும்பாலும் பங்கேற்கிறார்கள் என்று சொல்ல முடியாத அளவுக்கு ஒரு கலப்புக் குழு ஆகும். இந்த மாதிரி திட்டங்களின் மேல் இரு பாலினரும் சம அளவு ஆர்வம் கொண்டுள்ளது போல் தெரிகிறது. அவர்கள் ஃபேஸ்புக்கில் உறுப்பினரான பிறகு, தவறாமல் தம் உறவினர்களைஅந்தத் தளத்தில் தேட ஆரம்பிக்கின்றனர். ஃபேஸ்புக்கில் தாம் பல பத்தாண்டுகெளுக்கு முன் பார்த்த தனது இரண்டாவது ஒன்று விட்ட சகோதரரின் மகளையோ அல்லது தமது சொந்த கிராமத்திலிருந்து ஒரு தூரத்து சொந்தக்காரரையோ கண்டுபிடிப்பதில் பல வயதானவர்கள் பெருமை ெொள்கிறார்கள். 69 வயதான ஃபேஸ்புக் ஆர்வலர், திரு. ராஜாராம் கூறுவது போல 'இது கோயில் திருவிழாவில் தொலைந்த ஒருவரைக் கண்டுபிடிப்பதைப் போன்ற ஒன்று. 'ஜீனி'46 என்பது அவர்களுக்கு அது (தாமாகவே) தங்கள் குடும்ப மரத்தை நிர்மாணிக்க உதவுவதால், பெரியவர்களால் கூடப்படும் இன்னொரு தளம்.

பல பணி ஓய்வு பெற்றவர்கள் தங்கள் குடும்ப சரித்திரத்தை எழுதுவதற்காக தங்கள் சொந்த கிராமத்திற்கு திரும்பிச் செல்லுகிறார்கள், அவர்களில் பெரும்பாலானோர் தமது தொடர்புப் புள்ளிகளை அறிந்து கொள்வதற்காக ஃபேஸ்புக்கில் இருக்கின்றனர். ஆயினும், ஒரு தொடர்புப் புள்ளி (நீண்ட நாட்களாக சந்திக்காத உறவினர்) கிடைக்கும் பொழுது, ஒருவருடன் ஒருவருக்கான தொடர்பு, தீவிரமான சம்பாஷணைகளுக்காக ஒரு தொலைபேசி அல்லது வாட்ஸ் அப் உரையாடலாகவோ மாறி விடுகிறது, இது இந்தத் தேடலை மற்ற உறவினர்கள் வசம் ஃபேஸ்புக்கிலோ அல்லது திருவிழாவிலோ (இந்தத் தளத்தைப் பற்றிய உங்கள் பார்வையைப் பொறுத்து) தொடர விட்டு விடுகிறது. இப்பொழுது வாட்ஸ் அப் எழுத்து செய்திகளின் இடத்தைப் பிடித்து விட்டதால், இந்த விரிவு நிலைக் குடும்ப உறுப்பினர்களுக்கு இடையேயான தொடர்பு வாட்ஸ் அப் மூலமாகவும் நடை பெறுகிறது - சில நேரங்களில் குடும்பக் குழு தொடர்புகளாகவோ அல்லது சில நேரங்களில் தனிப்பட்டதாகவோ. மீண்டும் இது ஒருவர் ஆஃப்லைனில் கொண்டிருக்கும் உறவைப் பொறுத்தே அமைகிறது.

ஃபேஸ்புக் ஒரு விரிவு நிலைக் குடும்பத்துடன் தொடர்பு கொள்ளக் கூடிய சாதனம் எனப் பார்க்கப் படுவதால், ஒன்று விட்ட சகோதர - சகோதரிகளுக்கிடையேயான தொடர்பு, முக்கியமாகத் திருமணமாகி தொலைவில் வசிக்கக் கூடியவர்கள், வழக்கமாக ஃபேஸ்புக் மூலமே நிகழ்கிறது. ஸ்கைப் மற்றும் ஃபோன் அழைப்புகளும் நிகழ்கின்றன; ஆயினும் 
இது வளரும் பருவத்தில் இந்த உறவினர்கள் எவ்வளவு ஆஃப்லைன் தொடர்பில் இருந்தனர் என்பதைப் பொறுத்தே அமைகிறது. ஒன்று விட்ட சகோதர - சகோதரிகள் தமக்குள் ஃபேஸ்புக் ப்ரொஃபைலயையும் 'லைக்' செய்யும் மற்றும் அதன் மீது 'குறிப்பு எழுதும் பல நிகழ்வுகளும் நடக்கின்றன, மேலும் அவர்களுக்கு குழந்தைகள் உண்டானபின் இது மேலும்கணிசமாக அதிகரிக்கிறது. ஒரு சகோதரன்/சகோதரியின் குழந்தையின் படம் ஃபேஸ்புக்கில் அப்லோடு செய்யப்படும் ஒவ்வொரு முறையும், லைக் அல்லது குறிப்புகள் மூலம் அந்தக் குழந்தைக்கான ஆதரவு கொடுக்கப்படுகிறது. இந்த சகோதர சகோதரிகளின் குழுவால் நிகழ்ச்சிகளும் (தமது சொந்த கிராமத்தில் ஒரு திருவிழா போல) ஏற்பாடு செய்யப்பட்டன, ஆனால் அவை அவர்கள் ஒன்றாக வளர்ந்திருந்தாலோ அல்லது அவர்கள் பெற்றோர் மிகவும் நெருங்கியவர்களாக இருந்தால் மட்டும் நடந்தது.

விரிவு நிலைக் குடிம்ப உறுப்பினர்களுக்கிடையே தினசரித் தொடர்பை விட பிறப்பு, திருமணம் மற்றும் இறப்புப் போன்ற வாழ்க்கை நிகழ்வுகளே அதிகம் புலனாயின. இந்தப் புலன்பாடு ஃபேஸ்புக்கில் விரிவு நிலைக் குடும்ப வட்டங்களிலிருந்துப் பெறப்பட்ட அதிக எதிர் செயல்களின் விகிதத்தைப் (லைக் மற்றும் கமென்ட்டுகள் வடிவத்தில்) விளைவுதான். ஒரு குழந்தையின் பிறப்பு ஃபேஸ்புக்கில் அறிவிக்கப்பட்டாலும், அந்தக் குழந்தையின் படத்தை அப்லோடு செய்தல், அந்தக் குழந்தை பிறந்து இரு வாரங்கள் வரையிலோ அல்லது அதன் பிறப்புச் சம்பந்தப்பட்ட மத சடங்குகள் முடியும் வரையில், கண்திருஷ்டி பயத்தினால் ஒத்தி வைக்கப் படுகிறது - இதே அத்தியாயத்தில் நாம் முன்னர் பார்த்த திருமதி கீதா தியாகராஜனின் நிகழ்வைப் போலவே. இந்த மாதிரியான பழக்கங்கள், வீட்டில் பெரியவர்கள் இந்த மாதிரி விஷயங்களை முடிவு செய்யக்கூடும் இடங்களில் குறிப்பாக, வீடுகளில் மிகவும் உறுதியாகப் பின்பற்றப்பட்டன - முன்னர் கூறிய திருமதி கீதா தியாகராஜனின் நிகழ்வைப் போலவே.

பிறந்து ஒ ஒரு நாளைக்குள்ளாகவே குழந்தையின் படம் அப்லோடு செய்யப்பட்ட நிகழ்வுகளும் உண்டு, ஆனால் இது வீட்டில் உள்ள பெரியவர்கள் தம் மனதில் சமூக ஊடகங்களைப் பற்றி வைத்திருக்கும் எண்ணங்களைப் பொறுத்தது. விரிவு நிலைக் குடிம்ப உறுப்பினர்களின் குழு ஒரு நெருங்கிய பந்தத்துடனோ அல்லது ஒரு பொது வாட்ஸ் அப் குழுவின் உறுப்பினர்களாகவோ இருந்தால், இவ்வாறான பட்ங்கள் ஃபேஸ்புக்கைத் தவிர்த்து வாட்ஸ் அப்பில் பகிரப்படுகின்றன; ஏதோ 
ஒரு விதத்தில் ஃபேஸ்புக் வாட்ஸ் அப்பை விட இந்த மாதிரியானத் தொடர்புகளுக்கு பொருந்தாத ஒரு மக்கள் சாதனமாகக் காணப்படுகிறது. இந்தப் படங்கள் வாட்ஸ் அப்பில் பகிரப் பட்டாலும் உறவினர்கள் யாரும் இதை ஃபேஸ்புக்கில் அப்லோடு செய்வதில்லை. பதிலாக, அவர்கள் குழந்தையின் பெற்றோரோ அல்லது நெருங்கிய உறவினர்களில் ஒருவரோ இந்தப் படத்தை அப்லோடு செய்யக் காத்திருக்கின்றனர். இந்த மாதிரியான குடும்ப வட்டங்களில் ஒரு சில தொடர்புகளின் வடிவங்கள் (இந்த நிகழ்வில் பார்க்கக்கூடியது) ஒரு தளத்திலிருந்து இன்னொரு தளத்திற்கு மாறுவது குறித்து ஒரு அங்கீகரிக்கப்பட்ட நடைமுறை வழக்கம் இருக்கிறது. இதன் அர்த்தம் குழந்தையின் வருகைப் பற்றிய செய்தி குடும்ப வட்டத்திற்குள்ளேயே வைக்கப் படுகிறது என்பது அல்ல, ஆனால் இந்த செய்தி நெட்வொர்க்கை விட்டு வெளியே சென்றாலும், அது எழுத்திலோ அல்லது வாய்மொழியாகவோதான் செல்லும் என்பதுதான். இதன் வெளிப்பாடு என்னவென்றால், இந்த மாதிரியான செய்திகளைப் பற்றி தொடர்பு கொள்ள தளங்களின் மூலோபாயம் மட்டுமல்ல, ஆனால் செய்திகளின் எந்தப் பகுதிகள் எந்தெந்த தளங்களில் வர வேண்டும் என்பதும்தான்.

குழந்தைகளின் பிறந்த நாள் கொண்டாட்டங்களுக்கான அழைப்புகளும் ஃபேஸ்புக் மூலம் அனுப்பப்படுகின்றன. இது பொதுவாக அங்கீகரிக்கப் பட்டாலும், மற்ற நிகழ்வுகளில் - இந்த அத்தியாயத்தில் முன்னர் விவாதிக்கப்பட்ட திரு. ராகவன் அவர்களின் நிகழ்வைப் போல - சமூக ஊடகங்கள் வாயிலான அழைப்புகள் மரியாதை அற்றதாகக் கருதப்படுகின்றன; தனிப்பட்ட ஃபோன் அழைப்புகளே ஏற்றுக கொள்ளக் கூடியதாக இருந்தன. சில பிறந்த நாள் படங்களே வாட்ஸ் அப் மூலம் அனுப்பப்பட்டன, மற்றவை அவர்களின் நெட்வொர்க்கில் உள்ள எல்லோரும் பார்க்கும் வகையில் ஃபேஸ்புக்கில் அப்லோடு செய்யப்பட்டன, ஆனால் நோக்கம் விரிவு நிலைக் குடும்பம் மட்டும்தான். கமென்ட்களின் வடிவில் எதிர்செயல் விகிதங்கள் ஆல்பமில் உள்ள மற்றவற்றை ஒப்பிடிகையில், முதல் சில படங்களில் தெரிந்தன (பொதுவாக முதல் 12லிருந்து 15வரை). இவற்றின் ஒரே விலக்கு குழந்தை மற்றும் பெற்றோர்களின் போர்ட்ஃபோலியோ படங்கள், அல்லது குழந்தை கேக் கட் செய்பவை. இது விரிவு நிலைக் குடிம்ப உறுப்பினர்கள் நெருங்கிய குடும்பங்களில் பின்பற்ற வேண்டிய எதிர்பார்க்கப்படும் சமூக விதிமுறை ஆகும். மீண்டும் விரி நிலைக் குடும்பத்தாரால் தமது சமூக வட்டங்களில் மிக தூரத்து உறவினர்களுக்கு முன் ஒரு குறிப்பிட்ட குழுவுடனான தனது 
நெருக்கத்தை வெளிக்காட்டக் கூடிய செயலாக்கத்தின் ஒரு நிலையும் காணப்பட்டது. ${ }^{47}$

திருமணங்கள் தம்பதிகளின் ப்ரொஃபைலில் கவனிக்கத்தக்க மாற்றங்களைக் கொண்டு வரக் கூடிய முக்கிய வாழ்க்கை நிகழ்வு ஆகும். முதல் மாற்றம் அவர்களது உறவு நிலைகளில் ஏற்படும், அடுத்தது அவர்களது ஃபோட்டோ ஆல்பத்தில், அங்கு திருமண நிச்சயத்தின் காட்சிகள் அப்லோடு செய்யப்படும், இவை பல்வேறு நேர்மறை பதில்களைத் தோற்றுவிக்கும். திருமண நிகழ்ச்சியின் படங்கள் இரண்டு சுழற்சியில் வெளியிடப்படும், முதற் சுழற்சியில் திருமணம் முடிந்த உடனேயே அப்லோடு செய்யப்படும் மிகச் சிலப் படங்கள், இது குறிப்பாக இந்த நிகழ்ச்சியைத் தவற விட்ட வெளிநாடுகளில் வாழும் விரிநிலைக் குடும்ப உறுப்பினர்களுக்கானது. அடுத்த சுழற்சி, முழு ஆல்பமும் கொண்டது, இது திருமணம் முடிந்து ஏறக்குறைய ஒரு மாதத்தில் அப்லோடு செய்யப்படும்.

ஏறக்குறைய எதிர்பார்க்கப்படும் விதிமுறை இந்தியாவில் உள்ள உறவினர்கள் எல்லோரும் திருமணத்திற்கு வருவார்கள் என்பதுதான். நேரில் வர முடியாத உறவினர்களுக்கு நிகழ்நிலைப் படுத்துதல் மூலம் திருமணத்திற்கு வந்த ஒரு உணர்வைக் கொடுப்பதற்காக, மணமகன் மற்றும் மணமகளின் ஒன்று விட்ட சகோதர சகோதரிகள், மாமா-மாமி, சித்தப்பா-சித்தி, அத்தை-மாமா, பெரியப்பா-பெரியம்மா, போன்ற திருமணத்தில் உள்ளவர்கள், திருமண நிகழ்ச்சியின் சில படங்களை அப்லோடு செய்கிறார்கள். இம்மாதிரியான படங்கள் ஃபேஸ்புக் அல்லது வாட்ஸ் அப் மூலம் அனுப்பப் படலாம். மேற்கண்டவாறு, மணமகன் அல்லது மணமகள் திருமணம் முடிந்து ஏறக்குறைய ஒரு மாத காலத்தில் ஒரு தொழில் முறை புகைப்படக் கலைஞரால் எடுக்கப்பட்ட முழு திருமண படங்களின் ஆல்பத்தை அப்லோடு செய்வார்கள். இந்த கால அவகாசம் பொதுவாக குடும்ப வட்டத்தால் ஏற்றுக் கொள்ளப் படுகிறது, ஆனாலும் இந்த நேரத்தில் வாட்ஸ் அப் மூலம் இந்த நிகழ்வின் படங்களும், வீண் பேச்சுகளும் சுற்றி வரலாம். இரு நிகழ்வுகளில், இரண்டும் உச்ச மத்திய வகுப்புக் குடிம்பங்களைச் சேர்ந்தவை, ஒரு திருமண நிச்சயதார்த்த விழா, அதில் வருகை புரிய முடியாத விரிநிலைக் குடும்ப உறுப்பினர்களுக்காக, ஸ்கைப் மற்றும் மற்ற தொழில்முறை ஒளிபரப்பு அலைவரிசை ${ }^{48}$ மூலம் ஒளிபரப்பு செய்யப்பட்டது. ஆயினும், அவை அனைத்தும், இரு பக்கமுள்ள விரிநிலைக் குடும்ப உறுப்பினர்கள் மணமக்களின் குடும்பங்களுடன் கொண்டுள்ள நெருக்கத்தைப் பொறுத்தே அமைந்துள்ளது. 
நடுத்தரவயது தகவலர்களின் ப்ரொஃபைலில் வயதான உறவினர்களின் இறப்புச் செய்தி பற்றிய தொடர்புதான் அனைவற்றையும் விட வெளிப்படையாகத் தோற்றமளித்தது. மற்ற பாணிகளை விட இறப்பைப் பற்றிய போஸ்ட்கள் தான் அதிக எதிர் செயலைப் பெற்றன. இதன் காரணம் மற்ற நிகழ்ச்சிகளின் பல காட்சிகள் ஃபேஸ்புக்கில் அப்லோடு செய்யப்படுவதும், ஆனால் இறப்புச் செய்தி பொதுவாக ஒன்றிரண்டு படங்களுடனேயே வெளிவருவதுமாக இருக்கலாம், ஆகையால் இதற்கான பதில்கள் இந்த போஸ்ட்களின் மேலேயே குவிந்திருக்க வேண்டும்.

ஃபேஸ்புக்கில் எரியூட்டுதலைப் பற்றி ஒரு பொது அறிவிப்பு இருந்தாலும், வாட்ஸ் அப் குடும்பக் குழுக்களில் அதிகக் காலவட்ட நிகழ்நிலைப்படுத்துதல்கள் நடை பெறுகின்றன. இறந்த உடலுடனான செல்ஃபிக்கள் பொதுவாக ஊக்கமளிக்கப் படுவதில்லை, மற்றும் மத்திய வகுப்புக் குடும்பங்கள் சிலவற்றில் ஈமச்சடங்கின் புகைப்படங்களும் எடுக்க அனுமதிக்கப்படுவதில்லை, ஏனென்றால் அவர்கள் ஃபேஸ்புக்கில் இந்த மாதிரியான போஸ்ட்களுக்கு லைக்குகளைப் பெறுதல் உசிதமாக இருக்காது என நினைத்தார்கள்.

குடிம்ப வட்டங்களுக்குள் (ரத்த அல்லது திருமண பந்தங்களுக்குள்) சமூக நெட்வொர்க்கிங் உபகரணங்களின் உபயோகங்கள் மேலே விவாதிக்கப்பட்டன. ஆயினும், நாம் கோவிந்தனின் நிகழ்வில் கண்டவாறு, பஞ்சக்கிராமியில் உறவுமுறைச் சொந்தங்கள் நட்பு வட்டங்கள் வரையிலும் கற்பனை உறவு வடிவத்தில் விரிவடைகின்றன. உறவு வட்டத்திற்குள் சமூக நெட்வொர்க்கிங் உபகரணங்களின் உபயோகங்களை ஆய்வு செய்வதே இந்த அத்தியாயத்தின் முக்கிய நோக்கம் என்றாலும், அடுத்த பாகம், 'கற்பனை உறவுகள்' என்று சொல்லக்கூடிய நட்புச் சொந்தங்களைப் பற்றியதாகும்.

\section{ஃபேஸ்புக்கும் கற்பனை உறவுகளும்}

பஞ்சகிராமியில் நண்பர்களுக்கிடையேயானத் தொடர்புகள் கருத்தில் கொள்ள வேண்டிய முக்கிய உறவுகளாய் இருந்தாலும், இந்தப் பகுதி கற்பனை உறவு என்ற சொந்தமாகக்கூடிய நெருக்கமான நட்பின் ஒரு வடிவத்தைப் பற்றி கவனம் கொள்கிறது. அனைத்து உறவு முறைச் சொந்தங்களும் குறிப்பிட்ட நட்புகளைப் போல அத்தனை நெருக்கமாக இருப்பதில்லை, மற்றும் அனைத்துக் கற்பனை உறவுகளும் நெருக்கமான 
சொந்தங்களாக மாறுவதில்லை என்பதையும் உணர்ந்து கொள்ளுதல் அவசியமாகும். ${ }^{49}$ புதியவர்களுக்கு நடுவிலும் தினசரி வழக்கில் சில கற்பனை உறவுகளைக் குறிக்கக் கூடிய வார்த்தைகள் உபயோகிக்கப் படுகின்றன. உதாரணத்திற்கு அண்ணா, அக்கா போன்றவை. ஒருவர் மற்றொருவரை இந்த வார்த்தைகளைச் சொல்லி அழைப்பதனாலேயே, அது ஒரு நெருங்கிய சொந்தத்தையோ அல்லது எந்த வித நட்பையும் குறிப்பதாகாது. இவ்வாறு புதியவர்களையும் இந்த உறவு முறைகளைச் சொல்லிக் கூப்பிடுதல் தமிழ்நாட்டு வழக்கமாக இருந்தாலும், உறவு முறை இல்லாத சொற்களின், ‘சார், மேடம்/ மேம்' போன்றவை, உபயோகங்களும் காணப்படுகின்றன. இந்த அத்தியாயத்தின் தொடக்கத்தில் விவாதிக்கப்பட்ட கோவிந்தன் பற்றிய நிகழ்வு, இந்த மாதிரியான உறவு முறை உள்ளவை மற்றும் இல்லாதவை என வகைப்படுத்துதலின் ஒரு மாதிரி உதாரணமாகும். ஆயினும், இந்தப் பகுதி பரஸ்பரக் குறிப்பிடுதலுக்கு கற்பனை உறவுச் சொற்கள் உபயோகப்படுத்தப்படும் நெருங்கிய நட்பைப் ${ }^{50}$ பற்றியதாகும்.

ஒரு குறிப்பிட்ட சாதியால் ஆதிக்கம் செலுத்தப்படும் ஒரு குறிப்பிட்ட இடத்தில் வளர்ச்சியடையும் சொந்தங்களைப் புரிந்து கொள்ளுதல், உறவுகள் எப்படி நட்புகளாகின்றன, அவை மீண்டும் கற்பனை உறவு முறை சம்பந்தங்களின் மூலம் உறவுகளாகின்றன என்பதைப் புரிந்து கொள்ள அத்தியாவசியமானதாகும். இந்த அத்தியாயத்தில் முன்பு கூறப்பட்ட 120 வயதான கிராமத்தின் நிகழ்வை எடுத்துக் கொள்ளுங்கள், இது பஞ்சகிராமியின் ஒரு அங்கம், மேலும் முன்காலத்திலிருந்தே துரதிருஷ்ட வசமாகக் குறிப்பாக வேறுபடுத்தப்பட்ட ஒரு குறிப்பிட்ட சாதியின் ஆதிக்கத்தில் உள்ளது. இந்த கிராமத்தில் உள்ள பல இளைஞர்களுக்கு இந்த கிராமத்தில் உள்ள பெரும்பாலானவர்கள் (குறிப்பாக நீண்ட காலமாக வசிப்பவர்கள்) முற்காலத்தில் ஏதோ ஒரு தருணத்தில் உறவினர்களாக இருந்தவர்கள்தான் என்ற லேசான தெரிதல் இருந்தது. ஆயினும், கிறித்துவ மதத்திற்கு சமீப காலத்திய மத மாற்றத்திற்குப் பிறகும் (அவர்களுடைய மதம் மாறி விட்டாலும், சாதிக் குழு மாறவில்லை), முந்தைய தலைமுறையின் பல தார மணப் பழக்கத்தினாலும், அதிகக் குழப்பங்கள் ஏற்பட்டிருக்கின்றன. இதன் விளைவாக, வெகு சிலருக்கே தன் அண்டை வீட்டுக்காரருடன் அவர் சொந்தம் என்ன என்பது தெரியும். வெகு சில இளைஞர்களைத் தவிர மற்றவர்களுக்கு உறவுமுறையை அடையாளம் காணத் தெரியாது. அவர்கள் வயதையொத்த பிற இளைஞர்கள் 
பொதுவாக நண்பர்களாகவேக் கருதப்படுவொர்கள். ஆயினும் பரஸ்பரம் உறவுமுறை சொல்லிக் கூப்பிடிம் பழக்கத்தைக் கொண்ட ஒரு குறிப்பிட்ட முறையும் வழக்கில் உள்ளது, இதன் மூலம் மற்றவர்களை ஒரு உறவாக இல்லாமல் நட்பாகவே கொள்கிறார்கள். ஆகையால் கிராமவாசிகள் ஒருவரையொருவர், அண்ணா, மாமா, மச்சான், சகலை எனவே அழைக்கிறார்கள். இது ஏறக்குறைய பெண் நண்பர்களுக்கும் பொருந்தும். இது போன்ற நிகழ்வுகளில் உறவு-நட்பு-கற்பனை உறவின் ஒரு பரவளைய வளைவு காணப்படும். நிஜமாகவே மறக்கப்பட்ட சொந்தங்கள் நட்புகளாகப் பார்க்கப்பட்டு (குழப்பத்தைத் தீர்க்க) கற்பனை உறவுகளால் அழைக்கப்படுகிறது. இதை விவாதிக்க வேண்டிய காரணம், இந்த உறவு முறைகளுடன்தான் இவர்கள் ஃபேஸ்புக்கில் பரஸ்பரம் அழைத்துக் கொள்கிறார்கள், மேலும் இவர்களை ஒரு சமூகக் குழுவாக ஏற்றுக் கொள்ள இதைப் புரிந்து கொள்ளுதல் அவசியமாகிறது. பஞ்சகிராமியில் வெளிப்படுத்தப்படும் இன்னும் சில கற்பனை உறவுகளின் உதாரணங்கள் இந்த வகைச் சொந்தங்களை இன்னும் தெளிவாக வழங்க துணை புரிகின்றன.

26 வயதான ஸ்ரீதர் சாதி-அடிப்படையிலான உள்ளூர் அரசியல் கட்சியில் மிகவும் ஊக்கமுடன் தொடர்பில் இருக்கிறார். அவர் உள்ளூர் இளைஞர்களுக்கு பல உதவிகள் செய்வதால், எப்பொழுதுமே அண்ணன் எனவே அழைக்கப்படுகிறார். இன்னும் அதிக ஊக்கத்தையும், மற்றும் உறுப்பினர் சேர்க்கையும் ${ }^{51}$ அதிகப்படுத்தலில் அவருடைய உள்ளூர் கட்சி தலைவரின் ஆணையின் பேரில் ஸ்ரீதர் சமூக ஊடகங்கள் மூலம் தம் சாதியைச் சேர்ந்த மேல்நிலைப்பள்ளி மாணவர்களை தம் கட்சிக்குக் கவர ஒரு நுட்பத்தைப் பின்பற்றினார். அவர்கள் ஸ்ரீதரை ஆஃப்லைனில் பார்க்கும் பொழுது, இந்த மேல்நிலைப்பள்ளி மாணவர்கள் அவரை அண்ணன் என்று அழைக்கிறார்கள், அவர்கள் அவரைப் பற்றி ஃபேஸ்புக்கில் குறிப்பிடும் பொழுதும் அண்ணன் என்றேக் குறிப்பிடுகிறார்கள்.

பிரகாஷ், பஞ்சகிராமியில் வசிக்கும் ஒ ஒரு 21 வயது கல்லூரி மாணவர், அவரது அண்டைய சரியிணை ஆண்கள் குழுவில் நன்கு அறிமுகமானவர். அவருடைய புகழுக்கு அவர் ஃபேஸ்புக்கில் மற்ற மாநிலங்களில் இரருந்து 30 பெண்களுடன் (அறிமுகமில்லாதவர்கள்) நட்பு கொள்ளும் திறனே காரணம். அவர் தமது சரியிணைக் குழுவால் 'மச்சி' (மச்சினர்) என்று அழைக்கப் படுகிறார், ஆனால் அவர்கள் எப்பொழுது ஃபேஸ்புக்கில் புதியவர்களின் (குறிப்பாக பெண்கள்) நட்பைப் 
பெறுவதில் அவரது திறனையும், மேலும் குழு உறுப்பினர்களும் அவ்வாறே செய்ய உதவி செய்வதில் விருப்பத்தையும் பார்த்தார்களோ, அப்பொழுதே அவரை விளையாட்டாக மாமா என்று அழைக்க ஆரம்பித்தார்கள் (இதன் அர்த்தம் தமிழில் தாய்மாமனைக் குறித்தாலும், இது தமிழ் பேச்சு வழக்கில் காமத் தரகரையும் குறிக்கிறது). இந்த சரியிணைக் குழு பரஸ்பரம் வாட்ஸ் அப்பிலேயே உரையாடிக் கொண்டாலும், அவர்களால் ஃபேஸ்புக்கில் அப்லோடு செய்யப்படும் புகைப்படம் எப்பொழுதும் பிரகாஷை 'மாமா' என்றேக் குறிக்கிறது (அல்லது டேக் செய்யப்படிகிறது). அவர் தமது பல ஃபேஸ்புக் நண்பர்களுக்கு ஒரு விதத்தில் மாமாவாக இருந்தாலும், இது பொதுவாக மறந்து விடப்படுகிறது, ஏனென்றால் உண்மையில் அவர் அவர்களுக்கு எந்த விதத்தில் மற்றும் எப்படி மாமா ஆகிறார் என்பது யாருக்கும் தெரியாது. இங்கு நட்பு கற்பனை உறவாக விரிவடைகிறது, இந்தக் குழு உறுப்பினர்கள் பரஸ்பரம் இந்த வார்த்தைகள் மூலமே ஒருவரை ஒருவர் குறிப்பிடுகிறார்கள். முன்னர் கண்டவாறு, சில நேரங்களில் ஒருவரை ஒருவர் பங்காளி என அழைத்துக் கொண்டாலும், இவர்களில் சிலர் முன்னரே உறவுகளாக இருக்கிறார்கள், இருப்பினும், மாமா என்பது சமூக ஊடகத்தில் மற்றும் ஆஃப்லைனில் நேருக்கு நேர் பிரகாஷைக் குறிப்பிடும் மிக அதிகமான சாதாரண வார்த்தையாக உள்ளது,

கற்பனை உறவுகளைப் பற்றியக் குறிப்பிட்ட எண்ணம், தமிழ்நாட்டில் வகுப்பு மற்றும் சாதியைக் கடந்து உள்ளது. ஆனால், பஞ்சகிராமியில் இது, குறைந்தபட்சம் ஃபேஸ்புக்கில் மத்திய வகுப்பு மற்றும் உயர் மத்திய வகுப்பை விட கீழ் சமூகப் பொருளாதார வகுப்புகளில் மிக அதிகம் வெளிப்படையாக உள்ளது. ஆனால் இதன் பொருள் இந்த மாதிரியான கற்பனை உறவுகள் ஃபேஸ்புக்கில் மத்திய வகுப்பால் வெளிப்படுத்தப் படுவதில்லை என்பதில்லை. இதிலுள்ள வேறுபாடு எத்தனை முறை என்பதுதான். சில மத்திய வகுப்பு தகவலர்களின் டைம்லைனில் இந்த மாதிரியான கற்பனை உறவுச் சொந்தங்களை வெளிப்படுத்தும் பிறந்த நாள்/மண நாள் வாழ்த்துக்கள் காணப்படுகின்றன. எடுத்துக்காட்டிற்கு 23 வயதான சுநித்ரா, தமது நண்பரின் ஃபேஸ்புக் ப்ரொஃபைலில் பிறந்தநாள் வாழ்த்துக்களை எழுதுகிறார், இதில் இவர் கற்பனை உறவு சொந்தத்தைக் குறிப்பிடுகிறார்.

என்னுடைய இனிமையான நண்பருக்கு, நாம் ஒன்றாக வளர்ந்தோம், ஒருவரை ஒருவர் பாராட்டினோம். 
இதோ உங்களுக்காக இன்னும் ஒன்று. பிறந்தநாள் வாழ்த்துக்கள்!

சுநித்ராவின் செய்தி அவருடைய உண்மையான சகோதரருக்கு எழுதியது போல இருந்தாலும், இது உண்மையில் அவரது நெருங்கிய நண்பருக்கு எழுதியது, அவர் அந்த சுற்றுப்புறத்திலேயே அவருடன் வளர்ந்தவர். இன்னொரு உதாரணம், ஃபேஸ்புக்கில் தம்முடைய படத்திற்கு கமென்ட் எழுதிய தம் அன்னையின் தோழிக்கு பதிலளித்த பாரதியின் நிகழ்வு

அன்னையின் தோழி: அழகான உடை, பாரதிக் குட்டி மிகவும் அழகாக இருக்கிறாய்

பாரதியின் பதில்: நன்றி ஆன்ட்டி. இது போன வாரம் எக்ஸ்பிரஸ் அவென்யூவில் வாங்கியது. அம்மாவின் ${ }^{53}$ பரிசு.

இம்மாதிரியான செய்திகள் பல குடியிருப்பவர்களின் ஃபேஸ்புக் டைம்லைனில் காலம் காலமாகத் தென்பட்டுக் கொண்டிருக்கிறது. பல ஒரு நிகழ்ச்சியை அடிப்படையாகக் கொண்டவை (பிறந்த தினம் அல்லது திருமண தினம் மாதிரியான).

இம்மாதிரியானக் கற்பனை உறவுமுறைச் சொந்தங்களை வெளிப்படுத்தும் போஸ்ட்கள் கீழ் சமூகப் பொருளாதார வகுப்புத் தகவலர்களின் ஃபேஸ்புக் ப்ரொஃபைலில் தினந்தோறும் தோன்றுகின்றன. அவை நிக்ழ்ச்சிகளை அடிப்படையாகக் கொண்டவையாக இல்லை, அவை தினசரி நிகழ்வுகளாகவே இருக்கின்றன. கீழ் சமூகப் பொருளாதார வகுப்புகளில் ஆண்களே இந்த மாதிரியான செய்திகளை போஸ்ட் செய்கிறார்கள், ஆனால் மத்திய வகுப்பில் இது பெண்களிடையே அதிகமாகக் காணப்படுகிிறது.

மத்திய வகுப்பில் கற்பனை உறவுமுறைக்கு எதிரான முறையும் நடக்கிறது, ஆனால் இது கீழ் சமூகப் பொருளாதார வகுப்புகளில் தென்படுவதில்லை. இந்த நிகழ்வில் உண்மையான உறவினர் ஒருவர் நண்பர் எனக் கூறப்படலாம். இது குறிப்பாக நாத்தனார், கொழுந்தியாள் போன்ற பந்தங்களை உருவாக்கும் திருமணத்திலிருந்து உருவாகும் உறவுமுறைச் சொந்தங்களில் நடக்கிறது. இந்த செய்திகள் பொதுவாக ஒரு நிகழ்ச்சியின் அடிப்படையிலேயே இருக்கின்றன; உதாரணத்திற்கு, பெண்கள் பிறந்தநாள் வாழ்த்துக்கள் 
அனுப்பும் பொழுது அவர்கள் இந்த மாதிரியான உறவுகளை நட்பு எனக் குறிப்பிடுகிறார்கள். உதாரணத்திற்கு, 42 வயதான இல்லத்தரசி சரஸ்வதிக்கு அவரது நாத்தனாரிடமிருந்து இவ்வாறு ஒரு திருமணநாள் வாழ்த்து வந்தது:

'என்னுடைய அண்ணாவிற்கும், எனது மிக அன்பான தோழி சரஸ்க்கும், இந்த சிறப்பான நாளில் கடவுள் உங்களை ஆசிர்வதிக்கட்டும். உங்களுக்கு இ இந்த மாதிரியான அழகிய நாட்கள் அதிகம் உண்டாகட்டும்.'

இப்படிப்பட்ட செய்திகள் பல மத்திய வகுப்புத் தகவலர்களின் டைம்லைனில் காணப்படுகின்றன. ஆனால் உறவுமுறைச் சொந்தம் மற்றும் நட்பு இரண்டையும் வெளிப்படையாக வெளிப்படுத்தும் செய்திகளும் ஃபேஸ்புக் டைம்லைனில் நேரடியாகக் காணப்படுகின்றன. உதாரணத்திற்கு, 25 வயது ஐடி பணியாளரான அபிநயா, தன்னுடைய சித்தியின் பிறந்தநாளைக் கொண்டாட கீழ்க்கண்ட செய்தியை ஃபேஸ்புக்கில் போஸ்ட் செய்தார்

'என் மிக அன்பான சித்திக்குப் ${ }^{54}$ பிறந்த நாள் வாழ்த்துக்கள். நீங்கள் தான் என்னுடைய அனைத்து நேரத்தைய சிறந்த தோழி.'

ஒரு தமிழ் சமூகத்தில் படிநிலைக் கருத்துக்களே சித்தி மற்றும் மகளுக்கிடையே உள்ள தலைமுறைக்கிடையேயான உறவுகளையும், அல்லது நாத்தனாருடனானதைப் போன்ற ஒரே தலைமுறைக்குள்ளான உறவுகளையும் ஆளுகின்றன. ஆயினும், மேற்கூறிய இரண்டு உதாரணங்களைப் போல நட்பாக இருக்கும் எண்ணம், வெளி உலகத்திற்கு அவர்களது உறவு சமமானவர்களுக்கு இடையேயானது என்பதை வெளிக்காட்டுகிறது, இது படிநிலையால் ஆணையிடப்பட்டதற்கு பதிலாக எண்ணங்களின் சுதந்திர ஓட்டத்தையே அடிப்படையாகக் கொண்டது.

இதன் அர்த்தம் ஆண்கள் அவ்வாறான செய்திகளைப் போஸ்ட் செய்ய மாட்டார்கள் என்பதல்ல. அவர்களுடைய செய்திகள் அபிநயாவால் போஸ்ட் செய்யப்பட்டதைப் போலவே, அவர்கள் பிறருடன் கொண்டிருக்கும் உண்மையான உறவையும் மற்றும் இலட்சிய உறவையும் வெளிப்படுத்துவதாக இருக்கும். உதாரணத்திற்கு, 32 வயதான ஒரு ஐடி 
தொழிற்பண்பட்டவர் சர்வேஷின் பிறந்தநாள் அன்று அவரது டைம்லைனில் இப்படிப்பட்ட செய்திகளைப் பெற்றார்:

'என் குட்டித் தம்பிக்கு ${ }^{55}$... என் வாழ்க்கை ஆலோசகர், என் தோழர். பிறந்த நாள் வாழ்த்துக்கள் டா!'

'எனக்குப் பிரியமான ஒன்று விட்ட தம்பி, என் காலை 4 மணி தோழன், வேதாந்தி, வழிகாட்டி, இந்த நாள் உனக்கு மிகச் சிறந்த நாள் ஆக இருக்கட்டும்!'

சில நேரங்களில் இது போன்ற செய்திகள் ஒருவர் தனது ஃபேஸ்புக் நட்பு வட்டத்தில் மற்றவர்களுடன் கொண்டுள்ள உறவை தெளிவாகக் கூறுகின்றன. ஆனால் சர்வேஷுக்கு வந்த முதல் பிறந்தநாள் செய்தியை மீண்டும் ஒருமுறைப் பாருங்கள்; அது ஒரே நேரத்தில் உறவையும் நட்பையும் ஒன்றாகக் கூறுகின்ற செய்தி போலத் தெரிந்தாலும், அது உண்மையில் சர்வேஷின் ஒரு சகோதரியைப் போன்ற தோழியுடையது. அது ஒரு நிஜ உறவினருடைய செய்தி அல்ல. இதற்கு எதிராக, இரண்டாவது செய்தி, ஒரு ஒன்று விட்ட சகோதரரின் செய்தி. துரதிர்ஷ்ட வசமாக சர்வேஷాக்கு அவர்கள் ஏதோ ஒரு முறையில் உறவினர் என்பது மட்டுமேத் தெரியும்; ஆனால் அவர்கள் எவ்வாறு ஒன்று விட்டச் சகோதரர் என்பது தெரியாது, ஆகையால் 'சகோதரர்' என்பதை குறியீட்டுக்கானப் பொதுவான வார்த்தையாகக் கூறுகிறார்.

இத்தகைய வெளிப்பாட்டின் அ அடுக்கு நிகழ்வு பாலினத்தையும், வகுப்பையும் பொறுத்தே அமைந்தாலும், இந்த நட்பிலிருந்து கற்பனை உறவுமுறைக்கும் மற்றும் உறவுமுறையிலிருந்து நட்புக்கும் இடையிலான இடைவிடா மாறுதல் அடிக்கடி நடக்கிறது. இதற்கு ஒரு காரணம் தமிழ் சினிமாவிலிருந்து ெெளிப்படும் இடைவிடாது தூண்டும் தரநெறிசார் யோசனையும் மக்களுக்கு நட்பு உறவு முறையை விட எவ்வாறு மிகவும் முக்கியமாகிறது என்று கூறும் அறிவுரை மீமிக்களும்தான். ${ }^{56}$ இந்த நட்பைப் பற்றிய இலட்சிய உரைகள் நட்பை நெருக்கமான உறவுமுறைச் சொந்தத்திற்கு இணையாக நடத்த ஊக்கமளிக்கின்றன, இதற்கு நேர்மாறாகவும் நடக்கின்றன. ஆயினும் கீழ் சமூகப் பொருளாதார வகுப்புகளில் அனைத்து நட்புகளும் கற்பனை உறவுமுறைப் போலவே தென்படுகின்றன, ஆனால் மத்திய வகுப்பு போஸ்ட்டுகளில் உறவு முறையை அதற்கு பதிலாக நட்பாக வெளிப்படுத்தும் ஒரு மனோபாவம் காணப்படுகிறது. 


\section{முடிவுரை}

முடிவாக, இந்த அத்தியாயம் உறவு முறை சொந்தங்கள் வாயிலாக உறவு மற்றும் நெருக்கம் பற்றியக் கருத்துக்களை ஆய்வு ெய்துள்ளது. மானுடவியலின் ஒரு முக்கியமானக் கூறான உறவுமுறை பஞ்சகிராமியின் சமூகக் கட்டமைப்புகளுக்கு அடிப்படையாகும், இது தினசரி ஆஃப்லைன் உறவுகளின் மேல் மட்டுமல்லாமல், சமூக ஊடகங்களின் மேல் உள்ள உறவுகள் மீதும் தாக்கத்தை ஏற்படுத்துகிறது.

இந்த அத்தியாயம் தலைமுறைகளுக்கிடையேயான உறவுகளைப் பற்றி ஒரு ஆய்வுடன் தொடங்கியது. இங்கு வயது மற்றும் உறவுகளின் படிநிலைக் கொள்கைகள் அதிகாரத்தையும், தாக்கத்தையும் ஏற்படுத்தின, இதனால் ஒரு குடும்பத்தில் மூத்தவர்களாலேயே குடும்பத்திற்குள் தலைமுறைகளுக்கிடையே தொடர்புக்கான தொடர்பு அலைவரிசையின் தேர்வு ஊடகங்களின் வரிசையிலிருந்து சரியானதாக நிர்ணயிக்கப்படுகிறது என்பது உறுதி செய்யப் படுகிறது. ஊடகத்தின் தேர்வின் மீது இப்படிப்பட்ட தாக்கம் பல அடுக்குகளைக் கொண்டது மேலும் இவ்வாறான உறுதியான ஊடக இயற்பண்புகள் இருக்குமிடத்தில் பண்பாட்டுச் சூழலையொட்டி இவைப் புரிந்து கொள்ளப்பட வேண்டும்.

குடும்பத்திற்குள்ளானத் தொடர்புகளில் (குறிப்பாக பெற்றோருக்கும் பிள்ளைகளுக்கும் இடையேயான) கவலை மற்றும் அக்கறை போன்றவை காரணமாக மற்ற வடிவங்களை விட குரல் வழி தொடர்பே ஏறக்குறைய ஆதிக்கம் செலுத்தியது. உதாரணத்திற்கு, நாம் அத்தியாயம் 2ல் சந்தித்த இளம் தாய், லக்ஷ்மி, தம்முடைய காரியாலயத்திலிருந்து தம் குழந்தைகளுடன் பேச இயலவில்லை, ஆயினும் அவர் குரல் வழி செய்திகளைப் பதிவு செய்து அவற்றைத் தம் குழந்தைகளைக் கேட்கச் செய்ய வாட்ஸ் அப் மூலம் அனுப்பினார். ஆனால் ஒருவர் கல்வியறிவு மற்றும் பிற திறன்கள் போன்ற மாறிகளைக் குறைத்து எடை போட முடியாது, ஏனென்றால் மற்ற தொடர்பு வடிவங்களுக்கு பதில் குரல் மூலம் தொடர்பு கொள்ள தேர்வு செய்யும் கீழ் சமூகப் பொருளாதார வகுப்புக் குடும்பங்களில் இவை பங்கு வகிக்கின்றன. பாலிமீடியா மற்றும் ஊடகப் பன்முகமாக்கக் கொள்கைகளை ஆதரிக்கும் வலுவான பாங்குகள், மூத்தவர்கள் பல தொடர்பு ஊடகங்களைத் தேர்வு செய்யும் திறன் பெற்ற மத்திய மற்றும் மேல் மத்திய வகுப்புக் குடும்பங்களில் அமைந்துள்ளன. எப்படியிருந்தாலும், உண்மையான தூரம் போன்ற மற்ற காரணிகள் 
தொடர்பு ஊடகத்தின் தேர்வின் மீதுத் தாக்கத்தை ஏற்படுத்தினாலும், தலைமுறைகளுக்கிடையேயான உறவுகளின் விஷயத்தில், அனைத்து வகுப்புகளும் மற்றத் தொடர்பு வடிவங்களைக் காட்டிலும் குரல் வழித் தொடர்பையே அதிகம் விரும்பின.

விரிவுநிலைக் குடும்ப உறவுகளில் தரநெறி சார் இலட்சியங்கள், நெட்வொர்க்கின் எதிர்பார்ப்புகளுக்கு அனுசரித்தல் மற்றும் புலப்படும் தன்மை ஆகியவை ஒரு குடும்பம் ஃபேஸ்புக் போன்ற தளங்களில் செயல்திறன் நிலையை நிர்ணயிப்பதில் முக்கியப் பங்காற்றுகின்றன, இதன் மூலமே உலகம் (தயாராக இருக்கும் பார்வையாளர்களின் ஒரு பரந்த நெட்வொர்க்) அந்த குடும்பத்தின் நெருக்கத்தைக் காண முடியும். இது சில நெருங்கிய குடும்ப உறுப்பினர்கள் மற்றவர்களுக்காக செயல்படக் கூடிய விரிநிலை குடும்ப உறுப்பினர்களுக்கான வாட்ஸ் அப் சமூகத்திலும் தெளிவாகத் தெரிந்தது.

திருமணமான ஜோடிகளின் தினசரி தொடர்பில், எழுத்துச் செய்தி மற்றும் வாட்ஸ் அப் போன்ற பாதுகாப்பான தனிப்பட்ட அலைவரிசைகள் குரல் அடிப்படைத் தொடர்புகளோடு மாறி மாறி அமைந்தன. இது தலைமுறைக்கிடையேயான தொடர்புகளை விட தம்பதிகளிக்கிடையேயான தொடர்பு அடிக்கடி நிகழ்வதாலும் இருக்கலாம். உசிதமான ஊடகங்களின் வரிசையிலிருந்து தொடர்பு கொள்ள மிகச் சிறந்த வழியாக தேர்ந்தெடுக்கப்படும் கூட்டாளியின் விருப்பத்தில் ஒரு பரஸ்பர புரிதல் உணர்வு தெரிந்தது. இதன் மேல் மற்றக் கூட்டாளி இடம் பெற்றிருக்கும், நேரம் மற்றும் இடத்தைப் பற்றியப் பொது கவனத்தினாலும், அவர்களுக்கு கிடைத்திருக்கும் ஊடகங்களைப் பற்றிய விழிப்புணர்வினாலும் தாக்கம் ஏற்படுத்தப் படுகிறது.

திருமணமான தம்பதியரின் புலன்படுதல் மற்றும் தரநெறி சார் லட்சியங்கள் இவ்விதமான உறவுகளின் செயலாக்கம் மீது தாக்கத்தை ஏற்படுத்தக் கூடிய நிகழ்வுகள் இருந்தாலும், அவர்கள் அந்த செயலாக்கத்தை எதிர்காலத்திற்கான நினைவுகளின் உத்திபூர்வமான சேமிப்பு என்ற பகுப்புக் குறிப்பிடத் தக்கதாகும். திருமணமான தம்பதிகளால் அவ்வாறு வெளிப்படையான போஸ்ட்டுகள் பரந்த நெட்வொர்க்கின் பார்வைக்காக ஃபேஸ்புக்கில் செய்யப்பட்டாலும், அவை உத்திபூர்வமாகவும், தனது இலட்சியத்திற்கு பங்களிக்கக் கூடியதை மட்டும் காண்பிக்க விரும்புவதாகவும் இருக்கின்றன. அதே நேரம் வீணானது என நினைக்கின்ற தினசரித் தொடர்புகள், தனிப்பட்ட முறையில் வாட்ஸ் அப்பிலோ அல்லது எழுத்துச் செய்திகள் மூலமாகவோ நடைபெற்றன. 
இந்த அத்தியாயம் சமூக வகுப்புகள் அனைத்திலும் சகோதர உறவுகளுக்கு இடையேயான தொடர்புகள் பற்றியும் ஆய்வு செய்திருக்கிறது. இது பாலினம் மற்றும் தொடர்பு ஊடகங்களைப் பற்றிய முந்தைய விவாதத்துடன் சம்பந்தப்படுத்தப்படும் போது, மத்திய வகுப்புக்கு (பெண்களை சமூக ஊடகங்களை உபயோகிக்க அனுமதிக்கும் மேல் மத்திய வகுப்புக்கு பெரும்பாலாக), சகோதர பந்தம் பெண்கள் தனியாக இருக்கும் போது கூட ஏற்படலாம், கீழ் சமூகப் பொருளாதார வகுப்புகளில் (கீழ் மத்திய வகுப்புகளிலும் கூட) இது ஒரு சவாலாக இருக்கிறது. இந்தப் பின்புலத்தின் திருமணமாகாத இளம்பெண்கள் அடிப்படைத் தொடர்பு ஊடகத்தை உபயோகிக்க அனுமதிக்கப் படுவெதில்லை, ஆகையால் சமூக ஊடகத்தின் உபயோகத்திலிருந்தும் தடை செய்யப் பட்டிருக்கிறார்கள். ஆகையால் இந்த வகுப்புக்கு சமூக ஊடகத்தில் சகோதர பந்தம் திருமணத்திற்கு பின்போ அல்லது வேலைக்குச் சென்ற பின்போதான் சாத்தியமாகிறது, ஏனென்றால் அப்பொழுதுதான் அவர்களுக்கு தனக்கேயான ஃபோன் கிடைக்கிறது. ஒரு சகோதரியுடன் கூடிய சகோதர உறவில், சமூக வகுப்புகளைப் பொருட்படுத்தாமல், தொடர்பு மிகவும் அடிக்கடி நடை பெற்றது.

முடிவாக, இந்த அத்தியாயம் நட்பை கற்பனை உறவுமுறையின் நோக்கிலிருந்து பார்க்கத் தலைபட்டது, ஏனென்றால் இந்த அத்தியாயத்தின் அடிப்படை கவனம் உறவுதான். இதன் முக்கியமானக் கூறு என்னவென்றால், அனைத்து வகுப்புகளிலும் கற்பனை உறவுமுறையும் நட்பும் மாறி மாறி வந்த விதமும், அவை தம்மை ஃபேஸ்புக்கில் வெளிப்படுத்திக் கொள்ளும் முறையும்தான். கீழ் சமூகப் பொருளாதார வகுப்புகளில் இருப்பவர்கள் தம்மிடத்தில் இருக்கும் எல்லா நட்புகளையும் கற்பனை உறவுமுறையாகப் பார்க்கும் பொழுது, மத்திய வகுப்பினர், உறவுமுறை, கற்பனை உறவுமுறை மற்றும் நட்பு ஆகியவற்றில் மாறி மாறி இருந்தார்கள் - சில நேரங்களில் உண்மையான உறவுமுறையை நட்பாக, அதற்கு நேர்மாறாகவும், வெளிப்படுத்தினார்கள். சில நேரங்களில் உறவுகளின் இயல்பு உறுதியாக இல்லாத போது, உறவுமுறையிலிருந்து நட்புக்கும், பின் கற்பனை உறவுமுறைக்கும் ஒரு பரவளைய வளைவில் செல்லக்கூடிய பாவம் இருந்தது.

இந்த உதாரணங்களில் பாலிமீடியாவின் ஒரு சுத்தமான நிகழ்வும் தெளிவாக இருந்தது, அங்கு குறிப்பிட்ட உறவுகளுக்குள் தொடர்பு தடங்களுக்கிடையே மாறிக்கொண்டேயிருந்தது. அத்தியாயம் 3ல் நாம் நெட்வொர்க் எதிர்பார்ப்புகளுக்கு ஏற்ற காட்சித் தொடர்புகளைப் பார்த்தோம். இந்த அத்தியா- 
யத்தில் நாம் பல தளங்களின் வெளிப்படை உபயோகமும், தளங்களின் மேல் தொடர்புகளின் இயல்புகளும் ஒருவர் பராமரிக்கும் நெட்வொர்க்கின் எதிர்பார்ப்புகளிலேயே மையம் கொண்டிருக்கிறது என்பதை கவனித்தோம். ㅇபேஸ்புக்கை வெளித் தொடர்புக்கான ஒரு மக்கள் ஊடகமாகவும், வாட்ஸ் அப் அதிகம் தனிப்பட்ட தளம் என்று உணரப்பட்டக் கருத்துக்களும் மிகவும் முக்கியமானவை. பொது மற்றும் தனி என்ற வேறுபாட்டின் இன்னொரு அடுக்கு அதன் மேல் பராமரிக்கப்பட்ட சமூக வட்டத்தின் அடிப்படையில் வாட்ஸ் அப்பிலிருந்தும் வெளிப்பட்டது. ஃபேஸ்புக்கைப் போலவே சில வாட்ஸ் அப் குழுக்களும் பொதுவாக கருதப்பட்டாலும், மற்றவைத் தனியாகக் கருதப்படுகின்றன. குடிம்பத் தொடர்புகளிலிருந்து, அவற்றின் எதிர்பார்ப்புக்கு ஏற்றவாறு, வாட்ஸ் அப்பின் வெவ்வேறு நெட்வொர்க்குகளைச் சார்ந்த,தெளிவான உத்திபூர்வமான பரிவர்த்தனைப் பாங்குகள் வெளிப்படுகின்றன.

அனைத்து வகுப்புக்களுக்கு இடையிலுள்ள பல்வேறு உறவுமுறைச் சொந்தங்களுக்கு இடையேத் தோன்றும் தொடர்புகளின் வகைகளுக்குள்ளே, ஒரு மிகப் பெரிய சமூகக் குழுவைச் சார்ந்திருக்கும் உள்ளுணர்வு இருக்கிறது. இது இளைய தலைமுறை தனது ஊடக விருப்பத்திற்காக தம் மூத்த குடிம்ப உறுப்பினர்கள் அல்லது பெற்றோர்களுக்கு எதிராக பேரம் பேசுவதாக இருக்கலாம். தம்முடைய எண்ணம் தம் மகளைத் தொலைபேசியில் அழைப்பதன் மூலம் அவளுடைய நன்மையை காப்பதும் பத்திரப்படுத்துவதும் என்று உண்மையாக நம்பும் இந்தக் குழுவால் தடைகள் விதிக்கப்படலாம்; தம் சகோதரியை சில குறிப்பிட்ட ஊடகங்களை உபயோகிக்க அனுமதிக்காத சகோதரர்கள் கூட தாம் அவர்கள் பாதுகாப்புக்காகவே செய்ததாகக் கூறுவார்கள். குழுக்களின் இடையேயானத் தொடர்பின் இன்னொரு கூறு உறவுமுறை, நட்பு மற்றும் கற்பனை உறவுமுறையின் சிக்கலான, பர வளைய வளைவுதான். ஒரு இலட்சியக் குடும்பத்தைப் பேணுவதைப் பற்றிய அக்கறையைக் குறிப்பிடிவதின் மூலம், பயனாளிகள் ஒரு விதத்தில் தாம் பொருந்தி இருக்கின்ற மிகப் பெரிய சமூகக் குழுவின் இலட்சியங்களுக்கும் எதிர்பார்ப்புகளுக்கும் உண்மையில் ஏற்றவாறு இருக்கிறார்கள். இவ்வாறு, உறவுமுறைப் பற்றிய ஆய்வு மற்றும் சமூக ஊடகத்தில் வெவ்வேறு உறவுகளின் தொடர்புகள் மிகப் பெரிய சமுதாயத்தின் பிரதிபலிப்புகளாகவே இருக்கின்றன. 


\section{5}

\section{பணியில் வீட்டைக்}

\section{கொணர்தல்: பணி மற்றும்}

பணியல்லாதவைகளின் எல்லைகளை தெளிவற்றதாகச் செய்தல்

ஒரு வியாழக்கிழமை ட்டர் கோட் டெஸ்டர் அபிஜித், அன்று மாலை செயலாக்கலுக்காக சமர்ப்பிக்க வேண்டிய கோடை தமது தனியறையில் டெஸ்ட் செய்துக் கொண்டிருக்கிறார். அவர் பாலக்காட்டைச் (கேரளா) சேர்ந்தவர், ஆனால் பஞ்சக்கிராமியில் தம் வளர்ச்சி மையத்தை வைத்திருக்கும் ஒரு பெரிய ஐடி கம்பெனியில் பணி புரிந்து கொண்டிருக்கிறார். அவர் பணிக்காக கேரளாவிலிருந்து தம் குடிம்பத்தைப் பிரிந்து வந்த பிறகு, இந்த இடத்தில்தான் வசித்துக் கொண்டிருக்கிறார். திடீரென்று இந்த வாரக் கடைசியில் அவருடைய மனைவியாக வரக் கூடியவரின் குடும்பத்தை சந்திக்க அவரைப் பாலக்காட்டுக்கு வரப் பணிக்கும் தன் அன்னையின் எழுத்துச் செய்தி அவரைக் குறிக்கிடிகிறது. அவரது வயதில் உள்ள மற்ற இளைஞர்களைப் போலவே அபிஜித்தும் ஒரு நிச்சயிக்கப்பட்டத் திருமணத்திற்கான ${ }^{1}$ மிக நீண்ட நடைமுறையில் இருக்கிறார். இந்தச் செய்தியைப் பார்த்ததும் அவர் தமது தனியறையை விட்டு ஹாலுக்குச் சென்று தம் பயணத்தின் நோக்கத்தை முழுவதுமாக அறிந்து கொள்ள தம் அன்னையை அழைக்கிறார். அவர் அந்த வாரக் கடைசியில் பயணம் செய்ய ஒத்துக் கொண்டு, தம் தனிய- 
றைக்குத் திரும்புகிறார். பிறகு அவர் தமது ஸ்மார்ட் ஃபோன் மூலமாக இந்திய ரயில்வே இணைய தளத்தில் லாக்-ஆன் செய்து அந்த வெள்ளியன்று மாலை பாலக்காடு செல்வதற்கு ஒரு டிக்கெட் புக் செய்கிறார். பிறகு அபிஜித் தமக்கு வரக்கூடிய மனைவியின் படத்தைப் பார்க்க தமது வாட்ஸ் அப் பட ஃபோல்டரைப் பார்க்கிறார், அந்தப் படத்தை அவரின் சகோதரி தனது ஃபேஸ்புக் ப்ரொஃபைல் மூலமாக பதிவிறக்கம் செய்து அவருக்கு அன்று காலை அனுப்பியிருந்தார். அவர் தமக்குள் புன்னகைத்துக் கொண்டே, தாம் டெஸ்ட் செய்து கொண்டிருந்த கோடின் பக்கம் தம் கவனத்தைத் திருப்புகிறார்.

ஒரு வெள்ளிக்கிழமை மியில் ஒரு நிதி தரவுச் செயலாக்கக் கம்பெனியில் மனித வள இயக்குனராக இருக்கும் 37 வயது கவிதா, இவ்வாறு ஒரு மிருதுவான செய்தியை தமிழில் அனுப்புகிறார்: ' ‘இன்று இரவு உணவைச் சமைக்க வேண்டாம்... நாங்கள் உணவுக்கு வெளியே செல்கிறோம்.' இதற்குப் ப்தில் அவருக்கு ஒரு செய்தி வருகிறது (ஒரு பெண்ணிடமிருந்து குரல் மூலமாக): ‘சரி.... குழந்தைகளைத் தயார் செய்கிறேன்.' மூன்று பள்ளி செல்லும் குழந்தைகளுக்குத் தாயான கவிதா பணி மற்றும் வீட்டிற்கிடையே திறம்பட மாறிக் கொண்டே இருப்பதில் பெருமிதம் அடைகிறார். அவரும் அவரது கணவர் ராஜேஷமம் (இவர் ஒரு தொழில் முனைவர்) இப்பொழுது ஒரு சமையல் செய்பவர் மற்றும் ஆயாவைப் (கவிதாவின் குடிம்ப சாதியைச் சார்ந்த ஏறக்குறைய 50 வயது உள்ள ஒரு பெண்மணி) வீட்டு வேலைகளை கவனிக்கப் பணிக்கு அமர்த்தியுள்ளார்கள் ஏனென்றால் அவர்கள் பணி நீண்ட நேரத்திற்கு நீடிக்கிறது. ராஜேஷின் தாய் இறந்த பிறகு அவர்கள் வீட்டில் குழந்தைகளை கவனித்துக் கொள்ளவும், சமையலில் உதவவும் ஒரு ஆள் தேவை என்பதை முடிவு செய்தார்கள்.

இந்த வீட்டு வேலை உதவியாளரை தாம் வேலை செய்யும் விதத்திற்கு மாற்ற கவிதா அவருக்கு ஒரு விலை குறைந்த சாம்சங் ஸ்மார்ட்ஃபோனை வாங்கிக் கொடுத்தார். அவர்களுக்கிடையேயானத் தொடர்பு ஃபோன் அழைப்புகள் மூலம் ஆரம்பித்தாலும், இந்தப் பெண்கள் மெதுவாக வாட்ஸ் அப் மூலம் தொடர்பு கொள்தலுக்கு மாறினார்கள். அவர்களது ஆயாவிற்குக் வாட்ஸ் அப்பில் எழுத்து மூலத்தை விடக் குரல் மூலம் செய்திகளே வசதியாக இருப்பது சீக்கிரமே கவிதாவிற்குப் புலனாயிற்று. ஆகையால், வழக்கமாக வார நாட்களில் மாலை 5 முதல் 5.30க்குள் கவிதா தம் ஆயாவிற்கு இரவு உணவைப் பற்றிப் பணிப்பதையும், மற்றும் மளிகை மற்றும் 
பலசரக்குப் பொருள்களை வாங்குவதைப் பற்றி சரிபார்த்தலையும் செய்ய உறுதி செய்து கொள்கிறார். இந்த சடங்கு கவிதா பணியை விட்டு சுமார் மாலை 7 மணிக்குக் கிளம்பும் முன்னர் வாட்ஸ் அப் குரல் செய்தி மூலம் நடக்கிறது.

இந்த இரு உதாரணங்களும் பஞ்சக்கிராமியில் மக்கள் எவ்வாறு பணிக்கும் வீட்டிற்கும் இடையே தொடர்பு கொள்கிறார்கள் என்பதற்கு மாதிரிகளாகும். நவீன கம்பெனிகள் பணி மற்றும் பணியல்லாதவைகளுக்கு இடையேயான இடத்தில் ஒரு கடுமையான, முறைசார் எல்லையைப் பேண முயன்றாலும், அபிஜித் மற்றும் கவிதாவின் நிகழ்வுகளில் எடுத்துக்காட்டப்பட்ட எல்லை வரையறுப்பு சமூக ஊடகங்களால் தொடர்ச்சியாக வலுவிழக்கச் செய்யப்படுகிறது.

அத்தியாயம் 1ல் பணிக்களம் பஞ்சகிராமி நவீன இந்தியாவின் மிகப்பெரிய மாற்றத்தின் மீமிகை உதாரணத்தின் வேண்டுமென்றே தேர்ந்தெடுக்கப்பட்டது என்று அறிமுகம் செய்யப்பட்டது - இப்பொழுது அது உள்ளது போல் நாட்டுப்புற கிராமங்களுக்கு இடையே ஒரு ஐடி மண்டலத்தை அடுத்து வைப்பதை பிரதிபலிப்பதாய் உள்ளது. இந்த இடம் சமகால நவீன இந்தியாவுடன் சம்பந்தப்பட்டுள்ளதாக இருந்தாலும், இது இன்னமும் மிகவும் பழமையானதாகவே இருக்கிறது. ஆகையால் சமூக ஊடகங்கள் பற்றிய விவரங்கள், உறவுமுறை, வகுப்பு, மற்றும் சாதி ஆகியவற்றில் மூழ்கி இருக்கின்றன. எனினும், ஐடி துறையின் தோற்றம், சில விஷயங்களை முன்னே கொண்டு வருகின்றன, தெளிவாக அவற்றில் ஒன்று பணி மற்றும் மக்கள் வாழ்க்கையின் இதர அம்சங்களுக்கிடையேயான உறவு.

விவசாயத்துக்கு முரணாக, ஐடி வேலை பணி என்று தெளிவாக அமர்வு செய்யக்கூடிய அதிகம் முறை சார்ந்த மற்றும் தொழிற்பண்பட்டக் களமாகும். இது தெளிவாக, நாம் இது வரை விவாதித்த மற்ற வகை உறவுகளின் அதிகம் முறைசாராத மற்றும் சிக்கலான கலப்புடன் ஒரு சவாலை குறிக்கிறது. இதன் விளைவாக இந்த அத்தியாயம், நமது பணிக் களத்தின் நடுவே முறைசார்ந்த, பணி அடிப்படைக் கட்டமைப்பின் மிகப் பெரிய இருப்பை நாம் காணும்பொழுது சமூக ஊடகங்கள் என்னாகின்றன, என்பதிலேயே கவனம் செலுத்துகிறது. பணி மற்றும் பணியல்லாதவைகளின் எல்லைகள் இரு வழியிலும் எவ்வாறு மாறக்கூடும் என்ற அறிவு இருந்தாலும், (நீங்கள் அலுவலகப்பணியை வீட்டில் செய்ய எடுத்துச் செல்லலாம், அல்லது பணியில்லாத கூறுகளை பணியிடத்திற்கு எடுத்து வரலாம்), இந்த அத்தியாயம் பணி- 
யல்லாதக் கூறுகள் எவ்வாறு பணிக்குக் கொண்டு வரப்படுகின்றன என்பதில் கவனம் செலுத்துகிறது. வேறு விதமாக சொன்னால், நவீன பணி அமைப்புகள் மூலம் கடினமான பணி மற்றும் பணியல்லாதவைகளின் வரம்பைத் தெளிவற்றதாக்கி வீடு அலுவலகத்திற்குக் கொண்டு வரப்படுகிறது.

இதைத் திறம்படக் காட்ட வேண்டி, நாம் இந்த அத்தியாயத்தை, ஒரு சரித்திரச் சூழலில் இந்தியாவில் பணி என்பதைக் கருதத் தொடங்குமுன், மானுடவியலில் வரையறுக்கப்பட்ட பணியைப் பற்றியக் கருத்துடன் தொடங்குவோம். பிறகு நாம் ஐடி துறையின் பணிக் கலாசாரத்திற்கு சென்று சமூக ஊடகங்கள் அதில் எவ்வாறு பொருந்துகின்றன என்பது பற்றி பார்ப்போம், இது பஞ்சகிராமியின் பல நிகழ்வுகளால் எடுத்துக்காட்டப்படுகிறது. இந்த நிகழ்வு ஆய்வுகள் தனிப்பட்ட தொடர்பு தொழில் நுட்பங்கள் எவ்வாறு பணி மற்றும் பணியல்லாதவையின் கடினமான வரம்புகளை அடிக்கடித் தெளிவற்றதாக்குகின்றன என்பதைக் காட்டுகின்றன.

\section{பணி - ஒரு மானுடவியல் பார்வை}

'பணியைப்' பற்றியக் கருத்து சமூக அறிவியலால் விரிவாக ஆய்வு செய்யப்பட்டுள்ளது, மானுடவியலும் இதற்கு விதிவிலக்கல்ல. ${ }^{2}$ ஆரம்பத்தில் விவசாய வேலை போன்ற அதிகம் முறை சாராப் பணிகள் பற்றியே சம்பந்தப்பட்டிருந்தாலும், மானுடவியலாளர்கள் பிறகு முறை சார்ந்த நவீன தொழிற்பணி அமைப்புகளையும் கருத்தில் கொள்ள விழைந்தார்கள். ${ }^{3}$ மற்ற சமூக அறிவியல்கள் பணியை, பணி மற்றும் பணியல்லாத மற்றும் முறைசார் மற்றும் முறைசாராத பணி போன்று, இரு சமபக்கமுள்ளதாக நடத்த விரும்பினாலும், நடைமுறையில் பணியைப் பற்றிய இரட்டை வடிவமைப்புகள் ஆதாரமற்றவை என்பதை அது கண்டுணர்கிறது. மானுடவியல் பணியைப் பாகுப்படுத்துவதிலிருந்து தொடங்குவதில்லை, அது செயல்படக்கூடிய மண்டலம் அல்லது அதிகார எல்லையிலிருந்தே, இந்த மண்டலங்கள் மேற்பொருந்தியும் மற்றும் கலாசாரத்திற்கு ஏற்பவும் இருப்பதை ஏற்றுக் கொண்டு, தொடங்குகிறது, ${ }^{4}$ சமூகவியல் அல்லது உளவியல் போன்றே மானுடவியலும் பணியை பொருளாதாரத்தின் சம்பந்தத்திலேயே பார்ப்பதில்லை; இது பணியின் சமூகக் கூறுகளுக்கும், அவற்றிற்கிடையேயான உறவுக்கும் சமமான முக்கியத்துவத்தை அளிக்கிறது. 
பணி பற்றிய ஆய்வின் மேல் அதன் அணுகுமுறையில் மானுடவியல் மற்ற சமூக அறிவியல்களிடமிருந்து மாறு படுகிறது. உளவியல், சமூகவியல் மற்றும் பொருளாதாரம் போன்ற சமூக அறிவியல்கள் ஒரு தனிமனிதன், குழு அல்லது நிறுவனத்தின் பார்வையிலிருந்து முறையே பணியைப் பற்றியக் கருத்தை அணுக முற்பட்டாலும், மானுடவியல் இதை ஒரு முழுமையான பாணியிலேயே அறிய முற்படுகிறது. முறைசார் தொழிற்பணி அமைப்புகளைப் பற்றிய பல மானுடவியலாளர்களின் ஆய்வுகள் இந்த அணுகுமுறைக்கு சாட்சியாக இருக்கின்றன. ${ }^{7}$

மற்ற சமூக அறிவியல்களுடன் மானுடவியலும் பணி இரு சமபக்கமுடைய மாறி என்பதை ஏற்றுக் கொண்டாலும் (பணி மற்றும் பணியல்லாதவை), இது பரந்த சமூகச் சூழல் மற்றும் இடம் மற்றும் காலம் பற்றிய மாறுபடுகிறப் புரிதல் ஆகியவற்றுக்குள்ளேயேக் காணப்பட வேண்டும்.8 மானுடவியலாளர்கள் பண்டைய காலத்திலிருந்தே இந்த மாதிரிப் பணியைப் பற்றிய இரு சம பக்க பழக்கம் இல்லாத இடங்களிலும் பணிபுரிந்திருக்கிறார்கள் என்பதை அடையாளம் கண்டு கொள்வது அவசியம், இப்பொழுது அவர்கள் முறைசார் நவீன தொழில் அமைப்புச் சூழலை ஏற்றுக் கொள்கிறார்கள், இது பணி மற்றும் சொந்த உலகின் நடுவே உள்ள உறுதியான பிரிவை வரையறுக்க, பணி மற்றும் பணியல்லாதவை, அல்லது பணி மற்றும் வீடு போன்ற பணியின் இரு சம பக்க படிமங்களை வலியுறுத்தவும் மற்றும் உபயோகிக்கவும் முற்படுகிறது. மானுடவியல் ஆய்வுகள் டிஜிட்டல் தனித் தொடர்பு தொழில்நுட்பங்கள் போன்ற புத்தாக்கங்கள் ${ }^{10}$ இந்த இரு சம நோக்கைத் தெளிவற்றதாகவும், தொழில் மற்றும் சொந்த மண்டலங்களின் உறுதியானப் பிரிவை வலுவிழக்கவும் செய்கின்றன என்பதைக் கண்டு கொள்கின்றன.

இந்தியாவின் நவீன பணியிடங்கள் - குறிப்பாக அறிவு புற ஒப்படைப்பு (அவுட்சோர்சிங்) வளர்ச்சி மையமாக செயல்படும், ஐடி கம்பெனிகள் - பணியிடங்களைப் பற்றிய ஒரு மேற்கத்திய புரிதலுடனேயே வடிவமைக்கப் பட்டிருக்கின்றன; அவை இம்மாதிரியான இரு சம நோக்குகளை செயல்படுத்த கடுமையாக முயலுகின்றன, இவை உண்மையில் இந்தியாவில் பணியின் அதிகப் பாரம்பரிய வடிவங்களிலிருந்து வேறுபட்டவை. உதாரணத்திற்கு,அதிகம் சாதி அடிப்படையில் அமைந்திருக்கும் மிகப் பெரிய ஜவுளி உற்பத்தித் துறையில் நூற்றல், நெய்தல் மற்றும் சாயமேற்றுதல் போன்ற தொழில்கள் வீட்டை விட்டு வெளியே வசிக்க வேண்டிய நிர்ப்பந்தத்தை 
ஏற்படுத்தவில்லை; பல்லாண்டுகளாக இந்த இரு சம நோக்கு இருந்ததில்லை. ${ }^{11}$ ஆயினும், பெரிய அளவிலான தொழில்மயமாக்கலுக்கும் பிறகு அதன் விளைவாக நிறுவப்பட்ட முறைசார் தொழிற்சாலைகள் மற்றும் பணியிடங்களுக்குப் பிறகு, இவ்வாறான இரு சம நோக்குகள் தெளிவாகின்றன.12 இந்த அத்தியாயம் மக்கள் எவ்வாறு டிஜிட்டல் தொடர்பு சாதனங்கள் மூலம் இந்த நவீனப் பணிச்சூழலில் இருக்கும் இரு சம நோக்குகளைப் பற்றி மத்தியஸ்தம் மற்றும் முடிவு எடுக்கிறார்கள் என்பதையும், மேலும் அவ்வாறு செய்வதில் அம்மாதிரி எல்லைகளுக்கு எவ்விதமான சவால்களை அளிக்கிறார்கள் என்பது பற்றிப் பேசுகிறது.

ஆயினும், சமூக ஊடகங்கள் முறைசார் பணிகளின் எல்லையை பாதிக்கின்ற முறைகளின் மேல் கவனம் செலுத்தும் முன், நாம் பொதுவாக இந்தியாவின் தொழிலாளர் அமைப்பை ஏற்றுக் கொள்ள வேண்டும். இது இம்மாதிரியான எல்லைகளுக்கிடையே மத்தியஸ்தம் செய்வது புதிதல்ல என்பதைப் புரிந்து கொள்ள நமக்கு உதவும்; மக்கள் தொன்று தொட்டு பல நூற்றாண்டுகளாக இதைச் செய்து வருகிறார்கள். இது பஞ்சகிராமியின் முறைசார் தொழிலாளர் அமைப்பு வேலை-மையக் கொள்கைகளுடன் எவ்வாறு வெளிப்பட்ட முரண்பாடுகளைக் கொண்டுள்ளது என்பதை புரிந்து கொள்ள நமக்கு உதவுகிறது.

\section{பணி, சாதி மற்றும் உறவுமுறை - இந்தியாவின் ஒரு தொன்று தொட்டப் பார்வை}

மானுடவியல் மக்கள் பல மண்டலங்களில் பணி புரிகிறார்கள் என்பதை ஏற்றுக் கொள்கிறது. முறைசார் பணி என்பது, வீட்டு வேலை, பள்ளிப் படிப்பு போன்ற மற்றவற்றுடன் இணைந்து இருக்கும் அவ்வாறான ஒரு மண்டலம். இது இல்லத்தரசியின் (பாரம்பரியமாகப் பெண்) பணி, பொருள் ஈட்டுபெரின் பணியை (பாரம்பரியமாக ஆண்) என்று எப்பொழுதும் வாதிடப்படும் பெண்ணிய இலக்கியத்திற்கும் 13 இந்தக் கருத்து மையமாக உள்ளது. ஆக்க வளமுடைய மற்றும் ஆக்க வளமற்ற அல்லது பணியையே வரையறுக்கக்கூடியவைகளின் மேல் இடுகின்ற நேரடிப் பொருளாதார நன்மைகளின் அளவுகோல், இந்த மாதிரி இலக்கியங்களில் தலைகீழாக மாற்றப்பட்டுள்ளன. 
இது ஒரு இடத்திற்கு சம்பந்தமான பணி, அது செய்யப்படும் இடத்தை விட பணியின் இயல்பைப் பொறுத்தே (பொருளாதார அர்த்தத்தில் அல்ல) முக்கியத்துவம் அடைகிறது என்னும் உறுதிக்குக் காரணமாகிறது.

ஒரு இடத்தை மற்றதை விட அதிகம் முறைசார்ந்ததாக செய்வதில் கட்டுப்பாட்டைப் பற்றியக் கருத்து தொழில் மயமாக்கலுக்கு மிகவும் முன்பே பழக்கத்தில் இருந்தது; இதை இந்தியாவில் அரசாட்சி காலத்திலிருந்தே அடையாளம் கண்டு கொள்ளலாம்.14 ஆனால் தொழில்மயமாக்கலின் வருகைக்குப் பின்னே வகுப்பு மற்றும் சாதிக்கு நடுவில் இந்த மாதிரியான எண்ணம் அதிக வலுப் பெற்றது. 15 இடங்களைப் பிரிப்பதில் மட்டும் முறை சார்பு இருக்கவில்லை, ஆனால் படிநிலை, உரிமை, நிலைமை போன்ற பல காரணிகளைச் சார்ந்துள்ள அவர்களுடைய பங்கிற்கேற்ற இடத்தைப் பெற்றிருக்கும் அனைவருக்கும் தேவையான அனுமதிக்கப்பட்ட சமூக நடத்தையை சுமத்துவதிலும் இருக்கிறது. உதாரணத்திற்கு தலைமையாசிரியர் மற்றும் ஆசிரியர் மற்றும் மாணவன், தலைமை செயல் அலுவலர் மற்றும் மேலாளர் மற்றும் பாதுகாவலர். இது 4வது அத்தியாயத்தில் விவாதிக்கப்பட்டதைப் போல, இந்த வகை முறைசார்பு உறவுமுறையில் ஒருவரின் நிலை எவ்வாறு ஒருவருக்கொருவர் உறவுமுறையுடன் இருப்பவர்களின் சரியான நடத்தையை நிர்ணயிக்குமோ, அந்த வகையைப் போன்றே இருக்கிறது.

பஞ்சக்கிராமி தமிழ்நாட்டில் அமைந்திருப்பதால், தமிழ்நாடு மற்றும் பரவலாக இந்தியாவில் இருக்கும் பணியைப் பற்றியக் கருத்து புரிந்து கொள்ளப்பட வேண்டும். தொடர்ச்சி மற்றும் மாறுதல் ஆகிய எண்ணங்கள் இந்தியாவில் பிரயோகிக்கப்படக் கூடியவை, இவற்றை நிச்சயமாக இந்தியாவில் பணி பற்றிய எண்ணத்தையும் உள்ளடக்க விரிவு படுத்தலாம். இந்தியாவின் முந்தைய பணிக் கட்டுமானம் ஜாதி படிநிலைகளால் ஆன வேலைப் பிரிவு முறையின் அடிப்படையிலேயே இருந்தது. இந்த முறையில் ஒரு குறிப்பிட்ட சாதி சமூகம் ஹிந்து சாஸ்திரத்தால் தமக்குப் நியமிக்கப்பட்ட பணிகளையே செய்வதாக நம்பப்பட்டது. இது வர்ணாசிரம முறை என்று அழைக்கப்பட்டது, இது மதத் தலைவர்களால் ஜனத்தொகை நான்கு குழுக்களாகப் பிரிக்கப்பட்டதைப் பின்னோக்கிக் குறிக்கிறது. இது அவர்கள் செய்யும் பணியைப் பொறுத்தே, அதாவது, ஆச்சாரியர்கள், போர் வீரர்கள், வணிகர்கள் மற்றும் கடைநிலை ஊழியர்கள், அமைந்தது. முறையான அண்டவியல் அடிப்படையில் அறிந்து 
கொள்வதின் மூலம், இந்த வர்ணாசிரம முறைப் பின்பு மாறுதலுக்குட்பட்டது. இது வேறுபட்ட சாதிகளின் கூட்டங்களாக உடைந்து போனது, இங்கு ஒருவருடைய தொழிலும், அதனால் அடையும் நிலையும், ஒருவர் சமுதாயத்தில் தாம் செய்யத் தேர்வு செய்யும் தொழிலுக்கு முரணாக, அவர் பிறப்பால் ஒரு சாதியைச் சேர்ந்தவராக நியமிக்கப்படுகிறதது. ${ }^{17}$

சாதி ஒரு முக்கியத்துவம் வாய்ந்ததாகவே இருந்து வருகிறது, குயவர்கள், கொல்லர்களிடமிருநு நாவிதர்கள், வண்ணான் வரை பல பாரம்பரியத் தொழில் வல்லுநர்கள் அந்த வேலையைச் செய்ய நியமிக்கப்பட்ட சாதியிலேயேக் கட்டுப்பட்டிருந்தார்கள் - இது இந்தியா முழுவதையும் போலவே தமிழ்நாட்டிற்கும் உண்டான நிலைதான். சாதிகளுக்கிடையேயான பரிமாற்றச் சேவையாகவே பார்க்கப்பட்டது. ${ }^{19}$ சிறப்புப் பாரம்பரியத் தொழில்களை ஒரு குறிப்பிட்ட சாதியுடன் சம்பந்தப்படுத்துவது பரந்த உற்பத்தி மண்டலத்துக்கும் உண்மையாகவே இருந்தது; உதாரணத்திற்கு, காஞ்சிபுரத்தின் (இந்தப் பணிக்களம் அமைந்திருக்கும் இடம்) பட்டுத் தறி நெசவாளர்கள் முதலியார்/செட்டியார் ${ }^{20}$ போன்ற ஒரு குறிப்பிட்ட சாதியைச் சேர்ந்தவர்கள். தமிழ்நாட்டின் ஒரு பெரிய ஜவுளி உற்பத்தி மையமாக இருக்கும் திருப்பூரில் இந்தத் துறையில் வன்னியர்களும், வெள்ளாள கவுண்டர்களும் ${ }^{21}$ பணிக்கு அமர்த்தப் பட்டிருக்கிறார்கள். ${ }^{22}$

தொன்று தொட்டு இந்தியாவில் பணி என்பது குடிம்பம், உறவு அல்லது சாதியின் சூழலிலேயே செயலாக்கப்பட்டு வந்தது, இவை பணி மற்றும் வீட்டை ஒன்றிலிருந்து ஒன்று மாறுபட்டதாகவோ அல்லது இரு சம பக்கமுடையதாகவோ எண்ணவில்லை. இது தற்காலத் தமிழ்நாட்டிலும் மிகத் தெளிவாகக் காணப்படுகிறது. ${ }^{23}$ உதாரணத்திற்கு, ஜவுளி உற்பத்தி அமைப்பில், இழைமம் முதலில் ஒரு குறிப்பிட்டக் குழுவிடம் அவர்களது வீட்டிலேயே நூற்பதற்காகத் தரப்படலாம், பிறகு மற்றொரு குழுவினருக்கு அவர்களது வீட்டிலேயே நெய்வதற்காகத் தரப்படலாம். ${ }^{24}$ இதற்கு மேல் ஒரு இந்துப் பிரிக்கப்படாதக் குடிம்பத்தை ${ }^{25}$ அடிப்படையாகக் கொண்ட சட்டம் இந்தக் கூட்டு அல்லது விரிநிலைக் குடும்ப முறை மாதிரியின் அடிப்படையிலேயே இருக்கிறது, இது சொத்துக்கள் மற்றும் பணி பற்றிய உரிமை மேலும் விரிவாக்கப்பட்டு இருக்கிறது.

பதினெட்டு மற்றும் பத்தொன்பதாம் நூற்றாண்டின் தொழில் புரட்சி மற்றும் காலனித்துவ ஆட்சிக் காலம் இந்தியாவில் இருபதாம் நூற்றாண்டின் ஆரம்பத்தில் நிறுவப்பட்ட உற்பத்தித் துறை, இரும்பு மற்றும் எக்கு ஆலை மற்றும் 
சுரங்கங்களைத் தொழில்மயமாக்குதலின் பேரில் ஒரு தாக்கத்தை ஏற்படுத்தியது, இது மெதுவாக இந்தியாவின் தொழில்துறை அடிப்படை வசதிகளையும் ${ }^{26}$ அமைக்கத் தொடங்கியது. இந்தச் செயல்முறையில் பணி மற்றும் வீட்டிற்கு இடையே ஒருப் பிரிவினை எழுந்தது. ${ }^{27}$ இந்தியாவின் கிழக்கில் உள்ள சோட்டா நாக்பூர் பகுதியில் 29 மானுடவியல் ஆய்வுகள் ${ }^{28}$ மக்கள், குடிம்பம், உறவு மற்றும் சாதி கட்டமைப்புகளின் அடிப்படையில் அமைந்த பணிகளை விட்டு தொழில் கட்டமைப்புகளை நோக்கி குடிபெயர்ந்தாலும் அவர்கள் தம் குடிம்ப, உறவு மற்றும் சாதி உறுப்பினர்களுக்கு அதே தொழிற்சாலையில் பணி பெறவும், அதோடு அதே பகுதிகளுக்குக் குடி பெயரவும் உதவி செய்வது போலவும், இதன் மூலம் பின்னால் விட்டு வந்த பந்தத்தை மீண்டும் நிறுவுவது போலத் தோன்றினார்கள். ${ }^{30}$

திருப்பூர் பின்னலாடைத் தொழிற்சாலைப் பணியாளர்கள் அல்லது சோட்டா நாக்பூர் தொழிற்சாலைப் பணியாளர்களைப் பற்றிய மானுடவியல் ஆய்வுகள் ${ }^{31}$ முறை சார் கூலி சம்பந்தப்பட்ட உழைப்பு இடங்களில் வளர்ச்சியடைந்துள்ள சமூக உறவுகளை குறிப்பாக சுட்டிக்காட்டுகின்றன. அவைகள் குறிப்பாக மக்கள் எவ்வாறு தமது சமூக உறவுகளை பணிகளில் கொண்டு வருகிறார்கள் என்பதின் மேல் கவனம் செலுத்துகின்றன (உதாரணத்திற்கு திருப்பூர் பின்னலாடைத் தொழிற்சாலை பணியாளர்கள் விஷயத்தில் சாதி மர்றும் சோட்டா நாக்பூரில் உறவினர்கள் மற்றும் சுற்றங்கள்). கேரளாவின் ஈழவர்களைப் பற்றிய ஆய்விலும் இது போன்ற ஒரு நோக்கீடு ${ }^{32}$ செய்யப்பட்டிருக்கிறது. அங்கு சாதி உறுப்பினர் ஒருவர் மத்திய கிழக்குப் பகுதிக்குக் குடியேறுவது (குறிப்பாக யூஏஈக்கு) மற்ற உறவுக் குழுக்களுக்கும் சாதி உறுப்பினர்களுக்கும் அங்கு குடியேற உதவுகிறது, இதன் மூலம் உறவு முறையும் குடும்ப பந்தங்களும் மீண்டும் நிறுவப்படுகின்றன.

ஆகையால் நவீன தொழிற்பணி அமைப்புகளில் பணி மற்றும் வீட்டுக்கு இடையேயான உறுதியானப் பிரிவு முறை ஏற்பட்டிருந்தாலும், இவை இந்தியச் சூழலில் உறவு மற்றும் சாதியால் எப்பொழுதும் சமரசம் செய்யப்பட்டிருக்கின்றன என்பது தெளிவாகத் தெரிகிறது. ஆயினும் சாதி அடிப்படை நெட்வொர்க்குகள் மக்கள் ஒரே மதத்தினராகவும், சாதியினராகவும் இருக்கக்கூடிய குறிப்பிட்ட புவியியல் மண்டலத்தின் கூட்டுத் தொடக்கத்தின் அடிப்படையிலேயே இருந்தன. அவர்கள் ஒருவருக்கொருவர் உறவினராக இல்லாவிட்டா- 
லும், அவர்கள் ஒருவரை ஒருவர் விளக்கமான கற்பனை உறவு முறை வார்த்தைகளால் தெரிந்து வைத்திருந்தார்கள்; சில விதங்களில் உறவு முறை அடிப்படையிலான சமூகத்தின் மரபுவழியையேத் தொடர்ந்து வந்தன. ஆயினும் நகர்ப்புறத்திலிருந்து நகரத்திற்குக் குடியேறுதலைத் தொடர்ந்து இது மாற ஆரம்பிக்கிறது, ஏனென்றால் மக்கள் ஒரே சாதி பல மதங்களின் (இஸ்லாமியர்கள், கிறித்துவர்கள் மற்றும் இந்துக்கள்) குறுக்கே செல்வதைக் கண்டறிகிறார்கள். சாதி, சமகால இந்தியாவில் வகுப்புச் சமத்துவமின்மையின் மரபால் பின்னுக்குத் தள்ளப்பட்டாலும்,,33 ஒரு படிநிலை தகுதியைக் காட்டிலும் ஒரு கலாசாரப் பிரிவின் கூறாக விரைவாக மாறிக் கொண்டிருக்கிறது. ${ }^{34}$ அதிகம் சாதி அடிப்படையிலான நெட்வொர்க்குகள் இன்னும் இருக்கின்றன, ஆனால் அவைகள் அதிகமாக நவீனப் பணி அமைப்புகளில் போட்டியிடும் கட்டளைகள் மற்றும் வர்க்கங்களின் மிகவும் சிக்கலான மற்றும் தெளிவில்லாக் கட்டமைப்பின் ஒரு பகுதியாக மட்டுமே மாறிக் கொண்டு வருகின்றன. இவை பதிலுக்கு தொழிற்சங்கம் போன்ற அதிகம் பிரத்யேகமான குழுக்களாக மாறலாம். ${ }^{35}$

மேற்கண்டவாறு, நவீன தொழிற்பணி அமைப்புகளில் பணியிலிருந்து வீட்டை உறுதியாகப் பிரிக்கும் முறை உருவானாலும், இவ்வாறான இரு சமப் பக்கச் சூழல்களை மத்தியஸ்தம் செய்வது (வீடு மற்றும் பணி) சம்பளத்துடனான மேலாண்மை விட தொழில்துறைக்கு உள்ளேயும், வெளியேயும் இருக்கும் திறனற்ற, ஊதியம் சம்பந்தப்பட்ட பணிகளில் ${ }^{36}$ அதிகம் காணப்பட்டது. ஆயினும், இது மேலாண்மை உயர் படிமைகளிலும் ஒரு தொழிற்சாலையினுள் அதிகாரப் படிநிலை மற்றும் நிலையைப் பொறுத்துக் குறிப்பிட்ட சலுகைகள் வடிவத்தில் காணப்படுகிறது, இருந்தாலும், அவர்கள் இந்த மாதிரியான மத்யஸ்த வேலைகளில் ஈடுபடும்போது, மேலாண்மை உயர்ப் படிமைகளில் அவர்களது நிலைமை அவர்களின் புலனாகும் தன்மைக்கு அதிகம் வலு சேர்க்கிறது, இது அவர்கள் மீது உறவினர் ஆதரவுக் கொள்கைக் குற்றத்தை சுமத்த காரணமாகிறது. ஆயினும் குறைந்த ஊதியம் பணியாளர்களிடம் உறவினர் ஆதரவுக் கொள்கைத் தென்படுவதில்லை; தொழிற்சாலைகளில் தொழிலாளிகள் வந்துசெல்லுதல் மிக அதிகமாக இருக்கிறது ஆகையால் நெட்வொர்க்குகள் உதவியுடன் ஆட்சேர்ப்பு இந்த வந்துசெல் தொழிலாளிகள் மேலாண்மையில் மிகவும் பயனுள்ளதாக இருக்கும். 
ஆயினும், நாம் அடையாளம் கண்டு கொள்ள வேண்டியது என்னவென்றால், இந்தியாவில் தொன்று தொட்டு எல்லாப் பணிகளும் ஒரே இடத்தில் நடப்பதில்லை. ஒரு வெளியேற்றும் முறையும் ${ }^{37}$ இருந்தது, இதில் ஒரு தொழில்முறை செயலாக்கம் ஒரு குறிப்பிட்ட இடத்திற்குள் வரையறுக்கப் படுவதில்லை ஆனால் அவை செயலாக்கத்தின் பல நிலைகளைக் கடந்து செல்கின்றன. இவை ஒவ்வொன்றும் பணியாளரின் வடு போன்ற பல இடங்களில் நடை பெறலாம்.

தொழிற்சாலையின் உள்ளே பணி மற்றும் பணியல்லாதவைகளுக்கான எல்லைகளை மத்தியஸ்தம் செய்வதற்கான முழுச் செயலாக்கம், முழுத் தொழிலாளர் சக்தியின் உழைப்பாக இருந்தாலும், ஒரு விதத்தில் தொழிற்சாலைகளே இந்த மத்தியஸ்தத்தை நடக்க இயலச் செய்கின்றன. இவை அனைத்திலும் அமைப்பு முரண்பாடுகள் என விளக்கூடியவை இருக்கின்றன. தொழிற்சாலைகள் (சரியாகச் சொன்னால் பணியின் தொழிற்சாலை அமைப்பு) பணி மற்றும் பணியல்லாதவைகளுக்கு இடையே ஒரு உறுதியான பிரிவினையை நிறுவக் கடுமையாக முயற்சிக்கின்றன. ஆயினும் அதே நேரத்தில், குறைந்த ஊதியப் பணியாளர்களை தொழிற்சாலை அமைப்பில் பணியமர்த்த, அவர்கள் மற்றப் பணியாளர்களை பணியமர்த்த பரிந்துரைக்கவும், உதவி செய்யவும் ஊக்கப் படுத்துகிறார்கள். பணியாளர்கள் தம் பங்குக்கு, இந்த மாதிரியான விண்ணப்பங்களை ஆதரிப்பதன் மூலம், தம் குடும்ப அல்லது உறவுமுறை அல்லது சாதி அடிப்படையிலான உறுப்பினர்களை பணியமர்த்துகிறார்கள். ஒரு விதத்தில் இது, முறை சார் பணியிடங்களில், பணியல்லாதவை சம்பந்தப்பட்ட முறை சாராத் தொடர்புகளுக்கு ஒரு கிரியா ஊக்கி போல செயல்படுகிறது. இது பணியிடங்களின் சம்பிரதாயங்களை பலவீனப்படுத்துவதன் மூலம் பணியில் ஒரு பணியில்லாதச் சூழல் அமையக் காரணமாகிறது, ஆயினும், பணி முறை இந்தியாவில் பாரம்பரியமாகக் கடைப்பிடிக்கப் படுவதற்கேற்ப இருந்தாலும், முறைசார் பணி அமைப்பு இந்த ஏற்பாட்டை ஏற்காமல் இதன் மேல் பலக் கடுமையான தடைகளை விதிக்கிறது.

மேற்கூறிய எடுத்துக்காட்டு இ இந்த சுழல்முறைக் குழப்பத்தைக் காட்டுகிறது (படம் 5.1)

இந்த வகை பணி அமைப்புக்குள் குழப்ப சுழற்சியின் வெளிப்பாடு உற்பத்தித் துறையில் மட்டும் ஏற்படவில்லை. நாம் பார்க்கப்போவது போல, இது ஐடி போன்ற அறிவு செயலாக்கத் துறைகளின் பணி அமைப்புகளிலும் கூட இருக்கிறது; 
This brings in the

traditional idea of unity

between work and

non-work
Employees recruit their

own family/kids/caste

group

படம்: 5.1 பணியமைப்பு முரண்பாடு

ஆனால் இந்த முறை, சமூக ஊடகங்கள் மற்றும் தனிப்பட்ட டிஜிட்டல் தொடர்பு தொழில்நுட்பங்கள் சம்பந்தமாக.

\section{ஐடி பணிக் கலாசாரம்}

நாம் இந்தத் துறையினுள் கட்டமைப்புகளை மத்தியஸ்தம் செய்ய சமூக ஊடகங்கள் மற்றும் தனிப்பட்டத் தொடர்பு தொழில்நுட்பங்கள் உதவுகின்றன என ஆய்வு செய்வதற்கு முன்னால், இந்தியாவின் ஐடி துறையை ஆழ்ந்து கவனித்தல் உதவி செய்யலாம். இவ்வாறு செய்வதன் மூலம் நாம் பணி மற்றும் பணியல்லாதவற்றுக்கு இடையேயான எல்லைகளைத் தெளிவற்றதாக்குவதில் தனிப்பட்ட தொடர்பு தொழில்நுட்பங்களின் பங்கை நாம் நன்றாகப் புரிந்து கொள்ளலாம்.

1991ல் இந்தியப் பொருளாதார தாராளமயமாக்கலுக்குப் பின்பு, ஐடி/ஐடிஈஎஸ் ${ }^{38}$ துறையில் நாட்டின் இருப்பு படிப்படியாக முன்னேறியது. ${ }^{39}$ 1991க்குப் பின் அணுசக்தித் திறன் சோதனை மற்றும் அரசியல் நிலையுடன் இந்தத் துறையில் இதன் வளர்ச்சி, இந்தியப் பொருளாதாரத்தை ஒரு அறிவுப் பொருளாதாரமாகச் 40 சித்தரித்தது. இந்தத் துறையில் நுழைவது 
ஒருக் குறிப்பிடத்தக்க தேசிய கௌரவத்தின் அடையாளமாகக் கருதப்பட்டது. இது மக்களைக் கல்வி மூலதனத்தில் முதலீடு மூலம் இடுகுறி மூலதனத்தை ${ }^{41}$ நிர்மாணிக்க இயலுமைப் படுத்தியது மற்றும் சமூகத்தில் உயர்ந்த நிலைக்குச் செல்லும் அவாவையும் ${ }^{42}$ ஊக்கப்படுத்தியது. இவ்விதமானப் பொருளாதாரத்தின் இடுகுறியான ஐடி/ஐடிஈஎஸ் துறையின் வளர்ச்சி, பன்னாட்டு மற்றும் நாடு கடந்த வாடிக்கையாளர்களுக்கு உதவ மிகவும் திறன் வாய்ந்த அறிவானப் பணியாளர்களைப் பணியமர்த்தக் காரணமாகவும் ஆயிற்று. ${ }^{43}$ இவ்விதமானப் பணியமர்த்தல் செயல்கள் தகுதி அடிப்படையிலேயே நடப்பதாகச் சொல்லப்பட்டாலும், உறவுமுறை, குடும்பம் மற்றும் சாதிப் போன்ற விஷயங்களை விட ஒருவரின் கல்வி மற்றும் திறமைக்கே முன்னுரிமைக் கொடுக்கப்படும் காரணத்தால், திறமைத் தலைமையின் உண்மையான அளவுக் கேள்விக்குறியாகிறது. ${ }^{44}$ ஆயினும், ஆய்வுகள் ${ }^{45}$ காட்டுவெது போல், ஐடி/ ஐடிஈஎஸ் துறைப் பரிந்துரைகளை ஏற்றாலும், இது இன்னும் ஆங்கில அறிவு, கம்ப்யூட்டர் புரொகிராம்மிங் போன்ற குறிப்பிட்டத் திறன்களை அடிப்படையாகக் கருதுகிறது. இந்தத் துறை தன்னுடைய எதிர்காலம் அதிகம் திறன் வாய்ந்த பணியாளர்களிடமே இருக்கிறது என்பதை அடையாளம் கண்டு கொண்டிருக்கிறது, ஆகையால் அது நாம் சோட்டா நாக்பூர் மண்டலத்தின் திறனற்றப் பணியாளர்களினிடையேக் கண்ட குடும்ப உறுப்பினர்கள் மற்றும் அதே உறவுமுறை மற்றும் சாதியைச் சேர்ந்தவர்களை அறிமுகம் செய்வதைத் தடை செய்கிறது. இதைப் பற்றி மேல் ஆய்வு ${ }^{46}$ ஜவுளித் துறையிலும் உறவுமுறை அடிப்படையிலான பணியமர்த்தலுக்கான வரையறையைத் தெளிவாகக் குறிப்பிடுகிறது.

ஆயினும், ஐடி/ஐடிஈஎஸ் புரொகிராம்மிங், வணிக செயலாக்கம் புற ஒப்படைப்பு (BPO), அறிவு செயலாக்கம் புற ஒப்படைப்பு (KPO), அல்லது தகவல் அழைப்பு மையம் (call centres) போன்றவற்றில் ஈடுபட்டிருக்கும் பணியாளர்களைச் சூழ்ந்து கொள்வது மட்டுமில்லை என்பதை ஒருவர் நினைவில் கொள்ள வேண்டும். இந்தப் பிரிவுகளைக் காட்டிலும் இந்தத் துறை மிகவும் பெரியது. இது சுத்தம்/தரை ஊழியர்கள், உணவு விடுதி மற்றும் சிற்றுண்டிச் சாலைப் பணியாளர்கள், வாகன ஓட்டிகள், எலக்ட்ரீஷியன்கள், பாதுகாப்புப் பணியாளர்கள் போன்ற ஆதரவு அமைப்புகளை குறைந்த ஊதிய தரத்திலும், அத்துடன் ஆலோசகர்கள், மனித வளத்துறை மற்றும் இயக்கப் பணியாளர்களை நடு மற்றும் மூத்த நிலையிலும் பணியமர்த்துகிறது. குறைந்த ஊதியத் தரத்தினருக்கு அவசியமாக 
புரொகிராம்மிங் பற்றிய அறிவோ, ஒரு முழுமையான பாஷா ஞானமோ அல்லது ஆங்கில உச்சரிப்போ அவசியம் இல்லை. ஆகையால், ஐடி துறையினுள் இந்தக் கீழ் நிலைப் பணிகளுள் உறவு அல்லது குடும்ப இணைப்புகள் அடிப்படையிலான பரிந்துரைகள் இப்பொழுதும் நடக்கலாம். ஆயினும் மீண்டும் ஒரு முறை இவை எவ்வாறு ஒரு முறைசார் சூழலில் இயங்குகின்றன என்பதைக் காண விமர்சனப் பகுப்பாய்வு செய்யத் தேவைப்படலாம். மேலும் ஐடி/ஐடிஈஎஸ் துறையில் பணியாளர்கள் வந்து செல்லுதல் அதிகமாகக் காணப்படுகிறது, ${ }^{47}$ இது ஒரு பணியிடத்தில் உறவுமுறை, சாதி அல்லது குடும்ப பந்தங்களின் நெட்வொர்க்கில் ஸ்திரமற்ற தன்மையையும் உறுதி செய்கிறது..$^{48}$

இந்தியாவில் பெரும்பாலான ஐடி/ஐடிஈஎஸ் நிறுவனங்களால் பகிரப்படும் பணிக் கலாசாரம் தனித்தன்மையுடையதாகும். அது தொழிற்பண்பட்டப் பணியாளர்களின் சார்பு மதிப்பை அதிகப்படுத்துகிறது மற்றும் ஒரு 'பாலின சமநிலை' உருவத்தை சித்தரிப்பதின் மூலம் அதிகப் பெண் பணியாளர்களை ஈர்க்கிறது என்ற பெருமைகளைக் கொள்கிறது. ${ }^{49}$ இன்னொரு பக்கம், உடல் வணிகம் (body shopping) $)^{50}$ நிகழ்ச்சி பணியாளர்களை எப்பொழுதும் தாம் செய்வதில் கவனமாக இருக்கக் கட்டாயப் படுத்துகிறது, கம்பெனிகள் அவர்களை எப்பொழுதும் பணியிடங்களுடன் தொடர்பில் இருக்க ஊக்குவிக்கிறார்கள் என்பதையும் இந்த ஆய்வு சுட்டிக் காட்டுகிறது. இந்திய ஐடி/ஐடிஈஎஸ் துறையின் இன்னொரு கூறு அதனுடைய தனித்தன்மை வாய்ந்த நெட்வொர்க் கலாசாரமாகும். அதிகார பூர்வமான நெட்வொர்க்கிங் தளங்களின் தோற்றத்துடன், மக்கள் எப்பொழுதும் தமது பணியிடங்களுடன் தொடர்பிலேயே இருக்கிறார்கள். இது இந்திய ஐடி பணிக் கலாசாரத்தின் உண்மையாக இருக்கக் கூடிய நெட்வொர்க்குடனான நிறுவனங்களின் கலாசாரத்தைப் 51 பற்றியக் கருத்துடன் சம்பந்தப்பட்டிருக்கிறது, ஏனென்றால் இந்த கம்பெனிகளில் பல யுஎஸ்சில் இருக்கும் தமது வாடிக்கையாளர்களின் நிறுவனங்களைப் போல தமது பணியிடங்களையும் அமைத்திருக்கின்றன.

மேற்கத்திய வாடிக்கையாளர்களைத் திருப்தி படுத்துவதைப் பற்றிய எதிர்பார்ப்பு மற்றும் சர்வதேச மற்றும் நாடு கடந்த வாடிக்கையாளர்களுக்கேற்ப தமது பணிக் கலாசாரத்தை வடிவமைப்பது ஆகியவை ஐடி/ஐடிஈஎஸ் சூழலில் பணி மற்றும் வீட்டு மண்டலங்களுக்கு இடையே ஒரு திடமானப் பிரிவை உறுதி செய்துள்ளது.52 மேலும் இந்தியத் தொழிற்பணியாள- 
ர்களைப் பற்றிய ஆய்வில் உள்ள மிகப் பெரிய இடைவெளி என்னவென்றால் பணியிடங்களில் உறவு முறை-சமூக உறவுகளும் பரந்த நகர்ப்புற சுற்றுப்புறங்களில் சமூக உறவுகளும் ஆய்வு செய்யப்படும் தீவிரம்தான்..$^{53}$ இந்திய ஐடி/ஐடிஈஎஸ் துறையின் பணி அமைப்புகளைப் புரிந்து கொள்ள விரும்பும் எவருக்கும் உறவு முறைகளின் பொது உறவுகள், பணியிடங்களில் சமூக உறவுகள், தமது பரந்த சுற்றுப்புறத்துடன் ஐடி/ஐடிஈஎஸ் பணியாளர்களின் உறவுகள் ஆகியவை ஒரு குறிப்பிடத்தக்க பங்கை வகிக்கக் கூடிம்.

சமூக ஊடகங்கள் பணிக் கலாசாரத்தில் பொருந்துவதையும், பணி மற்றும் பணியல்லாதவற்றின் எல்லைகளை மத்தியஸ்தம் செய்ய உதவுவதில் அவற்றின் பங்கையும் ஆய்வு செய்யும் ஒரு பகுதியாக, ஐடி கம்பெனிகள் சமூக ஊடகங்களின் பயன்பாட்டின் மீது ஏன் குறிப்பிட்ட தடைகளை விதிக்கின்றன மற்றும் பணியாளர்கள் எவ்வாறு தொடர்ச்சியாக அவற்றை மீறுகிறார்கள் என்பதைக் கருத்தில் கொள்ளுதல் பயனுள்ளதாகும்.

\section{தடைகளை மீறுதல்}

இருபதாம் நூற்றாண்டின் கடைசிப் பத்தாண்டுகளும், இருபத்தொன்றாம் நூற்றாண்டின் ம முதல் பத்தாண்டுகெளும் முன்னேறிக் கொண்டிருக்கும் ஐடி துறைக்கு, முக்கியமான ஆண்டுகளாகும். இந்தத் துறை இந்தியாவில் உறுதியாகக் கால் பதிக்க முயன்று கொண்டிருக்கும் இந்த சமயத்தின் பல்வேறு ஆய்வுகள், நாட்டின் ஐடி துறை மற்றும் அதனால் வளர்ந்திருக்கும் அதன் பணிக் கலாசாரத்தின் பரிணாமத்தை விவரிக்கின்றன. ${ }^{54}$ ஒரு வட அமெரிக்க/ஐரோப்பிய வாடிக்கையாளர் குழுவுக்கு சேவை செய்வதன் மூலம் இந்த வணிக/ அறிவு செயலாக்க புற ஒப்படைப்புத் துறைகள் ${ }^{55}$ ஆங்கிலத்தை தமது பொது மொழியாக ஏற்றுக் கொண்டன. அவர்கள் தமது நுட்பமான வாடிக்கையாளர் குழுவிடம் தமது தொழில் சார்ந்த மனப்பான்மையை வலியுறுத்த வணிக முறை உடைகளுக்கான உடைக் குறியீட்டை ஏற்றுக் கொண்டார்கள், வாடிக்கையாளர்களும் இந்திய அலுவலகங்களில் பங்குதாரர் ஒப்பந்தங்களைக் கையெழுத்திட தமது மூத்தப் பணியாளர்களை அனுப்பினார்கள். ${ }^{56}$ இந்தச் செயல்முறை முந்தைய தொழிற்சாலை அமைப்புகளுக்கு எதிராக, ஒரு பெரிய அளவு தொழில் சார்ந்த மனப்பான்மையை வெளிப்படுத்துவதை 
நோக்கிய ஒரு கலாசாரத்தை ஏற்றுக் கொள்வதை அவசியமாக்கியது. திறனுடனான மற்றும் திறனற்ற உடல் உழைப்புத் தொழிலாளிகள் பணி செய்யக்கூடிய பணித்தளம் இப்பொழுது நன்கு படித்த, தொழில்நுட்பத் திறன் பெற்ற மற்றும் ஆங்கிலத்தில் உரையாடக் கூடிய பணியாளர்களுக்கான தனியறை ஆயிற்று. பொதுவாக, பாரம்பரியமான தொழிற்சாலைகள் தமது தனிப்பட்ட வாடிக்கையாளர்களின் ஒப்புதலுக்காக தமது பணியிடங்களை வெளிக்காட்ட வேண்டியக் கட்டாயத்தில் இல்லை. ஆயினும் ஐடி துறையின் வாடிக்கையாளர்கள், தகவல்களின் இரகசியம் மற்றும் பாதுகாப்பு அகியவற்றின் அக்கறையால், இந்திய அறிவு செயலாக்கத்துறையின் இந்த அமைப்பை மேற்பார்வை இட விரும்புகிறார்கள். இவ்வாறு செய்கையில் எப்பொழுதும் ஆர்வமுடனும், எச்சரிக்கையுடனும் இருக்கும் இந்த வாடிக்கையாளர் குழு இந்தத் துறை உயர்ந்த தரத்தைப் பேணுவதை உறுதி செய்தது.

ஆயினும் இம்மாதிரியான வளர்ச்சிகளும் ஒரு பரிணாம வளர்ச்சியின் செயல்முறைதான். மேற்கத்திய பணிக் கலாசாரத்தையும், பணியிடக் கொள்கைகளையும் தனதாக்கிக் கொள்வது (பொது மற்றும் தகவல் பாதுகாப்பு அமைப்புகள், பெரிய கம்பெனிகளில் தனியறை அமைப்புகள் மற்றும் சிறியவற்றில் பணி நிலையங்கள், செலவைக் கட்டுப்பாட்டுக்குள் வைத்திருக்கும் தொழில்முனைவோரால் நடத்தப் பெறும் தொடக்கநிலை நிறுவனங்கள் போன்றவை) அவர்களுடைய வாடிக்கையாளர்களின் கவலையைக் குறைக்க உதவி புரிந்தன. இது பதிலுக்கு 'முறை சார்ந்த உடைக்கு ${ }^{57}$ பதிலாக 'வணிக சாதாரணத்திற்கு' மெதுவான பரிணாமம், வெள்ளிக்கிழமை உடைகளின் தளர்த்தப்பட்ட விதிமுறை கலாசாரம், மற்றும் வார இறுதி வேலை விடுப்பு ஏற்பாடு ஆகியவற்றுக்கு உட்பட்ட உடைக் கோட்பாட்டை உள்ளடக்கிய மற்ற அம்சங்களின் மேலும் தாக்கத்தை ஏற்படுத்தியது. காரியாலய உடற்பயிற்சி சாலை, ஸ்னூக்கர் மேஜை மற்றும் டேபிள் டென்னிஸ் வசதிகள் இவற்றுடன் சிறிய சமையலறை, உணவு விடுதிகள் மற்றும் சிற்றுண்டிச் சாலைகள் ஆகியவை இந்தத் துறையில் தனக்குரியதாக்கப்பட்டன - இவை இரண்டிம் படித்த இந்திய இளைஞர்களை வசீகரிப்பதற்காக மற்றும் தமக்குச் சொந்தமான வணிகச் சூழலில் உலகளாவிய வாடிக்கைக் குழுவின் கலாசாரத்தை பிரதிபலிப்பதற்காக செய்யப்படுகின்றன. (இந்தத் ஏற்றுக் கொள்ளுதல் இன்னும் நிறைவு பெறவில்லை, ஆயினும் பல கம்பெனிகளில் இது நடப்பு வேலையாக இருந்து வருகிறது). இவை அனைத்தும் 
இருந்தாலும், இந்திய ஐடி கம்பெனிகளுக்கு பணி மற்றும் பணி அல்லாத மண்டலங்களுக்கு இடையே ஒரு திடமான பிரிவினைத் தேவைப்பட்டது. நவீன நடைமுறைகள் பணியாளர்களை பணியிடங்களுக்கு வெளியில் பணி அமைப்புகளை அணுக அனுமதித்தாலும், (வீட்டுக்கு மடிக்கணினிகளை எடுத்துச் செல்வதன் மூலம்) பணியல்லாதவற்றைப் பணியிடங்களுக்குக் கொண்டு வருவது பெரும்பாலான கம்பெனிகளில் இன்னும் ஏற்றுக்கொள்ளப்படாததாகவே இருந்தது.

ஒரு டிஜிட்டல் சுகாதாரப் பொருட்களின் விற்பனை இயக்குநரான 47 வயது சரத், ஒரு பன்னாட்டு ஐடி கம்பெனியில் பணி புரிந்தார் மற்றும் முறை சார் உடைகளையே அணிந்திருந்தார். அவர் முன்னே (ஏறக்குறையப் பத்தாண்டுகளுக்கு முன்) வாடிக்கையாளரிடமிருந்து கட்டணம் வசூலிக்க வேலை நேரங்கள் சரியாக அளக்கப்பட்டு அளவினை முடிவு செய்யப்பட்டு வரும் கலாசாரம் இருந்தது எனக் கூறுகிறார். கட்டணம் வசூலிக்கக்கூடிய பணியாளர் நேரம் குறைந்து விட்டதால், இது இயல்பாக பணியல்லவை சம்பந்தமான தொடர்புகளின் மேல் விருப்பமின்மையின் காரணமாக அமைந்தது. சென்ற சில வருடங்களில் இந்த மாதிரியான எண்ணம் குறைந்திருந்தாலும், இம்மாதிரியான கலாசாரம் இன்னும் சில கம்பெனிகளில் இருக்கின்றன.

34 வயதான கேசவ், ஷரத்தின் அணித் தோழராக இருக்கிறார் அவரிடம் நேராக அறிக்கைகளை சமர்ப்பிக்கிறார். ஒரு இஞ்ஜினீயர் மற்றும் அணித் தலைவராக இருக்கும் அவர், சில வருடங்களுக்கு முன்னால்யுஎஸ்சிலிருந்து ஒரு வாடிக்கையாளர் அவரது அணிக்கு வருகை தந்த போது நடந்த நிகழ்ச்சியை விவரிக்கிறார். வாடிக்கையாளரின் செயல் திட்டத் தணிக்கை வருகையின் போது, அவர் கேசவ் அணியின் உறுப்பினர்கள் தம் மேஜையில் ஸ்மார்ட்ஃபோன் உபயோகிப்பதைக் கண்டார். இந்த வாடிக்கையாளர் ஒரு அணி உறுப்பினரை ஃபோனின் காமரா செயல்பாட்டைப் பற்றி விசாரித்தார். அவர் வாடிக்கையாளரை திருப்தி செய்யும் ஆர்வத்தில், அணி உறுப்பினர் அவர் கேள்விக்கு பதில் அளித்ததோடு நில்லாமல், ஃபோனின் மிகச் சிறந்த காமரா செயல்பாட்டை உறுதி செய்ததுடன், அந்த மனிதருடன் ஒரு ஃபோட்டோவும் எடுத்துக் கொண்டார். வாடிக்கையாளர் மிகவும் கோபமடைந்து காமரா ஃபோனின் சட்டவிரோத உபயோகத்தினால் தகவல் திருட்டு ஏற்படலாம் என பயந்து, கேசவின் அறைக்குள் புயலென நுழைந்து, உடனடியாக செயல் திட்டத்தை ரத்து செய்வதாக அச்சுறுத்தினார். 
வழக்கமானக் கொள்கை என்னவென்றால் முக்கியமான செயல்திட்டங்களில் காமிரா ஃபோனுக்கு அனுமதி இல்லை (ராணுவ மற்றும் நிதி சம்பந்தமுள்ளவை போன்ற), இது போன்ற விதிகள் வாடிக்கையாளரின் குறிப்பிட்ட விசாரங்களுக்கு ப்திலாக சில நேரங்களில் முக்கிய ஐடி செயல் திட்டங்களுக்கும் பிரயோகிக்கப் படலாம். அந்த நாளிலிருந்து கேசவ்வின் அணி சுகாதாரம் சம்பந்தப்பட்ட ஐடி செயல் திட்டங்களின் போது காமிரா ஃபோனை உபயோகிப்பதைத் தடை செய்தது. இது ஸ்மார்ட்ஃபோனை (மற்றும் எந்த சமூக ஊடகத்தையும்) பணியில் கொண்டு வரத் தடை செய்தது; அவற்றை இடைவேளைகளில் மட்டும் சிறிது தூரத்தில் இருக்கும் பாதுகாப்புப் பெட்டகத்திலிருந்து எடுத்து உபயோகிக்கலாம். ஆனால், சமூக ஊடகத்தின் தோற்றத்திற்குப் பின் இந்த இடைவேளைகள் அடிக்கடி ஏற்படுவதாக கேசவ் குறிப்பிடுகிறார்; பணியாளர்கள் தமது வாட்ஸ் அப் அல்லது ஃபேஸ்புக் செய்திகளைக் காண ஒவ்வொரு அரை மணி நேரத்திற்கும் இடைவெளி எடுப்பதைக் காண்பது அசாதாரணமானதல்ல. சமூக ஊடகங்களின் உபயோகமும் கம்பெனியால் தடை செய்யப்பட்ட நிலையில் ஸ்மார்ட் ஃபோன் அணுகல் மீதான தடை தகவல் பாதுகாப்புக் காரணங்களுக்கான இந்தக் கட்டுப்பாட்டுக் கொள்கைஉடன் கூடியது.

ஆயினும், அனைத்து கம்பெனிகளும் ஸ்மார்ட்ஃபோன் அணுகலைத் தடை செய்யவில்லை என்பதைக் கூற வேண்டும்; இது செயல் திட்டத்தின் வகை, தனிப்பட்ட வாடிக்கையாளரின் கொள்கைகள் மற்றும் விசாரங்கள் போன்றவையை சார்ந்தே இருக்கிறது. ஃபேஸ்புக், யூட்யூப் மற்றும் ட்விட்டர் போன்ற தளங்களில் சமூக ஊடகங்களை அணுகுவதற்கான தடைபெரும்பாலான பெரிய அளவிலான ஐடி கம்பெனிகளின் நிலையானப் பழக்கம். அவைகள் இந்த முக்கிய சமூக நெட்வொர்க்கிங் தளங்களை பணியிலிருந்து ஒரு கவனச் சிதறலாகவே காண முற்படுகிறார்கள், இது அவற்றைப் பணியல்லாதவையாக வகைப் படுத்துகிறது. தொழில்சார் நெட்வொர்க்கிங் தளமாகிய லிங்க்ட் இன் இந்த கம்பெனிகளுள் சிலவற்றில் விதிவிலக்காக இருக்கிறது. இது தமது ஐடி சம்பந்தமான அறிவு குழுக்களுடன் ஏறக்குறைய ஒரு அறிவுப் பரிமாற்றத் தளமாகப் பார்க்கப்படுகிறது, ஆகையால் சில கம்பெனிகள் இந்த தளத்தை மட்டும் அணுக அனுமதி அளித்திருக்கின்றன. ஆயினும், மீண்டும் ஒரு முறை, இது ஒருவர் சார்ந்திருக்கும் செயல் திட்டத்தையும் அணியையும் பொறுத்ததே. 
எவ்வாறாயினும், இம்மாதிரி விதிமுறைகளுக்கு அவற்றின் வரம்புகள் உண்டு. ஒரு ஐடி கம்பெனியின் 20 ஆண்டு மனித வள அனுபவமுள்ளவர், ராஜகோபால் விவரிப்பது போல்:

இஞ்ஜினீயர்கள் விஷயத்தில் இந்தத் தடைகள் எப்பொழுதும் மீீறப்படுகின்றன. இந்த தடைகள் கண்டிப்பாகப் பின்பற்றப் படுகின்றன என நினைத்தால் நாம்தான் முட்டாள்கள். ஆயினும் நாம் இந்த இஞ்ஜினீயர்களின் நாணயத்தை நம்புகிறோம். ஒன்று நிச்சயம்; நமது தகவல்களை திருடினாலோ அல்லது அவற்றை பாதிக்கக் கூடியதாக்கினாலோ தமது வேலை பறி போகும் என அவர்களுக்குத் தெரியும், நமக்குத் தகவல் திருட்டைப் பற்றிய பிரச்சினை ஏற்பட்டால், நாம் அவர்கள் மேல் குற்றவியல் நடவடிக்கைகள் எடுக்கப்படுவெதை உறுதி செய்வோம், இது பதிலுக்கு அவர்கள் தொழிலையும் வாழ்வையும் பாதிக்கும்... ஆகையால், அவர்கள் இதைப் புரிந்து கொள்கிறார்கள்... அத்துடன் இ இந்தத் தடையை யார் மீறுகிறார்கள் என நம்மால் எளிதாகக் கண்டுபிடிக்க முடியும்.

24 வயதான திருமணமாகாத விஷாக் மற்றும் சுஜித் இருவரும் இஞ்ஜினீயர்கள். அவர்கள் தெலுங்கானா மாநிலத்திலிருந்து வருகிறார்கள், இப்பொழுது பஞ்சக்கிராமியில் ஒரு வணிக செயல் முறை புற ஒப்படைப்பு கம்பெனியில் பணி புரிகிறார்கள். அவர்கள் பணிக்களத்திற்கு அருகிலேயே வசிக்கிறார்கள். விஷாக்கும் சுஜித்தும் ஒரு ஐரோப்பிய கம்பெனிக்கான ஒரு வங்கி செயல் திட்டத்தில் பணி புரிகிறார்கள். தகவல் திருட்டு பற்றிய கவலைகளினால் அலுவலகக் கம்ப்யூட்டர்களிலிருந்து சமூக நெட்வொர்க் தளங்களை அணுகுதலின் மேல் கடுமையான தடைகள் உள்ளன. ஆனாலும், பணியாளர்கள் தமது வாடிக்கையாளரின் ஐரோப்பிய கணினியுள் லாக்-இன் செய்யும் பொழுது (இதை இவர்கள் தமது பணியின் ஒரு அங்கமாகச் செய்ய வேண்டும்), அவர்களுக்கு சமூக ஊடகங்களில் எல்லையில்லா அணுகுதல் கிட்டுகிறது. இவ்வாறே, வார இறுதியில் (தமது ஓய்வு நேரத்தில்) விஷாக்கும் சுஜித்தும் பழைய கிரிக்கெட் பந்தயங்களைப் பார்க்கவும், படங்களைப் பதிவிறக்கம் செய்யவும் யூட்யூப் வீடியோவை அணுகினார்கள். இவை அனைத்தும் அவர்களது வாடிக்கையாளரின் கணினி மூலமாகவே. இவை தெரிய வந்தால் இந்த மாதிரியான 
பழக்கங்கள் ஏற்றுக் கொள்ளப்படாது மற்றும் பணி முடிவுக்கும் காரணமாகி விடலாம். விஷாக்கும் சுஜித்தும் தமது சக பணியாளர்கள் பலரும் இவ்வாறே செய்வதைத் தெரிந்து கொண்டார்கள். அவர்களுடைய மேலாளரும் சில சமயங்களில் இவ்வாறே செய்வதாகவும், அவர்களது வேலை செய்து முடிக்கப்பட்டு மற்றும் தகவல் பாதுப்பு அம்சம் மீது சமரசம் செய்யப்படாமல் இருக்கும்போது, அல்லது வாடிக்கையாளரின் பக்கத்திலிருந்து எந்த வித புகாரும் வராமல் இருக்கும் பட்சத்தில் இந்த ஆன்லைன் சாகசங்களைப் பற்றிக் கவலைப்பட மாட்டார் எனவும் அவர்கள் துடுக்காகக் கூறினார்கள்.

விஷாக் மற்றும் சுஜித்தின் இந்த நிகழ்வும், ராஜகோபாலின் அறிக்கையும், இம்மாதிரியான எல்லைமீறல்கள் எப்போதும் இருப்பதை நிரூபிக்க துணை புரிகின்றன. மிகவும் தகுதி உள்ள இஞ்ஜினீயர்களுக்கு இந்த மாதிரியான திடமான அமைப்பு ஒரு கேலியைப் போல அமைந்து விடுகிறது. பணி அமைப்புக் குழப்பத்தின் இன்னொரு நிகழ்வும் வெளிப்படையாக இருக்கிறது; வாடிக்கையாளர் இந்தியப் பணியாளர்களை இந்தியக் கணினிகளின் மூலம் சமூக ஊடகங்களை அணுகுவதை தடை செய்யலாம், ஆனால் அந்த மாதிரி தடைகள் ஏதுமில்லாத தம் கணினிகள் மூலம் இந்த அணுகலை அனுமதிக்கலாம். ஐடி கம்பெனியின் நிலையிலிருந்து, அவர்கள் தடை விதித்தாலும் அதை எளிதில் மீறக் கூடிய உயர்ந்த தகுதி படைத்த இஞ்ஜினீயர்களுடன் வினை மேற்கொள்கிறார்கள் என்பதையும் அடையாளம் கண்டு கொள்கிறார்கள். அவர்கள் வேறு வழியின்றி தமது அமைப்பை விட தமது பணியாளர்களின் நாணயத்தை நம்பத் தயாராகிறார்கள். இந்த விதமானக் குழப்பம் அணுகலைத் தடை செய்வதன் மேல் மட்டுமல்லாமல், அலுவலக உட்புறத் தொடர்பு போன்ற மற்ற பணி பழக்கங்களிலும் காணப்படுகிறதது.

இன்னும் கம்பெனிகளின் ஒரு குழு முழுத் தடையிலிருந்து வரையறைப் படுத்தப்பட்ட அணுகல் வரைப் பயணிக்கிறது. நடுத்தர அளவு கம்பெனிகள் பணியாளர்களின் மக்களுடன் பழகுவதற்கான தேவையைப் புரிந்து கொள்கிறார்கள் ஆதலால் சமூக ஊடகங்களில் வரையறைக்குட்பட்ட அணுகல்களை அணுகலின் நேரங்களைக் கட்டுப்படுத்துவதின் மூலம் அனுமதிக்கிறார்கள் - நடு ம மதியத்திலிருந்து மதியம் 1 மணி வரை அல்லது மாலை 6 மணிக்குப் பிறகு, உதாரணத்திற்கு இதை சில தகவலர்கள் ‘ஃபேஸ்புக் நேரம்' என விவரித்தார்கள். இவற்றில் பல கம்பெனிகள் லிங்க்ட் இன்னிற்கு முழு அணுகலையும் வழங்கினார்கள், மற்ற சமூக ஊடகங்க- 
ளுக்குத் தடை விதித்தாலும், இதை இவர்கள் அறிவு சாதனமாகப் பார்த்தார்கள். ஆயினும் வளம் மிக்கப் பணியாளர்கள் லிங்க்ட் இன்னையும் தனிப்பட்ட செய்திகள் அனுப்ப பயன் படுத்த முடியும். உதாரணத்திற்கு 24 வயது ஐடி பணியாளரான சுசித்ரா ஒரு ஸ்மார்ட்ஃபோன் தடை செய்யப்பட்ட செயல்திட்டத்தில் பணி புரியும் போதே இன்னொரு ஐடி கம்பெனியில் இருக்கும் தனது தோழிக்கு லிங்க்ட் இன் மூலம் தொடர்பு கொண்டார்; அவர்கள் வெறுமனே லிங்க்ட் இன் செய்தி அமைப்பு மூலம் தமது தனிப்பட்ட செய்தியை அனுப்பினார். ஆயினும் பெரும்பாலான இந்த செய்தி அலைவரிசைகள், இம்மாதிரியான தடைகள் நீக்கப்படும் போது மாறுகின்றன. உதாரணத்திற்கு,சுசித்ரா பணியாளர்களை ஸ்மார்ட்ஃபோன் கொண்டு வர அனுமதிக்கும் செயல் திட்டத்திற்கு மாறிய போது அவருடையத் தனிப்பட்ட தொடர்பு வாட்ஸ் அப்பிற்கு மாறியது, இது கம்பெனியின் சொத்துக்களை தனிப்பட்ட உபயோகத்திற்கு பயன்படுத்துவதைக் குறைத்தது.

ஒப்பீட்டளவில் சிறிய மற்றும் புதிதாகத் தொடங்கிய தொழில்களில் இந்த மாதிரியானத் தடைகள் இருப்பதில்லை. பணியாளர்கள் ஸ்மார்ட்ஃபோன் மற்றும் தமது பணிக் கணினி மூலமும் சமூக ஊடகங்களை அணுக முற்பட்டார்கள், மேலும் இப்பொழுது வாட்ஸ் அப் உடனடி செய்தி சேவையின் இடத்தைப் பிடித்து விட்டதால், ஒரு பணியிடத்திலிருந்து பணியல்லாதவைகளிடம் அளவில்லா அணுகல்கள் வெளிப்படையாகக் காணப்படுகிறது. குறிப்பிடத்தக்க வகையில், கம்பெனி உபகரணங்களிலிருந்து சமூக ஊடகங்களை அணுகும் விஷயத்தில் ஃபேஸ்புக், லிங்க்ட் இன் மற்றும் யூட்யூப் ஆகியவை பணியிடங்களிலிருந்து மிக அதிகம் வருகை புரிந்த சமூக ஊடக தளங்கள் என்ற தகுதியைப் பெறுகின்றன - முக்கியமாக இந்த சிறிய கம்பெனிகள் மற்றும் புதிதாகத் தொடங்கிய தொழில்களின் பணியாளர்களால்.

ஒப்புக்கொண்டபடி, பெரும்பாலான இந்தப் புதிய தொழில்கள் இணையதளத்தை அடிப்படையாகக் கொண்டவை; அவர்களுடையப் பொருளாதாரப் படிமம் விளம்பரம் மற்றும் மொபைல் அப்ளிகேஷன்களை மையமாகக் கொண்டது. ஆனால் சமூக ஊடகங்கள் மூலமாக தனித் தொடர்பு இந்த வகையான பணிகளுடன் கணிசமாகக் குறைகிறது, ஏனென்றால் பெரும்பாலான சமூக ஊடக அணுகல்கள் இப்பொழுது பணியையே மையமாகக் கொண்டிருக்கின்றன. 22 வயது இயற்பியல் பட்டதாரி மற்றும் மொபைல் மற்றும் சமூக ஊடக ஆப்களை வடிவமைக்கும் ஒரு புதிய தொழிலின் 
சுயமாகக் கற்றுக்கொண்ட கம்ப்யூட்டர் புரோகிராமர், இவ்வாறு கூறுகிறார்:

தொடர்ச்சியான தனிப்பட்ட தொடர்புகள் தொல்லை தரக்கூடியனவாக இருக்குமாதலால், என்னுடைய கம்பெனியில் உள்ளவர்கள் ஆப்பை வடிவமைக்கும் போது ஃபேஸ்புக்கில் புலனாகா விதத்திற்கு சென்று விடுகிறார்கள் மேலும் நாங்கள் பெரும்பாலும் பணிக் கணக்கிலிருந்தே சமூக ஊடகங்களில் உள் நுழைகிறோம், இதை எதிர் கொள்ள தனிப்பட்ட கணக்குகளிலிருந்து செல்வதில்லை. என்னுடைய மேலதிகாரியும் எனக்கருகிலேயே அமர்ந்திருக்கிறார், ஏனென்றால் எங்களுக்கு மாறக்கூடிய பணியிடக் கொள்கை ${ }^{58}$ இருக்கிறது. பணியிடம் சிறியதாக இருக்கிறது, ஆகையால் நீங்கள் என்ன செய்து கொண்டிருக்கிறீர்கள் என்பது அனைவருக்கும் தெரியும். ஒருவரும் உடனடியாக உங்களுடைய தனிப்பட்ட கவனச் சிதறல்களை உங்களுக்குத் தெரியப்படுத்தா விட்டாலும், உங்களை நேராக்கக் கூடிய புலனாகும் குறிப்புகளை நிச்சயம் தருவார்கள். ஆகையால் நாங்கள் வாட்ஸ் அப் மூலம் தொடர்பு கொள்கிறோம்... இது எளிமையாகவும், ஜாக்கிரதையானதாகவும் இருக்கிறது, மேலும் ஒருவரையும் தொந்திரவு செய்வதுமில்லை. மேலும் இவ்வாறான சிறிய அமைப்புகளில் நீங்கள் என்ன செய்கிறீர்கள் மற்றும் எப்பொழுது ஆன்லைனில் ஏதும் செய்கிறீர்கள் என்பதை தெரிந்து கொள்வது எளிது. என்னுடைய நல்ல நண்பர்கள் என் பணியிடத்திலேயே இருந்தாலும், நான் நேருக்கு நேராகத் தொடர்பு கொள்கிறேன். எனக்கு வாட்ஸ் அப்பில் கல்லூரி நண்பர்கள் மட்டுமே இருக்கிறார்கள்... ஆகையால் பணிக்கு வெளியேயானவர்களுடன் பணி நேரத்தில் தொடர்பு கொள்வது சில நேரங்களில் மிகவும் குறைவாகவேக் காணப்படுகிறதது...

ரகு தமது வேலையை மற்றவருடைய சினிமாவைக் காணும் ஒரு படத்தயாரிப்பாளரின் வேலையுடன் ஒப்பிட்டார்; இதில் கவனம் தனிப்பட்ட பொழுதுபோக்குகளில் இருப்பதில்லை, ஆனால் படத்தின் தொழில்நுட்ப அம்சங்களின் மேலேயே இருக்கிறது.

சுசித்ரா மற்றும் ரகுவின் நிகழ்வுகள் மீண்டும் ஒரு முறை எவ்வாறு அமைப்பே ஒரு கம்பெனி தம் பணியாளர்கள் மீது விதிக்கும் தடையை மீற போதுமான சந்தர்ப்பங்களைக் 
கொடுக்கிறது என்பதற்கான உதாரணங்களாகும். ஆயினும் அது பகிரங்கமாக சந்தர்ப்பங்களை அளிக்கும்போது, இம்மாதிரியான தனிப்பட்ட தொடர்புக்கான தளம் ஏற்கனவே மாறி இருக்கிறது. பணியாளர்கள் இன்னும் கம்பெனி சொத்துக்கள் மூலம் சமூக ஊடகங்களை அணுகுவதில்லை, ஆனால் தங்கள் சொந்த உபகரணங்களுக்கே மாற விரும்புகிறார்கள்.

\section{அலுவலகத் தொடர்பாளர்}

ஒரு ஐடி பணிச் சூழலில் புதிய டிஜிட்டல் தொடர்பு தொழில்நுட்பங்களின் குறிப்பிட்ட பங்கை மேலும் ஆய்வு செய்ய,சூழல் முறைசார் பணியிடங்களை வரையறுத்தல் மட்டுமே இல்லை; அது இவை தொடர்புக்கான குறிப்பிட்ட நிலையை உருவாக்கிய விதத்துடன் சம்பந்தப்பட்டே இருந்தது. முறை சார் பணிகளில் முதல் நாளிரவுப் பார்த்த சினிமாவைப் பற்றி சக பணியாளரிடம் உரையாடல், அலுவலக உணவு விடுதி அல்லது குடிநீர் அருந்தும் இடத்தில் சாதாரண அரட்டைகள் போன்ற முறை சாராத் தொடர்புகளும் உள்ளடங்கியே இருந்தன. இந்த மாதிரியான இடங்கள், ஒரு முறைசார் பணியிடத்திற்கு உள்ளே இருந்தாலும், பணியல்லாதத் தொடர்புகளை வளர்க்கின்றன. சமீபத்திய வருடங்களில் இந்த இடங்களும் ஒரு ஆன்லைன் இடத்தை உள்ளடக்கும் வகையில் விரிவடைந்துள்ளன, இவற்றில் பணியல்லாதத் தொடர்புகளின் அலைவரிசையும் தோன்ற முடியும். உதாரணத்திற்கு, உடனடித் தொடர்பாளர் அல்லது அலுவலக உள்புற மன்றங்கள் போன்ற அலுவலக உடனடி செய்தி அமைப்பு இந்த மாதிரியான பணி சம்பந்தப்படாத தொடர்புகள் நடக்க அனுமதியளிக்கிறது. ஆகையால் ஆன்லைன் வசதிகளின் இந்த உபயோகம் - பணியல்லாதவை சம்பந்தப்பட்டத் தொடர்புகளுக்கான உள்புற அலுவலக செய்தி அமைப்பு உட்பட - பணியிலுள்ளவர்கள் முன்பு மற்ற விஷயங்களைப் பற்றி குடிநீருக்கருகோ ${ }^{59}$ அல்லது வேறு இடத்திலோ முன்பு நேருக்கு நேர் பேசியதைப் போன்ற ஏகப்பட்ட முன்னோடிகளுடன் உள்ளது.

சரத் மற்றும் கேசவின் கம்பெனியைப் போல, பல ஐடி கம்பெனிகள் புகழ் பெற்ற சமூக ஊடகத் தளங்களின் மேல் தடை விதிக்கின்றன. ஆயினும் தமது பணியாளர்களுக்கு இன்னும் ஒரு சமூக நெட்வொர்க் இருக்கிறது என்பதை உறுதி செய்ய, இந்த பெரிய கம்பெனிகளில் சில தொடர்புகளை எளிதாக்கவும், பணியாளர்களை கம்பெனிக்குள் மக்க- 
ளுடன் பழக அனுமதிக்கவும் தமது சமூக ஊடகத் தளங்களை நிறுவுகின்றன. பல பெரிய கம்பெனிகள் உள்புற கம்பெனி மன்றங்களை அணுகவும் அனுமதிக்கின்றன, அங்கு பணியாளர்கள் குழு சந்திப்பு மற்றும் உபயோகப்படுத்தப்பட்ட புத்தகங்கள், கடிகாரங்கள், வாகனங்கள் போன்றவற்றை விற்கவும் போஸ்ட் செய்யலாம். ஆயினும், அனைவரும் இந்த வசதிகளைப் பயனுள்ளதாக உபயோகிப்பதில்லை, ஏனென்றால் இந்த விதமான் சமூக நெட்வொர்க்கிங் இன்னும் பணி சம்பந்தப்பட்டதாகவும், மற்றும் பணியாளர்களின் செயல்பாடுகெை கவனிக்கக்கூடிய பணியிடங்களில் இயங்குவனவாகவும் இருக்கின்றன. ஒரு உள்புற கம்பெனி மன்றத்தில் உசிதமல்லாத நடத்தை ஒருவரின் பணியின் மீது குறிப்பிடத்தக்க தாக்கத்தை ஏற்படுத்துகிறது, ஆகையால் இந்த தளங்கள் இருக்கக்கூடிய இடங்களிலும், மக்கள் இவற்றை ஃபேஸ்புக், வாட்ஸ் அப் மற்றும் லிங்க்ட் இன்னிற்கு மாற்றுகளாக கருதவில்லை.

புதிய தொழில்கள் உள்புற சமூக ஊடகங்களை அளிக்கவில்லை என்றாலும் (வரவு செலவு திட்டக் கட்டுப்பாட்டினால்), அவர்கள் பணியாளர்களை கம்பெனியுடைய சிறியக் கட்டமைப்பைப் பூர்த்தி செய்ய மக்களுடன் பழகவும் மற்றும் ஒரு கல்லூரியைப் போன்ற சூழலை ஏற்படுத்தவும் கேட்டுக்கொள்ள விழைகிறார்கள். நிதர்சனத்தில், பணியாளர்கள் இதை ஃபேஸ்புக்கில் செய்வதில்லை (பணியிலிருந்து நண்பர்களைச் சேர்த்துக் கொண்டாலும்), ஆனால் இதை நேரிலோ அல்லது வாட்ஸ் அப் மூலமோ செய்கிறார்கள் (அவர்கள் பணியிடங்களுக்கு வெளியே இருந்தால்). இந்த உதாரணத்திற்கு பஞ்சகிராமியில் உள்ள பள்ளிக்கூடங்களின் அமைப்பைப் பற்றிய சரியான இணை அத்தியாயம் 6ல் விவாதிக்கப்பட்டுள்ளது.

இது அமைப்புக் குழப்பம் எவ்வாறு இயங்குகிறது என்பதைப் பற்றிய இன்னொரு உதாரணமாகும். பெரிய ஐடி கம்பெனிகள் அதைப் பயனுள்ளதாக உபயோகப்படுத்தும் தமது பணியாளர்களுக்காக தமது சொந்த சமூக ஊடகங்களை நிர்மாணிக்கின்றன. அதே நேரம் ஒருக் கல்லூரிச் சூழலை ஏற்படுத்த விரும்பும் புதிய தொழில்கள் அப்படிப்பட்ட பந்தத்தை உருவாக்க மிகவும் விரும்பக் கூடூம், ஆனால் நிதிக் கட்டுப்பாட்டினால் தடை பெற்றிருக்கின்றன, ஆகையால் இவற்றின் பணியாளர்கள் நேருக்கு நேரே தொடர்பு கொள்கிறார்கள்.

அணிச் சந்திப்புகள் முறை சார்ந்தவை என்று கூறப்பட்டாலும், பணி சம்பந்தமான சந்திப்புகள், மற்றும் பல சந்திப்புகள்/ கலந்துரையாடலகள் தமது நிலையில் அதிகம் ஸ்திரமற்ற- 
வையாகவே இருக்கின்றன; அவை முறையான பணி சம்பந்தப்பட்டத் தொடர்பாக ஆரம்பித்தாலும், வாழ்வின் வெவ்வேறு கோளங்களுக்கு இடையே பணியின் தொடர்ச்சி மூலமாகப் பயணிக்கின்றன. இது ஒரு முறை சார்ந்த பணி அமைப்பு/ பணியிடத்திற்குள்ளே ஏற்பட்டாலும், இம்மாதிரி தொடர்புகள் வழியாக வாழ்வின் வெவ்வேறு கோண்ஙகளுக்கிடையே ஒரு தடையில்லாத பிரவாகத்தின் வகை பேணப்படுகிறது. நாம் இப்பொழுது தொழிற்சாலை முறையில் இல்லாதிருந்த தொடர்பு அலைவரிசைகளைத் தருவதன் மூலம் எவ்வாறு சமூக ஊடகங்கள் இந்த விதமான மத்தியஸ்தத்தை அதிகரித்திருக்கின்றன என்பதை ஆய்வு செய்வோம்.

இந்தப் பகுதி பணியிடங்களில் வாட்ஸ் அப் மற்றும் உள்புற அலுவலகத் தொடர்பாளர் மூலம் நிகழும் தொடர்புகளின் மூன்று உதாரணங்களை ஆய்வு செய்வதிலிருந்து தொடங்குகிறது. அடுத்தப் பகுதியில் நாம் பரந்தச் சூழலுக்குள்ளேயான தொடர்பை, இங்கு பணித் தொடர்பு பணிக்கு வெளியே நடக்கும் தொடர்பிலிருந்து பிரிக்க முடியப்படுவெதில்லை, மதிப்பீடு செய்வோம். இதன் இறுதிப் பகுதி உள்ளூர் பணியல்லாதத் தொடர்புகள் தமது பணியிடங்களில் சமூக ஊடகத் தொடர்புகளைப் பணியமர்த்துவதன் மூலம் பணியில் பணியாளர் கட்டமப்பை மாற்றி விடும் இடத்தில் இவை ஒரு பொது சமூக ஊடக இடத்தை உருவாக்க ஒன்று சேர்கின்றன என்பதைக் காட்டுகிறது.

பின்வரும் நிகழ்வுகள் பஞ்சகிராமியில் வாழ்ந்து பணி புரிந்து ெொண்டிருக்கும் பெரும்பாலான மத்திய வகுப்பு ஐடி பணியாளர்களின் நடுவில் இவை மிகவும் பொதுவானதாக இருக்கின்றன. இந்த அனைத்து நிகழ்வுகளிலும், எந்த இடத்தில் இருந்து இந்த வெளிவருதல் நடை பெறுகிறதோ அதாவது பணியிடம், அதுதான் கருத்து முடிவுக்கான இடமும்.

ஒருவர் எவ்வளவு கடினமாக முயற்சித்தாலும், பின்வரும் நிகழ்வுகளுள் நடப்பன எவற்றையும் 'அலுவலகப் பணி' என வரையறுக்க முடியாது. இருப்பினும் இந்த நிகழ்வுகளில் இருந்து காண்பது என்னவென்றால் ஒரு கோளத்திலிருந்து மற்றொன்றுக்கு, மீண்டும் திரும்ப முதலுக்கு, தொடர்பு மற்றும் கருத்துக்களின் 'பெருக்கு’ ஆகும். இது பணியில் இருக்கும்போது பணி மட்டும் வீட்டு மண்டலங்களுக்கிடையேயான, பணி மற்றும் ஒரு குழுவைச் சேர்ந்த பொழுதுபோக்கு மண்டலங்களுக்கிடையேயான மற்றும் பணி மற்றும் ஒரு குழுவைச் சேர்ந்த விளையாட்டு மண்டலங்களுக்கிடையேயான, மீண்டும் பணி மண்டலத்தைச் சென்றடைகிற, தொடர்பின் 
பாங்குடன் சம்பந்தப்பட்டதாக இருந்தாலும், பொருந்துகிறது. இந்தப் பெருக்கின் உலகியல் தன்மை மாறி சார்புடையதாக மாறலாம், ஆனால் இம்மாதிரியானப் பெருக்குகள் தினமும் நிகழ்கின்றன, இவற்றை விலக்க முடிவதில்லை.

\section{அனிதா மற்றும் புருஷோத்தமன் - அலுவலக உள்புறத் தொடர்பாளரில் உள்ள தம்பதிகள்}

24 வயதான அனிதாவும், 27 வயதான புருஷோத்தமனும், சமீபத்தில் திருமணமானவர்கள், இருவரும் பஞ்சக்கிராமியில் உள்ள ஒரு ஐடி கம்பெனியில் பணி புரிகிறார்கள். புருஷோத்தமன் ஒரு செயல் திட்ட மேலாளர் மற்றும் அனிதா ஒரு வணிக ஆய்வாளர்; அவர்கள் இருவரும் வெவ்வேறு செயல் திட்டத்தில் பணி புரிகிறார்கள் மேலும் ஒரே கம்பெனியில் வெவ்வேறு தளங்களில் பணி புரிகிறார்கள். இந்த தம்பதிகள் ஒரு செடான் காரில் அலுவலகத்திற்கு சேர்ந்து வந்து சேர்ந்து கிளம்புகிறார்கள். ${ }^{60}$ இவர்கள் கம்பெனியின் அனைத்துப் பணியாளர்களையும் இன்ட்ராநெட் மூலம் இணைக்கக் கூடிய அலுவலக உள்புறச் தொடர்பாளர் மூலம் இரு மணி நேரங்களுக்கு ஒரு முறை பரஸ்பரம் செய்தி அனுப்பிக் கொள்கிறார்கள். அவர்கள் ஒருவருக்கொருவர் மதிய உணவு மற்றும் காஃபி இடைவேளைகளை ஒருங்கிணைக்கவும் ஒன்பது மணி பணி நேரக் காலத்தில் குறைந்தது நான்கு முறையாவது சந்தித்து விடவும் செய்தி அனுப்பிக் கொள்கிறார்கள். அலுவலகச் தொடர்பாளர் மூலம் அவர்கள் சக பணியாளர்களின் காரணத்தால் அனுபவிக்கும் விரக்தியையும் சில நேரம் இவர்களது திரையைப் பார்க்கும் யாரும் அவர்கள் யாரைக் குறிப்பிடுகிறார்கள் என்பதைப் புரிந்து கொள்ள முடியாதபடி குறிப்புகளின் மூலம் வெளிப்படுத்திக் கொள்கிறார்கள், இந்த தம்பதிகளின் காதல் செய்திகள் அலுவலக தொடர்பாளருக்கு பதிலாக வாட்ஸ் அப்புக்கு ஒதுக்கி வைக்கப் பட்டிருந்தாலும், - டெலிஃபோன் கட்டணம் செலுத்த வேண்டியதை ஞாபகப்படுத்துவது, இந்த நாளின் சந்திப்பதை ஏற்பாடு செய்வது போன்ற பொது செய்திகள் இந்த அலுவலக அலைவரிசை மூலமே பரிமாறிக் கொள்ளப்படுகின்றன. பார்வையாளருக்கு இது ஒருவர் தமது கம்ப்யூட்டர் திரையை உற்று நோக்கிக் கொண்டிருப்பது போலவும், ஒருவர் கடினமாக வேலை செய்வது 
போன்ற பிம்பத்தையும் கொடுக்கிறது. அனிதா சொல்வது போல் மொபைல் ஃபோன்களைக் கையாள்வது, ஒருவர் வேலை செய்யாதது போலவும், வேண்டாத பொருட்களுடன் விளையாடிக் கொண்டிருப்பது போலவும் ஒரு உணர்வைக் கொடுப்பதுடன் மேலாளருக்கு சிந்தனைக்கு உணவாகவும் ஆகிறது. இதற்கு முரணாக, அலுவலக தொடர்பாளர் மூலம் தொடர்பு கொள்ளுதல் ஒரு ஊக்கமுள்ளப் பணியாளர் என்ற பிம்பத்தைத் தக்க வைத்துக் கொள்ள உதவுகிறது, அதே நேரம் பணியாளர்களை அதே கம்பெனியில் பணி புரியும் நெருங்கிய குடிம்ப உறுப்பினர்களுடன் தொடர்பு கொள்ள வழி வகுக்கிறது. இவர்களுடைய நிகழ்வு அத்தியாயம் 4ல் விவாதிக்கப்பட்ட, ரங்கா மற்றும் சந்திராவின் நிகழ்வுக்கு ஒப்பானதுதான். தம்பதிகள் ஒரு கம்பெனியின் ஒரே வளாகத்தில் பணிபுரிந்து அதிகாரபூர்வ செய்தி அமைப்பின் மூலம் தனிப்பட்ட செய்திகளைப் பரிமாறிக்கொள்ளும் மற்ற நிகழ்வுகளும் நடந்துள்ளன.

\section{ரமணன் மற்றும் பாலசங்கர் - சைக்கிள் நண்பர்கள்}

24 வயதான திருமணமாகாத ரமணன், தமது 36 வயதான ஒன்று விட்ட சகோதரர் பாலசங்கரோடு ஒரே ஐடி கம்பெனியில் பணி புரிபவர். இருவரும் ஒரே கம்பெனியின் வெவ்வேறு கிளைகளில் பணி புரிந்தாலும், அவர்கள் இருவருக்கும் பொழுது போக்கு மற்றும் உடற்பயிற்சி என்ற முறையில் சைக்கிள் விடுதல் ஒரு பொது விருப்பமாக இருந்தது. அவர்கள் இருவரும் பரஸ்பரம் வாட்ஸ் அப் மற்றும் அலுவலக உள்புறத் தொடர்பாளர் மூலம் சைக்கிள் விடுதல் பற்றிய செய்திகளை அனுப்பிக் கொண்டார்கள், இவை அனைத்தும் அவர்கள் பணியில் உள்ள போதே செய்யப்பட்டன. இந்த இரு ஒன்று விட்ட சகோதரர்களும் ஒரு சைக்கிள் விடும் குழுவின் உறுப்பினர்கள், இக்குழுவுக்கு ஒரு குழு ஃபேஸ்புக் பக்கம் இருந்தது. இந்த பக்கத்தில் உள்ளப் பதிவுகள் அனைத்தும் ரமணனால் நாள் முழுவதும் தொடர்ச்சியாகப் பார்க்கப்பட்டு பாலசங்கருக்கு அனுப்பி வைக்கப்படும். அவர்கள் இருவரும் நாள் முழுவதும் அலுவலக உட்புறத் தொடர்பாளர் மற்றும் வாட்ஸ் அப் மூலம் பலமுறை உரையாடிக் கொள்கிறார்கள், இந்தப் பணி நேரத் தொடர்பு முக்கியமாக சைக்கிள் விடுதலைப் 
பற்றியே அமைந்துள்ளது. மீண்டும் ஒரு முறை, ரமணன் மற்றும் பாலசங்கரைப் பொறுத்தவரை இந்த உரையாடலுக்காக அவர்கள் ஹாலுக்கு சென்றுப் பேச வேண்டியதில்லை. அவர்கள் தமது பணி மேசையில் அமர்ந்தே உரையாடலாம், இதனால் இவர்கள் பணி புரிந்து கொண்டிருப்பது போலவே தோன்றும். ${ }^{61}$

இந்த உதாரணங்கள் ஐடி தொழிலாளர்கள் வாழ்வின் வெவ்வேறு கோணங்களுக்குச் செல்ல ஒரு குறிப்பிட்ட தொழில்நுட்பத்தின் செயல்பாட்டு சாத்தியத்தை உபயோகிக்கும் முறையை வெளிக்காட்டுகின்றன. இவை பணியிடங்களில் கௌரவத்தை அதிகரிக்கக் கூடிய, ஆனால் அதே நேரத்தில் பணி உலகுக்காக வடிவமைக்கப்பட்ட தொழில்நுட்பத்தின் மூலம் தனிப்பட்ட வாழ்க்கை அல்லது பொழுது போக்கு மண்டலங்களில் பணி தொழில்நுட்பத்தை விரிவு படுத்தும், நாடகபாணித் தொடர்பு சாத்தியங்களை அளிக்கின்றன. இவைகள் கம்பெனிகளுக்கு வெளிப்புறத் தொடர்பின் மீதுள்ள தடைகளை தக்க வைத்துக் கொள்ளுதல் ஏன் கடினமாக இருக்கிறது என்பதற்கு நிரூபணங்களைத் தருவதுடன், பணி மற்றும் பணியல்லாதவைகளின் தொடர்புக்கு இடையேயான திடமான எல்லைகளை வலுப்படுத்த விழையும் கம்பெனிகளின் பணி அமைப்புகளால் எதிர் கொள்ளப்படும் சவால்களுக்கும் செயல் விளக்கமளிக்கின்றன. திருமணமான தம்பதிகளையோ நெருங்கிய உறவினர்களையோ பணியில் அமர்த்தும் போது இதுக் குறிப்பாக உண்மையாகிறது.

\section{கிரிக்கெட் போட்டிகளும் அபிஷேக்கின் அலுவலகமும்}

இந்திய பிரிமியர் லீக் கிரிக்கெட் போட்டிகளின் போது, ஒரு 27 வயது ப்ரோகிராமர், அபிஷேக், அலுவலக உள்புறத் தொடர்பாளர் மூலமாக இன்னொரு செயல் திட்டத்தில் வணிக ஆய்வாளராக இருக்கும் தமது நண்பருக்கு ஒரு செய்தி அனுப்புகிறார். அவர் இந்தியாவின் புகழ் பெற்ற கிரிக்கெட் வீரர்களைப் பெற்றிருக்கும் ஒரு நாட்டுப்புற கிரிக்கெட் கிளப்பாகிய சென்னை சூப்பர் கிங்ஸின் ${ }^{62}$ மேம்படுத்தப்பட்ட ஸ்கோர்களைக் கேட்கிறார். இதற்கு பதிலாக அபிஷேக்கிற்கு தமது சகப் பணியாளர் வித்யாசங்கரிடமிருந்து ஸ்கோர்களைக் கேட்டு 
ஒரு செய்தி வருகிறது. விரைவிலேயே பெரும்பாலான அணி உறுப்பினர்கள் தமது ஸ்மார்ட் ஃபோனிலோ அல்லது இணைய தளத்திலோ ஸ்கோருக்காகத் தேடத் தொடங்குகிறார்கள், அதன் பின் அந்தத் தகவலை அலுவலக உள்புறத் தொடர்பாளர் மூலம் தமது சகப் பணியாளர்களுக்குத் தெரிவிக்கிறார்கள். மக்களுக்கு ஃபேஸ்புக் மற்றும் வாட்ஸ் அப் மூலமும் கிரிக்கெட் ஸ்கோர் மீது குறிப்புகளுடன் தமது நண்பர்களிடமிருந்து செய்திகள் வர ஆரம்பிக்கின்றன, மேலும் இவை அனைத்தும் அதே தளத்தில் பணி புரிந்து கொண்டிருக்கும் அணிகளைத் தொல்லை செய்யாமல் அலுவலகத் தொடர்பாளர் மூலம் அனுப்பப்படுகின்றன. வைஸ் பிரசிடென்ட்டிலிருந்து டேட்டா என்ட்ரி ஆபரேட்டர் வரை அனைவரும் இதில் தொடர்புடையவராக இருக்கிறார்கள். மறு நாள் அனைத்தும் இயல்பு நிலைக்கு மாறுகிறது, அலுவலக நேரத்தில் ஃபேஸ்புக்கைப் பார்ப்பது ஏற்றுக் கொள்ளப்படுவெதில்லை. இந்த நிகழ்வு 'வீட்டு' மண்டலம் பணி மண்டலத்துக்குள் செல்லும் ஒன்றாக ஏற்றுக் கொள்ளப்பட முடியாவிட்டாலும், இது தெளிவாக பணியில்லா மண்டலம் பணி மண்டலத்துள் செல்லும் ஒன்றாகும். இவ்வாறு செய்கையில் இது முதல்நிலையில் முறைசார் அலுவலகப் பணிக்காக உருவாக்கப்பட்ட குழுவினிடையில் ஒரு முறைசாரா தன்மையையும், ஒரு பந்தத்தையும் உருவாக்குகிறது.

ஆகையால் நாம் பணியிடங்களுக்கும் பணியல்லாத இடங்களுக்கும் இடையேயான பெருக்கைப் பற்றியப் பிரச்சினைகளைக் கருத்தில் கொள்வதற்கு முன்னமேயே, அலுவலக மற்றும் பணிச் சூழலில் எவற்றைத் தொடர்பின் பணி மற்றும் பணியல்லாத அம்சங்கள் எனக் கருதலாமோ, அவற்றின் பெருக்குகளின் சிக்கலான கலவை ஒன்று ஏற்கெனவே அங்கு இருக்கிறது என்பதைக் கண்டு கொள்ள வேண்டும்.

நமக்கு இப்பொழுது இந்தியாவின் ஐடி நிறுவனங்களின் தினசரிப் பணிக் கலாசாரத்தில் சமூக ஊடகங்கள் எவ்வாறு பொருந்துகின்றன என்பது பற்றி ஒரு மேல் நிலைக் காணல் இருக்கிறது எனக் கொள்ளும் பட்சத்தில், தனிப்பட்டத் தொடர்பு தொழில்நுட்பங்கள் மூலமாக இப்படிப்பட்ட கலாசாரத்தில் தினப்படி மத்தியஸ்தத்தின் மதிப்பு பின்வரும் நிகழ்வு ஆய்வு மூலம் அறியப்படலாம். இது பஞ்சக்கிராமியின் ஒரு பொதுவான மத்திய வகுப்புக் குடும்பத்தின் ஒரு பொதுவான வார நாளை விவரிக்கிறது. 


\section{பணி மற்றும் வீட்டுக் கலாசாரங்களின் மறு ஒருங்கிணைப்பு}

நமது முந்தைய உதாரணங்கள் ஒன்றில் நாம் ஒரே பணிச் சூழலை பகிர்ந்து கொள்ளும் திருமணமான தம்பதிகளான அனிதா மற்றும் புருஷோத்தமனைப் பற்றி விவாதித்தோம். இப்பொழுது கடிகாரத்தை முன்பக்கமாக சில வருடங்களுக்கு அவர்களுக்கு குழந்தைகள் இருக்குமாறுத் திருப்புங்கள். அப்பொழுது அவர்கள் ரவி மற்றும் ஸ்வேதாவைப் போல மாறி இருக்கலாம்.

36 வயதான ரவி மற்றும் 32 வயதான ஸ்வேதா இருவரும் பஞ்சக்கிராமியில் ஒரு பல மாடி அடுக்குக் குடியிருப்புக் கட்டடத்தில் ஒரு மூன்று படுக்கையறை அபார்ட்மென்ட்டில் வசிக்கும் தம்பதியினர். இவர்கள் இருவரும் நமது பணிக் களத்தில் உள்ள ஐடி கம்பெனிகளில் பணி புரிகிறார்கள். அவர்களது இரண்டு குழந்தைகளுடன் கூடிய ஒரு மாதிரி தனிக் குடும்பம். பெரியவன் 10 வயதான விஷால், அவன் அவர்களது அபார்ட்மென்ட் வளாகத்திலேயே இருக்கும் ஒரு புகழ் வாய்ந்த வளமான சர்வதேசப் பள்ளிக்கூடத்திற்கு செல்கிறான். அவர்களுடைய மகள் அனுவுக்கு இரண்டரை வயதாகிறது; அவள் அபார்ட்மென்ட் வளாகத்துக்கு அருகிலேயே இருக்கும் ஒரு மழலையர் பள்ளிக்குச் செல்கிறாள்.

ஒரு பொதுவான நாள் காலை 5 மணிக்கு ஸ்வேதாவும் ரவியும் தமது ஸ்மார்ட்ஃபோன் அமைக்கப்பட்டுள்ள அலாரத்திற்கு துயிலெழும்போது ஆரம்பமாகிறது. ரவி ஒரு விரைவான ஓட்டத்திற்கு வெளியே செல்லும் போது ஸ்வேதா தனது சமையலை ஆரம்பிக்கிறார். அவர்களது தினசரி வழக்கத்தின் ஒரு பாகம் தாம் ஏதாவது அலுவலக அல்லது தனிப்பட்ட தொடர்புகளைத் தவற விட்டிருக்கிறோமா என்பதைக் காண அவுட்லுக்கில் தமது அலுவலக இ-மெயில்களின் விரைவு சோதிப்பு, வாட்ஸ் அப்பின் ஒரு விரைவு சோதிப்பு, மற்றும் தத்தமது ஃபேஸ்புக் ப்ரொஃபைலின் மீது ஒரு மிக விரைவுப் பார்வை ஆகியனவாகும். இவை அனைத்தும் முடிந்த பிறகு, அவர்கள் நாளின் வீட்டு வேலைகளைத் தொடங்குகிறார்கள். விஷால் ஸ்கூலில் 7 மணிக்கு நடக்கும் கிரிக்கெட் பயிற்சிக்காக 6 மணிக்கு எழுப்பப் படுகிறான், அதற்குப் பிறகு அங்கு அவன் வகுப்புகளுக்குச் செல்கிறான். இந்த நேரத்திலேயே ரவி அவர் தமது ஐ-பாட்டில் தாம் சந்தாதாரராக இருக்கும் சில இ-செய்தித்தாள்களையும் தொழில்நுட்பப் பத்திரிகைகளைப் படிக்கவும் அதே நேரம் ஃபேஸ்புக்கில் தமது ஓட்டக் குழுவின் 
(மாரத்தான்) பக்கத்தைக் காணவும் சமயம் ஒதுக்குகிறார். அனு ரவி அவளை காலை 8 மணிக்கு மழலையர் பள்ளியில் விட்டு விட்டு தம் அலுவலகத்திற்கு செல்ல ஏதுவாக காலை 6.30 மணிக்கு எழுப்பப் படுகிறாள்.

இதே நேரத்தில் ஸ்வேதா அந்த நாளுக்கான தமது சமையலை முடித்து விட்டு தமது கம்பெனிக்கு காலை 7 மணிக்குக் கிளம்புகிறாள். இரண்டு கார்கள் இருப்பது ரவியின் குடும்பத்திற்கு எளிதாக இருக்கிறது, ஏனென்றால் அவர்கள் காலை நேரப் பயணத்திற்கு ஒருவரை ஒருவர் சார்ந்து இருக்க வேண்டியதில்லை. தாம் அலுவலகத்தை அடைந்ததும் ஸ்வேதா ரவிக்கு தாம் பத்திரமாக அலுவலகத்தை அடைந்ததைத் தெரியப்படுத்த ஒரு வழக்கமான வாட்ஸ் அப் செய்தியை அனுப்புகிறார். இந்தச் செய்தி சிறிது 'அன்பு தெரிவித்தலையும்' 'முத்தங்களையும்' உள்ளடக்கியதாக இருக்கிறது. ரவி இதையே இரு முறை செய்கிறார், முதல் முறை அனுவை மழலையர் பள்ளியில் விட்டவுடன், இரண்டாம் முறை அவர் அலுவலகத்தை அடைந்தவுடன். முதல் வாட்ஸ் அப் செய்தி பள்ளியில் விடிம் பொழுது அனு அழுதாளா என ஸ்வேதாவிற்குத் தெரிவிக்க, ஏனென்றால் இருவருமே சில சமயம் தங்களது மகளின் திடீர் உணர்ச்சி வெளிப்பாட்டைப் பற்றிக் கவலையுடன் இருந்தார்கள். இரண்டாவது செய்தி ரவி பாதுகாப்பாக அலுவலகம் வந்து சேர்ந்தது குறித்து ஸ்வேதாவுக்குத் தெரிவிக்க; இந்தக் குறிப்பில் 'அன்பு செய்கிறேன்' என்பதும் உண்டு.

ஏறக்குறைய காலை 10 மணிக்கு ஸ்வேதா தமது பணியாளை அவரது மொபைல் ஃபோனில் அவர் வீட்டை சுத்தம் செய்யும் பணிக்கு வந்து விட்டாரா எனத் தெரிந்து கொள்ளவும், சில ஆணைகளைக் கூறவும் அழைக்கிறார். பணியாளர் பதிலளிக்கவில்லை என்றால் ரவி தமது மனைவியிடமிருந்து பணியாளைத் திட்டும் மற்றும் அவர் நம்பிக்கைக்குப் பாத்திரமானவர் அல்ல எனக் கூறும் ஒரு விரக்தியான வாட்ஸ் அப் செய்தியைப் பெறுகிறார்.

ஏறக்குறைய நடு மதியத்தில் அனுவின் மழலையர் பள்ளி ரவி ஸ்வேதா இருவருக்கும் ஒரு வாட்ஸ் அப் செய்தியை அனுப்புகிறது, இது ஒரு அதிகப்படியானக் கட்டணத்திற்கு பள்ளி தரும் சேவை. இதைப் பெற விரும்பும் பெற்றோர்களுக்கு இது குழந்தை காலையில் மற்றும் மதியத்தில் என்ன செய்தது மற்றும் மதிய உணவைச் சரியாக உண்டதா என்பது பற்றி அனுப்பப்படும் தினசரி அறிக்கை. அனு சரியாக மதிய உணவை சாப்பிடாதது போலிருந்தால், ஸ்வேதா என்ன 
நடந்தது என்பதை அறிய மழலையர் பள்ளியை அழைக்கிறாள் மேலும் ரவிக்கு ஒரு வாட்ஸ் அப் செய்தியும் அனுப்புகிறாள். பணியாள் வந்தவுடன் அவர் ஸ்வேதாவுக்குத் தம்மைத் திரும்ப அழைக்கும்படிக் குறிக்கும் ஒரு மிஸ்டு கால் ${ }^{63}$ கொடுக்கிறார் ஃபோன் அழைப்புகளுக்கு பணம் செலவாகும், அதனால் அவரால் அவற்றை செய்ய இயலாது). அல்லது அவர் ஸ்வேதாவுக்கு எதிர்பாராத ஒரு கொரியர் அல்லது காஸ் வரவு பற்றி தெரிவிக்க வீட்டு லேண்ட்லைன் ஃபோனிலிருந்து அழைப்பு விடுக்கிறார். ஏதாவது எதிர்பாராதது நடக்கும் பொழுது ஸ்வேதாவும் ரவியும் விவாதித்து தெளிவு பெற வாட்ஸ் அப் செய்திகளைப் பரிமாறிக் கொள்கிறார்கள்.

மதிய உணவின் போதோ அல்லது உடனேயோ பிறகோ (வழக்கமாக மதியம் 1 மணிக்கு) ஸ்வேதா ரவி இருவரும் கட்டிக் கொணர்ந்த மதிய உணவை விரும்பினார்களா என்று தெரிவித்துக் கொள்ள பரஸ்பரம் வாட்ஸ் அப் செய்திகளைப் பரிமாறிக் கொள்கிறார்கள். ஸ்வேதா தமது கம்பெனி ஊக்குவிக்கும் 'வீட்டிலிருந்து பணி புரியும்' கொள்கையின்படி வழக்கமாக மதியம் 2.30 மணிக்கு வீட்டிற்குக் கிளம்புகிறார். அவர் அவளது ஆசிரியையுடன் ஒரு விரைவு உரையாடலுக்குப் பிறகு அனுவை பள்ளிக்கூடத்திலிருந்துக் கூட்டிச் செல்கிறார். அனுவை பள்ளியிலிருந்து கூட்டி வந்ததைத் தெரியப்படுத்த ரவிக்கு இன்னொரு விரைவுச் செய்தி அனுப்பப்படுகிறது.

அனு மதியம் தூங்கும் போது, ஸ்வேதா விஷாலுக்காக ஒரு சிற்றுண்டியைத் தயார் செய்கிறார், அவன் பள்ளியிலிருந்து மதியம் 3.30 மணிக்கு வீடு திரும்புகிறான். விஷால் வீடு வந்தவுடன், ஸ்வேதா சுமார் 4 மணிக்குத் தமது பணிக் கணினியுள் நுழைகிறார், அவர்களது எச்பி ஹோம் கம்ப்யூட்டரின் குரோம் ப்ரௌசரில் ம்யூசிக் வீடியோ திறக்கப் படுகிறது. விஷால் சுமார் 4.30 மணிக்கு அபார்ட்மென்ட் வளாகத்தின் மற்றக் குழந்தைகளுடன் விளையாடச் செல்கிறான் அல்லது எக்ஸ்பாக்ஸில் கேம் விளையாடுகிறான். இந்த நேரத்தில் அனு விழித்துக் கொள்கிறாள், ஸ்வேதா தமது ஐபாடில் கார்ட்டூன்கள் பார்க்க அவளுடன் சேர்ந்து கொள்கிறார், பிறகு தமதுப் பணிகளுக்குத் திரும்புகிறார். சுமார் 6 மணிக்கு ஸ்வேதா விஷாலை தமது வீட்டுப் பாடத்தை செய்யுமாறு முதல் முறை அழைக்கிறார், ஸ்வேதாவுக்கு வீட்டுப் பாடம் செய்ய தன் மகனை சமாதானப்படுத்த சிறிது நேரம் எடுக்கிறது - இது கேட்டல், கோருதல் முடிவில் கெஞ்சுதல் ஆகியவை உள்ளடக்கிய ஒரு செயல்முறை. அவன் வழக்கமாக சுமார் 6.30 மணிக்குத் தன் வீட்டுப் பாடத்தை ஆரம்பிக்கிறான், இந்த 
நேரத்தில் ஸ்வேதா தமது இன்னும் சில பணிகளை செய்து கொண்டே, விஷாலின் வீட்டுப் பாடத்திலும் உதவி செய்து கொண்டு அனுவின் மீதும் கவனம் வைத்துக் கொள்கிறார். இரவு சுமார் 7.30 மணிக்கு ரவி தாம் இன்னும் சில நிமிடங்களில் அலுவலகத்தை விட்டுக் கிளம்புவதாகவும், இரவு உணவு ஏற்பாடுகளைப் பற்றி விசாரித்தும் ஒரு வாட்ஸ் அப் செய்தி அனுப்புகிறார். ஸ்வேதா வழக்கமாக இரவு உணவு தயாரிக்கவும், இரவு உணவு நேரமான 8.30 மணிக்கு முன்னர் அனுவுக்கு உணவு தருவதற்காகவும் இரவு 7 மணிக்குப் பணியை விட்டு வெளியே வருகிறார். ஆயினும் இரவு உணவு தயாரிப்பது காலையில் காலை மற்றும் மதிய உணவு தயாரிப்பது போல் அத்தனை பரபரப்பு மிகுந்ததாக இல்லை. இந்த நேரத்தில் தம் பெற்றோர்களையோ அல்லது நண்பர்களையோ அழைப்பதை உறுதி செய்து கொள்கிறார்; அவர் தமது ப்ளூடூத் இயர் பீஸ் மூலமாக இயங்குகிறார் மேலும் விஷாலுக்கு வீட்டுப் பாடத்தை செய்ய உதவும் போதே உணவையும் சமைக்கிறார்.

இரவு உணவுக்குப் பிறகு, ரவி விஷாலுடன் அவன் வீட்டுப் பாடத்தில் சிறிது நேரத்தை செலவழிக்கும் போது, ஸ்வேதா அனுவை உறக்கத்திற்கு தயார் செய்கிறார். இரவு 9.30 மணியிலிருந்து 10 மணி வரை தமது மற்ற சமூக ஊடகங்களிலிருந்து பிற செய்திகளைக் காண ஸ்வேதாவின் நேரம்; இந்த நேரத்தில்தான் அவர் தாம் உறுப்பினராக இருக்கும் நான்கு வாட்ஸ் அப் குழுக்களின் செய்திகளைக் காணவும் தம்முடைய மற்ற தோழிகளுடன் (மற்ற பணி புரியும் தாய்கள் மற்றும் இல்லத்தரசிகள்), அவர்களில் பெரும்பாலானோர் அப்பொழுதுதான் ஓய்வாக இருப்பது போல் தோன்றுவதால், சாட் செய்கிறார். ரவி இதை தம் அலுவலகத்திலிருந்தே செய்கிறார், ஆகையால் பின் மாலையில் உறங்கச் செல்லும் முன் அவர் அலுவலக இ-மெயில்கள், வாட்ஸ் அப் மற்றும் ஃபேஸ்புக் ஆகியவற்றுக்கு மாறி மாறிச் செல்கிறார்.

இந்த நிகழ்வு ஒரு பொதுவான மத்திய வகுப்பு பணி புரியும் குடிம்பத்தின் தினசரி வழக்கத்தைக் குறிக்கிறது. பஞ்சக்கிராமியில் பல ஸ்வேதாக்கள், ரவிக்கள், அனுக்கள் மற்றும் விஷால்கள் இருக்கிறார்கள். இந்த நிகழ்வு ஆய்வின் மேல் ஒரு ஆழ்ந்த பார்வை இந்த மாதிரியான தம்பதிகள் அனுபவிக்கும் உணர்ச்சிகள் மற்றும் ஒரு தனிக்குடும்பம் என்ற விதத்தில் அவர்களால் நடத்தப்படும் வாழ்க்கை ஆகியவற்றைத் தெளிவாக்குகிறது. அவர்கள் பொதுவாக 'இரட்டை வருமானக் குடும்பங்கள்' என்று அழைக்கப் படுகிறார்கள், ஏனென்றால் கணவன் மனைவி இருவரும் உயர்ந்த 
தகுதியைப் பெற்றவர்கள் மற்றும் வழக்கமாக தனியார் துறை கம்பெனிகளில் பணி புரிபவர்கள். இவ்வாறானக் குடும்பங்கள் தமது அலுவலக வேலை எந்த வகையிலும் தடைப்படவில்லை என்பதை உறுதி செய்து கொள்கிறார்கள்; அவர்கள் ஆவலுள்ளவர்களாகவும், பணியில் பதவி உயர்வு லட்சியம் உடையவர்களாகவும் இருக்கையிலேயே தமது குடும்ப வாழ்க்கை அல்லது பணிக்கு வெளியேயான வாழ்க்கை எந்த விதத்திலும் பாதிக்கப்படக்கூடாது என்பதை உறுதி செய்து கொள்ள முயன்று கொண்டிருக்கிறார்கள். மேலே உள்ள நிகழ்வு ஆய்வு எவ்வாறு பணி புரியும் இளைய தம்பதிகள் தமது தினசரி குடும்ப வாழ்க்கையை மத்தியஸ்தம் செய்து கொள்கிறார்கள் என்பதின் தனிச் சிறப்பாக இருக்கும் பொழுது, மற்றவர்கள் சிறிது மாறு படுகின்றனர்; ஆயினும் பெரும்பாலானோர் பரந்த அளவில் இந்த மாதிரி பாங்கிலேயேப் பொருந்துகிறார்கள்.

இந்த விவரங்கள் பாலிமீடியாவையே விளக்குகின்றன ${ }^{64}$ ஸ்மார்ட் ஃபோன், வாட்ஸ் அப் மற்றும் ஃபேஸ்புக் போன்ற நிறைவை உண்டாக்குகின்ற தனிப்பட்ட தொடர்பு தொழில்நுட்பங்களின் பங்கு. இது வாட்ஸ் அப் எவ்வாறு பயனாளிகளுக்கு ஒரு நீண்ட வேலை நாளில் தோன்றும் அன்பு, குற்ற உணர்ச்சி, விரக்தி ஆகிய எண்ணற்ற உணர்ச்சிகளை வெளிப்படுத்தும்போது தனிப்பட்ட வாழ்க்கையுடன் மத்தியஸ்தம் செய்து கொள்ளவும் அதை அடையவும் உதவி செய்கிறது என்பதைக் காட்டுகிறது. பணி புரியும் மத்திய வகுப்பு இளைய தலைமுறையின் இந்தத் தலைமுறை அவர்கள் அன்னைகள் பெரும்பாலும் இல்லத்தரசிகளாக இருக்கும் ஒரு சகாப்தத்திலேயே வளர்ந்தார்கள், அவர்கள் தங்கள் குழந்தைகளின் மேல் கவனத்தைச் சொரியும் வல்லமை உடையவர்களாக இருந்தார்கள், அவர்களுடைய தந்தைமார்கள் பொருளீட்டும் பணிகளில் இருந்தார்கள். குறிப்பிடத்தக்கக் கல்வி முதலீட்டைச் சேர்த்ததனாலும், இந்தக் கல்வி முதலீட்டை பொருளாதார முதலீடாக மாற்றும் சந்தர்ப்பங்களைப் பெற்றிருந்ததாலும் இந்த புதிய மத்திய வகுப்பு ஒரு விதமானக் குழப்பத்தை எதிர் கொள்கிறது. பணிகளில் மூத்த நிலைகளின் மூலம் உயர்ந்த நிலைக்குச் செல்வதையும் பொருளாதார நன்மையை உறுதி செய்வதையும் தரக் கூடிய சந்தர்ப்பங்களை இழந்து விடக்கூடாது என இந்தத் தொழிற் பண்பட்டவர்கள் அறிந்து வைத்திருந்தனர்; ஆயினும் அதே நேரத்தில் அவர்கள் தாம் தம் பெற்றோர்களிடமிருந்து (முக்கியமாக அன்னைகளிடமிருந்து) பெற்ற கவனத்தை தம் பிள்ளைகளுக்கும் வழங்க வேண்டியதன் அவசியத்தை கண்டு கொண்டிருந்தனர். ${ }^{65}$ 
இது முக்கியமாக பணி புரியும் மத்திய வகுப்பு இளவயது அன்னைகள் விஷயத்தில் உண்மையாக இருந்தது. பணி புரியும் இள வயது அன்னைகள் மற்றும் சிறிய குழந்தைகளுடைய பணி புரியும் அன்னைகளுடனான நேர்காணல், அவர்கள் தமது தார்மீகக் கடமைகளாக நினைப்பவற்றை ஆற்றும் திறனுடையவர்களாக இல்லாமல் இருப்பதில் இருக்கும் ஒரு வித கையறு நிலை மற்றும் உணர்ச்சிகளின் கலவையை வெளிப்படுத்தினார்கள். இந்தக் குழப்பமான உணர்ச்சிகள் ஒரு பெரிய ஐடி கம்பெனியில் ப்ரோக்கிராமராக இருக்கும் 27 வயதான மற்றும் இரண்டு வயது மகனை உடைய பூர்ணிமாவின் வார்த்தைகளில் வெளிப்படுகிறது:

இந்த வயதில்தான் என் மகனுக்கு நான் தேவைப்படுகிறேன்; நான் தினசரி காலையில் அவனை மழலைக் காப்பகத்தில் விடும்போது மிகுந்தக் குற்ற உணர்ச்சிக்கு உள்ளாகிறேன். அவன் நான் அவனுடன் இருப்பேன் என எண்ணுகிறான். அவன் அழுகிறானா என எனக்குத் தெரியவில்லை, ஆனால் நான் தினமும் அழுகிறேன். நான் செய்வது தவறு என்று எனக்குத் தெரியும்... நாங்கள் இப்பொழுதுதான் ஒரு வீட்டிலும் காரிலும் முதலீடு செய்தோம்... நான் என் வேலையை விட முடியாது... இந்தக் கடன்களைக் கட்ட என் சம்பளம் அவசியம்... குறைந்த பட்சம் நான் என் குழந்தைக்கு ஒரு நல்ல எதிர்காலத்தை உறுதி செய்கிறேன்.

ஒரு நிமிடத்திற்குப் பிறகு அவர் கேட்டார்: 'நான் அவன் எதிர்காலத்தை பாதுகாப்பாக்குகிறேன் என்று என்னையே சமாதானப்படுத்திக் கொள்வதன் மூலம் என்னை நானே ஏமாற்றிக் கொள்கிறேனா? அவனுக்கு பிறகு எப்பொழுதையும் விட இப்பொழுது என் தேவை இல்லையா?'

சிறிய வயது குழந்தைகளை உடைய பணி புரியும் அன்னைகளுடனான நேர்காணல் வழக்கமாகப் பூர்ணிமா சொன்னதையே வெளிப்படுத்தும் என்பதால், மேலாக நடப்பதை விட இதில் அதிக விஷயம் உள்ளது. வழக்கமாக தமது முதல் குழந்தையை இப்போதுதான் பெற்றிருக்கும் அன்னையர் ஒரு சிறு இரண்டாவது குழந்தையை உடைய அன்னையரை விட தம் முதல் குழந்தையை விட்டு விட்டு பணிக்குச் செல்வதில் அதிக உணர்ச்சி வசப்பட்டவர்களாக இருந்தனர். இன்னும் சில நிகழ்வுகள் இதற்கு எதிர்மாறானதையேக் காட்டின. உதாரணத்திற்கு, ஒரு 30 வயது பணி புரியும் அன்னையான ஸ்ரீ- 
தேவிக்கு ஏழு மற்றும் இரண்டு வயதில் இரு குழந்தைகள் இருக்கிறார்கள். அவரது விதவைத் தாயார் தனது மரணத்திற்கு முன் மூன்று ஆண்டுகள் அவருடன் வாழ்ந்து வந்தார், அந்த சமயத்தில் அவர் ஸ்ரீதேவியின் முதல் குழந்தையை கவனித்துக் கொண்டார். இப்பொழுது அவரது இரண்டாவது குழந்தையை கவனித்துக் கொள்ள குடும்ப உறுப்பினர்கள் எவரும் இல்லை, ஆகையால் ஸ்ரீதேவி தமது குழந்தையை ஒரு பரிச்சயமான குடும்ப உறுப்பினருக்கு பதில் ஆயாவுடன் விட்டுச் செல்வதில் மிகுந்த குற்ற உணர்ச்சியுடன் இருக்கிறார். இந்தப் பகுதியில் இருக்கும் பலப் பெற்றோர்களைப் போல, ஸ்ரீதேவி தம் குழந்தையின் வாழ்வுடன் அதிகம் இணைந்து அதில் இடம் பெற வாட்ஸ் அப் குரல் செய்தியை அடைகிறார். வாட்ஸ் அப் தனிப்பட்ட வாழ்க்கையை எளிதாக்கும் இன்னொரு உதாரணத்தை சங்கர் மற்றும் ஜனனியின் நிகழ்வில் காணலாம். 32 வயதான சங்கரும் 30 வயதான ஜனனியும் பஞ்சகிராமியில் இரு வேறு ஐடி கம்பெனிகளில் பணி புரியும் தம்பதிகள். சங்கர் வழக்கமாக தமது மகளைப் பள்ளியிலும் ஜனனியை அலுவலகத்திலும் விடுகிறார். சங்கருடைய தாய் தனது 8 வயது பேத்தியான ஸ்ரவ்யாவை மாலை சுமார் 4 மணிக்கு பள்ளியின் நேரத்திற்குப் பிறகு அழைத்துக் கொள்கிறார் மேலும் சங்கர் ஜனனியை பணி முடிந்ததும் அழைத்துக் கொள்கிறார். அவர்கள் ஒருவருக்கொருவரும் சங்கருடைய தாய்க்கும் ஒரு நாளில் 10 முறைக்கு மேல் வாட்ஸ் அப் செய்திகளை அனுப்பிக் கொள்கிறார்கள். சங்கர் அலுவலகம் சென்றடையும் நேரத்திலிருந்து நாள் ஆரம்பமாகிறது, அப்பொழுது அவர் ஜனனிக்கு (சில சமயம் தமது தாய்க்கும்) தாம் பத்திரமாகப் பணிக்கு வந்து சேர்ந்ததையும் ஸ்ரவ்யாவை பள்ளியிலும் (ஜனனியையும் கூட) விட்டு விட்டதையும் தெரிவிக்கிறார். சங்கர் மதியம் உணவுக்குச் செல்லும் பொழுது இன்னொரு செய்தி அனுப்பப்படுகிறது, மேலும் நாளின் இரண்டு அல்லது மூன்று காஃஃபி இடைவேளைகளின் போது இன்னும் பல அனுப்பப்படுகின்றன. இந்தச் செய்திகளில் சில ஒத்த காலத்தில் நிகழ்வனவாக இல்லாததால், தம்பதிகள் இருவரும் அந்த சரியான நேரத்தில் அவர்கள் ஈடுபட்டிருக்கும் வேலையைப் பொறுத்து பதிலளிக்க அவகாசம் எடுத்துக் கொள்கிறார்கள். ஆயினும் பெரும்பாலான செய்திகள் வழக்கமாக அரை மணிக்குள்ளாகவே பதிலளிக்கப்பட்டு விடுகின்றன. ஸ்ரவ்யா வீடு வந்து சேர்ந்ததும் அவள் தனது பாட்டியின் ஃபோனிலிருந்து ஜனனிக்கு தாம் பத்திரமாக வீடு வந்து சேர்ந்ததைப் பற்றி வாட்ஸ் அப் செய்தி அனுப்புகிறாள். பாட்டி மாலை சுமார் 6 மணிக்கு சங்கருக்கும் ஜனனிக்கும் மாலை 
வீடு திரும்பும் போது அவர்கள் வாங்க வேண்டிய மளிகை சாமான்கள் பற்றியும் அன்றைய இரவு உணவுப் பட்டியல் பற்றியும் செய்தி அனுப்புகிறார்.

நாம் இந்த பாட்டியின் பாத்திரத்தை ஒரு பெண் சமையல்காரருடன் மாற்றினால், நீங்கள் இங்கு கவிதாவின் நிகழ்வு பிரதிபலிக்கப்படுவதைக் காணலாம். பஞ்சகிராமியில் ஒரு மூத்த உறுப்பினருடன் இருக்கும் பொதுவானக் குடும்பங்கள் இந்தப் பாணியில் தொடர்பு கொள்ள விழைகின்றன.

ஆயினும் தாத்தா பாட்டிகள் உடன் இருந்தாலும், விஷயங்கள் காண்பது போல் இருப்பதில்லை - இது நாம் 2ம் அத்தியாயத்தில் சந்தித்த லக்ஷ்மியின் நிகழ்வில் வெளிப்படையாகத் தெரிந்தது. ஒரு பணியில் தீவிரமாக இருக்கும் ஐடி மேலாளரான லக்ஷ்மி தமது பணியிடத்திலிருந்து தமது குழந்தைகளுடன் தொடர்பு கொள்ள வாட்ஸ் அப் குரல் செய்திகளை உபயோகித்தார். அவரால் ஒரு நல்ல 'பணிவாழ்க்கை-சமநிலையை' அடைய முடிந்ததா என வினவிய போது அவர் புன்சிரிப்புடன் இவ்வாறு பதிலளித்தார்:

'என்னால் முடிய வேண்டும் என நான் விரும்புகிறேன், அதனால்தான் நான் பேசுகிறேன். நான் இதை அடையப் போராடுகிறேன். நான் அணிச் சந்திப்புகளுடனும் காலக்கெடுக்களுடனும் பேரம் பேச என்னால் இயன்ற வரை முயலுகிறேன். நான் பணியில் இல்லாத பொழுதும் என் பிளாக்பெர்ரி தொலைபேசி எனக்கு உதவுகிறது. எனது அணி அனைவரும் ஆண்களாக இருக்கும் அணி என்றாலும், அவர்கள் நல்லவர்களாக இருப்பதால் புரிந்து கொள்கிறார்கள்.

தனிப்பட்ட வாழ்க்கை அவருடையப் பணியில் தலையிடுகிறது, ஆனால் வாட்ஸ் அப் போன்ற சமூக ஊடகங்கள் அதை சமாளிக்க அவருக்கு உதவுவதாக அவர் விவரிக்கிறார்.

எப்படியாயினும்,

குழந்தைகள்

தொடர்ச்சியாக முன்னுரிமை பெறுகின்றன, மேலும் பெற்றோர்கள் பணியில் இருக்கும் பொழுது தாத்தா பாட்டிகள் பேரக் குழந்தைகளை கவனித்துக் கொள்கிறார்கள். இதை மனதில் கொண்டு அத்தியாயம் 4ல் கண்டது போல, கூட்டுக் குடும்ப அமைப்பின் ஒரு சுவாரசியமான பரிமாணம் பஞ்சகிராமியில் காணப்பட்டது. மேற் கண்டாற் போல, பல எதிர்பார்ப்புகளை உடைய ஒரு பாரம்பரிய குடும்பத்தில் திருமணம் செய்தல் பணி புரியும் பெண்களுக்கு மிகுந்த அழுத்தத்தை ஏற்படுத்துவதால் 
அவர்கள் கூட்டுக் குடும்ப அமைப்பிலிருந்து தமது கணவர் மற்றும் குழந்தைகளுடன் பிரிந்து சென்று விடுகிறார்கள். ஆயினும் அவர்கள் பணி புரியும்போது இது குழந்தை பராமரிப்பு பற்றிய பிரச்சினையைக் கொணர்கிறது. இந்தப் பிரச்சினையைக் கடக்க, ஒரு சாத்தியமானத் தீர்வு - பெற்றோர் அல்லது புகுந்த வீட்டாரின் அருகில் வசித்தல் - மேற்கண்டாற் போலும் அத்தியாயம் 4ல் விவாதிக்கப்பட்டாற் போலும் இப்பொழுது ஒருு போக்காகியுள்ளது. இது அவர்கள் தனிக் குடும்பங்களாகப் பிரிந்து வாழ்வதையும், ஆனால் அண்டை வீட்டாராக அருகிலும், இதன் விளைவாகக் கூட்டுக் குடும்பமாகவும் வசிப்பதை உறுதி செய்கிறது. பணிபுரியும் பல குடும்பங்களுக்கு இது ஒரு சாத்தியமான தீர்வாகிவிட்டது, இது தனிமையை உறுதி செய்வதாக மட்டுமல்லாமல், பாதுகாப்பான, செலவு அதிகமில்லாத குழந்தை பராமரிப்புக்கும் உத்திரவாதம் அளிக்கிறது - சில நிகழ்வுகளில் உணவுக்கும்.

இந்த இளம் தாய்மார்களின் கணவர்கள் குழந்தை பராமரிப்பு நெறிகளைப் பற்றிய இந்த விஷயங்களையும் அவர்களின் செயல்முறை தேவைகளையும் புரிந்து வைத்திருக்கிறார்கள். அவர்கள் பல விதங்களில், உதாரணத்திற்கு குழந்தைகளை பள்ளிக்கூடத்தில் விடுவதிலும், ${ }^{66}$ சில நேரங்களில் சமையல் செய்வதிலும் ${ }^{67}$ உதவுகிறார்கள். பெரும்பாலான மத்திய வகுப்புக் குடிம்பங்களில் சுத்தம் செய்தல் போன்ற வேலைகளைச் செய்ய பெண் பணியாட்களும் இருக்கிறார்கள்.

அதே நேரத்தில் பஞ்சக்கிராமியின் மத்திய வகுப்பு ஒரு கூட்டுக் குழுவாக இருக்கிறது, ஆகையால் நண்பர்களுடனும், விரிநிலைக் குடும்பங்களுடனும் தொடர்பை நிறுவி அதில் இருப்பதும் முக்கியமானது. இது மக்களிடம் முக்கியமான தொடர்பு வட்டத்தின் பெயரைக் கேட்ட போது தெளிவாகத் தெரிந்தது. 96 சதவிகித தம்பதிகள் தமது முதல் முக்கிய வட்டமாக தமது கணவன்/மனைவி மற்றும் குழந்தைகளைக் கூறினாலும், மத்திய வகுப்பு தம்பதிகளில் 89 சதவிகிதத்தினர் தமது நண்பர்களையும், குடும்பத்தாரையும் இரண்டாவது முக்கிய வட்டம் என்றனர். .சிலர் உண்மையில் முதல் வட்டத்தில் தமது நாயையும் இரண்டாவதில் தமது மாமியாரையும் கூறினர். இதைச் செய்த ஆண்கள் மற்றும் பெண்கள் இடையே குறிப்பிட்ட புள்ளியியல் வேறுபாடுகெள் இருக்கவில்லை. தமது நண்பர்கள் மற்றும் குடும்பத்தினருடன் வாட்ஸ் அப் மற்றும் ஃபேஸ்புக் குழுக்கள் மூலம் எவ்வாறோ தொடர்பில் இருக்கும் ரவி மற்றும் சுஜாதா போன்றக் குடும்பங்களில் தொடர்பிலிருப்பதற்கான அவர்களுடைய தேவை தெளிவாகத் தெரிகிறது. 
பஞ்சகிராமியில் இது போன்ற பல நிகழ்வுகள் உள்ளன. மேல்தட்டுக்குச் செல்வதன் மேல் உள்ள அழுத்தமான அவாவுக்கும் தங்களை பாதிக்கக்கூடிய குழந்தைப் பராமரிப்பு நெறிகளை உறுதிப்படுத்த மற்றும் கையாள, அவர்கள் வழக்கமாகத் தமது அலுவலகப்பணிகளில் நீண்ட நேரம் பணி புரிந்தாலும், நாம் தம் அக வாழ்க்கையோடு எப்பொழுதும் தொடர்பில் இருக்க விரும்பும் ஒருவரின் இடையறா தேவையை பார்க்கலாம். இந்தப் பகுதிகளுக்குப் பாலம் அமைக்க தெரிந்து கொள்ளும் ஒரு வழி பணி மற்றும் அக வாழ்க்கை உலகு ஆகிய இரண்டு அம்சங்களுக்கும் இடையே தொடர்ச்சியான தொடர்பை உறுதி செய்வதன் மூலம்தான்.

இவை அனைத்துமே பிரத்தியட்சமாக இருந்தாலும், இவை நவீன பணியிடங்களில் நேராக இருப்பதில்லை குறிப்பாக தம் பணியிடங்களில் எவை அனுமதிக்கப்படுகின்றன, எவை அனுமதிக்கப்படுவதில்லை என்பதைப் பற்றிய அவர்களுடைய ஒழுங்குமுறைகளின் தொகுப்பைக் கொண்டிருக்கும் ஐடி கம்பெனிகளில். முன்னரேப் பார்த்தது போல, பணிக் கணினிகளில் இருந்து சமூக ஊடகங்களை அணுகல் அனுமதிக்கப் படாததினால், இவற்றை விலை மலிந்த ஸ்மார்ட்ஃபோன் மூலமே செய்யக்கூடும். ஆயினும் சமூக ஊடகத்தை பணி-வாழ்வு சமநிலையை சமாளிக்கும் ஆற்றலுடைய ஒரு உபகரணமாக நோக்கும் சில கம்பெனிகள் வரம்புக்குட்பட்ட அணுகலை அனுமதிக்கின்றன - உதாரணத்திற்கு, மதிய உணவு நேரத்தில் அல்லது வேலை நேரம் முடிந்து. வார இறுதி நாட்களும் அவ்விதமான தளங்களை அணுக திறந்து இருக்கின்றன, ஆனால் பிராட்பாண்ட் உபயோகத்தில் ஒரு பிடிப்பு வைக்கப்பட்டிருக்கிறது. ஒரு குறிப்பிட்ட அளவு உபயோகத்தைத் தாண்டுபவை (இந்த அளவு கம்பெனிகளுக்கிடையிலும், அணிகளுக்கிடையிலும் மாறுபடும்) கண்காணிக்கப்பட்டு கம்பெனியின் சொத்து தவறாக உபயோகிக்கப்படாத வண்ணம் அதன் மேல் நடவடிக்கை எடுக்கப்படுகிறது. தனிப்பட்ட ஸ்மார்ட்ஃபோன்கள் மூலம் இவ்வாறான தடைகள் மீறப்படும் பொழுது இவை குறைந்த முக்கியத்துவத்தையேப் பெறுகின்றன, இவை யாரும் பயனாளிகளைத் தொந்தரவு செய்யாத 24/7 தொடர்புக்கு உத்தரவாதம் அளிக்கிறது.

இந்தியாவில் பணியைப் பற்றிய பொதுக் கட்டுரைகளின் மதிப்பீட்டில் பணியிடங்களில் உறவு முறை தாமாகவே ஊடுருவும் வகைகளில்தான் கடுமையான 'பணி மற்றும் பணியல்லாதவைகளின்' பிரிவுகளின் லட்சியங்களின் அதிகப்படியான நீர்த்துப் போகுதல் காணப்பட்டது. ஒரு ஐடி கம்பெ- 
னியைப் பற்றிக் கருதும் போது இது எல்லைகளை அதிகம் தெளிவற்றதாக்கலாம். இந்த குறிப்பிட்ட நிகழ்ச்சி பஞ்சகிராமியில் கீழ் சமூகப் பொருளாதார வகுப்புகளிலிருந்து எடுக்கப்பட்ட ஒரு நிகழ்வின் மூலம் சிறந்த முறையில் விளக்கப்படலாம். இந்தப் பின்புலத்தைச் சேர்ந்த பணியாளர்கள் பொதுவாக இந்த ஐடி கம்பெனிகளில் கீழ் மேலாண்மை அல்லது அந்த நிலையுடன் சம்பந்தப்பட்ட வேலைகளில் பணி புரிந்தார்கள்.

\section{ஐடி துறையில் அமைப்புகளை மத்தியஸ்தம் செய்தல்: கீழ் சமூகப் பொருளாதார வகுப்புகளில் உறவு அடிப்படையிலான நெட்வொர்க்குகள்}

முந்தைய அத்தியாயங்களில் பார்த்தது போல, பஞ்சக்கிராமி சாதி அடிப்படையிலான பலமான நெட்வொர்க்குகள் கொண்ட கிராமங்களால் ஆனது. இந்த கிராமங்களில் ஒரே சாதியின் உறுப்பினர்களில் பெரும்பாலானோர் ஒருவருக்கொருவர் உறவினர்களாகவோ அல்லது குறைந்த பட்சம் ஒருவரை ஒருவர் கற்பனை உறவு முறைச் சொல்லி அழைப்பவராகவோ இருந்தனர். 'அண்ணா' போன்ற கற்பனை உறவு முறை வார்த்தைகளின் பொதுவாக்குதல் தமிழ்நாடு முழுவதிலும் இருக்கக்கூடிய சாதிகளுக்கிடையேயான அம்சமாக இருந்தாலும், இது பெரும்பாலும் சாதிகளுக்கிடையேயான குணாதிசயமாக இல்லாமல் சாதிக்குள்ளானதாகவே இருந்தது.

இந்த கிராமங்களில் இருக்கும் 18 வயதிலிருந்து 30 வயதுக்குள்ளான கீழ் சமூகப் பொருளாதார வகுப்பைச் சேர்ந்த பெரும்பாலான இளைஞர்கள் படிப்பைப் பாதியில் நிறுத்தியவர்கள் (மேல்நிலைப் பள்ளி அல்லது கல்லூரியிலிருந்து) அல்லது உள்ளூர் கல்லூரிகளிலிருந்து பட்டம் பெற்றவர்கள். இவர்களில் பெரும்பாலானோர் தமது கிராமத்திலிருக்கும் ஒரு ஐடி/ஐடிஈஎஸ் கம்பெனியில் பணி புரிபவர்கள். அவர்கள் கீழ் மேலாண்மைப் பதவிகளை வகிக்கிறார்கள் மற்றும் சிலர் கண்ணியமான நிலையை உடைய பணிகளுடன் கூடிய நிலைமையை அடைகிறார்கள், உதாரணத்திற்கு, காபி அசிஸ்டென்ட், ரெகார்ட்கீப்பர், ஹார்ட்வேர் டெக்னீஷியன் போன்றவை. இந்த இள வயதினர் தெரிந்தே பாரம்பரியமாக கீழ் சாதி நிலைமையுடன் சம்பந்தப்பட்ட அலுவலகத்தை சுத்தம் செய்தல் போன்ற வேலைகளைத் தவிர்க்கிறார்கள். ${ }^{68}$ அவர்கள் 
அனைவரும் பட்டம் பெறவில்லை என்றாலும், அவர்கள் தமது கல்வியறிவு, அறிவுப் பொருளாதாரத்தில் இது இவர்களுக்குக் கொணரும் முக்கியத்துவம் ஆகியவை இவர்களுக்கு பாரம்பரியமான சாதிக்கொள்கைகளுக்கு எதிராக கிளர்ந்தெழ உத்வேகம் அளிக்கின்றன என அவர்கள் நினைக்கிறார்கள். இந்த உணர்வு பூர்வமான முடிவு இள வயதினருக்கு இடையே சாதி விலங்குகளின் மீது குறிப்பிடத்தக்க மறுப்பை பிரதிபலிக்கிறது, மேலும் இது சமூகத்தில் உயர்ந்த நிலைக்குச் செல்வதை நோக்கிய இயக்கத்தைப் பிரகடனம் செய்யலாம். ${ }^{69}$

இந்த இள வயதினர் ஐடி/ஐடிஈஎஸ் கம்பெனியில் எவ்வாறு ஒரு நிலையை அடைகிறார்கள் - சிலர் பட்டப்படிப்பு கூட இல்லாமல்? இந்த ஐடி கம்பெனிகளில் எதுவும் இந்த கிராமத்து இளைஞர்களுக்கு திறனுள்ள பணியை வழங்குவதில் முறையான உடன்பாட்டு செயல் திட்டங்களுடன் இல்லை; ஆயினும் ஆதரவுப் பணியாளர் பணிக்கு (மற்றும் சுத்தம் செய்யும் பணிக்கு) இம்மாதிரியான கம்பெனிகள் பல உள்ளூரிலிருந்து பணியமர்த்துகிறார்கள்; மேலாண்மைப் பதவிகளை விட கீழ் நிலைப் பணிகளுக்கு உள்ளூர் கிராமத்தினரைப் பணியமர்த்துதல் எளிது. ஆகையால் ஐடி கம்பெனிகள் மேலாண்மைப் பதவிகளை இளைஞர்களுக்குப் பெற்றுத் தருவதில் செயலாக்கத்துடன் இல்லாவிட்டாலும், இளைஞர்கள் கீழ் மேலாண்மைப் பணிகளை ஒருவருக்கொருவர் பெற்றுத் தருவதில் உதவுகிறார்கள்.

தர்ஷன் மற்றும் நாகாவின் கதை ஐடி துறையில் ஆரம்ப நிலைப் பணிகளைப் பெறுவதில் இளைஞர்கள் எவ்வாறு ஒருவருக்கொருவர் உதவிக் கொள்கிறார்கள் என்பதற்கு உதாரணமானது. இப்பொழுது 30 வயதாகி இருக்கும் தர்ஷன் சில ஆண்டுகளுக்கு முன் தனது காதல் தோல்வி அடைந்ததால் உயர்நிலைப் பள்ளிப் படிப்பை பாதியில் நிறுத்தியவர். உண்மையான நட்பின் அடையாளமாக இப்பொழுது 29 வயதாகியிருக்கும், தர்ஷனின் நெருங்கிய நண்பரான நாகாவும், படிப்பை விட்டு விட்டார். செய்வதற்கு ஒன்றும் இல்லாததால், இருவரும் ஐடி கம்பெனிகளைச் சுற்றிக் கொண்டும், சலிப்பைத் தவிர்க்க தமது நேரத்தை அரட்டையில் போக்கிக் கொண்டும் இருந்தனர். பணி புரியும் அவர்களது பள்ளியில் முன் படித்தவர் ஒருவர் இந்த இருவரைக் கண்டு அவர்கள் என்ன செய்து கொண்டிருக்கிறார்கள் என விசாரித்தார். அவர்களுடைய கதையைக் கேட்ட பின், இந்த மூத்தவர் அவர்களுக்கு அறிவுரை கூறி இருவருக்கும் ஆங்கிலம் படிக்கவும் எழுதவும் தெரிவதால், தாம் 
பணி புரியும் கம்பெனியிலேயே அவர்களுக்கு ஆவணங்கள் பேணல் பணியை வாங்கித் தருவதாக உறுதி அளித்தார். தர்ஷனும் நாகாவும் இதற்கு சம்மதித்தார்கள், இந்த பணிகளுக்கு உடனடியாகப் பணியாளர்கள் தேவைப் பட்டதால் பணியிலமர்த்தும் கம்பெனி நேர்காணலில் அதிக கேள்விகள் கேட்கவில்லை, அவர்கள் இந்தப் பையன்களுக்கு எழுத, படிக்க தெரியும் என்பதை மட்டும் உறுதி செய்து கொண்டார்கள்.

அதற்குப் பிறகு விரைவிலேயே, அவர்கள் கடின உழைப்பு பலனளிக்கும் என்பதைத் தெரிந்து கொண்டார்கள். தர்ஷன் ஐடி கம்பெனியின் ஒரு குறிப்பிட்ட வாடிக்கையாளரிடம் ஆவணம் பேணுதலின் தலைவராக ஆகிவிட்டார், நாகா ஆவணம் சேகரித்தல் துறைக்குச் சென்று விட்டார். இருவரும் கம்பெனிகளில் நிரந்தரப் பணியைப் பெற்று விட்டார்கள். அவர்களுடையப் பள்ளித் தோழர் ஒருவர் வேலை தேடி அவர்களது வாசலுக்கு வந்த போது, தர்ஷனும் நாகாவும் அதே கம்பெனியில் தனது செல்வாக்கின் மூலம் அவருக்கு ஆவண நகலி பணியைப் பெற்றுத் தந்தனர். ஒரு நண்பருக்கு வேலை வாங்கித் தரும் சோதனைப் பலனளித்தவுடனேயே, இந்த இளைஞர்கள் இருவரும் தனது சமுதாய மற்றும் உறவு முறை மக்களுக்கு இதே மாதிரியானப் பணிகளைப் பெற்றுத் தருவதிலும் இவ்வாறு அவர்களை சமூகம் மற்றும் பொருளாதாரத்தில் உயர்ந்த நிலைக்குச் செல்ல தாம் ஏன் உதவக்கூடாது என விவாதித்தார்கள். ஆயினும் அவர்கள் இதை வெளியில் விளம்பரம் செய்யவில்லை, இதை வெறுமனே தமது ஃபேஸ்புக் ப்ரொஃபைலில் அதிகார பூர்வமின்றி தமது ஐடி கம்பெனியில் ஒரு பணியிடம் இருப்பதை அந்தப் பகுதி இளைஞர்கள் யாரும் பதிலளிக்கிறார்களா எனக் காண, போஸ்ட் செய்தனர். அவர்கள் செய்தார்கள்! இருவரும் பட்டப்படிப்பு படிக்காத நாகாவின் உறவினர் ஒருவரை அந்தப் பணியிலமர்த்துவதில் வெற்றிக் கண்டார்கள், அங்கு அவர் தளவாடங்கள் துறை பொறுப்பாளராக இருந்தார்.

தர்ஷன் மற்றும் நாகாவால் நல்ல வேலைகளைப் பெறுவதில் இளைஞர்களுக்கு உதவ முடியும் என்ற செய்தி பரவலாயிற்று. அந்தப் பகுதியின் இளைஞர்கள் அவர்கள் ஃபேஸ்புக்கில் விளம்பரங்களைப் போஸ்ட் செய்யும்வரைக் காத்திருந்தார்கள் அல்லது உதவி வேண்டி அவர்களை நேரடியாக நாடினார்கள். தர்ஷன் சொல்வது போல் இதன் திட்டம் அனைவரும் இதை அறிந்த பின்னர் செய்வதற்கு பதிலாக, இந்தப் பணியிடம் பற்றி உள்புறத்தில் விளம்பரப்படுத்தப்பட்ட உடனேயே இந்த சந்தர்ப்பத்தின் மீது 
பாய்வது, இவ்வாறு போட்டியை வெல்வது. அவர்களுடைய உத்தி இப்பொழுது பணியிடங்களைப் பற்றி ஃபேஸ்புக்கில் விளம்பரம் செய்வது கூட அல்ல - இதற்கு பதிலாக அவர்கள் ஒரு பொருத்தமான நபருக்கு, அவர் உடனடியாக விண்ணப்பிக்கக் கூடிய வகையில்,வாட்ஸ் அப் மூலம் செய்தி அனுப்புகிறார்கள், சில நேரங்களில் அவர்கள் நான்கு அல்லது ஐந்து ஆற்றல் மிக்க நபர்களுக்கு உள்ள பணியிடங்கள் பற்றி வாட்ஸ் அப் செய்திகளை அனுப்பி அந்தப் பணியிடத்திற்குப் பொருத்தமானவர் யார் எனச் சோதிக்கிறார்கள். முன்புப் பார்த்ததைப் போல இந்த நடத்தைப் புதியது இல்லை, ஆனால் இந்தியாவில் பணியின் முக்கிய குணாதிசயமாகவே இருக்கிறது. ஆயினும் முன் நாட்களில் உறவு முறைத் தொடர்புகள் வாய் வார்த்தைகளினாலேயேப் பணியிலமர்த்தப் பட்டிருப்பார்கள், இப்பொழுது சமூக ஊடகம் இந்தச் செயல் முறையை குடும்பத்திலிருந்து ஒருவரின் உள்ளூர் சமுதாயத்தின் பரந்தச் சூழல் வரை விஸ்தரிக்கத் திறனுள்ளதாயிருக்கிறது..

பணியில் அமர்த்தப்படுபவர்கள் கம்பெனியில் காலிப் பணியிடங்களைக் கவனிக்கிறார்கள், இதன் மூலம் தமது சமுதாயத்தில் உள்ள மற்றவர்களுக்கு வேலை கிடைக்கச் செய்வதில் உதவுகிறார்கள். தர்ஷன் இப்பொழுது தமதுப் பகுதியில் இருக்கும் பெண் பட்டதாரிகளுக்கு தமது ஐடி கம்பெனியில் பணி கிடைக்க உதவுகிறார். அப்பெண்களிடம் பொதுவாக மொபைல் ஃபோன்கள் இல்லாதக் காரணத்தால், அவரவர்களுடைய பெற்றோருடன் தொடர்பு கொண்டு, சமுதாயத்தில் அப்பெண்களின் நற்பெயரை பாதிக்காத வகையிலான நிலைகளைப் பெறுவதில் தம் செல்வாக்கை உபயோகிக்கிறார். உதாரணத்திற்கு ஆவணத்துறையிலும் போக்குவரத்துத் துறையிலும் பெரும்பாலும் ஆண்களே இருக்கிறார்கள். ஆகையால் தர்ஷன் தன்னுடைய சமுதாயப் பெண்கள் அங்கு பணி புரிவது உகந்தது என்று நம்பவில்லை. அவ்வாறு செய்தால் அவர்கள், அல்லது குறைந்த பட்சம் அவர்களின் பெற்றோர், மிகவும் சங்கடமாக உணரலாம் எனவும் மற்றும் பெண்களின் குணங்களையும் அவர்களுடைய திருமண வாய்ப்புகளையும் பாதிக்கக் கூடிய வதந்திகள் பரவலாம் எனவும் அவர் எண்ணினார். பதிலாக அவர் பெண் விண்ணப்பதாரர்களை ஆண்களை விடப் பெண்கள் அதிகம் பணிபுரியக் கூடிய தகவல் பதிவு பணிகளில் வைக்கிறார், இந்த இருவரும் ஆவண நகலி மற்றும் தகவல் ஆய்வாளர் போன்ற பட்டப் படிப்பு இல்லாதவர்களுக்கான, மற்றும் 
பட்டப் படிப்பு முடித்தவர்களுக்கான பணிகளைத் தெளிவாக அடையாளம் காணுகிறார்கள்.

இந்த நெட்வொர்க் அருகிலுள்ள மற்ற கம்பெனிகளுக்கும் பரவி விட்டதைப் போல் உள்ளது. கீழ் மேலாண்மை நிலைகளில் காலிப் பணியிடங்களைப் பற்றிய செய்தி ஒரு வாட்ஸ் அப் குழு மூலம் அனுப்பப்படுகிறது, மேலும் பணியமர்த்துதல் வழக்கமான செயல்முறையை விட வேகமாக நடை பெறுகிறது. கீழ் மேலாண்மை நிலைகளுக்கான இந்த உள்முறைக் குறிப்பிடுதல் முறை வேகமாகவும் பயனுள்ள விதத்திலும் வேலை செய்கிறது. இதன் இன்னொரு அம்சம் இந்த விண்ணப்பதாரர்களுக்கு அவர்கள் விண்ணப்பிக்கும் பணிக்கு ஏற்ப தர்ஷன் மற்றும்/ அல்லது நாகாவால், அல்லது அவர்களால் முன்னர் பணியமர்த்தப்பட்ட ஒருவரால், தரப்படும் முன் பயிற்சியாகும். இருவரும் இணைந்து 25 இளைஞர்களுக்கு பல்வேறு பணிகளைப் பெற்றுத் தந்துள்ளனர்; அவர்கள் தங்களது வெற்றி விகிதம் 95 சதவிகிதமாக உள்ளது எனப் பெருமை கொள்கிறார்கள்.

தர்ஷனுக்கும் நாகாவுக்கும் பதிலுக்கு என்னக் கிடைக்கிறது? அவர்கள் தமது சமுதாயத்தில் தனக்கென ஒரு பிம்பத்தை ஜாக்கிரதையாக உருவாக்கியிருக்கிறார்கள் மேலும் குறிப்பிடத்தக்க அளவில் சமூக முதலீட்டையும் பெற்றிருக்கிறார்கள். மேலாக, அவர்கள் சமுதாயத்தில் உள்ள மூத்தவர்கள் இவர்களை தமது சமுதாயத்தின் இளைய தலைமுறையினரின் எதிர்காலத்தை வடிவமைக்கக் கூடியவர்கள் எனக் கருதுகிறார்கள். தர்ஷன் மற்றும் நாகாவுக்கு அவர்களது பணியிடம் குறிப்பிடக் கூடிய அளவில் தமது சமுதாயத்தினர் இருக்கும் இடமாக மாறி விட்டது. நாகா மற்றும் தர்ஷனின் அழைப்பின் பேரில் அவர்களுடைய ஆவணங்கள் பகுதியின் பணித் தளத்திற்கு சென்ற போது இஙங்குள்ள பெரும்பான்மையான ஆண்கள் ஒருவரை ஒருவர் 'சார்' என்பதற்கு பதிலாக 'மாமா' அல்லது 'அண்ணா' எனப் பேச்சு வழக்கில் அழைப்பதைக் காண முடிந்தது. அவர்கள் வழக்கமாக சேர்ந்தே உணவருந்தினர் மேலும் ஒரு நவீன ஐடி/ஐடிஈஎஸ் கம்பெனியுள் ஒரு உறவுமுறை நெட்வொர்க்கை நிறுவி விட்டனர். அனைவரிடமும் வாட்ஸ் அப் நிறுவப்பட்ட ஒரு ஸ்மார்ட்ஃபோன் இருந்தது, இதன் மூலம் அவர்கள் வேலை வாய்ப்புகள் உருவாகும்போது ஒருவருடன் ஒருவர் தொடர்பு கொண்டு தகவல்களைப் பரிமாறி வந்தனர். சமுதாயத்தில் நடப்பவையும் வாட்ஸ் அப் மூலமாகப் பணி நேரத்தில் பரிமாறிக் கொள்ளப்பட்டன, மேலும் தமது குடும்பம் மற்றும் உறவினர்களுடனேயே பணி செய்து 
கொண்டிருப்பதால் எவரும் நீண்ட நேரம் பணி செய்வது போல் உணரவில்லை. அவர்கள் வீட்டை விட்டு வெளியே இருந்தாலும், அலுவலகத்தை விட்டு வெளியே இருந்தாலும், இதில் வேறுபாடு ஒன்றும் இல்லை; எல்லாம் ஒன்றாகவே இருந்தன.

தர்ஷனும் நாகாவும் தொழில்முறை அல்லாத பட்டம் பெற்றவர்களையும், பட்டம் பெறாதவர்களையும், அவர்களுக்குக் கீழ் மேலாண்மை நிலைகளில் வேலைகள் கிடைப்பதற்கு ஆதரவளிப்பதன் மூலலம், கவனித்துக் கொண்டனர், மறுபுறம் வாசு வாசு தமது சமுதாயத்திலுள்ள பட்டதாரிகளுக்கு மத்திய மேலாண்மை நிலைகளில் வேலைகள் கிடைக்க உதவுகிறார் - தாம் பணி புரியும் (தகவல் பாதுகாப்பு நிர்வாகியாக) ஐடி கம்பெனியிலோ அல்லது வேறு ஐடி கம்பெனிகளிலோ. 28 வயதான அவர் தர்ஷன் மற்றும் நாகாவின் சமுதாயத்தைச் சேர்ந்தவர் மற்றும் எஞ்ஜினீயரிங்கில் ஒரு தொழில்முறைப் பட்டம் பெற்றவர். வாசு, தர்ஷன் மற்றும் நாகா மூவரும் ஒரே சாதியைச் சேர்ந்தவர்கள்; அவர்கள் சமூகத்தில் உயர்ந்த நிலையை அடையப் போராடி இருக்கிறார்கள் மற்றும் அவர்களுடைய சமகாலத்தவர்கள் அனுபவிக்கும் சமூகத்தில் உயர்ந்த நிலைக்குச் செல்ல செய்யும் பிரயாசைகளை புரிந்து கொள்கிறார்கள். சமீப காலத்தில் பட்டம் பெற்ற எஞ்சினீயர்கள், அல்லது பணி மாற்றத்தை எதிர்பார்க்கும் எஞ்சினீயர்கள் வாசுவின் கதவைத் தட்டுகிறார்கள். அவர் இந்த தனது உறவுமுறை நெட்வொர்க்குடன் அமர்ந்து அவர்களது ஆளுமை, கல்வி மற்றும் தொழில் குறிப்பை தயாரிக்கும் செயல் முறையிலும், லிங்க்ட் இன் ப்ரொஃபைலை உருவாக்குவதிலும், நேர்காணல் கேள்விகளுக்கு பதில் அளிக்கவும், மற்றும் அவர்களை ஒரு வளமிக்க விண்ணப்பதாரராக தயார் செய்யவும் உதவுகிறார்கள், இவை அனைத்தும் அவர் அவர்களைப் பணியிடத்திற்கு பரிந்துரைப்பதற்கு முன்னேயே நடக்கின்றன. எனினும் உண்மையில், 'வாசுவுடன் அமருதல்' என்ற வாசகத்திற்கு வேறு அர்த்தம் உண்டு. அவர் வளமான விண்ணப்பதாரர்களை ஒரு முறைதான் சந்திக்கிறார், பிறகு அவர்களிடம் ஸ்கைப் மூலம் பேசவோ அல்லது வாட்ஸ் அப் மூலம் சாத்தியமான வேலை வாய்ப்புகளைப் பற்றி தொடர்பு கொள்கிறார்; இவை அனைத்தும் வாட்ஸ் அப் மூலமே செய்யப்படுகின்றன.

வாசு இதை தமது அலுவலகத்திலிருந்தோ அல்லது வீட்டிலிருந்தோ செய்கிறார், ஆனால் அவரிடம் உதவி கேட்டு வரும் உறவினர்கள் மற்ற தொடர்புக் கருவிகளைப் பற்றி ஒரு புரிதலை அடைகிறார்கள் என்பதை உறுதி செய்து கொள்கிறார், இது அவர்களுடைய எல்லைகளை சமூக ஊடகங்களின் மூலம் தனி- 
ப்பட்ட தொடர்புகளைத் தாண்டி மிகவும் விரிவானதாக்குகிறது. தர்ஷனும் நாகாவும் மக்களை லிங்க்ட் இன் ப்ரொஃபைலை உருவாக்கச் சொல்லி வற்புறுத்துவதில்லை, ஏனென்றால் அவர்களுக்கே அது இல்லை. மாறாக வாசு, அவரது வேலை தேடும் உறவினர்கள் இதை உருவாக்குவதோடு மட்டுமல்லாமல், அந்தத் தளத்தில் அறிவு அடிப்படையான இஞ்சினீயரிங் குழுவை எவ்வாறு அணுகுவது என்பதைப் புரிந்து கொள்வதையும் உறுதி செய்கிறார். இது நேர்காணலின் போது மட்டுமல்லாமல் பணியில் புதிய பங்குகளை ஏற்கும்போதும் உதவும் என அவர் வலியுறுத்துகிறார். வாசுவின் வெற்றி விகிதம் 70 சதவிகிதமாக இருக்கிறது, இது தமது உறவினர்களுக்கு ஐடி கம்பெனிகளில் மேலாண்மைப் பதவிகளைப் பெற்றுத் தர முயல்பவர்களுக்கு மோசமானதல்ல என வாசு எண்ணுகிறார். வாசுவின் அறிவுரையை நாடுபெர்கள் அவருடைய சாதி மற்றும் உறவுமுறை நெட்வொர்க்கைத் தாண்டி விரிந்து இப்பொழுது மற்ற சாதி மற்றும் சமுதாய மக்களும் இணைந்து இருக்கிறார்கள்.

ஆயினும் இங்கு கருத்தில் கொள்ளக் கூடியது உறவுமுறை நெட்வொர்க் ஒரு இடத்திலிருந்து மற்றொன்றுக்கு நகருவது மட்டுமல்ல, இந்த நிலைகளுக்கான தேர்வுக்குரியவர்களை அறிவு உருவாக்குதலின் மூலம் உருவாக்குதலும்தான். வேறு விதமாகக் கூறினால் இவை உறவுமுறை நெட்வொர்க்குகள் என்ற வேஷத்தில் இருக்கும் முறைசாரா அறிவு நெட்வொர்க்குகளாகும், இவை அனைத்தும் பணியமர்த்தல் அல்லது பந்தம் எதன் மேல் கவனமாயிருந்தாலும், பணியிடங்களை விட்டு வெளியேதான் இயங்குகின்றன. இந்த விதமான பணியமர்த்தல்கள் நாம் சோட்டா நாக்பூர், திருப்பூர் மற்றும் அது போன்ற இடங்களில் ஆய்வு செய்தது போல தொழிற்சாலை அடிப்படையிலான குறைந்த திறன் தொழிலாளர்களைப் பணியிலமர்த்துவதுடன் ஒப்பீடு செய்ய அனுமதி அளிக்கிறது. இந்த விதமான பணியமர்த்தல்களின் இயல்புகள் அடிப்படையில் ஒன்றாகவே இருந்தாலும், சமூக ஊடகத்தின் துணையுடன் இது நடக்கக்கூடிய வேகம் குறிப்பிடத்தக்க வகையில் வேறுபடுகிறது. சமூக ஊடகம், தர்ஷன் மற்றும் நாகாவின் நிகழ்வைப் போல, போட்டிகளைக் குறைப்பதன் விளைவுகளை அடைந்திருக்கலாம். தகவல்களை அனுப்புதல் மற்றும் கட்டுப்பாட்டுக்குள் வைத்திருத்தல் மூலம் ஒருவரைப் பணியிலமர்த்துவதில் உள்ள வேகம் இந்த இருவருக்கும் பணியிடத்திலும் மற்றும் அவரது சமுதாயத்திற்குள்ளும் அங்கீகாரத்தையும் அதிக சமூக அந்தஸ்தையும் பெற்றுத் தருகிறது. 
இங்கும் சமூக ஊடகங்களின் மேல் தடைகள் உள்ளன. உதாரணத்திற்கு, நாகாவின் நண்பர் ஆவண அறையில் அவரது படத்தை எடுத்து ஃபேஸ்புக்கில் போஸ்ட் செய்த பொழுது, அவரது ஃபேஸ்புக் நண்பரான அவரது மேலாளாருக்கு மேலாளர் அவர் உடனடியாக அந்தப் படத்தை நீக்காவிட்டால் பயங்கரமான விளைவுகள் ஏற்படும் என அச்சுறுத்தினார். கவனக்குறைவாக அது ஒரு 'பாதுகாப்பு அறையில்' எடுக்கப்பட்டு இரருந்தது - வாடிக்கையாளர்களின் கொள்கைகளின் படி காமிரா மற்றும் ஸ்மார்ட்ஃபோன்கள் தடை செய்யப்பட்ட பகுதி. நாகா மன்னிப்புக் கோரி படத்தை நீக்க வேண்டி இருந்தது. அவர் அந்த ஃபேஸ்புக் கணக்கை முடித்து ஒரு புதியக் கணக்கைத் துவங்கினார், மேலும் இந்த முறை அவர் நன்கு அறிந்த உள்ளூர் நண்பர்களுடனேயே இங்கு நட்பு கொண்டார், அவருடைய மேலதிகாரிகளுடன் இல்லை. இந்த மாதிரி சூழ்நிலைகளில் புலனாகுதல் தடைகளுக்குக் காரணமாய் அமைந்துள்ளது; ஒருவருடைய நட்பை விலக்குவது அல்லது இன்னொரு கணக்கைத் துவங்குவது ஒரு எளிய தீர்வாய் இருந்தது - மேலும் இது பணி அமைப்புக் குழப்பத்தை பிரதிபலிப்பதாகவும் இருந்தது. சமூக ஊடகம் பணியமர்த்தலுக்கு உபயோகப்படுத்தப்ப்டும் பொழுது பயனுள்ளதாகவும் பாராட்டத்தக்கதாகவும் இருக்கையில், இது ஒருவருடைய பணியிடத்தின் குறிப்பிட்ட அம்சங்களை வெளிக்காட்டும்போது ஏற்க முடியாததாக இருக்கிறது.

\section{முடிவுரை:}

மேலே விவாதிக்கப்பட்டது பணி அல்லது பணி அல்லாதவைகளின் பிரிவுகளில் மேற்படிந்த தொழிற் கட்டமைப்புகளின் மத்தியஸ்தம் மற்றும் கலந்தாய்வை உத்திபூர்வமாக அதிகரிப்பதில் தனிப்பட்ட தொடர்புக் கருவிகள் எவ்வாறு உபயோகப்படுத்தப்பட்டிருக்கின்றன என்பது பற்றிய ஒரு தெளிவான நிகழ்வு - வாழ்வின் வெவ்வேறு மண்டலங்களுக்கு இடையே கடினமானப் வரையறையை எப்பொழுதும் ஆதரிக்காத ஒரு சூழ்நிலையில். இந்த மத்தியஸ்தம் புதிதல்ல; இது இந்தியாவில் தொன்று தொட்டு வரும் ஒரு பழக்கம், இது சமகால இந்தியாவிலும் சில தொழில்களில் பழக்கத்தில் இருப்பதை நாம் காணலாம்.71 ஆயினும் டிஜிட்டல் தொழில்நுட்பத்தால் செயல்படுத்தப்பட்ட தனிப்பட்ட தொடர்பின் பங்குதான் புதியது, இது முன் காலங்களில் இல்லை. இவ்விதமான மத்தியஸ்தம் 
பணியாளர்களை தெளிவாகப் பணிக்காக ஒதுக்கப்பட்ட இடத்திற்குள் வருகை புரிவதுடன் சம்பந்தப்பட்ட சம்பிரதாயம் பற்றிய உணர்வைக் கடைப்பிடிக்க எதிர்பார்ப்பதன் மூலமே உருவாகிறது; அவ்விடத்தை அடைந்தவுடன் அ அனைத்துப் பணியாளர்களும் ஒரு குறிப்பிட்ட கால அளவுக்கு மற்ற எல்லா மண்டலங்களிடமிருந்து தமது தொடர்பை முறித்துக் கொள்ள ஊக்கமளிக்கப் படுகிறார்கள். ஆயினும் மண்டலங்களுக்கிடையேயான உறவுகளின் தொடர்ச்சி, தனிப்பட்ட இடங்கள் எதுவாக இருந்தாலும், அவ்வாறான உள்ளார்ந்த சம்பிரதாயத்தைப் பலவீனப்படுத்தியது. முந்தையக் கட்டுரைகள் உறவுமுறை பணியிடங்களை ஊடுருவும் முறை பற்றி கணிசமான ஆதாரங்களைத் தருகின்றன, இது குடும்பம் மூலம் மேலும் பணியமர்த்தலுக்குக் காரணமாகிறது. ${ }^{72}$ நாம் இங்கு சமூக ஊடகம் குடும்பத்திற்கு அப்பாற்பட்டு தொடர்பை பரந்ததாகச் செய்ய விழைவதைக் காண்கிறோம், இது நிறுவனங்கள் இழுத்துக் கொள்ளக்கூடிய பணியிலமர்த்துபவர்களின் பரந்த நெட்வொர்க்கில் பிரதிபலிக்கிறது.

நாம் மானுடவியல் பணியைப் பற்றியக் கருத்தை எவ்வாறு கையாள்கிறது என்பதைப் பற்றியும், இது பஞ்சக்கிராமியில் எவ்வாறு இயங்குகிறது என்பதைப் பற்றியும் ஆய்வு செய்துள்ளோம் மேலும் பணியில் உள்ளத் தொடர்புகள் என்று பணிக் கலாசாரத்தினுள் இருக்கும் பணியல்லாதவைகளின் தொடர்புகள் பற்றிய தலைப்புகளையும் உள்ளடக்கி இருக்கின்றன என்பது பற்றிய நிகழ்வுகளையும் கருத்தில் கொண்டுள்ளோம். நாம் காதல் உறவு மற்றும் திருமணம் பற்றிய ஒரு நிகழ்வைப் பற்றியும், அது எவ்வாறு பணி மற்றும் வீட்டிற்கிடையேயான தொடர்பின் ஒருங்கிணைந்த இயல்பு பற்றிய நமது அடுத்தப் பகுதிக்கு எவ்வாறு கூட்டிச் செல்கிறது என்பது பற்றியும் விவாதம் செய்துள்ளோம். ஆயினும் நவீன பணியிடங்களில், உதாரணத்திற்கு ஐடி துறை பணி மற்றும் பணியல்லாதவைகளின் எல்லைகளின் பல அடுக்குப் பாகுபாடுகளும் தெளிவாகத் தெரிந்தன. சில கம்பெனிகள், பெரும்பாலும் புதிதாகத் தொடங்கியவை, இவ்விதமான இரு கூறுகளாக இருப்பதைப் பொருட்படுத்தவில்லை என்றாலும், மற்றவை - வழக்கமாக தனது வாடிக்கையாளரின் வணிகத் தேவையைப் பூர்த்தி செய்ய - பொருட்படுத்தியே இருந்தன. ஐடி கம்பெனிகளில் மேலாண்மை அடுக்குகளுக்கிடையே ஒரு எழுதப்படாத ஒப்பந்தம் இருந்தது, அது ஒருவர் அலுவலகத்தில் இருக்கும்போது ஒரு வரம்புக்குள் பணியல்லாத செயல்களில் ஈடுபடலாம் என்பது, ஆனால் இதை வெளிப்படையானதாக்க 
முடியவில்லை. அனைவருக்கும் இந்த நிலையைப் பற்றித் தெரியும், ஆனால் யாரும் இதை வெளிப்படையாக ஒத்துக்கொள்ள மாட்டார்கள்.

தெளிவான மற்றொன்று என்னவென்றால் பணியாளர்கள் பணியிடக் கோட்பாடுகளுக்கேற்ப தம்மை உத்தி பூர்வமாக வெளிப்படுத்திக் கொள்வதுதான். ஒரு நிலையில், அலுவலகத்தில் இருக்கும் பொழுது பணியல்லாதவை சம்பந்தப்பட்ட வேலைகளில் அனைவரும் ஈடுபட வேண்டியிருக்கும் என அனைவரும் அறிந்தாலும், யாரும் இதைப் பற்றியக் கடுமையான பணியிடக் ெொள்கைகள் மேல் அதிருப்தியைத் தெரிவிக்கவில்லை; அவர்கள் இந்த பணியிட ஒழுங்குமுறைக்கேற்ப நடந்து கொள்வது போலவே தோற்றமளித்தார்கள். ஆயினும், இன்னொரு நிலையில், அவர்கள் அந்தத் தடைகளை மீறுவதன் மூலம் இடைவிடாது தமது அதிருப்தியை வெளிப்படுத்திக் கொண்டிருந்தனர், ஆனால் இது வெளிப்படையாகத் தெரியாத வண்ணம் இருப்பதிலும் கவனமாக இருந்தனர். எனினும், மேற்கண்டவாறு இவை அனைத்தும் பணியாளர்கள் உண்மையில் அனுசரித்த ஒரு எழுதப்படாத ஒப்பந்தத்தின் பகுதியாயின.

உறவுகளில் வெளிப்படுத்தப்பட்ட நெருக்கங்கள், அத்தியாயம் 4ல் விவரிக்கப்பட்டாற் போல, அதில் சம்பந்தப்பட்டுள்ள மக்களின் பல வகையான இடைவினைகளுக்கு அழைப்பு விடுக்கின்றன. சில எழுத்துச் செய்திகளை உள்ளடக்கியுள்ளன, சில குரல்களை; இன்னும் சில இவ்விரண்டின் சேர்க்கையை உள்ளடக்கி உள்ளன. ஆனால், முன்னே உள்ள அத்தியாயத்தில் கண்டது போல, தாய்மை இன்னும் குரல் வழித் தொடர்பையே உள்ளடக்கி இருந்தாலும், சமூக ஊடகம் ஒத்திசையாத குரல் வழித் தொடர்புக்கு வாய்ப்பளிக்க உதவி செய்கிறது, லக்ஷ்மி தான் அலுவலகத்தில் இருக்கும் போதும் தன் குழந்தைகளுக்குத் தாயாக இருக்க வாட்ஸ் அப்பை உபயோகப்படுத்தியது போல. பணிபுரியும் தம்பதிகளின் நிகழ்வில், சூழல் அடிப்படையில் அமைந்த தொடர்பின் வகை அவர்கள் பரஸ்பரம் தொடர்பு கொள்ள பல வகையான ஊடகங்களை உபயோகிக்க திறனுள்ளவர்களாக்குகிறது - இது பாலிமீடியாவின் ஒரு தெளிவான எடுத்துக்காட்டு. இந்த வீடு மற்றும் பணிக் கோளங்களின் இடையில் இந்த இடைவினையின் தொடர்ச்சியானப் பெருக்கின் காரணமாக, இந்தத் தம்பதிகளின் நிகழ்வில் பாலிமீடியா ஒழுங்குப்படுத்தப்பட்ட உள்புற அலுவலக அமைப்பு மூலமாக ஒரு பேராசையானத் தொடர்பை உள்ளடக்கியிருந்தது என்பது தெளிவாக வெளியானது. இவ்விதமானத் தொடர்புகளை மற்றப் பணி உறவுகளுக்கு 
விஸ்தரிப்பதன் மூலம், இது எவ்வாறு முந்தைய குடிநீருக்கு அருகிலான உரையாடலை அவரவர் பணி மேசையில் நடக்கும் ஒன்றாக மாற்றியது என்பதையும் நாம் பார்த்தோம்.

பணிபுரியும் தம்பதிகளின் நிகழ்வில், முக்கியமாக சிறு குழந்தைகளுடனிருப்பவர்கள், சமூக ஊடகத் தொடர்புகளின் அதிகரிப்பினால் அப்படிப்பட்டக் குடும்பங்களில் உள்ள பெண்களின் பொறுப்புகள் குறைந்து விட்டாற் போல் தோன்றலாம். எனினும், இது அவ்வாறு போல் காட்சியளிக்கவில்லை. இம்மாதிரியான தொடர்பு தளங்கள் வீடு மற்றும் அலுவலக மண்டலங்களை சிறப்பாக நிர்வகிப்பதில் பெண்களுக்கு உதவி புரிந்துள்ளன என வாதிக்க முடிந்தாலும், இது எந்த விதத்திலும் அவர்கள் வீட்டுப் பொறுப்பைக் குறைக்கவில்லை; ஒரு விதத்தில் இது அதிகமாக்கி இருக்கிறது. பணியில் எதிர்பார்ப்புகளை சமாளிக்க முயலும் போதே குழந்தைகளையும் தொடர்ச்சியாக கவனிக்க வேண்டிய தேவை அவர்களுடைய நிகழ்வில் வெளிப்படையாக தெரிகிறது.

மத்திய வகுப்பு ெெற முறைகளிலிருந்து இந்தப் பெண்களால் கிரகிக்கப்பட்ட ஒரு எதிர்பார்ப்பு நல்ல இல்லத்தரசியாக இருப்பதுதான், இதை அவர்கள் தர நெறி சார்ந்ததாகவே எண்ணி அதனுடன் வளர்ந்திருக்கிறார்கள். இந்த இளம் தாய்மார்கள் ஒரு நிரந்தரமானக் குற்ற உணர்ச்சியை அனுபவிக்கிறார்கள், அவர்கள் சில நேரங்களில் இம்மாதிரியான உணர்ச்சியைக் குறைக்க இந்த எதிர்பார்ப்புகளுக்கு அதிகமாக ஈடு கொடுக்க முயலுகிறார்கள் - அவர்களுடையப் பெற்றோரோ அல்லது புகுந்த வீட்டினரோ அருகே வசித்து குழந்தைகள் பராமரிப்பில் உதவும் போது கூட. சில தனிக் குடும்பங்களில், கணவர் வீட்டுப் பொறுப்புக்களைப் பகிர்ந்து கொள்ள முற்படுகிறார்கள், ஆனால் பல நிகழ்வுகளில் பகிரப்பட்ட பணியின் சுமை குறையவில்லை. ஆகையால் இளம் தாய்மார்கள் சமூக ஊடகங்களை உத்தி பூர்வமாக தம்மை போட்டி மிகுந்த கார்ப்பரேட் பணியிடங்களில் நிலை நிறுத்தவும், அதே நேரத்தில் குடும்பத்தில் தன் எதிர்பார்க்கப்பட்ட பங்கை பூர்த்தி செய்யவும் உபயோகிக்கிறார்கள். ${ }^{73}$

மேலும், உறவுமுறை நெட்வொர்க் மூலம் பணியமர்த்துதல் இந்திய தொழிலாளர் சந்தையின் ஒரு முகப்பாக இருந்தாலும், வாசு, தர்ஷன் மற்றும் நாகாவின் நிகழ்வில் இது மாறி உள்ளது. ஒரு உறவுமுறை அடிப்படையிலான நெட்வொர்க்காய் ஆரம்பித்தது இப்பொழுது சமூக ஊடகத்தால் உள்ளூர் அளவில் இணைக்கப்பட்ட மக்களின் பரந்த அடித்தளத்தைச் சூழும் வகையில் வளர்ச்சியடைந்துள்ளது. பணியமர்த்தலின் 
இந்தக் கூறுகள் அடிப்படையிலேயே புதியவை அல்ல, ஆனால் அவை இப்பொழுது, உற்பத்தித் தொழிற்சாலை நகரங்களில் பார்த்த பழமையான உதாரணங்கள் போல, ${ }^{74}$ வாய்மொழி மூலம் இயக்கப்பட்டதிலிருந்து ஐடி ஆதிக்கத்திலுள்ள ஒரு அறிவுப் பொருளாதாரத்தில் சமூக ஊடகங்களால் இயக்கப்படுபவையாக மாறி இருக்கின்றன. இம்மாதிரியான மாற்றம் இவ்வாறு இந்தியத் தொழிலாளர்களின் சில உள்நாட்டு குணாதிசயங்களை, பணி மற்றும் பணியல்லாத மண்டலங்களின் இடையே தெளிவான இரு கூறு பற்றிய சுருக்கமான மற்றும் அயல்நாட்டு கருத்துக்களை அறிமுகப்படுத்தும் ஒப்பீட்டு வகையில் சிறு காலத்திற்குப் பிறகு, மீண்டும் நிறுவ உதவி புரிந்திருக்கிறது.

உறவினர்கள் மற்றும் இதர பரிச்சயமானவர்களுக்குக் கீழ் மேலாண்மை நிலைகளில் காலிப் பணியிடங்கள் பற்றி நேராகத் தகவலளித்தல் மூலம் போட்டியை எதிர் கொள்ள சமூக ஊடகத்தை உபயோகிக்கும் நிகழ்வு, பரந்த உலகம் மற்றும் அதன் சம்பந்தப்பட்ட சமூக கலாசார பிரச்சினைகளின் சூழலில் புரிந்து கொள்ளப்பட வேண்டும். இது உறவினர் ஆதரவுக் கொள்கையை வளர்க்கும் காட்சி போலத் தோணலாம். நாம் இந்த சாதிக் குழுக்கள் எதிர் கொள்கிற சமூகக் கலாசாரப் பிரச்சினைகளையும் இந்தப் பகுதி ஐடி கம்பெனிகளிடமிருந்து உடன்பாட்டு செயல்முறை வேலை வாய்ப்புத் திட்டங்கள் இல்லாமல் இருப்பதையும் கருத்தில் கொள்ள வேண்டும். இந்தச் சூழலில் இம்மாதிரியான உயிர்ப்பான பணியமர்த்தல் உத்திகள் சமூகத்தில் உயர்ந்த இடத்துக்குச் செல்வதற்கான வழியாகவும், பெரிய அமைப்புகளால் அவற்றின் மீது சுமத்தப்படுகின்ற பழமையான சாதி விதிமுறைகளுக்கு எதிரான ஆர்ப்பாட்டமாகவும் காணப்பட வேண்டும். ${ }^{75}$

தர்ஷன் மற்றும் நாகாவின் ஐ ஐடி கம்பெனிகளுக்கானப் பணியமர்த்தல் எவ்வாறு அளவிடக்கூடிய சமூகத்தன்மைக்காக அழகாகப் பேசுகிறது என்பதையும் நாம் பார்க்கலாம். ${ }^{76}$ இங்கு காலிப் பணியிடங்கள் மற்றும் பணிகள் பற்றியத் தொடர்புகள் வெவ்வேறு அளவுள்ள குழுக்களினிடையே செல்கின்றன மேலும் சில தகவல்கள் பொதுவாக ஃபேஸ்புக்கில் அறிக்கையாக விடப்படுவதிலிருந்து மற்றவை தேர்ந்தெடுக்கப்பட்ட சிலருடன் வாட்ஸ் அப் மூலம் பரிமாறிக் கொள்ளப் படுகிறது. மறு புறம், பல தளங்களை உபயோகிக்கும் வாசுவின் நிகழ்வு, பாலிமீடியா செயல்படுவெதன் தெளிவான எடுத்துக்காட்டு. இந்த அனைத்து நிகழ்வுகளிலும், பணி மற்றும் பணியல்லாதவைகளின் எல்லைகளை மத்தியஸ்தம் செய்தல் தெளிவாக இருந்தது. 
ஆயினும், 'பணி - பணியல்லாதவை' ஆகிய இரு கூறுகள் எப்பொழுதும் பணியாளர்கள் மூலமாக மட்டுமே மீறப்பட்டது என்று சுட்டிக் காட்டுவது ஆசைகாட்டும் விஷயமாக இருந்தாலும், இது உண்மை இல்லை. நாம் கருத்தில் கொண்டிருக்கும் பணி அமைப்பு முரண்பாடுகெள் நிறைந்தது. இது ஒரு குறிப்பிட்ட பழக்கத்தை தடை செய்வது மற்றும் முறைப்படுத்துவது போல் தோற்றமளிக்கிறது, ஆனால் அதே நேரத்தில் ஒப்பீட்டளவில் எளிதாக தடை செய்யப்பட்டதை மீற போதுமான சந்தர்ப்பங்களை அளிக்கிறது.

அறிமுகத்திற்குத் திரும்பிச் செல்வோமானால், பணி மற்றும் பணியல்லாதவை என்ற தெளிவான இரு கூறுகளை ஒப்புக்கொள்ள மானுடவியலின் மறுப்பு பஞ்சகிராமி போன்ற இடங்களைப் பற்றிய ஆய்வுகளின் அடிப்படையிலேயே அமைந்துள்ளது என நாம் காணலாம். இம்மாதிரியான இடங்களில் பணி மற்றும் பணியல்லாதவைகளுக்கிடையே சாதாரண மற்றும் உறுதியான பிரிவுகள் எப்போதுமே இருந்ததில்லை என்பதை அடையாளம் காணுதல் அவசியமானது; எங்கு இநந் எல்லைகள் சுமத்தப்படுகின்றனவோ, அங்கு அவைகள் அடிக்கடி மீறப்படுகின்றன. எனினும் சமூக ஊடகத்தின் எழுச்சி இந்த விஷயத்தில் வெறுமனே பேசுவதை விட அதிகம் செயலாற்றி இருக்கிறது, இது இந்த அத்தியாயத்தில் விளக்கப்பட்ட கதைகளிலிருந்து தெளிவாகிறது. அவைகள் பணிக்குள்ளே, பணிக்கு வெளியே மற்றும் பணி மற்றும் பணியல்லாதவைகளுக்கு இடையே இடைவினையில் வளத்தைக் காண்பிக்கின்றன, இவை சில விதங்களில் முன்னுதாரணம் இல்லாதவையாக இருக்கலாம். 


\section{பரந்த உலகம்: அறிவார்ந்த பொருளாதாரத்தில் சமூக ஊடகங்கள் மற்றும் கல்வி}

\section{ரஞ்சித்தின் வாழ்வில் ஓர் நாள்}

ரஞ்சித் 15 வயதானவர். உயர்-மத்தியதர வகுப்பு பின்னணியில் இருந்து வருபவர். அவர் ஒரு சர்வதேச பள்ளியில் பயில்பவர்.

\section{புதன்கிழமை}

காலை 6 மணி - அலாரம் அடிக்கிறது....ஒத்திப்போடு..... இன்னொரு 5 நிமிட தூக்கம்.... அம்மா உச்சஸ்தாயியில் கத்துவது கேட்கிறது..... ஆனால் மீ்டும் தூங்கு...... இதெல்லாமே ஒரு கனவு...... 5 நிமிடங்கள் கழித்து சம்விராட்டிடம் இருந்து கூடைப்பந்து பயிற்சி காலை 7 மணிக்கு தானா என்று ஆங்கில கட்டுரை கொடுக்கவேண்டியது 11 மணிக்கு தானா என்றும் கேட்டு வரும் வாட்சப் தகவலுடன் எழுகை. கெட்டவார்த்தை பிரயோகம். பொருத்தமான ஸ்மைலி முகம் இட்டு வாட்சப் தகவல் அனுப்பு. [இங்கு ரஞ்சித் காலை முதல்வேலையாக மொபைல் போன் உபயோகிப்பதை பார்த்து அவனது தாயாரின் கத்தல்].

காலை 6:15முதல் - 6:45 வரை - தன்னைத்தானே சபித்துக்கொண்டு, பல் துலக்கும் போது அன்று கொடுக்கவேண்டிய கட்டுரையை ஆங்கில ஆசிரியர் மன்னிக்க வேண்டும் என்று 
பிரார்தனை. அரைத்தூக்கத்தில் காபி குடித்தல் - நடுநிசி 2 மணி வரை விழித்திருந்துவிட்டு தூங்கப்போயிருக்கக்கூடாது என்று அம்மாவின் உச்சஸ்தாயி கத்தல் கேட்கிறது. அவர் கூறுவது சரியே என்றுணர்ந்து, இரவு 10மணிக்கு மேல், கேமிங்கோ சாட்டிங்கோ கூடாது என்று தனக்குத்தானே உறுதியளித்தல். காலைக்கடன்களை முடித்தல். அவசர முகநூல் நோட்டம்....சௌம்யா தனது புது நாய்க்குட்டியுடன் இருக்கும் படத்திற்கு விருப்புத்தெரிவித்தல்....வாட்சப் நோட்டம்.... தானும் ஆங்கில வீட்டுப்பாடத்தை முடிக்கவில்லை என்ற சம்விராட்-டின் தகவலைப்பார்த்து மகிழ்தல்.....இந்த சமயம் முழுவதும் கார்ன்பிளேக்ஸ் உண்ணல்.....கூடைப்பந்து பயிற்சிக்கு ஓட்டம்.

காலை 7 முதல் 8 மணிவரை - கூடைப்பந்து.......மற்றவர்கள் விளையாடுவதை படமெடுத்து முகநூலில் பதிவிடல். பயிற்சியாளர் ஸ்மார்ட்போனை இகழ்கிறார். அடுத்தமுறை அதை வீட்டில் விட்டுவிட்டு வருவதாக பயிற்சியாளருக்கு வாக்குறுதி. பதிவை நோட்டம் விட்டு ஏற்கனவே 10 விருப்புகள் தெரிவிக்கப்பட்டிருப்பதை பார்த்தல்.

காலை 8 மணி - போனை விடுவதற்காக வீட்டிற்கு ஓட்டம்....அவசரக் குளியல்.....வாட்சப் நோட்டம்.....வகுப்பில் சிலர் மட்டுமே கட்டுரை இன்று கொடுக்கப்படவேண்டும் என்று ஞாபகம் வைத்திருப்பதை அறிந்து மகிழ்தல்....சம்விராட்டின் வாட்சப்பில் வகுப்புத்தோழர்களுடனான அவசர வீட்டுப்பாட நிலை சோதித்தலை பாராட்டுதல்....முகநூல் நோட்டம்.... கூடைப்பந்து பதிவிற்கு ஏற்கனவே 12 விருப்புகள். அநேகமாக அனைவரும் பள்ளி சென்றுகொண்டிருப்பர்.....பள்ளியில் மொபைல் போன்களுக்கு அனுமதியில்லை.....ஆகையால், அநேகமாக இந்தப்பதிவிற்கு அதிக விருப்புகள் மாலையில் கிடைக்கலாம்.

காலை 8:30 மணி - பள்ளி துவங்குகிறது - இயற்பியல் வகுப்பு...... 15 நிமிட விரிவுரையை தொடர்ந்து, யூட்யூப் காணொளியில் உள்ள டிஸ்கவரி சேனலின் ஆவணப்பட திரையிடல்......ஸ்மார்ட் வகுப்பறைகள் அற்புதம்.

காலை 9:15 மணி - கணித வகுப்பு - கால்குலஸ் தீர்வுகாணல்.....கான் அகாடமியில்' அளிக்கப்பட தேவையான பணிக்கடத்தை அனைவரும் பார்த்திருக்கிறோமா என்று ஆசிரியர் சோதித்தல்.

காலை 10 மணி - இடைவேளை - அனைவரிடமும் கட்டுரையைப்பற்றிய விரைவுச்சோதனை. அவர்களுக்கு முகநூல் படத்தைப்பற்றியும் தெரியப்படுத்துதல். 
காலை 11 மணி - ஆங்கில வகுப்பு - பெரும்பாலானோர் கட்டுரையை முடித்திராததால், ஆசிரியர் கட்டுரையின்மையை மன்னித்துவிட்டார். கட்டுரை வரையச்சொல்லி ஆசிரியர் கூறிய கணினி கூடத்திற்கு ஓட்டம். வகுப்புத்தோழன் விஜய் திருட்டுத்தனமாக மின்னஞ்சல் பார்க்க முயலுகிறான்....... இணையப்பாதுகாப்பு சுவர் அதை தடுக்கிறது........கணினி கூட ஒருங்கிணைப்பாளர் அவன் செயலைப் பார்த்து நுழைவுரிமையை தடுத்திருக்க வேண்டும்.

\section{மதியம் - மதிய உணவு - நண்பர்களுடன் அரட்டை}

மதியம் 12:45 மணி - உயிரியல் மற்றும் வேதியல் வகுப்புகளுக்கு செல்லுதல்.

மதியம் 2:30மணி - கணினிக்கூட வேளை. C ப்ரோக்ராம் எழுதுதல். $\quad$ C பழக்கவரிசைகளுக்கான (ருடீன்களுக்கான) கூகுள் தேடல்..... நிகழ்நிலை C வகுப்புகளை அணுகி இந்த பழக்கவரிசையின் (ருடீன்) கூறுகளை தேடுதல்......முகநூலை அணுக முயற்சி......வலைத்தளம் தடுக்கப்பட்டுள்ளது....யூட்யூபை அணுகி C பாடங்களை தேடுதெல்.

மாலை 3:15 மணி - வீட்டிற்கு செல்லுதல்.......வாட்சப் நோட்டம்.....முகநூல் நோட்டம்......14 விருப்புகள் மட்டுமே கிடைத்துள்ளன.....விரைவான சிற்றுண்டியுடன் கேமிங் துவக்கம்.

மாலை 4 முதல் 7:30 மணி வரை - நிகழ்நிலை கேமிங்..... வாட்சப் மற்றும் முகநூல் நோட்டம்........30 விருப்புகள்.... அற்புதம்......அம்மா வீட்டிற்கு வந்தாயிற்று......படிப்பை கவனிக்கச்சொல்லி கத்துதல்.

இரவு 8 மணி - பிளிப்க்கார்டில் 2 பூச்சாடி வாங்குவதில் உதவக்கோரி அம்மாவின் அழைப்பு......யூட்யூப் பார்த்தல்.... வாட்சப் மற்றும் முகநூலை நோட்டம் விட்டுக்கொண்டிருத்தல்.....தந்தை பணியிலிருந்து வீட்டிற்கு வருதல். இரவு உணவு உண்ணுதல்....தந்தைக்கு சில ஐபாட் இடுவுகளில் உதவுதல்.

இரவு 9 மணி - ஆங்கிலக்கட்டுரைக்காக நிகழ்நிலையில் ஆராய்தல்.....இறுதித்தேதி மறுநாளைக்கு நிச்சயிக்கப்பட்டிருக்கிறது. அதிதி மற்றும் சம்விராட்டுக்கு அவர்கள் முடித்துவிட்டார்களா என்று அறிந்துகொள்ள தகவல் அனுப்புதல்.....அனைவரும் நிகழ்நிலையில் இருக்கிறார்கள்.... 
கட்டுரையை எழுதும்/டைப் செய்யும் செயல்பாட்டில்..... சம்விராட்டை கட்டுரையை பற்றி பேசுவதற்காக அழைத்துவிட்டு இறுதியில் கூடைப்பந்து பற்றி அரட்டையடித்தல்.

இரவு 11 மணி - கட்டுரையை முடித்தல்....அதை பள்ளியின் நிகழ்நிலை பணிக்கட அமைப்பில் பதிவேற்றுதல்.....நிகழ்நிலை கேமிங் துவக்கம்....வாட்சப்பிலும் முகநூலிலும் மாறி மாறி நோட்டம் விடுதல்.

இரவு 11:30 மணி - தந்தை படுக்கையறையில் எட்டிப்பார்த்தல், தூங்கும் நேரம் ஆகிவிட்டது என்று கூறுதல்.

இரவு 11:45 மணி - இந்தமுறை அம்மா எட்டிப்பார்த்து தூங்கும் நேரமாகிவிட்டது என்று கத்துதல்.

நள்ளிரவு 00:30 மணி - அதிதிக்கு வாட்சப் குரல்வழி தகவல் பரிமாற்றத்தின் மூலம் நல்லிரவு முத்தங்களை அனுப்பியபின், இறுதியில் நேரத்தோடு உறங்க இயலுதல்.

\section{பாண்டியனின் வாழ்வில் ஓர் நாள்}

பாண்டியன் 15 வயதானவர். கீழ்மட்ட சமூகப்பொருளாதார பின்னணியில் இருந்து வருபவர். அவர் உள்ளூரில் ஒரு தனியார் பள்ளியில் பயிலுகிறார்.

\section{புதன்கிழமை}

அதிகாலை 4:30 மணி - துயிலெழுதல்......தமக்கையின் குரல் இது எழுவதற்கான நேரம் என்று கூறுகிறது....விரைந்து சென்று பல்துலக்கி காலைக்கடன்களை முடித்தல். நெல் வயலுக்கு செல்லவேண்டும்.....அங்கு மொபைல் சிக்னல் சுலபமாக கிடைக்கும். முகநூல் நோட்டம். வினோத்தும் சூரஜும் நேற்று எடுத்த படங்களை பதிவேற்றியிருக்கிறார்கள்.....அவற்றிற்கு விருப்பு தெரிவித்து கருத்துப் பதிவு செய்தல். ஏதேனும் புதிய நட்புதொடர்பு கோரிக்கைகள் வந்திருக்கிறதா என்று பார்த்தல். அரைமணி நேரம் ஆகப்போகிறது. மாட்டுக் கொட்டிலுக்கு ஓட வேண்டும்.

காலை 5 முதல் 7 மணி வரை - பால் கறப்பது, நெல் வயலில் வேலை செய்வது.

காலை 7 மணி - முகநூல் நோட்டம்.....தீபக்கும் கருத்து பதிவு செய்து விருப்பு தெரிவித்திருக்கிறான்..... பதிலுக்கு தீபக்கின் கருத்துக்கு விருப்பு தெரிவிக்கலாம்.....நண்பர்களின் 
ஊடகப்பக்கங்களில் உலாவல். தேநீர் அருந்தி விட்டு, காலை உணவுக்கு அரிசிக்கஞ்சி உண்ணுதல்.

காலை 7:30 மணி - விரைவாகக் குளித்துவிட்டு பள்ளிசெல்வதற்கு பேரூந்தை பிடிக்க ஓட்டம். பள்ளிக்கு செல்லும் வழியில் முகநூல் நோட்டம். நடிகர் விஜய்யின் “செல்பி புள்ள” பாடல் மற்றும் வேறுசில தமிழ்திரைப்படப் பாடல்களை கேட்டல். இன்னும் பல பாடல்களை பதிவிறக்க வேண்டும். இன்று மாலை புதிய தரவுத் தொகுப்பு வாங்கவேண்டும் என்பதை நினைவில் வைத்துக்கொள்ள வேண்டும்.

காலை 8:10 மணி - பள்ளிக்கு வந்து சேர்தல். சூரஜ் மற்றும் வினோத்துடன் அவர்களின் முகநூல் படங்கள் பற்றிய பேச்சு. வினோத்தின் மொபைல் போனில் புதிய பாடலைக் கேட்டல்.

காலை 8:30 முதல் 11 மணி வரை - தமிழ், ஆங்கிலம் மற்றும் கணித வகுப்புகள்.....கரும்பலகையில் உள்ள குறிப்புகளை வேகமாக எழுதிக்கொள்ள வேண்டும். ஆங்கில ஆசிரியர் அவற்றை கரும்பலகையில் இருந்து வெகு வேகமாக அழித்துவிடுவார்......கணித வகுப்பின் இறுதியில், வீட்டுப்பாட நோட்டுப்புத்தகத்தை சமர்ப்பிக்க வேண்டும்.

காலை 11:00 மணி - இடைவேளை - வினோத்தின் மொபைல் போனில் இருந்து சில புதிய பாடல்களை மாற்றிக்கொள்ளல்.

காலை 11:15 மணி - உடற்பயிற்சி கல்வி வகுப்பு - ஆசிரியரின் புதிய திரைப்பாடல்கள் இருக்கின்றனவா என்ற கேள்வி.....வினோத் புதிய பாடல்களை மாற்றுதல்.....இணையத்தில் இருந்து பதிவிறக்கப்பட்ட புதிய தமிழ்ப் படங்கள் இருக்கின்றனவா என்ற கேள்வி.....சூரஜின் போனில் இருந்து அவற்றை மாற்றுதல்....கால்பந்து விளையாட்டு....மதிய உணவு.

மதியம் 1:50 மணி - கம்ப்யூட்டர் சயின்ஸ் வகுப்பு. கம்ப்யூட்டர் சயின்ஸ் ஆசிரியர், கரும்பலகையில் எழுதப்பட்டுள்ள ஒரு கம்ப்யூட்டர் ப்ரோக்ராமை, அனைவரையும் எழுதிக்கொள்ள சொல்கிறார்.....கணினி கூடத்திற்கு செல்லுதல். ப்ரோக்ராமை இயக்கிப் பார்த்தல். விடை கிடைத்தல். நண்பர்களுடன் அரட்டை.....தீபக், ஒரு உடைந்த கம்ப்யூட்டரை சரிசெய்வதற்கு ஆசிரியருக்கு உதவுதல்.

மாலை 3:30 மணி - சாலையோரத்தில் உள்ள பங்க் கடை போன்ற மொபைல் போன் கடைக்கு சூரஜுடன் சென்று, ரூ. 15/- ற்கு, தரவுத் திட்டம் புதுப்பித்தல். கூடுதல் ரூ. 5/-ற்கு புதிய பாடல்களை போனில் ஏற்றிக்கொள்ளுதல்.

மாலை 5:00 மணி - விரைவான தேநீர்....நெல் வயலுக்கு ஓட்டம்....மாடுகளைக் கட்டுதல்...புதிய பாடல்களைக் கேட்டல்.... 
முகநூல் நோட்டம்....கூகுளில் இருந்து பதிவிறக்கிய தமிழ்த்திரைப்பட நடிகர் விஜய்யின் படத்தை பதிவிட்டால்....என்றும்-கைவிடா நண்பன் சூரஜ், எப்போதும்போல் முதல் விருப்பு தெரிவித்தல்.

மாலை 7:00 மணி - வீட்டுப்பாடம் செய்தல்......முகநூல் நோட்டம்.

இரவு 8:00 மணி - இரவு உணவு உண்ணுதல்....அன்னையையும் தமக்கையையும் நண்பன் சூரஜிடமிருந்து மாற்றிக்கொண்ட புதிய தமிழ்ப்படம் பார்ப்பதற்க்கு அழைத்தல். திரைப்படத்தை போனில் இருந்து ஒன்றுவிட்ட சகோதரனிடமிருந்து கடன் வாங்கிய அரசு அளித்த மடிக்கணினிக்கு மாற்றுதல். அன்னை மற்றும் தமக்கை, படத்தைப் பெறுவதில் மட்டுமல்லாமல் அதை சுலபமாக மாற்றுவதிலும் காட்டிய தொழில்நுட்ப நிபுணத்துவம் மற்றும் ஞானத்தைப் பாராட்டல்.

இரவு 10:30 மணி - திரைப்படம் நிறைவுறல்....மாட்டுக்கொட்டிலில், பசுக்கள் சரியாக இருக்கின்றனவா என்று சோதித்தல்....மீண்டும் முகநூல் நோட்டம்.

இரவு 11:00 மணி - உறக்கம்.

அவற்றிலேயே, முற்றிலும் மாறுபட்ட இந்த இரண்டு வழக்குகளும், இரண்டு 15 வயது பள்ளிக்குழந்தைகளின் அன்றாட வாழ்வையும் அவர்களின் சமூக ஊடகத்தொடர்பையும், எடுத்துரைப்பதாக இருக்கின்றன. இருப்பினும், இவை, இரு வேறு சமூகங்களில் நிகழும் இரண்டு முற்றிலும் மாறுபட்ட காட்சிகள் அல்ல. இவை, பஞ்சக்கிராமியில் ஒன்றுக்கொன்று அருகிலேயே நடப்பவை. இவற்றிலிருந்து நாம் என்ன அறிந்துகொள்ளலாம் என்று பார்ப்பதற்காக, நாம் ஓரடி தள்ளி நின்று, ஒரே பகுதியில் இந்த இரண்டு பதிப்புரு காட்சிகளையும் நடக்க உதவும் பேரளவிலான சமூக கட்டமைப்புகளை பின்னணியாகக் கருதவேண்டும். இந்த அத்தியாயத்தின் துல்லியமான பணியே அதுதான்.

\section{அறிமுகவுரை}

இந்த வரிசையில் உள்ள ஒவ்வொரு புத்தகமும், ஆறாம் அத்தியாயத்தை போன்றதான தலைப்பைக் கொண்டிருக்கிறது. இவை ஒரு பொதுவான குறிக்கோளை குறிப்பிடுகிறது. இந்த வரிசையில் உள்ள இந்த அத்தியாயங்களின் பணி, எவ்வாறு பெரிய சமூக கட்டமைப்புகளும் உட்கட்டமைப்புகளும் சமூக ஊடகங்களின் மீது பாதிப்பை ஏற்படுத்துகின்றன 
அல்லது சமூக ஊடகங்களால் மாற்றப்படுகின்றன என்பதை அலசுவது தான். ஒருசில வழக்குகளில், நூலாசிரியர்கள், இதுபோன்ற பல கட்டமைப்புகளை கருதியுள்ளனர். அரசியல், அரசாங்கம், சமயம் மற்றும் வணிகம் 5 போன்றவை அவற்றில் சில. ஆனால் பஞ்சக்கிராமியின் வழக்கில், அரசியல், இனம் போன்ற இந்த பரந்த விசாரங்களில் பல இந்த புத்தகம் முழுவதும் கலந்தாய்வு செய்யப்பட்டுள்ளன. அவற்றை மீண்டும் முழுவதுமாக கையாள்வது என்பது மிகவும் சிக்கலானதாகும். அதற்கு பதிலாக, இந்த அத்தியாயம், கல்வி என்ற ஒரு குறிப்பிட்ட சூழலைப் பற்றிய ஆழமான அலசலை அளிக்க தலைப்படுகிறது. கல்வி, பேரார்வம் என்ற பரந்த அரங்கையும், வளமை அல்லது குறைந்தபட்சம் பண்பார்ந்த வாழ்க்கைக்கான போராட்டத்தையும் தன்னுடன் கொண்டு வருகிறது. இது ஐ.டி துறையைச் சுற்றி வளர்ந்துள்ள குறிப்பிட்ட வேலை நடைமுறைகளுக்கு மிகவும் பொருத்தமாக இருப்பதால், இது முந்தைய அத்தியாயத்தின் கருப்பொருளில் இருந்து இயல்பாகத் தொடர்கிறது. இந்த ஒற்றைக்களத்தில் குவியம் வைப்பதன் மூலம், பரந்த அளவிலான அணுகுமுறை அனுமதிக்காத ஆழமான அலசலை அளிப்பது சாத்தியமாகிறது.

கல்விக்கான உட்கட்டமைப்புகளின் வளர்ச்சி, எவ்வாறு ஒருவகையில், இந்தப்பகுதி “அறிவார்ந்த பொருளாதாரமாக" கருதப்படுவதற்கான குறியீடாக மாறி, அனைத்து சமூகப்பொருளாதார வகுப்புகளுக்கு இடையேயும் பேரார்வத்தை தூண்டி, பதிலுக்கு இந்தப்பகுதியில் கல்விக்கான ஒதுக்கீட்டில் ஆக்கத் திரிபுகள் உண்டாக்குகிறது என்பதை பற்றிய கண்ணோட்டத்தை பின்வரும் பகுதிகள் அளிக்கின்றன. பின்னர் நாங்கள், இணையதள நுழைவுரிமை, சமூக ஊடகங்கள் மற்றும் மொபைல் போன்கள் ஆகியவை முக்கிய பங்கு வகிக்கக்கூடிய, பள்ளிகளில் தகவல்தொழில்நுட்ப சாதனங்களின் பயன்பாடுகள் பற்றி கருத்தாய்வு செய்கிறோம். அடுத்து, பஞ்சக்கிராமியில் உள்ள பல்வேறு பள்ளி அமைப்புகள், சமூக ஊடகங்களை எவ்வாறு புரிந்துகொள்கின்றன என்பதை ஆராய்ந்துவிட்டு ஆசிரியர்கள், மாணவர்களுடன் சமூக ஊடகங்களில் நண்பர்களாக ஆவது பற்றிய கலந்தாய்வுக்கு நகர்கிறோம். இறுதியில் இந்த அத்தியாயம், ஒருசில வளமான பள்ளிகள் எவ்வாறு, தங்கள் மாணவர்கள் பிரபலமான சமூக ஊடகத்தளங்களை அணுகுவதை தவிர்ப்பதற்காக தங்களின் சொந்த சமூக ஊடகத்தளத்தை உருவாக்குகின்றன என்பது பற்றிய வழக்காய்வுடன் நிறைவுபெறுகிறது. 
கல்வி மீதான மற்ற சமூகப்பொருளாதார காரணிகளின் பாதிப்பும் விவாதிக்கப்படுகிறது. மேற்கூறிய இரண்டு வழக்குகளிலும் கொடுக்கப்பட்டுள்ளது போல, பேரளவிலான கட்டமைப்புகள் பின்னணியில் இருந்து செயல்பட்டுக்கொண்டு, சமூூக ஊடகங்களின் வெளித்தோற்றத்தில் மாறுபடும் அன்றாட உபயோகத்தை பாதிக்கிறது என்பதை எடுத்துக்காட்ட ஒரு முயற்சி செய்யப்பட்டிருக்கிறது.

\section{அறிவார்ந்த பொருளாதாரம்: பேரார்வங்களுக்கு ஊக்கமளிக்கும் ஒரு அடையாளம்}

ஆராய்ச்சியாளர்கள், அறிவார்ந்த பொருளாதாரம் இந்தியாவிற்கு7 பொருந்துமா என்று விவாதித்துக் கொண்டிருக்கும் வேளையில், இந்திய அரசாங்கம் இந்த எண்ணத்தை ஆவலுடன் தத்தெடுத்துக் கொண்டது. இந்தக் கோட்பாடு, பஞ்சக்கிராமியின் உள்ளூர் அரசாங்கம் மற்றும் இந்தப் பிராந்தியத்தின் தனியார் ஐ.டி நிறுவனங்கள், இந்தப் பகுதி அறிவார்ந்த பொருளாதாரம் என்னும் தோற்றத்தை முன்னிறுத்தவேண்டும் என்பதை உறுதிசெய்வதற்காக பத்தாண்டுகளுக்கும் மேலாக எவ்வாறெல்லாம் போராடியிருக்கின்றன என்பதிலும் பிரதிபலிக்கப்படுகிறது. அவர்கள், ஐ.டி நிறுவனங்களை அமைத்து, திறன் வாய்ந்த மற்றும் கல்வியறிவுள்ள பணியாளர்களை கவர்வதிலும் வெற்றிபெற்றுள்ளனர். அவர்கள், தங்களது நோக்கத்தில் ஓரளவு வெற்றியும் பெற்றுள்ளனர், ஏனெனில், வேலையாட்களும் இந்தப் பகுதியின் மீது இதுபோன்ற அடையாளத்தை சுமத்துகிறார்கள். பஞ்சக்கிராமியின் குடியிருப்பாளர், அறிவார்ந்த பொருளாதாரம் என்னும் சொற்கூற்றை புரிந்துகொள்ள விட்டாலும், இந்தப் பகுதி, அறிவுஞானத்தை அனைத்திற்கும் மேலாக மதிக்கும் ஒரு பொருளாதாரமாக மாறிவருகிறது என்பதை அவர் கண்டிப்பாக புரிந்து கொள்வார். இது அவர்களின் கல்வி தொடர்பான பேரார்வங்களிலும் ${ }^{8}$ பிரதிபலிக்கிறது.

முதலாம் அத்தியாயத்தில் பார்த்தபடி, ஒரு தகவல் தொழில்நுட்ப பூங்கா அமைத்தல், பல உட்கட்டமைப்பு மாறுதல்களை கொண்டுவந்தது. ஐ.டி நிறுவனங்களின் வளர்ந்து வரும் திறன்வாய்ந்த பணியாளர்களுக்கான தேவைகளை கல்வி நிறுவனங்கள் பூர்த்தி செய்வது அவற்றில் ஒன்று. பஞ்சக்கிராமியின் உள்ளும் அதைச்சுற்றியும், பள்ளி மற்றும் 
கல்லூரிகளின் எண்ணிக்கையில் எழுச்சி, மற்றவகையான விரிவாக்கங்களுடன் இணைந்து நடந்தது. அதிநவீன குடியிருப்புகள், வணிகவளாகங்கள் அமைத்தல் மற்றும் உள்வரும் திறன்வாய்ந்த மக்கட்தொகையின் தேவைகளை பூர்த்தி செய்யும் வகையிலான இதர இணைக்கப்பட்ட சேவைகள் போன்றவை இவற்றுள் அடக்கம். நீண்டகால பஞ்சக்கிராமி குடியிருப்பாளருக்கு, மடிக்கணினி மற்றும் மொபைல் போன்களுடன் சேர்ந்த இந்த அனைத்து மாறுதல்களும், ஞானம் மற்றும் திறன் ஆகியவற்றில் அதீத மதிப்பு அளிக்கும் பொருளாதாரத்திற்கான அடையாளமாக தெரிந்தன.

பஞ்சக்கிராமியில் உள்ள கீழ்மட்ட சமூகப்பொருளாதார வகுப்புகளுக்கு, ஐ.டி என்ற வார்த்தை, வளமை என்பதற்கான இணைச்சொல் ஆகிவிட்டது. அவர்கள் ஐ.டி என்பதை, கல்வி மற்றும் அறிவுத்திறன் ஆகியவற்றின் மூலம் மட்டுமே அடையக்கூடிய வளமை மற்றும் வளர்ச்சியின் அடையாளமான, அச்சுறுத்தும் கட்டுமான அமைப்புகளைக் கொண்ட கணினிகள் உள்ள மாபெரும் கட்டிடங்களாகத் தான் பார்க்கிறார்கள். ஆகவே, கீழ்மட்ட சமூகப்பொருளாதார வகுப்பினருக்கு, ஐ.டி நிறுவனங்களில் பணிபுரிவது என்பது, நேரடியாக சமூக இயங்குதிறன் என்று அர்த்தமாகிறது. இந்த இயங்குதிறனுக்கான மக்களின் பேரார்வம், இந்தப்பகுதியின் கட்டுமான அமைப்புகளால் மட்டுமல்லாமல், இந்தப்பகுதியின் புதிய வளஆதாரங்களின் உபயோகத்தாலும் (உயர்மட்ட வீடுகள், விலையுயர்ந்த உணவகங்கள், புதிய ஸ்மார்ட்போன்கள் போன்றவை) இங்கு புதிதாக குடியேறியிருக்கும் ${ }^{10}$ கல்வியறிவு பெற்ற மத்தியதர வகுப்பைச்சேர்ந்த மக்கட்தொகையால் அன்றாடம் மறு உறுதிசெய்யப்படுகிறது.

கீழ்மட்ட சமூகப்பொருளாதார வகுப்பைச்சேர்ந்த பெற்றோர்கள், சமூக இயங்குதிறன் பற்றிய இந்த பேரார்வத்தை தங்கள் பிள்ளைகள் மூலம் அடைய முயலுகின்றனர். இதற்காக அவர்கள், தாங்கள் அறிவுத்திறனின் அடையாளமாகக் கருதும் கணினிகளை தங்கள் பிள்ளைகளுக்கு வாங்கித்தந்திருக்கின்றனர். இந்தக் கணினிகளின் மூலம், இணையதளம் மற்றும் முகநூல் போன்ற சமூக ஊடகத்தளங்களை அணுகுவது, சமூக இயங்குதிறனை அடைவதற்கான சரியான பாதையில் தங்கள் பிள்ளைகள் போவதற்கான மற்றொரு ஆதாரமாக பார்க்கிறார்கள். அடுத்த நிலை, தங்கள் பிள்ளைகளை சரியான பள்ளிக்கு அனுப்புவது தான். அவர்களை பொறுத்தவரை, சரியான பள்ளி என்பது, கணினிகள் மூலம் கல்வி பயிற்றுவிக்கும் பள்ளி தான். 
ராணி என்கிற 36 வயது பெண்மணி வீட்டு உதவியாளராக பணிபுரிபவர், பஞ்சக்கிராமியின் நீண்டகால குடியிருப்பாளர். இந்தப்பகுதி சந்தித்திருக்கும், மாபெரும் உட்கட்டமைப்பு மாற்றங்களின் அடையாளமாக விளங்கும், அடுக்குமாடி குடியிருப்பு வளாகங்களில் ஒன்றில் பணிபுரிகிறார். ராணியை பொறுத்தமட்டில், ஐ.டி என்பது, பிரம்மாண்டமான குளிரூட்டப்பட்ட அலுவலகங்களும், அவற்றில் பணிபுரியும், நன்கு படித்து, நாகரீக உடையணிந்து, நாள் முழுவதும் கணினியில் பணி செய்து, அதற்காக ஏராளமான சம்பளம் பெறும் மக்களும் தான். எட்டு வயதில் மூன்றாம் வகுப்பு படிக்கும் போதே, பள்ளியில் இருந்து விலகிய அவருக்கு, இப்போது ஒன்பதாம் வகுப்பில் இருக்கும் அவரது இரட்டையரான மகனும் மகளும், நன்கு படித்து எதிர்காலத்தில் ஐ.டி துறையில் நல்ல பணியில் அமரவேண்டும் என்பது லட்சியமாக இருக்கிறது. அவர் அவர்களுக்காக இரண்டாம் உபயோக மேசைக் கணினியும், அரசு அளித்த மடிக்கணினியும் ஒரு சில வருடங்களுக்கு முன் வாங்கித்தந்திருக்கிறார். இது அவர்களை கடினமாக உழைத்து நன்கு படிக்கத் தூண்டி, தனது இலட்சியத்தை நிறைவேற்ற உதவும் என்று அவர் நினைக்கிறார்.

கீழ்மட்ட சமூகப்பொருளாதார வகுப்பினரின் பேரார்வங்களைப் போலவே, உயர்-மத்தியதர வகுப்பை சேர்ந்த குடும்பங்களும் (பெரும்பாலும் இரட்டைவருமானமுள்ள ஐ.டி பணியாளர்கள்) தங்கள் பிள்ளைகளை, சரியான பள்ளிகளுக்கு அனுப்ப விழைகிறார்கள். இந்தக்குழுவினருக்கு, சரியான பள்ளி என்பது, தேசீய அல்லது சர்வதேச அளவிலான பாடத்திட்டம் கொண்ட, ஆங்கிலமுறையில் பயிற்றுவிக்கும் பள்ளிகள் தான். இந்தப்பகுதியில், இதுபோன்ற பள்ளிகளின் பெருக்கத்தினால், இந்தப் பள்ளிகள் தங்களை தனித்தன்மை உடையவையாக காட்டிக்கொள்வதற்காக எடுத்துக்காட்டும் ஒரு விஷயம் தான் ஸ்மார்ட் வகுப்பறைகள். அதாவது, இந்த வகுப்பறைகள், இணையதள தொடர்புடன், தகவல் தொழில்நுட்ப சாதனங்களைக் கொண்டு (ஐசிடி) பாடம் கற்பிக்கின்றன. அதுமட்டுமல்லாமல், அவர்களால் புதிய கல்வித்தளங்களான “கான் அகாடமி"11 மற்றும் "ஓபன் கோர்ஸ்வேர்"12 போன்றவற்றையும் யூட்ூபில் காணொளி ஆவணங்களையும் கூட அணுக முடியும்.

அஸ்வின் என்பவர் 41 வயதானவர். பன்னாட்டு ஐ.டி நிறுவனம் ஒன்றில் பணிபுரிபவர். நான்கு வருடங்களுக்கும் மேலாக, அவர் அமெரிக்காவின் அரிசோனா மாகாணத்தில் உள்ள அவரது வாடிக்கையாளர் ஒருவரின் அலுவலகத்தில் 
ஆலோசகராக பணிபுரிந்திருக்கிறார். அவர் பணிபுரியும் நிறுவனம், சில காலத்திற்கு அவர் இந்தியா வரவேண்டும் என்று கூறியதால், அவர் தனக்கென்று பஞ்சக்கிராமியில் ஒரு அடுக்குமாடி குடியிருப்பில் ஒரு வீடு வாங்கிக்கொண்டிருக்கிறார். அவருக்கு இந்தியாவில் அவரது நீண்டகால திட்டங்களை பற்றிய நிச்சயம் இல்லாததால் (நிறுவனத்தின் திட்டங்களையும் அவரது பணியின் போக்கையும் பொறுத்து, ஒரு சந்தர்ப்பத்தில் அவர் அமெரிக்காவுக்கு திரும்பவும் கூடும்) ஆறாவது வகுப்பில் படிக்கும் அவரது மகன், சர்வதேச பாடத்திட்டமுள்ள பள்ளிக்கு செல்லவேண்டும் என்று அவர் விரும்புகிறார். இது அவர் மகனுக்கு, இணையதள தொடர்பையும், பாடத்திட்டத்தில் ஐசிடி மீது போதுமான முக்கியத்துவத்தையும் கொண்டதாக இருக்கும். குடும்பம் மீண்டும் அமெரிக்கா திரும்புவதற்கு முடிவு செய்யும் பட்சத்தில், இது மிகவும் உதவிகரமானதாக இருக்கும் என்பது அஸ்வினின் கருத்து. அப்படியே அவர்கள் திரும்பிப்போகாவிட்டாலும், அவருடைய மகன் ஒரு உயர்தர பொறியியல் கல்லூரில் சேர விரும்பினால், இது அதற்கு மிகவும் உதவியாக இருக்கும்.

பஞ்சக்கிராமியில், ராணி மற்றும் அஸ்வின் போன்ற பலரைக் காண முடியும். இவர்கள் அனைவருமே, தங்கள் குழந்தைகளுக்கு பாதுகாப்பான எதிர்காலத்தை ஏற்படுத்திக் கொடுப்பதற்காக பெற்றோர் எவ்வாறெல்லாம் தங்கள் குழந்தைகளின் கல்வியை சாமர்த்தியமாக திட்டமிடுகிறார்கள் என்பதற்கான உதாரணங்கள். கீழ்-மத்தியதர வகுப்பில் இருந்து வரும் மற்றொரு வகையான பெற்றோர், மாநில அளவிலான பாடத்திட்டம் கொண்ட, ஆங்கில முறையில் பயிற்றுவிக்கக்கூடிய தனியார் பள்ளிகளுக்கு அனுப்புவதில் மகிழ்ச்சியடைகிறார்கள். அவ்வாறு செய்வதன் மூலம், அந்தக் குழந்தை நன்கு படித்து, பொறியியல், அறிவியல் அல்லது வணிகத்துறையில் பட்டம் பெற்று அதன் மூலமாக ஐ.டி துறையில் நுழையக்கூடும் என்பது அவர்களின் எண்ணம்.

ஆகவே, பெற்றோரின் பேரார்வங்கள், ஒரு பாணியில் அமைந்திருப்பதை பார்க்க முடிகிறது. குழந்தைகள் எந்தப்பள்ளியில் படித்தாலும், குடும்பம் எந்த சமூக வகுப்பை சேர்ந்ததாக இருந்தாலும், இந்தக் குழந்தைகளின் இலக்கு ஐ.டி துறையாகத்தான் தெரிகிறது. சுருக்கமாகச்சொன்னால், இதுபோன்ற பெற்றோர்களுக்கு, தங்கள் பிள்ளைகள் ஐ.டி துறையில் கௌரவமான பதவிகளில் சேர்வது என்பது, அவர்கள் வாழ்வில் ஒரு நல்ல நிலைமைக்கு வருவது என்பதுடன், சமூக இயங்குதிறனையும் சாதிப்பதாகும். இதை சாதி- 
ப்பதற்கு, இந்தக் குழந்தைகள் ஆங்கில பயிற்றுவிப்பு முறை உள்ள பள்ளிகளில் கல்வி பயில வேண்டும். இவை, தொழில்நுட்பங்களுக்கு முன்னுரிமை கொடுக்கும் (கற்கும் தளமாகவேனும்). இந்தப்பள்ளிகளில் உள்ள ஐசிடி-க்கள் ${ }^{13}$ இதை அடையாளப்படுத்துகின்றன.

\section{பஞ்சக்கிராமியில் பள்ளி அமைப்பு}

பஞ்சக்கிராமியில் உள்ள பள்ளிகள் ${ }^{14}$, மூன்று வெவ்வேறு அமைப்புகளை சேர்ந்தவையாக இருக்கின்றன. மாநில அளவிலான பாடத்திட்டத்தை பின்பற்றும் மாநிலப்பள்ளிகள் ${ }^{15}$; தேசீய அளவிலான பாடத்திட்டத்தை பின்பற்றும் சிபிஎஸ்இ பள்ளிகள் ${ }^{16}$ மற்றும் சர்வதேச பாடத்திட்டத்தை பின்பற்றும் ஐஜிசிஎஸ்இ பள்ளிகள் ${ }^{17}$. பஞ்சக்கிராமிக்கு உள்ளும் அதைச் சுற்றியும் இரண்டு சர்வதேசப் பள்ளிகள், அரை டஜன் சிபிஎஸ்இ பள்ளிகள் மற்றும் குறைந்தபட்சம் ஒரு டஜன் தனியார் மற்றும் அரசாங்கம் நடத்தும் மாநிலப்பள்ளிகள் ஆகியவற்றைக் காணலாம். சுலபமான புரிதலுக்காக, இந்த அத்தியாயம், "வளமான பள்ளிகள்", "குறைந்த வளமுள்ள பள்ளிகள்" போன்ற சொற்கூறுகளை, உண்மையான பள்ளி வாரியங்களுக்கு பதிலாக உபயோகிக்கும். பொதுவாக, வளமான பள்ளிகள் என்பவை, மத்தியதர மற்றும் உயர்-மத்தியதர வகுப்பைச்சேர்ந்த பிள்ளைகள் பயிலும் பள்ளிகளாகும். (அவை பெரும்பாலும் ஐஜிசிஎஸ்இ அல்லது சிபிஎஸ்இ பாடத்திட்டம் கொண்ட பள்ளிகளாக இருக்கும். மாநில பாடத்திட்டம் கொண்ட ஒருசில தனியார் பள்ளிகளும் சில சமயங்களில் இந்த வகையைச் சேர்ந்தவையாக இருக்கும்). குறைந்த வளமுள்ள பள்ளிகள் என்பவை, அரசாங்கம் மற்றும் தனியார் நடத்தும் மாநிலப்பள்ளிகளைக் குறிக்கும். இவற்றில் பிந்தையது, கீழ்-மத்தியதர வகுப்பு மற்றும் கீழ்மட்ட சமூகப்பொருளாதார வகுப்பைச்சேர்ந்த பிள்ளைகளின் கல்வித்தேவைகளை பூர்த்திசெய்வதாகவும், அதற்காக அவர்களின் வளமான எதிரிணையாளர்களை விட, மிகவும் குறைந்த அளவிலான கட்டணத்தை ${ }^{18}$ சுமத்துபவையாகவும் இருக்கிறது. இருப்பினும், இந்தப்பள்ளிகளில், உட்கட்டமைப்பு வசதிகள் பலசமயங்களில் குறைவாக இருக்கின்றன.

அனைத்து வளமான பள்ளிகளும் ஆங்கிலத்தை பயிற்றுவிக்கும் மொழியாக கொண்டிருக்கும்போது, குறைந்த வளமுள்ள மாநிலப்பள்ளிகள், தமிழ் அல்லது ஆங்கிலத்தை தங்கள் பயிற்றுவிக்கும் மொழிகளாக கொண்டிருக்கின்றன ${ }^{19}$. 
இருப்பினும், இந்தப்பகுதியில், ஆங்கிலக்கல்விக்கான ஆவல் 30 வருடங்களுக்கு முன்னரே நுழையதுவங்கி விட்டாலும், தமிழ் வழி கல்வியளிக்கும் பள்ளிகளை விட, ஆங்கில வழி கல்வியளிக்கும் பள்ளிகளுக்கான கிராக்கி ${ }^{20}$, பெருமளவிற்கு, ஐ.டி துறையின் பணியமர்த்தும் நடைமுறைகளால் உந்தப்பட்டவையாக இருக்கிறது. இங்கு முரண்பாடான விஷயம் என்னவென்றால், தமிழ் வழி கல்வி அளிக்கும் அரசுப்பள்ளிகளில் தொடக்கநிலை வகுப்புகளில் பயிலும் குழந்தைகளில் பெரும்பாலானோர், இந்தியாவின் பல்வேறு மாநிலங்களில் இருந்து, பஞ்சக்கிராமிக்கு கட்டுமானப்பணிகளுக்காக புலம்பெயர்ந்த ஏழைத் தொழிலாளிகளின் பிள்ளைகள் தான்.

பஞ்சக்கிராமியில் உள்ள வளமான மற்றும் குறைந்த வளமுள்ள பள்ளிகள் தங்கள் தகவல் தொழில்நுட்ப உட்கட்டமைப்புகளை விளம்பரப்படுத்தினாலும், இந்தப்பள்ளிகளுக்கிடையேயான குறிப்பிடத்தக்க வித்தியாசம், ஸ்மார்ட் வகுப்பறைகள் மற்றும் இணையதள தொடர்பு ஆகியவற்றின் மூலம் கோடிட்டுக் காட்டப்படுகிறது. இந்தக்காரணி, ஒரு பள்ளியின் பிராபல்யத்தை கூட்டவோ குறைக்கவோ செய்கிறது. பல வளமான பள்ளிகள் தங்கள் அதீதமான கட்டணங்களை, ஸ்மார்ட் வகுப்பறைகளை காரணம் காட்டி நியாயப்படுத்தினாலும், குறைந்த வளமுள்ள பள்ளிகள், கணினி கூடத்துடன் சமாளித்துக்கொள்கின்றன.

\section{பள்ளிகளில் ஐசிடி மற்றும் சமூக ஊடகங்கள்}

ஆச்சரியத்திற்கிடமின்றி, இந்தப்பகுதியில் உள்ள வளமான பள்ளிகள், மிகச்சிறந்த ஐசிடி மற்றும் ஸ்மார்ட் வகுப்பறை வசதிகளைக் கொண்டுள்ளன. அவர்களின் கணினிகளில் பல இ இணையதள தொடர்பு உள்ளவை. இ இந்தப்பள்ளிகளில் பின்பற்றப்படும் பிரபலமான வழிகாட்டும் முறை யூட்யூப் காணொளி ஆவணங்களை காட்டுவது. இங்குள்ள ஆசிரியர்கள் பயன்படுத்தும் மற்றொரு தோற்றுவாய், கான் அகாடமி ${ }^{21}$. கூகுள் தேடல்கள் மற்றும் கூகுள் படத்தொகுப்புகள் ஆகியவையும் வகுப்பின் செயல்திட்டங்கள் மற்றும் முன்னிலைப்படுத்தல்களுக்கு ஆராய்வதில் இங்குள்ள ஆசிரியர்களுக்கு கூடுதல் வழிமுறைகளாக உதவுகின்றன. இவை தவிர, இந்த வளமான பள்ளிகள், ஆசிரியர்களின் பொது அறையில், தடையற்ற இணையதள அணுகலுடனான சில கணினிகளையும் கொண்டிருக்கின்றன. சில பள்ளிகள், நடவடிக்கை 
அடிப்படையிலான செயல்திட்டங்களில் ஈடுபடும் சமயங்களில் அதற்கு தேவையானவற்றை பதிவிறக்குவதற்கு ஆசிரியரின் உதவியை நாடுமாறு மாணவர்களை ஊக்குவிக்கின்றன.

இருப்பினும், மாணவர்கள் என்று வரும்போது, பல வளமான பள்ளிகளில், இணையதள அணுகலை தடுக்கும், கடுமையான சட்டதிட்டங்கள் இடப்பபட்டிருக்கின்றன. இந்தப்பள்ளிகளில் அநேகமாக அனைத்துப்பள்ளிகளுமே தங்கள் கணினிகளில் சமூக ஊடக அணுகலை தடை செய்திருக்கின்றன. இங்கு ஐந்தாம் வகுப்பு மற்றும் அதற்கு மேற்பட்ட வகுப்பைச்சேர்ந்த குழந்தைகள், ஆசிரியர்கள் மற்றும் கணினிக்கூட பயிற்றுவிப்பாளர் ஆகியோரின் வழிகாட்டலில் இணையதளத்தை உபயோகிக்குமாறு ஊக்குவிக்கப்பட்டாலும், ஆசிரியர்கள், உயர்வகுப்பில் இருக்கும் ஒருசில கீழ்படியாத மாணவர்களை (வழக்கமாக பத்து அல்லது பதினோராம் வகுப்பை சேர்ந்தவர்கள்) அவர்கள் முன்பின் அறிந்திராத கட்டுப்படுத்தப்பட்ட வலைத்தளங்களை அணுக முயற்சிப்பதாக புகார் செய்கின்றனர். இணையதளம் மற்றும் சமூக ஊடகத்தளங்கள் 22 மீதான தங்கள் அணுகுமுறையை தொடர்ந்து ஆராய்ந்துகொண்டிருக்கும் இந்தப்பள்ளிகளுக்கு, இது மிகவும் புதிய விஷயம் என்பது இதிலிருந்து தெரிகிறது.

இந்த வளமான பள்ளிகள், இணையதள இயக்கத்துடன் கூடிய ஐசிடி உட்கட்டமைப்புடன் ஒரு உடன்படிக்கைக்கு வந்துகொண்டிருக்கும் அதே சமயத்தில், குழந்தைகளுக்கான பாதுகாப்பான இணையதள உலாவல் பழக்கங்கள் குறித்த நடைமுறை பாடங்கள் பற்றிய பெற்றோரின் கோரிக்கையையும் திருப்தி படுத்த முனைகின்றனர். இதற்கான ஒரு பகுதிக்காரணம், இந்தக்குழந்தைகள் பெரும்பாலும் பெற்றோர் இருவரும் பணியில் இருக்கும், இரட்டை வருமானமுள்ள குடும்பங்களை சேர்ந்தவர்கள். பெற்றோரில் ஒருவரேனும் வீடு திரும்பும் வரை, இவர்கள் வீட்டில் தனித்திருக்கிறார்கள். ஆசிரியர்கள் மற்றும் பெற்றோர்கள் இருசாராருமே, வீட்டில் தனிமையான சூழல், ஐபாட் அல்லது மடிக்கணினி போன்றவற்றின் இருப்பு, மற்றும் தடையற்ற இணையதள தொடர்பு ஆகியவற்றின் கூட்டு ஆபத்தான ஒன்று என்று கருதுகிறார்கள். ஆகவே பாதுகாப்பான இணையதள உலாவல் பற்றி குழந்தைகளுக்கு எடுத்துக்கூறுவது ஆசிரியர்களின் பொறுப்பாகிப் போனது. பெற்றோர், பெற்றோருக்குரிய கட்டுப்பாடுகளை விதித்தாலும், இதுபோன்ற கட்டுப்பாடுகளை சுற்றிச்செல்வது குழந்தைகளுக்கு கடினமானதல்ல என்பது அவர்களுக்கும் தெரியும். எனினும், பல பள்ளிகளில் இந்தப்பாடங்கள், ஒரே 
நாளில் பயிற்றுவிக்கப்படுவெது இல்லை. மாறாக, வகுப்பறைகளில் தொடர்ச்சியான முறையில் பயிற்றுவிக்கப்படுகிறது.

மாணவர்களுக்கு இணையதள உலாவல் பழக்கங்களைப்பற்றி அறிவுரைகூறவும் கையாளவும் அவர்களை தயார்படுத்தும் வகையில், இந்த வளமான பள்ளிகளில் சில, தங்களின் ஆசிரியர்களுக்கும் பாதுகாப்பான இணையதள உலாவல் பற்றிய வகுப்புகளை நடத்துகின்றன. தங்கள் பிள்ளைகளை குறிப்பாக 14 வயதுக்கு உட்பட்டவர்களை ${ }^{23}$ சமூக ஊடக வலைத்தளங்களில் குறிப்பாக முகநூலில் உறுப்பினர்கள் ஆவதற்கு அனுமதிக்கக்கூடாது என்று பெற்றோர்களுக்கு இந்த வளமான பள்ளிகள் வலியுறுத்துகின்றன. இவை முகநூலை ஒரு கவனச்சிதறலாக மட்டுமல்லாமல், பிள்ளைகள் தம்மை, சமூக விரோத சக்திகள் மற்றும் பள்ளியின் அடாவடிகளிடம் வெளிப்படுத்திக்கொள்ளும் சாத்தியக்கூறுள்ள ஒரு ஆபத்தான இடப்பரப்பாகவும் பார்க்கிறார்கள். எது எப்படியிருப்பினும், அவர்களின் மாணவர்களில் பலர் சமூக ஊடகங்களில் குறிப்பாக முகநூலில் 24 இருக்கிறார்கள் என்பது அவர்களுக்கு நிச்சயம். இந்த மாணவர்கள் முகநூலில் இருப்பதோடல்லாமல், அதிலுள்ள தங்களின் ஆசிரியர்களுடனும் நட்புதொடர்பு ஏற்படுத்திக் கொள்கிறார்கள். இருப்பினும், சமூக ஊடகங்கள் மற்றும் இணையதளம் பற்றிய இதுபோன்ற விசாரங்கள் வாய்வழி செய்தியாக தான் குறிப்பிடப்படுகெறதே தவிர, இந்தப்பள்ளிகளால், அதிகார பூர்வ நெறிப்படுத்தும் கொள்கைகளாக அறிவிக்கப்படுவெதில்லை. பல சமயங்களில், இந்த விசாரங்கள், பெற்றோரிடம், பெற்றோர்-ஆசிரியர் கழக கூட்டத்தில் தெரிவிக்கப்படுகின்றன.

இந்தப்பள்ளிகள், சமூக ஊடகங்களை பற்றி கொண்டுள்ள விசாரங்கள், அவர்கள் இணையதளம் மற்றும் சமூக ஊடகங்களை வகைப்படுத்துவதையும் பாதிக்கின்றன. ஆசிரியர்கள், யூட்யூப் மற்றும் இணையதளத்தை அறிவார்த்த ஆதாரமாக 25 பார்க்கிறார்கள். ஆனால் முகநூல் மற்றும் இதர சமூக ஊடகங்களை வித்தியாசப்படுத்துகிறார்கள். அவர்களை பொறுத்தவரை, இவை எந்தவொரு கல்விப்பயனும் அல்லாத வெறும் அற்பப்பொழுதுபோக்குக்கானவை என்பது எண்ணம். ஆகையால் இவை கவனச்சிதறல்கள் ${ }^{26}$ என்று ஒதுக்கப்படுகின்றன. இது போன்ற நம்பிக்கைகள், பள்ளிகளில் இருந்து இணையதளத்தை அணுகுவது பற்றிய விதிமுறைகளையும் பாதிக்கின்றன. இவை மிகவும் எளிதானவை. கூகுள் மற்றும் யூட்யூப் தவிர, வேறு வலைத்தளங்களை அனுமதிப்பதில்லை. யூட்யூப் காணொளிகளும் நாம் சாதாரணமாக அவற்றை 
பார்க்கும் போது நமக்களிக்கும் மற்ற காணொளிகளைப் பற்றிய ஆலோசனைகளை அளிப்பதில்லை. கூடுதலாக, இந்தப் பள்ளிகள் விளம்பரங்கள் எதுவும் வராமலும் தடுத்துவிடிகின்றன. பெற்றோருக்குரிய கட்டுப்பாடுகளும், மற்ற பாதுகாப்பு அம்சங்களும் பள்ளிகளின் கணினிக்கூடங்களில் நிறுவப்பட்டிருக்கின்றன. ஆனாலும், இவை மேலும் இறுக்கப்படுவது மதுவந்தியின் அனுபவம் போன்ற ஒன்று ஏற்பட்ட பிறகு தான்.

L. மதுவந்தி என்பவர் ஒரு வளமான பள்ளியில், 38 வயதான மேல்நிலைப்பள்ளி அறிவியல் ஆசிரியை. அவருடைய மாணவர்களுடனான ஒரு கணினிக்கூட அமர்வு வேளையின் போது, அவர், தனது மாணவர்கள் ஒரு யூட்யூப் ஆவணப்படத்தை பார்க்கவேண்டும் என்று விரும்பினார். ஆனால் ஒருசில மாணவர்கள் பள்ளிக் கணினிகளில் இருந்து முகநூலை பயன்படுத்துவதை கண்டறிந்தார். உடனே அவர், அந்த மாணவர்களின் நுழைவுரிமையை ரத்து செய்து அவர்களுக்கு அறிவுரை கூறலானார். இதுபோன்ற நுழைவுரிமைகளை தடுக்கும் இணைய பாதுகாப்புச்சுவர் எதுவும் பள்ளியில் இல்லாத காரணத்தினால் தான், மாணவர்கள் கணினிக்கூட அமர்வு வேளைகளில் முகநூல் போன்ற சமூக ஊடகத்தளங்களை அணுகுவதற்கு தூண்டப்படுகின்றனர் என்று அவர் கருதினார். இது போன்ற பல தொடர்ச்சியான புகார்களுக்கு பிறகே, பள்ளி நிர்வாகம், சில வலைத்தளங்களுக்கான நுழைவுரிமையை தடுக்கும் இணையப்பாதுகாப்பு சுவரை நிறுவியது. மேலும், இதுபோன்ற பாதுகாப்புகளை சுற்றிச்செல்லக்கூடிய மாணவர்கள் மீது ஒரு கவனிக்கும் கண் வைத்துக்கொள்வதற்காக தகுதியுள்ள கணினிக்கூட நிர்வாகியையும், பணியில் அமர்த்தியது. இது போன்ற பள்ளிகளில் உள்ள பல கணினிக்கூட பயிற்சியாளர்கள், தங்கள் கணினிகளில் உள்ள இணைய பாதுகாப்புச்சுவர் தடுப்புகள், சிலசமயம் தளர்வாக இருப்பதாக, குறிப்பாக அவற்றின் சமூக ஊடக நுழைவுரிமை கட்டுப்பாட்டில், ஒத்துக்கொண்டார்கள். இவை சரியாக இருக்கும்போதே, சில மாணவர்கள், அவற்றை எப்படி கடந்து செல்வது என்பதை அறிந்திருந்ததாக அவர்கள் கூறுகிறார்கள்.

மறுபுறம், குறைந்த வளமுடைய பள்ளிகளில் பல, தங்கள் கணினிகளுக்கு இணையதள தொடர்பு கொடுக்கவேயில்லை. அப்படியே கொடுத்திருந்தாலும், ஒன்றிரண்டு கணினிகளுக்கு மட்டுமே இணையதள தொடர்பு கொடுக்கப்பட்டிருக்கும். அவையும், கணினிக்கூட நிர்வாகி அல்லது பொறுப்பில் இருக்கும் ஆசிரியர் ஆகியோரின் கண்காணிப்பில் தான் வைக்கப்பட்டிருக்கும். 
இந்த குறைந்த வளமுடைய பள்ளிகளில் உள்ள மாணவர்களுக்கு, தொழில்நுட்ப சாதனங்களை வைத்திருப்பது என்பதே ஒருவித ஆடம்பரம் தான். அரசாங்கத்தின், "ஒரு மாணவருக்கு ஒரு மடிக்கணினி" என்ற திட்டத்தின் கீழ், கீழ்மட்ட சமூகப்பொருளாதார வகுப்புகளில் இருந்து வரும் மேல்நிலைப்பள்ளி மாணவர்கள் பலர் இப்போது மடிக்கணினி வைத்திருக்கிறார்கள். இந்த மாணவர்கள் இணையதள தொடர்பு இல்லாமலோ அல்லது, இணையத்தொடர்புள்ள மலிவான ஸ்மார்ட்போன்களில் இருந்து இணைய இணைப்பை கொடுத்தோ வைத்திருந்தனர். இருப்பினும், இது ஆண் மாணவர்களுக்கு ${ }^{27}$ மட்டுமே சாத்தியமாக இருந்தது. பெண் மாணவர்கள், சிறிது நேரத்திற்கு மட்டும் கடன் வாங்கிய இணையத்தொடர்புள்ள போன்களுடன் (பெரும்பாலும் அவர்களுடைய மூத்த சகோதரர்கள் அல்லது மற்ற ஆண் உறவினர்களிடம் இருந்து பெற்றவை) மடிக்கணினியை இணைத்து குறுகிய காலங்களுக்கு மட்டும் இணையதளத்தை பயன்படுத்திக்கொண்டனர். யுஎஸ்பி டாங்கிள் இணையத் தொடர்புகள், உடனடியாக இணையதள தொடர்பளிப்பதால் ${ }^{28}$, இப்போது அறிவுத்திறனின் அடையாளமாகவே மாறிவருகிறது. இருப்பினும் இது போன்ற சாதனங்களின் உபயோகத்திலும், பாலின அடிப்படையிலான கட்டுப்பாடுகள் தெளிவாக தெரிகின்றன. அத்தியாயம் 2-ல் விவாதித்தது போல, கீழ்மட்ட சமூகப்பொருளாதார வகுப்பைச்சேர்ந்த பெண் மாணவர்களுக்கான சமூக ஊடக நுழைவுரிமை, அவர்களின் மற்ற குடும்ப உறுப்பினர்களால், கட்டுப்படுத்தவோ அல்லது தடை செய்யவோ படுகிறது.

சமூக ஊடகங்கள், இந்த குறைந்த வளமுடைய பள்ளிகளில், அன்றாடம் கையாளப்படவேண்டிய விசாரமாக இல்லாததால், அவர்கள், இதை பெற்றோருடன் விவாதிக்கவே இல்லை. இதன் விளைவாக, இந்தக்குழந்தைகளின் பெற்றோர்கள், (குறிப்பாக ஆண் குழந்தைகள்) தங்கள் குழந்தைகளை சமூக ஊடகங்களை உபயோகிப்பதற்கு அனுமதிக்கிறார்கள். அவர்களைப்பொறுத்தவரை, சமூக ஊடகங்கள், அறிவார்ந்த பொருளாதாரத்தில் நிபுணத்துவம் தேவைப்படும் தொழில்நுட்ப முன்னேற்றத்தின் வெளிப்படையான அடையாளங்களில் ஒன்று.

வளமான பள்ளிகளில் உள்ள ஐசிடி வசதிகள், யூட்யூப் ஆவணப்படங்களை பார்ப்பதற்கு ஆவன செய்தாலும், இது ஏறத்தாழ குறைந்த வளமுடைய பள்ளிகளில் இணையதளத்துடன் இணைக்கப்படாத டிவிடி 29 பார்ப்பதற்கு சமமானதாகவே இருக்கிறது. பல குறைந்த வளமுள்ள பள்ளிகளில் உள்ள 
ஆசிரியர்களுக்கு கான் அகாடமி போன்ற திறவுநிலை ஆதாரவளங்களின் இருப்பு பற்றிய அறிதல் இல்லாவிட்டாலும், யூட்யூபில் கல்விக்கான கூறுகள் இருப்பது பற்றி அவர்கள் அறிந்தே இருந்தார்கள். ஆகவே அவர்கள் பல சமயங்களில், தங்கள் மாணவர்களை, மொபைல் போனில், யூட்யூபில் கல்விக் காணொளிகள் பார்ப்பதற்கு ஊக்குவித்தார்கள். இருப்பினும், இது அவர்கள் பாடத்திட்டத்தின் அங்கமாக இல்லாததால், வளமான பள்ளிகளைப் போல, யூட்யூப் தொடர்பான வீட்டுப்பாடங்களோ அல்லது பாட செயல்திட்டங்களோ இந்தப் பள்ளிகளில் கொடுக்கப்படவில்லை.

இதுபோன்ற குறைந்த வளமுள்ள பள்ளிகளில் உள்ள பல ஆசிரியர்கள், தங்கள் மாணவர்களுக்கும் இதுபோன்ற வாய்ப்புகள் கிடைத்தால், அவர்களும் சிறப்பாக செயல்படுவார்கள் என்று குறிப்பிட்டனர். இநந்த மனப்பாங்கு, சமூக ஊடகங்கள் பற்றிய அவர்களது கண்ணோட்டத்தையும் பாதிக்கும் வாய்ப்பிருக்கிறது. இந்தப் பள்ளிகளில் உள்ள பல ஆசிரியர்கள், சமூக ஊடகங்களுக்கும், பரந்த இணையதளத்திற்கும் இடையே உள்ள வித்தியாசத்தை உணரவில்லை என்பது தெளிவாக தெரிகிறது. அவர்கள் இவ்விரண்டையுமே, பொருளாதார சூழ்நிலைகளால், தங்கள் மாணவர்களுக்கு மறுக்கப்பட்ட அறிவுத்திறனை அலசுவதற்கான சமமான வாய்ப்பாகத்தான் பார்த்தார்கள். இது, இந்த ஆசிரியர்கள், மிகக்குறைந்த அளவிலான இணைய மற்றும் சமூக ஊடக ஞானமும் ஆற்றலும் கொண்ட மாணவர்களை, அது அவர்களின் பாடத்திட்டத்திற்கு தொடர்புடையதாக இல்லாவிட்டாலும், ஊக்குவிப்பதில், நடத்தும் விதத்திலும் தெளிவாகத் தெரிகிறது. இது வளமான பள்ளிகளின், கவனச்சிதறல் என்ற கருத்திற்கு முற்றிலும் மாறுபட்டதாக இருக்கிறது.

இந்த வித்தியாசம், ரஞ்சித் மற்றும் பாண்டியன் ஆகியோரின் வழக்காய்வுகளில் கண்கூடாகத் தெரிகிறது. அவர்களுடைய உடற்பயிற்சி நடவடிக்கை சமயங்களில், (ரஞ்சித்தின்) "பயிற்சியாளர்" மற்றும் (பாண்டியனின்) உடற்பயிற்சி ஆசிரியர் தத்தம் மாணவர்களின் கைகளில் ஸ்மார்ட்போன்களை பார்த்தபின், எதிர்வினைகள் முற்றலும் மாறுபட்டவையாக இருந்தன. பயிற்சியாளர், ரஞ்சித்தின் பயிற்சி வேளையில், இந்தப் போன் விளைவிக்கக்கூடிய கவனச்சிதறலை சுட்டிக்காட்டி முகம் சுளிக்கிறார். ஆனால் அதுவே, பாண்டியன் பயிலும் உள்ளூர் பள்ளியின் ஆசிரியர், அவனிடமே புதிய திரைப்பட பாடல்களை கேட்கிறார். அவர்களுடைய எதிர்வினைகள் வெவ்வேறாக இருந்தாலும், இருவருமே 
இதுபோன்ற சாதனங்களின், ஊடுருவும் இயல்பையும், சமூக ஊடகங்கள் தங்கள் மாணவர்கள் மீது கொண்டுள்ள தாக்கத்தையும் ஒத்துக்கொள்வதாக தெரிகிறது.

\section{மொபைல் தளங்களில் சமூக ஊடகங்களை உபயோகித்தல்}

பெரும்பாலான பள்ளி மாணவர்கள், சமூக ஊடகங்களை, பல்வேறு சாதனங்களின் மூலம் உபயோகித்தாலும் (அவர்களுடைய சமூகப்பொருளாதார பின்னணிக்கு ஏ ஏற்றவாறு), சமூக ஊடகங்களையும், இணையதளத்தையும் அணுகுவதற்கான பிரபலமான ஊடகமாக, ஸ்மார்ட்போன்கள் இருப்பதாக காணப்படுகிறது.

பஞ்சக்கிராமியில் உள்ள அனைத்து பள்ளிகளிலும், அவரவர் பள்ளி வளாகங்களில், கடுமையான "மொபைல் போன் கூடாது" என்ற கொள்கை இருக்கிறது. இதனால், மாணவர்கள், பள்ளிகளுக்கு மொபைல் போன் கொண்டுவருவதில் இருந்து தடுக்கப்படுகிறார்கள். இருப்பினும், வளமான பள்ளிகள் மற்றும் குறைந்த வளமுள்ள பள்ளிகள் ஆகிய இரண்டிலுமே, ஆசிரியர்கள், பள்ளி வளாகத்தினுள் ஏகப்பட்ட மாணவர்களை, மொபைல் போனுடன் பிடித்திருப்பதாக குறிப்பிட்டிருக்கின்றார்.

ஒழுங்கு நடவடிக்கையாக, அவர்களின் மொபைல் போனை, பள்ளி நிர்வாகமே வைத்துக்கொண்டு, அது, அவர்களின் பெற்றோர்களிடம் தான் கொடுக்கப்படும் என்று மாணவர்களிடம் தெரிவித்துவிடுகின்றனர். இது, இந்தக் கொள்கைகளைப் பற்றியும், அவை உடைவதால் ஏற்படும் பிரச்சினைகளை பற்றியும் பெற்றோர்களிடம் பேசுவதற்கு, ஒரு சந்தர்ப்பத்தையும் ஏ ஏற்படுத்திக்கொடுக்கிறது. பல சந்தர்ப்பங்களில், 10-12 வயது மாணவர்களுக்கு மொபைல் போன் கொடுப்பதற்காக, ஆசிரியர்கள் பெற்றோரை தான் குற்றம் சாட்டுகிறார்கள். இந்தப் புத்தகத்திற்கான பேட்டியின் போது, பல ஆசிரியர்கள் குறிப்பிட்டது என்னவென்றால், இதுபோன்ற விதிமீறல்கள், இரண்டு பெற்றோரும் (தாய், தந்தை இருவரும்) பணிக்கு செல்லும் குழந்தைகளால் தான் செய்யப்படுகிறது என்று குறிப்பிட்டனர். ஆசிரியர்களை பொறுத்தவரை, அள்ளியிறைக்கக்கூடிய வருமானமுள்ள பெற்றோர்கள், தங்கள் பிள்ளைகளுக்கு அவர்கள் கேட்கும் தொழில்நுட்ப சாதனங்களையெல்லாம், அவர்களுடன் செலவிடமுடியாத நேரத்தி- 
ற்கு ஈடுகெட்டுவது போல வாங்கிக்கொடுத்துவிடுகிறார்கள். உண்மையில், குறைந்த வளமுள்ள பள்ளிகளில் இருக்கும் ஆசிரியர்கள் கூட, இதுபோன்ற புகார்களை கூறுகிறார்கள். ஆனால் இது பெருமளவு ஆண் மாணவர்களை குறித்ததாக தான் இருக்கிறது. இவர்கள் பல சமயங்களில் இதுபோன்ற போன்களை, அவர்களின் பெற்றோரிடமிருந்து நேரடியாக பெறுவதைவிட, தங்கள் நீட்டித்த குடும்பத்து உறவினர்களிடமிருந்து பரிசாகப் பெறுகிறார்கள் (மாமா, சிற்றப்பா போன்ற வயதில் மூத்த ஆண் உறவினர்). இந்த மாணவர்களை வளமான பின்னணியில் இருந்து வரும் மாணவர்களிடம் இருந்து வேறுபடுத்திக்காட்டுவது என்னவென்றால், இவர்கள் பெரும்பாலும், இரண்டாம் உபயோகமாக கைமாறிய போன்களையே வைத்திருக்கிறார்கள். வளமான பள்ளிகளில் பயிலும் மாணவர்கள், புத்தம்புதிய ஸ்மார்ட்போன்களை வைத்திருக்கிறார்கள். குறைந்த வளமுள்ள பள்ளிகளில், ஆண் மாணவர்கள் தான் பெரும்பாலும் அகப்பட்டுக்கொள்கிறார்கள். வளமான பள்ளிகளின் மாணவர்களில், ஆண், பெண் இருபாலாரும் அகப்பட்டுக்கொள்கிறார்கள். வளத்தைப் பற்றிய பொருட்டில்லாமல், அனைத்துப்பள்ளிகளிலும், மொபைல் போன் வைத்திருக்கும் மாணவர்கள் அகப்பட்டுக்கொள்கிறார்கள் என்று தெளிவாகத்தெரிகிறது.

வளமான பள்ளிகளில் பயிலும் மாணவர்களின் விஷயத்தில், ஆசிரியர்களின் நியாயப்படுத்தல் என்னவென்றால், இதுபோன்று ஒரு சாதனத்துடன் ஒட்டுதலாக இருப்பது, தனிமையான குழந்தையாக இருப்பதன் ஒரு வகையான தீர்வு; பெற்றோர் இருவரும் நீண்ட நேர பணிநேரங்களைக் கொண்டிருப்பதால், இந்தக்குழந்தைகளுடன், அவர்களின் பெற்றோரை விட, மொபைல் போன் தான் அதிக நேரம் உரையாடுகிறது என்பது தான். இவர்களுக்கு போன் அல்லது ஐபாட் ஒரு உண்மையான துணையாக ஆகிவிடுகிறது. நிகழ்நிலை விளையாட்டுக்கள் விளையாடும் பல இளம் குழந்தைகள் குறிப்பிட்டது என்னவென்றால், அவர்கள் பெற்றோர் பணியில் இருக்கும் சமயம், இந்த விளையாட்டுக்கள் தான் அவர்களுக்கு துணையாக இருக்கின்றன என்பது தான்.

ஆசிரியர்களை பொறுத்தவரை, இந்த விளையாட்டுக்கள் தான் இந்தக் குழந்தைகள் அவர்களின் மொபைல் தொழில்நுட்ப சாதனங்களுடன் இவ்வளவு ஒட்டுதலாக இருப்பதற்கான முதன்மை காரணம். இந்த விளையாட்டுக்கள் பல வழிகளில் நடைபெறுகிறது. பல விளையாட்டுக்கள் செயலிகள் வடிவில், கூகுள் அங்காடி, ஆப்பிளின் செயலி அங்காடி அல்லது 
சாம்சங் அங்காடி ஆகியவற்றில் இருந்து பதிவிறக்க முடியும். இருப்பினும், பல பெற்றோர்கள், தங்கள் குழந்தைகளை, முகநூலில் சேர அனுமதிக்கின்றனர். இதன்மூலம் அவர்கள் முகநூலில் உள்ள விளையாட்டுக்களையும் விளையாடுகிறார்கள். முகநூலில் சேரும் இளம் குழந்தைகள் பெரும்பாலும், அதை விளையாட்டுக்கள் விளையாடுவதத்காக மட்டுமே பயன்படுத்துகிறார்கள் என்று ஆசிரியர்கள் குறிப்பிடுகின்றனர். இதுபோன்ற சமூக ஊடக உறுப்பினராக இருத்தல், அவர்களை தேவையற்ற கவனச்சிதறல்களுக்கு ஆட்படுத்துகிறது என்று அவர்கள் கருதினாலும், பெற்றோர், தங்கள் குழந்தைகள் முகநூலில் இருப்பதைப்பற்றி பொருட்படுத்துவதாக தெரியவில்லை.

ஒரு வளமான பள்ளி, மாணவர்கள் இடைவேளைகளின் போது மொபைல் போன்களை அமைதி நிலையில் வைத்துக்கொண்டு விளையாட்டுக்கள் விளையாடுவதற்காகவும், சமூக ஊடகங்களை, குறிப்பாக முகநூல் மற்றும் வாட்சப் ஆகியவற்றை உபயோகப்படுத்துவதற்காகவும் பயன்படுத்துகின்றனர் என்ற வதந்தியை தொடர்ந்து, யாரேனும் மொபைல் போன்கள் வைத்திருக்கிறார்களா என்று கண்டறிவதற்காக, ஒவ்வொரு மாணவரின் புத்தகப்பைகளையும் திடீர் சோதனைக்கு ஆட்படுத்தியது. இந்த சோதனை, 17 வயது வரையானவர்கள் மட்டும் உள்ள பதினோராம் வகுப்பில் இருந்து மட்டும், 21 மொபைல் போன்கள் கைப்பற்றப்பட்டதுடன் நிறைவுற்றது. இது மேலும் கடுமையான கொள்கைகள் விதிக்கப்படுவதற்கு வழிவகுத்தது. பள்ளிகள், பெற்றோர் ஆசிரியர் கழக கூட்டத்திற்கு ஏற்பாடு செய்து, இதைப்பற்றி பெற்றோர்களுக்கு தெரியப்படுத்தினாலும், ஒரு சில பெற்றோர் எந்த அக்கறையும் காட்டவில்லை என்று அவர்கள் குறிப்பிட்டனர்.

குறைந்த வளமுள்ள பள்ளிகளிலும், மொபைல் போன் ஒழுங்கு முறைகள் நடத்தப்படுகின்றன. அங்கும் “மொபைல் போன் கூடாது" என்ற கொள்கை இருக்கிறது. முன்பே பார்த்தபடி, இந்தப்பள்ளிகளிலும் மாணவர்கள் மொபைல் போனுடன் அகப்பட்டுக்கொண்டனர். இருப்பினும், ஒருசில குறைந்த வளமுள்ள பள்ளிகளில், மாணவர்கள் மொபைல் போன் வைத்திருப்பதை ஆசிரியர்கள் பார்த்தாலும், அதை அவர்கள் பெரிதாக பொருட்படுத்தவில்லை. இந்தப்பள்ளிகளில், பொதுவாக பத்தாவது முதல் பன்னிரெண்டாவது வகுப்பு வரை படிக்கும் மாணவர்கள், மொபைல் போன்களை எடுத்துவருகிறார்கள். இவர்கள் சோதனையின் போது ஆசிரியர்களிடம் அகப்பட்டுக்கொள்கிறார்கள். ஒருசில ஆசிரியர்கள், 
இதுபோன்ற திடீர் சோதனைகளின் போது, ஒரு எச்சரிக்கையாகத் தான், இந்த போன்களை கைப்பற்றுகிறார்கள். இவர்கள், அபராதக் கட்டணம் விதிக்கும் முன்போ அல்லது வேறு ஏதேனும் ஒழுங்குமுறை நடவடிக்கை எடுக்குமுன்போ, இவர்கள் இந்த மாணவர்களின் பொருளாதார பின்னணியையும் கருத்தில் கொள்கிறார்கள்.

இந்தப்பள்ளிகளில் ஒன்றில் ஆசிரியராக இருக்கும் சுஜாதா என்பவர், பெற்றோர் ஆசிரியர் கழக கூட்டத்தில் நடந்த ஒரு சம்பவத்தை குறிப்பிடுகிறார். இந்த சமயத்தில், பள்ளி நிர்வாகம், பள்ளி வளாகத்தினுள், மொபைல் போன் உபயோகிப்பதில் விதிக்கப்பட்டுள்ள தடை பற்றி பெற்றோரை எச்சரித்திருக்கிறது. பெற்றோர்கள், தங்கள் பிள்ளைகளின் புத்தகப்பைகளை மொபைல் போன் இருக்கிறதா என்று பார்ப்பதற்காக சோதனையிடவும், தங்கள் மொபைல் போனை பள்ளிக்கு எடுத்துவரும் குழந்தைகள் மீது ஒருகண் வைத்துக்கொள்ளவும் ஊக்குவிக்கப்பட்டார்கள். அநேகமாக அனைத்துப் பெற்றோரும், பள்ளியின் இந்த முடிவிற்கு ஆதரவாக இருப்பது போல் தான் தெரிந்தது. இருந்தாலும், இரண்டு நாட்களுக்கு உள்ளாகவே, இரண்டு மாணவர்கள், காலை இடைவேளை சமயம் தங்கள் மொபைல் போனில் பேசிக்கொண்டிருக்கும்போது அகப்பட்டுக்கொண்டார்கள். அவர்களிடமிருந்து போன்கள் கைப்பற்றப்பட்ட போது, அவர்கள் தங்கள் அன்னையரிடம், மதிய உணவை பள்ளிக்கு அ அனுப்பிவைக்கும்படி கேட்டுக்கொண்டிருப்பது தெரியவந்தது. இதற்காக பெற்றோர்கள் அழைக்கப்பட்டபோது, அவர்கள் அப்படிப்பட்ட கொள்கையைப் பற்றி அறிந்திருக்கவில்லை என்று கூறினர். தங்களதையும் சேர்த்து பல பள்ளிகள், இதுபோன்ற கொள்கைகளை வாய்வழி தகவலாக தான் வெளிப்படுத்தியுள்ளன என்பதை சுஜாதா குறிப்பிட்டார். இது எங்கும் எழுத்தில் குறிப்பிடப்படாததால், இதைப்பற்றி அறியவில்லை என்றுரைப்பது சுலபமாகிவிடுகிறது. ஒருசில பள்ளிகள் மொபைல் போன் உபயோகம் பற்றி எழுதுவடிவ கொள்கைகள் வைத்திருந்தாலும் (பள்ளி வளாகத்தினுள் மொபைல் போன் உபயோகம் தடை செய்யப்பட்டிருப்பது), பல பள்ளிகள் அவ்வாறு குறிப்பிடுவது இல்லை. அவை சமூக ஊடகங்கள் பற்றியும் எந்தக்கருத்தும் தெரிவிப்பதில்லை. மொபைல் போன்களுக்கான தடை என்பது, எழுதப்படாத/பேசப்படாத விதியாகத்தான் இருக்கிறது. பல ஆசிரியர்கள், இது எழுத்தில் இருக்கவேண்டிய அவசியமில்லை, இது ஒரு பகுத்தறிவுப் புரிதல் தான் என்றெல்லாம் 
கூறினாலும், இவற்றை பின்பற்றுவது என்று வரும்போது, பெற்றோரின் ஒத்துழைப்பைப்பற்றிய தங்களது சந்தேகங்களையும் அவர்கள் தெரியப்படுத்தினர்.

வளமான பள்ளிகளின் வழக்குகளிலும் கூட, பள்ளி வளாகங்களில், மொபைல் போன் உபயோகத்தை தடை செய்யும் ஒழுங்குமுறை கொள்கைகள் நிறுவப்பட்டிருக்கும் போது கூட, சில சமயங்களில் இவற்றால் எந்த பயனும் இல்லை என்று ஆசிரியர்கள் அடிக்கடி புகார் கூறுகின்றனர். இது, தடைசெய்யப்பட்டிருந்தாலும், பள்ளிக்கு மொபைல் போன் ஏன் எடுத்துவருகின்றனர் என்ற, குழந்தைகளின் தன்னாட்சியைப் பற்றிய கேள்வியை எழுப்புகிறது. இதற்கு பல காரணங்கள் புலப்படுகின்றன. பல குழந்தைகள், தங்களுக்கு மொபைல் போன் வேண்டுமென்றும், இது மட்டுமே, தாங்கள் தங்கள் பெற்றோருடனும் (இருவரும் பணிக்கு செல்பவர்கள்) அல்லது பொறுப்பான வேறு பெரியவர்களிடம் தேவைப்படும் சமயத்தில் தொடர்பு கொள்வதற்கான ஒரே வழி என்றும் குறிப்பிட்டனர். மேலும், அவர்கள் வைத்திருக்கும் போனின் வகை, தொடர்ச்சியான விளையாட்டுகளுக்கான வாய்ப்பை தாண்டிய சமூக அந்தஸ்தை, தங்களையொத்தவர்களுடன் அமைத்துக்கொள்ள உதவுவதாகவும் கூறினர். ஒருசில மேல்நிலைப்பள்ளி குழந்தைகள், இது தங்கள் பெற்றோர் மற்றும் ஆசிரியர்களின் அதிகாரத்திற்கு எதிரான எதிர்ப்புச்செயல் என்றும் குறிப்பிட்டனர்.

நேர்மறை பக்கத்தை பார்த்தால், இதுபோன்ற மொபைல் போன்களுடனான ஒட்டுதல், ஒருவகையில், இந்த குழந்தைகளுக்கு, ஸ்மார்ட்போன்களின் தொழில்நுட்ப நுணுக்கங்களைப் பற்றி புரிந்துகொள்ள உதவுகிறது என்பதை ஆசிரியர்கள் ஒத்துக்கொள்கின்றனர்.

வளமான பள்ளிகளில் ஒன்றில், கம்ப்யூட்டர் சயின்ஸ் ஆசிரியையாகவும், கணினி நிர்வாகியாகவும் இருக்கும் கல்பனா என்பவர் குறிப்பிட்டது. ஒருமுறை அவரது புதிய ஐபோனில் அவர் எதோ பிரச்சினையை சந்தித்தபோது, அதைப்பற்றி, ஒன்பதாம் வகுப்பில் உள்ள சுமார் 15 வயது மாணவரிடம் எதேச்சையாக குறிப்பிட்டுள்ளார். அந்த மாணவர், சில நிமிடங்களிலேயே அந்தப் பிரச்சினையை தீர்த்து வைத்துவிட்டார். அவர் அந்த மாணவரைப் பற்றி, ஒரே சமயத்தில் ஆச்சரியமும் பெருமிதமும் அடைந்தார். அந்தக்குழந்தையின் பெற்றோர் வீட்டில் ஐபோன் வைத்திருப்பது அவருக்கு தெரியவந்தது. அவர், இதைப்பற்றி மற்ற ஆசிரியர்களிடம் தற்செயலாக குறிப்பிட்டிருக்கிறார். இந்த விஷயம் மெல்ல மெல்ல பரவி, பள்ளி நிர்வா- 
கத்திற்கு தெரியவந்திருக்கிறது. பள்ளியின் தலைவர், இது போல மாணவர்களின் உதவியை, சொந்த சாதனங்களை சரிசெய்வதற்கு எதிராக அவரை எச்சரித்தாலும், இந்தக்கால குழந்தைகள் போன்கள் மற்றும் இதர தொழில்நுட்ப சாதனங்களை பற்றி, அதிக அளவில் தெரிந்து வைத்திருக்கிறார்கள் என்பதை ஆசிரியர்கள் ஒத்துக்கொள்ளவேண்டும் என்று கல்பனா கருதுகிறார். மொபைல் போனோ அல்லது இணையதள அணுகலோ இருப்பது குழந்தைகளுக்கு கெடுதலான ஒன்றல்ல என்றும், இவையிரண்டுமே தவிர்க்கப்பட முடியாதது என்பதை ஆசிரியர்கள் புரிந்துகொள்ள வேண்டும் என்றும் அவர் கூறுகிறார். அவர், குழந்தைகளின் தேவைகள் மற்றும் பின்னணியை பற்றிய முழுமையான புரிதலை விரும்புகிறார். அப்போது தான் தொழில்நுட்பத்தை பொறுப்புடன் பயன்படுத்த அவர்களுக்கு கற்பிக்க முடியும் என்பது அவர் கருத்து. இது அவரது சொந்த கருத்து. வேறு எந்த ஆசிரியரும் இதுபோல் கருதவில்லை, குறிப்பாக அவரது பள்ளியில் கூட.

வளமான மற்றும் வளம் குறைந்த பள்ளிகளில் உள்ள ஆசிரியர்கள், பலவகையான ஸ்மார்ட்போன்கள் மற்றும் இதர போன்களை, கையாளவும் பழுது பார்க்கவும் தெரிந்த தொழில்நுட்பத்திறன் வாய்ந்த மாணவர்களை பற்றி குறிப்பிடுகின்றனர். இருப்பினும், தொழில்நுட்பத்திறன் வாய்ந்த மாணவர்களை பற்றி குறிப்பிடும் போது, குறைந்த வளமுள்ள பள்ளிகளில், அது பத்தாவது அல்லது பன்னிரெண்டாவது படிக்கும் 16 முதல் 18 வயதுக்குட்பட்ட மாணவர்களை குறிப்பிட்டாலும், வளமான பள்ளிகளில், இது ஏழு அல்லது எட்டாம் வகுப்பைச்சேர்ந்த 12 முதல் 14 வயதிற்குள் இ இருக்கும் மாணவர்களை குறிப்பிடுகிறது. இது போன்ற கதைகள், இந்த மாணவர்களிடையே உள்ள தொழில்நுட்ப வெளிப்பாடு தொடர்பான அறிவார்த்த இடைவெளியை தெளிவாகக் காட்டுகிறது. வளமான பள்ளிகளில், தனிப்பட்ட ஆசிரியர்கள் இந்த ஆற்றலை ஊக்குவித்தாலும், இதுபோன்ற தொடர்ந்த செயல், முகம் சுளிக்கப்பட்டு, கவனச்சிதறலாக பார்க்கப்படுகிறது. இருப்பினும், குறைந்த வளமுள்ள அதிகப்படியான தொழில்நுட்ப உட்கட்டமைப்பு இல்லாத பள்ளிகளில், இந்த தொழில்நுட்ப ஆற்றல் நவீன பஞ்சக்கிராமியில் வாழ்வதற்கு தேவையான தொழில்நுட்ப அறிவாகப் பார்க்கப்படும். உதாரணத்திற்கு, இந்த அத்தியாயத்தின் துவக்கத்தில் விவாதிக்கப்பட்ட வழக்காய்வில், பாண்டியனிடம் பாடல்கள் கேட்ட ஆசிரியர், பாடல்களை பதிவிறக்குவதில் அவனுக்குள்ள திறனை, தொழில்நுட்ப ஆற்றலாகப் பார்க்கிறார். அதேபோல, 
ஒரு கம்ப்யூட்டரை சரிசெய்வதற்கு பாண்டியனின் நண்பன் தீபக்கின் உதவியைப்பெற்ற கம்ப்யூட்டர் சயின்ஸ் ஆசிரியர், இந்த உதவியை தொழில்நுட்ப ஆற்றலாக பார்க்கிறார். இந்தப்பள்ளிகளில் ஆசிரியர்கள் மாணவர்களின் உதவியை பெற்றால், யாரும் அதைப்பற்றி முகம் சுளிப்பதில்லை.

பள்ளிகளின் அமைப்புகள் அல்லது மொபைல் போன், இணையதளம் மற்றும் சமூக ஊடகங்கள் ஆகியவற்றைப்பற்றிய அவர்களது கண்ணோட்டம் ஆகிய எதையும் பொருட்படுத்தாமலேயே, இந்தப்பள்ளிகள் அனைத்திற்கும், தங்கள் மாணவர்களின் மீது பொதுவாக தொழில்நுட்பத்தின் பாதிப்பு பற்றியும், குறிப்பாக சமூக ஊடகங்களின் ஆதிக்கத்தைப் பற்றியும் ${ }^{30}$, தெளிவாக தெரிந்திருந்தது. பள்ளிகள் சம்பிரதாயப்படி சமூக ஊடகங்களை எப்படி பார்க்கின்றன என்பதை மேலுள்ளவாறு விவாதித்த பின்பு, மாணவர்கள் தங்கள் ஆசிரியர்களுடனான உறவை அவர்களை முகநூலில் நண்பர்களாக்கிக்கொள்வதன் மூலம் நீட்டித்தால் என்னவாகும் என்று பார்ப்பதற்கு நகரலாம். பின்வரும் பகுதி, இதுபோன்ற நட்புறவுகளை ${ }^{31}$ ஆசிரியர்கள் எவ்வாறு பார்க்கிறார்கள் என்பதை அலசுகிறது. வேறு வார்த்தைகளில் சொல்லப்போனால், ஆசிரியர்கள் மாணவர்களின் நட்புறவு கோரிக்கைகளை, பாரம்பரியமான ஆசிரியர்-மாணவர் என்ற அதிகாரப் படிநிலையை அவமதிப்பதாக கருதி அவற்றை மறுக்கிறார்களா அல்லது இதுபோன்ற செயல்களை தங்கள் மாணவர்களின் தொழில்நுட்ப ஆற்றலுக்கான அடையாளமாகக் கருதி இது போன்ற நட்புறவுகளை ஊக்குவிக்கிறார்களா என்பது பற்றி ஆராய்கிறது.

\section{சமூக ஊடகங்கள்: ஆசிரியர்களை நண்பர்களாக்கிக் கொள்வது}

முந்தைய பகுதிகளில் பார்த்தது போல, பதின்பருவத்தின் துவக்கத்தில் இருக்கும் தங்களது மாணவர்கள் பலர் முகநூலில் இருக்கிறார்கள் என்பது பல ஆசிரியர்களுக்கு தெரியும். இந்த நிலை அவர்களுக்கு உறுத்தலாக இருந்தது ஏனென்றால், அவர்கள் முகநூலை குழந்தைகளுக்கான வலைத்தளமாக கருதவில்லை. 18 வயதுக்குட்பட்ட குழந்தைகள் முகநூல் ${ }^{32}$ பயன்படுத்த அனுமதிக்கப்படக்கூடாது என்ற இந்திய சட்டரீதியான விதிமுறைகளை அவர்கள் வலியுறுத்திக்கொண்டிருந்தாலும், இந்தத்தளத்தை குழந்தைகளுக்கு முற்றிலுமாகத்தடை 
செய்வது என்பது இயலாத காரியம் என்பதை அவர்கள் ஒத்துக்கொண்டனர். பலர் அவர்களை எதிர்நோக்கியிருக்கும் நிதர்சனத்தை, அரைமனத்துடன் ஒத்துக்கொள்ள தயாராகி விட்டனர்.

இருப்பினும், ஒருசில முக்கிய கூறுகள் விரைவில் தெளிவாயின. இதுபோன்ற விசாரங்களை வெளிப்படுத்திய ஆசிரியர்களில் பலர், முகநூலில் தங்கள் மாணவர்களுடன் நண்பர்களாக இருந்தனர். பள்ளிக்கு வெளியே, ஆசிரியர்களும் மாணவர்களும் நண்பர்களாக இருப்பதை ஊக்குவிக்க கூடாது என்ற தளர்வான கொள்கையை பள்ளிகள் கொண்டிருந்தாலும், நிஜத்தில் எந்தப்பள்ளிகளுமே, பள்ளிக்கு வெளியே என்ன நடக்கிறது என்பதைப்பற்றி அதிகம் கவலைப்படுவதாக தெரியவில்லை. எது எப்படியிருப்பினும், தங்கள் மாணவர்களுடனான முகநூல் நட்பைப்பற்றி பள்ளி வளாகத்தினுள் விவாதிப்பதை ஆசிரியர்கள் விரும்பவில்லை. ஆனால், அதையே மாணவர்கள் பல்வேறு காரணங்களுக்காக செய்தார்கள். ஒருசிலர், அதிகார மையத்துடனான தங்களது நெருக்கத்தை தங்களை ஒத்தவர்களுக்கு காட்டிக்கொள்வதற்காக அப்படிச்செய்தனர். வேறுசிலர், தங்கள் ஆசிரியர்களின் வாழ்வில் என்னநடக்கிறது என்றறிந்துகொள்ள நிஜமாகவே ஆவலாக இருந்தனர். சில மாணவர்களுடன் நடத்தப்பட்ட பேட்டி இதை தெளிவாக்கியது.

\section{உதாரணத்திற்கு, ஒரு வளமான பள்ளியின் 14 வயது மாணவன் ராஜீவ் கூறியது:}

“நான் பிரேமா மேடத்துடன் முகநூலில் நண்பனாக இருக்கிறேன்.......வகுப்புத்தோழர்களுக்கு சொன்னேன்....அவர்களும் கோரிக்கைகள் அனுப்பி நண்பர்களாகிவிட்டார்கள். அவர் குறைவாகத்தான் பதிவிடுகிறார். அவர் ஒரு மலேசிய பயணம் சென்றிருந்தார்.....அவருடைய தொகுப்பில் நான் புகைப்படங்களை பார்த்தேன்.....எங்கள் வகுப்பிலுள்ள பெண்களுக்கு அவருடைய உடை பிடித்திருந்தது....பையன்களுக்கு கேளிக்கை பூங்கா பிடித்திருந்தது."

மற்றொரு வளமான பள்ளியின் வாரெண்யா என்ற ஒன்பதாம் வகுப்பு மாணவர் விளக்கியது:

“நான் எனது ஆசிரியருடன் முகநூலில் நட்புத்தொடர்பு கொண்டபோது அவரது பிறந்தநாளுக்காக வாழ்த்தி- 
னேன். அவர் அனைத்து கருத்துப்பதிவிற்கு விருப்பு தெரிவித்திருந்தார்....நான் இதை வகுப்புத்தோழர்களுக்கு சொன்னேன்.....நான் அவரை பள்ளியில் பார்த்தபோது வாழ்த்து எதுவும் சொல்லவில்லை"

குறைந்த வளமுள்ள பள்ளியில் பயிலும் தண்டபாணி என்ற பத்தாம் வகுப்பு மாணவர் குறிப்பிட்டது:

“என்னுடைய கம்ப்யூட்டர் சயின்ஸ் ஆசிரியர் மட்டுமே முகநூலில் இருக்கிறார். நான் முகநூலில் சேர்ந்தவுடன் அவருக்கு நட்புக்கோரிக்கை அனுப்பினேன். எனது நண்பன் அருள்ராஜ் அவரை நட்பாக்கிக்கொள்ளக் கூறினான். ஏனெனில் அவன் அவருடன் முகநூலில் நட்புதொடர்பில் இருக்கிறான். என்னுடைய ஆசிரியர் உடனே கோரிக்கையை ஏற்றுக்கொண்டார். மறுநாள் அவரை பள்ளியில் பார்த்ததும் அவருக்கு நன்றி கூறினேன்..அவர் வெறுமே புன்னகை மட்டும் செய்தார்."

தங்கள் மாணவர்களுடன் முகநூலில் நண்பர்களாக இருந்த சில ஆசிரியர்கள் அந்தத்தளத்தில் தங்கள் நடவடிக்கைகளை பற்றி மிகவும் கவனமாக இருந்தாலும், ஒரு சிலர் அதைப்பற்றி கவலைப்படுவதாக தெரியவில்லை. ஒருசிலர், தங்களின் முகநூல் நண்பர்கள் வலைத்தொடர்பு தங்கள் மாணவர்களையும் உள்ளடக்கியதாக இருப்பதை மறந்தே விட்டதாக கூறினார்கள். உதாரணத்திற்கு, மஞ்சுளா என்கிற பதினோராம் வகுப்பு ஆசிரியை, சிபிஎஸ்இ பள்ளி ஒன்றில் பணி புரிபவர், ஒரு மாணவர் தன்னை, சமீபத்தில் பதிவுகள் எதுவும் இடாததை பற்றி விசாரித்த பின்பு தான் தன்னுடைய மாணவர்களில் ஒருவரை முகநூலில் நண்பராக்கிக் கொண்டுள்ளதே தனக்கு நினைவுக்கு வந்ததாக கூறினார். மற்றவர்கள், தங்கள் இளம் மாணவர்களுடன் (14 வயதுக்கு உட்பட்ட) அந்தத்தளத்தில் நண்பர்களாக ஆவதன் மூலம் அந்த தளத்தில் அவர்களின் நடவடிக்கைகளின் மீது ஒரு நெருக்கமான கண்காணிப்பு வைத்திருப்பதாக கூறினர். இது தங்கள் மாணவர்கள் தாங்கள் என்ன பதிவிடுகிறோம் என்பதில் (படங்கள் மற்றும் பயன்படுத்தும் மொழி) ஓரளவிலான கட்டுப்பாட்டை கொண்டுவரும் என்று அவர்கள் நம்பினர். அவர்கள் பொருத்தமற்றதாக எதையாவது பார்த்தால், அதைப்பற்றி தங்கள் மாணவர்களிடம் நேரடியாகவோ அல்லது மறைமுகமாகவோ கேட்டுவிடுவெதாக ஒத்துக்கொண்டனர். 
இந்தவகையான கண்காணிப்பும் சமூக கட்டுப்பாடும் தான் உயர் வகுப்புகளில் இருக்கும் மாணவர்கள், தங்கள் ஆசிரியர்களுடன் முகநூலில் நண்பர்களாக இருப்பதை தவிர்ப்பதற்கான காரணம். பலர், குறிப்பாக ஆண் மாணவர்கள், அதுபோன்ற நட்புறவுகள், தங்கள் அந்தரங்கத்தின் மீதான வரம்பு மீறலாக கருதினர். இந்த வகுப்புகளில் உள்ள பெண் மாணவர்கள், தங்கள் அபிமான ஆசிரியருடன் நட்புறவு கொள்வதைப் பற்றி நிச்சிந்தையாக இருந்தனர். ஒருசிலர், ஆசிரியர்கள் தங்களுக்கு நட்புதொடர்பு கோரிக்கை அனுப்பியதாகவும், தாங்கள் அதை ஏற்றுக்கொண்டதாகவும் கூறினர். மற்ற மாணவர்கள், பள்ளியில் ஏற்பாடு செய்யப்படும் ஏதாவது ஒரு நிகழ்ச்சியில் அவர்கள் பங்கெடுத்துக்கொண்டிருந்தால், தங்கள் ஆசிரியர்களுடன் நட்புதொடர்பு கொள்ள வலியுறுத்தப்பட்டனர். ஏனெனில் சம்பிரதாயமற்ற ஒருங்கிணைவு முகநூல் மூலம் நடந்தது. இருப்பினும், ஒரு ஆசிரியருடன் ஒரு மாணவர் பகிர்ந்துகொண்ட உறவுமுறை, அபிமான ஆசிரியராக யார் இருப்பார்கள், முகநூல் குறித்த அவரது மனப்பாங்கு என்னவாக இருக்கும், தங்கள் ஆசிரியருடன் முகநூலில் நண்பர்களாக இருக்கும் மற்ற மாணவர்கள் அல்லது வகுப்புத்தோழர்களின் எண்ணிக்கை போன்ற அனைத்துமே, பள்ளியைப் பொறுத்ததாக இருக்கிறது. ஒருசில வழக்குகளில் மாணவர்கள், தாங்கள் ஆசிரியருடன் நட்புதொடர்பு கொள்வதற்கு, தங்கள் வலைதொடர்பில் உள்ள மற்ற நண்பர்கள் அவருடன் முகநூல் நண்பர்களாக இருந்தது தான் காரணம். இதனால் தாங்கள் சரியிணை அழுத்தத்தை உணர்ந்ததாக ஒத்துக்கொண்டனர். இந்தப்போக்கு பல பள்ளிகளிடையே தெளிவாகத் தெரிந்தது.

ஒருசில ஆசிரியர்கள், தங்கள் மாணவர்கள் அனைவருடனும் நட்பாக இருப்பதில் சௌகரியமாக உணர்ந்தாலும், மற்றவர்கள் இதில் மிகவும் கவனத்துடன் இருந்தார்கள். குறிப்பாக, உயர் வகுப்பில் இருப்பவர்கள். பல ஆசிரியர்கள் தாங்கள் நன்கறிந்த மாணவர்களுடன் (அதாவது அந்த மாணவரை பலவருடங்களான அறிந்தோ அல்லது அவருக்கு பயிற்றுவித்தோ இருத்தல்) மட்டுமே சௌகரியமாக இருப்பதாக கருதினர். மற்ற சில வழக்குகளில், மாணவர்களின் சுயவிவரத்தை பார்த்துவிட்டு பின்பு இந்த கோரிக்கைகளை ஏற்றுக்கொள்கின்றனர். பல ஆசிரியர்கள், தெளிவான சுயவிவரப்பக்க புகைப்படம் இல்லாத மாணவர்களை நண்பர்களாக்கிக்கொள்வதில் அவர்கள் மிகவும் எச்சரிக்கையாக இருப்பதாக குறிப்பிட்டுள்ளனர். 
கருணா என்கிற 49 வயதானவர், ஒரு வளமான பள்ளியில் பன்னிரண்டாம் வகுப்பு ஆங்கில ஆசிரியர். அவர், தம் பள்ளியில் உள்ள எந்த மாணவரையும் நண்பராக்கிக் கொள்ளுமுன் கருதுவதாக ஒருசில விதிமுறைகளை தொகுத்து வைத்திருக்கிறார். அவருக்கு அவர்கள் யார் என்று தெளிவாகத் தெரிய வேண்டும். அவர் அவர்களின் மனப்பாங்கை கணிக்க வேண்டிம். கருணா, பள்ளியில் புதிதாக பதினோராம் வகுப்பில் சேர்ந்திருக்கும் ஒரு ஆண் மாணவர் தொடர்பான சம்பவத்திற்கு பிறகு மிகவும் கவனமாக இருப்பதாக தெரிகிறது. அவர், தம் பள்ளி மாணவர்களை, தன்னுடன் முகநூலில் நண்பர்களாக இருக்க ஊக்குவித்திருக்கிறார். இதன் விளைவாக பலர் அவ்வாறு செய்திருக்கின்றனர். இந்த குறிப்பிட்ட மாணவரும் அவ்வாறே செய்திருக்கிறார். ஒருசில மாதங்களுக்கு பிறகு, இந்த குறிப்பிட்ட மாணவர், கருணாவின் கணக்கின் மூலம், பத்தாம் வகுப்பில் படிக்கும் அவரது மகளுடனும் நட்புதொடர்பில் இருந்திருக்கிறார். தனது மகளின் படத்திற்கான இ இந்த மாணவரின் கருத்துப்பதிவை பார்த்தபின்பு தான் கருணா இதுபற்றி கண்டறிந்திருக்கிறார். இந்த மாணவர், அவரது மகளின் படங்களுக்கு விருப்பு தெரிவிப்பது மற்றும் கருத்து பதிவு செய்வது என்றிருந்தாலும், கருணா இதை முதலில் தீங்கற்ற நட்பாகத்தான் கருதியிருக்கிறார். இருப்பினும், இதற்கு சிலநாட்களுக்குள்ளாகவே, அந்த மாணவர், கருணாவுடனான நட்புதொடர்பை துண்டித்துவிட்டு அவரது மகளுடன் மட்டும் நட்புதொடர்பில் இ இருந்திருக்கிறார். கருணா, தனது மகளின் ஊடகப்பக்கத்தில், இந்த மாணவரின் ஆபாசம் என்ற எல்லையைத்தொடும் வகையிலான மற்றொரு கருத்துபதிவைப் பார்த்து அதிர்ந்து போய்விட்டார். அவர் உடனே தனது மகளிடம் அந்த மாணவருடனான நட்புதொடர்பை துண்டிக்குமாறு கூறினார். அவர் இந்த விஷயத்தை பள்ளித்தலைமையிடமும் கொண்டுசெல்ல முடியாது. ஏனெனில், பள்ளி மாணவர்களுக்கும் ஆசிரியர்களும் இடையே தனிப்பட்ட சமூக ஊடக தொடர்புகளை ஊக்குவிப்பதில்லை.

இது ஒரு அபூர்வமான வழக்காக இருந்தாலும், உயர்வகுப்புகளில் உள்ள மாணவர்களுடன் நட்புதொடர்பு கொள்வது என்று வரும்போது, அந்தரங்கம் பற்றிய விசாரங்கள், இருபுறமும் எழக்கூடிய பிரச்சினைகள் ஆகியவற்றால், ஆசிரியர்கள் சற்று கூடுதல் கவனமாகவும் சந்தேகத்துடனும் தான் செயல்படுகிறார்கள். இருப்பினும் கீழ் வகுப்புகளை சேர்ந்த ஆசிரியர்கள், ஆர்வமிகுந்தவர்களாகவும், தங்கள் மாணவ- 
ர்களை தங்களுடன் நட்புதொடர்பு கொள்ளுமாறு முனைப்புடன் ஊக்குவிப்பவர்களாகவும் இருக்கிறார்கள். இது அவர்கள் வகையில், மாணவர்கள் முகநூலில் எந்தப்பிரச்சினையிலும் சிக்கிக்கொள்ளாமலும் இணைய உலக அடாவடித்தனத்திற்கு ஆளாகாமல் இருப்பதையும் உறுதிப்படுத்திக் கொள்வதற்காகவும் அவர்கள் மீது ஒரு கண் வைத்துக்கொள்வதற்காகவும் தான்.

இந்தப்பகுதியில் உள்ள வளமான பள்ளிகளில் படிக்கும் குழந்தைகள், பெரும்பாலும், பெற்றோர் இருவரும் பணிக்கு செல்லும் இரட்டை வருமானமுள்ள குடும்பங்களில் இருந்து வருபவர்களாக இருக்கிறார்கள். பள்ளிநேரம் முடிந்த பிறகு அவர்கள் தங்கள் பாட்டி, தாத்தாவுடன் இருக்கிறார்கள் அல்லது ஒரு சில வழக்குகளில், தனிமையான வீட்டுக்கு திரும்புகிறார்கள். தடையற்ற இணைய தொடர்பு மற்றும் ஏகப்பட்ட தொழில்நுட்ப சாதனங்கள் ஆகியவை இருப்பதால், குழந்தைகள் இளம் வயதிலேயே முகநூலில் சேருவதில் ஆச்சரியம் ஒன்றும் இல்லை. பெரும்பாலானோர், தங்கள் முகநூல் பயணத்தை, நிகழ்நிலை விளையாட்டுக்கள் விளையாடுவதன் மூலம் துவக்கினாலும், வெகு விரைவில் அவர்கள் முகநூலில் தங்களை ஒத்தவர்களின் குழுக்களுடன் தொடர்பு கொள்ள துவங்கி விடுகிறார்கள். இந்த சமயத்தில் தான் இவர்கள் தங்கள் ஆசிரியர்களையும் நண்பர்களாக்கிக் கொள்கிறார்கள். ராகுலின் வழக்கு இதை தெளிவாக எடுத்துக்காட்டுகிறது.

அத்தியாயம் 4-ல் விவாதித்தது போல, ஒரே குடியிருப்பு வளாகத்தில் இருவேறு குடியிருப்பு தளங்களில் குடியிருக்கும் குடும்பத்தைப்பற்றிய மற்றொரு உதாரணம் இது. ராகுல் என்கிற 15 வயதான மாணவர், பஞ்சக்கிராமியில் ஒரு வளமான பள்ளியில் பயிலுகிறார். பள்ளி முடிந்தபின் அவர் பொதுவாக அவரது தாத்தாபாட்டி வீட்டில் அவரது பெற்றோர் இரவு நேரம் கழித்து வரும் வரை காத்திருப்பார். அந்த சமயத்தில் தான் மிகவும் தனிமையாக உணர்வதாக அவர் கூறுகிறார். அந்தக் குடியிருப்பு வளாகத்தில் அவருக்கு நண்பர்கள் யாரும் இல்லாததும் இதற்கு ஒரு காரணம். தனக்கு நண்பர்கள் இல்லாததற்கு தனது பல்வேறு ஆர்வங்களே காரணம் என்று ராகுல் குறிப்பிடுகிறார். தனக்கு கணினியில் இருக்கும் ஆர்வம் விளையாட்டுக்களில் இல்லையென்றும், தன்னுடைய ஆர்வத்தை வேறு யாரும் பகிர்ந்துகொள்ளவில்லை என்றும் அவர் குறிப்பிடுகிறார்.மேலும், அவர், குடியிருப்பு வளாகத்தில் உள்ள பல குழந்தைகள் செல்லும் பள்ளிக்கு செல்லவில்லை (இந்த வழக்கில், குடியிருப்பு வளாகத்தாலேயே நடத்தப்படும் 
பள்ளி). இதனால் அவர் ஒரு வெளியாள் போல் உணர்கிறார். ராகுலின் தனிமையும், நண்பர்கள் இல்லாமையும் அவரது தாத்தா பாட்டியை கவலைக்குள்ளாக்கியது. அவர்கள் ராகுலின் பெற்றோரிடம், ராகுலை, குடியிருப்பு வளாகம் நடத்தும் பள்ளிக்கு மாற்றும்படி வலியுறுத்திக்கொண்டிருக்கின்றனர். இதனால் ராகுலுக்கு அதிக நண்பர்களும், கொஞ்சமேனும் உடற்பயிற்சியும் கிடைக்கும் என்பது அவர்களது நம்பிக்கை.

ராகுலின் பாட்டி, பள்ளியிலிருந்து திரும்பி வந்த பிறகான ராகுலின் நடவடிக்கைகளை பற்றி வெளிப்படையாக பேசினார். அவரது முதல் வேலை, தனது மடிக்கணினி மற்றும் சாம்சங் காலக்சி நோட்பேட்-ஐ உயிர்ப்பித்து, விளையாட்டுக்கள் விளையாட துவங்குவது. இது அவரது பெற்றோர் வீடு திரும்பும் வரை தொடர்கிறது. நடப்பில் பார்த்தல், ராகுல், கேமிங் தளங்களில் ஒவ்வொரு மாலையும் குறைந்தது நான்கு மணிநேரங்களை செலவிடுகிறார். மற்றபடி, முகநூலில் தனது பள்ளி நண்பர்களுடன் அரட்டையடித்துக்கொண்டே அதிலுள்ள விளையாட்டுக்களை விளையாடுகிறார்.

ராகுலும், ஒரேசமயத்தில் கேமிங் மற்றும் முகநூலில் நட்புதொடர்பு ஏற்படுத்திக்கொள்வது ஆகியவற்றில் தனக்கு உள்ள ஆர்வங்களை பற்றி மிகவும் வெளிப்படையாக இருக்கிறார். அவர் தனது நண்பர்களுக்கு தனது கேமிங் புள்ளிகளின் விவரங்களை எடுத்துக்கூறுகிறார். அதோடு பல சமயங்களில், தனது மற்ற பள்ளி நண்பர்களுடன் (இவரதை போலவே அவர்களின் பெற்றோரும் பணியில் இருப்பவர்கள்) நிகழ்நிலையில் விளையாட்டுக்கள் விளையாடுகிறார். அவரது சாம்சங் ஸ்மார்ட்போனில் இருக்கும் வாட்சப் மூலம், தனது கேமிங் நண்பர்கள் குழுவுடன் தகவல்கள் பரிமாறிக்கொள்கிறார். ராகுல், ஒரு வருடத்திற்கு முன்பாக தனக்கென முகநூல் கணக்கு உருவாக்கிக்கொண்டார். அதை செய்வதற்கு தனது பள்ளித்தோழன் உதவியதாக அவர் கூறினார். முகநூலில் அவர் செய்த முதல் காரியம், தனது பள்ளித்தோழர்களை தேடுவது. அதில் பெரும்பாலானோரை இந்தத்தளத்தில் கண்டு அவர் மகிழ்ச்சியடைந்தார். தனது நண்பர்களுடன் தங்களது ஆசிரியர்கள் பலரும் முகநூலில் நண்பர்களாக இருப்பது கண்டு அவர்களுக்கும் நட்புக்கோரிக்கைகள் அனுப்பதுவங்கினார். ஒன்பதாம் வகுப்பில் இருந்தாலும், பன்னிரெண்டாம் வகுப்பு ஆசிரியருக்கு நட்புக்கோரிக்கை அனுப்பியிருந்தார். அந்த ஆசிரியர் இவருக்கு வகுப்புகள் எதுவும் எடுத்திராவிட்டாலும், அந்த ஆசிரியரை 
இவருக்கு தனிப்படத்தெரிந்திரா விட்டாலும் இவரது நட்புக்கோரிக்கை உடனே ஏற்றுக்கொள்ளப்பட்டது. தனது பள்ளித்தோழர்கள் மற்றும் ஆசிரியர்கள் அநேகமாக அனைவருமே தான் நட்புக்கோரிக்கை அனுப்பிய சில மணிநேரங்களிலேயே அதை ஏற்றுக்கொண்டுவिட்டதாகவும், இப்போது அவர் அவர்கள் அனைவருடன் முகநூலில் நட்புதொடர்பில் இருப்பதாகவும், அவர்கள் அனைவரும் இவரை விட அதிக நேரம் முகநூலில் செலவிடுவதாகவும், ராகுல் குறிப்பிடுகிறார். ஆதலால் அவர், தன் பெற்றோரையும், தாத்தாபாட்டியையும், மற்றவர்கள் அனைவரும் முகநூலில் இருக்கும்போது தான் மட்டும் ஏன் அதிலிருந்து விலகவேண்டும் என்று கேட்டுக்கொண்டே இருக்கிறார். அவரது அன்னை ஆசிரியர்களை பாசாங்குக்காரர்கள் என்றழைப்பது வரை போய்விட்டார். அவர்கள் குழந்தைகளின் முகநூல் உபயோகத்தை தடுக்கவேண்டும் என்று பெற்றோருக்கு அறிவுரை கூறுகின்றனர். ஆனால் அதே சமயம், தங்கள் மாணவர்களை அதில் நண்பர்களாக்கிக் கொள்கின்றனர். ராகுலின் தாயார் இதை "குழந்தையையும் கிள்ளிவிட்டு தொட்டிலையம் ஆட்டிவிடும்" அணுகுமுறை என்று குற்றம்சாட்டினார்.

இருப்பினும், விரைவிலேயே ராகுலின் பெற்றோர் தான் அவரை முகநூல் உபயோகிக்கச்சொல்லி ஊக்குவிப்பது தெரியவந்தது. வெளிநாட்டில் இருக்கும் ராகுலின் ஒன்றுவிட்ட சகோதர சகோதரிகள் அனைவரும் அதை உபயோகிப்பதாகவும், ராகுலும் அதை உபயோகிக்க வேண்டும் என்றும், அப்போது தான் அவர்களின் நீட்டித்த குடும்பத்தின் அங்கமாக அவரால் உணரமுடியும் என்றும் அவர்கள் கூறுகின்றனர். அவரது அன்னை, இந்தியப்பள்ளிகள் சற்று வளரவேண்டும் என்றே குறிப்பிட்டார். அவை மாணவர்கள் சமூக ஊடகங்களை உபயோகிப்பதை இ இந்தப்பள்ளிகள் ஒத்துக்கொள்ளவேண்டும் அல்லது உறுதியான முடிவெடுத்து அதை முற்றிலுமாக தடை செய்யவேண்டும் என்று அவர் வெளிப்படையாக குறிப்பிட்டார். அவர், குழந்தைகளின் முகநூல் உபயோகத்தை தடுக்கவேண்டும் என்று பெற்றோருக்கு அறிவுரை கூறும் அதே சமயம், தங்கள் மாணவர்களை அதில் நண்பர்களாக்கிக் கொள்ளும் ஆசிரியர்களின் கொள்கையை ஏற்கவில்லை. ராகுலின் தந்தையும், பள்ளிகள் தங்கள் வளாகங்களில் என்ன நடக்கிறது என்பது பற்றி மட்டும் தான் கவலைப்படுகிறாார்கள் என்றும், இதன்மூலம் முழுமையான கல்வி பற்றிய தங்களின் சீர்மை கருத்துக்களுடன் தாங்களே முரண்படுகிறார்கள் என்றும் வெளிப்படையாக குறிப்பிட்டார். 
இருப்பினும், குறைந்த வளமுடைய பள்ளிகளில் உள்ள வழக்குகள் இதற்கு மாறாக உள்ளது. இங்கு உயர் வகுப்பில் உள்ள ஆசிரியர்களும், தங்களை முகநூலில் நண்பர்களாக்கிக்கொள்ளுமாறு தங்கள் மாணவர்களை முனைப்புடன் ஊக்குவித்தனர். இது புதிய எல்லைகளை ஆராயுமாறு மாணவர்களை ஊக்குவிப்பதாக கருதப்பட்டது. மீண்டும் பாலின பாகுபாடு பற்றிய விசாரம் எழுந்தது. பெரும்பாலும் ஆண் மாணவர்கள் தான் அதிகமாக சமூக ஊடகங்களில் குறிப்பாக முகநூலில் இருந்தனர். குறைந்த வளமுள்ள பள்ளிகளில் பயிலும் எட்டு முதல் பன்னிரெண்டாம் வகுப்பு வரை உள்ள பெண் மாணவர்களிடம் எடுக்கப்பட்ட பேட்டிகளில், அவர்கள் தாங்கள் சமூக ஊடகங்களிலோ முகநூலிலோ இல்லை என்றும், அதற்கான காரணம் பொருளாதார சூழ்நிலைகளும், குடும்பத்தால் விதிக்கப்பட்ட கட்டுப்பாடுகளும் தான் என்றும் அவர்கள் தெரிவித்தனர்.

ரமேஷ் என்ற, பஞ்சக்கிராமியில் உள்ள தமிழ் பயிற்றுவிப்பு முறை பள்ளியில் பயிலும் பன்னிரெண்டாம் வகுப்பு மாணவர், மூன்று வருடங்களுக்கு முன், தன்னுடைய முகநூல் கணக்கை துவக்கினார். அவர் தனது வகுப்பு தோழர்கள் சிலருக்கும் முகநூல் கணக்குகளை துவங்குவதில் உதவியிருக்கிறார். அவர், தன்னுடைய முகநூல் கணக்கைப் பற்றி தன்னுடைய ஆண் ஆசிரியர்கள் சிலரிடம் குறிப்பிட்டபோது, அவர்கள், தங்களுக்கும் முகநூல் கணக்குகள் துவங்கித்தருமாறு ரமேஷிடம் கேட்டுக்கொண்டனர். ரமேஷின் இந்தவகையான முகநூல் தொழில்நுட்ப ஆற்றல் பற்றிய செய்தி பரவத்துவங்கியது. மற்ற ஆசிரியர்களிடம் இருந்தும் முகநூல் கணக்குகள் துவங்கித்தர வேண்டி கோரிக்கைகள் பொழியத்துவங்கின. விரைவில் தொழில்நுட்ப அறிவாளியாக ரமேஷின் பிம்பம் வடிவெடுக்கத்துவங்கியது. அவர், இணையதளத்தையும் கணினிகளையும் பற்றி அவரது ஆசிரியர்களை விட அதிகம் அறிந்தவராக பார்க்கப்பட்டார். முதலில் அவர் தமிழறிஞராக வேண்டும் என்று தான் விரும்பியிருந்தார். ஆனால் முகநூலுடனான அவரது வெற்றியும், தொழில்நுட்ப வல்லுனராக வளர்ந்து வரும் அவரது அந்தஸ்தும், ரமேஷை, தனது வாழ்வின் தேர்வை பாதித்து கம்ப்யூட்டர் சயின்ஸ்-ற்கு மாற்றி விட்டது.

வளமான பள்ளிகளில், முகநூலில் நண்பர்களாக்கிக்கொள்வது ஒத்துக்கொள்ளப்பட்டாலும், வாட்சப் என்று வரும்போது, ஆசிரியர்களும் மாணவர்களும் எச்சரிக்கையை கடைபிடித்தனர். ஆசிரியர்கள் தங்கள் போன் எண்களை மாணவர்க- 
ளுடன் பரிமாறிக்கொள்வதில் அதிக ஆர்வம் காட்டவில்லை. இந்த உணர்வு பரஸ்பரமான ஒன்றாக இருந்தது. ஆசிரியர்கள், மாணவர்கள் மொபைல் போன் உபயோகிப்பது பற்றி மட்டுமல்லாமல், பெருமளவு, பெற்றோர்கள் தங்களை அடிக்கடி அழைத்து பள்ளிப்பாடங்களில் தங்கள் பிள்ளைகளின் செயல்திறன் பற்றி கேட்டு தொந்திரவு செய்யக்கூடும் என்று அச்சப்பட்டனர். ஆகவே ஆசிரியர்கள் மற்றும் மாணவர்களிடையே வாட்சப் விருப்பமான தகவல் பரிமாற்ற வழிமுறையாக காணப்படவில்லை.

கீழ்மட்ட சமூூகப்பொருளாதார வகுப்பு பின்னணியில் இருந்து வரும் மாணவர்களுக்கு, அவர்களின் ஆசிரியர்கள் (பெரும்பாலும் ஆண் ஆசிரியர்கள்) போன் எண்களை பரிமாறிக்கொள்வதில் வெளிப்படையாக இருந்தாலும், மொபைல் இணையதள அணுகல் கட்டணங்களால், வாட்சப் உபயோகம் மிகவும் குறைவாகவே இருந்தது. மொபைல் இணைய தொடர்பு சந்தாதாரராக இருப்பவர்களின் வாட்சப் உபயோகம் கூட, அவர்களது தொடர்பில் யார்யார் உபயோகிக்கிறார்கள் என்பதை பெருமளவு பொறுத்திருந்தது. இந்த மாணவர்கள் கல்லூரிக்கு சென்றபின் தான் அது மிகப்பெரிய தகவல் பரிமாற்ற வழிமுறையாக ஆனது. இருப்பினும் களப்பணியில் இறுதிக்கட்டங்களில், இது மாறுவதாக தெரிந்தது. கீழ்மட்ட வகுப்புகளில் இருந்து வரும் ஆண் மாணவர்கள் பலர், வாட்சப்பை தகவல் பரிமாற்ற தளமாக தத்தெடுத்துக்கொண்டனர்.

சமூக ஊடகங்களில், ஆசிரியர்-மாணவர் உறவுமுறையை அலசுவது, மிகவும் முக்கியமானது. ஏனெனில், முதன்முறையாக இப்போது தான் பாரம்பரிய அதிகாரப்படிநிலையை தகர்க்கும் இடப்பரப்பில் இந்த உறவுமுறை சோதிக்கப்படுகிறது. மேலே பார்த்தபடி, இது இன்னும் சோதிப்பு செய்முறையில் தான் இருக்கிறது. ஆசிரியர்கள் மற்றும் மாணவர்கள் இருவருமே, தங்கள் தனிப்பட்ட வாழ்வைப்பற்றிய விவரங்களை வெளிப்படுத்துவதில் எச்சரிக்கையாக இருக்கின்றனர் என்பது தெளிவாகிறது. இது சம்பிரதாயமான பள்ளிச்சூழலில் அபூர்வமாக தான் காணக்கிடைக்கிறது. குழந்தைகளின் சமூக ஊடக நடவடிக்கைகள் என்று வரும்போது, ஆசிரியர்கள் மற்றும் பெற்றோர்களிடையே ஒரு இயல்பான பதட்டம் இருப்பதை காண முடிகிறது. இருப்பினும், பெற்றோர்கள் மற்றும் பள்ளிகளின் சமூக ஊடகங்கள் மீதான உறவுமுறையும் முக்கியமானதே. ஏனெனில், தங்கள் குழந்தைகளின் சமூக ஊடக நடவடிக்கை பற்றிய விசாரத்தை வெளிப்படுத்திய அதே பெற்றோர்கள் தான், 
பள்ளிகளுடன் தொடர்பில் இருப்பதற்கான சௌகரியமான தளமாக முகநூலை கருதுகிறார்கள், அவர்களை பாதிக்கவும் செய்கிறார்கள்.

\section{சமூக ஊடகங்களும் பெற்றோர் ஆசிரியர் கழகங்களும்}

சுபாஷினி என்பவர் 37 வயதானவர். பஞ்சக்கிராமியில் உள்ள வளமான பள்ளி ஒன்றில் பயிலும் இரண்டு குழந்தைகளின் அன்னை. 11 வயதான மூத்த குழந்தை ஆறாவது வகுப்பிலும், 8 வயதான இளைய குழந்தை மூன்றாவது வகுப்பிலும் படிக்கின்றனர். சுபாஷினியும் அவரது கணவரும், எட்டு ஆண்டுகள் அமெரிக்காவில் வாழ்ந்த பிறகு, சில வருடங்களுக்கு முன் தான் இந்திய திரும்பியுள்ளனர். அவரது குழந்தைகள் பஞ்சக்கிராமியில் உள்ள பள்ளியில் சேர்க்கப்பட்ட பிறகு, அதன் பெற்றோர் ஆசிரியர் கழகத்தில் தான் ஒரு சுறுசுறுப்பான பங்கு வகிக்க வேண்டும் என்று அவர் விரும்பினார். இருப்பினும் அமெரிக்கப்பள்ளிகளில் உள்ள பெற்றோர் ஆசிரியர் கழகங்களில் அவர் பங்கெடுத்துக்கொண்டதுடன் ஒப்பிடும் போது, இந்தியாவில் நிறைய கட்டுப்பாடுகள் இருப்பதை அவர் கண்டறிந்தார். பெற்றோர் தங்கள் குழந்தைகளின் கல்வி வளர்ச்சியில் தனித்தனியே ஈடுபட்டிருந்தாலும், ஒன்றுபட்ட கழகமாக அது இல்லாததையும் அவர் கண்டுகொண்டார்.

பதிலாக சுபாஷினி, இரண்டு முகநூல் குழுக்களை அமைத்தார். ஒவ்வொன்றும் அவரது ஒவ்வொரு குழந்தையின் வகுப்பில் உள்ள மற்ற குழந்தைகளின் பெற்றோரை உள்ளடக்கியிருந்தது. இந்தப்பள்ளியில் உள்ள கல்விமுறையில் ஏதேனும் மாற்றங்கள் செய்யப்படவேண்டுமா என்று விவாதிப்பது தான் இந்தக் குழுக்களின் குறிப்பிட்ட நோக்கமாக இருந்தது. மேலும் அவர்கள் குழந்தைகளின் வீட்டுப்பாடங்களை விவாதிக்கவும், எதிர்கால நிகழ்ச்சிகள் மற்றும் ஒன்றாகக் கூடுவெது பற்றிய உரையாடல்களுக்காகவும் இந்த குழுக்களை உபயோகிக்க கருதியிருந்தனர். இந்தக்குழு தொடக்கத்தில் மேற்கூறிய காரணங்களுக்காக சுறுசுறுப்பாக இயங்கினாலும், விரைவில் தாய்மார்கள் சமையல் மற்றும் புடவைகளை பற்றி கிசுகிசுக்கும் இடப்பரப்பாக மாறிவிட்டது. இந்தப்பள்ளியில் உள்ள சில ஆசிரியர்களைப்பற்றி புறம்பேசுவதும் நடந்தது. இது அன்னையர்கள் மட்டுமான குழுவாக இருப்பதால், இதன் குவியம் மாறிவிட்டதாக சுபாஷினி 
கருதினார். பெற்றோர் இருவரும் பங்குபெறும் முகநூல் குழுக்கள் இருப்பதை சுபாஷினி அறிவார். அவை சரியாக செயல்படுவெதாக தெரிந்தது.

பள்ளியின் தலைமை ஆசிரியர், பல குழுக்களிலான பெற்றோர்கள் தங்கள் பிள்ளைகளின் பாடங்களைப் பற்றி விவாதிப்பதற்காக முகநூல் குழுக்களை அமைத்திருப்பதையும் அவர்களில் சிலர் உண்மையான அக்கறை கொண்டிருப்பதையும் கண்டுகொண்டார். இதுபோன்ற விசாரங்கள் வெவ்வேறு வழிகளில் வெளிப்படுத்தப்படும்போது அவற்றை கவனிப்பது கடினமாகையால், இதுபோன்ற குழுக்களை ஒழுங்குபடுத்துவது அவசியமாகிப்போனது. அவர், சுபாஷினி போன்ற பெற்றோர்களை, உயர்ந்த அளவிலான பெற்றோர் ஆசிரியர் செயற்குழுவாக செயல்படக்கூடிய முகநூல் குழு ஒன்றை அமைக்குமாறு ஊக்குவித்தார். அதில் பள்ளியின் பிரதிநிதிகளாக பல ஆசிரியர்களையும் பங்கெடுக்க வைத்தார். இந்தவகையில் தகவல் பரிமாற்றங்கள் நெறிப்படுத்தப்பட்டு, விசாரங்கள் உயிர்ப்புடன் கவனிக்கப்பட்டன. விரைவில் சுபாஷினி குழு நிர்வாகிகளில் ஒருவராக பொறுப்பேற்றுக்கொண்டதுடன் இது நிதர்சனமாயிற்று. இநந்க்குழு, அதிக ஒழுங்குமுறையுடன் செயல்படுவொகவும், ஒரு சமூக உணர்வை உருவாக்கி இருப்பதாகவும் அவர் கருதுகிறார். பல உறுப்பினர்கள் முகநூல் வழியாக மட்டுமல்லாமல், வாட்சப் மூலமாகவும் தகவல் அனுப்பத் துவங்கியிருக்கிறார்கள். இந்தக்குழு பல உப குழுக்களை உருவாக்கியிருக்கிறது. தங்கள் குழந்தைகளுக்கு பள்ளியில் மத்திய உணவு கொண்டுவரும் அன்னையர் (குறிப்பாக இல்லத்தரசிகள்) குழு, தந்தையர்களின் கிரிக்கெட் குழு (ஒவ்வொரு சனிக்கிழமையும் குழந்தைகளுடனான கிரிக்கெட் விளையாட்டு பற்றியது), மற்றும் வழிகாட்டிகள் குழு (பெருநிறுவனங்களின் பணிபுரியும் தந்தையர்கள், குழந்தைகளுக்கு வாழ்வியல் திறன்கள் பற்றி வழிகாட்டும் குழு) போன்றவை இவற்றில் அடக்கம். இந்தக்குழுக்கள் முகநூல் மற்றும் வாட்சப் ஆகிய இரண்டிலுமே சுறுசுறுப்பாக செயல்பட்டுக்கொண்டிருக்கின்றன.

பள்ளி, அதன் பங்காக, ஒரே ஒரு நிகழ்நிலை தகவல் பரிமாற்ற வழி மற்றும் விசாரங்களை கவனிப்பதற்காக மாதாந்திர நேருக்கு நேர் கூட்டங்கள் ஆகியவற்றை மட்டுமே ஊக்குவிக்கிறது. இது முகநூல் மூலமான தகவல் பரிமாற்றத்தை ஊக்குவித்தாலும், வாட்சப் மூலமான தகவல் பரிமாற்றத்தை வலுவாக தவிர்க்கிறது. ஏனெனில், இதற்கு ஆசிரியர்களின் தனிப்பட்ட மொபைல் எண்கள் பகிரப்பட 
வேண்டும். இதனனால் பள்ளி நேரம் தாண்டிய பெற்றோர்களின் கேள்விகளுக்கு ஆசிரியர்கள் பதில் சொல்லவேண்டிய அவசியம் ஏற்படக்கூடும். பள்ளியின் கண்ணோட்டத்தில், இதுபோன்ற நிகழ்நிலை தகவல் பரிமாற்ற வழிமுறைகளை நெறிப்படுத்துவது கல்விமுறைகளை பற்றிய ஆரோக்கியமான விவாதங்களுக்கும் பாடத்திட்டம் பற்றிய கலந்துரையாடல்களும் வழிவகுக்கும். இது தனிப்பட்ட ஆசிரியர்களை கேள்வி கேட்பதையும், வேறு வகையான பழிபோடும் வழக்கங்களையும் கட்டுப்படுத்தி நெறிப்படுத்தும்.

\section{மாற்று சமூக ஊடகம் - ஒரு வளமான பள்ளியில் இருந்து வந்துள்ள வழக்காய்வு.}

பள்ளிகள் எவ்வாறு குழந்தைகளை சமூக ஊடகங்களை பயன்படுத்துவதில் இருந்து ஊக்கங்கெடுக்கிறது என்ற வழக்குகளை அலசும் சமயம், அதிக வளமுள்ள பள்ளிகளை கருதும் போது நிலைமை சிக்கலாகிறது. இந்தப்பள்ளிகள், குழந்தைகளை சமூக ஊடகங்களில் இருந்து தள்ளி வைக்க வேண்டாம் என்ற நவீனத்தேவையை ஒத்துக்கொள்கின்றன. ஆனால் அதேசமயம், அவர்கள் சங்கடமான, தடுமொற்றமான, ஆரோக்கியமற்ற மற்றும் ஆபத்தான சந்தர்ப்பங்களில் சிக்கிக்கொள்ளாமல் இருப்பதற்காக அவற்றை பொறுப்பாக பயன்படுத்த வேண்டியதன் அவசியத்தை உறுதிப்படுத்துகின்றன. இந்தப்பள்ளிகள் அவற்றின் தொகுதிகளிடையே உரையாடலுக்கான பொதுவான அமைப்பு இருப்பதன் சாதகங்களை உணர்ந்துகொண்டு, இதுபோன்ற ஊடாடு அமைப்பை உருவாக்குவதற்கு பலவகைகளில் முயன்று வருகின்றன. இது ஆய்வுக்களத்திற்கு வெகு அருகில் அமைந்திருக்கும் அதிக வளமான பள்ளி ஒன்றின் வழக்காய்வின் மூலம் எடுத்துரைக்கப்பட முடியும். பஞ்சக்கிராமியில் உள்ள பல வளமான குடும்பங்களில் உள்ள குழந்தைகள் அங்கு பயிலுகிறார்கள்.

இந்தப்பகுதியில் உருவாகியுள்ள வளமான சர்வதேசப் பள்ளிகளில் டிஎம்ஜி-யும் ஒன்று. இதன் மூத்த நிர்வாகத்தை அமைத்திருக்கும் தொழில்முனைவர் குடும்பத்தின் மூளைக்குழந்தையான இந்தப்பள்ளி, இருபதாண்டுகளுக்கு மேலாக செயல்பட்டுக்கொண்டிருக்கிறது. இந்த காலகட்டத்தில், இது உள்ளூர் பாடத்திட்டத்தை பின்பற்றும் வழக்கமான பள்ளியில் இருந்து அதன் இப்போதைய வடிவான சர்வதேச பாடத்திட்டத்தை பின்பற்றும் பள்ளியாக உருமாறியுள்ளது. இந்தியாவில் 
உள்ள சர்வதேச பள்ளிகள், வழக்கமான பள்ளிகளில் இருந்து அவர்களின் பாடத்திட்டத்தில் மாறுபடுகின்றன. இது நாடு குறித்ததாகவோ (உதாரணத்திற்கு அமெரிக்க சர்வதேச பள்ளி) அல்லது உலகளாவியதாகவோ (உதாரணத்திற்கு ஐஜிசிஎஸ்இ அல்லது ஐபி)இருக்கக்கூடும். இந்த பாடத்திட்டத்தின் சவால்களை சந்திப்பதற்கு, மற்ற உள்ளூர் பள்ளிகளிலிருந்து மாறுபட்ட அணுகுமுறையில் கல்வியை அணுக வேண்டும். இதன் ஒட்டுமொத்த அணுகுமுறையும், ஒரு குழந்தையின் வளர்ச்சியில் அறிவுக்கூறு, உணர்வுபூர்வமான பதில்கள், புலன்கள் சார்ந்த வளர்ச்சி, அசைவு உய்த்துணர்வு, தனிமனித தகவல் பரிமாற்றம் உள்ளிட்ட பல கூறுகளையும் போஷிக்கும் வகையில் செலுத்தப்படுகிறது. இதுபோன்ற பன்முக வளர்ச்சியை சாதிப்பதென்பது, நடவடிக்கை அடிப்படையிலான கற்றல் (வகுப்பு நடவடிக்கைகள், ம்ன்னிலைப்படுத்தல்கள் மற்றும் காட்சிப்படுத்தல்கள் மூலம் கற்றல்), ஒத்தவர்களுக்கிடையேயான வழிகாட்டல் (மூத்த மாணவர்கள், இளையவர்களுக்கு அடாவடித்தனம், கழிவு மேலாண்மை போன்ற சமூக மற்றும் குடிமை நல விசாரங்களைப் பற்றி எடுத்துரைப்பது) மற்றும் ஐசிடி-க்களின் விரிவான பயன்பாடு போன்ற பலவகையான பயிற்றுவிக்கும் அணுகுமுறைகளை சார்ந்திருக்கிறது.

ஐசிடி-க்களின் பயன்பாடு, இந்தப்பள்ளிகளில் குறிப்பிடத்தக்க வகையில் தெரிகிறது. அனைத்து வகுப்பறைகளும், ஸ்மார்ட் பலகைகள் மற்றும் கணினிகள் மூலம், இணையதள தொடர்பு கொண்டிருக்கிறது. முழுமையாக ஆயத்தப்படுத்தப்பட்ட கணினிக்கூடமும் இருக்கிறது (குழந்தைகள் நேரடியாக கையாள்வதற்கு ஏ ஏற்ப). அதோடு, ஆசிரியர் ஓய்வறைகளில் இணையதள தொடர்புடனான கணினிகள் (ஆசிரியர்களின் சொந்த ஆராய்ச்சிக்காகவும் மாணவர்களின் சிறிய குழுக்களுக்கு எப்போதாவது பயிற்றுவிப்பதற்காகவும்). மாணவர்கள் தங்களது வகுப்பறை பாட செயல்திட்டங்களுக்காக ஆராய்ச்சி செய்வது போல, ஆசிரியர்களும், தங்களது பாட திட்டங்களை வரைவதற்காகவும், தேர்வுக்கான வினாத்தாள்களை தயாரிப்பதற்காகவும், வகுப்பறையில் செய்ய வேண்டிய பயிற்சித்தாள்களை தயாரிப்பதற்காகவும், நிகழ்நிலையில் ஆராய்ச்சி செய்வதற்கு ஊக்குவிக்கப்படுகிறார்கள். பெற்றோருக்கு தெரிவிக்கப்படவேண்டிய முக்கியமான விஷயங்கள், தனிப்பட்ட முறையில் மாணவர்களின் பள்ளி மின்னஞ்சலுக்கு அனுப்பப்படுகிறது (ஒவ்வொரு மாணவருக்கும் அவருக்கென தனிப்பட்ட 
பள்ளி மின்னஞ்சல் கணக்கு உண்டு), ஆசிரியர்களுக்கான குழு அஞ்சலும், மின்னஞ்சல் மூலமே அனுப்பப்படுகிறது.

இந்தப்பள்ளியின் ஐசிடி வட்டத்தில் உள்ள ஒரு புதிய முயற்சி, நிகழ்நிலை கணிப்பீடு உபகரணம் (பள்ளியின் இயக்குனர் மற்றும் வெளியில் உள்ள சகபணியாளர்களால் பள்ளியினுள்ளேயே உருவாக்கப்பட்டது). இது நிகழ்நிலை தேர்வுகள் மற்றும் உருவாக்க கணிப்பீடுகளை, ஆசிரியர்கள் மற்றும் மாணவர்கள் இருவருக்குமே சுலபமானதாகவும் உள்ளுணர்வு சார்ந்ததாகவும் ஆக்க முயல்கிறது. முழுமையாக அலசி ஆராயும், ஒரு ஆசிரியர் குழு தான், மாறுபாடான தேவைகள் உள்ள குழந்தைகளுக்கு வழக்கமான வகுப்பறைகளிலும் சிறப்புத்தேவை இருக்கும் குழந்தைகளுக்கான சிறப்பு வளஆதார மையங்களிலும் உதவும் சிறப்பு பயிற்றுவிப்பாளர்கள். இந்த ஆசிரியர்கள் ஐசிடி-க்களை, பாரம்பரிய வாய்வழி பயிற்றுவிப்பு முறைகளுக்கு பிரதிபலிக்காத குழந்தைகளுக்கு வெவ்வேறு புலன்சார்ந்த அனுபவங்களை, அளிப்பதற்கு ஏற்ற சிறந்த தளமாக பார்க்கிறார்கள். இது போன்ற ஆசிரியர்களுக்கு ஐசிடி-க்கள், அவர்களின் திறன் சார்ந்த துறைகளில், சமீபத்திய நிகழ்வுகளை அறிந்துகொள்ளவும், வழக்கமான பாடத்திட்டம் ஒத்துவராத மாணவர்களுக்கு ஏற்ற பயிற்றுவிப்பு முறைகளை பற்றி ஆராய்வதற்கும் உதவுகிறது.

பள்ளியினுள்ளேயே, கற்றல் மற்றும் பிரதிபலித்தலை தூண்டுவதற்கு நிறைய நடவடிக்கைகளை சார்ந்திருக்கும் வகுப்பறை அடிப்படையிலான பயிற்றுவிப்புமுறையை ஐசிடி-க்கள் பூர்த்திசெய்கின்றன. இந்த நடவடிக்கைகள், அந்த வகுப்பு நேரம் முழுதும் வகுப்பினுள்ளான நடவடிக்கைகளில் (விவாதங்கள், முன்னிலைப்படுத்தல்கள் போன்றவை) இருந்து கண்காட்சிகள், பாட செயல்திட்டங்கள் போன்ற நீண்டகால (இரண்டு நாள் முதல் ஒரு மாதம் வரையிலானவை) நடவடிக்கைகள் வரை பல வகைப்படும். இதுபோன்ற முக்கிய நடவடிக்கைகளில் ஒன்று, பொதுவான கருப்பொருள் கொண்ட, பள்ளி முழுமைக்குமான இரண்டு நாள் கண்காட்சி. இதற்கான தயாரிப்புகள், பல வாரங்களுக்கு முன்பிருந்தே துவங்கிவிடுகின்றன. இந்த நடவடிக்கைகள், பள்ளியின் முகநூல் பக்கத்தில் இடப்படும் தொடர்ச்சியான பதிவுகளின் மூலம் வெளியுலகுக்கு காட்சிப்படுத்தப்படுகிறது. இது பள்ளியின் முன்னாள் மாணவர்கள் மற்றும் ஆசிரியர்கள் போன்ற வழக்கமான பார்வையாளர்களிடம் இருந்து அதிக அளவில், விருப்புக்களும் கருத்துப் பதிவுகளும் பெறுகிறது. 
இந்த சர்வதேசப் பள்ளி, தனது சொந்த அகஇணைய சமூக வலைத்தொடர்பு வலைத்தளத்தை உருவாக்கியுள்ளது. இது பொதுநோக்கிய சமூக வலைத்தொடர்புத் தளங்களின் அனுபவத்தை, கட்டுப்பாடான சூழலில் அளிப்பதை குறிக்கோளாக கொண்டு செயல்படுகிறது. "ரிலேட்" என்ற பெயர் கொண்ட இந்த வலைத்தளம், பள்ளியின் பணியாளர்களுக்கும் மாணவர்களுக்கும் மட்டுமானது. இதற்கான நுழைவுரிமை, பள்ளியினால், உள்நுழைவு உபயோகிப்பாளர் பெயர்கள் மற்றும் கடவுச்சொற்களால் கட்டுப்படுத்தப்படுகிறது. மேலும், இந்தப்பள்ளி, ஒவ்வொரு மாணவருக்கும் பள்ளியில் சேரும் சமயம் ஒரு மின்னஞ்சல் கணக்கு உருவாக்கி அளிப்பதால், இந்த சமூக வலைத்தொடர்பு வலைத்தளம் அதன் மூலமாகவே அணுகுமாறு அமைக்கப்பட்டிருக்கிறது. இது ஆசிரியர்களும் மாணவர்களும் கல்வி மற்றும் சமூக காரணங்களுக்காக ஒருவரோடொருவர் உரையாடிக்கொள்வதற்கான மன்றமாக விளங்குகிறது. இந்த வலைதளத்தின் அமைவு, முகநூலை ஒட்டியதாக, ஒவ்வொரு உபயோகிப்பாளரையும், தங்கள் சுவரில் பதிவிட, மற்ற உறுப்பினர்களுடன் நட்புதொடர்பு ஏற்படுத்திக்கொள்ள, குழுக்கள் மற்றும் மன்றங்கள் போன்றவற்றை உருவாக்க, உரையாடல் தொடர்களை துவங்க அனுமதிப்பதாகவும் இருக்கிறது. இருப்பினும், இந்த வலைத்தளத்தில் கடைபிடிக்கப்படவேண்டிய பொருத்தமான நடத்தை முறைகள் மற்றும் ஒழுங்குமுறை விதிகளை மீறினால் வரக்கூடிய பின்விளைவுகள் ஆகியவை பற்றிய விரிவான மற்றும் தெளிவான அறிவிப்போடு, இதன் உபயோகம் கவனமாக கண்காணிக்கப்படுகிறது.

இந்த அகஇணைய சமூக வலைத்தொடர்பு வலைதளத்தின் முக்கிய நோக்கமே, குழந்தைகளுக்கு, குறிப்பாக ஐந்து முதல் ஒன்பதாவது வரையிலான வகுப்புகளில் படிப்பவர்களுக்கு, முகநூல் போன்ற வெளிநோக்கிய சமூக வலைத்தொடர்பு வலைத்தளத்தை எவ்வாறு பொறுப்புடன் கையாள வேண்டும் என்று பயிற்சியும் குறிப்புரைகளும் அளிப்பதற்காக தான். மேலும், நிர்வாகமும் ஆசிரியர்களும், இந்த சமூக வலைத்தொடர்பு வலைதளத்தின் ஒப்புருவாக்கத்தை இரு-வழி கற்றல் அனுபவமாக அதாவது, இருபக்கத்தாரும் ஐசிடி-க்கள் மூலம் அறிவுத்திறன் பெறுவது, பார்க்கிறார்கள்.

மாணவர்களும், ஆசிரியர்களும், இந்த வலைத்தளத்தை, பலவிதங்களிலும் பல நோக்கங்களுக்காகவும் பயன்படுத்துகிறார்கள். விழாக்கள் அல்லது விடிமுறைகளுக்கான சமூக வாழ்த்துச் செய்திகள் மாணவர்களிடையே பொதுவான ஒன்று. பள்ளிசிப்பந்திகளும், மற்ற சிப்பந்திகளுடன் நட்புதொ- 
டர்பு ஏற்படுத்திக்கொண்டு, இநந்த வலைத்தளத்தில் அவர்களுடன் சமூக உரையாடல்களை வைத்துக்கொள்கிறார்கள். ஆசிரியர்களும் இந்த வலைத்தளத்தை, பாட செயல்திட்டங்கள், மாணவர்கள் நடத்தும் கண்காட்சிகள் போன்ற கல்விசார் நிகழ்ச்சிகளைப் பற்றி கருத்துதெரிவிப்பதற்காக பயன்படுத்துகிறார்கள். பதிலுக்கு மாணவர்கள், மற்ற மாணவர்கள் மற்றும் ஆசிரியர்களிடையே ஆர்வத்தை தூண்டக்கூடிய விசாரங்களைப் பற்றிய தேர்தலை நடத்துகிறார்கள். இதன் மூலம் ஜனநாயக நடைமுறை பற்றிய ஒரு முன்னோட்டத்தை அவர்களுக்கு அளிக்கிறார்கள். இறுதியாக வீட்டுப்பாடங்களும் இந்த வலைத்தளத்தில், மாணவர்கள் செய்து முடிப்பதற்காக பதிவிடப்படுகின்றன. இவற்றுக்கான உடனடி தெளிவாக்கங்கள், அவற்றுக்குரிய ஆசிரியர்களுடனான அளவளாவல்களின் மூலம் சாத்தியமாக்கப்படுகிறதது. உறுப்பினர்களுக்கு இடையேயான உரையாடல்களை எளிதாக்குவதோடு, "ரிலேட்" உபயோகிப்பாளர்களை காணொளிகள் (பெரும்பாலும், கல்வி சார்ந்தவை. ஆசிரியர்களால் மட்டுமே பதிவிடப்படக்கூடியவை), புகைப்படங்கள் (ஆசிரியர்கள் மற்றும் மாணவர்களால் பதிவிடப்பட்டவை) மற்றும் வலைத்தளங்கள் மற்றும் வலைப்பதிவுகள் போன்ற வெளி தோற்றுவாய்களுக்கான தொடர்புச்சுட்டிகள் போன்றவற்றை பதிவிடிவதற்கும் அனுமதிக்கிறது.

மேலும், நிர்வாகத்தினர் ஒத்துக்கொள்வது போல, “ரிலேட்" ஐந்தாம் வகுப்பு மற்றும் அதற்கு மேற்பட்ட அனைத்து மாணவர்களுக்கும் பொதுவானது மற்றும் தன்விருப்பார்ந்தது என்றாலும், இது மூத்த மாணவர்களை விட, அதிகமாக நடுநிலைப்பள்ளி மாணவர்களால் மட்டுமே பயன்படுத்தப்படுகிறது. மேலும், ஆண்களோடு ஒப்பிடும் போது, பெண்கள் இந்த வலைத்தளத்தை உபயோகிக்கும் பாணியில் ஒரு வித்தியாசம் காணப்படுகிறது. ரிலேட்ல் பெண் மாணவர்களின் பெரும்பாலான பதிவுகள், ஓவியங்கள் மற்றும் ஆக்கபூர்வ வடிவமைப்பு பற்றியதாக இருக்கிறது. அதே சமயம், ஆண் மாணவர்களின் பதிவுகள், கால் பந்து, பேஸ்பால் போன்ற விளையாட்டுக்களை பற்றியதாக இருக்கிறது.

இந்த வலைத்தொடர்பு வலைதளத்தின் உள்நுழைவு பக்கத்தின் படம் கீழுள்ள படம்: 6.1-ல் காட்டப்பட்டுள்ளது.

ஐசிடி-க்களின் விரிவான பயன்பாடு, பள்ளியின் தகவல்தொழில்நுட்ப (ஐடி) குழுவுக்கும் சவாலாக இருக்கிறது. இந்தக்குழு இப்போது ஐசிடிதொடர்பான பிரச்சினைகளான இணையவெளி அடாவடித்தனம், பொருத்தமற்ற படங்களை பதிவிடுதல், பொதுவான வலைத்தொடர்பு தளத்தில் பிற 


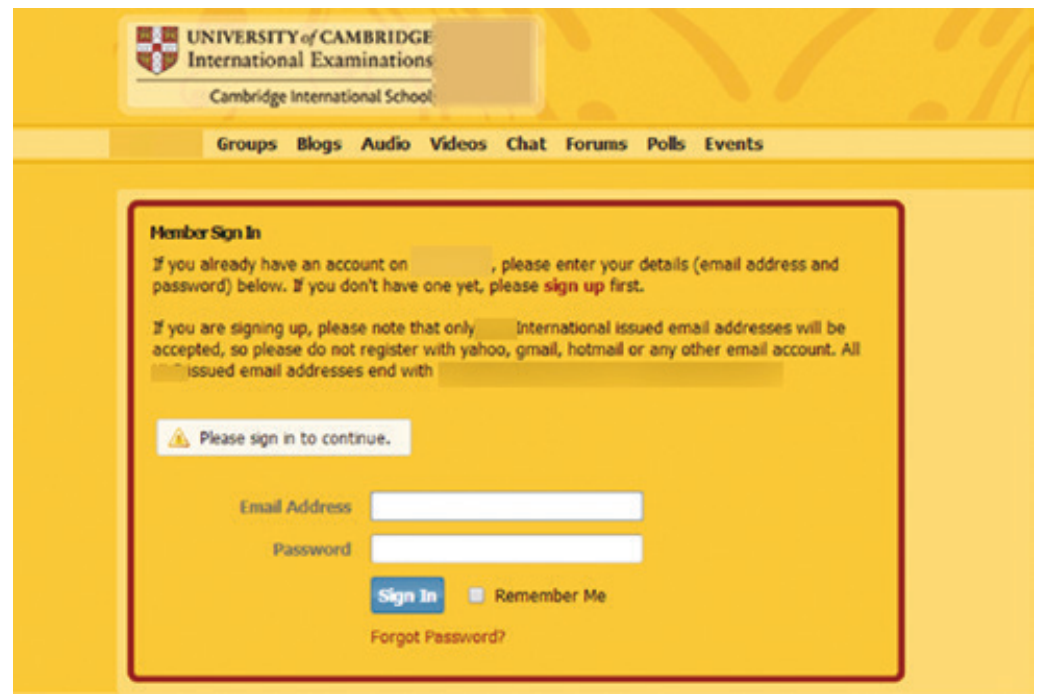

படம்: 6.1 அகஇணைய பள்ளி வலைத்தொடர்பு வலைதளத்தின் உள்நுழைவுப் பக்கம்

ஊடகங்கள், வகுப்பறை அல்லது கல்வி தொடர்பான ஆராய்ச்சிகள் தவிர மற்ற நோக்கங்களுக்காக இணையதளத்தை பிரயோகிப்பது போன்றவற்றுக்கு ஈடுகொடுக்க திணறிக்கொண்டிருக்கிறது.

\section{முடிவுரை}

இந்த அத்தியாயம், ரஞ்சித் மற்றும் பாண்டியன் என்ற இரு முற்றிலும் வேறான வழக்காய்வுகளை நோக்குவதன் மூலம் துவங்கியது. இருப்பினும், அன்றாட வாழ்வில் சமூக ஊடகங்களின் சிறிய அளவிலான உபயோகத்தை, கல்வி மற்றும் பிற சமூகப்பொருளாதார காரணிகளின் பெரிய அளவிலான கட்டமைப்புகள் பாதிக்கின்றன என்பது பற்றிய ஆழமான புரிதலுடன் அதே வழக்காய்வுகளை மீண்டும் நோக்கும்போது, இந்த அலசலின் கீழிருக்கும் பிரச்சினைகள் வெளிப்படுகின்றன. இவற்றை முழுமையாக புரிந்துகொள்வதற்கு, இந்தப்பகுதியில் உள்ள பள்ளிகளின் பன்முகத்தன்மையை, விலையுர்ந்த கட்டணமுள்ள வளமான பள்ளிகளில் இருந்து, குறைந்த வருமானமுள்ள மற்ற மாநிலங்களில் இருந்து வந்த புலப்பெயர்வு தொழிலாளர்களின் கல்வித் தேவைகளை பூர்த்திசெய்யும் 
இலவச அரசாங்க பள்ளிகள் வரை அனைத்தும் மதிப்பீடு செய்யப்படவேண்டும். இந்த அறிமுகம் ஏன் முக்கியமானது என்று எங்களால் இப்போது பார்க்க முடிகிறது. பள்ளி அமைப்புகளில் உள்ள சமூக ஊடக பயன்பாட்டின் ஒவ்வொரு கூறும், இந்த வட்டத்தின் நெடுகிலும், பெருநகரப்பள்ளிகளில் இருந்து உள்ளூர் பள்ளிகள் வரை, முறையாக வித்தியாசப்படிவதாக தெரிகிறது. இந்தச்சூழலில், சமூக ஊடக பயன்பாடு, ஆசிரியர்களின் மனப்பாங்கை மட்டுமல்லாமல், மாணவர்களின் மனப்பாங்கையும், அத்தோடு பெற்றோரின் பேரார்வங்கள் மற்றும் எதிர்பார்ப்புகள் ஆகியவற்றையும் சேர்த்துப் பிரதிபலிக்கிறது. புதிய ஐடி வளாகத்தின் வருகை, கருத்துக்களின் மேலிருந்து கீழ் சுமத்துதலை பிரதிபலிப்பது போல - "அறிவார்ந்த பொருளாதாரம்" உண்மையில் செய்வது போல, இது பதிலுக்கு ஒரு ஆய்வுக்களத்தின் பரந்த சூழலை பிரதிபலிக்கிறது. இவை சில சமயங்களில் பற்றாக்குறையாக புரிந்துகொள்ளப்பட்டாலும், மக்கட்தொகையின் அனைத்து துறைகளும், மிகக்குறைந்த வருமானமுள்ளவை உட்பட, கல்வி என்பது தொழில்நுட்பத்திற்கான வாய்ப்பாகவும் ஐடி தொடர்பான திறன்களாகவும் கருதப்பட்டால், தங்கள் குழந்தைகளின் எதிர்காலங்கள் மேம்படும் என்பதை அங்கீகரிக்கின்றன என்பது தெளிவாகிறது. ஒரு வகையில் பஞ்சக்கிராமியில் உள்ள ஒவ்வொருவரும் அறிவார்ந்த பொருளாதாரம் என்ற கருத்தை, எதோ ஒரு நிலையில் தழுவிக்கொண்டுவிட்டனர்.

இந்த அத்தியாயத்தில் நாங்கள், இது பள்ளிகளில் சமூக ஊடகங்களின் இருப்பின் மீது கொண்டுள்ள விளைவுகளையும் அலசியிருக்கிறோம். மொபைல் போன்கள் மற்றும் தொழில்நுட்பங்களின் அணுகல் மீதான பள்ளிகளின் மனப்பாங்கில் துவங்கி, ஆசிரியர்கள் மற்றும் மாணவர்களின் இடையே சமூக ஊடகங்களில் உள்ள தனிப்பட்ட தொடர்புகள் நோக்கி முன்னேறியிருக்கிறோம். சமூக ஊடகங்கள் மற்றும் தொழில்நுட்பங்களை பயனுள்ள கல்வி சாதனங்களாகவும், கவனச்சிதறல்களாகவும் வகைப்படுத்துதலும் கருதப்படவேண்டும். வளமுள்ள பள்ளிகளில் உள்ள ஆசிரியர்கள் ஏன் ஒருசில சமூக ஊடகங்களை பார்த்து முகம் சுளிக்கிறார்கள் என்றும் குறைந்த வளமுள்ள பள்ளிகளில் பணிபுரியும் ஆசிரியர்கள் அவற்றின் உபயோகத்தை ஊக்குவிக்கிறார்கள் என்றும் புரிந்துகொள்வதற்கு இது மிகவும் அவசியம் ${ }^{33}$. இந்த மாதிரியான வகைப்படுத்துதல், மாணவர்களின் பெற்றோர்கள், சமூக ஊடகங்களை எவ்வாறு பார்த்தார்கள் என்பதிலும் தெளிவாகிறது. கீழ்மட்ட சமூகப்பொருளாதார வகுப்பைச்சார்ந்த, 
தங்கள் பிள்ளைகளை குறைந்த வளமுள்ள பள்ளிகளுக்கு அனுப்பிய பெற்றோர்கள், தங்கள் பிள்ளைகளின் சமூக ஊடக ஈடுபாட்டை, பஞ்சக்கிராமி உருமாறியிருக்கும் அறிவார்ந்த பொருளாதாரத்தில் தொடர்ந்து வாழ தேவைப்படும் தொழில்நுட்ப ஆற்றலின் காட்சிப்படுத்தலாக பார்க்கிறார்கள். ஆனால் உயர்-மத்தியதர வகுப்பைச்சேர்ந்த பெற்றோர், சமூக ஊடகங்களை, கல்விக்கான கவனச்சிதறலாகத்தான் பார்க்கிறார்கள். ஒரு வகையில், இது அந்தப் பிள்ளைகள் படிக்கும் பள்ளிகள் சமூக ஊடகங்களை பார்த்த விதத்திற்கு ஒத்ததாக இருக்கிறது.

இருப்பினும், சமூக ஊடகங்களை புரிந்துகொள்ளுதல் மற்றும் கையாளுதல் என்ற கருத்து, பள்ளியமைப்புகள் முழுமையிலும் குழப்பமான கலவை தான் என்பது தெளிவாகிறது. எந்த பள்ளிகளிலும் தெளிவான சமூக ஊடக கொள்கைகள் காணப்படவில்லை. ஒருசிலவற்றில் இணையதள கொள்கைகள் இருந்தாலும், அவற்றில் சமூக ஊடகங்களை பற்றிய எந்த குறிப்புகளும் இல்லை. சமூக ஊடகங்களை பற்றிய விதிமுறைகள் எப்போதும் தளர்வாகவே இருந்தன ஏனெனில் பள்ளிகள், அவை எழுப்பிய மாறிக்கொண்டேயிருக்கும் விசாரங்களுடன் மன்றாடிக்கொண்டிருந்தது தான் காரணம். இறுதியாக அனைத்துப் பள்ளிகளும், சமூக ஊடகங்களின் தப்பமுடியாத ஊடுருவும் இருப்பை அங்கீகரித்தாலும், இந்தப் பள்ளிகள் எதையும் முன்கூட்டியே கலந்தாய்வு செய்து தேவையான நடவடிக்கைகளை எடுக்கவில்லை.

ஒருசில மாணவர்கள் முகநூலில் ஆசிரியர்களுடன் நண்பர்களாகிக்கொண்டிருந்த சமயத்தில், வளமான பள்ளிகளில், ஆசிரியர்களிடையே மாணவர்களை முகநூலில் நண்பர்களாக்கிக்கொள்வதை பற்றியும், அவர்களிடம் தொழில்நுட்ப உதவிகள் கேட்பது பற்றியும் பதட்டம், குழப்பம் எச்சரிக்கை ஆகியவை நிலவியது. மறுபுறம், குறைந்த வளமுள்ள பள்ளிகளில், சமூக ஊடகங்களில் நட்புதொடர்பு கொள்வது ஊக்குவிக்கப்பட்டு, செயல்படுத்தவும் பட்டது. ஆசிரியர்களை நண்பர்களாக்கிக் கொள்வது என்பது, 12 முதல் 15 வயதுள்ள நடுநிலைப்பள்ளி மாணவர்களிடையே காணப்பட்டாலும், மூத்த மாணவர்கள், தொடர்ந்த கண்காணிப்பு ${ }^{34}$ பற்றிய விசாரங்களால், அவ்வாறு செய்வதற்கு தயங்கினார்கள். இதுபோன்ற விசாரங்கள், வாட்சப் வழக்கில் மேலும் தெளிவாகத் தெரிந்தது. ஏனெனில் அது தனிப்பட்ட மொபைல் எண்களை பரிமாறிக்கொள்ளுவது சம்பந்தப்பட்டதாகையால் இருசாராருமே தொடர்பு கொள்ள தயங்கினர். இதுபோன்ற 
விசாரங்கள், பள்ளிகளில் ஆசிரியர்களின் போன் எண்களை பெற்றோருடன் பகிர்ந்து கொள்வதிலும் காணப்பட்டது. பள்ளி நேரம் தாண்டிய தொந்திரவு பற்றிய சாத்தியக்கூறுகளும், அந்தரங்கத்தின் மீதான குறுக்கீடும் இதத்கான காரணங்களாக இருக்கலாம்.

அந்தரங்கம் என்பது இந்த வழக்குகளில், கருதப்படும் சமூக ஊடகத்தின் அடிப்படையில் அடுக்குகளில் இருக்கிறது. முகநூல் அதிகாரபூர்வ நிறுவன தகவல்பரிமாற்றத்திற்கான முறையான தளமாக பார்க்கப்பட்டாலும், வாட்சப் பிரத்யேக வழிமுறையாகத்தான் பார்க்கப்பட்டது. இதன் விளைவாகவும், ஆசிரியர்களுடன் ${ }^{35}$ ஒருவருக்கொருவரான இடைவினைக்கு வழிவகுத்ததாலும் இது தடைவிதிக்கப்பட்டது. இந்த வட்டங்களில் உள்ளவர்கள் அதிகம் விவாதித்த தளங்களாக முகநூலும் வாட்சப்பும் காணப்பட்டாலும், டுவிட்டர் அனேகமாக கண்ணுக்கு புலப்படாமல் போய்விட்டது, விதிவிலக்கான ஒரே ஒரு வளமான சர்வதேச பள்ளியைத்தவிர. இருப்பினும், இந்தப்பள்ளியும், டுவிட்டர மற்ற தளங்களுடன் சேர்த்துதான் உபயோகப்படுத்தியது. வளமான பள்ளிகளில் உள்ள மாணவர்களிடையே, டிவிட்டர், முகநூல் மற்றும் வாட்சப் அளவிற்கு பிரபலமாக இல்லை. இது, இங்கிலாந்தில் உள்ள ஆய்வுக்களமான "தி க்ளேட்ஸ்"36 -ல் உள்ள மாணவர்களின் டுவிட்டர் உபயோகத்திலிருந்து முற்றிலும் மாறுபட்ட போக்காக இருந்தது.

கூடுதலாக, பாலினங்களுக்கிடையேயான நட்புதொடர்புகள் வளமான பள்ளிகளில் காணப்பட்டாலும், குறைந்த வளமுள்ள பள்ளிகளில், ஆண் ஆசிரியர்கள் மட்டுமே ஆண் மாணவர்களுடன் நட்புதொடர்பு கொண்டனர். இது, பெண் மாணவர்களுக்கான சமூக ஊடக மற்றும் மொபைல் போன் உபயோகம் குறித்த சமூக-கலாசார விசாரங்களின் விளைவாகும். நாம் ஆழமாக தோண்டத் தோண்ட, சமூக ஊடகங்களைப் பற்றிய பள்ளிகளின் கருத்துக்கள், பரந்த சமூகத்தின் சமூக-கலாசார கூறுகளுக்கு ஒத்ததாக இருப்பதையும், அவை பதிலுக்கு பள்ளிகளையே பாதிப்பதையம் நாம் உணரமுடிகிறது.

இதில் குறிப்பிடத்தக்கது என்னவென்றால், சமூக ஊடகங்களைப்பற்றிய இந்த தொடர்புடைய கருத்துக்களை பள்ளிகள் முரணான நடைமுறைகளை பின்பற்றி கையாளும் வழிமுறைகள் தான். ஒரு வளமான பள்ளி, தன் மாணவர்களுக்காக உருவாக்கிய சொந்த சமூக ஊடகத்தளத்தைப் பற்றிய கடைசியான வழக்காய்வு குறிப்பிடத்தக்கது. இந்த 
சந்தர்ப்பத்தில், தன் இளம் மாணவர்களின் சமூக ஊடக பயன்பாட்டை முகச்சுளிப்புடன் பார்த்து, அவர்களை எச்சரித்து, ஊக்கங்கெடுக்கும் வளமான பள்ளி, உண்மையில் அவர்களுக்காகவே, வேறொரு சமூக ஊடகத்தளத்தை புத்திசாலித்தனமாக உருவாக்கி, தன் மாணவர்களை, முகநூலில் இருந்து தூரமாக கொண்டு சென்றிருக்கிறது. இதற்கு மாறாக குறைந்த வளமுள்ள பள்ளிகள், சமூக ஊடகங்களை ஊக்குவித்து ஆதரித்தாலும், அவர்களின் உபயோகத்திற்கென்று புதிய தளத்தை உருவாக்கும் அளவிற்கு தேவையான பொருளாதார வழிவகைகளை பெற்றிருக்கவில்லை. இங்கு வெளிப்படும் தனித்து தெரியக்கூடிய முரண்பாடு என்னவென்றால், சமூக ஊடக பயன்பாட்டை பார்த்து முகம் சுளிக்கும் கல்வியமைப்பு, அதை ஆதரிக்கும் ஆனால் சமூக ஊடகத்தை பயன்படுத்த முடியாத கல்வியமைப்பிற்கு அ அடுத்தாற்போல் அமைந்திருப்பது தான்.

முடிவாக, சமூக ஊடகங்கள் என்பவை முன்னெப்போதும் இல்லாத ஒரு வளர்ச்சி. பள்ளிகள் அவற்றை தொடர்ந்து சோதித்து பரிசோதிப்பதன் மூலம் மெல்லமெல்ல அவற்றுடன் ஒரு உடன்படிக்கைக்கு வந்துகொண்டிருக்கின்றன. அவர்கள் அனைவருமே இதை தங்கள் சொந்த வகையில் கையாண்டு கொண்டிருக்கின்றனர். இது, ரஞ்சித் மற்றும் பாண்டியனின் வழக்குகளில் தெளிவாக தெரிந்தது போல, நிஜத்தில் அவர்கள் இணைந்திருக்கும் சமூக கலாச்சார சூழலுக்கு தொடர்புடையதாக இருக்கிறது. 


\section{7 \\ முடிவுரை: சமூக ஊடகங்களும் அவற்றின் தொடரும் சிக்கற்பாடுகளும்}

சுந்தர்ராஜன் என்பவர் 57 வயதானவர். வாகன உதிரி பாகங்களின் வியாபாரி. இவர் உயர்குலத்தை சேர்ந்தவர். உயர்-மத்தியதர வகுப்பின் இந்து சமய பின்னணியை உடையவர். இவர் பஞ்சக்கிராமியின் அடுக்குமாடி குடியிருப்பு வளாகங்கள் ஒன்றில் இரண்டு, இரண்டு படுக்கையறை வீடுகளுக்கு சொந்தக்காரர். அவரது நோக்கம் அவற்றில் ஒன்றை வாடகைக்கு விடுவது. ஆனால், உயர்குலத்தை சேர்ந்த, இந்து சமயத்தை சேர்ந்த சைவம் சாப்பிடுபவர்களுக்கு மட்டுமே. முதலில் சுந்தர்ராஜன் உள்ளூர் செய்தித்தாள்களில் உள்ள கிளாசிபைட் பகுதியை முயற்சித்தார். ஆனால் அவர் பெற்ற பதில்கள் அவருக்கு திருப்தியாக இல்லை. பின்னர் அவர், அதே குடியிருப்பு வளாகத்தில் இருக்கும் உயர்குலத்தை சேர்ந்த இந்துசமய அண்டை வீட்டவர்களை சந்தித்தார். அவர்களிடம் தனது வாடகை வீடு பற்றிய விளம்பரத்தை அவர்களின் முகநூல் மற்றும் வாட்சப் வலைத்தொடர்புகளில் பகிர்ந்து கொள்ளுமாறு கேட்டுக்கொண்டார். ஒரு சில மாதங்களுக்கு பின்னர், சுந்தர்ராஜன் ஒரு பேட்டியில், தன் அண்டை வீட்டாரின் பதிவுகளால், உயர்குலத்து-சைவம் உண்ணும்-இந்துசமயத்தவரிடம் இருந்து தான் சுமார் பத்து தகவல் விசாரிப்புகளை பெற்றதாகவும், தனது வீட்டை தனக்கு திருப்தியாக இருந்த ஒருவருக்கு வெற்றிகரமாக வாடகைக்கு விட்டிருப்பதாகவும் குறிப்பிட்டார். மேலும் அவர், தான் பெற்ற பதில்களால் தான் ஆச்சரியப்படவில்லை என்றும், தனது அண்டைவீட்டார் பெரும்பாலும், உயர்குலத்து இந்துசமயத்தை சேர்ந்தவர்களுடன் தான் பழகுகிறார்கள் என்பதை தான் அறி- 
ந்திருந்ததாகவும், முகநூலிலும் வாட்சப்பிலும் உள்ள அவர்களின் வலைத்தொடர்புகளும் அதேபோலத்தான் இருக்கும் என்று தான் கணக்கிட்டதாகவும் குறிப்பிட்டார்.

சுந்தர்ராஜனின் தற்செயலான கூர்நோக்கல், பேட்டிகள் மூலமாகவும், உயர்குலத்து இந்துசமயத்தவரின் சுயவிவரப்பக்கங்களின் நிகழ்நிலை காட்சியமைப்பு அலசல்களின் மூலமாகவும் உறுதிப்படுத்தப்பட்டது. சராசரியாக அவர்கள் நண்பர்கள் மற்றும் தொடர்பில் உள்ளவர்களில் 58 சதவிகிதத்தினர் அவர்களின் குலச்சமூகத்தை சேர்ந்தவர்கள். மற்றவர்களுடனான நிகழ்நிலை நட்புறவுகள் இருந்தாலும், பெரும்பாலானவை அவர்கள் இயல்புநிலையில் ஏற்கனவே அறிந்திருந்த மக்களுடன் தான். இது பேட்டிகளில் குறிப்பிடப்பட்டபோது, சிலர் ஆச்சரியப்பட்டனர், வேறுசிலர் அதிர்ச்சியடைந்தனர், மேலும் பலர் இந்தப்பாணியை நியாயப்படுத்தினர். ஆனால் அநேகமாக அனைவருமே, தங்களது இயல்புநிலை சமூக வட்டம் மற்ற குலத்தை சேர்ந்த நண்பர்களையும் கொண்டிருந்தாலும், பெரும்பாலானோர் தங்களது சொந்த குலச்சமூகத்தை சேர்ந்தவர்கள் தான் என்பதை ஒத்துக்கொண்டனர்.

இந்தத்தொடர்புகள், ஒரு குலம் சார்ந்த சமூகத்தை, அவை வரிசைப்படுத்தப்பட்ட வளர்ந்துவரும் வர்க்க காரணியுடன் இணைத்துவிட்டது. பேட்டிகள் வெளிப்படுத்தியது என்னவென்றால், அவர்களின் சொந்த குலத்திற்குள்ளாகவே அவர்களது முதன்மை தொடர்புகள் நடுத்தர வர்க்கத்தை சேர்ந்த மற்றவர்களுடன் தான் என்பதைத்தான். கீழ் வர்க்கத்தவருடன் தொடர்புகள் இருந்தாலும், அவை குறைவானதாகவும், சமூகச்சார்பை விட பெரும்பாலும் செயல்பாடு சார்ந்ததாகவும் தான் இருந்தன. உதாரணத்திற்கு, ஒருவர், தன் சொந்த குலத்தை சேர்ந்தவரான தனது சமையல்காரருடன், வாட்சப் தொடர்பில் இருந்தால், (அத்தியாயம் 5-ல் தனது சமையல்கார/ குழந்தை பராமரிப்பாளருக்கு வாட்சப் மூலம் குரல்வழி தகவல்கள் அனுப்பிய கவிதாவை போல) அது, அன்றைய இரவு உணவிற்கு என்ன சமைக்க வேண்டும் என்பதை விவாதிப்பதற்காகத்தான் இருக்கும். இது நிஜத்தில் சமூகத்தன்மையை நிறுவக்கூடிய வகையில், வேடிக்கைகள், மீம்கள் மற்றும் பிற காட்சியமைப்புகளை முன்னனுப்பிக்கொள்ளக்கூடிய ஒத்தவர்களுடனான நட்புறவை அமைத்துக்கொள்ளும் சமூக நடைமுறை அல்ல.

இந்தப்பாணி, பஞ்சக்கிராமியின் நீண்டகால குடியிருப்பாளர்களின், சமூக ஊடகத்தொடர்புகளிலும் காணப்பட்டது. 
அவர்களின் சொந்த கிராமத்தில் இருந்து வந்தவர்கள் கூட, அவர்களின் சமூகப்பொருளாதார வர்க்கத்தை சேர்ந்தவர்களாகவே இருந்தனர். சுந்தர்ராஜனின் வழக்கை போல தன்னுடைய வலைத்தொடர்புகளை சாமர்த்தியமாக உபயோகிப்பதும் காணப்படுகிறது. உதாரணத்திற்கு அத்தியாயம் 5-ல் தர்ஷன் மற்றும் நாகா என்ற கீழ்மட்ட சமூகப்பொருளாதார வகுப்பைச்சேர்ந்த இரண்டு இளம் ஆண்கள், தங்கள் நிகழ்நிலை வலைத்தொடர்புகளை, தங்கள் குலத்தைச்சேர்ந்த இளைஞர்களுக்கு ஐடி துறையின் கீழ்மட்ட படியணியில் உள்ள பணிகளைப் பெறுவதற்கு பயன்படுத்தினர் என்பதை நாம் அலசினோம். சுந்தர்ராஜன் தனது வலைத்தொடர்பை தனது தனிப்பட்ட தேவைகளுக்காக பயன்படுத்தினாலும், தர்ஷனும் நாகாவும் தங்கள் வலைத்தொடர்புகளை, தங்கள் குலத்தவரின் ${ }^{2}$ வளர்ச்சிக்காகவும் அவர்களின் இலட்சியங்களை நிறைவேற்றுவதற்காகவும், உபயோகித்தனர். இருந்தாலும், இறுதியில் அவர்களது வலைத்தொடர்புகளை தங்கள் நோக்கம் நிறைவேறுவதற்காகத்தான் அவர்களும் பயன்படுத்தியிருக்கின்றனர்.

வர்க்கம் குலம் இவற்றைத்தவிர, மற்றவகையான நிகழ்நிலை தன்குலச்சார்பு வ வலைத்தொடர்புகளும் இருக்கின்றன. இருப்பினும், இவையெல்லாம், இயல்புநிலை தொடர்புகளின் எதேச்சையான தேர்வின் பாதிப்பால் விளைந்தவையாக இருக்கின்றன. இதுபோன்ற வலைத்தொடர்பு தன்குலச்சார்பு, ஒருவகையான, "இயல்புநிலை மற்றவை" என்ற உணர்வை வலியுறுத்துகின்றன. அதாவது, ஒவ்வொரு குழுவும், மற்ற ஒவ்வொருவரையும், "மற்றதாக" பார்க்கிறது. பஞ்சக்கிராமியின் சமூககலாச்சார இயக்கவியல், அத்தியாயம் 1-ல் பார்த்தது போல, அநேகமான இடைவினைகள், குழுக்குள்ளான தொடர்புகளால் விளையும் சூழலில், இந்த மற்றவை என்ற கருத்திற்கு தானாகவே பங்களிக்கிறது. உயர்மதியதர ஐடி/ஐடி-அல்லாத பணியாளர்கள் மற்றும் பஞ்சக்கிராமியின் நீண்டகாலக்குடியிருப்பாளர்கள் ஆகியோரிடையே நடைபெறும் இடைவினைகள் (வர்க்கத்தைப்பற்றிய பொருட்டில்லாமல்), பரிமாற்றம் தொடர்பானவையாக இருக்கின்றன. உதாரணத்திற்கு, வணிகச்சந்தையில் வாங்குவது விற்பது தொடர்பாக நிகழும் பரிமாற்றங்கள் பற்றிய இடைவினைகள். இந்தப்பாணி, நிகழ்நிலையிலும் தொடர்கிறது. மற்றவை என்பது, வெளிப்படையாக செய்யப்படவில்லை என்றாலும், குழுவிற்குள்ளான தொடர்புகள், தானாகவே அதற்கு வழிவகுத்தது.

இந்தியாவில் சமூகச்சார்பு ${ }^{4}$ (அத்தியாயம் 1-ல் பார்த்தது போல) கிராமப்புற மற்றும் நகர்ப்புற இடப்பரப்பில் ஆராயப்பட்டி- 
ருந்தாலும், இந்தப்புத்தகம், நிகழ்நிலை மற்றும் இயல்புநிலை இடப்பரப்புகளுக்கிடையே உள்ள அதுபோன்ற சார்பை, அலசுகிறது. இந்த அலசலுக்கான சூழல், கிராமப்புறத்திலிருந்து நகர்ப்புற சூழலுக்கு மாறிக்கொண்டிருக்கும் ஒரு நிலப்பரப்பாகும்.

ஒருவகையில் இது, புதிய ஐடி துறை குடியிருப்பாளர்கள் மற்றும் நீண்டகால குடியிருப்பாளர்கள் என்ற இருவேறு வகைகளை அளித்த ஆய்வுக்களத்தின் ஆரம்பகால அமைப்பிற்கு ஒத்ததாக இருக்கிறது.

முதல் அத்தியாயம், விவசாயத்தின் ஆதிக்கம் நிறைந்த பாரம்பரிய கிராமிய இடப்பரப்பில் அறிவார்ந்த பொருளாதாரத்தை பேணுவதற்காக, உட்கட்டமைப்புகளின் பக்க அணிமைநிலையைக் கொண்ட ஒரு இடமான ஆய்வு நடத்தப்பட்ட பகுதியின் பின்னணியை விவரித்தது. பஞ்சக்கிராமி இப்போது, உட்கட்டமைப்புகளில் மட்டுமல்லாமல், மற்ற சமூகப்பொருளாதார காரணிகளிலும் பிரம்மாண்ட மாற்றங்களை சந்தித்து வருகிறது. இது, பழையன புதியனவற்றையும், உள்ளூர் உலகளாவலையும் சந்திக்கக்கூடிய இயக்கவியல் மற்றும் மாற்றம் ஆகியவற்றால் வகைப்படுத்தப்பட்ட, சிக்கலான நிலைமைக்கு அடித்தளம் அமைக்கிறது.

பஞ்சக்கிராமியின் நீண்டகால குடியிருப்பாளர்கள் மற்றும் குடியிருப்பாளர்களான ஐடி நிறுவனங்கள் மற்றும் அவை சார்ந்த இதர சேவை துறைகளில் பணிபுரிபவர்களான உள்வரும் குடியிருப்பாளர்களிடையே வர்க்கம் அடிப்படையிலான முறைசாரா மற்றும் மறைமுக வகைப்பாடுகள் இருந்தாலும், பஞ்சக்கிராமியில் உள்ள மக்கள் பழமையான அகமண உறவு வகையான குலத்தாலும் ${ }^{5}$ வகைப்படுத்தப்பட்டிருக்கிறார்கள். முதல் அத்தியாயத்தில் காட்டப்பட்டது போல், இந்த குலம் என்ற வகை, பல்வேறு பரிமாணங்களை கொண்டது. உதாரணத்திற்கு, மானுடவியலாளர்களால் 6 நீண்டகாலமாக ஆராயப்பட்டுவரும் அகமண நடைமுறைகள், தொழில்முறை அதிகாரப்படிநிலைகள், போன்றவை. இன்றைய நிலையில், குலமானது, பழமையான வகைகள் மற்றும் அடையாளங்களுடன் அரசாங்க அடையாள (மற்ற வகுப்புகள், பின்தங்கிய வகுப்புகள், மிகவும் பின்தங்கிய வகுப்புகள், ஆதிதிராவிட வகுப்புகள் மற்றும் ஆதிதிராவிட பழங்குடியினர்) மற்றும் வரிசைப்படுத்தும் கோட்பாடுகெளையும், உள்ளடக்கியதாக இருக்கிறது.

இதுபோன்ற சமூக வகைகளும் பிரிவினைகளும் தங்களை பிரகடனப்படுத்திக்கொள்ளும் தள வரிசையாக 
சமூக ஊடகங்கள் மாறிவிட்டன என்று மக்கள் இன அமைப்பியல் குறிப்பிடுகிறது. இதுபோன்ற சமூக வகைகளில் இருந்து முன்னேற்றமளிக்கும் விடுதலை நோக்கிற்கு பதிலாக, இவற்றின் மீளுறுதியைத்தான் நிகழ்நிலையில் பார்க்க முடிகிறது.

முதல் அத்தியாயம், இந்த கிராமத்தின் நீண்டகால குடியிருப்பாளர்களின் பழமையான சமூக வகைகளை கையாள்கிறது. இந்த நீண்டகால குடியிருப்பாளர்களின் பொருளாதார வளமையின் பொதுவான உயர்வு (நிலம் மற்றும் வீட்டுமனைகள் வணிக ஒப்பந்தங்களால்), அவர்களின் ஐடி துறையின் அருகாமை, மாறிவரும் இலட்சியங்கள், கட்டுப்படியாகக்கூடிய தகவல் தொழில்நுட்பங்கள் ஆகியவற்றுடன் சேர்ந்து, இந்தக்குழுவின் போன்கள் ${ }^{7}$ (சாதாரண போன்கள் மற்றும் ஸ்மார்ட்போன்கள்) மற்றும் சமூக ஊடகங்களின் பயன்பாட்டை அதிகரித்திருக்கிறது. அவர்களின் இந்த பயன்பாடு, மற்ற பிராந்தியங்களிலுள்ள கிராமத்தவர்களைக் காட்டிலும், ஐடி துறையினரின் நடைமுறைக்கு ஒத்ததாக இருந்தது.

முதல் அத்தியாயம் ஐடி துறையை, அதிக திறன்வாய்ந்த ஐடி பணியாளர்கள் முதல், குறைந்த திறனுள்ள பணியாளர்கள், வாகன ஓட்டினர்கள், நிர்வாக சிப்பந்திகள் வரையிலான பல சமூகப்பொருளாதார குழுக்களுக்கு வேலைவாய்ப்பு வழங்கக்கூடிய பல தொழில்முறை வகைகளை உள்ளடக்கிய சூழல்மண்டலமாக ${ }^{8}$ காட்சிப்படுத்துகிறது. இந்த பன்முக தொழில் வகைகளை சேர்ந்த மக்கள், இந்தப் பகுதிக்கு புலம்பெயர்ந்து, நீண்டகால குடியிருப்பாளர்களுக்கு அருகிலேயே குடியேறும் போது, அதன் விளைவு, பல்வேறு சமூக வகைகள் மற்றும் அவற்றின் சமூக ஊடக பயன்பாடுகள் ஆகியவற்றுக்கிடையேயான ஆற்றல்வாய்ந்த இடைவினையாக இருக்கிறது. அந்தவகையில், அத்தியாயம் 2, சமூகஊடகங்கள் மற்றும் பஞ்சக்கிராமியில் உள்ள பல்வேறு குழுக்களின் சமூக ஊடக பயன்பாடு ஆகியவற்றின் வரலாறு பற்றிய விவரங்களை நமக்களித்தது.

இந்தத்தருணத்தில், ஏற்கனவே தெளிவான விஷயம் என்னவென்றால், பஞ்சக்கிராமியில் சமூகஊடகங்கள் பற்றிய எந்தவொரு விவாதமும், குறைந்தபட்சம் குலம் மற்றும் வர்க்கம் போன்றவற்றின் சிக்கலான கலவையை உள்ளடக்கியதாக இருக்கும் என்பது தான். சமூக ஊடகங்களை, சமத்துவத்தை, குறைந்தபட்சம் டிஜிட்டல் சமத்துவத்தை உருவாக்கக்கூடிய சர்வரோகநிவாரணியாக பார்த்த எதிர்பார்ப்புகளுக்கு மாறாக, பஞ்சக்கிராமியில், சமூக ஊடகங்கள், டிஜிட்டல் 
அல்லது வெறெந்தவகையான சமத்துவத்தையும் அளித்ததற்கான சான்றுகள் அபூர்வமாகத்தான் காணப்பட்டன. தகவல் தொழில்நுட்பங்களின் பெருகிவரும் கட்டுப்படியாகும் தன்மை, பலருக்கு (குறிப்பாக ஆண்களுக்கு) சமூக ஊடகங்களில் இருக்கக்கூடிய வாய்ப்பை ஏற்படுத்திக்கொடுத்தாலும், வெறுமே சமூக ஊடகங்களில் இருத்தல் என்பது மட்டுமே பரந்த சமத்துவத்திற்கு வழிவகுத்திடாது. நிகழ்நிலை சமத்துவம், இயல்புநிலை சமத்துவத்திற்கு வழிவகுத்திடாது என்ற பொதுவான கூர்நோக்குதல், எங்களின் பல ஆய்வுக்களங்களுக்கும் பொதுவான ஒன்று.

இருப்பினும், வேறு சில ஆய்வுக்களங்களைப் போலல்லாமல், கட்டுப்படியாதல் பற்றிய விசாரம், இந்த களத்திற்கு பொருத்தமானதாக இருக்கிறது. அத்தியாயம் 2-ல் விவாதித்தது போல, கட்டுப்படியாகக் கூடிய விலையில் கிடைக்கும் முன்கட்டணம் செலுத்தப்பட்ட தரவு அட்டைகள், கீழ்மட்ட சமூகப்பொருளாதார வகுப்பில் உள்ளவர்களையும், சமூக ஊடகங்களை அணுக அனுமதித்தாலும், நடைமுறையில், இவர்கள் தங்களது முன்கட்டணம் செலுத்தப்பட்ட மாதாந்திர இணைய அலைவரிசையை, முதல் 20-22 நாட்களிலேயே செலவு செய்துவிடுகிறார்கள். இதனால் மாதத்தின் இறுதியில் 8-10 நாட்கள் எந்தவித இணைய தொடர்பும் இல்லாமலே இருக்க நேரிடுகிறது. அதேபோல, உத்தேசமாக இவர்களின் பதிவுகளில் 84 சதவிகிதம் அவர்கள் தங்களின் முன்கட்டணம் செலுத்திய இணையத் தொடர்பை செயலூக்கிய முதல் 15 நாட்களில் செய்யப்படுகிறது. அவர்களின் இ இணையதள தரவு புதுப்பித்தல் சுழற்சி, அவர்களின் மாதாந்திர ஊதிய தேதிகளுடன் ஒத்திருப்பதில் ஆச்சரியம் ஒன்றும் இல்லை. ஆகவே, கீழ்மட்ட சமூகப்பொருளாதார வகுப்பினருக்கு, மாதத்தின் அனைத்து நாட்களிலும் சமூக ஊடகங்களில் இருத்தல் என்பது இயலாத ஒன்றாகும்.

அத்தியாயங்கள் 2-6, சமூக ஊடகங்களின் மூலம் நடைபெறும் இடைவினைகள் பலவும், அந்தந்த சமூகப்பொருளாதார வகுப்புகளுக்கு உள்ளேயே நடைபெறுகிறதே தவிர, அவற்றுக்கு இடையே அல்ல என்பதற்கான தெளிவான ஆதாரங்களை ம முன்வைத்திருக்கின்றன. இது மத்தியதர, உயர்மத்தியதர மற்றும் கீழ்மட்ட வகுப்பினருக்கிடையே நடக்கும் இடைவினைகளை கூர்ந்து நோக்கினால் நமக்கு புலப்படும். முன்பு விவாதித்தது போல, இந்த இடைவினைகள் அனைத்தும் பரிமாற்றம் அல்லது செயல்பாடு தொடர்புடையவையே தவிர சமூகத்தன்மை கொண்டவை 
அல்ல. உயர்-மத்தியதர வகுப்பைச்சேர்ந்த நபர்கள் பலர், தங்கள் ஓட்டுனெரையோ சமையல்காரரையோ, அவர்கள் எந்தக் குலத்தை சேர்ந்தவராக இருந்தாலும், தனக்கு வேடிக்கைகள் போன்றவற்றை முன்னனுப்பக்கூறி ஊக்குவிப்பதில்லை. இது தனது வீட்டில் வேலைக்கு இருந்த ஆண் வேலையாளிடமிருந்து தகவல் பெற்றதில் சிந்து அடைந்த அச்சப்பாட்டில் இருந்து தெளிவாகிறது (அத்தியாயம் - 2). இது துவக்கத்தில் பாலின பிரச்சினை போல தெரிந்தாலும், இதில் வர்க்கம் முக்கிய பங்கு வகிக்கிறது. கவிதாவின் வழக்கில், அவரது குலத்தைச் சேர்ந்த சமையல்காரர்/ குழந்தை பராமரிப்பாளர் உடனான அவரது உரையாடல்கள் குலத்திற்குள்ளான தகவல் பரிமாற்றத்தையும் வர்க்க பேதம் பாதிக்கிறது என்பதை எடுத்துக்காட்டுகிறது. குலக்குழுவைப்பற்றிய பொருட்டில்லாமல் வகுப்புகளுக்கிடையேயான இடைவெளி அதிகரிக்கும் போது, சமூக ஊடகங்களில் தகவல் பரிமாற்றங்கள் சமூகச்சார்பை விட, செயல்பாடு சார்ந்ததாக ஆகிவிடுகிறது.

இது, குலம் மற்றும் வர்க்கத்தினால் பாதிக்கப்பட்டு, வலைத்தொடர்பு தன்குலச்சார்பால் முக்கியத்துவம் பெற்று, இயல்புநிலையில் இருந்து நிகழ்நிலை இடப்பரப்பிற்கு ஏற்பட்ட தொடர்ச்சி போல் தோன்றினாலும், இதே சமூக வகைகள், உறவுமுறையுடன் இணைந்து, பஞ்சக்கிராமி மக்களின் சமூக ஊடகங்களில் உள்ள பொதுவான நடவடிக்கைகள் மற்றும் பதில்களை (அவர்களது காட்சியமைப்பு கலாச்சாரம், வலைத்தொடர்பு அனுசரித்தல் போன்றவை) பார்க்க உதவுகிறது. ஒரு வகையில், இது ஆழமான தமிழ்கலாச்சாரத்தால் பாதிக்கப்பட்டு, பல்வேறு சமூக வகைகளுக்கிடையேயோ அல்லது ஐடி பணியாளர்கள் மற்றும் நீண்டகால கிராமக் குடியிருப்பாளர்கள் ஆகியோரிடையேயோ உள்ள ஒத்ததன்மைகளை விட, பொதுத்தன்மைகளை பார்க்க வழிவகுக்கிறது. ஆகவே, சமூக ஊடகங்களில் மக்கள் தாங்கள் ஏற்கனவே அமைத்துக்கொண்ட சமூக குழுக்களுடன் மட்டுமே பெருமளவு தொடர்பு வைத்துக்கொள்கிறார்கள் என்கிற முடிவால், குலம், வர்க்கம் போன்ற அனைத்து வட்டங்களிலும், மக்கள் சமூக ஊடகங்களால் பாதிக்கப் பட்டிருப்பதால் உள்ள பொதுத்தன்மையை பற்றிய தனிப்பட்ட முடிவை விலக்க முடியாது. இதுபோல, மக்கள் இன அமைப்பியலும் ஐடி பணியாளர்கள் மற்றும் உள்ளூர் கிராமவாசிகளிடையே சமூக ஊடக பயன்பாட்டில் எதிர்பார்த்ததை விட குறைந்த அளவான வித்தியாசங்களையே கண்டிருக்கிறது. 
இந்தக் குழுக்களிடையே சமூக ஊடக பதிவுகளில் காணப்படும் ஒரு தெளிவான பொதுத்தன்மை என்னவென்றால், தமிழ்நாட்டின் பிரசித்திபெற்ற பொதுவகைகளான சினிமா மற்றும் அரசியல் ஆகியவற்றின் மீது இவர்களுக்கு இருக்கும் பக்தி தான். இது அத்தியாயம் 3-ல் அவர்களது முகநூல் பக்கங்களில் உள்ள காட்சிக்குரிய பதிவுகளை அலசுவதில் தெள்ளத்தெளிவாக தெரிகிறது. உயர் சமூகப்பொருளாதார வகுப்புகள், சினிமா பற்றிய பதிவுகளை, தங்கள் ஊடக பக்கங்களில் சினிமா பற்றிய புத்திசாலித்தனமான கட்டுரை என்ற விளக்கத்துடன் பகிர்ந்து கொள்கிறார்கள். மேலும், அரசியல் செய்திகள் அரசியல் நையாண்டி போன்றவற்றையும் பகிர்ந்து கொள்கிறார்கள். கீழ்-மத்தியதர மற்றும் கீழ்மட்ட சமூகப்பொருளாதார வகுப்பினர், சினிமா மற்றும் அரசியல் மீதான தங்கள் அபிமானத்தை, தங்கள் அபிமான நட்சத்திரங்களின் படங்கள் மற்றும் தாங்கள் ஆதரிக்கும் அரசியல் கட்சிகளின் படங்கள் ஆகியவற்றை முகநூலில் பதிவிடுவதன் மூலம் இன்னும் வெளிப்படையாக காட்டுகிறார்கள். மேலும் இந்தக்குழுக்கள், அரசியல் நையாண்டிகளை, பழித்துக்காட்டும் மீம்கள் உருவத்தில் முகநூல் பக்கங்களில் பதிவிடுவதில் இன்னும் கூட வெளிப்படையாக இருக்கிறார்கள்.

அத்தியாயம் 3, முந்தைய இயல்புநிலை நிகழ்வுகளில் இருந்து புலம்பெயர்ந்த மற்ற காட்சிக்குரிய வகைகளையும் வெளிப்படுத்துகிறது. உதாரணத்திற்கு, தங்களின் புகைப்படங்களை பெருமளவு பகிர்ந்துகொள்வதில் உள்ள பொதுத்தன்மை, அனைத்து சமூக வகுப்புகளிலும் ${ }^{10}$ பெருமளவு பரவி இருக்கிறது. இது தனிப்பட்ட படங்கள் மற்றும் குழுப் படங்கள் ஆகியவற்றை அலசுவதன் மூலம் தெளிவாக்கப்பட்டது. வகுப்பு பேதமில்லாமல், பெண்கள் பதிவிட்ட புகைப்படங்கள், உடைகள் மற்றும் படங்களுக்கு காட்சியளிக்கும் முறை பற்றிய ஒருசில பாரம்பரிய விதிமுறைகளை அனுசரித்தே இருந்தன. இருபாலரும் இருக்கும் படங்களை சமூக ஊடகங்களில் பதிவிடுவெது, அது ஆணாக இருந்தாலும் சரி, பெண்ணாக இருந்தாலும் சரி, குழுப்படங்களை உள்ளடக்கியதாக இருந்தது. உயர்-மத்தியதர வகுப்புகளிலும் கூட, ஆண்-பெண் இருவரும் சேர்ந்திருக்கும் படங்களை பதிவிடுவது, அது இயல்புநிலை வாழ்வில், அவரவர்களின் பெற்றோருக்கு தெரிந்திருந்தாலும், அது காதல் சம்பந்தப்பட்ட உறவல்ல என்று பெற்றோருக்கு நிச்சயமான பின்னரே பதிவிடப்பட்டது.

இயல்புநிலையில் இருந்து நிகழ்நிலைக்கு வந்திருக்கும் மற்றொரு பொதுவான கரு, காலை/மாலை வணக்கங்கள் 
போன்ற அன்றாட வாழ்த்துச்செய்திகள் தெரிவிப்பது. நிகழ்நிலையில் இது பொதுவாக, எதாவது ஒரு இயற்கை காட்சி அல்லது கடவுளின் படத்துடன் சேர்ந்திருக்கும். ஒருசிலர், இதை நிகழ்நிலையில் சமூக தன்மையை அமைப்பதாக கருதுகின்றனர். வேறுசிலர் இதை தங்களுக்கான நல்ல கர்மா புள்ளிகளை சேகரித்துக்கொள்வதாகவும், தங்களில் வாழ்வில் நேர்மறை எண்ணங்களை விதைப்பதாகவும் கருதுகின்றனர்.

தெளிவாகத்தெரிந்த மற்றொரு விஷயம் என்னவென்றால், அவர்கள் பதிவிடும் காட்சியமைப்புகள் எந்தளவிற்கு, தங்கள் வலைத்தொடர்புகளின் எதிர்பார்ப்புகளுடன் அனுசரித்துப்போகின்றன என்பது தான். எந்தவித எதிர்ப்பையும் வெளிப்படுத்தாத அன்றாட வாழ்த்துச்செய்திகள் போன்ற பொதுப்படையான படங்கள், உபயோகப்பாளர்களின் முகநூல் பக்கங்களிலும், அவர்களின் வாட்சப் தகவல் பரிமாற்றங்களும் அடிக்கடி காணப்பட்டன. சமூக அனுசரிப்பு மற்றும் தங்கள் வலைத்தொடர்புகளுடன் எதிர்ப்படாமல் இருத்தல் ஆகியவற்றின் முக்கியத்துவத்தை புரிந்துகொண்டு மக்கள் தங்கள் காட்சிக்குரிய தகவல் பதிவுகளை சாமர்த்தியமாக வடிவமைப்பதை ஜோத்ஸ்னா மற்றும் சகாயம் ஆகியோரின் வழக்குகளில் பார்த்தோம். அவர்கள் தங்கள் எதிர்ப்பை தெரிவிக்க வேண்டும் என்றாலும், அதை தனிப்பட்ட உரையாடல் அல்லது தகவலின் மூலமாகவோ அல்லது அமைதி காப்பதின் (அதாவது விருப்பு தெரிவிப்பதோ அல்லது கருத்து பதிவு பண்ணுவதோ செய்யாமல் இருப்பது) மூலமாகவோ தெரியப்படுத்தினர். அத்தியாயம் 2-ல் பார்த்தது போல, தரநெறிசார் எதிர்பார்ப்புகளுக்கு எதிர்ப்போ அல்லது எதிர்மறை கருத்தோ தெரிவிப்பது என்பதன் மற்றொரு வகை, பொய்யான பல ஊடக கணக்குகள் மூலம் பதிவுகள் இடிவது என்பதாகும். இந்த கணக்குகளில் உள்ள பன்முகமுள்ள தான் எனும் தன்மை, அவர்களைப்பொறுத்தவரை நம்பகமானதும் அவர்களின் அடையாளத்தை முழுமைப்படுத்துவதும் ஆகும்.

பஞ்சக்கிராமியில் ஆதிக்கம் நிறைந்ததும், அனைத்து குழுக்களிடையேயும் புகுந்து செல்வதுமான ஒரு தகவல் பரிமாற்ற களம், உறவினர்கள். இது அத்தியாயம் 5 மற்றும் 6-ல் தெளிவாகத்தெரிந்தது. எனினும், உடனடி மற்றும் நீட்டித்த குடும்பங்களுக்குள் தகவல் பரிமாற்றம் என்பதின் மீது குவியம் கொண்ட அத்தியாயம் 4-ல் அதிக தெளிவாக காட்டப்பட்டது. இநந் அத்தியாயத்தில் காட்டப்பட்டுள்ள பன்முக ஊடகங்களின் உண்மையான சாரம், புதிய சாதனங்களின் மூலம் பாரம்பரியம் தன்னை பிரகடனப்படுத்தி- 
க்கொள்ளும் மற்றொரு உதாரணத்தை, வெளிப்படுத்தியது. வயதின் அடிப்படையிலான அதிகாரப்படிநிலை, குடும்ப உறுப்பினர் ஒருவரோடொருவர் வைத்திருக்கும் சம்பிரதாயமான உறவுமுறை, ஊடகத்தின் இயல்பு, உறவுமுறை அமைப்பில், ஒவ்வொரு உறுப்பினரின் இடம், இவையனைத்தும், ஒரு குடும்ப வட்டத்தில் தகவல் பரிமாறிக்கொள்வதற்காக ஒருவர் தேர்ந்தெடுக்க வேண்டிய தகவல் பரிமாற்ற முறையை தீர்மானிக்கும் காரணிகளாகும். நெருக்கமான குடும்பங்களுக்குள்ளான, குறிப்பாக பெரியவர்களுடனான தகவல் பரிமாற்றங்கள், குரல்வழி தகவல் பரிமாற்றத்தை விரும்பினாலும், (வயது, அதிகாரப்படிநிலை, கல்வி, பாசம் போன்ற பல காரணங்களால்), ராகவன், ரவியின் தாய், ஷோபனா, லட்சுமி மற்றும் ராகுல், மற்றும் சுக்ரிதியின் பெற்றோர் ஆகிய வழக்குகளில், நீட்டித்த குடும்பங்களுடனான தகவல் பரிமாற்றம், குடிம்ப அடிப்படையிலான வாட்சப் குழுக்களுக்கு மாறிவிட்டதை நமக்கு எடுத்துக்காட்டுகிறது. இன்னும் பரந்திருக்கும் உறவுகளுக்கு குடும்ப நிகழ்ச்சிகளை தெரியப்படுத்த, இது முகநூல் வழி தகவல் பரிமாற்றமாக மாறவும் கூடும். ராகவன் போன்றவர்கள், இதுமாதிரியான தகவல் பரிமாற்றங்கள் தனிமனித சார்பற்றவையாகவும் பெரியவர்களுக்கு மரியாதையை அளிக்காததாகவும் இருக்கிறது என்று கருதினாலும் கூடத்தான்.

உறவு வலைத்தொடர்புகளுக்கிடையே என்ன நடக்கிறது என்பது, அத்தியாயம் 4-ல் பார்த்தது போல் பன்னூடகங்கள் ${ }^{11}$, அளவீட்டுச் சமுதாயம் ${ }^{12}$ மற்றும் ஊடக பன்முகத்தன்மை ${ }^{13}$ ஆகிய கருத்துக்களின் சிக்கலான இருதிசை பயன்விளைவு ஆகும். சமூக ஊடகங்கள், குறிப்பாக முகநூல், செயல்பாட்டு ஊடகமாக காணப்படுகிறது (விருப்புகளை மற்றும் கருத்துபதிவுகளின் மூலம்). இது குடும்ப உறுப்பினர்கள் ஒற்றுமையான விழுமிய குடும்பம் என்ற தோற்றத்தை, முகநூலில் உள்ள பரந்த வலைத்தொடர்புகளுக்கு எடுத்துக்காட்டுவெதற்காக ஒன்றுகூடி செயல்படும் தளமாக இருக்கிறது. இருப்பினும் உண்மையில், இது சமூக ஊடகங்களின் குடும்ப பயன்பாட்டின் பிரதிபலிப்பு அல்ல. முகநூல், ஒரு சாமர்த்தியமான, செயல்பாட்டு பங்குள்ள வெளிநோக்கிய சாதனமாக பயன்படுத்தப்படுகிறது. அது, குடும்பத்திலுள்ள தகவல்பரிமாற்றத்திற்காக தளமாக வாட்சப் போன்ற முக்கியத்துவத்தை பெற்றிருக்கவில்லை. ஆகவே, குடும்பத்திற்குள்ளான தகவல் பரிமாற்றத்திற்கு ம்கூல் உபயோகப்படுத்தப்பட்டபோது ராகவன் கோபப்பட்டதில் எந்த ஆச்சரியமும் இல்லை. 
குடும்ப வட்டங்களுக்குள், குடும்பத்திற்குள்ளான தகவல் பரிமாற்றத்திற்கு, சரிவராத, குறைந்த அந்தரஙகமான, பொதுவான ஒன்றாக கருதப்படும் முகநூலின் பங்கு, வேறுசில அ அந்தரங்கமான பதிவுகளோடு பார்க்கும்போது வேறுமாதிரியான கொள்கையை வெளிப்படுத்துகிறது. உதாரணத்திற்கு, பிறந்த குழந்தையின் படங்களை, கண்ணேறு படக்கூடும் என்பதற்காக முகநூலில் ஒ ஒருசில மாதங்களுக்கு பதிவிடுவதை தவிர்த்தல் போன்ற ஒருசில பதிவுகள் அதிக பாரம்பரியமான கோட்பாட்டை வெளிப்படுத்துகின்றன. ஆனால், இந்த சமயத்தில், இதுபோன்ற படங்கள் வாட்சப் மூலம் தனிப்பட பகிர்ந்துகொள்ளப்படுகின்றன. முகநூல் நீட்டித்த உறவினரை சென்றடைந்தாலும், அது ஒரு பொதுப்பார்வைக்கான ஊடகம். ஆகவே, இந்தப்படங்கள் குடும்பத்தினர் அல்லாதவர் பார்வையில் படக்கூடிய வாய்ப்பு பிரச்சினைக்குரியது. இந்தபயம் வாட்சப்பில் இல்லாததால் இது விருப்பத்தேர்வாக இருக்கிறது.

புதிய ஊடகங்களின் மூலம், பாரம்பரிய மற்றும் தரநெறிசார் ஒழுங்கு மீண்டும் நிறுவப்பட்டிருக்கிறது என்பதை, நாம் பார்க்கமுடிகிறது. இது பாலின விதிமுறைகளின் மீதான தாக்கத்தில் குறிப்பாக தெளிவாகிறது. தொடர்ச்சியான கண்காணிப்பு அல்லது பெண்கள் சமூக ஊடகங்கள் உபயோகிப்பதில் உறவினர் மற்றும் குடும்ப வட்டங்கள் தடை விதிப்பது குல அடிப்படையிலான பண்புகள் மற்றும் தரநெறிசார்பு பற்றிய பிரசங்கங்களின் தாக்கத்தினால் விளைந்தது. குலத்தினிடை திருமணங்கள் மற்றும் காதல் உறவுகள் பற்றிய பயம், கீழ்மத்தியதர மற்றும் கீழ்மட்ட சமூகப்பொருளாதார வகுப்பினரிடையே நிலவுவதால், இது அவர்களை தங்கள் குலத்திலுள்ள, திருமணமாகாத இளம் பெண்களை மொபைல் போன் மற்றும் சமூக ஊடகங்கள் உபயோகிப்பதில் இருந்து தடை விதிக்கச்செய்கிறது. இந்தத்தடைகள் பலவகையானவை. ஒருசில கண்காணிப்பு மற்றும் மொபைல் போன்கள் மற்றும் சமூக ஊடகங்கள் உபயோகிப்பதில் ஒட்டுமொத்த தடை போன்ற கடின வடிவங்கள் எடுத்தது. மற்றவை, சமூகஊடகங்களின் பயன்பாட்டை வீட்டின் பாதுகாப்பான சூழலில் மட்டுமே அனுமதித்தது. அதன் மீது நேரக்கட்டுப்பாடும் விதித்தது.

இயல்புநிலை மற்றும் நிகழ்நிலை இடப்பரப்பு, இங்கு ஆணுக்குரியதாகக் கருதப்படுகிறது. சமூக ஊடகங்களை தடைசெய்வதன் கருத்து, அவற்றை ஆணுக்குரிய இடப்பரப்பாக ${ }^{14}$ கருதுவதன் அடிப்படையில் விளைந்தது. அவற்றை உபயோகிப்பது இளம்பெண்களை தேவையற்ற கவனச்சித- 
றல்களுக்கு ஆட்படுத்தி, அதன் மூலம் குடும்ப கௌரவத்திற்கு கேடுவிளைவிக்க கூடும். இதே கருத்து இயல்புநிலை இடப்பரப்பிற்கும் பொருந்தும். ஒருவரின் வீட்டிற்குள்ளான சூழல் பாதுகாப்பானதாக கருதப்படுகிறது. அதுவே வீட்டுக்கு வெளியேயான இடப்பரப்பு ஆணுக்குரியதாகவும், ஆதலால் சமூக ஊடகங்களை பயன்படுத்த விரும்பும் பெண்களுக்கு ஆபத்து விளைவிக்க கூடியதாகவும் கருதப்படுகிறது. இதே கருத்து, சமூக ஊடகங்களின் பயன்பாட்டில் நேரம் அடிப்படையிலான கட்டுப்பாடுகளுக்கும் பொருந்தும். இளளம்பெண்கள் 8 அல்லது 9 மணிக்கு மேல் சமூக ஊடகங்கள் பயன்படுத்தக்கூடாது என்ற கருத்து, அந்த நேரத்திற்கு மேல் அவர்கள் தவறாக பயன்படுத்திக்கொள்ளப்படக்கூடும் என்ற கண்ணோட்டத்தினால் விளைந்தது தான். இங்கு நேரம் மற்றும் இடத்தின் சந்திப்பு ஆணுரு எடுத்துக்கொள்கிறது.

இது போன்ற கட்டுப்பாடுகள், இந்த சமூக குழுக்களில் இருந்து வரும் பெண்களை சமூக ஊடகங்களுக்கு செல்ல அனுமதிப்பதில்லை. இது இந்த குழுக்களின் இளம் ஆண்களின் மீது மறைமுக பாதிப்பை ஏற்படுத்தியிருக்கிறது. ஒரு விதத்தில் இது வலைத்தொடர்பு கலப்புமரபுத்தன்மையை செலுத்தி, குலம் மற்றும் வர்க்கங்களுக்கிடையே நட்புறவுகளை அதிகரித்திருக்கிறது. பாரம்பரியமாக பாலினங்களுக்கிடையேயான தொடர்புகள் மற்றும் உறவுமுறைகள் பெருமளவு வட்டாரப்படுத்தப்பட்டதாகவும், அன்றாட இயல்பு வாழ்வில் சந்திக்கும் மக்கள் சம்பந்தப்பட்டதாகவும் இருந்தது. ஆனால் சமூக ஊடகங்களின் விளைவு, ஒரு வகையான சக்திப்புலனை உருவாக்கி, இந்த ஆண்களை பாரம்பரிய மையத்தில் இருந்து, தொடர்புகளின் பரந்த வட்டத்துள் தள்ளிவிட்டிருக்கிறது. உள்ளூர் பெண்களுடன் நட்புதொடர்பு ஏற்படுத்திக்கொள்வது தோற்றதால், இளம் ஆண்கள், இன்னும் மேலே சென்று, தங்கள் கல்வி நிறுவனங்கள் அல்லது பணியிடங்கள், மற்ற மாநிலங்கள் மற்றும் நாடுகள் போன்ற இடங்களில் பழைமை குறைவான பெண்களுடன் நட்புதொடர்பு கொண்டனர். இவர்கள் அந்தப்பெண்களுடன் இயல்புநிலையில் ஒரு வார்த்தை கூட பேசாமலிருக்கக்கூடிம். ஆனால் அவர்கள் வெட்கப்படாமல் தங்கள் நட்புறவை, சமூக ஊடகங்கள் என்ற இந்த தனிப்பட்ட இடப்பரப்பில் வெளிப்படையாகவும் சுதந்திரமாகவும் காட்டக்கூடும்.

சமூக ஊடகங்களில் வெவ்வேறு வகுப்பினரின் நடவடிக்கை, ஒன்றுக்கொன்று வித்தியாசமாக இருந்தாலும், இவையனைத்தும் சமூக ஊடகத்தை, மதிப்பு மற்றும் மரியாதையை 
பெறவும், தங்கள் குடும்ப கௌரவத்தை பேணவும், பொதுவாக பயன்படுத்துகின்றனர். உயர்-மத்தியதர வகுப்பினர், இதை விழுமிய குடிம்ப அமைப்பாக காட்டக்கூடிய செயல்களை சமூக ஊடகங்களில் செய்வதன் மூலம், சாதித்தாலும், கீழ்மத்தியதர மற்றும் கீழ்மட்ட சமூகப்பொருளாதார வகுப்பினர், தங்கள் குடிம்பத்தின் இளம்பெண் உறுப்பினர்களை, சமூக ஊடக அணுகலில் இருந்து தடுப்பதன் மூலம் குடும்ப கௌரவத்தை பேண முடியும் என்று நம்புகின்றனர்.

மக்கள் ஒருவரையொருவர், சமூக ஊடகங்களில் விளிக்கும் முறையில் குறிப்பிடத்தக்கது என்னவென்றால், அவர்கள் பயன்படுத்தும் கற்பனையான உறவுமுறைச்சொற்கள் தான். கற்பனையான உறவு என்ற கருத்து அனைத்து சமூகப்பொருளாதார குழுக்களிலும் படர்ந்திருக்கிறது. நண்பர்களை கற்பனையான உறவுமுறைச்சொற்களிட்டு அழைப்பது நிகழ்நிலை மற்றும் இயல்புநிலை இடப்பரப்புகளில் பொதுவாக காணப்பட்டாலும், உறவினர்களை நண்பர்கள் போல விளிப்பதும் மத்தியதர வகுப்பினரிடையே காணப்படுகிறது. இரண்டுமே பரந்த தமிழ் சமுதாயத்தில் காணப்படும் உறவு மற்றும் நட்புமுறைகளைப் பற்றிய பிரசாங்கங்களினால் ஏற்பட்ட பாதிப்பின் விளைவாக தெரிகிறது.

அத்தியாயம் 5, இந்த ஆய்வின் அடிப்படை கருப்பொருளை வெளிப்படுத்துகிறது. பணி சார்ந்த மற்றும் பணிசாரா எல்லைகளை, சமூக ஊடகங்கள் எவ்வாறு தீவிரமாக மறுதலித்து, தொடர்ந்து அவற்றை கீழறுக்கிறது என்பதை காட்சிப்படுத்துவது தான் அந்த அடிப்படை கருப்பொருள். பணியை வீட்டிற்கு எடுத்துச்செல்வது என்பது நவீன பணியிடத்தில் எதிர்பார்ப்புகளை அனுசரிக்கும் சமூக நடைமுறையாக பார்க்கப்பட்டாலும், பணிசாரா விஷயங்களை பணிநேரத்தில் கவனிப்பது என்பது, கண்டனத்துடன் பார்க்கப்பட்டது. இருப்பினும் இது போன்ற எதிர்ப்புகள், உண்மையில், பாரம்பரியமாக இதுபோன்ற எல்லைகள் இல்லாத தென்னிந்தியாவின் பணி பற்றிய வரலாற்று கருத்தியலை ஒத்ததாக இருக்கிறது. பணிசாரா இடப்பரப்புடனான தொடர்ச்சியான இடைவினைகள், அன்றாட சமூகத்தன்மையின் அங்கமாக கருதப்பட்டது. பாரம்பரியமாக இதுபோன்ற எந்தவொரு பிரிவினையும், இந்தியச்சூழலில் உள்ள பணிவுலகில் மற்ற பிராந்தியங்களில் இருந்ததைப்போல கடுமையாக விதிக்கப்படவில்லை. இது பணியமைப்பில் உள்ள, பணி மற்றும் பணிசாரா எல்லைகளை, அவற்றை சுற்றிச்செல்ல அனுமதிக்கக்கூடிய வாய்ப்புகளின் மூலம் மீண்டும் மீண்டும் 
மறு-வரையறுக்க அனுமதிக்கும் ஒரு முரண்பாட்டின் மூலம் வெளிப்படுத்தப்பட்டது.

பணி மற்றும் பணிசாரா எல்லைகளை பற்றிய இந்த விவாதம், பஞ்சக்கிராமியின் திடீர் மாற்றத்தைக்குறிக்கும் சூழலில் அமைந்திருக்கிறது. பாரம்பரிய விவசாய அமைப்பாக இருந்த ஒன்று, நவீன சம்பிரதாயமான பணியிடத்தின் தேவைகளை பூர்த்திசெய்யும் வகையில் விருத்தி செய்யப்பட்டது. நவீன பணிச்சூழல்களில், கீழ்மட்ட வேலைவாய்ப்புகளுக்கான தேடலில் குலம் மற்றும் உறவுமுறைகளுக்கிடையே (கற்பனை உறவுமுறை) ஒரு சிக்கலான இருதிசை பயன் விளைவு நடைபெறுகிறது. இந்த நடைமுறைகளில் சமூக ஊடகங்களின் பங்கு (பணியமர்த்தம், தகவல் பரிமாற்றம் போன்றவை), அத்தியாயம் 5-ல் விவாதிக்கப்பட்டது. இது குறிப்பாக, கீழ்மட்ட சமூகப்பொருளாதார வகுப்பினரின் வழக்காக இருந்தாலும், பணி மற்றும் பணிசாரா வட்டங்களுக்கிடையே தொடர்ந்த தகவல் பரிமாற்றத்தை ஏற்படுத்தி, அதன்மூலம், உயர்மட்ட சமூகப்பொருளாதார குழுக்களின் பணிசார்ந்த மற்றும் பணிசாரா எல்லைகளை கீழறுப்பதில் சமூக ஊடகங்களின் பங்கும் அத்தியாயம் 5-ல் விவாதிக்கப்பட்டது. இது நீண்டகால கிராமத்தவருக்கும் தொழில்நெறிசார் ஐடி பணியாளர்களுக்கும் வேறுவகையான வேலைசெய்தாலும், பணிசார்ந்தவற்றை பணிசாராதவற்றிலிருந்து பிரிப்பதற்கு எதிர்ப்புத்தெரிவிக்க சமூக ஊடகங்களை மக்கள் பயன்படுத்தும் பொதுவான முறை அனைத்து சமூகங்களுக்கும் பொதுவானது தான்.

அத்தியாயம் 4-ல் முன்னணியில் காணப்பட்ட அதிகாரப்படிநிலை பற்றிய விசாரங்கள், பள்ளிக்கல்வி பற்றிய அத்தியாயம் 6-லும் தங்களை வெளிப்படுத்திக்கொண்டன. ஆசிரியர்களும் மாணவர்களும் சமூக தகவல் பரிமாற்றத்திற்காக, எவ்வாறு இந்த புதிய ஊடகங்களை புரிந்துகொண்டும், தங்களை அவற்றுக்கேற்ப திசையமைத்துக்கொண்டும், உள்ளனர் என்பதில் உள்ள பதட்டங்களை சமூக ஊடகங்கள் வெளிப்படுத்துவதை நாம் கண்டோம். பாரம்பரிய அதிகாரப்படிநிலையான ஆசிரியர் மாணவர் உறவுமுறையை எவ்வாறு இந்தப்புதிய “முகநூல் நண்பர்கள்" என்ற நிலைக்கு வரிசைப்படுத்துவது என்பது பற்றிய நிச்சயமின்மையும் இருமனப்போக்கும், பஞ்சக்கிராமியின் பள்ளிகளில் உள்ள விசாரங்களாகும். இதுகுறித்த மனப்பாங்கும் பதில்களும், பல்வேறு சமூகப்பொருளாதார குழுக்கள் மற்றும் பல்வேறு பள்ளி நிர்வாகங்கள் ஆகியோரிடையேபெரிதும் வித்தியாசப்ப- 
டுகிறது. உயர்மட்ட சமூகப்பொருளாதார வகுப்பினர் பயிலும் வளமான பள்ளிகளில் உள்ள விசாரம் என்னவென்றால், சமூக ஊடகங்கள் தங்கள் மாணவர்களுக்கு கவனச்சிதறலாக இருக்கக்கூடும் என்பது தான். கீழ்மட்ட சமூகப்பொருளாதார வகுப்பினர் பயிலும் குறைந்த வளமான பள்ளிகளில், சமூக ஊடகங்களும் தொழில்நுட்பமும் சமூக இயங்குதிறனுக்கான அடையாளமாக பார்க்கப்படுகின்றன. அறிவார்ந்த பொருளாதாரத்தில் இவ்விரண்டிலுமான ஆற்றல், வளத்தைப் பெற்றுத்தரும் என்று அவர்கள் நம்புகிறார்கள்.

இதன் விளைவாக, குறைந்த வளமுள்ள பள்ளிகளில் உள்ள ஆசிரியர்கள், சமூக ஊடகங்களில் மாணவர்களுடன் நட்புதொடர்பு ஏற்படுத்திக்கொள்வதை, தங்கள் மாணவர்களின் லட்சியங்களை வெளிப்படையாக ஊக்கமளித்தலாக பார்க்கிறார்கள். கீழ்மட்ட சமூகப்பொருளாதார வகுப்பிலிருந்து வரும் மாணவர்களுக்கு, ஆசிரியரை நண்பராக்கிக் கொள்வது என்பது, தங்களது சொந்த புத்திசாலித்தனத்தை வெளிக்காட்டிக்கொள்வதற்கு வழிவகுக்கக்கூடும். இது வகுப்பறையில் அவரது அங்கீகாரத்தையும்.பெற்றுத்தரக்கூடும். இருப்பினும், வளமான பள்ளிகளில் உள்ள ஆசிரியர்கள் மற்றும் மாணவர்களுக்கு ஒருவரோடொருவர் நட்புதொடர்பு ஏற்படுத்திக்கொள்வது என்பது, நிறுவப்பட்ட அதிகாரப்படிநிலையின் முறிவால் விளைந்த அந்தரங்க குறைவுக்கு வழிவகுக்கக்கூடும். இரண்டு பள்ளிகளுக்கும் இது, இருபுறமும் தொடர்ச்சியான பரிசோதனைகளுக்கு வழிவகுக்கும் விஷயமாகவும் தீவிரமாக மாறிவரும் ஒரு விஷயமாகவும் இருக்கிறது.

கல்வியை பற்றிய அத்தியாயம், மக்கள் இன அமைப்பியல் முழுமையிலும் கண்டறியப்பட்ட கண்டுபிடிப்புகளை திறம்பட வலியுறுத்துகிறது. ஒருபக்கம் இது குலம் மற்றும் வர்க்கத்தில் உள்ள பிடிவாதமான நிகழ்நிலை வித்தியாசங்களை வெளிக்காட்டுவதன் மூலம் பொதுவான சமத்துவமின்மையை வெளிப்படுத்துகிறது. இது அகமண நடைமுறைகளை மக்கள் கடைபிடிக்கும் வரையிலும் நிலைத்திருக்கக்கூடிய குல வித்தியாசங்களையும், அளவிடக்கூடிய வர்க்க பேதங்களையும் இணைக்கிறது. அதேசமயம், முந்தைய அத்தியாயங்களில் உறவுமுறைகளாலும் அத்தியாயம் 6-ல் ஆசிரியர்கள், மாணவர்கள் மற்றும் பெற்றோர்கள் என்ற முக்கோணத்திலும் வெளிப்படுத்தபட்ட சமூக ஊடக பயன்பாடு பற்றிய விசாரங்கள் அனைவருக்கும் பொதுவானவையே.

இதை முழுமையாக பார்க்கும் போது, நாம் இன்னும் ஒரு முக்கிய கேள்வியால் எதிர்கொள்ளப்படுகிறோம். சமூக ஊடக- 
ங்கள் முக்கியம் தானா? இந்த கேள்விக்கான பதில் அத்தியாயம் 4-ல் உள்ள முக்கிய கண்டுபிடிப்பை எதிர்கொள்வதன் மூலம் தொடங்கப்படவேண்டும்.

பஞ்சக்கிராமியில் உள்ள பெரும்பாலான மக்களுக்கு அவர்களுக்கு மிகவும் முக்கியமான உறவுமுறைகள், உதாரணத்திற்கு அவர்கள் வாழும் குடும்பம், என்று வரும்போது, அனைத்து வகையான சமூக ஊடகங்களும் முக்கியத்துவம் பெறுவதில்லை. நிலைமை பற்றிய பொருட்டில்லாமல், சமூக ஊடகங்களை ஆராய்ச்சிக்கான பொருளாக தேர்ந்தெடுத்த பிறகு, சமூக ஊடகங்களுக்கு மாபெரும் பங்கு மற்றும் முக்கியத்துவத்தை சுமத்தக்கூடிய இயல்பான போக்கை திருத்தும் இதுபோன்ற மெய் உணர்தல் மதிப்புவாய்ந்ததாகும். சமூக ஊடகங்களை பற்றிய நாநலமிக்க சான்றுரை என்னவென்றால், முகநூலை, குடிம்பங்களுக்குள் இருக்கும் நேசத்தை, மற்ற நீட்டித்த குடும்ப வட்டங்களுக்கும், குடும்பத்தில் அல்லாதவருக்கும் வெளிப்படையாக எடுத்துக்காட்ட பயன்படுத்த முடியும், ஆனால் அந்த நேசத்தை குடும்பத்திற்குள் வெளிப்படுத்த பயன்படுத்தப்பட்டதில்லை என்பது தான். இது குடும்பங்களின் வாட்சப் பயன்பாட்டின் மூலம் முரண்படுத்தப்பட்டதாக தோன்றக்கூடும். ஆனால், உண்மையில் இதற்கான காரணம், அத்தியாயம் 2-ல் குறிப்பிட்டுள்ளது போல வாட்சப் பெரும்பாலும், சமூக ஊடகமாக பார்க்கப்படுவதை விட, நீட்டித்த தகவலனுப்பும் சேவையாகத்தான் பார்க்கப்படுகிறது. இது ஒருவகையில், பஞ்சக்கிராமியில் சமூக ஊடகங்கள் எவ்வாறு வகைப்படுத்தப்படுகின்றன என்பதை வெளிக்காட்டுகிறது. டிவிட்டர் மிகவும் உயர்ந்த நிலையில் இருக்கும் பெருமளவு பொதுப்பார்வைக்கான தளமாக கருதப்படுகிறது. அதைத்தொடர்ந்து முகநூல், பரிச்சயமற்றவர்களை நண்பர்களாக்கிக்கொள்ள அனுமதிக்கும் தளமாக பார்க்கப்படுகிறது. அதன்பின் வாட்சப், அளவிடக்கூடியது என்றாலும், இன்னமும் தனிப்பட்ட தளமாக, பொது ஆர்வக்குழு உறுப்பினர்கள் பரிச்சயமானவர்களாக பார்க்கப்படும் தளமாக கருதப்படுகிறது. இது பன்னூடகங்கள் ${ }^{15}$ மற்றும் அளவீட்டு சமுதாயம் ${ }^{16}$ என்ற இரண்டு கருத்துக்களுடனும் நேரடியாக பேசுகிறது.

ஒட்டுமொத்தமாக பார்த்தால், சமூக ஊடகங்கள் என்ற சொற்றொடர் கூட, பல ஆய்வுகளில் இருந்து வந்த இந்த இந்திய வழக்கில், மாறுபட்ட பொருளுடையதாக இருக்கிறது. இன்றளவில், ஒருசில நாடுகளில் சமூக ஊடகங்கள் என்பவை தனிப்பட்ட மற்றும் இருவினைசார் தகவல் பரிமாற்றத்தில் 
இருந்து நீட்டிக்கப்பட்ட, பரவலான அங்கத்தினர் குழுவுடனான தகவல் பரிமாற்றத்தை கையாளக்கூடிய ஆற்றலை உள்ளடக்கிய ஒன்றாகத்தான் பார்க்கப்படுகிறது. இருப்பினும் இந்தியாவில் "சமூக" என்ற சொல், உறவுமுறை, வயது, பாலினம், குலம், வர்க்கம் போன்ற பாரம்பரிய கோட்பாடுகளால் இறுக்கமாக அமைக்கப்பட்ட சமூகம் என்பதன் இணைச்சொல்லாக கருதப்படுகிறது. வேறு வார்த்தைகளில் சொல்லவேண்டுமென்றால், சமூகம் என்பது வகைகளுக்கேற்ப இறுக்கமாக வரிசைப்படுத்தப்பட்டிருக்கிறது. இங்கு சமூக ஊடகங்கள் எவ்வாறு பயன்படுத்தப்படுகின்றன என்பதை புரிந்துகொள்வது என்பது, இந்த முன்வரிசைப்படுத்தப்பட்ட குழுக்களில் எந்த ஊடகமும் எவ்வாறு சமூகப்படுத்தப்பட்டிருக்கிறது என்பதையும் புரிந்துகொள்வதாகும். அளவீட்டு சமூகத்தன்மையின் கொள்கை அறிவுறுத்துவது என்னவென்றால், ஆரம்பகால சமூக ஊடகங்கள், பொது அலைபரப்புகளில் இருந்து குழுக்களாக அளவிறக்கம் செய்யப்பட்டவை. ஆனால், சமீபத்திய சமூக ஊடகங்கள், இருவினைசார் தகவல் பரிமாற்றத்தில் இருந்து குழுக்களுக்கு அளவேற்றம் செய்யப்பட்டவை. ஆனால், தென்னிந்தியாவின் இந்த பிராந்தியத்தில், சமூக ஊடகங்கள் முதன்மையாக குழு தகவல் பரிமாற்றம் பற்றியவை. ஏனெனில், இங்கு சமூக வலைத்தொடர்பு என்பது எப்போதுமே, குழு தகவல் பரிமாற்றம் பற்றியதாக தான் இருந்திருக்கிறது.

இந்த ஆராய்ச்சி செயல்திட்டத்தின், உண்மையான நோக்கங்களுடனும், அதற்கான இடம் பற்றிய முதல் விருப்பத்தேர்வுடன் தொடர்புடைய வளாகத்துடனும் முரண்படுவதாலேயே இந்தக்குறிப்பு மிகுந்த சக்தியுடன் ஸ்தாபிக்கப்படுகிறது. இது இந்தியாவில் பரவலாக நடந்துகொண்டிருக்கும், பாரம்பரியம் மற்றும் நவீனத்தின் பக்க அணிமை நிலை மட்டுமல்ல. இது, பத்தே வருட காலகட்டத்தில் 200,000 ஐடி பணியாளர்களை கிராமங்களுக்கும் வயல்களுக்கும் இடையே வாழ்வதற்காக அழைத்துவரும் செயலாகும். இதன் அளவு, இது நடத்தப்பட்ட வேகம், மற்றும் இந்த இரண்டு சமூகங்களுக்கும் இடையேயான வித்தியாசம் அனைத்துமே அசாதாரணமானது. இந்த பக்க அணிமை நிலை, இந்த புத்தகம் முழுமையும் ஆதிக்கம் செலுத்துவது என்பது, தவிர்க்கமுடியாததாகவும் கண்கூடானதாகவும் தெரிகிறது. இ இருந்தாலும், இது இந்தியா. மக்கள் இன அமைப்பியல் முறையின் வெளிப்படைத்தன்மையை பார்க்கும்போது, சமூக ஊடகங்களின் உண்மையான பயன்பாடு மற்றும் விளைவுகள் பற்றிய எங்களது கண்ணோட்டம் விரைவில் மாறியது. 
பாரம்பரிய கிராமிய அமைப்புகளுடனான இந்த அதிநவீன அமைப்புகளின் பக்க அணிமை நிலை எப்படி இருந்தபோதிலும், இந்த இரண்டு குழுக்களுக்கும் பொதுவான விஷயங்கள் தான் முக்கியமானவை என்பது தெளிவாகிறது. பஞ்சக்கிராமியின் அனைத்து மக்கட்தொகையும், அதாவது நீண்டகால குடியிருப்பாளர்கள் மற்றும் சமீபத்தில் குடியேறியவர்கள் ஆகியவை, குலம், வர்க்கம், குடிம்பம் முதல், வயது மற்றும் பாலினத்தின் அதிகாரப்படிநிலை வரையிலான அனைத்து தீர்க்கமான இந்திய அமைப்புகளான ஒழுங்குமுறை, பண்புகள், சமூக வகைகள் ஆகியவற்றால் நெறிப்படுத்தப்பட்டுள்ளன. இங்குள்ள அனைத்தும் ஏற்கனவே தீவிரமாக சமூகப்படுத்தப்பட்டுவிட்டது. இவையனைத்தும் இந்த சமூக ஊடகங்களை, மற்ற சமூகங்களில் காணப்படுவெனவற்றில் இருந்து வித்தியாசமானதாக இருக்கும் இந்த பரந்த இந்திய உணர்திறன் மற்றும் அமைப்புகளின் பிரதிபலிப்பாக ஆக்குவதற்கு ஒன்றுகூடி செயலாற்றுகின்றன. 


\section{குறிப்புகள்}

\section{அத்தியாயம் 1}

1. குலம் என்பது ஒரு தனிநபருக்கு பிறப்பிலேயே சமூகஅளவில் தெளிவான குழு அடையாளத்தை சுமத்தும் அகமண உறவு அமைப்பு. தமிழ்நாட்டில் குல அமைப்பு பற்றி மேலும் தகவலுக்கு, see Pillay, K. P. K. 1977. The Caste System in Tamil Nadu. Chennai: University of Madras.

2. http://www.discoveranthropology.org.uk/about-anthropology/fieldwork/ethnography.html

3. ஐந்து கிராமங்களின் குழுவிற்கான புனைபெயர்

4. இந்த எண்ணிக்கை, ஐடி பணியாளர்கள், சிறு வணிகர்கள், கட்டுமானப் பணியாளர்கள் போன்றவர்களை உள்ளடக்கிய மிதக்கும் மக்கட்தொகையின் தோரயமாக்கம். இது, ஐடி பணியாளர்கள், பஞ்சாயத்து அலுவலர்கள், கல்லூரி பேராசிரியர்கள், மற்றும் இந்தப்பகுதியின் குடியிருப்பாளர்கள் ஆகியோருடனான பெட்டியின் அடிப்படையில் அமைந்தது.

5. "அதீத-ஆண்மை" என்பது, ஆண்மையைப்பற்றிய மிகைப்படுத்தப்பட்ட கருத்து. இந்த உதாரணத்தில் இது பெண்களெல்லாம் பொதுவாகவே பலவீனமானவர்கள், மற்ற ஆண்களிடம் இருந்து காக்கப்பட வேண்டியவர்கள் என்பதையும் குறிக்கிறது. பார்க்க: Parrott, D. J. and Zeichner, A. 2003. 'Effects of hypermasculinity on physical aggression against women.' Psychology of Men \& Masculinity 4(1): 70 and Spencer, M. B., Fegley, S., Harpalani, V. and Seaton, G. 2004. 'Understanding hypermasculinity in context: A theory- driven analysis of urban adolescent males' coping responses.' Research in Human Development 1(4): 229-57.

6. தொடர்ச்சி என்பது இடம் மற்றும் நேரம் கடந்த உடைக்கப்படாத நிரந்தரமான தொடர்பு

7. இந்தியாவில் பிரபலமான சோப்பு

8. இந்தியாவில் பிரபலமான வாசனைதிரவியம்

9. Mandelbaum, D. G. 1970. Society in India: Continuity and Change (vol.1). Oakland, CA:University of California Press.

10. McPherson, M., Smith- Lovin, L. and Cook, J. M. 2001. 'Birds of a feather: Homophily in social networks.' Annual Review of Sociology: 415-44.

11. Why We Post' - https://www.ucl.ac.uk/why-we-post

12. இது பிரேசிலிலும் கூர்நோக்கப்பட்டது. ஆலோசிக்க: Spyer, J. Forthcoming. Social Media in Brazil. London: UCL Press.

13. இது "நாம் ஏன் பதிவிடுகிறோம்" என்ற பெரிய செயல்திட்டத்தை கண்டுபிடிப்புகளிலும் ஒன்றாகும். - https://www.ucl.ac.uk/why-we-post/discoveries/why-we-post/discoveries/7-weused-to-just-talk-now-we-talk-photos

14. அத்தியாயம் 3 பொதுவான மற்றும் தனிப்பட்ட வட்டங்களுக்கு இடையே விழும் காட்சியமைப்புகளை இடைப்பட்ட காட்சியமைப்புகள் என்று இயல்புநிலை இடப்பரப்பில் அறிமுகப்படுத்துகிறது. பின் அதற்கான இணையை சமூக ஊடக தகவல் பரிமாற்றங்களில் வரைகிறது

15. Cialdini, R. B. and Goldstein, N. J. 2004. 'Social influence: Compliance and conformity.'Annual Review of Psychology. 55: 591-621.

16. இந்்ியாவில் உறவுமுறை பற்றிய மேலும் தகவலுக்கு பார்க்க: Uberoi, P., ed. 1993. Family, kinship and marriage in India. New Delhi: Oxford University Press. For Dravidian and Tamil kinship please see Dumont, L. 1953-4. 'The Dravidian Kinship Termi- 
nology as an Expression of Marriage.' Man 53: 34-9; Trautmann, T. R. 1981. Dravidian Kinship. New Delhi: Sage Publications Pvt Limited; Trawick, M. 1990. Notes on love in a Tamil family. Oakland, CA: University of California Press.; Gough, E. K. 1956. 'Brahman kinship in a Tamil village.' American Anthropologist 58(5): 826-53; Kapadia, K. 1994. “"Kinship burns!":kinship discourses and gender in Tamil South India.' Social Anthropology 2(3): 281-97.

17. குல அகமண உறவு பற்றி மேலும் தகவலுக்கு பார்க்க: https://www.umanitoba.ca/ faculties/arts/anthropology/tutor/marriage/caste_endogamy.html

18. Laughey, D. 2007. Key themes in media theory. Berkshire: McGraw- Hill Education;Gottdiener, M., Budd, L. and Lehtovuori, P. 2015. Key concepts in urban studies. London: Sage Publications.

19. Laughey, D., 2007; Gottdiener, M. et al., 2015.

20. Reddy, P. G. 2010. 'Some Problems Faced By The Software Professionals In India: An Overview' in Lakshmansamy, T., ed. Population Dynamics and Human Development Opportunities and Challenges. New Delhi: Bookwell, 451-71; Upadhya, C. and Vasavi, A. R. 2006. Work, culture, and sociality in the Indian IT industry: a sociological study. Bangalore: National Institute of Advanced Studies, Indian Institute of Science Campus, Bangalore.

21. நகர தொழிற்சாலைகளில் உறவுமுறையில் பங்கு இந்தியாவில் சாதாரமானது. நெசவு துறையில் உறவுமுறை பற்றிய மேலும் தகவலுக்கு பார்க்க:De Neve, G. 2008.' "We are all sondukarar (relatives)!": kinship and its morality in an urban industry of Tamilnadu, South India.' Modern Asian Studies 42(1): 211-46; Vidyarthi, L. P. 1984, in Vidyarthi, L. P., ed. Applied anthropology in India: principles, problems and case studies. New Delhi: Kitab Mahal.

22. Dahlman, C. J. and Utz, A. 2005. India and the knowledge economy: leveraging strengths and opportunities. Washington D. C.: World Bank Publications.

23. http://www.firstpost.com/india/celebrating-madras-day-so-many-reasons-to-lovechennai-m odern-indias-first-city-1669189.html

24. காஞ்சிபுரம் அல்லது காஞ்சி என்றழைக்கப்படிம் புராதன நகரம், சென்னையிலி ருந்து சுமார் 72 கிமீ தூரத்தில் உள்ளது. பஞ்சக்கிராமி காஞ்சிபுரம் மாவட்டத்தை சேர்ந்தது, காஞ்சிபுரம் நகரில் இருந்து சுமார் 75 கிமீ தூரத்தில் இருக்கிறது.

25. கால்வாயை சுத்தமாக வைத்துக்கொள்வது பற்றிய விசாரங்கள், நீர்பாதைகளின் பொதுவான நிர்வாகம் மற்றும் இதர போக்குவரத்து முறைகளின் வளர்ந்து வரும் பிராபல்யம் ஆகியவை இந்த மூடலுக்கான சில காரணங்கள்

26. ஐக்கிய நாடுகள் கல்வி அறிவியல் பண்பாட்டு வளர்ச்சிக் கழகம்

27. Nagaswamy, R. and Nakacami, I. 2008. Mahabalipuram. New York: Oxford University Press.

28. Dubreuil, G. J. 1917. The Pallavas. New Delhi: Asian Educational Services.

29. Muthiah, S. 2011. Madras Miscellany: A decade of People, Places and Potpourri. Chennai: Westland; Lakshman, N. 2013. Degree Coffee by the Yard: A Short Biography of Madras. New Delhi: Aleph Book Company.

30. Pillay, K. P. K. 1977. The Caste System in Tamil Nadu. Chennai: University of Madras; Thurston, E. and Rangachari, K. 1909. 'Castes and tribes of South India.' New Delhi: Asian Educational Services.

31. Rabe, M. D. 2001. The great penance at Māmallapuram: deciphering a visual text. Generaleditor G. John Samuel. Chennai: Institute of Asian Studies; Iyengar, P. S. 1929. History of the Tamils from the Earliest Times to 600 AD. New Delhi: Asian Educational Services.

32. இந்துசமய முமூர்த்திகளில் ஒருவர்

33. இந்துசமய முமூர்த்திகளில் ஒருவர்

34. Madhavan, C. 2007. Vishnu Temples of South India: vol.I: Tamil Nadu. Chennai: Alphaland Books.

35. Pedersen, J. D. 2000. ‘Explaining economic liberalization in India: state and society perspectives.' World Development 28(2): 265-82.

36. தகவல் தொழில்நுட்பம் மற்றும் தகவல் தொழில்நுட்பம் அனுமதிக்கும் சேவைகள்.

37. தமிழை தாய்மொழியாக பேசுபவர்களாக இங்கு விளக்கப்பட்டிருப்பவர்கள்

38. மலையாளத்தவரால் கொண்டாடப்படும் ஒரு பண்டிகை. பார்க்க: http://www. onamfestival.org/what-is-onam.html

39. ஏப்ரல் மாதத்தின் மத்தியில் கொண்டாடப்படும் ஒரு பண்டிகை. 
40. இந்துசமயத்தை சேர்ந்த வடக்கிந்திய பெண்களால் கொண்டாடப்படும் ஒரு பண்டிகை http://www.karwachauth.com/

41. Ambedkar, B. R. 1944. 'Annihilation of caste, with a reply to Mahatma Gandhi.' - http:// www.ambedkar.org/ambcd/02.Annihilation\%20of\%20Caste.htm - Undelivered speech prepared in 1936; the third edition was released in 1944.

42. Veeramani, K. 2005. Collected works of Periyar EVR. Chennai: The Periyar Self- Respect Propaganda Institution.

43. தலித் என்ற சொற்றொடர் அரசால் வகைப்படித்தப்பட்ட ஆதிதிராவிட வகுப்பை சேர்ந்த சில குலக்குழுக்களை குறிப்பிட்டாலும் பஞ்சக்கிராமியில் உள்ளவர்கள் தலித் என்ற சொற்றொடரையே பயன்படுத்துகிறார்கள் பார்க்க: https://en.wikipedia.org/wiki/Dalit and http://socialjustice.nic.in/UserView/index?mid=76750

44. உதாரணத்திற்கு பிராம்மணர்கள் இதர வகுப்புகள் என்றும் தலித்துகள் ஆதிதிராவிட வகுப்புகள் என்றும் இருளர்கள் ஆதிதிராவிட பழங்குடியினர் என்றும் வகைப்படுத்தப்பட்டுள்ளனர்

45. Fuller, C. J., ed. 1996. Caste today. New York: Oxford University Press; Gough, E. K.1955. 'The Social Structure of a Tanjore Village', in Marriot, M., ed. Village India. Chicago, IL: University of Chicago Press; Gough, E. K. 1960. 'Caste in a Tanjore Village', in Leach, E., ed. Aspects of Caste in South India, Ceylon and Northwest Pakistan. Cambridge:Cambridge University Press; Beteille, A. 1965. Caste, class, and power: Changing patterns of stratification in a Tanjore village. Los Angeles, CA: University of California Press; Srinivas, M. N. 1960. The social structure of a Mysore village. Indianapolis, IN:Bobbs- Merrill.

46. கிராம நிர்வாக குழு. இந்தப்பள்ளிகளெல்லாம் இன்னும் கிராம பஞ்சாயத்துக்கள் ஆதரவில் தான் இருக்கின்றன

47. Thurston, E. and Rangachari, K. 1909. Castes and tribes of South India. New Delhi: Asian Educational Services.

48. http://censusindia.gov.in/2011-prov-results/data_files/india/Final_PPT_2011_chapter6. pdf - As of 2011, the literacy rate of Tamil Nadu was higher than the national literacy rate.

49. Picherit, D. 2012. 'Migrant Labourers' Struggles Between Village and Urban Migration Sites: Labour Standards, Rural Development and Politics in South India.' Global Labour Journal 3(1): 143-62.

50. Gough, E. K. 1955; Gough, E. K. 1960; Beteille, A. 1965; Srinivas, M. N. 1960.

51. ஒரு ஸ்டெர்லிங் பவுண்ட் உத்தேசமாக இந்தியப்பணம் 100 ரூபாய்களுக்கு ஒத்ததாகும்

52. http://dmk.in/english

53. http://aiadmk.com/en/home/

54. http://mdmk.org.in/

55. http://www.dmdkparty.com/

56. http://www.thiruma.in/

57. http://www.pmkparty.in/

58. தமிழ்நாட்டு அரசாங்கம், காலம்காலமாக தவறாக நடத்தப்பட்டுவரும் குலக்குழுக்களுக்கு ஆதரவாக கோட்டா அடிப்படையிலான ஒதுக்கீட்டு கொள்கையை உருவாக்கியிருக்கிறது குறிப்பாக வேலைவாய்ப்புகள் (அரசாங்க வேலைகள்) மற்றும் கல்வி (அரசு மற்றும் தனியார் பள்ளிகள்)

59. Hammersley, M. and Atkinson, P. 2007. Ethnography: Principles in practice. New York: Routledge; Van Maanen, J. 2011. Tales of the field: On writing ethnography. Chicago, IL: University of Chicago Press.

60. Boellstorff, T. 2012. Ethnography and virtual worlds: A handbook of method. Princeton, NJ:Princeton University Press; Pink, S., Horst, H., Postill, J., Hjorth, L., Lewis, T. and Tacchi, J. 2015. Digital ethnography: principles and practice. Los Angeles, CA: Sage Publications; Hine, C. 2015. Ethnography for the internet: embedded, embodied and everyday. London:Bloomsbury Publishing.

61. இந்த கேள்வித்தாள்கள் அனைத்து ஒன்பது ஆய்வுக்களங்களுக்கும் பொதுவானவை. Q2 வின் முடிவுகள் ஒப்பிடப்பட்டு அத்தியாயமாக பிரசுரிக்கப்பட்டன. பார்க்க: Miller, D., Costa, E., Haynes, N., McDonald, T., Nicolescu, R., Sinanan, J., Spyer, J., Venkatraman, S. and Wang, X. 2016. How the World Changed Social Media. London: UCL Press, 286.

62. Miller et al. 2016. 


\section{அத்தியாயம் 2}

1. http://www.deccanchronicle.com/141016/nation-current-affairs/article/ chennai-fifth-number-facebook-users

2. இந்த துரிதமான இயக்கம் இந்தியாவில் ஐசிடி-க்களின் வளர்ச்சியுடன் தொடர்புடையது. தவளைப்பாய்ச்சல் மற்றும் தொழில்நுட்ப விரவல் ஆகியவை வளரும் நாடுகளுக்கான வளர்ச்சி இலக்கியங்களுக்காக ஐசிடியில் தீவிரமாக விவாதிக்கப்படுகின்றன. இருப்பினும், பஞ்சக்கிராமியின் வரிசையான பாய்ச்சலுக்கு காரணம், கட்டுப்படியாகும் தொழில்நுட்பங்களின் வணிகச்சந்தை ஊடுருவலும் டிஜிட்டல் பிரிவின் ஒருங்கிணைப்பு மற்றும் வளர்ந்து வரும் ஐடி துறை ஆகியவற்றால் இ இந்தப்பகுதியில் ஏற்பட்ட சமூூகப்பொருளாதார மாற்றமும் தான். See Friedman, T. L. 2005. The world is flat: A brief history of the twenty- first century. New York: Farrar, Straus and Giroux; Jeffrey, R. and Doron, A. 2013. Cell phone nation: How mobile phones have revolutionized

3. business, politics and ordinary life in India. New Delhi: Hachette; Soete, L. 1985.'International diffusion of technology, industrial development and technological leapfrogging.'World Development 13(3): 409-22; Pentland, A., Fletcher, R. and Hasson, A. 2004.'Daknet: Rethinking connectivity in developing nations.' Computer 37(1): 78-83; Mani, S.2007. 'Revolution in India's Telecommunications Industry.' Economic and Political Weekly:578-80; Mani, S. 2012. 'Bridging the digital divide: the Indian experience in increasing the access to telecommunications services.' International Journal of technological Learning, Innovation and Development 5(1-2): 184-203; Rangaswamy, N. and Cutrell, E. 2013. 'Anthropology, Development and ICTs: Slums, Youth and the Mobile Internet in Urban India', Special Issue, Reflections at the Nexus of Theory and Practice, Information Technology and International Development, Cambridge, MA: MIT Press; Toyama, K., Kiri, K., Maithreyi,L., Nileshwar, A., Vedashree, R. and MacGregor, R. 2004. 'Rural kiosks in India.' MSR Technical Report. For discussions on further growth of ICTs please see Steinmueller, W.E. 2001. 'ICTs and the possibilities for leapfrogging by developing countries.' International Labour Review 140(2): 193-210; Mathur, A. and Ambani, D. 2005. 'ICT and rural societies:Opportunities for growth.' The International Information \& Library Review 37(4): 345-51. For the diffusion of ICT in India and its associated development see Vijaybaskar,M. and Gayathri, V. 2003. 'ICT and Indian Development: Processes, Prognoses, Policies.'Economic and Political Weekly 38(24): 2360-4; Bajwa, S. B. 2003. 'ICT Policy in India in the era of liberalization: its impact and consequences.' Global Built Environment Review 3(2):49-61; Kumar, P. 2014. 'ICT and Its Development in India.' http://www.irjcjournals.org/ijieasr/Feb2014/2.pdf. For a critique on bridging the digital divide, see Wade, R. H. 2002.'Bridging the digital divide: new route to development or new form of dependency?' Global Governance 8(4): 443-66.

4. Kumar, K. J. 2014. Mass communication in India. New Delhi: Jaico Publishing House; Rangaswamy, N. Pocket Social Networking in India - SMS GupShup Expands, Asia Pacific Memo, November 2012. http://www.asiapacificmemo.ca/pocket-social-networkin g-inindia-sms-gupshup-expands

5. இயற்கையான அமைப்புகளில் நேருக்கு நேரான தகவல் பரிமாற்றங்கள் இன்றும் பஞ்சக்கிராமியின் ஆதிக்கம் நிறைந்த தகவல் பரிமாற்ற வகையாக கருதப்படுகிறது. நேருக்கு நேர் இடைவினைகளைப் பற்றிய புரிதலுக்கு பார்க்க: Goffman, E. 2005. Interaction ritual: Essays in face to face behavior. Piscataway,NJ: AldineTransaction.

6. இது ஒருவகையில் ஊடகம் தான் தகவலே என்ற மெக்லுஹானின் கருத்துக்கு தொடர்புடையதாக இருக்கலாம் McLuhan, M.1964. Understanding Media. The extensions of man. London: MIT Press. See also Rogers, E.M. and Bhowmik, D. K. 1970. 'Homophily- heterophily: Relational concepts for communication research.' Public Opinion Quarterly 34(4): 523-38.

7. ஒருசில தகவல்களை தெரிவிப்பதற்காக சரியான தகவல் தெரிவிப்பாளரை கண்டுபிடிப்பது சில சமயங்களில் சவாலாக இருக்கக்கூடும். உதாரணத்திற்கு, J.F மார்ஷல் என்பவற்றின் கட்டுரை இந்தியகிராமப்புறங்களில் குடும்பக்கட்டுப்பாடு திட்டங்களை பற்றி பேசுவதற்கு அதிக அளவில் பெண்களை பணியில் அமர்த்த வேண்டிய தேவையை பற்றி விவாதிக்கிறது.இந்தக்கட்டுரை 1971 ஆம் வருடத்தை சேர்ந்ததாக இருந்தாலும், குறிப்பிட்ட தகவல்களை தெரிவிப்பத- 
ற்கு, குறிப்பிட்ட வகையான தகவல் தெரிவிப்பாளர்கள் தேவைப்படும் என்ற வரலாற்று உணர்வை நமக்கு அளிக்கிறது. Marshall, J. F. 1971. 'Topics and networks in intra- village communication', in Polgar, S. A., ed. Culture and population: a collection of current studies. Cambridge, MA: Schenkman, 160-6. For an understanding of communication in Indian villages see Rao, Y. L. 1966. Communication and development:A study of two Indian villages. Minneapolis, MN: University of Minnesota Press; Damle, Y. B.1956. 'Communication of modern ideas and knowledge in Indian villages.' Public Opinion Quarterly 20(1): 257-70. Epstein, A. L. 1969. 'Gossip, Norms and Social Network', in Mitchell, J. C., ed. Social networks in urban situations: analyses of personal relationships in Central African towns. Manchester: Manchester University Press.

8. பஞ்சக்கிராமியில், இந்த பொது இடப்பரப்பு, சமூகத்தில் குலத்தின் அடி ப்படையில் தனிப்படுத்தப்படவில்லை. இதை அறிந்துகொள்வது முக்கியம் ஏனென்றால், இந்தியாவில் பொது இடங்கள் பலசமயங்களில் குலம் மற்றும் தீண்டாமையோடு தொடர்புபடுத்தப்படுகிறது. பார்க்க: Alex, G. 2008. 'A Sense of Belonging and Exclusion: "Touchability" and "Untouchability" in Tamil Nadu.' Ethnos 73(4): 523-43; Bros, C. and Couttenier, M. 2010. Untouchability and public infrastructure. https://hal.archives-ouvertes.fr/halshs-00542235/; Shah, G.2006. Untouchability in rural India. New Delhi: Sage Publications; Sooryamoorthy, R.2008. 'Untouchability in modern India.' International Sociology 23(2): 283-93; Alexander,K. C. 1968. 'Changing Status of Pulaya Harijans of Kerala.' Economic and Political Weekly: 1071-4.

9. ஆங்கிலத்தில் டெலிக்ராம் என்றும் தமிழில் தந்தி என்றும் கூறப்படுகிறது

10. http://www.bbc.co.uk/news/world-asia-india-23304251

11. இந்தியாவில் பெருந்திரள் தகவல் பரிமாற்றம் பற்றிய பொதுவான வரலாற்று விவரங்களுக்கு பார்க்க: Kumar, K. J. 2014; Vilanilam, J. V.2005. Mass communication in India: A Sociological perspective. New Delhi. Sage Publications.

12. http://www.suryanfm.in/chennai/; http://www.radiomirchi.com/chennai; http://www. 927bigfm.com/city.php?id=11

13. http://www.firstpost.com/politics/freebies-culture-in-tamil-nadu-reeks-of-a-guiltyconscious-neta-who-doesnt-really-care-2781472.html; http://www.livemint.com/Opinion/GGMQFv1iFGJiKMzPZWWVLN/Why-Tamil-Nadus-freebie-culture-works. html

14. http://www.sunnetwork.in/, http://jayatvnetwork.com/default.aspx, http://www.in.com/tv/ channel/star-vijay-164.html

15. http://www.dailythanthi.com/, http://www.dinamalar.com/

16. http://www.thehindu.com/, http://www.newindianexpress.com/, http://www.deccanchronicle.com/, http://timesofindia.indiatimes.com/, http://economictimes.indiatimes.com/

17. https://www.kumudam.com/, http://www.vikatan.com/

18. http://indiatoday.intoday.in/, http://www.businesstoday.in/, http://www.femina.in/,http:// www.vogue.in/

19. http://www.dqindia.com/, http://www.digit.in/. These were accessed both online and in print versions. A few IT managers even said that their companies had subsidised subscription fees for tech magazines.

20. Jeffrey, R. and Doron, A. 2013; Pitroda, S. 1993. 'Development, democracy, and the village telephone.' Harvard Business Review 71(6): 66-8.

21. இது தற்காலிகமானது. அண்டை வீட்டார், தொலைபேசி இருக்கும் வீட்டாரை அணுகி, தங்களது நெருங்கிய உறவினருக்கோ அல்லது நண்பருக்கோ தொலைபேசி அழைப்பு விடுக்கவோ அல்லது அவர்களிடமிருந்து அழைப்பு பெறவோ அவர்களது உதவியை நாடுவார்கள். அப்போதெல்லாம், மாநிலங்களுக்கு இடையேயான நீண்ட தூர அழைப்புகளுக்கு நெடுந்தொலைப்பேச்சு என்கிற ட்ரங்க் கால் பதிவு செய்ய வேண்டி இருந்தது.

22. Kumar, K. J. 2014. STD is an abbreviation of Subscriber Trunk Dialling; ISD stands for International Subscriber Dialling.

23. http://scroll.in/article/744579/what-happened-to-india-when-the-landline-telephon efell-terminally-ill-20-years-ago

24. ஸ்மார்ட்போன் அல்லாத என்கிற சொற்றொடர், செயல்பாட்டு போன் என்பதற்கான இ இணைச்சொல்லாக பயன்படுத்தப்பட்டிருக்கிறது. இந்தியாவில் மொபைல் போன்களால் விளைந்த பொதுவான மற்றும் தீவிரமான மாற்றங்களைப் பற்றிய விரிவான அலசலுக்கு பார்க்க:Jeffrey, R. and Doron, A. 2013; Kavoori, 
A. and Chadha, K. 2006. 'The cell phone as a cultural technology: Lessons from the Indian case', in Kavoori, A. and Arceneaux, N., eds. The cell phone reader: Essays in social transformation. New York: Peter Lang; Katz, J. E. 2008. Handbook of mobile communication studies. Cambridge, MA: MIT Press. For a detailed understanding of mobile use in the developing world see Donner, J.2008. 'Research approaches to mobile use in the developing world: A review of the literature.'The Information Society 24(3): 140-59. For a general idea of mobile phone use from an anthropological perspective see Horst, H. and Miller, D. 2006. The cell phone: An anthropology of communication. Oxford: Berg.

25. தர அடையாளம் இல்லாத, உள்ளூர் வன்பொருள் தொழில்நுட்ப பணியாளரால் பாகங்கள் ஒன்றிணைக்கப்பட்ட மேசைக் கணினிகள் மிகவும் பிரபலமாயின. அவற்றின் எண்ணிக்கையும் அதிகரிக்க துவங்கியது.

26. Nisbett, N. 2009. Growing Up in the Knowledge Society: Living the IT Dream in Bangalore. New Delhi: Routledge.

27. இது இந்தப்பகுதியின் குடியிருப்பு வளர்ச்சியுடன் நெருங்கிய தொடர்புடையது

28. Sreekumar, T. T. 15-19 July 2014. 'New media, space and marginality: A comparative perspective on cyber cafe use in small and medium towns in Asia.' Paper presented at the International Association for Media and Communication Research (IAMCR) Annual Conference, Hyderabad, India; Rangaswamy, N. and Bombay, L. S. I. 2007. 'ICT for development and commerce: A case study of internet cafés in India' (research in progress paper) in Proceedings of the 9th International Conference on Social Implications of Computers in Developing Countries, São Paulo, Brazil; Donner, J. September 2006. 'Internet use (and non- use) among urban microenterprises in the developing world: an update from India', in Conference of the Association of Internet Researchers (AoIR): 28-30.

29. இந்தியாவின் முதல் மற்றும் மிகப்பிரபலமான சமூக ஊடகமான ஆர்குட் பற்றிய பல ஆராய்ச்சிகள் இருக்கின்றன. பார்க்க Ahmad, A. 2011. 'Rising of social network websites in India overview.'International Journal of Computer Science and Network Security 11(2): 155-8; Pillai, 2012.'User acceptance of social networking websites in India: Orkut vs. Facebook.' International Journal of Indian Culture and Business Management 5(4): 405-14; Goyal, S. 2012. 'Social networks on the Web', in Peitz, M. and Waldfogel. J., eds. The Oxford handbook of the digital economy. Oxford: Oxford University Press, 434-59; Mishra, S. 2010. Participation of youth in social networking sites in India.' International Journal of Business Innovation and Research 4(4): 358-75; Mahajan, P. 2009. 'Use of social networking in a linguistically and culturally rich India.' The International Information \& Library Review 41(3): 129-36;Das, A. 2010. 'Social interaction process analysis of Bengalis on Orkut', in Tiwai, R.,ed. Handbook of Research on Discourse Behavior and Digital Communication: Language Structures and Social Interaction. Hershey, PA: IGI Global, 66-84; Das, A. 2012. 'Impression Management on Facebook and Orkut: A Cross Cultural Study of Brazilians and Indians.'Internet Research 13.0 Conference, University of Salford, UK, 18-22 October 2012; Das, A. and Herring, S. C. 2016. 'Greetings and interpersonal closeness: The case of Bengalis onOrkut.' Language \& Communication 47: 53-65.

30. http://www.alexa.com/siteinfo/orkut.com; Peterson, M. 2011. Orkut Dissected: Social Networking in India \& Brazil. http://www.aimclearblog.com/2011/06/27/orkut-dissectedsocia 1-networking-in-india-brazil/

31. குடியுரிமை அல்லாத இந்தியர்கள் (NRIs) என்பவர்கள், ஒரு வருடத்தின் 182 நாட்களுக்கு மேற்பட்டு இந்தியாவிற்கு வெளியே வாழும் இந்தியர்களைக் குறிக்கும். இது ஒரு வரி அந்தஸ்தாக இருந்தாலும், இது போன்ற தனிநபர் குறிப்புகள் பஞ்சக்கிராமியில் மிகவும் பொதுவாக ஆகிவிட்டது. ஐடி துறையினரின் தொடர்ச்சியான புலம்பெயர்வு மற்றும், அவர்களின் வாடிக்கையாளர்களை தொடர்புகொள்வதற்கான அவர்களின் வெளிநாட்டு பயணங்கள் ஆகியவை தான் இது போன்ற குறிப்புகளுக்கு அடிப்படை. https://en.wikipedia. org/wiki/Non-resident_Indian_and_person_of_Indian_origin. NRI as a reference term is also commonly used in Indian urban centres as well.

32. கூகுளால் பெறப்பட்ட ஆர்குட், 2014ஆம் ஆண்டு கலைக்கப்பட்டது. http://orkut.com/

33. Jeffrey, R. and Doron, A. 2013; Smyth, T. N., Kumar, S., Medhi, I. and Toyama, K. April 2010. 'Where there's a will, there's a way: mobile media sharing in urban India', in Proceedings of the SIGCHI conference on Human Factors in computing systems. Atlanta, 
GA: ACM, 753-62; Kumar, K. J. and Thomas, A. O. 2006. 'Telecommunications and Development: The Cellular Mobile "Revolution" in India and China.' Journal of Creative Communications 1(3): 297-309. For a view of the smartphone industry see Woyke, E. 2014. 'The Smartphone: Anatomy of an industry.' New York: The New Press.

34. Rangaswamy, N. and Yamsani, S. 2011. "Mental Kartha Hai" or "It's Blowing my Mind": Evolution of the Mobile Internet in an Indian Slum.' EPIC, The Ethnographic Praxis in Industry Conference, Boulder, CO, 18-21 September; Kumar, N. and Rangaswamy, N. 2013. 'The Mobile Media Actor- Network in Urban India.' ACM Conference on Human Factors in Computing Systems (CHI 2013), Paris, France, April 2013; Donner, J. 2015. After access: Inclusion, development, and a more mobile Internet. Cambridge, MA: MIT Press.

35. Jeffrey, R. and Doron, A. 2013.

36. இது ஐபோன்கள் முதல் மலிவு விலை ஸ்மார்ட்போன்கள் வரை அனைத்தையும் உள்ளடக்கியது.

37. http://www.statista.com/statistics/257048/smartphone-user-penetration-in-india/

38. இது நீண்டகால குடியிருப்பாளர்கள் (கிராமங்களில் இருந்து வந்தவர்கள்), மத்தியதர வகுப்பை சேர்ந்த ஐடி பணியாளர்கள் மற்றும் இந்த பகுதியில் இப்போது குடியேறியிருக்கும் மற்றவர்கள் ஆகிய அனைவரையும் உள்ளடக்கியது.

39. அவர்கள், இந்தப்பகுதியில் குடியிருப்பாளர்கள் அல்ல. அநேகமாக சென்னையில் இருந்து வருபவர்கள்.

40. தமிழ்நாட்டின் வெற்றிகரமான போன் வணிகர் பற்றிய குறிப்புக்கு பார்க்க: Jeffrey, R. and Doron, A. 2013.

41. ஒரு ஸ்டெர்லிங் பவுண்டு சுமார் நூறு இந்திய ரூபாய்கள் என மதிப்பிடப்படுகிறது.

42. Fuller, C. 2011. 'Timepass and boredom in modern India.' Anthropology of this Century 1 - a review of Jeffrey, C. 2011. 'Timepass: Youth, Class and The Politics of Waiting in India.'

43. குறிப்பாக உயர்-மத்தியதர குடும்பங்களில் காணப்படுகிறது

44. இந்த அத்தியாயம், இதை மேலும் முகநூல் பகுதியில் விவாதிக்கிறது. இது கல்வியைப்பற்றியதான அத்தியாயம் 6-லும் விவாதிக்கப்படுகிறது.

45. http://www.dqchannels.com/laptops-freebies/

46. http://en.wikipedia.org/wiki/Tethering

47. For more on pirate modernity in India see Sundaram, R. 2009. Pirate modernity: Delhi's media urbanism. New York. Routledge.

48. http://timesofindia.indiatimes.com/tech/how-to/Assemble-a-PC-within-Rs-30000-budget/ articleshow/19797394.cms; http://www.icmrindia.org/free\%20resources/Articles/Indian\%20 PC\%20Market2.htm

49. http://www.firstpost.com/politics/phones-wi-fi-electricity-aiadmks-manifesto-fortamilnadu-polls-is-full-of-freebies-2767768.html; http://aiadmk.com/en/tn-election-2016/tamilnad u-election-manifesto-2016/; http://aiadmk.com/en/tn-election-2016/manifestoinfographics/\#

50. இதன் ஒரு வடிவம் முன்னிலைப்படுத்தப்பட்டது Venkatraman, S., Rangaswamy N. and Arora, P. 2014.'Polymedia: A perspective through filial relationships at Panchagrami.' Paper for Panel,Reconstituting Marginality and Publics in the Digital Age, Annual International Conferenceof Media and Communication Research, 15-19 July 2014. Hyderabad.

51. குறுந்தகவல் சேவை. எழுதுதல் என்றும் கூறப்படுகிறது.

52. தவறும் அழைப்புகளை தகவல் பரிமாற்ற முறையாக பயன்படுத்துதல் வேறு பல சூழல்களிலும் காணப்படுகிறது. இது இந்தியாவிற்கு மட்டும் உரித்தானதல்ல. பார்க்க Donner, J. 2007. 'The rules of beeping: exchanging messages via intentional "missed calls" on mobile phones.' Journal of Computer- Mediated Communication 13(1): 1-22; Donner, J. 2005. 'What can be said with a missed call? Beeping via mobile phones in sub- Saharan Africa.' Proc. Seeing, Understanding, Learning in the Mobile Age, Institute for Philosophical Research of the Hungarian Academy of Sciences and T- Mobile Hungary Co Ltd, Budapest: 267-76.

53. http://ww w.independent.co.uk/news/world/asia/nirbhaya-case-anger-and-protests-asjuvenile-delhi-gang-rapist-freed-after-three-years-in-reform-a6780601. html; http://www.bbc.co.uk/news/world-asia-3511597454 
54. Baym, N. K. 2015. Personal connections in the digital age. Chichester: John Wiley \& Sons;Papacharissi, Z., ed. 2010. A networked self: Identity, community, and culture on social networksites. New York: Routledge.

55. இந்த கணக்கெடுப்பின் நோக்கம் சமூக ஊடக உபயோகிப்பாளர்களிடையே ஒரு சில சமூக ஊடகத்தளங்களின் பிராபல்யத்தை கணிப்பது தான். ஒரு ஊடகத்தளத்தின் மீது மற்றதன் பிராபல்யத்தை கணிப்பதற்கு இது மிகவும் முக்கியம். ஆகவே, இந்த கணக்கெடுப்பின் முடிவுகள், இது நடத்தப்பட்ட மாதிரி மக்கட்தொகைக்கானதே தவிர, பொதுவான மக்கட்தொகைக்கு இது நீட்டிக்கப்படமாட்டாது. இந்த கணக்கெடிப்பு, மக்கத்தொகையை, நீண்டகால குடியிருப்பாளர்கள் (n=62) மற்றும் அடுக்குமாடி குடியிருப்புகளில் வசிக்கும் சமீபத்திய குடியிருப்பாளர்கள் (n=68) என்று இரண்டு வகையாக பிரிக்கப்பட்டிருக்கிறது. பெரும்பாலான நீண்டகால குடியிருப்பாளர்கள் கீழ்மட்ட சமூகப்பொருளாதார வகுப்பு மற்றும் மத்தியதர வகுப்பை சேர்ந்தவர்கள். ஐடி யில் பணிபுரியும் புதிய குடியிருப்பாளர்கள், மத்தியதர மற்றும் உயர்-மத்தியதர வகுப்பை சேர்ந்தவர்கள். கணக்கெடுப்பின் மாதிரியில், நீண்ட கால குடியிருப்பாளர்கள் 14-38 வயதுக்குட்பட்ட ஆண்களாகவும், புதிய குடியிருப்பாளர்கள் 15-70 வயது வரம்பிலுள்ள ஆண்-பெண் இருபாலாராகவும் இருக்கிறார்கள்.

56. தளங்களாக இரண்டும் வெவ்வேறாக இருந்தாலும், வணிகநிறுவனமாக இரண்டும் ஒன்றே தான். வாட்சப் மற்றும் இன்ஸ்டாகிராம் இரண்டுமே முகநூலுக்கு தான் சொந்தமானது: http://www.bbc.co.uk/news/business-26266689. Facebook also owns Instagram: http://www.bbc.co.uk/news/technology-17658264

57. இன்ஸ்டாகிராமின் இடைப்பட்ட பயன்பாடு, மத்தியதர வகுப்பைச்சேர்ந்த பதின்பருவத்தினர் மற்றும் கல்லூரி மாணவர்களிடையே காணப்பட்டது.

58. இந்த எண்ணிக்கை, முகநூலை மொபைலில் உபயோகிப்பவர்கள் எண்ணிக்கையோடு ஏறத்தாழ ஒத்துப்போகிறது (socialbakers.com) on 30 April 2015.

59. இது பஞ்சக்கிராமியில் மட்டுமல்லாமல் வடஇந்தியாவில் உள்ள கிராமங்களிலும் காணப்பட்டது. பார்க்க http://www.independent.co.uk/news/world/asia/ girls-and-unmarried-women-in-indiaforbidden-from-using-mobile-phones-to-preven t-disturbance-in-a6888911.html; http://mashable.com/2016/02/22/india-villages-ban-mobil e-phones/\#iCfdPBCP.ZqJ

60. உயர்மட்ட மற்றும் கீழ்மட்ட குலத்தை சேர்ந்தவர்களிடையேயான திருமணமும் உறவுக்கலப்பும், மாசுபட்டதாக பார்க்கப்படுகிறது. மாசுபாடு மற்றும் தூய்மை பற்றிய கருத்துக்கள் மற்றும் இவற்றின் குலத்துடனான தொடர்புகள் பற்றிய அறிமுக விவரங்களுக்கு பார்க்க http://rohitshrawagi.blogspot.co.uk/. For an anthropological exploration and critique of this concept see Fuller, C. J. 1979.'Gods, priests and purity: on the relation between Hinduism and the caste system.' Man:459-76; Marglin, F. A. 1977. 'Power, purity and pollution: Aspects of the caste system reconsidered.'Contribution to Indian Sociology Delhi 11(2): 245-70; Gough, E. K. 1955. 'The social structure of a Tanjore village.' in McKim, M. ed. Village India, Chicago, IL: University of Chicago Press; Gough, K. 1973. 'Harijans in Thanjavur.' Imperialism and revolution in South Asia. New York: Monthly Review Press, 222-45.

61. இதை மொபைல் போனை பகிர்ந்து கொள்வது என்று கூறமுடியாது ஏனெனில், இங்கு ஒரு குறிப்பிட்ட நேரத்துக்கு மொபைல் போன் கடன் பெற்றுக்கொள்ளப்படுகிறது. இருப்பினும், மொபைல் போன்களை பகிர்ந்து கொள்வது இந்தியாவில் நாடாகும் ஒரு செயல் தான். பார்க்க Steenson, M. and Donner, J. 2009. 'Beyond the personal and private: Modes of mobile phone sharing in urban India', in Ling, R. and Campbell, S. eds. The reconstruction of space and time: Mobile communication practices, New Brunswick, NJ: Transaction, 231-50.

62. கிராமப்புறங்களிலும், கீழ்-மத்தியதர மற்றும் கீழ்மட்ட சமூகப்பொருளாதார வகுப்பினரிடையேயும் பெண்கள் மொபைல் போன் உபயோகப்படுத்துவது வேறுபடுகிறது. இவற்றில் ஒரு சில சமூகங்கள், அவர்களை சுதந்திரமாக போன் உபயோகிக்க அனுமதிக்கின்றன. ஒருசில, இடம் மற்றும் நேரக்கட்டுப்பாடு விதிக்கின்றன. வேறுசில ஒட்டுமொத்த தடை விதிக்கின்றன. பார்க்க Doron, A. 2012. 'Mobile persons: Cell phones, gender and the self in North India.' The Asia Pacific journal of Anthropology 13(5): 414-33; Jeffrey, R. and Doron, A. 2013; Mehta, B. S. 2013. 'Capabilities,costs, networks and innovations: impact of mobile phones in rural India.' Available at SSRN 2259650; Jouhki, J. 2013. 'A Phone of One's Own? Social Value, Cultural Meaning and Gendered Use of the Mobile Phone in South India.' Journal of the Finnish Anthro- 
pological Society 38(1): 37-58; Gurumurthy, A. and Menon, N. 2009. 'Violence against women via cyberspace.' Economic and Political Weekly: 19-21; Tenhunen, S. 2014. 'Mobile telephony,mediation, and gender in rural India.' Contemporary South Asia 22(2): 157-70. The associations of a mobile phone with romance and sexual liaisons were not unique to India but were also found in the context of Jamaica; see Miller, D. and Slater, D. 2005. 'Comparative Ethnography of New Media', in Curran, J. P. and Gurevitch, M., eds. Mass Media and Society, 4th edition. London: Hodder Arnold.

63. Laughey, D. 2007; Gottdiener, M. 2015.

64. அவர்கள் அனைவரும், என்னுடன் நட்புதொடர்பை மறுத்துவிட்டனர். என்னுடைய ஆராய்ச்சி முகநூல் பக்கம் மூலமாக மற்ற ஆண்களுக்கு வெளிப்பட்டுவிடுவோமோ என்ற பயம் தான் இதற்கு காரணம்.

65. ஐடி துறை பணியாளர்கள், தொழில் முனைவோர்கள் மற்றும் இதர திறன்வாய்ந்த பணிகளில் இருப்போரின் குடும்பத்தினர்.

66. Rangaswamy, N. and Arora, P. 2015. 'Digital Romance in the Indian City', in The City and South Asia. Cambridge, MA: Harvard South Asia Institute.

67. மிகக்குறைந்த தொழில்நுட்ப சாத்தியக்கூறுகள் உள்ள மொபைல் போன்களிலும் கூட ஊடகத்தளங்களின் பகிர்தலும் காணப்படுகிறது. Please refer to Smyth, T. N. et al. 2010.

68. இவற்றின் வழக்காய்வுகள் அத்தியாயம் 4-ல் குடும்பங்களைப் பற்றிய கலந்தாய்வின் ஒரு அங்கம் வகிக்கின்றன.

69. உறவுமுறைகள் பற்றிய பல தரநெறிசார் பிரசங்கங்கள் இந்த முதியவர்களின் குழுவில் நிகழ்ந்துகொண்டிருந்தன.

70. http://blogs.ucl.ac.uk/global-social-media/2013/12/15/non-resident-indians/

71. பஞ்சக்கிராமியில் உள்ள பொய்யான முகநூல் கணக்குகள் பற்றிய எண்ணிக்கையை கணக்கிடுவது மிகவும் கடினமாக இருந்தது. ஏனெனில், யாரும் அத்தகைய தகவலை பகிர்ந்துகொள்வதில் விருப்பம் காட்டவில்லை. இரு வேறு சமூக வலைத்தொடர்புகளில் ஒரு நபர் தனக்கான அடையாளத்தை நிறுவுவதற்காக உருவாக்கக்கூடிய பல கணக்குகள் பற்றிய நம்பகமான விவரங்களுக்கு, பார்க்க: http://blogs.ucl.ac.uk/global-social-media/2014/04/11/who-am-i-the-case-of-caste-r elated-profiles-on-facebook/

72. இந்த செயல்திட்டம் இன்னும் வாட்சப்பை சமூக ஊடகத்தளமாக பார்க்கிறது.

73. Q1 கணக்கெடுப்பில் விவாதித்தது போல்.

74. அத்தியாயம் 1-ல் விவாதித்த அளவீட்டு சமுதாயம் பற்றிய கருத்துடன் நெருங்கிய தொடர்புடையது. பார்க்க Miller, D. et al. 2016.

75. Madianou, M. and Miller, D. 2013. 'Polymedia: Towards a new theory of digital media in interpersonal communication.' International Journal of Cultural Studies 16(2): 169-87.

76. இருப்பினும் அவர்கள் டுவிட்டர் அல்லது லிங்க்ட்-இன் போன்ற மற்ற தளங்களையும் பயன்படுத்தினர். ஒரே தளத்தை மட்டும் பயன்படுத்துவது என்பது வெகு அபூர்வமாக தான் இருந்தது. பஞ்சக்கிராமியில் பன்னூடகங்கள் என்ற கலாச்சாரம் மிகவும் உறுதியாக இருந்தது. Madianou, M. and Miller, D. 2013.

77. ஒருவகையில் இது அளவீட்டு சமுதாயம் என்ற எண்ணத்தை குறிப்பிடுகிறது இது விளக்கப்பட்டுள்ள இடம்: Miller, D. et al. 2016.

78. http://www.dnaindia.com/scitech/report-whatsapp-user-base-crosses-70-milli on-inindia-2031465

79. இந்த குழுக்கள் அளவு மற்றும் வகையில் மாறுபட்டிருந்தன. உதாரணத்திற்கு, அவை தனிப்பட்டவை, தொழில் நெறி சார்ந்தவை, ஆன்மிகம், பொழுதுபோக்கு என்று பலவகைப்படலாம்.

80. Miller, D. et al. 2016.

81. இதைப்பற்றிய மேலும் விவரங்கள் குடும்பத்தின் மீது வாட்சப்பின் தாக்கம் பற்றிய அத்தியாயம் 4-ல் விவாதிக்கப்பட்டுள்ளது.

82. லட்சுமி, இதை தன் மாமியாரிடம் செய்வதில்லை. அவர் ஏற்கனவே லக்ஷ்மியை தாய்மையின் கவனிப்பில்லாமல் தனது குழந்தையை விட்டுவிட்டு வேலைக்கு செல்லும் தாய் என்று விமர்சித்துக்கொண்டிருக்கிறார்.

83. See Appadurai, A. 1988. The social life of things: Commodities in cultural perspective. Cambridge: Cambridge University Press.

84. கருதப்பட்டது ஏனெனில், பலர், தங்கள் மொபைல் போன் வாட்சப் மட்டுமல்லாமல் இத்தகைய இயங்குதிறனையும் அளிக்கிறது என்பதை அங்கீகரிக்க தவறிவிட்டார்கள். 
85. இருப்பினும், முன்பே குறிப்பிட்டபடி, அவர்கள் வயதையொத்த ஆண்களை விட அவர்கள் முகநூலில் அதிக சுறுசுறுப்பாக செயல்பட்டுக்கொண்டிருந்தனர்.

86. ஒரு பெண்ணின் கண்ணோட்டத்திலிருந்து வாட்சப் மூலமான காதல் பற்றிய மேலும் தகவலுக்கு பார்க்க Costa,E. 2016. Social Media in Southeast Turkey. London: UCL Press, 206.

87. https://en.wikipedia.org/wiki/Kitty_party

88. http://blogs.ucl.ac.uk/global-social-media/2015/04/17/women-entrepreneurs-whatsapp/

89. இது பொதுவாக பொறாமை அல்லது வெறுப்பு போன்ற உணர்ச்சிகளால் கேடுவிளைவிக்கக்கூடிய பார்வையால் வரக்கூடிய சாபமாக கருதப்படுகிறது. இது யார் மேல் விழுகிறதோ அவர்களுக்கு கேடுகள் பல விளையும் என்று நம்பப்படுகிறது. பலசமயம் இதுபற்றிய விஷயங்கள் தெளிவாக இல்லாததால், முன்னெச்சரிக்கையாக இருத்தல் நல்லது என்று கருதப்படுகிறது. See https:// en.wikipedia.org/wiki/Evil_eye

90. http://blogs.ucl.ac.uk/global-social-media/2014/07/25/its-ok-to-send-myboss-a-whats app-message/

91. ஜனவரி 2016க்கு முன்பாக, பஞ்சக்கிராமியில் உள்ள வாட்சப் பயன்பாட்டாளர்கள் கட்டணம் செலுத்தவேண்டியிருந்தது ஒரு பொருட்டாகவே கருதப்படவில்லை.

92. http://thehackernews.com/2016/01/whatsapp-free-lifetime.html

93. தென்கிழக்கு துருக்கியிலும் இதுவே தான் வழக்காக இருந்தது. பார்க்க Costa, E. 2016.

94. கோலிவுட் என்பது தமிழ்த்திரையுலகின் இருப்பிடமான கோடம்பாக்கம் என்ற சொல்லுக்கும் ஹாலிவுட் என்ற சொல்லுக்குமான இணைமருவல் தான் பார்க்க https://en.wikipedia.org/wiki/Tamil_cinema

95. அத்தியாயம் 3, சமூக ஊடகங்களின் மீது திரைப்படங்களின் தாக்கம் பற்றி மேலும் விரிவாக விவாதிக்கிறது.

96. அவர்கள் களப்பணி காலத்தில் செயல்பாட்டில் இருக்கவில்லை. இந்த காலம் ஒருசில மாதங்களில் இருந்து பல வருடங்கள் வரையிலான வரம்பு கொண்டது.

97. தங்கள் பெண்களின் சமூக ஊடக பயன்பாட்டின் மீது கண்காணிப்பு போன்ற கட்டுப்பாடுகளை வைத்திருக்கும் இவர்கள் தாங்கள் பழமைவாதிகள் அல்ல என்று கூறுவது முரண்பாடாக தெரிகிறது. ஆனால், இவர்கள் ஒட்டுமொத்த தடை விதித்தவர்களோடு ஓத்திட்டு தங்களை பழமைவாதிகள் அல்ல என்று கூறிக்கொள்கிறார்கள்.

98. Miller, D. et al. 2016.

99. Madianou, M. and Miller, D. 2013.

100. Miller, D. et al. 2016.

\section{அத்தியாயம் 3}

1. இந்த மாற்றம் வடிவேலு கட்டுப்படியாகும் விலையில் முன்பக்க காமெராவுடன் கூடிய சாம்சங் ஸ்மார்ட்போன் வாங்கியதுடன் ஒத்து இருந்தது.

2. Nakassis, C.V. 2014. 'Suspended Kinship and Youth Sociality in Tamil Nadu, India.' Current Anthropology 55(2): 175-99; Nisbett, N. 2007. 'Friendship, consumption, morality: practising identity, negotiating hierarchy in middle- class Bangalore.' Journal of the Royal Anthropological Institute 13(4): 935-50.

3. அவர்களது சமூக வலைத்தொடர்புகளின் விழுமங்களுடன் அனுசரித்துப்போவது, அதுவும் இந்த வழக்கில் அவர்களின் சமூக வலைதொடர்பின் எதிர்பார்ப்புகளுக்கு ஏற்ப செயல்படுவது என்பது இந்த இளைஞர்களுக்கு மிகவும் முக்கியமானதாக இருக்கிறது. இதற்கான காரணம் வலைத்தொடர்பு ஒத்தியல்பாக இருக்கலாம். See McPherson, M. 2001. For an introduction to social network analysis, its research and applications see Marin, A. and Wellman, B. 2011. 'Social network analysis: An introduction', in Carrington, P., and Scott, J., eds. The SAGE handbook of social network analysis. London. Sage Publications, 11-25; Mitchell, J. C., ed. 1969. Social networks in urban situations: analyses of personal relationships in Central African towns. Manchester: Manchester University Press; Wasserman, S. and Galaskiewicz, J. eds. 1994. Advances in social network analysis: Research in the social and behavioral sciences. Thousand Oaks, CA: Sage Publications, 171; Christakis, N. A. 2010. Connected: The amaz- 
ing power of social networks and how they shape our lives. London: HarperCollins; Watts, D. 2003. Six Degrees: The Science of a Connected Age. New York: W. W. Norton \& Co.; Scott, J. 2012. Social network analysis. London: Sage Publications. For more on compliance in social networks see Brass, D. J. 1992. 'Power in organizations: A social network perspective.' Research in Politics and Society 4(1): 295-323.

4. Nakassis, C.V. 2013. "Youth masculinity, "style" and the peer group in Tamil Nadu, India.'Contributions to Indian Sociology 47(2): 245-69; Nakassis, C. V. 2010. 'Youth and Status in Tamil Nadu, India.' Publicly Accessible Penn Dissertations. 227. http://repository.upenn.edu/edissertations/227. UPenn Repository. For a comparative view of friendship in Kerala see Osella, C. and Osella, F. 1998. 'Friendship and flirting: micro- politics in Kerala, South India.' Journal of the Royal Anthropological Institute 4(2): 189-206.

5. Christakis, N.A. 2010.

6. Pushpa, S. 1996. 'Women and philanthropy in India.' VOLUNTAS: International Journal of Voluntary and Nonprofit Organizations 7(4): 412-27.

7. மத்தியவர்க்க இனநலப்பண்பின் இந்த கூறு கீழ்கண்டவற்றில் விவாதிக்கப்பட்டிருக்கிறது Varma, P. K. 2007. The great Indian middle class. New Delhi: Penguin Books, India, and Mathur, N. 2010. 'Shopping malls, credit cards and global brands: consumer culture and lifestyle of India's new middle class.' South Asia Research 30(3): 211-31. Irrespective of whether participants engage in charitable works to avoid feelings of guilt or whether it is true altruism, this remains a powerful part of their ideology, at least at Panchagrami. Middle- class women in Panchagrami still tend to participate in such philanthropic activities.

8. ஸ்மார்ட்போன்களின் அதிகமான சேமிப்பு இடப்பரப்பு மற்றும் வாட்சப் போன்றவற்றின் மூலம் இந்த புகைப்படங்களை நிகழ்நிலையில் பகிர்ந்து கொள்ளுதல் ஆகியவை, மக்களை மலரும் நினைவுகளில் திளைப்பதன் மூலம் இவற்றின் வாழ்நாளை நீட்டித்திருக்கின்றன பார்க்க Van Dijck, J. 2008. 'Digital photography: communication,identity, memory.' Visual Communication 7(1): 57-76.

9. கற்பனையான உறவுமுறைகளைப் பற்றிய விரிவான விவாதங்களுக்கு பார்க்க அத்தியாயம் 4

10. மரபு கண்காணிப்பாளர்களாக மீம்களின் பங்கு, மற்ற பல ஆய்வுக்களங்களிலும் காணப்பட்டது. இதுவும் நாம் ஏன் பதிவிடுகிறோம் வரிசையின் சமூக ஊடக பயன்பாட்டைப்பற்றிய பல கண்டுபிடிப்புகளில் ஒன்று. பார்க்க https://www.ucl. ac.uk/why-we-post/discoveries/14-memes-have-become-the-moral-police-of-online-life

11. Trawick, M. 1990. Notes on love in a Tamil family. Los Angeles, CA: University of California Press.

12. இது ஒருவரின் சமூக வலைதொடர்பின் மூலம் ஆதரவு கோருவதாகவும் பார்க்கப்படக்கூடும். பார்க்க Gottlieb, B.H. 1981. Social networks and social support, vol. 4. Thousand Oaks, CA:Sage Publications, Inc.

13. திரைநட்சத்திரங்களுக்கான ரசிகர் மன்றங்கள் குறிப்பாக தமிழ் திரை நட்சத்திரங்களிடையே மிகவும் பிரபலம். ஒரு சில நட்சத்திரங்கள் இவற்றை தங்களது அரசியல் ஆதாயத்துக்காகவும் பயன்படுத்திக்கொண்டிள்ளனர். பார்க்க Dickey, S. 1993. 'The politics of adulation:Cinema and the production of politicians in South India.' The Journal of Asian Studies 52(2): 340-72; Dickey, S. 1993. Cinema and the Urban Poor in South Asia. Cambridge: Cambridge University Press; Rogers, M. 2009. 'Between fantasy and "reality": Tamil film star fan club networks and the political economy of film fandom.' South Asia: Journal of South Asian Studies 32(1): 63-85; Rajanayagam, S. 2015. Popular Cinema and Politics in South India: The Films of MGR and Rajinikanth. New Delhi: Routledge; Pandian, M. S. S. 2015. The image trap: MG Ramachandran in film and politics. New Delhi: Sage Publications India; Srinivas, S.V. 1996. 'Devotion and defiance in fan activity.' Journal of Arts and Ideas 29(1): 67-83.

14. https://www.ucl.ac.uk/why-we-post/discoveries/why-we-post/discoveries/7-we-usedto-just-talk-now-we-talk-photos; Miller, D. 2015. 'Photography in the Age of Snapchat.'Anthropology and Photography Pamphlet Series. London: Royal Anthropological Institute [Photographic Committee] Web https://www.therai.org.uk/images/stories/photography/AnthandPhotoVol1B.pdf; Miller, D. and Sinanan, J. 2017. Visualising Facebook. London: UCL Press.

15. Pinney, C. 2008. The coming of photography in India. London: The British Library; Dwyer, R. 2006. Filming the gods: Religion and Indian cinema. Oxford and New York: Routledge; 
Pinney, C. 2004. 'Photos of the Gods': The Printed Image and Political Struggle in India. London: Reaktion Books; Jain, K. 2007. Gods in the bazaar: The economies of Indian calendar art. Durham, NC and London: Duke University Press.

16. Mazzarella, W. 2003. Shoveling smoke: Advertising and globalization in contemporary India. Durham, NC and London: Duke University Press.

17. Khanduri, R. G. 2014. Caricaturing Culture in India: Cartoons and History in the Modern World. Cambridge: Cambridge University Press. The Amul advertisements that are famous in India are examples of caricatures and cartoons on public billboards. See Murray, L. C.2014. Advertising Amul: On Meaning, Materiality, and Dairy in India. Web. $\quad$ http://static1.squarespace.com/static/534587eae4b0fb5fdfb963aa/t/54a9f38ce4b08424e6a8c019/1420424106572/Milk+In+India+Part+II.pdf

18. Velayutham, S. ed. 2008. Tamil cinema: the cultural politics of India's other film industry, vol.10. Oxford and New York: Routledge. Pandian, A. 2015. Reel world: an anthropology of creation. Durham, NC: Duke University Press; Dickey, S. and Jacob, P. 2008. Celluloid Deities: The Visual Culture of Cinema and Politics in South India. Lanham: MD: Lexington Books.

19. Pinney, C. 2004.

20. Ibid.

21. Zeff, A. 1999. Marriage, film, and video in Tamilnadu: Narrative, image, and ideologies of love. A dissertation submitted to University of Pennsylvania. Web: http://repository. upenn.edu/dissertations/AAI9926222/?pagewanted=all

22. தமிழ் மாதங்கள் பொதுவாக ஆங்கில மாதத்தின் 15 அல்லது 16 தேதி வாக்கில் துவங்கும். பார்க்க https://en.wikipedia.org/wiki/Tamil_calendar for more information on Tamil months.

23. அம்மனின் பெயர், இடத்திற்கு இடம் வித்தியாசப்படும். உதாரணத்திற்கு பாலாண்டியம்மன் அங்காளம்மன் போன்றவை பார்க்க: Beck, B. E. 1981. 'The Goddess and the Demon. A local South Indian festival and its wider context', in Biardeau, M. ed. Autour de la déesse hindoue. Paris: Purusartha; Sciences Sociales en Asie du Sud, 83-136. Good, A. 1985. 'The annual goddess festival in a South Indian village.' South Asian Social Scientist 1(2): 119-67. Hiltebeitel, A. 1991. The Cult of Draupadi: On Hindu Ritual and the Goddess, vol.2. Chicago, IL: University of Chicago Press. Fuller, C. J. and Logan, P. 1985. 'The Navarātri Festival in Madurai.' Bulletin of the School of Oriental and African Studies 48(1): 79-105.

24. Fuller, C. J. 2001. 'The "Vinayaka Chaturthi" Festival and Hindutva in Tamil Nadu.'Economic and Political Weekly. 1607-16.

25. கோவில் திருவிழாக்களில் சமூகங்கள் மற்றும் இனங்களின் பங்கை புரிந்துகொள்ள பார்க்க Neve, G. D.2000. 'Patronage and "community": the role of a Tamil "village" festival in the integration of a town.' Journal of the Royal Anthropological Institute 6(3): 501-19. Mosse, D. 1997.'Honour, caste and conflict: The ethnohistory of a Catholic festival in rural Tamil Nadu (1730-1990).' Purusārtha 19: 71-120.

26. Hardgrave, R. L. 1975. When stars displace the gods: The folk culture of cinema in Tamil Nadu.Austin, Texas: Center for Asian Studies, University of Texas; Dickey, S. and Jacob, P. 2008;Velayutham, S. ed. 2008; Pandian, A. 2016.

27. பாலிவுட் என்ற சொற்றொடர் ஹிந்தி திரையுலகை குறிப்பது போல கோலிவுட் என்பது தமிழ் திரையுலகை குறிக்கும்.

28. தமிழ்நாட்டில் சினிமாவை பற்றிய தெளிவான புரிதலுக்கு பார்க்க: Hardgrave, R. L. and Neidhart,A. C. 1975. 'Films and political consciousness in Tamil Nadu.' Economic and Political Weekly. 27-35; Hardgrave, R. L. 1973. 'Politics and the Film in Tamilnadu: The Stars and the DMK.' Asian Survey 13(3): 288-305; Dickey, S. 1993. 'The politics of adulation: Cinema and the production of politicians in South India.' The Journal of Asian Studies 52(2): 340-72; Pandian, M. S. S. 1992. M G Ramachandran in Film and Politics: The Image Trap. New Delhi: Sage Publications.

29. தமிழ்நாட்டின் முதலமைச்சர் செல்வி ஜே.ஜெயலலிதா அஇஅதிமுக கட்சியை சேர்ந்தவர், முன்பு பிரபலமான திரைநட்சத்திரமாக இருந்தவர். எதிர்க்கட்சி தலைவரான மு. கருணாநிதி அவர்கள் பிரபலமான திரைக்கதாசிரியர் மற்றும் வசனகர்த்தாவாக இருந்தவர். பார்க்க: Jacob, P. 1997. 'From co- star to deity: Popular representations of Ms. Jayalalitha Jayaram.' Women: A Cultural Review 8(3): 327-37. For the success of Mr Karunanidhi and his party see Hardgrave, R. L. 1973.

30. Pinney, C. 2004. 
31. புதிய படங்கள் பொதுவாக வெள்ளிக்கிழமைகளில் வெளியிடப்படுகின்றன. இதன் மூல வாரஇறுதி கூட்டத்தை பெறமுடியும் என்ற எண்ணமே காரணம்.

32. Bate, B. 2013. Tamil Oratory and the Dravidian Aesthetic: Democratic Practice in South India. New York: Columbia University Press.

33. பஞ்சக்கிராமியில் உள்ள கோயில் படிமவியல் மற்றும் கட்டமைப்பு வேறு வகையை சேர்ந்தது. பல்லாண்டுகளாக நிலைத்திருக்கிறது.

34. Pinney, C. 2008.

35. அத்தியாயம் 1-ல் குறிப்பிட்டுள்ளது போல இந்த களப்பணி காலகட்டத்தில் வெகுசில இஸ்லாமிய குடும்பங்களே பஞ்சக்கிராமியின் கிராமப்புறங்களிலும் அடுக்குமாடி குடியிருப்புகளிலும் வாழ்ந்து வந்தன. அவர்களை சந்திக்கக்கூடிய வாய்ப்பு வெகு குறைவாகவே இருந்தது. சீக்கிய குடும்பங்களுக்கும் அதே கதைதான்.

36. இந்துசமயத்தில், ஒருவரின் மூதாதைதயரை நினைவுகூருவது என்பது மிகவும் குறிப்பிடத்தக்க ஒன்றாகும். கலாசார மற்றும் சமயம் சார்ந்த நடவடிக்கையாக இது இந்துசமயத்தவரின் இல்லங்களில் இணைந்திருக்கும் ஒன்றாகும். பஞ்சக்கிராமியில் உள்ள கிறிஸ்தவ குடும்பங்களிலும் அவர்களது காலஞ்சென்ற பெற்றோர் அல்லது தாத்தாபாட்டி ஆகியோரின் படங்கள் வரவேற்பறை சுவர்களை அலங்கரித்தது. அவர்களுக்காக அவர்களின் இறந்த நாளில் இவர்கள் பிரார்த்தனை நடத்துகிறார்கள். பொதுவாக இதுபோன்ற புகைப்படங்கள் பெற்றோர் மற்றும் தாத்தாபாட்டி ஆகிய இரண்டு தலைமுறையை மட்டுமே கொண்டதாக இருக்கின்றன.

37. உயர்மத்தியதர வகுப்பை சேர்ந்தவர்கள் சில சமயங்களில் தங்கள் வரவேற்பறை புகைப்படங்களின் சட்டங்களை ஏதேனும் நிகழ்ச்சி அல்லது கொண்டாட்டத்துக்கு ஏற்ப மாற்றினர்.

38. ஒரு சில பெண்கள், தங்கள் குடும்பங்கள் மற்றும் நீட்டித்த குடும்பங்களின் பழைய புகைப்படங்களை தங்களின் பிணைப்பை வெளிப்படுத்துவதாகவும், மலரும் நினைவுகளாகவும் பதிவிடிகின்றனர்.

39. இதையே வாட்ஸப்பில் செய்வதென்பது சாத்தியமில்லை ஏனெனில், முகநூலில் செய்வது போல இதிலுள்ள காட்சியமைப்பு கூறுகளை பிரித்து வைக்க முடியாது.

40. தமிழ்நாட்டில் சினிமா தான் மிகவும் பிரபலமான பொழுதுபோக்குவகை என்று வாதிடமுடியும். பார்க்க: Dickey, S. 1993, Velayudham, S. 2008 and Pandian, A. 2015.

41. Pandian, M. S. S. 1992; Dickey, S. 2008. 'The nurturing hero: changing images of MGR', in Velayudham, S. ed. Tamil Cinema: The Cultural Politics of India's Other Film Industry, vol.10. Oxford and New York: Routledge. 77-94. Note: The pictures of MGR presented in this chapter have been taken from different Facebook groups on MGR.

42. Rajanayagam, S. 2015. Note: Rajinikanth, the current superstar of Tamil cinema, though not yet active in politics, continues with a similar style of image display.

43. இங்கு இயல்புநிலை என்பது அத்தியாயத்தின் துவக்கத்தில் விவாதித்திருந்த படி நடிகரின் படங்களை பதாகைகள் மற்றும் கட்-அவுட்டுகளாக பொதுப்பார்வைக்கு வைப்பது என்பதை குறிக்கும்.

44. நடிகைகள், குறிப்பாக தமிழ் திரைப்படங்களில் கதாநாயகிகளாக இருப்பவர்களுக்கு, தங்கள் திறமைகளை வெளிப்படுத்த வெகு குறைந்த காலமே கிடைக்கிறது. ஏனெனில் கதாநாயகர்களின் ஆதிக்கம் தமிழ் சினிமாவில் அதிகமாக இருப்பது தான். பலசமயங்களில் இவர்கள் பிற மாநிலங்களை சேர்ந்தவர்களாக இருக்கிறார்கள். இவர்களின் தோற்றம், தற்போதைய திரையுலகின் போக்கு, நாகரீகத்துடன் கூடிய புத்திகூர்மை ஆகியவை கதாநாயகிகளின் தேர்வை எம்ஜிஆர் காலத்திலிருந்தே பாதித்து வருகிறது. பார்க்க: Chinniah, S. 2008. 'The Tamil film heroine: from a passive subject to a pleasurable object', in Velayudham, S. ed. Tamil Cinema: The Cultural Politics of India's Other Film Industry, vol. 10. Oxford and New York: Routledge. 77-94; Lakshmi, C. S. 2008. 'A good woman, a very good woman: Tamil cinema's women' in Velayudham, S. ed. 2008; Nakassis, C.V. 2015. 'A Tamilspeaking Heroine.' BioScope: South Asian Screen Studies 6(2): 165-86.

45. மாநில அளவிலான அரசியல் கட்சிகளான அஇஅதிமுக (அனைத்திந்திய அண்ணா திராவிட முன்னேற்ற கழகம்), திமுக (திராவிட முன்னேற்ற கழகம்), பாமக(பாட்டாளி மக்கள் கட்சி), தேமுதிக (தேசிய முற்போக்கு திராவிட கழகம்), விசிக (விடுதலை சிறுத்தைகள் கழகம்), மதிமுக (மறுமலர்ச்சி திராவிட முன்னேற்ற கழகம்) ஆகியவையும், தமாகா (தமிழ் மாநில காங்கிரஸ்) மற்றும் பிஜேபி (பாரதீய ஜனதா பார்ட்டி) போன்ற தேசீய அளவிலான அரசியல் கட்சிகளின் தமிழ்நாட்டு கிளைகள் ஆகியவை இங்கு காணப்படுகின்றன. இந்தப்பகு- 
தியில் அரசியல் காட்சிகள் பற்றிய விரிவான தகவல்களுக்கு அத்தியாயம் 1-ஐ பார்க்கவும்

46. இந்தப்பகுதியில் அரசியல் ஆண்களின் ஆதிக்கம் நிறைந்ததாக இருக்கிறது. தேர்தல் சமயங்களில் ஆதரவிற்காக பெண்கள் அழைத்துக்கொள்ளப்படுகிறார்கள்.

47. அஇஅதிமுக தலைவி செல்வி ஜெயலலிதாவின் படங்கள் மட்டும் இதற்கு விதிவிலக்கு.

48. திமுக (திராவிட முன்னேற்ற கழகம்) தமிழ்நாட்டின் மாபெரும் தமிழ் அரசியல் கட்சி.

49. https://twitter.com/stalin_offl. For further discussion on the effective conversion of social networks to leadership see Balkundi, P. and Kilduff, M. 2006. 'The ties that lead: A social network approach to leadership.' The Leadership Quarterly 17(4), 419-39; on using social capital see Burt, R. S. 2000. 'The network structure of social capital.' Research in organizational behavior 22: 345-423; Lin, N., Cook, K. S. and Burt, R. S. eds. 2001. Social capital: Theory and research. Piscataway: NJ: Transaction Publishers.

50. அஇஅதிமுக (அனைத்திந்திய அண்ணா திராவிட முன்னேற்ற கழகம்) தமிழ்நாட்டின் மற்றொரு மாபெரும் தமிழ் அரசியல் கட்சி மற்றும் தற்போதைய ஆளும்கட்சி. தமிழ்நாட்டின் அதிகார மையங்களாக அஇஅதிமுக மற்றும் திமுக ஆகிய இரண்டும் தான் 40 ஆண்டுகளாக கோலோச்சி வருகின்றன. Perumal, C. A. and Padmanabhan, V. K. 1987. 'Political Alliances in Tamil Nadu.' The Indian Journal of Political Science 48(4):618-24; Suresh, V. 1992. 'The DMK debacle: Causes and portents.' Economic and Political Weekly. 2313-21; Thirunavukkarasu, R. 2001. 'Election 2001: Changing Equations.'Economic and Political Weekly. 2486-9.

51. அரசியல்வாதிகளின் கட் -அவுட் மற்றும் ஓவியங்களை பற்றிய மற்றும் பல சுவாரசியமான தகவல்களுக்கு பார்க்க: the work of Dr Roos Gerritsen at http:// www.materialworldblog.com/2010/04/the-unanticipated-city-shifting-urban-landscapes-a nd-the-politics-of-spectacle-in-chennai/

52. கேலிச்சித்திரங்கள் இ இந்தியாவில் நீண்ட வரலாற்றை கொண்டிருக்கின்றன. பார்க்க: Khanduri, R. G. 2014.

53. https://www.youtube.com/watch?v=vtAmdUOfuFQ\&index=14\&list=PLVwGSavjGgExtI65BTxuE4CuclQqjA7fu

54. இது பிரபலமான அரசியல் தலைவர்களை தங்களின் முகநூல் வலைத்தொடர்பு பக்கங்களுக்குள்ளாகவே பழித்துப்பேசிக்கொள்வது பற்றியது. இருப்பினும், பொதுநோக்கிய முகநூல் பக்கங்களில் அவ்வாறு செய்வது, பழிப்போடு மட்டுமல்லாமல் வசைபாடுவதாகவும் ஆகிவிடக்கூடும். தற்சமயம் பிஜேபி-யின் கொள்கைகள் பற்றிய பழித்தல்கள் இருப்பதைப்போல. பார்க்க an Udupa, S. 2015 E- seminar on Mediaanth Listserv at http://www.media-anthropology.net/file/udupa_abusive _exchange_final2.pdf. Further, though caste- related trolling does also occur, most examples are seen on public pages rather than on individual profiles.

55. Khanduri, R. G. 2014.

56. Miller, D. et al. 2016.

57. https://en.wikipedia.org/wiki/Ganesha. The Hindu deity Lord Ganesha is a symbolic representation of victory in Hindu theology. Greeting people with an image of Lord Ganesha here signifies a wish for a good day with no obstacles.

58. வணக்கம் என்பது ஆங்கிலத்தில் ஹலோ போல ஒரு வாழ்த்துச்சொல். இது மற்றவரை அங்கீகரிக்கவும், அவர்கள் மீீதா மரியாதையை தெரிவிக்கவும் பயன்படுத்தப்படுகிறது. http://test-ie.cfsites.org/custom.php?pageid=363

59. Bourdieu, P. 1980. The Logic of Practice. Cambridge: Polity Press.

60. Sivananda, S. 2004. Practice of Karma Yoga. Rishikesh: Divine Life Society.

61. Similar to Appadurai, A. 1988. The social life of things: Commodities in cultural perspective.Cambridge: Cambridge University Press.

62. புளிச்சாற்றை அடிப்படையாக கொண்டு செய்யப்பட ஒரு குழம்பு. அரிசிச்சோற்றுடன் உண்ணப்படுவது.

63. Miller, D. et al. 2016.

64. பஞ்சக்கிராமி பல்வகையான மக்கட்தொகையை கொண்டிருந்தாலும், அத்தியாயம் 2-ல் பார்த்தது போல ஒவ்வொன்றிற்கும் உள்ளிருக்கும் வலைத்தொடர்புகள் இறுக்கமாக கட்டப்பட்டவை.

65. Miller, D. et al. 2016.

66. Noelle- Neumann, E. 1974. 'The spiral of silence: a theory of public opinion.' Journal of Communication 24(2): 43-51. 


\section{அத்தியாயம் 4}

1. De Neve, G. 2008. ““We are all sondukarar (relatives)!": kinship and its morality in an urban industry of Tamilnadu, south India.' Modern Asian Studies 42(1): pp.211-46; Nakassis, C.V.2014. 'Suspended Kinship and Youth Sociality in Tamil Nadu, India.' Current Anthropology 55(2): 175-99; Freed, S. A. 1963. 'Fictive kinship in a north Indian village.' Ethnology 2(1): 86-103.

2. சகோதரி என்ற வார்த்தை தமிழில் அக்காள் அல்லது தங்கை யாரை வேண்டுமானாலும் குறிக்கும். கோவிந்தனின் இந்த வார்த்தை பிரயோகம் பொதுவானது.

3. தங்கள் ஒன்று விட்ட சகோதரிகளை, சகோதரிகள் என்று குறிப்பிடிவது பஞ்சக்கிராமியில் மிகவும் சகஜமானது. உறவுமுறை பற்றிய பொதுவான கருத்துக்களுக்கு பார்க்க: Dumont, L. 1953-4. 'The Dravidian Kinship Terminology as an Expression of Marriage.' Man 53: 34-9; Trautmann, T. 1982. Dravidian Kinship. Berkeley, CA: University of California Press.

4. இந்த முகநூல் நண்பர்கள் வேறு சமூகப்பொருளாதார வகுப்பிலிருந்து வந்தவர்கள்.

5. Chithiraputhiran, H. 1999. 'Semantic study of Tamil kinship terms.' Journal of Tamil Studies, 55-6; Trawick, M. 1990. Notes on love in a Tamil family. Berkeley, CA: University of California Press.

6. உதாரணத்திற்கு, அந்நியர்கள் கூட சமயங்களில் அண்ணா என்றோ அக்கா என்றோ கற்பனை உறவுமுறையிட்டு அழைக்கப்படுகிறார்கள். இது அந்நியர்களுடன் பேசுவதற்கான ஒருவித பிணைப்பை உருவாக்கித்தருகிறது என்று எண்ணப்படுகிறது.

7. இந்தியாவில் இனம் மற்றும் உறவுமுறைகளைப்பற்றிய பல்வேறு நூல்கள் இருக்கின்றன பார்க்க: Parry, J. P. 2013. Caste and Kinship in Kangra. Oxford: Routledge; Mayor, A. C. 1960. Caste and Kinship in Central India: A Village and its Region. Oxford: Routledge; Uyl, M. D. 1995. Invisible barriers: gender, caste and kinship in a southern Indian village. Utrecht: International Books. For examples of Tamil-specific notions of caste and kinship see Gough, E. K. 1956.'Brahman kinship in a Tamil village.' American Anthropologist 58(5): 826-53; Rudner, D.W. 1994. Caste and capitalism in colonial India: the Nattukottai Chettiars. Berkeley, CA:University of California Press.

8. தமிழ்நாட்டில் பாலினம் மற்றும் உறவுமுறைகள் பற்றி தெரிந்துகொள்ள பார்க்க: Kapadia, K. 1994. '“Kinship burns!": kinship discourses and gender in Tamil South India.' Social Anthropology 2(3): 281-97; Dube, L. 1997.'Women and kinship: Comparative perspectives on gender in South and South- East Asia.'Tokyo: UNU Press; Dube, L. 1988. 'On the construction of gender: Hindu girls in patrilineal India.' Economic and Political Weekly, WS11- WS19.

9. Kolenda, P. 1967. 'Regional differences in Indian family structure', in Crane, R. I. ed. Regions and Regionalism in South Asian Studies: An Exploratory Study. Durham, NC: Duke University Monographs and Occasional Papers Series (5).

10. Kolenda, P. M. 1968. 'Region, Caste and Family Structure: a Comparative Study of the Indian "Joint" Family.' Singer, M. and Cohn, B. S. eds. Structure and change in Indian society. Chicago, IL: Aldine Press. 339-96.

11. Shah, A. M. 1998. The family in India: critical essays. New Delhi: Orient Longman; Shah, A.M. 1973. The household dimension of the family in India. New Delhi: Orient Longman; Karve, I. 1965. Kinship organization in India. Bombay: Asia Publishing House. 569-70.

12. Säävälä, M. 2014. The "Hindu Joint Family": past and present.' Studia Orientalia Electronica 84: 61-74.

13. Fuller, C. J. and Narasimhan, H. 2007. 'Information technology professionals and the newrich middle class in Chennai (Madras).' Modern Asian Studies 41(1): 121-50; Gough, K. 1955. 'The social structure of a Tanjore village', in Marriot, M. ed. Village India. Chicago, IL: University of Chicago Press.

14. இந்தியாவில் குடும்ப அமைப்புகள் பற்றிய ஆழமான புரிதலுக்கு பார்க்க: Uberoi, P. ed. 1993. Family, kinship and marriage in India. New Delhi: Oxford University Press; Uberoi, P. 2005. 'The Family in India.' Writing the women's movement: a reader. New Delhi: Zubaan. 361-96.

15. அடுக்குமாடி குடியிருப்பு வளாகத்தின் அளவைப்பொறுத்து அதில் பல்வேறு அடுக்குகள் இருக்கக்கூடும். ஒவ்வொரு அடுக்கிலும் பல்வேறு தனித்தனி வீடுகள் இருக்கக்கூடும் சுமார் 4 முதல் 100 வரையிலும் கூட. 
16. சில சமயங்களில் இளைய தலைமுறையினர் ஏதேனும் உணவகங்களில் பெற்றோரையோ மற்ற குடும்பத்தினரையோ அழைக்காமல் தனியே உணவருந்த விழையக்கூடும். .

17. இந்தியாவில் நகர்ப்புற குடும்பங்கள் மற்றும் குடும்ப அமைப்புகளில் உள்ள மாறுதல்களை பற்றிய பொதுவான விவரங்களுக்கு பார்க்க: Abbi, B. L. 1969. 'Urban Family in India: A Review Article.' Contributions to Indian Sociology 3(1): 116-27.

18. Stern, H. 1977. 'Power in traditional India: territory, caste and kinship in Rajasthan', in Fox, R. G. ed. Realm and region in traditional India. New Delhi: Vikas Publishing House.52-78; Ishii, H. 1995. 'Caste and kinship in a Newar village', in Gellner, D. N. and Quigley, D. eds. Contested Hierarchies: A collaborative ethnography of caste among the Newars of the Kathmandu Valley, Nepal. Oxford: Clarendon Press. 109-57; Ramu, G. N. 1977. Family and caste in urban India: a case study. New Delhi: Vikas Publishing House.

19. இந்திய கிராமங்களில் கற்பனை உறவுமுறைகளை பற்றிய மேலும் விவரங்களுக்கு பார்க்க: Freed, S. A. 1963; Vatuk, S. 1969. 'Reference, address and fictive kinship in urban north India.' Ethnology 8(3): 255-72.

20. பல உப-இனங்கள் தங்களை தனிப்பட்ட இனங்களாக அடையாளம் காட்டிக்கொள்கின்றன. இருப்பினும், இந்த சூழலில், இனக்குழு என்பது பல்வேறு உப-இனங்களை சார்ந்தவர்கள் இருக்கக்கூடிய ஒரு பெரிய குழுவாக தான் பார்க்கப்படுகிறது. மேலும் விவரங்களுக்கு அத்தியாயம் 1ஐ பார்க்கவும்.

21. பாலினம் மற்றும் வயது போன்றவை தகவல் பரிமாற்றத்தை பாதிக்கும் முக்கியமான காரணிகளாகும். அதேபோல, ஆணாதிக்கமுள்ள அதிகாரப்படிநிலை அமைப்புகள் உள்ள குடும்பங்களில் வர்க்கமும் இனமும் கூட இது போன்ற முக்கிய காரணிகள் தான். .

22. இந்தியாவில் மொபைல் போன்களின் பங்கு பற்றிய விவரமான புரிதலுக்கு பார்க்க Doron, A. and Jeffrey,R. 2013. The great Indian phone book. Cambridge, MA: Harvard University Press.

23. ஒரு சில வழக்குகளில் முந்தைய அத்தியாயங்களில் பார்த்தது போல தாய், மிகக்குறைந்த வருமானம் வாங்கும் ஒரு கட்டுமானப்பணியாளராகவோ, கைவினை தொழிலாளியாகவோ அல்லது வீட்டு வேலை செய்பவராகவோ அல்லது உள்ளூர் தொழிற்சாலை அல்லது கடைகளில் பணிபுரிபவராகவோ இருக்கக்கூடிம்.

24. இந்தக்கட்டுரையில் குறிப்பிட்டிள்ளது போல, மணமாகாத பெண்கள் மொபைல் போன்கள் உபயோகிப்பதிலிருந்து தடை செய்யப்படுவது வடக்கிந்திய கிராமங்களிலும் காணப்படுகிறது. http://www.independent.co.uk/news/world/asia/ girls-and-unmarried-women-in-india-forbidden-from-using-mobilephones-to-prevent-di sturbance-in-a6888911.html

25. இந்தியாவில் குலம். மேலும் விவரங்களுக்கு பார்க்க அத்தியாயம் 1. மேலும் பார்க்க Heesterman, J. C. 1964. Brahmin,ritual and renouncer. Vienna: Universität Wien. Indologisch Institut für die Kunde Süd- und Ostasiens 8: 1-31; Fuller, C. J. and Narasimhan, H. 2010. 'Traditional vocations and modern professions among Tamil Brahmans in colonial and post- colonial south India.' Indian Economic \& Social History Review 47(4): 473-96; Pandian, M. S. S. 2007. Brahmin and non-Brahmin: Genealogies of the Tamil political present. New Delhi: Permanent Black.

26. https://en.wikipedia.org/wiki/Thanjavur_painting

27. http://www.airtel.com/

28. 'Voice Over Internet Protocol'

29. ஏதேனும் அபூர்வமான தருணத்தில் தங்களின் இருப்பை உறுதிப்படுத்துவதற்காக மின்னஞ்சலும் அனுப்பக்கூடும்.

30. Rainie, L. and Wellman, B. 2012. Networked: The new social operating system. Cambridge,MA: MIT Press; Haythornthwaite, C. 2002. 'Strong, weak, and latent ties and the impact of new media.' The Information Society 18(5): 385-401.

31. Xiang, B. 2007. Global 'body shopping': an Indian labor system in the information technology industry. Princeton: NJ: Princeton University Press.

32. மற்ற வழக்குகளில் வீடுகள், மற்ற தொழில்நெறிஞர்கள் அல்லது மாணவர்களுக்கு வாடகைக்கு விடப்படிகிறது. இதற்கான விளம்பரம் நிறுவனத்திற்குள்ளான குழுக்களில் காணப்படுகிறது.

33. Madianou, M. and Miller, D. 2013. Migration and new media: Transnational families and polymedia. Oxford: Routledge; Haythornthwaite, C. 2002. 
34. Miller, D. and Sinanan, J. 2014. Webcam. Cambridge: Polity Press.

35. இந்தியாவில் கொள்ளிக்கண் என்பது, ஒருவரின் வளம் அல்லது சந்தோஷத்தை பார்த்து மற்றவருக்கு தோன்றும் பொறாமையை குறிக்கிறது. அது குடும்பத்திற்கு கேடு விளைவிக்க கூடும் என்று நம்பப்படுகிறது.

36. இங்கு பொது என்ற சொற்றொடர் ஒருவர் முகநூலில் நண்பர்களாக சேகரிக்கும் சமூக வட்டத்தை குறிக்கும்

37. IT Enabled Services (IT support services).

38. ஆர்த்தியின் அன்னை, அவரது கணவர் அறியாமல் ஆர்த்தியை வந்து பார்த்துவிட்டு போகிறார். ஏனெனில் ஆர்த்தியின் தந்தை அவர்களது திருமணத்தை இன்னமும் ஏற்கவில்லை.

39. களப்பணியின் போது

40. பின்குறிப்பிடப்பட்டுள்ளவற்றில் காணப்படும் கலாச்சார மற்றும் சமூக செயல்பாடுகள் பற்றிய கருத்துக்களுடன் இதை தொடர்புபடுத்த முடியும். Turner, V.W. 1982. From ritual to theatre: The human seriousness of play. New York: PAJ Publications; Turner, V. W. and Schechner, R. 1988. The anthropology of performance. New York: PAJ Publications; Goffman, E. 1978. The presentation of self in everyday life.Harmondsworth: Penguin.

41. தமிழ் திரையுலகம்

42. ஹிந்தி திரைத்துறை.

43. அவர்களுடைய முகநூலில் உள்ள காட்சிப்படுத்தல் ஒருவகையில் தங்களுக்கு தாங்களே பார்த்துக்கொள்வது போலாகும். இது பள்ளியில் உள்ள சேவற்சண்டை காட்சிகளை தங்களை பற்றிய கதையை தங்களுக்கே சொல்வது போன்ற காட்சிப்படுத்துவதற்கு இணையானது. அவ்வாறு செய்வது ஒரு அடையாளத்தை உருவாக்கும். இந்த வழக்கில் சீர்மையான நெருக்கமான தம்பதியினர் என்ற அடையாளம். பார்க்க: Clifford, G. 1973. The interpretation of cultures. New York: Basic Books.

44. சீர்மையான தமிழ்ப்பெண் என்ற கருத்து தமிழ் சினிமா கலாச்சாரத்திலும் மீண்டும் மீண்டும் சொல்லப்படுகிறது ஒருவகையில் இதுபோன்ற கருத்துக்கள் தமிழ் ஆண்மைத்தனத்தை விளக்க முற்படுகின்றன. பார்க்க: Pandian,M. S. S. 2015. The image trap: MG Ramachandran in film and politics. New Delhi: Sage Publications.

45. Bott, E. and Spillius, E. B. 2014. Family and social network: Roles, norms and external relationships in ordinary urban families. Oxford: Routledge.

46. www.geni.com

47. ஒரு குழு செயல்பாட்டை செயல்படுத்துவதன் மூலம் அமைக்கப்படும் குழு அடையாளமாக இது கருதப்படலாம். இங்கு அங்கத்தினர் என்பவர்கள், சமூக முறையில் அமைக்கப்பட்டு இந்த செயல்பாட்டிற்கு பங்களிக்கிறார்கள். இதை பற்றி மேலும் விவரங்களுக்கு பார்க்க: Desmond, J. 1999. Staging tourism: Bodies on display from Waikiki to Sea World. Chicago, IL: University of Chicago Press.

48. இந்த வசதி தொழில்முறை புகைப்படக்கலைஞர்களால் ஏற்பாடு செய்யப்பட்டது.

49. Freed, S. A. 1963; Vatuk, S. 1969.

50. Bell, S. and Coleman, S. eds. 1999. The Anthropology of Friendship. Oxford: Berg;Killick, E. and Desai, A. 2010. 'Introduction: Valuing Friendship', in Desai, A. and Killick,E. eds. 2010. The Ways of Friendship. Oxford: Berghahn; Nisbett, N. 2007. 'Friendship, consumption,morality: practising identity, negotiating hierarchy in middle- class Bangalore.Journal of the Royal Anthropological Institute 13(4): 935-50; Osella, C. and Osella, F.1998. 'Friendship and flirting: micro- politics in Kerala, South India.' Journal of the Royal Anthropological Institute 4(2): 189-206; Nakassis, C. V. 2013. 'Youth masculinity, "style" and the peer group in Tamil Nadu, India.' Contributions to Indian Sociology 47(2): 245-69.

51. Alm, B. 2010. 'Creating followers, gaining patrons: Leadership strategies in a Tamil Nadu village', in Price, P. and Rudd, E. eds. Power and Influence in India. Bosses, Lords and Captains: 1-19. New Delhi: Routledge.

52. தமிழில் குட்டி என்பது நெருக்கமான வட்டங்களில் சிறியவர் என்பதை குறிக்கும்

53. தமிழில் அன்னை

54. தமிழில் அன்னையின் தங்கையை குறிக்கும்.

55. இந்த தமிழ் வார்த்தை சிறிய தம்பி என்ற பொருள்படும்

56. Nakassis, C. V. and Dean, M. A. 2007. 'Desire, youth, and realism in Tamil cinema.' Journal of Linguistic Anthropology 17(1): 77-104 


\section{அத்தியாயம் 5}

1. பெற்றோரால் ஏற்பாடு செய்யப்பட்ட திருமணம் என்பது இந்தியாவில் காலங்காலமாக வழக்கத்தில் இருக்கும் ஒன்று. ஆண்களும் பெண்களும் திருமணவயதை அடைந்தவுடன், அவர்களது பெற்றோர்கள் அல்லது காப்பாளர்கள் அவர்களுக்கான சரியான துணையை சமயம், இனம், வர்க்கம் போன்ற அனைத்து கூறுகளையும் அலசி ஆராய்ந்து அதன் அடிப்படையில் தேர்ந்தெடுக்கின்றனர். பார்க்க: Caldwell, J. C., Reddy, P. H. and Caldwell, P. 1983. 'The causes of marriage change in South India.'Population Studies 37(3): 343-61; de González, L. T. 2013. 'Modern Arranged Marriage in Mumbai.' Teaching Anthropology: SACC Notes 34.

2. Wallman, S. ed. 1979. Social anthropology of work 19. London: Academic Press.

3. Baba, M. L. 1998. 'The anthropology of work in the Fortune 1000: a critical retrospective.'Anthropology of Work Review 18(4): 17-28; Nash, J. 1998. "Twenty Years of the Anthropology of Work: Changes in the State of the World and the State of the Arts.' Anthropology of Work Review 18(4): 1-6.

4. Wallman, S. ed. 1979; Ortiz, S. 1994. 'Work, the division of labour and co-operation', in Ingold, T. ed. Companion encyclopaedia of anthropology. Oxford: Routledge.

5. Wallman, S. ed. 1979.

6. Wallman, S., ed. 1979; Baba, M. L. 1998; Jordan, A. T. 2012. Business anthropology. Long Grove, IL: Waveland Press.

7. Parry, J. P. 2001. 'Ankalu's errant wife: sex, marriage and industry in contemporary Chhattisgarh.' Modern Asian Studies 35(4): 783-820; Parry, J. P. 1999. Introduction. In Parry, J. P., Breman, J. and Kapadia, K. The worlds of Indian industrial labour. London: Sage Publications; Freeman, C. 2000. 'High tech and high heels in the global economy: Women, work, and pink- collar identities in the Caribbean.' Durham, NC: Duke University Press; De Neve, G. 2005. The everyday politics of labour: Working lives in India's informal economy. New Delhi: Social Science Press; Moeran, B. 2005. The business of ethnography: Strategic exchanges, people and organizations. London: Bloomsbury Academic; Broadbent, S. 2011. L'intimité au travail. Limoges, France: FYP éditions.

8. Grint, K. 2005. The sociology of work: introduction. Cambridge: Polity Press.

9. Broadbent, S. 2011.

10. Broadbent, S. 2011.

11. Swallow, D. A. 1982. 'Production and control in the Indian garment export industry', in Goody, E. N. ed. From Craft to Industry: the Ethnography of Proto- industrial Cloth Production. Cambridge: Cambridge University Press, 1982. 133-65.

12. De Neve, G. 2005.

13. Ortiz, S. 1994; Mirchandani, K. 1999. 'Feminist insight on gendered work: New directions in research on women and entrepreneurship.' Gender, Work \& Organization 6(4): 224-35; Williams, J. 2001. Unbending gender: Why family and work conflict and what to do about it. New York: Oxford University Press.

14. Choudhary, R. 1971. Kautilya's political ideas and institutions. New Delhi: Chowkhamba Sanskrit Series Office; Buhler, G. 2004. The laws of manu. New Delhi: Genesis Publishing Pvt Ltd.; Gopal, T. B. 1976. Niti Shastra. Agra: Ratan Prakashan Mandir. The idea of formal spaces in these texts was specific to Brahmins' ritual spaces and to court halls, which were treated as holy and formal spaces and were cordoned off from other nonreligious and informal activities.

15. இந்தியாவில் தொழிற்சாலைமயமாக்கலின் துவக்கம் பத்தொன்பதாம் நூற்றாண்டின் இறுதிக்கு சுவடு காணமுடியும்.

16. Fuller, C. J. 1996. In Fuller, C. J. ed. Caste today. New York: Oxford University Press. 1-31.

17. Ambedkar, B. R. 1944; Pillay, K. P. K. 1977. The Caste System in Tamil Nadu. Chennai: University of Madras.

18. Ambedkar, B. R. 1944; Pillay, K. P. K. 1977.

19. Wiser, W. H. 1988. The Hindu jajmani system. New Delhi: Munshiram Manoharlal Publishers Pvt Ltd; Gould, H. A. 1986. 'The Hindu jajmani system: a case of economic particularism.' Journal of anthropological research 42(3): 269-78; Kolenda, P. 1963. 'Toward a model of the Hindu Jajmani system.' Human Organization 22(1): 11-31; Commander, S. 1983. 'The jajmani system in North India: an examination of its logic and status across two centuries.' Modern Asian Studies 17(2): 283-311. 
20. உத்தேசமாக பின்தங்கிய வகுப்பு என்று வகைப்படுத்தப்படமுடியும் அல்லது சிலசமயம் உப-இனங்களில் அடிப்படையில் மற்ற வகுப்புகள் என்று வகைப்படுத்தமுடியும்

21. De Neve, G. 2005. The Vanniyars and Vellala Gounder castes may also be classified as belonging to Most Backward Castes and Backward Castes respectively.

22. வர்த்தக வாய்ப்புகள் மற்றும் அவற்றுடன் இணைந்த சமூகத்திற்கான தங்களின் கடமை பற்றிய உலகயியல் புரிதல் ஆகிய இரண்டும் ஒன்றிணைந்து ஒரு சில குலக்குழுக்களை ஒரு சில பணிகளை செய்யவைத்திருக்கிறது. இருப்பினும், தலித்துக்கள் போல சமூக அளவில் பேதப்படுத்தப்படாத குழுக்களால் மட்டுமே இது சாத்தியம். இந்தியாவில் சம்பிரதாய மற்றும் முறைசாரா பணி சூழல்களில் குடிம்பம், உறவுமுறை மற்றும் குலம் ஆகியவற்றை பற்றிய புரிதலுக்கு பார்க்க: Niehoff, A. H. 1959. Factory workers in India. PhD dissertation. Board of Trustees, Milwaukee Public Museum; Vidyarthi, L. P. 1984. In Vidyarthi, L. P. ed. Applied anthropology in India: principles, problems, and case studies. New Delhi: Kitab Mahal; Swallow. D. A. 2008; Holmström, M. 1976. South Indian factory workers: Their life and their world. Cambridge: Cambridge University Press; Parry, J. 1999; Harriss- White, B. 2003. India working: Essays on society and economy 8. Cambridge: Cambridge University Press; De Neve, G. 2005; Bear, L. 2007. Lines of the nation: Indian Railway workers, bureaucracy, and the intimate historical self. New York: Columbia University Press; Breman, J. 2013. At work in the informal economy of India: a perspective from the bottom up. Oxford: Oxford University Press. There is a large amount of literature concerned with understanding work in the midst of caste and class in Indian rural and urban life. Of particular interest for South Indian contexts see Srinivas, M. N. 1962. Caste in modern India and other essays. New Delhi: Asia Publishing House; Fuller, C. J. 1996; Reiniche, M. L. 1996. 'The urban dynamics of caste: a case study from Tamil Nadu', in Fuller, C. J. ed. Caste today. New York: Oxford University Press. 124-49; Dirks, N. 1996. 'Recasting Tamil society: The politics of caste and race in contemporary southern India', in Fuller, C. J. ed. Caste today. New York: Oxford University Press. 263-95; Beteille, A. 1965. Caste, class, and power: Changing patterns of stratification in a Tanjore village. Berkeley, CA: University of California Press. Osella, F. and Osella, C. 2000. Social mobility in Kerala: modernity and identity in conflict. London: Pluto Press.

23. Swallow, D. A. 2008. De Neve, G. 2005.

24. இந்த இரண்டு மக்கட்குழுக்களும் வெவ்வேறு குலத்தை சேர்ந்தவை

25. https://en.wikipedia.org/wiki/Hindu_joint_family

26. Vidyarthi, L. P. 1984. Bear, L. 2007.

27. Niehoff, A. H. 1959; Vidyarthi, L. P. 1984; Holmstrom, M. 1976; Parry, J. 1999; De Neve,G. 2005.

28. Vidyarthi, L. P. 1984.

29. https://en.wikipedia.org/wiki/Chota_Nagpur_Plateau

30. Holmstrom, M. 1976.

31. De Neve, G. 2005; Vidyarthi, L. P. 1984.

32. Osella, F. and Osella, C. 2000.

33. Fuller. C. J. 1996; Fanselow, F. S. 1996. 'The disinvention of caste among Tamil Muslims',in Fuller, C. J. ed. Caste today. New York: Oxford University Press. 202-26; Vatuk, S. 1996. 'Identity and difference or equality and inequality in South Asian Muslim society', in Fuller, C. J. ed. Caste today. New York: Oxford University Press. $227-62$.

34. Fuller, C. J. 1996; Beteille, A. 1996. 'Caste in contemporary India', in Fuller, C. J. ed. Caste today. New York: Oxford University Press. 150-79; Reiniche, M. L. 1996.

35. Bear, L. 2007.

36. Holmstrom, M. 1976.

37. Swallow, D. A. 2008.

38. IT: Information Technology; ITES: Information Technology Enabled Services.

39. Fuller, C. J. and Narasimhan, H. 2007.

40. Nisbett, N. 2009.

41. https://en.wikipedia.org/wiki/Symbolic_capital

42. https://en.wikipedia.org/wiki/Symbolic_capital 
43. Arora, A. and Athreye, S. 2002. 'The software industry and India's economic development.' Information Economics and Policy 14(2): 253-73; Upadhya, C. and Vasavi, A. R. 2006. Work,culture, and sociality in the Indian IT industry: a sociological study. Bangalore: National Institute of Advanced Studies, Indian Institute of Science Campus, Bangalore; Nisbett, N. 2009.

44. Das, G. 2002. India Unbound. London: Profile; Fuller, C. J. and Narasimhan, H. 2007; Nisbett, N. 2009.

45. Das, G. 2002. Arora, A. and Athreye, S. 2002; Upadhya, C. and Vasavi, A. R. 2006.

46. De Neve, G. 2005.

47. De Neve, G. 2005.

48. Arora, A. and Athreye, S. 2002; Upadhya, C. and Vasavi, A. R. 2006; Fuller, C. J. and Narasimhan, H. 2007; Nisbett, N. 2009.

49. Annapoorna, S. and Bagalkoti, S. T. 2011. 'Increasing Women Employment in IT Industry: An Analysis of Reasons.' International Journal of Research in Commerce, IT and Management 1(5): 87-9; Arora, A. and Athreye, S. 2002.

50. Body shopping (https://en.wikipedia.org/wiki/Body_shopping) for IT workers in India involves a third-party company recruiting IT workers for short- term contract services. See Bhatnagar, S. C. and Madon, S. 1997. 'The Indian software industry: moving towards maturity.'Journal of Information Technology 12, 277-88; Mir, A., Mathew, B. and Raza, M. 2000.'The Codes Of Migration.' Cultural Dynamics 12: 5-33; Kumar, N. 2001. 'Indian Software Industry Development: International And National Perspective.' Economic and Political Weekly 36, 10 November, 4278-90; Fuller, C. J. and Narasimhan, H. 2007.

51. அமெரிக்காவில் உள்ள நிறுவனங்களில் குவியம் கொண்டுள்ள ஆராய்ச்சிகளுக்கு பார்க்க: Rainie, H. and Wellman, B. 2012. Networked: The new social operating system. Cambridge, MA: MIT Press. 358.

52. Friedman, T. 2005. The world is flat. New York: Farrar, Straus and Giroux; Upadhya, C. and Vasavi, A. R. 2006; Fuller, C. J. and Narasimhan, H. 2007; Nisbett, N. 2009.

53. Parry, J. 2001.

54. Athreye, S. S. 2005. 'The Indian software industry and its evolving service capability.' Industrial and Corporate Change,14(3): 393-418; Reddy, P. G. 2010. 'Some Problems Faced By The Software Professionals In India: An Overview.' Population Dynamics and Human Development Opportunities and Challenges, Lakshmansamy, T. ed. New Delhi: Bookwell. 451-71; Fuller, C. J. and Narasimhan, H. 2007; Nisbett, N. 2009.

55. https://en.wikipedia.org/wiki/Knowledge_process_outsourcing

56. விமானநிலையத்தில் இருந்து மேற்கத்திய வாடிக்கையாளர்களை அழைத்துவர அனுப்பப்படும் வாகன ஓட்டுனர்களுக்கு கூட சில சமயங்களில் அடிப்படையான ஆங்கில போதனை அளிக்கப்படுகிறது. அழைத்துவரும் போது உரையாட உதவியாக இருக்கும் என்பதற்காக.

57. வாடிக்கையாளர்களுடன் ஏதேனும் சம்பிரதாயமான கூட்டங்கள் இருந்தால் மட்டுமே பணியாளர்கள் சம்பிரதாயமான உடை அணிந்து வரச்சொல்லி கோரப்பட்டனர்.

58. இந்த முறை பணியாளர்களை பல்வேறு பணி தலங்களுக்கு இடையே சுழற்சிமுறையில் பணிபுரிய செய்கிறது. அவர்கள் ஒரு பணி அறையில் கட்டுப்படுத்தப்படிவதில்லை. Joroff, M. L., Porter, W. L., Feinberg, B. and Kukla, C. 2003. 'The agile workplace.' Journal of Corporate Real Estate 5(4): 293-311.

59. Fayard, A.L. and Weeks, J. 2007. 'Photocopiers and water- coolers: The affordances of informal interaction.' Organization studies 28(5): 605-34.

60. https://en.wikipedia.org/wiki/Sedan_(automobile)

61. இது அன்றாட பெருநிறுவன வாழ்வில் ஒருவர் தன்னைப்பற்றி அளித்த முன்னிலைப்படுத்தலாக தோன்றக்கூடும். See Goffman, E. 2002. The presentation of self in everyday life. 1959. New York: Garden City.

62. https://en.wikipedia.org/wiki/Chennai_Super_Kings

63. Donner, J. 2007. 'The rules of beeping: exchanging messages via intentional "missed calls" on mobile phones.' Journal of Computer- Mediated Communication 13(1): 1-22.

64. Madianou, M. and Miller, D. 2013.

65. Säävälä, M. 2010. Middle- class moralities: Everyday struggle over belonging and prestige in India. New Delhi: Orient Blackswan. 
66. இது மூன்று குழந்தைகள் பள்ளி ஒருங்கிணைப்பாளர்களால் உறுதிப்படுத்தப்பட்டது. மேலும் இரண்டுவார காலமாக, குழந்தைகளை பள்ளிக்கு அழைத்துவரும் தந்தையரின் எண்ணிக்கையை கணக்கிட்டதின் மூலமும் தெரியவந்தது. 41 சதவிகித இளம் குழந்தைகள் தந்தையராலும், 27 சதவிகிதத்தினர் தங்களது அன்னையராலும், 24 சதவிகிதத்தினர் தங்களது தாத்தாபாட்டியாலும், 8 சதவிகிதத்தினர் குழந்தைகாப்பாளர்களாலும் அழைத்துவரப்படுகின்றனர்.

67. வேலைக்கு செல்லும் அன்னையர் தந்தையர் மத்தியில் வீட்டு வேலைகளை சரிசமமாக பிரித்துக்கொள்வது என்பது இன்னும் நெடுந்தூரத்தில் தான் இருக்கிறது.

68. கீழ்மட்ட வகுப்பை சேர்ந்தவர்கள் என்றழைக்கப்படுபவரின் பணிகள் மற்றும் அவர்கள் சந்திக்கும் பாகுபாடுகள் பற்றிய மேலும் விவரங்களுக்கு பார்க்க: https:// www.hrw.org/reports/2001/globalcaste/caste0801-03.htm and https://www.britannica.com/ topic/untouchable. For more on caste and occupations see http://countrystudies.us/ india/89.htm

69. Ambedkar, B. R. 1944.

70. Fuller, C. 2011. 'Timepass and boredom in modern India.' Anthropology of this Century 1.

71. Swallow, D. A. 2008; Vidyarthi, L. P. 1984; De Neve, G. 2005.

72. Vidyarthi, L. P. 1984.

73. Fuller, C. and Narasimhan, H. 2008. 'Empowerment and Constraint: Women, Work and the Family in the Software Industry in Chennai', in Upadhya, C. and Vasavi, A. R. eds.,In an Outpost of the Global Economy: Work and Workers in India s Information Technology Industry. New Delhi: Routledge. 190-210.; Kelkar, G., Shrestha, G. and Veena, N. 2002. 'IT industry and women's agency: Explorations in Bangalore and Delhi, India.' Gender, Technology and Development 6(1): 63-84; Nath, D. 2000. 'Gently shattering the glass ceiling: experiences of Indian women managers.' Women in Management Review 15(1): 44-52. Aziz, M. 2004. 'Role stress among women in the Indian information technology sector.'Women in Management Review 19(7): 356-63; Pleck, J. H. 1977. 'The workfamily role system.' Social problems 24(4): 417-27; Baruch, G. K., Biener, L. and Barnett, R. C. 1987. 'Women and gender in research on work and family stress.' American Psychologist 42(2): 130; Myrdal, A. and Klein, V. 2003. Women's two roles: Home and work. Oxford: Routledge/ Psychology Press; Sandberg, S. 2013. Lean In: Women, Work, and the Will to Lead. New York: Knopf.

74. Vidyarthi, L. P. 1984; De Neve, G. 2005.

75. Ambedkar, B. R. 1944.

76. Miller, D. et al. 2016.

\section{அத்தியாயம் 6}

1. லாபமில்லா கல்வி நிறுவனத்தின் யூட்யூபில் உள்ள சிறிய விரிவுரைகள். இது இணையதள தொடர்புடைய எவராலும் அணுகப்பட முடியும்.

2. amazon.com போன்ற நிகழ்நிலை வணிகர்

3. மக்களின் சுய-புகைப்படம் தேடிதலை பற்றிய பிரபலமான தமிழ் திரைப்பாடல்

4. தமிழ் திரையுலகில் பிரபலமான நடிகர் (see also Chapter 3).

5. அரசாங்கமும் அரசியலும் போன்ற தலைப்புகளுக்கு பார்க்க: Costa, E. 2016. Social Media in South East Turkey. London: UCL Press, and Haynes, N. 2016. Social Media in Northern Chile. London: UCL Press. For religion and commerce see Miller, D. 2016. Social Media in an English Village. London: UCL Press.

6. Dahlman, C. J. and Utz, A. 2005. India and the knowledge economy: leveraging strengths and opportunities. Washington DC: World Bank Publications; Powell, W. W. and Snellman, K. 2004. 'The knowledge economy', in Cook, K. S. and Hagan, J. eds. Annual review of sociology 1(30): 199-220. Palo Alto, CA: Annual Reviews.

7. உதாரணத்திற்கு பார்க்க: Konana, P. and Balasubramanian, S. 2002. 'India as a knowledge economy: aspirations versus reality.' Frontline 19(2), 65-9.

8. மலேசியாவில் அறிவார்ந்த பொருளாதாரத்தில் இலட்சியங்கள் பற்றிய இதே போன்ற வழக்கு விதிக்கப்பட்டுள்ள இடம் Lepawsky, J. 2005. 'Digital Aspirations: Malaysia and the Multimedia Super Corridor.' FOCUS on Geography 48(3): 10-18. 
9. Berg, P. O. and Kreiner, K. 1990. 'Corporate architecture: Turning physical settings into symbolic resources', in Gagliardi, P. ed. Symbols and artifacts: Views of the corporate landscape.New York, NY: Aldine de Gruyter. 41-67.

10. அத்தியாயம் 1-ல் பார்த்தது போல இங்குள்ள அனைத்து புதிய மத்தியதர வகுப்பை சேர்ந்த மக்கட்தொகையினரும் ஐடி பணியில் இல்லாவிட்டாலும் அவர்கள் அனைவரும் எதோ ஒரு வகையில் ஐடி நிறுவனத்துடன் தொடர்புடையவர்களாக இருக்கிறார்கள். இதுவே அவர்களது வளத்திற்கு காரணம்.

11. https://www.khanacademy.org/

12. Open courseware refers to course lessons created by universities and made available for free over the internet. https://en.wikipedia.org/wiki/OpenCourseWare

13. பள்ளிகளில் உள்ள தகவல் தொழில்நுட்பங்கள் ஸ்மார்ட் வகுப்பறைகள் கணினி கூடங்கள் போன்றவையாக இருக்கக்கூடும்

14. இந்திய பள்ளி அமைப்புகள் மற்றும் அவற்றின் வளர்ச்சி பற்றிய ஒரு கண்ணோட்டத்திற்கு பார்க்க: Kingdon, G. G . 2007. 'The progress of school education in India.' Oxford Review of Economic Policy 23(2): 168-95; Venkatanarayanan, S. 2015. 'Economic Liberalization in 1991 and Its Impact on Elementary Education in India.' SAGE Open 5(2): DOI: 10.1177/ 2158244015579517; Asadullah, M. N.,Kambhampati, U. and Boo, F. L. 2014. 'Social divisions in school participation and attainment in India: 1983-2004.' Cambridge Journal of Economics 38(4): 869-93; Desai, S.,Dubey, A., Vanneman, R. and Banerji, R. 2009, April. 'Private schooling in India: A new educational landscape', in India Policy Forum 5(1): 1-38. New Delhi: National Council ofApplied Economic Research.

15. http://www.samacheerkalvi.in/

16. http://cbse.nic.in/

17. http://www.cie.org.uk/programmes-and-qualifications/cambridge-secondary-2/ cambridge-igcse/

18. வளமான பள்ளியில் மூன்றாம் வகுப்பு பயிலும் மாணவர் சுமார் ரூ. 150000/வருடாந்திர கட்டணமாக செலுத்தக்கூடும். இது இந்தியாவில் உள்ள சாதாரண மத்தியதர வகுப்பினரின் வருமானத்தை பார்க்கும் போது மிகவும் அதிகம்.

19. இது பள்ளிகளில் உள்ள பயிற்றுவிக்கும் மொழியின் அடிப்படையில் ஆனது.

20. Annamalai, E. 2004. 'Medium of power: The question of English in education in India', in Tollefson, J. W. and Tsui, A. B. M. eds. Medium of Instruction Policies - Which Agenda? Whose Agenda. Mahwah, NJ: Lawrence Erlbaum Associates. 177-94.

21. Thompson, C. 2011. 'How Khan Academy is changing the rules of education.' Wired Magazine 126: 1-5.

22. For an examination of research into use of SNS in UK Formal Educational Settings see Facer, K. and Selwyn, N. 2010. 'Social Networking - Key messages from the Research.' Sharpe, R., Beetham, H. and de Freitas, S. eds. Rethinking Learning for a Digital Age. 31-42.

23. இந்தியாவில் 14 வயது வரை குழந்தைகளுக்கு கட்டாயக்கல்வி என்பது வலியு றுத்தப்பட்டிருக்கிறது. இந்தப்பள்ளிகள் இந்த வயதை முகநூல் பயன்பாட்டுடன் தொடர்புபடுத்தி பார்க்கின்றன. முவநூல் குழந்தைகளை 13 வயதில் இருந்தே உறுப்பினர்களாக அனுமதிக்கிறது.

24. For an examination of why students are on social networks see Cheung, C. M., Chiu, P. Y. and Lee, M. K. 2011. 'Online social networks: Why do students use Facebook?' Computers in Human Behavior 27(4): 1337-43.

25. For an examination of YouTube as a knowledge resource in different contexts, see Burke, S. C. and Snyder, S. L. 2008. 'YouTube: An Innovative Learning Resource for College Health Education Courses.' International Electronic Journal of Health Education 11: 39-46; Duffy, P. 2008. 'Engaging the YouTube Google- eyed generation: Strategies for using Web 2.0 in teaching and learning.' The Electronic Journal of e- Learning 6(2): 119-30.

26. A similar finding was discussed by Roblyer, M. D. et al. 2010. 'Findings on Facebook in higher education: A comparison of college faculty and student uses and perceptions of social networking sites.' The Internet and Higher Education 13(3): 134-40. Here the college faculty believed social networking sites to have only a social function and no instructionalpurpose. 
27. முந்தைய அத்தியாயங்களில் விவாதித்தது போல ஒரு சில குலத்தை சேர்ந்த மணமாகாத இளம் பெண்கள் சமூக ஊடகங்களை அணுகுவதற்கான முதன்மை வழியான மொபைல் போன்களை பயன்படுத்துவதில் இருந்து தடை செய்யப்பட்டிருந்தனர். இந்தியாவில் கல்வி மற்றும் ஆண்மை பற்றிய பொதுவான கருத்துக்களுக்கு பார்க்க: Jeffrey, C., Jeffery,P. and Jeffery, R. 2008. Degrees without freedom? Education, masculinities and unemployment in north India. Stanford, CA: Stanford University Press.

28. கீழ்மட்ட சமூகப்பொருளாதார வகுப்பை சேர்ந்தவர்கள் இணையதளத்தை அறிவுத்திறனின் சின்னமாக பார்த்தனர். அவர்களைப்பொறுத்தவரை அதை பயன்படுத்துவதற்கான திறனை பெறுவது என்பது அன்றாட போராட்டங்களில் இருந்து விடுதை பெறுவது போலாகும். இணையத்தளம் எப்படி சுதந்திரத்தின் சின்னமாக பார்க்கப்படக்கூடும் என்பது பற்றிய கருத்துக்கு பார்க்க: Miller, D. and Slater, D. 2000. The Internet: an ethnographic approach. Oxford: Berg.

29. Digital Video Disc - https://en.wikipedia.org/wiki/DVD

30. Buckingham, D. 2013. 'Digital Childhoods? New Media and Children's Culture.' Beyond technology: children's learning in the age of digital culture. Cambridge: Polity Press.

31. ஒரு பல்கலைக்கழக சூழ்நிலையில் ஆசிரியரின் கண்ணோட்டத்தில் இருந்து மாணவர்களை நண்பர்களாக்கிக் கொள்வதில் உள்ள விசாரங்கள் பற்றிய விவாதத்திற்கு பார்க்க: Raynes- Goldie, K. and Lloyd, C. 2014. 'Unfriending Facebook? Challenges From an Educator's Perspective', in Kent, M. and Leaver, T., eds. An education in Facebook? Higher education and the world's largest social network. Oxford: Routledge. 153-61.

32. The teachers referred to articles such as http://archive.indianexpress.com/news/explainho w-children-open-facebook-other-accounts-delhi-high-court-to-govt/1107592/, in which 18 seems to be the stipulated age to open a Facebook account. However, the association of Facebook activity with the concluding age for compulsory education, namely 14 years, was also apparent, as discussed earlier in this chapter.

33. இது போன்ற கருத்துக்கள், சமூக ஊடகங்களின் மூலம் பெறக்கூடிய முறைசாரா கல்வியுடன் தொடர்புடையதாகவும் இருக்கலாம். This is discussed in a comparative perspective by Miller, D. et al. 2016.

34. More about surveillance is discussed by Miller, D. et al. 2016. This work also explores why in certain countries social media was seen as a platform for surveillance by schools, concluding that in China it was apparently so, due to the functionalities offered by Chinese social media platforms.

35. களப்பணியின் இறுதியில், ஒரு சில பள்ளிகளுக்கான முறையான வாட்சப் குழு உருவாக்கப்பட்டிருப்பதாக தெரிகிறது. ஒரு பள்ளியின் அதிகாரபூர்வ தொலைபேசி என்னுடன்.

36. Miller, D. et al. 2016.

\section{அத்தியாயம் 7}

1. இது போன்ற செயல்முறை உரையாடல்கள் சமூகத்தன்மையின் ஒரு அங்கமாக கருதப்பட்டாலும், இது போன்ற பரிமாற்றங்கள் சமூகத்தன்மையை கட்டமைப்பதில் ஒத்தவர்களிடையேயான நடவடிக்கையாக பார்க்கப்படுவெதில்லை என்பது தான் இங்குள்ள நோக்கமே.

2. இது இறுதியில் தர்ஷனும் நாகாவும் தங்களது சமூகத்தில் தங்களுக்கான மரியாதையையும் அந்தஸ்தையும் பெறுவதில் முடிந்தாலும், அவர்களது நோக்கமென்னவோ தங்களது சமூகத்தின் நன்மை தான்.

3. McPherson, M. 2001.

4. Mandelbaum, D. G. 1970. Society in India: Continuity and change, vol.1. Los Angeles, CA:University of California Press; Mayer, A. 1996. 'Caste in an Indian village: change and continuity1954-1992', in Fuller, C. J. ed. Caste today. New York: Oxford University Press. 32-64.

5. Beteille, A. 1996; Fuller, C. J., ed. 1996.

6. Fuller, C. J. ed. 1996. 
7. Jeffrey, R. and Doron, A. 2013. Cell phone nation: How mobile phones have revolutionized business, politics and ordinary life in India. New Delhi: Hachette.

8. அத்தியாயம் 5, பஞ்சக்கிராமியின் ஐடி துறையை பற்றிய ஆழமான விவரங்களை கொடுக்கிறது.

9. Miller, D. et al. 2016.

10. இது சுயபுகைப்படங்களாகவோ அல்லது காட்சி கொடுத்து மற்றவர்களால் எடுக்கப்பட்ட புகைப்படங்களாகவோ வடிவெடுக்கக்கூடிம். சுயபுகைப்படம் இயல்புநிலையில் இல்லாவிட்டாலும், தன்னுடைய புகைப்படங்களையே பதிவிடுவது இயல்புநிலையில் உள்ள நடைமுறையின் தொடர்ச்சியே ஆகும்.

11. Madianou, M. and Miller, D. 2012. Migration and new media: Transnational families and polymedia. Oxford: Routledge.

12. Miller, D. et al. 2016.

13. Rainie, H. and Wellman, B. 2012. Networked: The new social operating system. Cambridge, MA: MIT Press. 58.

14. Laughey, D. 2007; Gottdiener, M. et al. 2015

15. Madianou, M. and Miller, D. 2013.

16. Miller, D. et al. 2016. 


\section{சான்றாதாரம்}

Abbi, B. L. 1969. 'Urban Family in India. A Review Article.' Contributions to Indian Sociology 3(1): $116-27$.

Ahmad, A. 2011. 'Rising of social network websites in India overview.' International Journal of Computer Science and Network Security 11(2): 155-8.

Alex, G. 2008. 'A Sense of Belonging and Exclusion: "Touchability" and "Untouchability" in Tamil Nadu.' Ethnos 73(4): 523-43.

Alexander, K. C. 1968. 'Changing Status of Pulaya Harijans of Kerala.' Economic and Political Weekly 3(26/28): 1071-4.

Alm, B. 2010. 'Creating followers, gaining patrons: Leadership strategies in a Tamil Nadu village. Power and influence in India.' Bosses, Lords and Captains. New Delhi: Routledge. 1-19.

Ambedkar, B. R. 1944. 'Annihilation of caste, with a reply to Mahatma Gandhi.' http://www. ambedkar.org/ambcd/02.Annihilation\%20of\%20Caste.htm - Undelivered speech prepared in 1936; the third edition was released in 1944.

Annamalai, E. 2004. 'Medium of power: The question of English in education in India.' Medium of Instruction Policies-Which Agenda? Whose Agenda. Mahwah, NJ: Lawrence Erlbaum Associates. 177-94.

Annapoorna, S. and Bagalkoti, S. T. 2011. 'Increasing Women Employment in IT Industry: An Analysis of Reasons.' International Journal of Research in Commerce, IT and Management 1(5): 87-9.

Appadurai, A. 1988. The social life of things: Commodities in cultural perspective. Cambridge: Cambridge University Press.

Arora, A. and Athreye, S. 2002. 'The software industry and India's economic development.' Information Economics and Policy 14(2): 253-73.

Asadullah, M. N., Kambhampati, U. and Boo, F.L. 2013. 'Social divisions in school participation and attainment in India: 1983-2004.' Cambridge Journal of Economics 38(4): 869-93.

Athreye, S. S. 2005. 'The Indian software industry and its evolving service capability.' Industrial and Corporate Change 14(3): 393-418.

Aziz, M. 2004. 'Role stress among women in the Indian information technology sector.' Women in Management Review 19(7): 356-63.

Baba, M. L. 1998. 'The anthropology of work in the Fortune 1000: a critical retrospective.' Anthropology of Work Review 18(4): 17-28.

Bajwa, S. B. 2003. 'ICT Policy in India in the era of liberalization: its impact and consequences.' Global Built Environment Review 3(2): 49-61.

Balkundi, P. and Kilduff, M. 2006. 'The ties that lead: A social network approach to leadership.' The Leadership Quarterly 17(4): 419-39.

Baruch, G. K., Biener, L. and Barnett, R. C. 1987. 'Women and gender in research on work and family stress.' American Psychologist 42(2): 130.

Bate, B. 2013. Tamil Oratory and the Dravidian Aesthetic: Democratic Practice in South India. New York: Columbia University Press.

Baym, N. K. 2015. Personal connections in the digital age. Chichester: John Wiley \& Sons.

Bear, L. 2007. Lines of the nation: Indian Railway workers, bureaucracy, and the intimate historical self. New York: Columbia University Press.

Beck, B. E. 1981. 'The Goddess and the Demon. A Local South Indian Festival and its Wider Context' in Biardeau, M. ed. Autour de la Déesse hindoue. Purusartha: Sciences Sociales en Asie du Sud 5: 83-136. 
Bell, S. and Coleman, S. eds. 1999. The Anthropology of Friendship. Oxford: Berg.

Berg, P.O. and Kreiner, K. 1990. 'Corporate architecture: Turning physical settings into symbolic resources', in Gagliardi, P., ed. Symbols and artifacts: View of the corporate landscape. New York: Aldine de Gruyter. 41-67.

Beteille, A. 1965. Caste, class, and power: Changing patterns of stratification in a Tanjore village. Los Angeles, CA: University of California Press.

Beteille, A. 1996. 'Caste in contemporary India', in Fuller, C. J. ed. Caste today. New York: Oxford University Press. 150-79.

Bhatnagar, S. C. and Madon, S. 1997. 'The Indian software industry: moving towards maturity'. Journal of Information Technology 12: 277-88.

Boellstorff, T. 2012. Ethnography and virtual worlds: A handbook of method. Princeton, NJ: Princeton University Press.

Bott, E. and Spillius, E. B. 2014. Family and social network: Roles, norms and external relationships in ordinary urban families. Oxford: Routledge.

Bourdieu, P. 1980. The Logic of Practice. Cambridge: Polity Press.

Brass, D. J. 1992. 'Power in organizations: A social network perspective.' Research in politics and society 4(1):295-323.

Breman, J. 2013. At work in the informal economy of India: a perspective from the bottom up. Oxford: Oxford University Press.

Broadbent, S. 2011. L'intimité au travail. Limoges, France: FYP éditions.

Bros, C. and Couttenier, M. 2010. Untouchability and public infrastructure. https://hal. archives-ouvertes.fr/halshs-00542235/

Buckingham, D. 2013. 'Digital Childhoods? New Media and Children's Culture.' Beyond technology: Children's learning in the age of digital culture. Cambridge: Polity Press.

Buhler, G. 2004. The laws of manu. New Delhi: Genesis Publishing Pvt Ltd.

Burke, S. C. and Snyder, S. L. 2008. 'YouTube: An Innovative Learning Resource for College Health Education Courses.' International Electronic Journal of Health Education 11: 39-46.

Burt, R. S. 2000. 'The network structure of social capital.' Research in organizational behavior 22: $345-423$.

Caldwell, J. C., Reddy, P. H. and Caldwell, P. 1983. 'The causes of marriage change in South India.' Population Studies 37(3): 343-61.

Cheung, C. M., Chiu, P. Y. and Lee, M. K. 2011. 'Online social networks: Why do students use Facebook?' Computers in Human Behavior 27(4): 1337-43.

Chinniah, S. 2008. 'The Tamil film heroine: from a passive subject to a pleasurable object', in Velayutham, S. ed. Tamil cinema: the cultural politics of India's other film industry, vol.10. Oxford: Routledge.

Chithiraputhiran, H. 1999. 'Semantic study of Tamil kinship terms.' Journal of Tamil Studies 55-56: 163-180.

Choudhary, R. 1971. Kautilya's political ideas and institutions (vol.73). New Delhi: Chowkhamba Sanskrit Series Office.

Christakis, N. A. 2010. Connected: The amazing power of social networks and how they shape our lives. London: HarperCollins.

Cialdini, R. B. and Goldstein, N. J. 2004. 'Social influence: Compliance and conformity.' Annual Review of Psychology 55: 591-621.

Clifford, G. 1973. The interpretation of cultures. New York: Basic Books.

Commander, S. 1983. 'The jajmani system in North India: an examination of its logic and status across two centuries.' Modern Asian Studies 17(2): 283-311.

Costa, E. 2016. Social Media in Southeast Turkey. London: UCL Press. 206.

Dahlman, C. J. and Utz, A. 2005. 'India and the knowledge economy: leveraging strengths and opportunities.' Washington. DC: World Bank Publications.

Damle, Y. B. 1956. 'Communication of modern ideas and knowledge in Indian villages.' The Public Opinion Quarterly 20(1): 257-70.

Das, A. and Herring, S. C. 2016. 'Greetings and interpersonal closeness: The case of Bengalis on Orkut.' Language \& Communication 47: 53-65.

Das, A. 2010. 'Social interaction process analysis of Bengalis on Orkut', in Tiwai, R. ed., Handbook of Research on Discourse Behavior and Digital Communication: Language Structures and Social Interaction. Hershey, PA: IGI Global. 66-84. 
Das, A. 2012. 'Impression Management on Facebook and Orkut: A Cross Cultural Study of Brazilians and Indians.' Internet Research 13.0 Conference, University of Salford, 18-22 October 2012.

Das, G. 2002. India Unbound. London: Profile Books.

De González, L. T. 2013. 'Modern Arranged Marriage in Mumbai.' Teaching Anthropology: SACC Notes 19 (1 and 2): 34-49.

De Neve, G. 2000. 'Patronage and "community": the role of a Tamil "village"festival in the integration of a town.' Journal of the Royal Anthropological Institute 6(3): 501-19.

De Neve, G. 2005. 'The everyday politics of labour: Working lives in India's informal economy.' New Delhi: Social Science Press.

De Neve, G. 2008. "“We are all sondukarar (relatives)!": kinship and its morality in an urban industry of Tamilnadu, South India.' Modern Asian Studies 42(1): 211-46.

Desai, S., Dubey, A., Vanneman, R. and Banerji, R. April 2009. 'Private schooling in India: A new educational landscape.' India Policy Forum 5(1): 1-38. National Council of Applied Economic Research.

Desmond, J. 1999. Staging tourism: Bodies on display from Waikiki to Sea World. Chicago, IL: University of Chicago Press.

Dickey, S. 1993a. Cinema and the Urban Poor in South Asia. Cambridge: Cambridge University Press.

Dickey, S. 1993b. 'The politics of adulation: Cinema and the production of politicians in South India.' The Journal of Asian Studies 52(02): 340-72.

Dickey, S. and Jacob, P. 2008. Celluloid Deities: The Visual Culture of Cinema and Politics in South India. Lanham, MD: Lexington Books.

Dirks, N. 1996. 'Recasting Tamil society: The politics of caste and race in contemporary southern India', in Fuller, C. J. ed. Caste today. New York: Oxford University Press. 263-95.

Donner, J. 2005. 'What can be said with a missed call? Beeping via mobile phones in sub-Saharan Africa.' Proc. Seeing, Understanding, Learning in the Mobile Age. Budapest: Institute for Philosophical Research of the Hungarian Academy of Sciences and T-Mobile Hungary Co Ltd. 267-76.

Donner, J. 2006. 'Internet use (and non-use) among urban microenterprises in the developing world: an update from India', in Conference of the Association of Internet Researchers (AoIR), September 2006. 28-30.

Donner, J. 2007. 'The rules of beeping: exchanging messages via intentional "missed calls" on mobile phones.' Journal of Computer-Mediated Communication 13(1): 1-22.

Donner, J. 2008. 'Research approaches to mobile use in the developing world: A review of the literature.' The Information Society 24(3): 140-59.

Donner, J. 2015. After access: Inclusion, development, and a more mobile Internet. Cambridge, MA: MIT Press.

Doron, A. 2012. 'Mobile persons: Cell phones, gender and the self in North India.' The Asia Pacific Journal of Anthropology 13(5): 414-33.

Doron, A. 2013. The great Indian phone book. Cambridge, MA: Harvard University Press.

Dube, L. 1988. 'On the construction of gender: Hindu girls in patrilineal India.' Economic and Political Weekly, 30 April 1988. WS11-WS19.

Dube, L. 1997. Women and kinship: Comparative perspectives on gender in South and South-East Asia. Tokyo: UNU Press.

Dubreuil, G. J. 1917. The Pallavas. New Delhi: Asian educational services.

Duffy, P. 2008. 'Engaging the YouTube Google-eyed generation: Strategies for using Web 2.0 in teaching and learning.' The Electronic Journal of e-Learning 6(2): 119-30.

Dumont, L. 1953-4. 'The Dravidian Kinship Terminology as an Expression of Marriage.' Man 53: 34-9.

Dwyer, R. 2006. Filming the gods: Religion and Indian cinema. Oxford and NewYork: Routledge.

Epstein, A. L. 1969. 'Gossip, Norms and Social Network', in Mitchell, J. C. ed. Social networks in urban situations: analyses of personal relationships in Central African towns. Manchester: Manchester University Press.

Facer, K. and Selwyn, N. 2010. 'Social Networking - Key messages from the Research,' in Sharpe R., Beetham, $\mathrm{H}$ and de Freitas, S. eds. Rethinking Learning for a Digital Age. New York: Routledge. 31-42.

Fanselow, F. S. 1996. 'The disinvention of caste among Tamil Muslims,' in Fuller, C. J. ed. Caste today. New York: Oxford University Press. 202-26. 
Fayard, A. L. and Weeks, J. 2007. 'Photocopiers and water-coolers: The affordances of informal interaction.' Organization Studies 28(5): 605-34.

Freed, S. A. 1963. 'Fictive kinship in a north Indian village.' Ethnology 2(1): 86-103.

Freeman, C. 2000. 'High tech and high heels in the global economy: Women, work, and pink-collar identities in the Caribbean.' Durham, NC: Duke University Press Books.

Friedman, T. L. 2005. The world is flat: A brief history of the twenty-first century. New York: Farrar, Straus and Giroux.

Fuller, C. J. 1996. In Fuller, C. J. ed. Caste today. New York: Oxford University Press. 1-31.

Fuller, C. J. and Logan, P. 1985. 'The Navarātri Festival in Madurai.' Bulletin of the school of Oriental and African Studies 48(01): 79-105.

Fuller, C. J. and Narasimhan, H. 2007a. 'Information technology professionals and the new-rich middle class in Chennai (Madras).' Modern Asian Studies 41(1): 121-50.

Fuller, C. J. and Narasimhan, H. 2007b. 'Empowerment and constraint: women, work and the family in Chennai's software industry.' Upadhya, C. and Vasavi, A. R., eds., In an Outpost of the Global Economy: Work and Workers in India's Information Technology Industry. New Delhi: Routledge India. 190-210.

Fuller, C. J. and Narasimhan, H. 2010. 'Traditional vocations and modern professions among Tamil Brahmans in colonial and post-colonial south India.' Indian Economic and Social History Review 47(4): 473-96.

Fuller, C. J. 1979. 'Gods, priests and purity: on the relation between Hinduism and the caste system.' Man 14(3): 459-76.

Fuller, C. J. 2001. 'The "Vinayaka Chaturthi" Festival and Hindutva in Tamil Nadu.' Economic and Political Weekly 36(19): 1607-16.

Fuller, C. J. 2011. 'Timepass and boredom in modern India.' Anthropology of this Century 1.

Goffman, E. 1978. The presentation of self in everyday life. Harmondsworth: Penguin.

Goffman, E. 2005. Interaction ritual: Essays in face to face behavior. Piscataway, NJ: AldineTransaction.

Good, A. 1985. 'The annual goddess festival in a South Indian village.' South Asian Social Scientist 1(2): 119-67.

Gopal, T. B. 1976. Niti Shastra. Agra: Ratan Prakashan Mandir.

Gottlieb, B. H. 1981. Social networks and social support, vol.4. Thousand Oaks, CA: Sage Publications, Inc.

Gough, E. K. 1955. 'The social structure of a Tanjore village.' in Marriot, M. ed. Village India, Chicago, IL: University of Chicago Press

Gough, E. K. 1956. 'Brahman kinship in a Tamil village.' American Anthropologist 58(5): 826-53.

Gough, E. K. 1969. 'Caste in a Tanjore village', in Leach, E. ed. Aspects of Caste in South India, Ceylon and Northwest Pakistan. Cambridge: Cambridge University Press.

Gough, K. 1973. 'Harijans in Thanjavur.' Imperialism and revolution in South Asia. New York: Monthly Review Press. 222-45.

Gould, H. A. 1986. 'The Hindu jajmani system: a case of economic particularism.' Journal of Anthropological Research 42(3): 269-78.

Goyal, S. 2012. 'Social networks on the Web.' The Oxford handbook of the digital economy. Oxford: Oxford University Press. 434-59.

Grint, K. 2005. The sociology of work: introduction. Cambridge: Polity Press.

Gurumurthy, A. and Menon, N. 2009. 'Violence against women via cyberspace.' Economic and Political Weekly, 3 October 2009. 19-21.

Hammersley, M. and Atkinson, P. 2007. Ethnography: Principles in practice. New York: Routledge.

Hardgrave, R. L. and Neidhart, A. C. 1975. 'Films and political consciousness in Tamil Nadu.' Economic and Political Weekly, 11 January 1975. 27-35.

Hardgrave, R. L. 1973. 'Politics and the Film in Tamilnadu: The Stars and the DMK.' Asian Survey 13(3): 288-305.

Hardgrave, R. L. 1975. When stars displace the gods: The folk culture of cinema in Tamil Nadu. Austin, TX: Center for Asian Studies, University of Texas.

Harriss-White, B. 2003.Indiaworking:Essaysonsociety andeconomy, vol.8. Cambridge: Cambridge University Press.

Haynes, N. 2016. Social Media in Northern Chile. London: UCL Press.

Haythornthwaite, C. 2002. 'Strong, weak, and latent ties and the impact of new media.' The Information Society 18(5): 385-401.

Heesterman, J. C. 1964. Brahmin, ritual and renouncer. Vienna: Universität Wien. Indologisch Institut. 
Hiltebeitel, A. 1991. The Cult of Draupadi: On Hindu Ritual and the Goddess, vol.2. Chicago, IL: University of Chicago Press.

Hine, C. 2015. Ethnography for the internet: embedded, embodied and everyday. London: Bloomsbury Publishing.

Holmström, M. 1976. South Indian factory workers: Their life and their world. Cambridge: Cambridge University Press.

Horst, H. and Miller, D. 2006. The cell phone: An anthropology of communication. Oxford: Berg.

Ishii, H. 1995. 'Caste and kinship in a Newar village', in Gellner, D. N. and Quigley, D., eds. Contested Hierarchies: A collaborative ethnography of caste among the Newars of the Kathmandu Valley, Nepal. Oxford: Clarendon Press. 109-57.

Iyengar, P. S. 1929. History of the Tamils from the Earliest Times to 600 AD. New Delhi: Asian Educational Services.

Jacob, P. 1997. 'From co-star to deity: Popular representations of Ms. Jayalalitha Jayaram.' Women: A Cultural Review 8(3): 327-37

Jain, K. 2007. Gods in the bazaar: The economies of Indian calendar art. Durham, NC and London: Duke University Press.

Jeffrey, C. 2010. Timepass: Youth, class, and the politics of waiting in India. Stanford, CA: Stanford University Press.

Jeffrey, C., Jeffery, P. and Jeffery, R. 2010. Education, Unemployment, and Masculinities in India. Stanford, CA: Stanford University Press.

Jeffrey, R. and Doron, A. 2013. Cell phone nation: How mobile phones have revolutionized business, politics and ordinary life in India. New Delhi: Hachette.

Jordan, A.T. 2012. Business anthropology. Long Grove, IL: Waveland Press.

Joroff, M. L., Porter, W. L., Feinberg, B. and Kukla, C. 2003. 'The agile workplace.' Journal of Corporate Real Estate 5(4): 293-311.

Jouhki, J. 2013. 'A Phone of One's Own? Social Value, Cultural Meaning and Gendered Use of the Mobile Phone in South India.' Journal of the Finnish Anthropological Society 38(1): 37-58.

Kapadia, K. 1994. “"Kinship burns!”: kinship discourses and gender in Tamil South India.' Social Anthropology 2(3): 281-97.

Karve, I. 1965. Kinship organization in India. Bombay: Asia Publishing House.

Katz, J. E. 2008. Handbook of mobile communication studies. Cambridge, MA: The MIT Press.

Kavoori, A., and Chadha, K. 2006. 'The cell phone as a cultural technology: Lessons from the Indian case', in Kavoori, A. and Arceneaux, N., eds. The cell phone reader: Essays in social transformation. New York: Peter Lang.

Kelkar, G., Shrestha, G. and Veena, N. 2002. 'IT industry and women's agency: Explorations in Bangalore and Delhi, India.' Gender, Technology and Development 6(1): 63-84.

Khanduri, R. G. 2014. Caricaturing Culture in India: Cartoons and History in the Modern World. Cambridge: Cambridge University Press.

Killick, E and Desai, A. 2010 'Introduction: Valuing Friendship', in Desai, A. and Killick, E. eds. The Ways of Friendship. Oxford: Berghahn.

Kingdon, G. G. 2007. 'The progress of school education in India.' Oxford Review of Economic Policy 23(2): 168-95.

Kolenda, P. 1963. 'Toward a model of the Hindu Jajmani system.' Human Organization 22(1): 11-31.

Kolenda, P. 1967. 'Regional differences in Indian family structure', in Crane, R. I. ed. Regions and Regionalism in South Asian Studies: An Exploratory Study. Durham, NC: Duke University Monographs and Occasional Papers Series, 5.

Kolenda, P. M. 1968. 'Region, Caste and Family Structure: a comparative Study of the Indian "Joint" Family', in Singer, M. and Cohn, B. S. eds. Structure and change in Indian society. Chicago, IL: Aldine Press. 339-96.

Konana, P. and Balasubramanian, S., 2002. 'India as a knowledge economy: aspirations versus reality.' Frontline 19(2): 65-9.

Kumar, K. J. and Thomas, A. O. 2006. 'Telecommunications and Development: The Cellular Mobile "Revolution" in India and China.' Journal of Creative Communications 1(3): 297-309.

Kumar, K. J. 2014. Mass communication in India. New Delhi: Jaico Publishing House.

Kumar, N. 2001. 'Indian Software Industry Development: International And National Perspective.' Economic and Political Weekly 36, 10 November 2001, 4278-90.

Kumar, N and Rangaswamy, N. 2013. The Mobile Media Actor-Network in Urban India. Paris: ACM Conference on Human Factors in Computing Systems (CHI 2013). 
Kumar, P. 2014. 'ICT and Its Development in India.' http://www.irjcjournals.org/ijieasr/ Feb2014/2.pdf

Lakshman, N. 2013. Degree Coffee by the Yard: A Short Biography of Madras. New Delhi: Aleph Book Company.

Lakshmi, C. S. 2008. 'A good woman, a very good woman: Tamil cinema's women', in Velayutham, S. ed. Tamil cinema: the cultural politics of India's other film industry, vol.10. Oxford and New York: Routledge.

Laughey, D. 2007. Key themes in media theory. Berkshire (UK): McGraw-Hill Education.

Lepawsky, J. 2005. 'Digital Aspirations: Malaysia and the Multimedia Super Corridor.' FOCUS on Geography 48(3): 10-18.

Lin, N., Cook, K. S. and Burt, R. S. eds. 2001. Social capital: Theory and research. Piscataway: NJ: Transaction Publishers.

Madhavan, C. 2007. Vishnu Temples of South India, vol.1. Chennai, Tamil Nadu: Alphaland Books.

Madianou, M. and Miller, D. 2013a. Migration and new media: Transnational families and polymedia. Oxford: Routledge.

Madianou, M. and Miller, D. 2013b. 'Polymedia: Towards a new theory of digital media in interpersonal communication.' International Journal of Cultural Studies 16(2): 169-87.

Mahajan, P. 2009. 'Use of social networking in a linguistically and culturally rich India.' The International Information \& Library Review 41(3): 129-36.

Mandelbaum, D. G. 1970. Society in India: Continuity and change, vol.1. Los Angeles, CA: University of California Press.

Mayer, A. 1996. 'Caste in an Indian village: change and continuity 1954-1992,' in Fuller, C. J. ed. Caste today. New York: Oxford University Press. 32-64.

Mani, S. 2007. 'Revolution in India's Telecommunications Industry.' Economic and Political Weekly 42(7): 578-80.

Mani, S. 2012. 'Bridging the digital divide: the Indian experience in increasing the access to telecommunications services.' International Journal of Technological Learning, Innovation and Development 5(1-2): 184-203.

Marglin, F. A. 1977. 'Power, purity and pollution: Aspects of the caste system reconsidered.' Contribution to Indian Sociology 11(2): 245-70.

Marin, A. and Wellman, B. 2011. 'Social network analysis: An introduction.' The SAGE handbook of social network analysis. London: Sage Publications. 11-25.

Mathur, A. and Ambani, D. 2005. 'ICT and rural societies: Opportunities for growth.' The International Information \& Library Review 37(4): 345-51.

Mathur, N. 2010. 'Shopping malls, credit cards and global brands consumer culture and lifestyle of India's new middle class.' South Asia Research 30(3): 211-31.

Mayor, A. C. 1960. Caste and Kinship in Central India: A Village and its Region. London: Routledge.

Mazzarella, W. 2003. Shoveling smoke: Advertising and globalization in contemporary India. Durham, NC and London: Duke University Press.

McLuhan, M. 1964. Understanding Media. The extensions of man. London: MIT Press.

McPherson, M., Smith-Lovin, L. and Cook, J. M. 2001. 'Birds of a feather: Homophily in social networks.' Annual Review of Sociology. 27(1): 415-44.

Mehta, B. S. 2013. 'Capabilities, costs, networks and innovations: impact of mobile phones in rural India.' Available at SSRN Electronic Journal 2259650, May 2013.

Miller, D. and Slater, D. 2000. The Internet: an ethnographic approach. Oxford: Berg.

Miller, D. and Slater, D. 2005. 'Comparative Ethnography of New Media', in Curran, J. P. and Gurevitch, M. eds. Mass Media and Society 4th edition. London: Hodder Arnold.

Miller, D. 2015. 'Photography in the Age of Snapchat.' Anthropology and Photography Pamphlet Series. London: Royal Anthropological Institute [Photographic Committee] Web. https:// www.therai.org.uk/images/stories/photography/AnthandPhotoVol1B.pdf

Miller, D., Costa, E., Haynes, N., McDonald, T., Nicolescu, R., Sinanan, J., Spyer, J., Venkatraman, S. and Wang, X. 2016. How the World Changed Social Media. London: UCL Press.

Miller, D. 2016. Social Media in an English Village. London: UCL Press.

Miller, D. and Sinanan, J. 2017. Visualising Facebook. London: UCL Press.

Mir, A., Mathew, B. and Raza, M. 2000. 'The Codes Of Migration.' Cultural Dynamics 12, 5-33.

Mirchandani, K. 1999. 'Feminist insight on gendered work: New directions in research on women and entrepreneurship.' Gender, Work \& Organization 6(4): 224-35.

Mishra, S. 2010. 'Participation of youth in social networking sites in India.' International Journal of Business Innovation and Research 4(4): 358-75. 
Mitchell, J. C. ed. 1969. Social networks in urban situations: analyses of personal relationships in Central African towns. Manchester: Manchester University Press.

Moeran, B. 2005. The business of ethnography: Strategic exchanges, people and organizations. London: Berg Publishers.

Mosse, D. 1997. 'Honour, caste and conflict: The ethnohistory of a Catholic festival in rural Tamil Nadu (1730-1990).' Purusārtha (19): 71-120.

Murray, L. C. 2014. Advertising Amul: On Meaning, Materiality, and Dairy in India. Web http://static1.squarespace.com/static/534587eae4b0fb5fdfb963aa/t/54a9f38ce4b08424e6a8c019/1420424106572/Milk+In+India+Part+II.pdf

Muthiah, S. 2011. Madras Miscellany: A Decade of People, Places and Potpurri. Chennai: Tranquebar Press.

Myrdal, A. and Klein, V. 2003. Women's two roles: Home and work, vol.137. Oxford: Routledge / Psychology Press.

Nagaswamy, R. and Nakacami, I. 2008. Mahabalipuram. New York: Oxford University Press.

Nakassis, C.V. and Dean, M. A. 2007. 'Desire, youth, and realism in Tamil cinema.' Journal of Linguistic Anthropology 17(1): 77-104.

Nakassis, C.V. 2010. Youth and Status in Tamil Nadu, India. Publicly Accessible Penn Dissertations web http://repository.upenn.edu/cgi/viewcontent. cgi article $=1247 \&$ context $=$ edissertations.

Nakassis, C. V. 2013. 'Youth masculinity, "style" and the peer group in Tamil Nadu, India.' Contributions to Indian Sociology 47(2): 245-69.

Nakassis, C. V. 2014. 'Suspended Kinship and Youth Sociality in Tamil Nadu, India.' Current Anthropology 55(2): 175-99.

Nakassis, C. V. 2015. 'A Tamil-speaking Heroine.' BioScope: South Asian Screen Studies 6(2): 165-86.

Nash, J. 1998. 'Twenty Years of the Anthropology of Work: Changes in the State of the World and the State of the Arts.' Anthropology of Work Review 18(4): 1-6.

Nath, D. 2000. 'Gently shattering the glass ceiling: experiences of Indian women managers.' Women in Management Review 15(1): 44-52.

Niehoff, A. H. 1959. Factory workers in India. PhD dissertation: Board of Trustees, Milwaukee Public Museum.

Nisbett, N. 2007. 'Friendship, consumption, morality: practising identity, negotiating hierarchy in middle-class Bangalore.' Journal of the Royal Anthropological Institute 13(4): 935-50.

Nisbett, N. 2009. Growing Up in the Knowledge Society: Living the IT Dream in Bangalore. New Delhi: Routledge.

Noelle-Neumann, E. 1974. 'The spiral of silence a theory of public opinion.' Journal of Communication 24(2): 43-51.

Ortiz, S. 1994. 'Work, the Division of Labour and Co-operation', in Ingold, T. ed. Companion encyclopaedia of anthropology. Oxford: Routledge.

Osella, C. and Osella, F. 1998. 'Friendship and flirting: micro-politics in Kerala, South India.' Journal of the Royal Anthropological Institute: 4(2) 189-206.

Osella, F. and Osella, C. 2000. Social mobility in Kerala: modernity and identity in conflict. London: Pluto Press.

Pandian, A. 2015. Reel world: an anthropology of creation. Durham, NC: Duke University Press.

Pandian, M. S. S. 1992. M G Ramachandran in Film and Politics: The Image Trap. New Delhi: Sage Publications India.

Pandian, M. S. S. 2007. Brahmin and non-Brahmin: Genealogies of the Tamil political present. New Delhi: Permanent Black.

Papacharissi, Z. ed. 2010. A networked self: Identity, community, and culture on social network sites. New York: Routledge.

Parrott, D. J. and Zeichner, A. 2003. 'Effects of hypermasculinity on physical aggression against women.' Psychology of Men \& Masculinity 4(1): 70.

Parry, J. P. 1999. 'Introduction', in Parry, J. P., Breman, J. and Kapadia, K., eds. The worlds of Indian industrial labour. London: Sage Publications.

Parry, J. P. 2001. 'Ankalu's errant wife: sex, marriage and industry in contemporary Chhattisgarh.' Modern Asian Studies 35(4): 783-820.

Parry, J. P. 2013. Caste and kinship in Kangra. Oxford: Routledge.

Pedersen, J. D. 2000. 'Explaining economic liberalization in India: state and society perspectives.' World Development 28(2): 265-82. 
Pentland, A., Fletcher, R. and Hasson, A. 2004. 'Daknet: Rethinking connectivity in developing nations.' Computer 37(1): 78-83.

Perumal, C. A. and Padmanabhan, V. K. 1987. 'Political Alliances in Tamil Nadu.' The Indian Journal of Political Science 48(4): 618-24.

Peterson, M. 2011. 'Orkut Dissected: Social Networking in India \& Brazil.' http://www.aimclearblog.com/2011/06/27/orkut-dissected-social-networking-in-india-brazil/

Picherit, D. 2012. 'Migrant Labourers' Struggles Between Village and Urban Migration Sites: Labour Standards, Rural Development and Politics in South India.' Global Labour Journal 3(1): 143-62.

Pillai, A. 2012. 'User acceptance of social networking websites in India: Orkut vs. Facebook.' International Journal of Indian Culture and Business Management 5(4): 405-14.

Pillay, K. P. K. 1977. The Caste System in Tamil Nadu. Chennai: University of Madras.

Pink, S., Horst, H., Postill, J., Hjorth, L., Lewis, T. and Tacchi, J. 2015. Digital ethnography: principles and practice. Los Angeles, CA: Sage Publications.

Pinney, C. 2004. 'Photos of the Gods': The Printed Image and Political Struggle in India. London: Reaktion Books.

Pinney, C. 2008. The coming of photography in India. London: British Library.

Pitroda, S. 1993. 'Development, democracy, and the village telephone.' Harvard Business Review 71(6): 66-8.

Pleck, J. H. 1977. 'The work-family role system.' Social Problems 24(4): 417-27.

Powell, W. W. and Kaisa, S. 2004. 'The Knowledge Economy.' Annual Review of Sociology 30: 199-220. Palo Alto, CA: Annual Reviews.

Pushpa, S. 1996. 'Women and philanthropy in India.' VOLUNTAS: International Journal of Voluntary and Nonprofit Organizations 7(4): 412-27.

Rabe, M. D. 2001. The great penance at Māmallapuram: deciphering a visual text, vol.1. General editor: G. John Samuel. Chennai, Tamil Nadu: Institute of Asian Studies.

Rainie, H. and Wellman, B. 2012. Networked: The new social operating system. Cambridge, MA: The MIT Press.

Rajanayagam, S. 2015. Popular Cinema and Politics in South India: The Films of MGR and Rajinikanth. New Delhi: Routledge.

Ramu, G. N. 1977. Family and caste in urban India: a case study. New Delhi : Vikas Publishing House.

Rangaswamy, N. and Arora, P. 2015. 'Digital Romance in the Indian City', in The City and South Asia. Cambridge, MA: Harvard South Asia Institute.

Rangaswamy, N. and Cutrell, E. 2013. 'Anthropology, Development and ICTs: Slums, Youth and the Mobile Internet in Urban India.' Special Issue, Reflections at the Nexus of Theory and Practice, Information Technology and International Development. Cambridge, MA: The MIT Press.

Rangaswamy, N. and Yamsani, S. 2011. 'Mental Kartha Hai or Its Blowing my Mind: Evolution of the Mobile Internet in an Indian Slum.' EPIC, The Ethnographic Praxis in Industry Conference, Boulder, CO, 18-21 September 2011.

Rangaswamy, N. and Bombay, L. S. I. 2007. 'ICT for development and commerce: A case study of internet cafés in India' (Research in progress paper), in Proceedings of the 9th International Conference on Social Implications of Computers in Developing Countries. São Paulo, Brazil.

Rangaswamy, N. 2012. 'Pocket Social Networking in India - SMS GupShup Expands.' Asia Pacific Memo, November 2012. web http://www.asiapacificmemo.ca/pocket-socialnetworking-in-india-sms-gupshup-expands

Rao, Y. L. 1966. Communication and development: A study of two Indian villages. Minneapolis, MI: University of Minnesota Press.

Raynes-Goldie, K. and Lloyd, C. 2014. 'Unfriending Facebook? Challenges From an Educator's Perspective.' An Education in Facebook, 153-61. Oxford: Routledge

Reddy, P. G. 2010. 'Some Problems Faced By The Software Professionals In India: An Overview,' in Lakshmansamy, ed. Population Dynamics and Human Development Opportunities and Challenges. New Delhi: Bookwell. 451-71.

Reiniche, M. L. 1996. 'The urban dynamics of caste: a case study from Tamil Nadu', in Fuller, C. J, ed. Caste today. New York: Oxford University Press. 124-49.

Roblyer, M. D., McDaniel, M., Webb, M., Herman, J. and Witty, J. V. 2010. 'Findings on Facebook in higher education: A comparison of college faculty and student uses and perceptions of social networking sites.' The Internet and Higher Education 13(3): 134-40. 
Rogers, E. M. and Bhowmik, D. K. 1970. 'Homophily-heterophily: Relational concepts for communication research.' Public Opinion Quarterly 34(4): 523-38.

Rogers, M. 2009. 'Between fantasy and "reality": Tamil film star fan club networks and the political economy of film fandom.' Journal of South Asian Studies 32(1): 63-85.

Rudner, D. W. 1994. 'Caste and capitalism in colonial India: the Nattukottai Chettiars.' Berkeley, CA: University of California Press.

Säävälä, M. 2010. Middle-class moralities: Everyday struggle over belonging and prestige in India. New Delhi: Orient Blackswan.

Säävälä, M. 2014. 'The "Hindu Joint Family": Past and present.' Studia Orientalia Electronica 84: 61-74.

Sandberg, S. 2013. Lean in: Women, work, and the will to lead. New York: Knopf.

Scott, J. 2012. Social network analysis. Thousand Oaks, CA: Sage Publications.

Shah, A. M. 1973. The household dimension of the family in India. Delhi: Orient Longman.

Shah, A. M. 1998. The family in India: critical essays. New Delhi: Orient Blackswan.

Shah, G. 2006. Untouchability in rural India. New Delhi: Sage Publications.

Sivananda, S. 2004. Practice of Karma Yoga. Rishikesh: Divine Life Society.

Smyth, T. N., Kumar, S., Medhi, I. and Toyama, K. 2010. “"Where there's a will there's a way": mobile media sharing in urban India', in Proceedings of the SIGCHI conference on Human Factors in computing systems. ACM Digital Library: 753-62.

Soete, L. 1985. 'International diffusion of technology, industrial development and technological leapfrogging.' World Development 13(3): 409-22.

Sooryamoorthy, R. 2008. 'Untouchability in modern India.' International Sociology 23(2): 283-93.

Spencer, M. B., Fegley, S., Harpalani, V. and Seaton, G. 2004. 'Understanding hypermasculinity in context: A theory-driven analysis of urban adolescent males' coping responses.' Research in Human Development 1(4): 229-57.

Spyer, J. Forthcoming. Social Media in Brazil. London: UCL Press.

Sreekumar, T. T. 2014. 'New media, space and marginality: A comparative perspective on cyber cafe use in small and medium towns in Asia.' Paper presented at the International Association for Media and Communication Research (IAMCR) Annual Conference, Hyderabad, India, 15-19 July 2014.

Srinivas, M. N. 1962. Caste in modern India and other essays. New Delhi: Asia Publishing House.

Srinivas, M. N. 1960. The social structure of a Mysore village. Indianapolis, IN: Bobbs-Merrill.

Srinivas, S. V. 1996. 'Devotion and defiance in fan activity.' Journal of Arts and Ideas 29(1): 67-83.

Steenson, M. and Donner, J. 2009. 'Beyond the personal and private: Modes of mobile phone sharing in urban India.' The reconstruction of space and time: Mobile communication practices 1: 231-50.

Steinmueller, W. E. 2001. 'ICTs and the possibilities for leapfrogging by developing countries.' International Labour Review 140(2): 193-210.

Stern, H. 1977. 'Power in traditional India: territory, caste and kinship in Rajasthan', in Fox, R. G. ed. Realm and region in traditional India. New Delhi: Vikas Publishing House. 52-78.

Suresh, V. 1992. 'The DMK debacle: Causes and portents.' Economic and Political Weekly, 17 October 1992. 2313-21.

Swallow D.A. 1982. Production and control in the Indian garment export industry. Cambridge: Cambridge University Press. 133-65.

Tenhunen, S. 2014. 'Mobile telephony, mediation, and gender in rural India.' Contemporary South Asia 22(2): 157-70.

Thirunavukkarasu, R. 2001. 'Election 2001: Changing Equations.' Economic and Political Weekly, July 7-13, 2001. 2486-9.

Thompson, C. 2011. 'How Khan Academy is changing the rules of education.' Wired Magazine 126: $1-5$.

Thurston, E. and Rangachari, K. 1909. Castes and tribes of South India. New Delhi. Asian Educational Services.

Toyama, K., Kiri, K., Maithreyi, L., Nileshwar, A., Vedashree, R. and MacGregor, R. 2004. 'Rural kiosks in India.' MSR Techincal Report. 1 July 2004.

Trautmann, T. R. 1981. Dravidian kinship. Berkeley, CA: Sage Publications.

Trawick, M. 1990. Notes on love in a Tamil family. Los Angeles, CA: University of California Press. 
Turner, V. W. and Schechner, R. 1988. The anthropology of performance. New York: PAJ Publications.

Turner, V. W. 1982. From ritual to theatre: The human seriousness of play. New York: Paj Publications.

Uberoi, P. ed. 1993. Family, kinship and marriage in India. New Delhi: Oxford University Press.

Uberoi, P. 2005. 'The Family in India', in Khullar, M. ed. Writing the women's movement: a reader. New Delhi: Zubaan. 361-96.

Upadhya, C. and Vasavi, A. R. 2006. 'Work, culture, and sociality in the Indian IT industry: a sociological study.' Bangalore: National Institute of Advanced Studies, Indian Institute of Science Campus, Bangalore.

Uyl, M. D. 1995. Invisible barriers: gender, caste and kinship in a southern Indian village. Utrecht: International Books.

Van Dijck, J. 2008. 'Digital photography: communication, identity, memory.' Visual Communication 7(1): 57-76.

Van Maanen, J. 2011. Tales of the field: On writing ethnography. Chicago, IL: University of Chicago Press.

Varma, P. K. 2007. The great Indian middle class. New Delhi: Penguin Books India.

Vatuk, S. 1996. 'Identity and difference or equality and inequality in South Asian Muslim society', in Fuller, C. J. ed. Caste today. New York: Oxford University Press. 227-62.

Vatuk, S. 1969. 'Reference, address, and fictive kinship in urban north India.' Ethnology 8(3): 255-72.

Veeramani, K. 2005. Collected works of Periyar EVR. Chennai: The Periyar Self-Respect Propaganda Institution, Chennai.

Velayutham, S. ed. 2008. Tamil cinema: the cultural politics of India's other film industry, vol.10. Oxford and New York: Routledge.

Venkatanarayanan, S. 2015. 'Economic Liberalization in 1991 and Its Impact on Elementary Education in India.' SAGE Open 5(2): DOI: 10.1177/2158244015579517.

Venkatraman, S , Rangaswamy, N. and Arora, P. 'Polymedia: A perspective through filial relationships at Panchagrami.' Paper for Panel, Reconstituting Marginality and Publics in the Digital Age, Annual International Conference of Media and Communication Research, Hyderabad, 15-19 July 2014.

Vidyarthi, L. P. 1984. In Vidyarthi, L. P. ed. Applied anthropology in India: principles, problems, and case studies. New Delhi: Kitab Mahal.

Vijaybaskar, M. and Gayathri, V. 2003. 'ICT and Indian Development: Processes, Prognoses, Policies.' Economic and Political Weekly 38(24): 2360-4. 14-20, 2003.

Vilanilam, J. V. 2005. Mass communication in India: A sociological perspective. New Delhi: Sage Publications.

Wade, R. H. 2002. 'Bridging the digital divide: new route to development or new form of dependency?' Global Governance 8(4): 443-66.

Wallman, S. ed. 1979. Social anthropology of work, vol.19. London: Academic Press.

Wasserman, S. and Galaskiewicz, J., eds. 1994. Advances in social network analysis: Research in the social and behavioral sciences. vol.171. Thousand Oaks, CA: Sage Publications.

Watts, D. 2003. Six Degrees: The Science of a Connected Age. New York: W. W. Norton \& Company.

Why We Post - https://www.ucl.ac.uk/why-we-post

Williams, J. 2001. Unbending gender: Why family and work conflict and what to do about it. New York: Oxford University Press.

Wiser, W. H. 1988. The Hindu jajmani system. New Delhi: Munshiram Manoharlal Publishers Pvt Ltd.

Woyke, E. 2014. The Smartphone: Anatomy of an industry. New York: The New Press.

Xiang, B. 2007. 'Global 'body shopping': an Indian labor system in the information technology industry. Princeton, NJ: Princeton University Press.

Zeff, A. 1999. Marriage, film, and video in Tamilnadu: Narrative, image, and ideologies of love. Dissertation submitted to University of Pennsylvania. Web: http://repository.upenn.edu/ dissertations/AAI9926222/?pagewanted=all 


\section{Internet sources}

http://aiadmk.com/en/home/

http://aiadmk.com/en/tn-election-2016/manifesto-infographics/

http://aiadmk.com/en/tn-election-2016/tamilnadu-election-manifesto-2016/

http://blogs.ucl.ac.uk/global-social-media/2013/12/15/non-resident-indians/

http://blogs.ucl.ac.uk/global-social-media/2014/07/25/its-ok-to-send-my-boss-

a-whatsapp-message/

http://blogs.ucl.ac.uk/global-social-media/2015/04/17/women-entrepreneurs-whatsapp/

http://cbse.nic.in/

http://censusindia.gov.in/2011-prov-results/data_files/india/Final_PPT_2011_chapter6.pdf

http://dmk.in/english

http://economictimes.indiatimes.com/

http://en.wikipedia.org/wiki/Tethering

http://indiatoday.intoday.in/

http://jayatvnetwork.com/default.aspx

http://mashable.com/2016/02/22/india-villages-ban-mobile-phones/\#iCfdPBCP.ZqJ

http://mdmk.org.in/

http://scroll.in/article/744579/what-happened-to-india-when-the-landline-telephon

e-fell-terminally-ill-20-years-ago

http://thehackernews.com/2016/01/whatsapp-free-lifetime.html

http://timesofindia.indiatimes.com/

http://timesofindia.indiatimes.com/tech/how-to/Assemble-a-PC-within-Rs-30000-budget/

articleshow/19797394.cms

http://www.927bigfm.com/city.php?id=11

http://www.airtel.com/

http://www.alexa.com/siteinfo/orkut.com

http://www.bbc.co.uk/news/business-26266689

http://www.bbc.co.uk/news/technology-17658264

http://www.bbc.co.uk/news/world-asia-35115974

http://www.bbc.co.uk/news/world-asia-india-23304251

http://www.businesstoday.in/

http://www.cie.org.uk/programmes-and-qualifications/cambridge-secondary-2/

cambridge-igcse/

http://www.dailythanthi.com/

http://www.deccanchronicle.com/

http://www.deccanchronicle.com/141016/nation-current-affairs/article/

chennai-fifth-number-facebook-users

http://www.digit.in/

http://www.dinamalar.com/

http://www.discoveranthropology.org.uk/about-anthropology/fieldwork/ethnography.html

http://www.dmdkparty.com/

http://www.dnaindia.com/scitech/report-whatsapp-user-base-crosses-70-million-in-india-2031465

http://www.dqchannels.com/laptops-freebies/

http://www.dqindia.com/

http://www.femina.in/

http://www.firstpost.com/politics/freebies-culture-in-tamil-nadu-reeks-of-a-guilty-conscious-neta-who-doesnt-really-care-2781472.html

http://www.firstpost.com/politics/phones-wi-fi-electricity-aiadmks-manifesto-for-tamil-nadu-polls-is-full-of-freebies-2767768.html

http://www.icmrindia.org/free\%20resources/Articles/Indian\%20PC\%20Market2.htm

http://www.in.com/tv/channel/star-vijay-164.html

http://www.independent.co.uk/news/world/asia/girls-and-unmarried-women-in-india-forbidden-from-using-mobile-phones-to-prevent-disturbance-in-a6888911.html 
ht tp://www.independent.co.uk/news/world/asia/nirbhaya-case-anger-and-protests-as-juvenile-delhi-gang-rapist-freed-after-three-years-in-ref orm-a6780601.html

http://www.geni.com

http://www.livemint.com/Opinion/GGMQFvliFGJiKMzPZWWVLN/Why-Tamil-Nadus-freebi e-culture-works.html

http://www.materialworldblog.com/2010/04/the-unanticipated-city-shiftin g-urban-landscapes-and-the-politics-of-spectacle-in-chennai/

http://www.newindianexpress.com/

http://www.pmkparty.in/

http://www.radiomirchi.com/chennai,

http://www.samacheerkalvi.in/

http://www.statista.com/statistics/257048/smartphone-user-penetration-in-india/

http://www.sunnetwork.in/

http://www.suryanfm.in/chennai/

http://www.thehindu.com/

http://www.thiruma.in/

http://www.vikatan.com/

http://www.vogue.in/

https://en.wikipedia.org/wiki/Chennai_Super_Kings

https://en.wikipedia.org/wiki/Ganesha

https://en.wikipedia.org/wiki/Thanjavur_painting

https://twitter.com/stalin_offl

https://www.khanacademy.org/

https://www.kumudam.com/

https://www.ucl.ac.uk/why-we-post/discoveries/why-we-post/discoveries/7-we-usedto-just-talk-now-we-talk-photos

https://www.youtube.com/watch?v=vtAmdUOfuFQ\&index=14\&list=PLVwGSavjGgExtI65BTxuE4CuclQqjA7fU 


\section{அட்டவணை}

அகமண உறவு 157

அமைப்பு முரண்பாடுகெள் 213

அந்தரங்க அமைப்பை 69

அரசியல் காட்சியமைப்புகள் 119

அரசியல் செய்திகள் 308

அளவீட்டுச் சமுதாயத்திற்கான 89

அறிவார்ந்த பொருளாதாரத்தின்- 37

அன்றாட வாழ்த்துச்செய்திகள் 309

ஆடி மாதத்தில் 97

ஆண்மை 124

ஆணாதிக்க முறைகளை 155

இரரட்டை வருமானக் குடும்பங்கள் 235

இறப்பு 189

உணர்வுபூர்வ விசாரங்களால் 86

உறவினர் ஆதரவுக் கொள்கைக் குற்றத்தை 212

உறவினர்கள் போல் கருதும் நண்பர்கள் 93

உறவுமுறை 8

ஊக்கமளிக்கக்கூடிய 133

எழுத்தறிவு 26

ஒப்புதல் செயல்திட்ட 33

கட்டிப்படியாகக்கூடிய தகவல்

தொழில்நுட்பங்கள் 305

கட்டுப்பாடுகெள் 289

கட்டுமானத் தொழிலாளர்கள் 22

கண்காணிப்பு 86

கல்வி 10

காட்சிக்குரிய பதிவுகளை 8

காட்சியமைப்பு தகவல் பரிமாற்றத்தின் வகையிலக்கணங்கள் 146

குரல்வழி தொடர்பே 199

குலம் 312

குலத்தை 15

கூட்டுச் சீர்மைகளை 95

சமத்துவமின்மையை 315

சமூக அந்தஸ்தை 127

சமூக அனுசரித்தலை 89

சமூக இயங்குதிறன் 263

சமூக ஊடகங்கள் 231

சமூக ஊடக பதிவுகளில்

பொதுத்தன்மை 307

சமூகக் கட்டமைப்புகளுக்கு 199

சமூகத்தன்மை 306

சமூக நீதிக்கும் 115

சமூக நோக்கங்கொண்ட 116

சமூக வகை 4

சமூக விதிமுறைகளையும் 130
சார்ந்திருக்கும் உள்ளுணர்வு 202

சிறப்புப் பொருளாதார

மண்டலம் 15

டாங்கிள் 50

தகவல் தொழில்நுட்ப

சாதனங்களை 264

தகவல் பாதுகாப்பு 220

தரும காரியங்கள் 91

தலைமுறைகளுக்கு இடையேயான தகவல் பரிமாற்ற 57

திறமைத் தலைமை 215

தொடர்பு அலைவரிசைகள் 165

தொழில் சார்ந்த

மனப்பான்மையை 217

தொழில்நுட்பத்திறன் 278

தொழில்மயமாக்கலுக்கும் 208

தொழிலாளர் அமைப்பு 208

தொழிலாளிகள் வந்துசெல்லுதல் 212

தொழிற்பணி அமைப்புகளில் 211

நெருக்கமான 183

பணிக் கலாச்சாரம் 10

பணிபுரியும் அன்னைகள் 237

பணியமர்த்தலுக்கு 249

பலதார மணப்பழக்கத்தினாலும் 193

பன்னாட்டு நிறுவனங்களான 16

பாகுபாடு 69

பாகுபாட்டினால் 23

பாரம்பரியம் 4

பாலினம் 37

பிரபலமான கலாச்சாரத்தில் 147

பிறப்பு 189

புலம்பெயர்ந்திருக்கும்

தொழிலாளர்கள் 22

பெண்ணியல்பைப் 185

பெண் பிரதிநிதிகள் 113

பெண்மை 124

பெற்றோருக்குரிய கட்டுப்பாடுகளை 268

பொருளாதார வர்க்கங்களை 21

பொருளுடையதாக 316

மக்கள் இன அமைப்பியல் 305

மதம் 23

மானுடவியலின் 199

மின்னஞ்சல் 57

முகநூல் 1

வர்ணாசிரம முறை 209

வீணுரை 40

ஜவுளி உற்பத்தி 207 

தென்னிந்தியாவில் சமூக ஊடகங்கள், தமிழ்நாட்டு மக்களின் அன்றாட வாழ்வில் சமூக ஊடகங்களின் பயன்பாடு பற்றிய புரிதலை தீவிர மாற்றமடைந்து வரும் ஒரு பிராந்தியத்தில் நடத்தப்பட்ட முதல் மக்கள் இன அமைப்பியல் ஆய்வின் மூலம் நமக்களித்திருக்கிறது. கடந்த பத்தாண்டுகளில் ஐடி நிறுவனங்களின் வருகை, முன்பு விவசாய பகுதியாக இருந்த ஒரு இடத்தை வளர்ந்து கொண்டேயிருக்கும் அறிவார்ந்த பொருளாதாரம் மற்றும் பாரம்பரிய கிராம வாழ்வு ஆகியவற்றின் பக்க அணிமை நிலையாக ஆக்கியிருக்கிறது. இந்த பக்க அணிமை நிலையால் ஒருசில வர்க்க பேதங்கள் காணப்பட்டபோதும், இந்த பிராந்தியத்தில் நடத்தப்பட்ட சமூக ஊடக ஆய்வு, ஒற்றுமைகளும் இருந்திருப்பதற்கான சான்றளிக்கிறது. குறிப்பாக பழைய மற்றும் புதிய குடியிருப்பாளர்களிடையே பணி மற்றும் வாழ்வின் இடையேயான தேய்ந்து வரும் எல்லைகளில் இது காணப்படுகிறது.

வெங்கட்ராமன், வீடுகள், பணியிடங்கள் மற்றும் பள்ளிகளில் சமூக ஊடகங்களின் பாதிப்புகளை ஆராய்ந்து, இனம், வர்க்கம், வயது பாலினம் ஆகியவற்றின் தாக்கத்தையும் அலசி, எந்த வகையான சமூக ஊடகத்தளங்கள் எந்தெந்த சூழல்களில் பயன்படுத்தப்படுகின்றன என்பதை ஆராய்ந்திருக்கிறார்.

இந்த காரணிகள், சமூக ஊடக பயன்பாட்டின் மீது குறிப்பிடத்தக்க பாதிப்பை ஏற்படுத்தியிருப்பதாக கூறும் அவர், தென்னிந்தியாவில் சமூக ஊடகங்கள், சமுதாய மாற்றத்தை தூண்டியிருப்பது போல தோன்றினாலும், அவை உண்மையில், உள்ளூர் பாரம்பரியங்கள் மற்றும் நடைமுறைகளால் கட்டுண்டு இருக்கின்றன என்றும் குறிப்பிடுகிறார்.

ஸ்ரீராம் வெங்கட்ராமன் யுசிஎல்-ல் இருந்து மானுடவியலில் முனைவர் பட்டம் பெற்று, தற்போது, டெல்லியில் உள்ள இந்திரப்ரஸ்தா இன்ஸ்டிடியூட் ஆப் இன்போர்மேஷன் டெக்னாலஜி-யில் பேராசிரியராக பணிபுரிகிறார். அவர் பயிற்சி பெற்ற தொழில் முறை கணக்கெடுப்பாளர். அவருடைய முனைவர் பட்டபடிப்புக்கு முன் அமெரிக்காவில் வால்மார்ட்டில் பல தலைமை பதவிகளை வகித்துள்ளார். அவருடைய ஆராய்ச்சி ஆர்வங்கள், பணியிடத்து தொழில்நுட்பங்கள், நிறுவன கலாச்சாரம் மற்றும் தொழில்முனைவு ஆகியவற்றை உள்ளடக்கியதாகும். 